ULRICH RING

ÖFFENTLICHE

PLANUNGSZIELE UND STAATLICHE BUDGETS 


\section{ULRICH RING}

\section{ÖFFENTLICHE PLANUNGSZIELE UND STAATLICHE BUDGETS}

In der Bundesrepublik Deutschland wirken neben dem Staat als Hauptträger der öffentlichen Leistungserstellung verschiedene nicht-staatliche Entscheidungseinheiten an der Erfüllung öffentlicher Aufgaben mit und beeinflussen so die Realisation der öffentlichen Planungsziele. Obgleich die entsprechenden Aktivitäten dieser Institutionen weitgehend außerhalb des staatlichen Budgets ablaufen, sollte eine zielorientierte öffentliche Aufgabenplanung sie nicht ignorieren. Die Arbeit untersucht deshalb die Wirkungen, die von den Handlungen nicht-staatlicher Entscheidungseinheiten auf die budgetären Ausgabenschwerpunkte sowie auf verschiedene Leistungs- bzw. Zielebenen ausgehen.

Ulrich Ring wurde 1952 in Stuttgart geboren. Studium der Volkswirtschaftslehre von 1972 bis 1977 an den Universitäten Stuttgart, Nürnberg/Erlangen und Mannheim. Von 1977 bis 1984 wissenschaftlicher Mitarbeiter am Lehrstuhl für Volkswirtschaftslehre IV und im Sonderforschungsbereich 5 „Staatliche Allokationspolitik im marktwirtschaftlichen System" an der Universität Mannheim. 
Öffentliche Planungsziele und staatliche Budgets

Ulrich Ring - 978-3-631-75590-7

Downloaded from PubFactory at 01/11/2019 03:20:37AM

via free access 


\section{STAATLICHE ALLOKATIONSPOLITIK IM MARKTWIRTSCHAFTLICHEN SYSTEM}

Herausgegeben von

Klaus Conrad, Heinz König, Hans-Heinrich Nachtkamp, Rudiger Pethig, Ulrich Schlieper, Horst Siebert, Eberhard Wille

Band 12

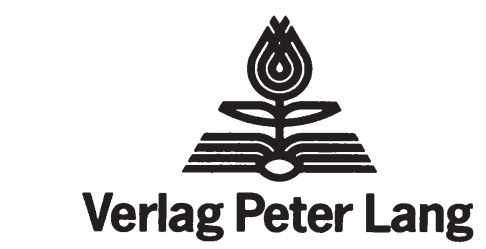

Frankfurt am Main · Bern · New York 


\section{ULRICHRING}

\section{ÖFFENTLICHE PLANUNGSZIELE UND STAATLICHE BUDGETS Zur Erfüllung öffentlicher Aufgaben durch nicht-staatliche Entscheidungseinheiten}

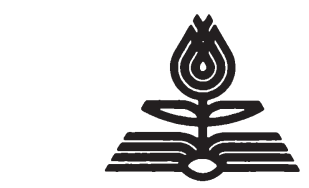

Verlag Peter Lang

Frankfurt am Main · Bern · New York 
CIP-Kurztitelaufnahme der Deutschen Bibliothek

\section{Ring, Ulrich:}

Öffentliche Planungsziele und staatliche Budgets:

zur Erfüllung öffentl. Aufgaben durch nicht-staatl.

Entscheidungseinheiten / Ulrich Ring. -

Frankfurt am Main ; Bern ; New York :

Lang, 1985.

(Staatliche Allokationspolitik im marktwirt-

schaftlichen System ; Bd. 12)

ISBN 3-8204-8292-X

Open Access: The online version of this publication is published on www.peterlang.com and www.econstor.eu under the international Creative Commons License CC-BY 4.0. Learn more on how you can use and share this work: http://creativecommons. org/licenses/by/4.0.

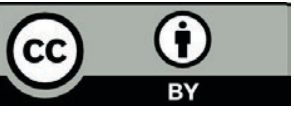

This book is available Open Access thanks to the kind support of ZBW - Leibniz-Informationszentrum Wirtschaft.

NE: GT

ISSN 0721-2860

ISBN 3-8204-8292-X

ISBN 978-3-631-75590-7 (eBook)

(c) Verlag Peter Lang GmbH, Frankfurt am Main 1985

Alle Rechte vorbehalten.

Nachdruck oder Vervielfältigung, auch auszugsweise, in allen Formen wie Mikrofilm, Xerographie, Mikrofiche, Mikrocard, Offset verboten.

Druck und Bindung: Weihert-Druck GmbH, Darmstadt 


\section{Vorwort}

Die vorliegende Arbeit entstand während meiner Tätigkeit am Mannheimer Sonderforschungsbereich 5 "Staatliche Allokationspolitik im marktwirtschaftlichen system". Sie wurde im Sommersemester 1984 von der Fakultät für Volkswirtschaftslehre und Statistik der Universität Mannheim als Dissertation angenommen.

Die Studie geht zurück auf eine Anregung meines geschätzten akademischen Lehrers, Herrn Prof. Dr. Eberhard Wille. Ihm möchte ich an dieser stelle nachdrücklich für die kontinuierliche, konstruktive Kritik während aller Phasen der Entstehung, Reifung und Verfeinerung der Arbeit danken. Wertvolle Hinweise und Verbesserungsvorschläge wurden mir durch Seminare und Einzeldiskussionen mit meinen Kollegen im Sonderforschungsbereich 5 zuteil. Last und keineswegs least danke ich Frau Sylvia Dreischer für die fast schon heroisch anmutende Bewältigung des Manuskripts.

Mannheim, im oktober 1984

Ulrich Ring 
Ulrich Ring - 978-3-631-75590-7

Downloaded from PubFactory at 01/11/2019 03:20:37AM

via free access 


\section{INHALTSVERZE ICHNIS}

Seite

Kapitel I: రFFENTLICHE AUFGABENERFULLUNG DURCH NICHTSTAATLICHE ENTSCHEIDUNGSEINHEITEN

1. Zum Verhältnis zwischen öffentlichen

Ausgaben und Aufgaben

2 .

Die nicht-staatlichen Entscheidungseinheiten

2.1 . Abgrenzung

2.2 .

Bezüge zur öffentlichen Planung

3.

Disposition

Kapitel II: BUDGETÄRE AUSGABENSCHWERPUNKTE UND STAATLICHE ALLOKATIONSZIELE

1.

Budgetäre Ausgabenschwerpunkte als

Indikatoren der staatstätigkeit

1.1 .

Die Kennziffern

1.1 .1 .

Typen

1.1.2.

Interpretationsprobleme

1. 2 .

Verzerrungen durch endogene störgrößen

1.2.1.

offentliches Vermögen und Staatsausgaben

1.2 .2 . Staatliche Normsetzung

1.2.3.

Verlagerung von Aufgaben auf nichtstaatliche Entscheidungseinheiten

1.2.3.1.

Intermediäre Entscheidungseinheiten

$1.2 \cdot 3 \cdot 1.1$.

Parafisci

$1.2 \cdot 3 \cdot 1.2$.

Öffentliche Unternehmen

$1.2 \cdot 3 \cdot 1 \cdot 3$.

Rundfunkanstalten

$1.2 \cdot 3 \cdot 2$.

Private Entscheidungseinheiten

1.2 .4 . Aufgabenerfüllung über öffentliche Einnahmen

$1.2 \cdot 5$ Zur Ausgabenintensität der öffentlichen Aufgabenerfüllung 
Seite

1.3 .

Verzerrungen durch exogene Störgrößen

1.3.1.

Relativpreisveränderungen

2.

Budgetäre Ausgabenschwerpunkte und

Angebot an öffentlichen Gütern

3.

Verbesserungsmöglichkeiten auf der

Inputebene

3.1 .

Die Notwendigkeit von Korrekturen

3. 2 .

Korrekturmöglichkeiten

3.2.1.

Zur Bestimmung öffentlicher Aufgaben

3.2 .2 .

Spezielle Korrekturansätze für

öffentliche Unternehmen

3.2 .3 .

Die Kosten der Erfüllung öffentlicher

Aufgaben

3.2 .4 .

Die Aussagefähigkeit der korrigierten

Daten

110

Kapitel III: DIE AKTIVITÄTEN NICHT-STAATLICHER ENTSCHEIDUNGSEINHEITEN IN DEN VOLKSWIRTSCHAFTLICHEN GESAMTRECHNUNGEN

1.

Die Zuordnung der nicht-staatlichen Entscheidungseinheiten auf die sektoren der Volkswirtschaftlichen Gesamtrechnungen

2.

Produktionskonto und Bewertung der Aktivitäten der nicht-staatlichen Entscheidungseinheiten 
Kapitel IV: ANSÄTZE ZUR MESSUNG DER OFFENTLICHEN

LEISTUNGEN NICHT-STAATLICHER ENTSCHEIDUNGSEINHEITEN

1.

Zum Leistungsbegriff bei nicht-staatlichen

Entscheidungseinheiten

2.

Eindimensionale MeBverfahren zur

Bestimmung des Leistungsumfangs

2.1 .

Die Willingness-to-Pay

2.2 .

Marktpreise

163

2.3 .

Kosten der Leistungserstellung

170

2.4 .

Nicht-marktmäßige Einnahmen

177

2.4.1.

Der Payment-Price Approach

2.4 .2 .

Freiwillig entrichtete Beiträge und spenden

2.5 .

Wirkungen auf privaten Konsum und

private Investitionen: Verfahren

der Nutzen-Kosten-Analyse

$2 \cdot 5 \cdot 1$

Zur konzeptionellen Eignung der Nutzen-

Kosten-Analyse für die Leistungsmessung

186

2.5.2. Qualitätsverbesserungen privater

Produktionsfaktoren

191

2.5.3. Qualitätsverbesserungen privater

Konsumgüter

196

2.5.4. Kostensenkungen durch öffentliche

Aktivitäten

$2 \cdot 5 \cdot 5$

Nachfrageschätzungen für öffentliche

Leistungen

199

2.5 .6 .

Kritische würdigung

200

2.6 .

Die Messung der Leistungsabgabe von

Vermögensbeständen

202

2.7 .

Indizes der realen Leistungen des Staates

208 
Seite

2.8 .

Spezielle Verfahren der Leistungsmessung

für Unternehmen

2.8.1. Die gesellschaftsbezogene Unternehmensrechnung

2.8.2. Exkurs: Die gesellschaftsbezogene Berichterstattung der Deutschen Bundesbahn

3.

Mehrdimensionale Meßverfahren zur

Bestimmung des Leistungsumfangs

228

3.1.

Soziale Indikatoren

228

3.1 .1 .

Quellen und Ansatzpunkte der Sozialindikatorenforschung

3.1 .2 .

Zum Selektionsproblem

3.1 .3 .

objektive und subjektive Indikatoren

3.1 .4 . Leistungsmessung mit Hilfe sozialer Indikatoren

$3 \cdot 1 \cdot 5$.

Gesellschaftsbezogene Berichterstattung von Unternehmen mit Hilfe sozialer Indikatoren

3. 2 .

Kosten-Wirksamkeits-Analysen

3. 3 .

Das Planning-Programming-Budgeting system

3.3.1. ziele und Charakteristika

4. Ausgewählte Verfahren zur Messung der öffentlichen Leistungen nicht-staatlicher Entscheidungseinheiten

4.1. Verfahren zur Messung der von nichtstaatlichen Entscheidungseinheiten produzierten Leistungen

4.2. Von nicht-staatlichen Entscheidungseinheiten erbrachte Transfers 
Kapitel V: LEISTUNGEN NICHT-STAATLICHER ENTSCHEIDUNGSEINHEITEN UND OCFFENTLICHE PLANUNG

1. Leistungen nicht-staatlicher Entscheidungseinheiten und des staates im Vergleich

2 .

Leistungen nicht-staatlicher Entscheidungseinheiten und ziele der öffentlichen

planung

Kapitel VI: ZUSAMMENFASSUNG

ANHANG :

Katalog von Erfolgskomponenten einer

gesellschaftsbezogenen Erfolgsrechnung

für öffentliche Unternehmen des

Personen-Nahverkehrs 
Ulrich Ring - 978-3-631-75590-7

Downloaded from PubFactory at 01/11/2019 03:20:37AM

via free access 
Kapitel I: ÖFFENTLICHE AUFGABENERFULLUNG DURCH NICHT-STAATLICHE ENTSCHEIDUNGSEINHEITEN

1. Zum Verhältnis zwischen öffentlichen Ausgaben und Aufgaben

Staatsausgaben stellen heute in den meisten Fällen eine notwendige Voraussetzung dar, um öffentliche Aufgaben zu verwirklichen ${ }^{1)}$. Die öffentlichen Budgets ordnen die in ihnen ausgewiesenen öffentlichen Ausgaben einem bestimmten Verwendungszweck zu. Die politischen Entscheidungsträger treffen mit den Budgetansätzen in den einzelnen Aufgabenbereichen Entscheidungen über Art, Umfang und Qualität der in einem bestimmten zeitraum geplanten Aktivitäten. In den Staatsausgaben schlagen sich im Prinzip die Kosten der Durchführung dieser Entscheidungen nieder. Daher scheinen budgetäre Ausgaben prima facie zur Feststellung von Schwerpunkten der öffentlichen Aktivität geeignet ${ }^{2}$. Demgegenüber dienen die öffentlichen Einnahmen vorwiegend zur Finanzierung dieser Ausgaben.

Budgetäre Ausgaben bilden somit Instrumente zur Erfüllung öffentlicher Aufgaben bzw. (in normativer Hinsicht) Mittel zur Wohlfahrtssteigerung. Jedoch lassen sich zwischen den Ausgaben und den mit ihnen angestrebten öffentlichen Auf-

1) Zu den wichtigsten Ausnahmen in Staatswesen der Gegenwart siehe Kapitel II, Abschnitte 1.2. und 2. dieser Arbeit sowie H. Zimmermann: Die Ausgabenintensität der öffentlichen Aufgabenerfüllung, in: Finanzarchiv N.F., Bd. 32 $(1973 / 74)$, S. 1-20. In historischer Betrachtungsweise ist insbesondere auf die oikenmäßige und die leiturgische Erfüllung öffentlicher Aufgaben hinzuweisen. Vgl. hierzu H. Kolms: Finanzwissenschaft, Bd. I, Grundlegung. రffentliche Ausgaben, 4. Aufl., Berlin, New York 1974, S. 12 ff.

2) Vgl. H. Fecher: Ausgaben, öffentliche, I: Ansätze zu ihrer Analyse, in: Handwörterbuch der Wirtschaftswissenschaft (HdWW), hrsg. von W. Albers et al., Bd. 1, stuttgart et al. 1977, S. 334-349, S. 335; C.V. Brown und P.M. Jackson: Public Sector Economics, 2nd Ed., Oxford 1982, S. 93. 
gaben bzw. wohlfahrtsrelevanten zielen mehrere ziel- bzw. Mittelebenen unterscheiden ${ }^{1}$ ).

Das folgende Ziel-Mittel-Schema (vgl. Schaubild 1) soll lediglich den ziel- bzw. Mittelcharakter und die operationalität der einzelnen Ebenen verdeutlichen; Aussagen über die Art und Weise der zielbildung enthält es nicht. In diese Hierarchie, die für den Bereich der staatlichen Allokationspolitik entwickelt wurde, lassen sich auch andere Anbieter öffentlicher Leistungen als der staat integrieren.

Die staatlichen Realausgaben (bzw. Leistungsausgaben) ${ }^{2}$ dienen zur Beschaffung von Produktionsmitteln, sie sind daher in dieser Ziel-Mittel-Hierarchie als monetäre Inputs zur Erfüllung der öffentlichen Aufgaben bzw. Planungsziele auf der untersten Ebene angesiedelt. Es bleibt auf dieser Stufe im Prinzip noch völlig offen, inwieweit eine Erhöhung der nominellen Ausgabenplafonds $\mathrm{zu}$ einem Zugang an sachlichen und personellen Inputs führt oder sich in Preisniveausteigerungen verliert.

1) Vgl. zum Folgenden E. Wille: Offentlicher Haushalt IV: Finanz- und Aufgabenplanung, in: Handwörterbuch der Wirtschaftswissenschaft (HdWW), hrsg. von W. Albers et al., Bd. 5, Stuttgart et al. 1980, S. 591-620, S. 605 ff. sowie E. Wille: Soziale Indikatoren als Ansatzpunkte wirtschaftspolitischer Zielbildung und Kontrolle - Verbesserung oder Verschleierung politischer Entscheidungsgrundlagen?, in: ORDO, Bd. 31 (1980), S. 127-151, S. $138 \mathrm{ff}$. Eine ähnliche Aufgliederung verwendet C. Leipert: Gesellschaftliche Berichterstattung. Eine Einführung in Theorie und Praxis sozialer Indikatoren, Berlin, Heidelberg, New York 1976, S. $102 \mathrm{ff}$.

2) $\mathrm{Zu}$ dieser Terminologie vgl. H. Haller: Einige Gedanken zum Thema: Öffentliche Finanzen im Wirtschaftswachstum, in: E. Schneider (Hrsg.): Wirtschaftskreislauf und Wirtschaftswachstum. Carl Föhl zum 65. Geburtstag, Tübingen 1966, S. 57-74, S. 59; W. Ehrlicher: Öffentliche Sachausgaben, in: Handbuch der Finanzwissenschaft, hrsg. von F. Neumark, Bd. 1, 3. Aufl., Tübingen 1977, S. 753-795, S. 754 ff. 
Bei den Produktionsmitteln handelt es sich um physische Inputs (z.B. Krankenhäuser, Ärzte, Verkehrspolizisten), die in den Proze $\beta$ der öffentlichen Leistungserstellung eingehen. Im marktwirtschaftlichen Produktionsproze $\beta$ entsprechen sie den Arbeitern, Angestellten und Fabriken, die als Produktionsfaktoren die eigentliche Produktionsleistung erst erzeugen sollen ${ }^{1)}$.

Die Art und Weise der Kombination dieser Produktionsfaktoren im staatlichen ProduktionsprozeB bestimmt Art, Umfang und Qualität der öffentlichen Produkte. Hierbei handelt es sich, da der staat überwiegend Dienstleistungen anbietet, um ein Leistungspotential im Sinne eines (noch) nicht konsumierten öffentlichen Angebotes (z.B. das Angebot der öffentlichen Krankenhäuser zur Verabreichung bestimmter Behandlungen).

Die Existenz eines öffentlichen Angebotes erlaubt noch keinerlei Schlüsse auf seine Beurteilung durch die (potentiellen) Empfänger und damit die Wohlfahrtsrelevanz der betreffenden öffentlichen Leistungen. Die Angebots- und die Nachfragebzw. Empfängerseite treffen erst auf der Ebene der outputobjectives zusammen. Diese Ausbringungsziele markieren die Nutzung - sei es als aktive, sei es als passive Konsumtion eines öffentlichen Angebotes (z.B. die Anzahl der nachgefragten stationären Behandlungen in Krankenhäusern) .

Im Marktbereich könnte die Wohlfahrtsanalyse im Prinzip, entsprechend dem individualistischen Ansatz, der von den geäuBerten Präferenzen der Wirtschaftssubjekte ausgeht, mit der freiwilligen und aktiven Nutzung eines Angebotes bzw. dem

1) In der Literatur zur öffentlichen Planung firmieren die physischen Produktionsmittel teilweise unter der irreführenden Bezeichnung "outputs". Vgl. beispielsweise B. Rürup: Die Programmfunktion des Bundeshaushaltsplanes. Die deutsche Haushaltsreform im Lichte der amerikanischen Erfahrungen mit dem Planning-Programming-Budgeting system, Berlin 1971, S. $59 \mathrm{f}$. 
Schaubild 1: zur Hierarchie zwischen Ressourceninputs im öffentlichen Sektor und wirtschaftspolitischen zielen

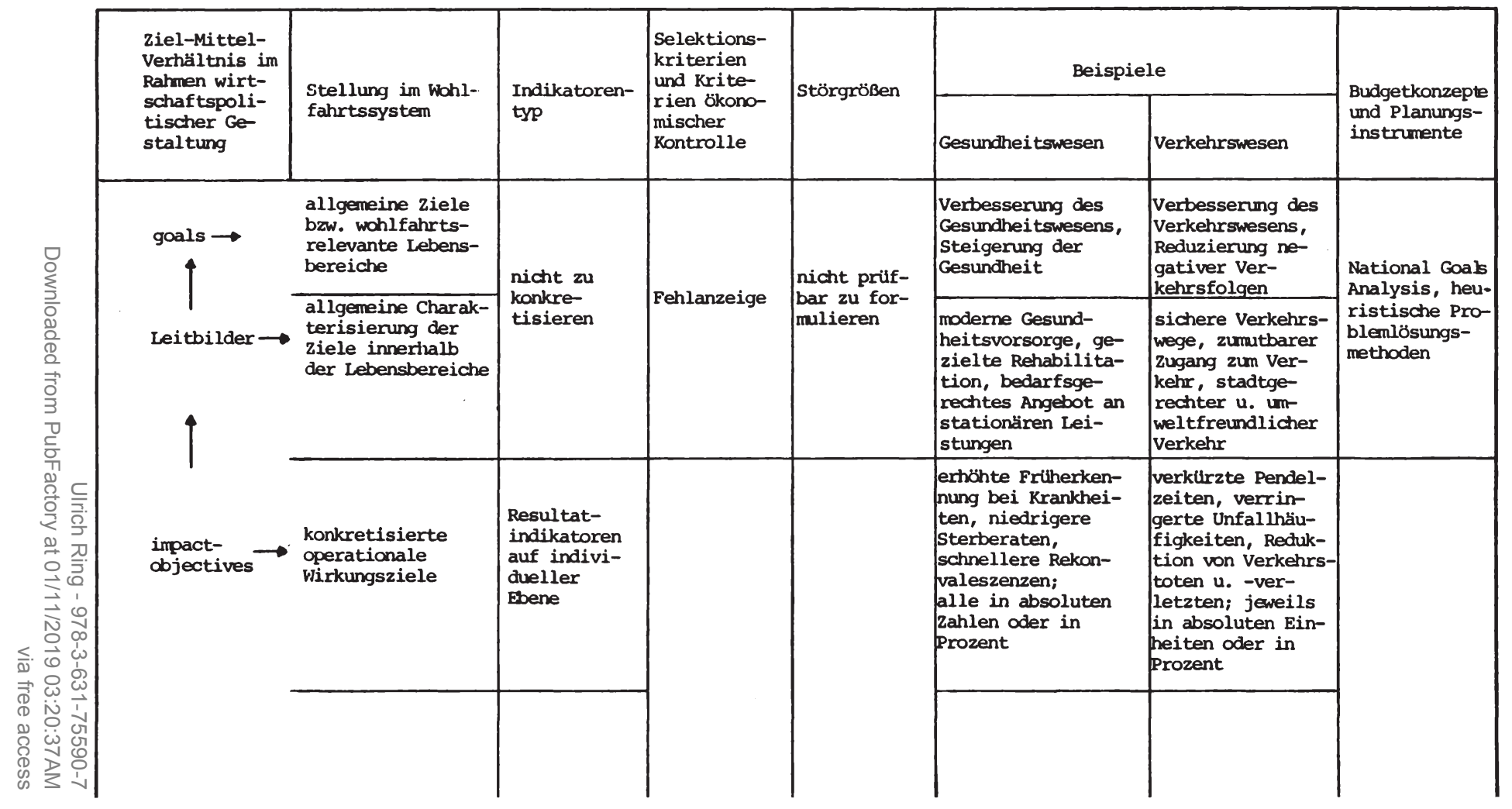




\begin{tabular}{|c|c|c|c|c|c|c|c|c|}
\hline$\prod_{8}^{4}$ & $\begin{array}{l}\text { output- } \rightarrow \\
\text { objectives }\end{array}$ & $\begin{array}{l}\text { konkretisierte } \\
\text { operationale } \\
\text { Ausbringungs- } \\
\text { ziele }\end{array}$ & $\begin{array}{l}\text { Nutzungsin- } \\
\text { dikatoren }\end{array}$ & \multirow[t]{2}{*}{$\begin{array}{l}\text { Effektivität= } \\
\text { (Wirksankeit) } \\
\text { objectives } \\
\text { Ausgaben }\end{array}$} & \multirow{2}{*}{$\begin{array}{l}\text { fehlender } \\
\text { zielbezug } \\
\text { der Maßnah- } \\
\text { men, mangeln- } \\
\text { der Zieler- } \\
\text { reichungs- } \\
\text { grad der- } \\
\text { selben }\end{array}$} & $\begin{array}{l}\text { Anzahl der Impfun- } \\
\text { gen, diagnostischen } \\
\text { Untersuchungen so- } \\
\text { wie ambulanten und } \\
\text { stationären Behand- } \\
\text { lungen }\end{array}$ & $\begin{array}{l}\text { Anzahl der Be- } \\
\text { nutzer offentli- } \\
\text { cher Verkehrs- } \\
\text { mittel, Nutzungs- } \\
\text { haufigkelt der } \\
\text { StraBen }\end{array}$ & \multirow{2}{*}{$\begin{array}{l}\text { Programbud- } \\
\text { get; Pro- } \\
\text { grammstruk- } \\
\text { turen, sozi- } \\
\text { ale Indika- } \\
\text { toren als } \\
\text { Ansatzpunk- } \\
\text { te einer } \\
\text { Programi- } \\
\text { planung }\end{array}$} \\
\hline \multirow[t]{4}{*}{ 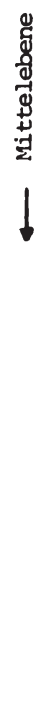 } & $\begin{array}{l}\text { quasi-End- } \\
\text { produkte }\end{array}$ & $\begin{array}{l}\text { konkretisier- } \\
\text { bare Lei- } \\
\text { stungen mit } \\
\text { wechselndem } \\
\text { Ziel-/Mittel- } \\
\text { charakter }\end{array}$ & $\begin{array}{l}\text { Erreichbar- } \\
\text { keitsindi- } \\
\text { katoren }\end{array}$ & & & $\begin{array}{l}\text { Entfernung bis zum } \\
\text { nächsten Arzt, Dif- } \\
\text { ferenz zwischen } \\
\text { Eintritt eines Not- } \\
\text { falles u. mögli- } \\
\text { cher Behandlung; } \\
\text { jeweils in zeit- } \\
\text { einheiten }\end{array}$ & $\begin{array}{l}\text { zugang zu offent- } \\
\text { lichen Verkehrs- } \\
\text { mitteln, Erreich- } \\
\text { barkeit zentraler } \\
\text { Einrichtungen; } \\
\text { jeveils in zeit- } \\
\text { einheiten }\end{array}$ & \\
\hline & $\begin{array}{l}\text { Öffent- } \\
\text { liche } \\
\text { Produkte }\end{array}$ & $\begin{array}{l}\text { (noch) nicht kon- } \\
\text { sumiertes öf- } \\
\text { fentliches } \\
\text { Angebot }\end{array}$ & $\begin{array}{l}\text { Angebots- } \\
\text { bzw. } \\
\text { Leistungs- } \\
\text { indikatoren }\end{array}$ & \multirow{2}{*}{$\begin{array}{l}\text { Effizienz = } \\
\text { (Wirtschaft- } \\
\text { lichkeit) } \\
\text { "outputs" } \\
\text { Ausgaben }\end{array}$} & \multirow{2}{*}{$\begin{array}{l}\text { unwirtschaft- } \\
\text { licher Ein- } \\
\text { satz der mo- } \\
\text { netăren Mit- } \\
\text { tel, Infla- } \\
\text { tion, Preis- } \\
\text { struktur- } \\
\text { effekte }\end{array}$} & $\begin{array}{l}\text { Angebot an Impfun- } \\
\text { gen, diagnosti- } \\
\text { schen Untersuchun- } \\
\text { gen sowie ambulan- } \\
\text { ten und stationären } \\
\text { Behandlungen }\end{array}$ & $\begin{array}{l}\text { Angebot an (be- } \\
\text { nutzbaren) neuen } \\
\text { und ausgebesser- } \\
\text { ten Straßen }\end{array}$ & \multirow{2}{*}{$\begin{array}{l}\text { performance } \\
\text { budget, Ro- } \\
\text { sten- bzw. } \\
\text { Ausgaben- } \\
\text { stellen- } \\
\text { rechnung }\end{array}$} \\
\hline & $\begin{array}{l}\text { Produk- } \\
\text { tions- } \\
\text { mittel }\end{array} \longrightarrow$ & $\begin{array}{l}\text { sachliche und } \\
\text { personelle } \\
\text { Kapazitäten } \\
\text { bzw. Inputs }\end{array}$ & $\begin{array}{l}\text { physische In- } \\
\text { put- oder } \\
\text { Austat- } \\
\text { tungsindi- } \\
\text { katoren }\end{array}$ & & & $\begin{array}{l}\text { Krankenhäuser, Diag- } \\
\text { nosezentren, Rönt- } \\
\text { gengeräte, Arzte, } \\
\text { Krankenschwestern }\end{array}$ & $\begin{array}{l}\text { neue Straßenki- } \\
\text { lameter, Ver- } \\
\text { kehrsampln, } \\
\text { Polizisten }\end{array}$ & \\
\hline & Ausgaben $\longrightarrow$ & $\begin{array}{l}\text { monetäre } \\
\text { Inputs }\end{array}$ & $\begin{array}{l}\text { monetăre } \\
\text { Inputin- } \\
\text { dikatoren }\end{array}$ & $\begin{array}{l}\text { juristische } \\
\text { Ordnungsmäs- } \\
\text { sigkeit }\end{array}$ & $\begin{array}{l}\text { Zuwiderhand- } \\
\text { lungen beim } \\
\text { Vollzug, } \\
\text { strafbare } \\
\text { Verfehlungen }\end{array}$ & $\begin{array}{l}\text { Ausgaben flur Gesund- } \\
\text { heitswesen im Sinne } \\
\text { des Funktional- } \\
\text { budgets }\end{array}$ & $\begin{array}{l}\text { Ausgaben fur Ver- } \\
\text { kehrswesen im } \\
\text { Sinne des Funk- } \\
\text { tionalbudgets }\end{array}$ & $\begin{array}{l}\text { Einjahres- } \\
\text { und Zweijah- } \\
\text { resbudget, } \\
\text { Nationalbud- } \\
\text { get, mehrjath- } \\
\text { riger (ausga- } \\
\text { benorientier- } \\
\text { ter) Finanz- } \\
\text { plan }\end{array}$ \\
\hline
\end{tabular}

Quelle: E. Wille: Soziale Indikatoren als Ansatzpunkte wirtschaftspolitischer zielbildung und Kontrolle... a.a.O., S. 138 f. 
Kauf einer Leistung enden. Die Verkaufswerte informieren sowohl über die abgenommenen Mengen als auch über die (Untergrenze der) Bewertung durch die Käufer und insoweit über die Wohlfahrtsrelevanz der betreffenden Leistungen. Bei den meisten öffentlichen Gütern hingegen vermittelt die Nutzung noch kaum Informationen über die Beurteilung einer Leistung durch die Empfänger, da die Nachfrager bzw. Nutznießer für diese i.d.R. ohne spezielles Entgelt abgegebenen Leistungen ihre Zahlungsbereitschaft nicht offenbaren müssen ${ }^{1)}$. Die bloße Nutzung eines öffentlichen Angebotes, auf die der von der Ebene der output-objectives repräsentierte "konventionelle outputbegriff"2) primär abstellt, garantiert daher, vor allem bei passiver Konsumtion, noch keine Verbesserung der individuellen Wohlfahrt. Infolgedessen muB die öffentliche Planung versuchen, die Wohlfahrtsrelevanz der meisten öffentlichen Güter auf der Ebene der impact-objectives zu begründen. Diese Wirkungsziele repräsentieren nicht lediglich die Nutzung einer öffentlichen Leistung, sondern deren (i.d.R. in physischen Größen definierte) Wirkungen auf die Empfänger in operationaler und prüfbarer Form. $\mathrm{Zu}$ ihrer operationalisierung dienen soziale Resultatindikatoren auf individueller Ebene, über deren Endproduktcharakter und Wohlfahrtsrelevanz allgemeiner Konsens besteht (z.B. die Lebenserwartung, Mütter- und Säuglingssterblichkeiten). Sie sollen anzeigen, "wie die Leistungen auf der Ebene der Individuen verarbeitet werden, was von der Leistungsverbesserung wohlfahrtsmäßig auf der individuellen Ebene 'ankommt" "3).

1) Zudem erschwert der Umstand, daß es sich bei öffentlichen Gütern größtenteils um Dienstleistungen handelt, Aussagen über die Mengenkomponente.

2) H. Brüngger und C. Orga: Ansätze zur Messung des Outputs des Staates, in: Schweizerische Zeitschrift für Volkswirtschaft und statistik, 114. Jg. (1978), S. 357-388, S. 367 .

3) C. Leipert: Gesellschaftliche Berichterstattung...a.a.0., S. 104. Zu einer ausführlicheren Analyse siehe die Abschnitte 3.1.4. in Kapitel IV und 2. in Kapitel V dieser Arbeit. 
Auf den beiden höchsten Ebenen des Ziel-Mittel-Schemas rangieren die goals, die die relevanten Wohlfahrtskomponenten auflisten, und die gesellschaftlichen Leitbilder. Im Gegensatz zu diesen zielebenen erlauben die impact-objectives eine Operationalisierung und Prüfbarkeit. Daher siedelt die öffentliche Aufgabenplanung die staatlichen Planungsziele tendenziell auf der Ebene dieser Wirkungsziele an und wählt anstelle der Effizienz die Effektivität als Selektionskriterium. Falls sich in bestimmten Aufgabenbereichen keine impactobjectives formulieren lassen oder die empirische Datenbasis (noch) zu schwach ist, müssen output-objectives oder gar öffentliche Angebotswerte als Hilfsgrößen an ihre Stelle treten.

Die quasi-Endprodukte sind per se keiner Ebene in diesem ZielMittel-Schema eindeutig zuzuordnen, sondern spiegeln ein bestimmtes Wohlfahrtskonzept wider. So stellen gewisse Infrastrukturinvestitionen zunächst lediglich ein öffentliches Angebot dar. Auch ohne eine tatsächliche Nutzung läßt sich jedoch schon die bloße Existenz dieses Angebotes als Verbesserung der Zugangsmöglichkeiten $z u$ den entsprechenden öffentlichen Leistungen auffassen. Je nach den Präferenzen eines Individuums mag es diese Investitionen als von ihm nicht genutztes staatliches Angebot werten oder aber "das Wissen um die jederzeitige Nutzungsmöglichkeit" ${ }^{1)}$ im Sinne einer beruhigenden Option wie ein wohlfahrtsmehrendes Endprodukt konsumieren.

1) C. Leipert: Gesellschaftliche Berichterstattung...a.a.0., s. 103. Zur Optionsgutproblematik siehe bereits B.A. Weisbrod: Collective-Consumption Services of IndividualConsumption Goods, in: Quarterly Journal of Economics, Vol. $78(1964)$, S. 471-477. 
Die Qualifizierung einer Ebene als Input oder Output hängt von der Betrachtungsweise ab. Der hierarchische Aufbau des Ziel-Mittel-Schemas impliziert, daß im Prinzip jede Ebene im Verhältnis zur jeweils tiefer (höher) angesiedelten Ebene den Charakter eines outputs (Inputs) aufweist ${ }^{1}$. Erweitert man den Blickwinkel von einem einzelnen auf mehrere Aufgabenbereiche, lassen sich weitere Input-Output-Relationen konstruieren ${ }^{2)}$. So können die Ergebnisse eines Aufgabenbereiches positive oder negative Auswirkungen auf die Resultate in anderen Aufgabenbereichen ausüben. Die Einführung des Zeitaspektes schließlich gestattet es, ähnliche Beziehungen auch in intertemporaler Hinsicht zu knüpfen. Beispielsweise kann ein hohes Bildungsniveau auf dem Wege von Produktivitätssteigerungen die Leistungsvoraussetzungen in den folgenden Perioden verbessern.

2. Die nicht-staatlichen Entscheidungseinheiten

\subsection{Abgrenzung}

Führt man sich die Verhältnisse in der Bundesrepublik Deutschland vor Augen, so stellt man fest, daß hier ein quasi arbeitsteiliges, in seiner struktur heterogenes system der öffentlichen Aufgabenerfüllung vorliegt, das über den öffentlichen Sektor (selbst in der Abgrenzung der Volkswirtschaftlichen Gesamtrechnungen) hinausreicht. Neben den Gebietskörperschaften als Hauptträger der öffentlichen Leistungserstellung treten nicht-staatliche Entscheidungseinheiten als

1) Henke bringt dies auch terminologisch zum Ausdruck, indem er in einem Modell für das Gesundheitswesen zwischen Ebenen des final output und des intermediate output unterscheidet. Vgl. K.-D. Henke: Öffentliche Gesundheitsausgaben und Verteilung. Ein Beitrag zur Messung und Beeinflussung des gruppenspezifischen Versorgungsniveaus im Gesundheitsbereich, Göttingen 1977, S. $35 \mathrm{ff}$.

2) Vgl. C. Leipert: Gesellschaftliche Berichterstattung... a.a.o., S. $104 \mathrm{ff}$. 
Anbieter öffentlicher Leistungen auf und üben dadurch Wirkungen auf die Erfüllung von Aufgaben und Zielen der öffentlichen planung aus.

Die nicht-staatlichen Entscheidungseinheiten, die wir an dieser stelle lediglich in institutioneller Hinsicht abgrenzen, aber noch nicht näher definieren und erläutern wollen ${ }^{1)}$, umfassen intermediäre und private Entscheidungseinheiten. Bei den intermediären Entscheidungseinheiten handelt es sich um den weiten Bereich der Parafisci und der öffentlichen Unternehmen, die in der Bundesrepublik wie auch in mehreren westlichen Industrienationen ausschließlich oder in Teilbereichen ihrer Aktivitäten mit der Wahrnehmung wichtiger öffentlicher Aufgaben betraut sind, sowie um die Rundfunkanstalten. Die privaten Entscheidungseinheiten erstrecken sich über private Unternehmen und private Haushalte bis hin zu bestimmten Organisationen ohne Erwerbscharakter wie Kirchen, Wohlfahrtsverbände etc. Sie wirken teils freiwillig (vor allem die karitativen Organisationen), teils aufgrund staatlichen Zwangs (man denke etwa an den "versteckten Staatsbedarf") an der Erfüllung öffentlicher Aufgaben mit.

Integriert man die nicht-staatlichen Entscheidungseinheiten in das im vorhergehenden Abschnitt beschriebene Ziel-MittelSchema, so läßt sich dessen "institutioneller Unterbau" folgendermaßen darstellen:

1) Eine ausführliche Darstellung erfolgt im Kapitel II dieser Arbeit. 
Schaubild 2: Mitwirkende bei der Erfüllung öffentlicher Aufgaben

Offentliche Aufgaben bzw. Ziele der öffentlichen Planung

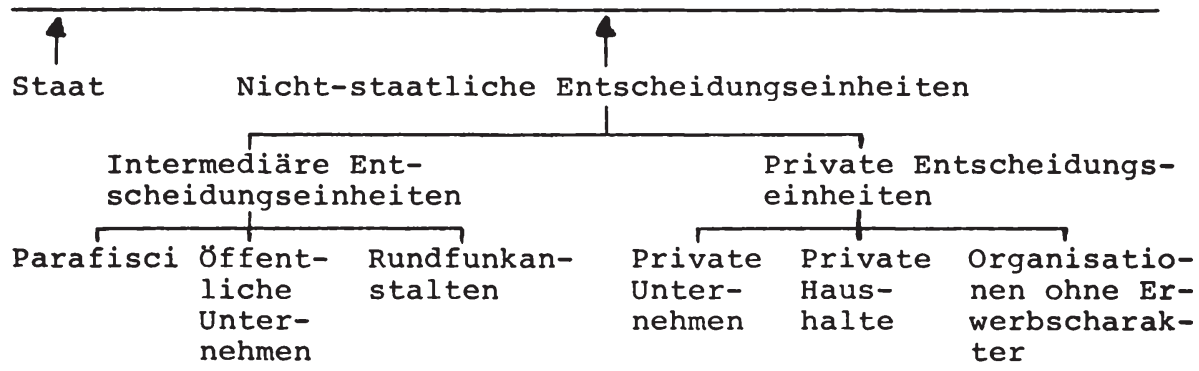

Diese rein institutionelle Darstellung bringt allerdings zwei wesentliche Tatbestände nicht zum Ausdruck. Zum einen ist neben dem Staat - für die Erfüllung öffentlicher Aufgaben nicht, wie Schaubild 2 zunächst vermuten läßt, die Gesamtheit der aufgeführten Institutionen verantwortlich; diese decken ja den privaten sektor einer Volkswirtschaft vollständig ab. Vielmehr wollen wir für unser weiteres Vorgehen eine funktionale Abgrenzung wählen: Es dürfen hier lediglich diejenigen Aktivitäten der nicht-staatlichen Entscheidungseinheiten Berücksichtigung finden, die sich ausschlieBlich auf die Erfüllung öffentlicher Aufgaben bzw. die Erreichung öffentlicher Planungsziele richten. Soweit diese Institutionen private - nicht aber meritorische - Leistungen erbringen, beeinflußt ihr Angebot die öffentliche Aufgabenerfüllung nicht bzw. nicht unmittelbar. Darüber hinaus sind diejenigen Aktivitäten nicht-staatlicher Entscheidungseinheiten nicht zu berücksichtigen, die zwar auf die Erstellung öffentlicher Güter (im Sinne der Theorie der öffentlichen Güter) gerichtet sind, indes keine Ziele der öffentlichen Planung tangieren.

Zum anderen laufen die Aktivitäten von Staat und nicht-staatlichen Entscheidungseinheiten zur Erfüllung öffentlicher Aufgaben nicht völlig isoliert voneinander $a b$, sondern es be- 
steht hier eine Fülle von teilweise interdependenten Querbeziehungen, auf die wir im folgenden Abschnitt eingehen wollen.

\subsection{Bezüge zur öffentlichen Planung}

Dłe auf die Erfüllung öffentlicher Aufgaben gerichteten Aktivitäten nicht-staatlicher Entscheidungseinheiten tangieren die öffentliche Planung in mehrfacher Hinsicht. Auf der Ebene der monetären Inputs betrifft der "diagonale Finanzausgleich" die öffentliche Planung direkt. Der staat unterstützt einen Teil dieser Aktivitäten finanziell, vor allem auf dem Wege von Zuschüssen und steuervergünstigungen.

Wesentliche Bedeutung für die öffentliche Programmplanung und -evaluation besitzen die Einflüsse auf den verschiedenen Outputebenen. Falls die staatlichen Entscheidungsträger die Aktivitäten nicht-staatlicher Entscheidungseinheiten bei der Verfolgung ihres zielsystems ignorieren, besteht zunächst die Gefahr, daß sie öffentliche Projekte - selbst bei anspruchsvoller Programm- bzw. Aufgabenplanung - im Planungsstadium falsch dimensionieren. Sodann ist zu erwarten, daß bei der Evaluation Projekterfolge mit Maßnahmen in Verbindung gebracht werden, die dafür zumindest nicht allein. ursächlich sind. Das bedeutet, daß die, heute häufig geforderte, ökonomische Erfolgskontrolle auf einer unzutreffenden Bezugsbasis aufbaut.

Dies sei an einem einfachen Beispiel erläutert. Sofern die staatlichen Entscheidungseinheiten bei der Planung eines bestimmten Gesundheitsprogramms nicht berücksichtigen, daß nicht-staatliche Entscheidungseinheiten gleichzeitig ihre Aktivitäten in demselben Bereich verstärken (reduzieren), hätte dies zur Folge, daß das staatliche Programm im Verhältnis zum angestrebten $\mathrm{Zielerreichungsgrad} \mathrm{überdimensioniert} \mathrm{(unter-}$ dimensioniert) ist. Die isolierte Betrachtung der staatlichen 
Maßnahmen führt außerdem $z u$ einer unzutreffenden Bewertung des Programmerfolges, was wiederum für die Zukunft weitere Fehlplanungen befürchten läßt.

Eine anspruchsvolle öffentliche Aufgabenplanung gründet sich, will sie die Aktivitäten nicht-staatlicher Entscheidungseinheiten in angemessener Form berücksichtigen, auf eine Effizienz- und darüber hinaus auf eine Wirksamkeitsanalyse ${ }^{1)}$. Die Effizienzanalyse erfordert neben der Erfassung der Inputs vor allem die Messung der Outputs der nicht-staatlichen Entscheidungseinheiten auf der Ebene der öffentlichen Produkte. Dies erlaubt eine Beurteilung der Effizienz der Produktion nicht-staatlicher Entscheidungseinheiten, auch im Vergleich zum staat, läßt jedoch den Bezug zu den auf der Ebene der impact-objectives angesiedelten öffentlichen Planungszielen noch offen. Ausgehend von den auf dieser Ebene realisierten zielen fragt die Wirksamkeitsanalyse nach dem Einfluß der Leistungen der nicht-staatlichen Entscheidungseinheiten. Sie ist erforderlich, weil die Gegenüberstellung der realisierten und der angestrebten Ziele der öffentlichen Planung zwar Aussagen über den Grad der zielerreichung, jedoch nicht über die Wirksamkeit eines bestimmten Einflußfaktors zuläßt. Die Realisierung der öffentlichen planungsziele ist regelmäßig das Resultat eines komplexen "sozialen Produktionsprozesses", innerhalb dessen die Aktivitäten der nicht-staatlichen Entscheidungseinheiten nur einen Faktor darstellen. So hängt die Lebenserwartung als Indikator des Gesundheitsstandes von der staatlichen Gesundheitsfür- und -vorsorge, entsprechenden Leistungen nicht-staatlicher Entscheidungseinheiten, den Konsumgewohnheiten, dem Bildungsniveau, der Umweltqualität, dem Gesundheitsbewußtsein etc. ab. Aufgabe der Wirksamkeitsanalyse ist es, den Anteil am Gesamtergebnis zu isolieren,

1) Siehe hierzu Kapitel V dieser Arbeit. 
der auf die Aktivitäten der nicht-staatlichen Entscheidungseinheiten zurückgeht ${ }^{1)}$. Einer eindeutigen Identifizierung des kausalen Prozesses zwischen ihren Leistungen und den gemessenen Ergebnisses im zielbereich steht allerdings die Komplexität des "sozialen Produktionsprozesses" entgegen ${ }^{2)}$.

Unübersehbar ist die ordnungspolitische Brisanz der Aktivitäten nicht-staatlicher Entscheidungseinheiten. Bei positiver Betrachtungsweise fällt auf, daß die in der finanzwissenschaftlichen Literatur übliche Aufteilung einer Volkswirtschaft in einen privaten und einen öffentlichen sektor der Realität nicht gerecht wird. In diesem idealtypischen Schema dient im privaten Sektor der Markt- und Preismechanismus als Allokationsinstrument, während im öffentlichen sektor die (öffentliche) Planung koordiniert. Die Finanzpolitik übernimmt zunächst die Aufgabe, den Umfang des öffentlichen Sektors abzustecken, womit zugleich auch das relative Gewicht des Koordinationsinstrumentes öffentliche Planung bestimmt wird. Sodann ist sie unmittelbar für die versorgung mit öffentlichen Gütern zuständig. Mit Hilfe der öffentlichen Planung werden somit die nationalen Ressourcen auf den öffentlichen und den privaten sektor sowie innerhalb des öffentlichen Sektors aufgeteilt. Die auf die Erfüllung öffentlicher Aufgaben gerichteten Aktivitäten nicht-staatlicher Entscheidungseinheiten finden in diesem vereinfachenden Schema kei-

1) Ähnlich C. Leipert: Gesellschaftliche Berichterstattung... a.a.0., S. $123 \mathrm{ff}$.

2) Vgl. hierzu Abschnitt 2 in Kapitel V dieser Arbeit. 
nen Platz ${ }^{1)}$. Sie implizieren zumindest, daß im privaten Sektor nicht allein der Marktmechanismus zum zuge kommt. Daraus läßt sich allerdings nicht der Umkehrschluß ziehen, bei allen Aktivitäten nicht-staatlicher Entscheidungseinheiten zur Erfüllung öffentlicher Aufgaben erfolge die Koordination mit Hilfe der öffentlichen Planung. Diese Auffassung erscheint prima facie nur in jenen Fällen als berechtigt, in denen der Staat den nicht-staatlichen Entscheidungseinheiten bestimmte Aktivitäten detailliert vorschreibt oder dies gar noch mit der Gründung eigenständiger Institutionen zur Erfüllung öffentlicher Aufgaben (z.B. die Träger der gesetzlichen Rentenversicherung) verbindet. Soweit nicht-staatliche Entscheidungseinheiten hingegen aus eigenem Antrieb Aktivitäten zur Erfüllung öffentlicher Aufgaben entfalten, bleibt noch weitgehend offen, ob mittels Konkordanz oder einer Teilüberschneidung von Marktmechanismus und öffentlicher Planung koordiniert wird oder ein gewisses Koordinationsvakuum herrscht.

Auch in normativer Hinsicht läBt sich eine umfassende Zuständigkeit der öffentlichen Planung für die Koordination der Aktivitäten des Staates und derjenigen nicht-staatlicher Entscheidungseinheiten zur Erfüllung öffentlicher Aufgaben kaum ableiten bzw. rechtfertigen. Die oben geforderte Integration dieser Aktivitäten in die öffentliche Aufgabenplanung bedeutet nicht, daB der Staat sie auch vollständig determinieren muB. Zwar ist derzeit noch weitgehend ungeklärt, ob die Erfüllung öffentlicher Aufgaben durch nicht-staatliche Entscheidungseinheiten die öffentliche

1) Formal läßt sich dieses Problem durch die Einführung eines dritten Sektors lösen. Vgl. etwa B.A. Weisbrod: Toward a Theory of the Voluntary Nonprofit Sector in a Three-sector Economy, in: M. Pfaff (Ed.): Grants and Exchange, Amsterdam, New York, Oxford 1976, S. 227-243; E. Wille: Offentlicher Haushalt. IV... a.a.O., S. 592 ff.; E. Wille: Gesamtwirtschaftliche Allokation zwischen "Markt- und Staatsversagen" - ein ordnungspolitischer Uberblick, in: E. Wille (Hrsg.): Beiträge zur gesamtwirtschaftlichen Allokation. Allokationsprobleme im intermediären Bereich zwischen öffentlichem und privatem Wirtschaftssektor. Frankfurt et al. 1983, S. $1-28$, S. $1 \mathrm{ff}$. 
Planung auf effektive Weise entlastet oder Fehlallokationen verursacht und zusätzliche Abstimmungsprobleme erzeugt ${ }^{1)}$, a priori kann ein Koordinationsbedarf jedoch nur dort postuliert werden, wo das Angebot der nicht-staatlichen Entscheidungseinheiten aus der Sicht der öffentlichen Planung zu klein oder qualitativ unzureichend ist. Eine eher aktive Koordination, bei der der staat gezielt EinfluB auf die nichtstaatlichen Entscheidungseinheiten nimmt, indem er ihnen bestimmte Aktivitäten vorschreibt, läBt sich damit allein freilich nicht rechtfertigen. Für staatliche Eingriffe mit Zwangscharakter bedarf es der Voraussetzung einer Verbesserung der allokativen Effizienz ${ }^{2)}$. Ist dies nicht gewährleistet, muB sich die öffentliche Planung im prinzip mit einer eher passiven Koordination begnügen: Der staat nimmt die geplanten Aktivitäten der nicht-staatlichen Entscheidungseinheiten zur Kenntnis und dimensioniert seine eigenen in quantitativer und qualitativer Hinsicht kompensatorisch in bezug auf seine ziele.

In der Literatur zur öffentlichen Planung wurden die Aktivitäten nicht-staatlicher Entscheidungseinheiten zur Erfüllung öffentlicher Aufgaben bisher kaum als Teilproblem öffentlicher Planung gesehen oder gar behandelt. Die meisten Beiträge beschränken sich auf eine ausgewählte Gruppe der nicht-staatlichen Entscheidungseinheiten und befassen sich mit engum-

1) Bei bestimmten Mischgütern scheint einiges dafür zu sprechen, daß private Organisationen ohne Erwerbscharakter ein den heterogenen Präferenzen der Nachfrager besser entsprechendes Angebot bereitstellen können als der Staat. Vgl. U. Schmoltzi: Zur Finanzierung privater Organisationen ohne Erwerbscharakter. Monolithisches staatliches Güterangebot versus vielfältiges Angebot, in: E. Wille (Hrsg.): Beiträge zur gesamtwirtschaftlichen Allokation. Allokationsprobleme im intermediären Bereich zwischen öffentlichem und privatem Wirtschaftssektor, Frankfurt et al. 1983, S. 65-87, S. $67 \mathrm{ff}$.

2) Unter distributionspolitischen zielsetzungen, die etwa bei der gesetzlichen Krankenversicherung eine wesentliche Rolle spielen, ergeben sich andere Bedingungen für staatliche Eingriffe. 
rissenen Fragestellungen. So existiert zu den öffentlichen Unternehmen eine Fülle von Literatur ${ }^{1)}$. Bezüglich der Parafisci liegen die empirischen und theoretischen Pionierarbeiten von Mann und Herrmann schon über 40 Jahre zurück; sie zielen überdies in erster Linie darauf ab, die finanzielle Belastung der Zensiten durch die Beitragszahlungen an Parafisci zu ermitteln ${ }^{2)}$. Einige Arbeiten jüngeren Datums behandeln spezielle Probleme der Sozialversicherungsträger ${ }^{3)}$ oder beziehen sich auf die Verhältnisse bei den Parafisci in Österreich ${ }^{4}$. Erst in den letzten Jahren läßt sich im Zuge einer eher funktionalen, sich am Paradigma des öffentlichen Gutes orientierenden, Betrachtungsweise eine rudimentäre Ausdehnung des Forschungsinteresses auf private Anbieter öffentlicher Leistungen ohne Erwerbsmotive feststellen ${ }^{5)}$. Auch die Theorie des Föderalismus

1) Stellvertretend seien hier genannt: D. Bös: Öffentliche Unternehmungen, in: Handbuch der Finanzwissenschaft, hrsg. von F. Neumark, Bd. 2, 3. Aufl., Tübingen 1980, S. 1-60; C.B. Blankart: Okonomie der öffentlichen Unternehmen. Eine institutionelle Analyse der Staatswirtschaft, München 1980.

2) Vgl. F.K. Mann: Die intermediären Finanzgewalten und ihr Einfluß auf Deutschlands finanzielle Belastung, in: Jahrbücher für Nationalökonomie und Statistik, Bd. 129 (1928), S. 219-237 sowie W. Herrmann: Intermediäre Finanzgewalten. Eine Analyse deutscher hilfsfiskalischer Gebilde im ersten Jahrzehnt nach der Stabilisierung, Jena 1936.

3) Vgl. etwa H. Meinhold: Fiskalpolitik durch sozialpolitische Parafisci, Tübingen 1976; L. Hajen: Bestimmungsgründe für die parafiskalische Organisation von Krankenversicherung, Frankfurt, Bern, Cirencester 1979; D. Lepelmeier: Soziale Sicherung und Parafiskalität. Zur Einkommensumverteilungsproblematik im Bereich der Sozialversicherung, Frankfurt 1979.

4) Vgl. C.A. Andreae: Die parafiskalischen Gebilde in finanzwissenschaftlicher Schau, in: Festschrift Walter Heinrich. Ein Beitrag zur Ganzheitsforschung, Graz 1963, S. 333-344; C. Smekal: Die Flucht aus dem Budget, Wien 1977.

5) Siehe vor allem B.A. Weisbrod: The Voluntary Nonprofit Sector. An Economic Analysis, Lexington 1977; W. Kirberger: Staatsentlastung durch private Verbände. Die finanzpolitische Bedeutung der Mitwirkung privater Verbände bei der Erfüllung öffentlicher Aufgaben, Baden-Baden 1978. 
in Verbindung mit der ökonomischen Theorie der Politik erweitert inzwischen ansatzweise ihren Blickwinkel von den regionalen zu den funktionalen sowie von den staatlichen zu den nichtstaatlichen Kollektiven, was in den Begriff "funktionaler Föderalismus" einmündete" ${ }^{1)}$. Ferner spielen die Aktivitäten nicht-staatlicher Entscheidungseinheiten eine wesentliche Rolle bei der Definition von (funktionalen) staatsquoten ${ }^{2}$.

\section{Disposition}

Die vorliegende Arbeit beschäftigt sich mit den staatlich veranlaBten und den freiwillig entfalteten Aktivitäten nichtstaatlicher Entscheidungseinheiten zur Erfüllung öffentlicher Aufgaben in der Bundesrepublik Deutschland als Teilproblem öffentlicher Planung. Diese Aktivitäten stellen - unabhängig davon, ob die politischen Entscheidungsträger ihnen im Vergleich zu staatlichen Maßnahmen substitutiven oder eher subsidiären Charakter beimessen - ein in wirkungsanalytischer Hinsicht mit den Aktivitäten des staates selbst im Prinzip gleichrangiges Mittel zur Realisation der öffentlichen Planungsziele dar. Die öffentlichen Planungen bzw. ihre Träger sollten diese Art der Aufgabenerfüllung zumindest zur Kennt-

1) Vgl. G. Kirsch: Einleitung, in: G. Kirsch (Hrsg. unter Mitarbeit von J. Theiler): Föderalismus, Stuttgart, New York 1977, S. 1-14, S. 12 ff. und speziell zum Gesundheitswesen K.-D. Henke: Dezentralisierung im Gesundheitswesen. Föderalismustheoretische und andere Ansätze zur Bestimmung und Messung des Zentralitätsgrades im Gesundheitswesen, in: K. $-D$. Henke und U. Reinhardt (Hrsg.): Steuerung im Gesundheitswesen, Gerlingen 1983, S. 13-56.

2) Vgl. vor allem K. Littmann unter Mitarbeit von B. Krüger: Definition und Entwicklung der Staatsquote. Abgrenzung, Aussagekraft und Anwendungsbereiche unterschiedlicher Typen von Staatsquoten, Göttingen 1975 , S. $17 \mathrm{ff}$. und S. $100 \mathrm{ff}$., im folgenden zitiert als: K. Littmann: Definition und Entwicklung der Staatsquote..., sowie Wissenschaftlicher Beirat beim Bundesministerium der Finanzen: Gutachten zur Aussagefähigkeit staatswirtschaftlicher Quoten, in: Bulletin des Presse- und Informationsamtes der Bundesregierung, Bonn, $\mathrm{Nr} .90$ vom 30.7.1976, S. $849-862$, S. 852 f. und S. 857 f. 
nis nehmen. Unter einem weniger entscheidungs- als vielmehr produktionstheoretischen Blickwinkel begreift unsere Untersuchung die Aktivitäten nicht-staatlicher Entscheidungseinheiten als Bestandteil einer erweiterten öffentlichen Allokationsabteilung im Musgraveschen Sinne, bezieht allerdings auch teilweise distributionspolitische Aspekte ein, da hier in der Realität häufig Utberschneidungen existieren. Als Orientierungsrahmen dient die oben dargestellte ziel-Mittel-Hierarchie. Von unten nach oben bis hin zu den konkretisierten öffentlichen Planungszielen bzw. zur Ebene der impact-objectives fortschreitend soll die Bedeutung der Aktivitäten nicht-staatlicher Entscheidungseinheiten für die Erfüllung öffentlicher Aufgaben aufgezeigt werden.

Im nächsten Kapitel (II), das primär die Ebene der Ausgaben bzw. monetären Inputs betrifft, untersuchen wir zunächst, auf welche Weise sich die Aktivitäten nicht-staatlicher Entscheidungseinheiten im staatlichen Budget niederschlagen können. Dabei steht weniger der Umfang als die Struktur der Aktivitäten zur Erfüllung öffentlicher Aufgaben im Mittelpunkt. Im ersten Schritt wird analysiert, inwieweit sich auf der Basis des Funktionalbudgets gebildete Ausgabenschwerpunkte zur Kennzeichnung der struktur der Staatstätigkeit eignen. Im Anschluß an eine Diskussion der Kriterien, die üblicherweise zur Bestimmung budgetärer Ausgabenschwerpunkte dienen, zeigen wir Einflußgrößen auf, die in der Realität Verzerrungen in bezug auf die schwerpunkte der staatstätigkeit hervorrufen können. Hier wirken zunächst endogene, von der öffentlichen Planung unmittelbar beeinflußbare, Faktoren als Störgrößen. Neben anderen Verzerrungsfaktoren (u.a. öffentliche Darlehen und Gewährleistungen sowie Steuervergünstigungen) gehören $z u$ dieser Gruppe auch die staatlich veranlaßten Aktivitäten nicht-staatlicher Entscheidungseinheiten. Um die Aktivitäten der nicht-staatlichen Entscheidungseinheiten im Sinne unseres Themas besonders herauszustellen, untersuchen wir den Niederschlag ihrer staatlich erzwungenen Aktivitäten im staatlichen Budget zunächst 
getrennt von den übrigen Einflußgrößen bzw. staatlichen Instrumentvariablen zur Erfüllung öffentlicher Aufgaben. Da der Staat seine Instrumentvariablen häufig insbesondere mit der Ubertragung von Aktivitäten auf nicht-staatliche Entscheidungseinheiten kombiniert, nehmen wir anschließend eine zusammenfassende Analyse des Einflusses der endogenen Faktoren auf die Kennziffern zur Bestimmung von Ausgabenschwerpunkten mit Hilfe des Konzeptes der Ausgabenintensität der öffentlichen Aufgabenerfüllung vor. Ferner problematisieren wir den Einfluß exogener, d.h. vom staat nicht unmittelbar beeinflußbarer, Störgrößen (Veränderungen von Relativpreisen auf den staatlichen Beschaffungsmärkten und die Bevölkerungsentwicklung) auf die budgetären Ausgabenschwerpunkte.

Im zweiten Schritt erfolgt die Erweiterung von der staatstätigkeit auf die in einer Volkswirtschaft insgesamt anfallenden Aktivitäten zur Erfüllung öffentlicher Aufgaben. Unter diesem Aspekt rücken die freiwillig entfalteten Aktivitäten nichtstaatlicher Entscheidungseinheiten in den Brennpunkt des Interesses. Wir versuchen Anhaltspunkte dafür herauszuarbeiten, daß die Schwerpunkte dieser Aktivitäten in der Realität anders verteilt sind als diejenigen der staatstätigkeit.

Der dritte Abschnitt des Kapitels II ist der Suche nach Verbesserungsmöglichkeiten bei einer Schwerpunktbestimmung auf der Inputebene gewidmet. Nicht die budgetären Ausgaben, sondern die für die Erfüllung öffentlicher Aufgaben anfallenden Kosten im Sinne von Opportunitätskosten geben hierfür grundsätzlich die geeignete Basis ab. Dies erfordert die Ermittlung und periodengerechte Zurechnung der zur Erfüllung einer Aufgabe eingesetzten Inputs, um so die verzerrenden wirkungen der meisten endogenen Faktoren $\mathrm{zu}$ vermeiden. $\mathrm{zu}$ diesem $\mathrm{zweck}$ suchen wir nach Wegen, um die Aktivitäten nicht-staatlicher Entscheidungseinheiten zur Erfüllung öffentlicher Aufgaben von ihren übrigen Handlungen zu trennen sowie ihre Inputs mit denjenigen des Staates zu aggregieren. Für den Bereich der öffent- 
lichen Unternehmen existieren entsprechende spezielle Korrekturansätze. Den Abschluß des Kapitels bildet eine Diskussion der Aussagefähigkeit der ermittelten Kosten der öffentlichen Aufgabenerfüllung.

Kapitel III thematisiert, wie die Volkswirtschaftlichen Gesamtrechnungen die Aktivitäten nicht-staatlicher Entscheidungseinheiten erfassen. An eine Darstellung der Regelungen, die nach der Revision 1982 bezüglich der sektoralen zuordnung und des Nachweises von In- und Outputs der nicht-staatlichen Entscheidungseinheiten gelten, knüpft eine kritische würdigung an. zum einen prüfen wir, ob die Volkswirtschaftlichen Gesamtrechnungen sich besser als das staatliche Budget zur Bestimmung von Schwerpunkten der Aktivitäten zur Erfüllung öffentlicher Aufgaben eignen. zum anderen konzentrieren wir uns auf die Frage, inwieweit sie Informationen über den output nicht-staatlicher Entscheidungseinheiten zu liefern vermögen.

Kapitel IV visiert ausschließlich die Outputebenen des zielMittel-Schemas an. Da das staatliche Budget grundsätzlich nicht und die Volkswirtschaftlichen Gesamtrechnungen i.d.R. nur unzureichend über den mit den Aktivitäten nicht-staatlicher Entscheidungseinheiten erzielten Output informieren, kristallisiert sich als zentrales Problem die Leistungsmessung heraus. Grundlage für die Einordnung und Beurteilung der untersuchten Verfahren bildet eine outputbezogene Abgrenzung des Begriffs "öffentliche Leistungen", die auf die betroffenen Individuen als Bewertungsinstanz abstellt. Auf dieser Basis werden nach der Diskussion einer Reihe von ein- und mehrdimensionalen Ansätzen überwiegend Verfahren, die die Ebene der output-objectives repräsentieren, zur Messung der öffentlichen Leistungen nicht-staatlicher Entscheidungseinheiten ausgewählt. Daneben analysieren wir Möglichkeiten zur Erfassung der von diesen Institutionen erbrachten (und sich mit ihren Leistungen teilweise überschneidenden) Transfers. 
In Kapitel $\mathrm{V}$ steht die Integration der Aktivitäten nichtstaatlicher Entscheidungseinheiten in die öffentliche Planung auf den Outputebenen im Mittelpunkt. Diese Integration (zumindest im Sinne der oben beschriebenen passiven Koordination) dient vornehmlich dazu, Fehlallokationen zu vermeiden. Wir verbleiben im ersten Schritt auf der Ebene der output-objectives, indem wir Probleme des Vergleichs der Leistungen nicht-staatlicher Entscheidungseinheiten mit jenen des Staates, die Aussagefähigkeit ihrer Leistungen im Hinblick auf die Erfüllung öffentlicher Aufgaben sowie Kriterien für ihren zielgerechten Einsatz diskutieren. Da eine auf der Basis ihrer Leistungen ermittelte Effektivität sich als unzureichendes Kriterium für die Beurteilung der allokativen Vor- und Nachteile der Aktivitäten nicht-staatlicher Entscheidungseinheiten erweist, muß die öffentliche Planung ihre ziele auf der übergeordneten Ebene der impact-objectives ansiedeln. Zunächst gehen wir den Implikationen der Operationalisierung dieser Wirkungsziele mit Hilfe von sozialen Resultatindikatoren im Hinblick auf die Bewertungsinstanz und die Messung eines Endergebnisses nach und prüfen dann, welche Probleme für die öffentliche Planung aus der Mitwirkung nicht-staatlicher Entscheidungseinheiten an der Erfüllung öffentlicher Aufgaben bei der Zielerreichungskontrolle einerseits und der Wirksamkeitskontrolle (Evaluation) andererseits erwachsen. Die im zuge einer Evaluation angestrebten Erkenntnisse über den EinfluB nicht-staatlicher Entscheidungseinheiten auf die öffentlichen Planungsziele bilden eine unerläBliche Voraussetzung für die Beurteilung ihrer Aktivitäten und ggf. für die Entscheidung über einen zukünftigen staatlichen Handlungsbedarf. Schließlich untersuchen wir, welche Hemmnisse einer Bestimmung von Schwerpunkten der öffentlichen Aufgabenerfüllung sowie einem Effektivitätsvergleich über alle Aufgabenbereiche entgegenstehen. Das abschließende Kapitel VI enthält eine Zusammenfassung der wichtigsten Ergebnisse der Arbeit. 
Kapitel II: BUDGETÄRE AUSGABENSCHWERPUNKTE UND STAATLICHE ALLOKATIONSZIELE

1. Budgetäre Ausgabenschwerpunkte als Indikatoren der Staatstätigkeit

1.1. Die Kennziffern

1.1.1. Typen

In jedem Budget setzen die politischen Entscheidungsträger notwendigerweise schwerpunkte ${ }^{1)}$ im intra- und interministeriellen Bereich, da die Ausgabenwünsche der Ressorts die verfügbaren finanziellen Mittel regelmäßig übersteigen. Diese faktische Schwerpunktsetzung auf der Ebene der monetären Inputs resultiert zwangsläufig aus der Koordinations-bzw. Ausschlußfunktion des Budgets, läßt jedoch keine Schlüsse auf das angewandte Selektionskriterium $\mathrm{zu}^{2}$ ). Es kann hier offen bleiben, ob die Programmselektion auf einer anspruchsvollen Aufgabenplanung beruht, sich an Vorjahresquoten oder -plafonds orientiert oder die politische Hausmacht der Ressortchefs widerspiegelt. Die im Budget vorgesehene Verteilung der finanziellen Mittel auf die verschiedenen Aufgabenbereiche stellt unabhängig vom Selektionskriterium und von den Argumenten der Zielfunktion der politischen Entschei-

1) Vgl. zum Folgenden auch U. Ring: Die Aussagefähigkeit budgetärer Ausgabenschwerpunkte - ein Uberblick, in: E. Wille (Hrsg.): Konzeptionelle Probleme öffentlicher Planung, Frankfurt et al. 1983, S. 21-84, S. $26 \mathrm{ff}$.

In der Literatur findet sich im Hinblick auf mehrjährige Finanzpläne teilweise die Unterscheidung in (sachliche) Schwerpunkte und (zeitliche) Prioritäten. Vgl. etwa G. Hagemann: Beziehungen zwischen mittelfristiger Finanzplanung und Finanzverfassung im föderativen staat unter besonderer Berücksichtigung der Verhältnisse in der Bundesrepublik Deutschland, in: Mittelfristige Finanzplanung, Beihefte der Konjunkturpolitik, Heft 15, Berlin 1968, S. 47-56, S. 53 sowie E. Wille: Offentlicher Haushalt IV...a.a.O., S. 598. Da jedoch eine Aufgabe mit Schwerpunktcharakter in der Regel auch in zeitlicher Hinsicht Priorität genießt und die Unterscheidung beim Einoder Zwei-Jahresbudget an Bedeutung verliert, wollen wir in dieser Arbeit beide Begriffe synonym verwenden.

2) Vgl. E. Wille: Öffentlicher Haushlwilt RIV.-97a-3a6.30-7,5590-7598 . 
dungsträger eine Resultante der Handlungen letzterer dar.

Die übliche Gliederung der Ausgaben nach Ressorts läßt indes die zu den Ausgaben gehörenden Aufgaben und Programme nicht sichtbar werden. Für eine Beurteilung der Dringlichkeit von Ausgaben scheinen die nach funktionalen Gesichtspunkten gegliederten Haushaltspläne besser geeignet zu sein. Die Staatsausgaben werden dabei nach Aufgabenbereichen wie Bildung, Wissenschaft, Forschung, Soziale sicherung, Gesundheit etc. aufgeschlüsselt ${ }^{1)}$. Bei der zuordnung von Staatsaufgaben auf Funktionsbereiche können allerdings Uberschneidungen auftreten. Beispielsweise werden die öffentlichen Ausgaben für die Ausbildung von Ärzten, Krankenschwestern etc. dem Funktionsbereich Bildung zugerechnet, besitzen aber ebenso Bedeutung für den Bereich Gesundheitswesen. Derartige Uberschneidungen kommen im Prinzip um so häufiger vor, je mehr Funktionsbereiche unterschieden werden ${ }^{2}$.

Der Funktionenplan bietet nach verbreiteter Auffassung jenes Zahlenwerk, an dem ein AuBenstehender am ehesten die Prioritätsvorstellungen der Regierung ablesen kann. Dies dokumentiert auch pars pro toto die Feststellung, die funktionale Haushaltsgliederung diene der "Erfüllung der politischen Budgetfunktion" 3 ).

1) Vgl. P. Senf: Die Reform der öffentlichen Haushaltsgebarung zur Erhöhung der Transparenz, in: H. Haller (Hrsg.): Probleme der Haushalts- und Finanzplanung, Schriften des Vereins für Socialpolitik N.F., Bd. 52, Berlin 1969, S. 143-174, S. $144 \mathrm{ff}$. und $S .155 \mathrm{ff}$. sowie P. Senf: Kurzfristige Haushaltsplanung, in: Handbuch der Finanzwissenschaft, hrsg. von F. Neumark, Bd. 1, 3. Aufl., Tübingen 1977, S. 371-425, S. $402 \mathrm{f}$.

2) Vgl. K. Littmann: Definition und Entwicklung...a.a.o., s. $104 \mathrm{f} . ;$ K.-D. Henke: రffentliche Gesundheitsausgaben...a.a.0., S. $27 \mathrm{ff}$.

3) H. Klaas: Aufgaben der funktionalen Haushaltsgliederung im Rahmen des Funktionenplanes, in: Der öffentliche Haushalt, 10. Jg. (1969/70), S. 150-158, S. 156, ähnlich auch (allerdings mit Bezug auf den mehrjährigen Finanzplan) L. Wolkersdorf: Beziehungen zwischen Haushaltsplan und Finanzplanung unter besonderer Berücksichtigung politischer und administrativer Aspekte, in: Mittelfristige Finanzplanung, Beihefte der Konjunkturpolitik, Heft 15, Berlin 1968, S. 31-46, S. 41. 
Zunächst scheinen alle Aufgabenbereiche, für die das Budget uberhaupt Ausgaben vorsieht, Priorität im Verhältnis zu jenen Aufgaben zu genießen, für die es keine Ausgaben bereitstellt. zudem neigen die verantwortlichen Politiker dazu, nahezu alle Aufgabenbereiche als Schwerpunkte zu deklarieren. Sowohl zur Differenzierung $z w i s c h e n$ den verschiedenen Ausgaben im Funktionalbudget als auch zur Uberprüfung, ob die Verteilung der finanziellen Mittel den verbal formulierten Prioritäten entspricht, sind daher objektiv kontrollierbare Kriterien erforderlich ${ }^{1)}$.

In der Literatur werden im wesentlichen fünf Kennziffern zur Feststellung von Schwerpunkten diskutiert ${ }^{2}$ ) (Es seien A die Gesamtausgaben eines Budgets und $a_{i}$ die Ausgaben in einem Aufgabenbereich $i$, so daß gilt: $\left.\sum a_{i}=A_{\text {. }}\right)$

Kennziffer 1: Die absolute Höhe der Ausgaben eines Aufgabenbereiches

$\mathrm{K}_{1}=\mathrm{a}_{\mathrm{i}}$

Kennziffer 2: Die absolute Veränderung der Ausgaben eines Aufgabenbereiches

$\mathrm{K}_{2}=\Delta \mathrm{a}_{\mathrm{i}}$

Kennziffer 3: Der Anteil der Ausgaben für einen Aufgabenbereich an den Gesamtausgaben

$$
K_{3}=\frac{a_{i}}{A}
$$

1) Vgl. K. Schmidt und E. Wille: Die mehrjährige Finanzplanung. Wunsch und Wirklichkeit, Tübingen 1970, S. $79 \mathrm{ff}$.

2) Vgl. ebenda, S. 84 ff.; K. Gresser: Probleme der mehrjährigen öffentlichen Finanzplanung, Berlin 1974, S. $78 \mathrm{ff}$.; W. Kitterer: Das moderne Budget. Eine vergleichende Analyse der zentralen Staatshaushalte Frankreichs und der BRD, Frankfurt, Bern 1976, S. $146 \mathrm{ff}$. sowie die jährlichen Analysen der Vorlage des US-Bundesbudgets durch die Brookings Institution, zuletzt J.A. Pechman (Ed.): Setting National Priorities. The 1984 Budget, Washington D.C. 1983. 
Kennziffer 4: Die Zuwachsrate der Ausgaben für einen Aufgabenbereich

$k_{4}=\frac{\Delta a_{i}}{a_{i}}$

Kennziffer 5: Der Anteil der Ausgaben für einen Aufgabenbereich an den Gesamtausgaben, multipliziert mit der $\mathrm{Zu}-$ wachsrate der Ausgaben dieses Aufgabenbereiches $k_{5}=\frac{a_{i}}{A} \cdot \frac{\Delta a_{i}}{a_{i}}$

Die Kennziffern 1 und 3 stellen rein statische Kriterien dar. Als Schwerpunkte gelten die Aufgabenbereiche mit den größten (Anteilen an den) Gesamtausgaben. Dabei bleibt die zuwachsrate der Ausgaben unberücksichtigt, die eventuell verstärkte Aktivitäten in den betreffenden Aufgabenbereichen ausdrücken kann ${ }^{1}$. Bereiche mit (noch) niedrigen Ausgaben bzw. kleinem Anteil an den Gesamtausgaben, aber hoher Dringlichkeit, sind so nicht zu erkennen.

Kennziffer 2 weist den Aufgabenbereichen den höchsten Rang $z u$, die gegenüber dem Vorjahr die größte zuweisung an zusätzlichen finanziellen Mitteln erhalten. Bei diesem Kriterium bleibt jedoch die absolute Höhe der Ausgaben, zu denen sich die zusätzlichen Ausgaben addieren, unberücksichtigt. Aufgabenbereiche mit großen Anteilen an den Gesamtausgaben bedürfen schon dann hoher Ausgabenzuwächse, wenn sie wie bisher weitergeführt werden. Dagegen können neue oder kleine Aufgabenbereiche trotz absolut kleiner Ausgabenzuwächse von der Regierung als Schwerpunkte angesehen werden.

1) Vgl. K. Schmidt und E. Wille: Die mehrjährige Finanzplanung ...a.a.O., S. 84 f.; W. Kitterer: Das moderne Budget... a.a.o., s. 148 ff. 
Nach Kennziffer 4 rangieren die Aufgabenbereiche mit den höchsten Zuwachsraten als Schwerpunkte. Ein Mangel dieses Kriteriums besteht darin, daß es das relative Gewicht der Aufgabenbereiche nicht berücksichtigt. Bei Bereichen mit geringen Bestandswerten im Ausgangszeitpunkt kann mit einer geringen zuweisung an Mitteln leicht eine hohe Zuwachsrate erzielt werden. Umgekehrt können Aufgabenbereiche mit absolut hohem Ausgangsniveau und absolut hohen Zuweisungen an Mitteln als negative Schwerpunkte rangieren, obwohl ihr Anteil an den Gesamtausgaben erheblich ist ${ }^{1)}$.

Da die Kennziffer 4 nur die Zuwachsrate der Aufgabenbereiche, nicht aber deren relatives Gewicht zum Ausdruck bringt, und da es bei der Kennziffer 3 gerade umgekehrt ist, schlagen $\mathrm{K}$. Schmidt und E. Wille vor, beide Kennziffern miteinander zu verknüpfen (Kennziffer 5) ${ }^{2}$ ). Hier soll das Produkt aus dem Anteil eines Aufgabenbereiches an den Gesamtausgaben und der Zuwachsrate der Ausgaben des betreffenden Bereichs den Stellenwert der einzelnen Aufgabenbereiche anzeigen. Die Verknüpfung gleicht zwar die Mängel der beiden enthaltenen Kennziffern teilweise aus ${ }^{3)}$, die Mischzahl enthält jedoch keine wesentlich neuen Informationen. Sie verbirgt sogar teilweise Aussagen, die in einem getrennten Ausweis ihrer Komponenten zum Ausdruck kommen ${ }^{4}$ ).

Unabhängig davon, welcher Kennziffer man den Vorzug gibt, erscheint es sinnvoll, sich bei der Bestimmung von Schwerpunkten nicht auf ein einzelnes Budget zu beschränken, sondern derartige Analysen über mehrere Jahre hinweg durchzuführen. zum einen existieren in verschiedenen Aufgabenbereichen auch mittelfristig unterschiedlich weite und unterschiedlich rasch ausdehn-

1) Vgl. K. Schmidt und E. Wille: Die mehrjährige Finanzplanung... a.a.O., S. 84 .

2) Vgl. ebenda, S. 85 .

3) Vgl. K. Gresser: Probleme der mehrjährigen öffentlichen Finanzplanung...a.a.0., S. 82 .

4) Vgl. W. Kitterer: Das moderne Budget... a.a.O., S. $152 \mathrm{f}$. 
bare Kapazitätsgrenzen. So erfordert etwa ein sprunghaft erhöhter Bedarf an Lehrern zunächst den Neubau von Ausbildungsstätten, zudem läßt er sich erst nach Ablauf der Ausbildungszeit von Lehrern decken ${ }^{1)}$. Zum anderen sind gravierende Umstrukturierungen der Staatsausgaben im Sinne eines Zero-Base Budgeting kurzfristig kaum durchführbar, da die jährlich für neue Aufgaben oder für verstärkte Aktivitäten in bestehenden Aufgabenbereichen frei verfügbaren Mittel in praxi relativ gering sind ${ }^{2}$. Der größte Teil der zusätzlichen Ausgaben eines Budgets resultiert aus "built-in-Ausgabensteigerungen", die sich automatisch durch gesetzliche Verpflichtungen und bereits laufende Programme (Folgekostenproblematik) ergeben ${ }^{3)}$. Daher besteht die Möglichkeit, daß bei Finanzierungsengpässen dringliche, aber relativ teure Aufgaben zugunsten von weniger dringlichen, aber relativ billigen Aufgaben zurückgestellt werden ${ }^{4)}$. Dieser Aspekt spricht tendenziell für die marginal orientierten Kennziffern 2 und 4. Die mittelfristig angelegte Analyse setzt allerdings voraus, daß sich die Abgrenzung der Aufgabenbereiche, auf die sich die Schwerpunktbildung bezieht, im Laufe der zeit nicht verändert ${ }^{5)}$. zudem ist darauf zu achten,

1) Vgl. K. Gresser: Probleme...a.a.O., S. 80.

2) Selbst beim mehrjährigen Finanzplan der Bundesregierung scheint der durch Gesetze, Verträge oder auch Tradition festgeschriebene "Kern" einen wesentlichen Teil des Gesamtvolumens auszumachen. Vgl. F. Neumark: Planung in der öffentlichen Finanzwirtschaft, in: E. Schneider (Hrsg.) : Rationale Wirtschaftspolitik und Planung in der Wirtschaft von heute, Schriften des Vereins für Socialpolitik N.F., Bd. 45, Berlin 1967, S. 173-205, (Diskussion: S. 206-238), S. 237 .

3) Vgl. C.L. Schultze et al.: Setting National Priorities. The 1972 Budget, Washington D.C. 1971, S. $12 \mathrm{ff.}$ sowie B.M. Blechman et al.: Setting National Priorities. The 1976 Budget, Washington D.C. 1975, S. $190 \mathrm{ff}$.

4) Vgl. K. Gresser: Probleme...a.a.O., S. 80 f.

5) Vgl. K. Schmidt und E. Wille: Die mehrjährige Finanzplanung... a.a.o., s. 87 . 
ob im Betrachtungszeitraum in einem Aufgabenbereich möglicherweise Kompetenzverlagerungen $z w i s c h e n$ den verschiedenen Ebenen der Gebietskörperschaften auftreten, die das Ergebnis verzerren ${ }^{11}$.

\subsubsection{Interpretationsprobleme}

Jede der diskutierten Kennziffern gestattet die Aufstellung einer vollständigen Rangordnung der im Funktionalbudget unterschiedenen Aufgabenbereiche ${ }^{2)}$. Diese zeigt zunächst einmal nur an, welche Aufgabenbereiche die politischen Entscheidungsträger bevorzugt mit staatsausgaben bedenken und welche sie in finanzieller Hinsicht vernachlässigen. Einige Autoren gehen in ihrer Interpretation wesentlich weiter, indem sie diese Rangordnung als identisch mit der Rangordnung der Aufgabenbereiche in den Augen der politischen Entscheidungsträger ansehen. Demnach halten die Politiker diejenigen Aufgabenbereiche für die wichtigsten, die die ersten Plätze in einer Kennziffernreihe belegen ${ }^{3)}$. Das bedeutet allerdings nicht unbedingt, daß sie in den Aufgabenbereichen mit höchster Priorität auch die objektiv besten Ergebnisse bzw. den höchsten Grad der Aufgabenerfüllung im Sinne von output- oder impact-objectives anstreben oder gar erreichen. Wenn die Schwerpunktbildung im Budget oder im mehrjährigen Finanzplan "den politisch bestimmten zielen entsprechen"4) soll und "die Werthaltung der Entscheidenden im

1) Vgl. K. Gresser: Probleme...a.a.O., S. 79 ff. Eine Zusammenfassung der Ausgaben aller an der Erfüllung einer Aufgabe beteiligten Gebietskörperschaften ist unter entscheidungstheoretischen Gesichtspunkten nicht sinnvoll.

2) Als Grenze zwischen positiven und negativen Schwerpunkten bietet sich dabei der Durchschnittswert der jeweiligen Kennziffer an. Vgl. K. Schmidt und E. Wille: Die mehrjährige Finanzplanung...a.a.0., S. 84 .

3) Vgl. ebenda, S. $82 \mathrm{ff.;}$ K. Gresser: Probleme... a.a.O., S. $78 \mathrm{ff}$.

4) K. Schmidt und E. Wille: Die mehrjährige Finanzplanung... a.a.0., s. 81 . 
Zeitpunkt der Entscheidung (ausdrückt)" "1), so handelt es sich um die persönlichen oder parteipolitischen ziele der Politiker. Sie bewerten bzw. gewichten die verschiedenen öffentlichen Aufgabenbereiche in der Regel unterschiedlich, gemäß ihrer ideologischen Einstellung einer Aufgabe gegenüber oder, bei eher zweckrationaler Handlungsweise, z.B. unter dem Aspekt des erwarteten Gewinns an Wählerstimmen, und setzen entsprechende Prioritäten (und zwangsläufig auch Posterioritäten) bei den Staatsausgaben. So mag eine tendenziell konservative Regierung der Inneren Sicherheit a priori einen höheren Wert beimessen und daher höhere Ausgaben hierfür vorsehen als ein eher liberales Kabinett. Aus der Entscheidungsfindung in demokratisch organisierten Gemeinwesen folgt, daß die auf der Basis der Staatsausgaben ermittelte Prioritätenliste allenfalls die politischen zielvorstellungen der Regierung bzw. des Gesetzgebers insgesamt, jedoch nicht notwendigerweise auch einzelner Politiker widerspiegelt. Die Entscheidung im Kabinett über den Haushaltsentwurf stellt grundsätzlich einen Kompromiß zwischen den beteiligten Politikern dar, bei dem im Grenzfall kein einziger Minister seine individuellen Präferenzen voll verwirklicht sieht.

Die Interpretation einer Kennziffernreihe als Rangordnung der Aufgabenbereiche, die den (im obigen sinne definierten) zielvorstellungen der politischen Entscheidungsträger entspricht, entbehrt gleichwohl nicht der Plausibilität und der Realitätsnähe. Die Entscheidung für ein bestimmtes Budget und damit eine bestimmte Aufgabenstruktur impliziert, daB die politischen Entscheidungsträger diesen Haushalt insgesamt gegenüber jeder anderen Alternative präferieren, insofern ist er Ausdruck ihrer Zielvorstellungen. Der UmkehrschluB von den budgetären Ausgaben

1) K. Gresser: Probleme... a.a.0., S. 76. Gresser nennt allerdings (ebenda) eine zweite Determinante der (Ausgaben-) Plafonds: Sie spiegeln neben der Priorität einer Aufgabe auch den EinfluB aufgabenspezifischer Faktoren wider. 
auf die zielvorstellungen der Politiker erscheint zwar prima vista als vermessen ${ }^{1)}$, doch sprechen Plausibilitätsüberlegungen für die Brauchbarkeit der Kennziffern zumindest zum Nachweis grober Widersprüche. So wirkt es wenig glaubhaft, daß ein von den politischen Entscheidungsträgern als äuBerst wichtig bezeichneter, jedoch finanziell vernachlässigter Aufgabenbereich tatsächlich Priorität genieBt. Derlei Betrachtungen weisen indes umso geringere Treffsicherheit auf, je weiter man sich von den oberen und unteren Extremen einer Kennziffernreihe in Richtung auf die Mitte zu entfernt; sie legen daher den Verzicht auf den Nachweis einer durchgehenden Rangordnung nahe. Die Realitätsbezogenheit dieser Methode der Kennzeichnung von Schwerpunkten erfährt Unterstützung, vergegenwärtigt man sich den hohen stellenwert, den die staatsausgaben in der politischen Diskussion einnehmen. Dabei entsteht des öfteren der Eindruck, Opportunitätskosten würden, etwa beim Vergleich zweier zur Auswahl stehender staatlicher Projekte, lediglich auf der Ausgabenebene in Betracht gezogen.

Andererseits zeigen empirische Untersuchungen unter Verwendung der Kennziffern 3 bis 5 , daß sich je nach benutzter Kennziffer ganz unterschiedliche Rangordnungen ergeben ${ }^{2)}$. Zweifel an der Interpretation einer Kennziffernreihe als Prioritätenliste nährt

1) Siehe in diesem Zusammenhang auch Kitterers Kritik an der Interpretation einer Kennziffernreihe als Ausdruck der Zielvorstellungen der Regierung. Kitterer stützt sich allerdings auf einen Vergleich der Entscheidung eines privaten Haushalts über die Verwendung einer vorgegebenen Geldsumme für den Kauf zweier Güter mit den Budgetentscheidungen der Regierung. Dies bedeutet faktisch die Ubertragung von Erkenntnissen der individuenbezogenen Entscheidungstheorie auf Entscheidungen im Kollektiv. Vgl. W. Kitterer: Das moderne Budget... a.a.0., S. $153 \mathrm{ff}$. Zur Theorie der Entscheidungsfindung in Gruppen siehe etwa W. Kirsch: Entscheidungsprozesse. Band 3: Entscheidungen in Organisationen, Wiesbaden 1971, S. $52 \mathrm{ff}$.;

G. Gäfgen: Theorie der wirtschaftlichen Entscheidung . Untersuchungen zur Logik und Bedeutung des rationalen Handelns, 3. Auf 1., Tübingen 1974, S. $176 \mathrm{ff}$.

2) Vgl. K. Schmidt und E. Wille: Die mehrjährige Finanzplanung... a.a.0., S. $82 \mathrm{ff.}$ sowie $\mathrm{W}$. Kitterer: Das moderne Budget... a.a.0., S. $146 \mathrm{ff}$. 
auch ein Blick auf den Ablauf der Budgetberatungen in der Realität. Sowohl im Kabinett als auch im Parlament beraten die Politiker nicht über einen funktional, sondern über einen nach Ressorts gegliederten Haushaltsentwurf ${ }^{1)}$. Die Erstellung des Funktionalbudgets, an dem die Prioritäten offenbar werden sollen, erfolgt erst im AnschluB an die haushaltspolitischen Entscheidungen.

Wesentlich gravierender als die beiden letztgenannten Argumente wiegt jedoch der Umstand, daß die Interpretation einer Kennziffernreihe als Prioritätenliste grundsätzlich nur dann vertretbar ist, wenn sich die Politiker bei ihrer Entscheidungsfindung ausschlieBlich auf die staatlichen Ausgaben konzentrieren. Diese implizite Voraussetzung ist nicht notwendigerweise immer erfüllt. Zum einen steht dem Staat zur Erfüllung seiner Aufgaben neben budgetären Ausgaben eine Reihe weiterer Instrumente zur Verfugung. Falls die politischen Entscheidungsträger bei der Festlegung der staatlichen Aktivitäten bewuBt auch Instrumente mit geringen oder gar keinen Ausgabenwirkungen einsetzen und somit ihre ziele auf ganz unterschiedlichen Wegen zu verwirklichen trachten, erfährt die Aussagekraft von allein auf budgetären Ausgaben beruhenden Kennziffern eine wesentliche Einschränkung, was wir unten noch ausführlich erläutern.

Zum anderen ist es denkbar, und nur dies würde auch den Erfordernissen einer anspruchsvollen öffentlichen Aufgabenplanung genügen, daB sich die politischen. Entscheidungsträger bei der Determinierung der staatlichen Aktivitäten weniger an den Staatsausgaben als vielmehr an den staatlichen Outputs, gemessen auf den Ebenen der output-oder der impact-objectives, orientieren. In diesem Falle betrachten die Politiker die Grade der zielerreichung bzw. Aufgabenerfüllung in den einzelnen Aufgabenbereichen als bloBe Mittel zur verfolgung ihrer persönlichen

1) Vgl. P. Senf: Kurzfristige Haushaltsplanung ... a.a.o., S. $381 \mathrm{ff}$. 
oder parteipolitischen zielvorstellungen und bewerten bzw. gewichten nicht Ausgaben, sondern staatliche Outputs im Hinblick auf ihre ziele. So mag ein Politiker einen relativ niedrigen. Zielerreichungsgrad im Bildungswesen für ausreichend erachten und andererseits großen Wert auf einen hohen zielerreichungsgrad bei der Inneren sicherheit legen ${ }^{1)}$.

Vordergründig betrachtet lassen sich auch in diesem Falle die Zielvorstellungen der politischen Entscheidungsträger an einer Kennziffernreihe ablesen, denn die budgetären Ausgaben sind eine Folge ihrer Entscheidungen über die angestrebten staatlichen Outputs. Dem stehen jedoch zwei Umstände entgegen. Zunächst gilt auch hier derselbe Einwand wie bei der ausgabenorientierten Haltung der Politiker: Es können auch andere Instrumente als Ausgaben zur Erzielung staatlichen Outputs zum Zuge kommen. Selbst wenn das nicht der Fall ist, ergibt sich eine zusätzliche Schwierigkeit: Bei der outputorientierten Haltung der politischen Entscheidungsträger ist in jedem staatlichen Aufgabenbereich eine "Produktionsfunktion"2), die ihnen zumindest in groben zügen bekannt ist, zwischen die staatsausgaben und ihre Zielvorstellungen geschaltet. Dies bedeutet aber, daß hier ein zweiter Grund für die unterschiedliche zuweisung finanzieller Mittel auf die staatlichen Aufgabenbereiche ins spiel kommt. Die Bildung einer vollständigen Rangordnung auf der Basis der Ausgaben setzt zumindest voraus, daB in den verschiedenen Aufgabenbereichen die "Produktionsfunktionen", d.h. der Zusammen-

1) Derzeit ist es allerdings wenig wahrscheinlich, daß sich die politischen Entscheidungsträger tatsächlich an den verschiedenen Zielerreichungsgraden in den öffentlichen Aufgabenbereichen orientieren. So weist etwa olson darauf hin, daB die Regierung häufig keinerlei Informationen über den staatlichen Output besitzt. Vgl. M. Olson: Evaluating Performance in the Public Sector, in: M. Moss (Ed.): The Measurement of Economic and Social Performance, Studies in Income and Wealth, Vol. 38 , New York 1973, S. 355-384 (Diskussion: S. 384-409), S. $405 \mathrm{ff}$.

2) Diese hängt u.a. von der Entwicklung der relativen Preise der Produktionsfaktoren ab. 
hang zwischen staatsausgaben und den mit ihrer Hilfe angestrebten Veränderungen bei den staatlichen Outputs, identisch oder wenigstens soweit ähnlich sind, daß von daher keine Veränderungen der Rangordnung entstehen. Dies wiederum würde in allen Aufgabenbereichen eine gleich hohe Produktivität erfordern. Solange nicht garantiert ist, daß jede ausgegebene Geldeinheit dieselbe marginale Veränderung beim staatlichen output bewirkt, gleichgültig in welchen Aufgabenbereich sie flieBt, läßt sich nicht zweifelsfrei feststellen, ob der hohe Rang einer Aufgabe gemäB einer Kennziffernreihe Ausdruck der von den politischen Entscheidungsträgern gesetzten Prioritäten oder aber Folge unzureichender Effizienz oder geringeren Produktivitätsanstiegs in diesem Aufgabenbereich ist. Darüber hinaus setzt der Schluß von den budgetären Ausgaben auf die zielvorstellungen der Politiker die völlige Unabhängigkeit der "Produktionsfunktionen" in den verschiedenen Aufgabenbereichen voneinander voraus, so daB weder synergieeffekte auftreten noch Aktivitäten in einem Aufgabenbereich negative Auswirkungen auf die Outputs in anderen Bereichen besitzen.

Als wesentliche Voraussetzung für die Interpretation einer auf der Basis budgetärer Ausgaben gebildeten Kennziffernreihe als Ausdruck der Ziel-bzw. Prioritätsvorstellungen der politischen Entscheidungsträger kristallisiert sich damit heraus, daß diese sich bei der Planung der staatlichen Aktivitäten auf das Instrument Staatsausgaben beschränken. Wenngleich Politiker Staatsausgaben offenbar als das wichtigste Mittel ansehen, benutzen sie in der Regel doch das gesamte Instrumentarium zur Erfüllung öffentlicher Aufgaben. Das Setzen von Schwerpunkten bedeutet bei weniger entscheidungstheoretischer als vielmehr produktionstheoretischer Betrachtungsweise, daB in bestimmte öffentliche Aufgabenbereiche bevorzugt Inputs 
fließen 1)2). Fatal für die schwerpunktbestimmung anhand der Staatsausgaben ist indes der Umstand, daB sich budgetäre Ausgaben lediglich teilweise mit den Inputs zur Erfüllung öffentlicher Aufgaben decken. Abweichungen können in zweierlei Hinsicht auftreten:

Beschränkt man sich zunächst auf die staatsausgaben, so ist festzustellen, $d a B$ die im Budget enthaltenen Ausgaben die dahinterstehenden Inputs zur Erfüllung öffentlicher Aufgaben nicht mit gleicher Gewichtung repräsentieren. Wie anhand verschiedener Formen der Subventionierung sowie der Zusammenhänge zwischen dem öffentlichen Vermögen und den Staatsausgaben noch zu zeigen ist ${ }^{3)}$, enthält das staatliche Budget - in betriebswirtschaftlicher Terminologie ausgedrückt 4 ) sowohl Auszahlungen (z.B. gewährte Darlehen) als auch Kosten (z.B. Zinssubventionen) und gewichtet beide gleich.

Zweitens sind die Staatsausgaben als monetäre Inputs nicht vollständig in dem Sinne, daß sie sämtliche Inputs zur Erfüllung öffentlicher Aufgaben widerspiegeln. Erweitert man den Blickwinkel von den staatsausgaben auf die staatstätigkeit, wobei insbesondere die zwangsweise tbertragung öffentlicher Aufgaben auf nicht-staatliche Entscheidungseinheiten sowie

1) Vor allem bei den Aktivitäten nicht-staatlicher Entscheidungseinheiten können diese beiden Betrachtungsweisen zu unterschiedlichen Ergebnissen bezüglich der Aussagekraft der Kennziffern führen. (Vgl. die Abschnitte 1.2.5. und 2. in diesem Kapitel.) Insgesamt wollen wir primär auf die produktionstheoretische Betrachtungsweise abstellen.

2) Dies besagt jedoch noch nichts über den mit Hilfe der Inputs erzielten Output.

3) Siehe Abschnitt 1.2.1. in diesem Kapitel.

4) Vgl. dazu etwa G. Wöhe: Bilanzierung und Bilanzpolitik. Betriebswirtschaftlich-Handelsrechtlich-S teuerrechtlich. Mit einer Einführung in die verrechnungstechnischen Grundlagen, 4. Aufl., München 1976, S. 8 ff. 
die Einnahmenseite des staatlichen Budgets einzubeziehen sind, treten beträchtliche Divergenzen zwischen Staatsausgaben und Inputs bzw. Aktivitäten zur Erfüllung öffentlicher Aufgaben zutage.

Wenn demnach das Bild, das die Staatsausgaben bzw. das Funktionalbudget von der Allokation der Inputs zur Erfüllung öffentlicher Aufgaben vermitteln, strukturelle Verzerrungen aufweist, sind die Schwerpunkte der zu berücksichtigenden Aktivitäten möglicherweise anders verteilt als es eine Kennziffernreihe signalisiert. Der folgende Abschnitt 1.2. dieses Kapitels behandelt staatliche Instrumentvariablen, deren Auswirkungen auf das Budget sich - bei weiter Auslegung - unter das von Zimmermann entwickelte Konzept der Ausgabenintensität der öffentlichen Aufgabenerfüllung ${ }^{1)}$ subsumieren lassen. Während Zimmermann bei seiner Argumentation jedoch im wesentlichen auf das Niveau der Staatstätigkeit bzw. der öffentlichen Aufgabenerfüllung abstellt, geht es hier nicht um die Aussagefähigkeit der staatsquote ${ }^{2)}$. Die qualitative Analyse

1) Vgl. zu diesem Konzept H. Zimmermann: Die Ausgabenintensität... a.a.0., sowie Abschnitt 1.2.5. in diesem Kapitel.

2) Zu den Problemen im Zusammenhang mit der Berechnung von Staatsquoten siehe vor allem $\mathrm{K}$. Littmann: Definition und Entwicklung der Staatsquote... a.a.o. sowie Wissenschaftlicher Beirat beim Bundesministerium der Finanzen: Gutachten zur Aussagefähigkeit staatswirtschaftlicher Quoten, a.a.o. In den Volkswirtschaftlichen Gesamtrechnungen entsteht eine systematische Verzerrung zwischen dem privaten und dem öffentlichen Sektor dadurch, daß diese den Produktionswert der Unternehmen als Summe der (überwiegend) zu Marktpreisen bewerteten Outputeinheiten ermitteln, während derjenige des Staates sich größtenteils aus Inputs zusammensetzt. Dieser Aspekt, aus dem möglicherweise auch strukturelle Verzerrungen innerhalb des Staatssektors resultieren, bleibt im folgenden unberücksichtigt. Zum einen beschränkt sich die Untersuchung auf den budgetären Niederschlag verschiedener Aktivitäten zur Erfüllung öffentlicher Aufgaben, zum anderen ist nicht einmal die Richtung dieses Niveaueffektes bekannt. 
der dem staat zur Verfügung stehenden Palette von Instrumenten zur Erfüllung öffentlicher Aufgaben dient vielmehr dazu, Möglichkeiten struktureller Verschiebungen zwischen den verschiedenen staatlichen Aufgabenbereichen aufzuzeigen, die einer Schwerpunktbestimmung auf der Basis der budgetären Ausgaben im Wege stehen. Ein exakter Nachweis von Verzerrungen und daraus möglicherweise resultierenden Veränderungen der Rangordnung, zumindest aber der Abstände zwischen den Aufgabenbereichen, ließe sich letztlich nur auf empirischem Wege erbringen, was hier nicht zu leisten bzw. beabsichtigt ist. Die $\mathrm{Z}$ ahlenangaben in Abschnitt 1.2. dienen lediglich zur Vermittlung einer Vorstellung uber die quantitative Bedeutung eines Instrumentes.

Im Gegensatz zu den staatlichen Realausgaben scheinen bei den Transferzahlungen im Hinblick auf die Schwerpunktbestimmung keinerlei Probleme aufutreten, da hier der Entscheidungsspielraum der Politiker auf das Gewähren oder Verweigern einer Subvention zusammenschrumpft. Staatliche Transferzahlungen stellen jedoch nur eine aus einer Reihe von Subventionsformen mit unterschiedlichem Niederschlag im Budget dar. Da der staat die Subventionsformen sowohl innerhalb als auch zwischen seinen Aufgabenbereichen variieren kann, lassen sich die staatlichen Transferzahlungen aus der folgenden Analyse nicht ausklammern.

Die Schwerpunktbestimmung anhand der Staatsausgaben sieht sich mit zwei zentralen Hemmissen konfrontiert. Während die in $\mathrm{Ab}-$ schnitt 1.2. erörterten möglichen strukturellen Verzerrungen auf den Einsatz der staatlichen Instrumentvariablen und damit unmittelbar auf den staat selbst zurückzuführen sind, ist dies bei den im AnschluB daran aufgeführten Faktoren nicht der Fall. Abschnitt 1.3. untersucht den Einfluß von veränderungen von Relativpreisen sowie der Bevölkerung auf das staatliche Budget. Diese Faktoren stellen, da der staat allenfalls beschränkten EinfluB darauf hat, exogene Störgrößen im Hinblick auf die 
Schwerpunktbestimmung dar. Ihre Wirkungen sind zwar - soweit sie die politischen Entscheidungsträger antizipieren - im Prinzip in den staatsausgaben enthalten, jedoch nicht ohne weiteres als solche zu erkennen. Sowohl die endogenen als auch die exogenen Faktoren können Verzerrungen bei der Bestimmung von Schwerpunkten der Staatstätigkeit auf der Basis budgetärer Ausgaben bewirken. Demgegenüber markieren die in Abschnitt 2 beschriebenen freiwilligen Aktivitäten nicht-staatlicher Entscheidungseinheiten zur Erfüllung öffentlicher Aufgaben den Ubergang von der staatstätigkeit auf das (umfassendere) Angebot an öffentlichen Gütern. Diesen Faktor vermag der staat in Grenzen, etwa mittels Finanzhilfen und steuervergünstigungen, zu beeinflussen, gleichwohl handeln die nicht-staatlichen Entscheidungseinheiten im Prinzip autonom, so daß es sich hierbei aus seiner sicht zwar ebenfalls um einen weitgehend exogenen Faktor, jedoch allenfalls teilweise auch um einen Bestandteil der staatstätigkeit handelt. Für den staat resultiert aus Veränderungen von Relativpreisen und der Bevölkerung sowie den freiwilligen Aktivitäten nicht-staatlicher Entscheidungseinheiten zur Erfüllung öffentlicher Aufgaben primär nicht ein Steuerungs-, sondern ein Informationsproblem: Er muß diese Faktoren bei der Planung seiner Aktivitäten als (weitgehend) vorgegebene Daten hinnehmen bzw. einbeziehen.

1.2. Verzerrungen durch endogene Störgrößen

1.2.1. Öffentliches Vermögen und Staatsausgaben

Der Unterschied zwischen Auszahlungen und Kosten tritt besonders deutlich an den zusammenhängen zwischen dem öffentlichen Vermögen und den staatsausgaben zutage. Das öffentliche Vermögen dient in erster Linie der Leistungserstellung; sofern 
Erträge anfallen, ist ihre Erzielung zumeist nur Nebenzweck ${ }^{1)}$.

Der Realvermögensbestand des öffentlichen Sektors übt laufend Wirkungen auf die Ziele der öffentlichen Planung aus, indem er Leistungen abgibt. Das Budget hält jedoch nur Vermögenszugänge und -abgänge im Zeitpunkt der Anschaffung bzw. des Ausscheidens eines Wirtschaftsgutes fest. Eine periodengerechte Verteilung der Anschaffungsausgaben bzw. -kosten beispielsweise eines Schulgebäudes auf die Jahre der Nutzung könnte durch die Vornahme von Abschreibungen erfolgen. Das staatliche Budget verbucht jedoch die Anschaffungskosten für öffentliche Investitionen im Anschaffungsjahr in voller Höhe ${ }^{2)}$.

Je nachdem, ob der staat zur Verfolgung seiner ziele eigene Vermögensbestandteile heranzieht oder private Vermögensobjekte mietet oder pachtet, können sich zudem Unterschiede im Hinblick auf die Verzinsung des eingesetzten Kapitals ergeben. Mietet der Staat beispielsweise ein Gebăude, so schließen die entsprechenden Ausgaben ein Äquivalent für die Verzinsung des

1) Vgl. A. Meier: రffentliches Vermögen II: Umfang und Funktionen, in: Handwörterbuch der Wirtschaftswissenschaft (HdWW), hrsg. von W. Albers et al., Bd. 5, stuttgart et al. 1980 , S. 623-633, S. 629. Dies gilt auch für manche Beteiligungen des Staates an privaten Unternehmen, die hier unter die öffentlichen Unternehmen subsumiert werden. Siehe dazu unten Abschnitt 1.2.3.1.2.

2) In den Volkswirtschaftlichen Gesamtrechnungen, die im Prinzip die Kosten der staatlichen Leistungserstellung ausweisen, werden dagegen im Wege der Schätzung Abschreibungen auf den öffentlichen Kapitalstock mit Ausnahme von Straßen, Brücken, Wasserwegen und ähnlichen Gütern mit schwer bestimmbarer Nutzungsdauer vorgenommen. Vgl. Statistisches Bundesamt: Fachserie 18 Volkswirtschaftliche Gesamtrechnungen, Reihe 1 Konten und Standardtabellen 1981, stuttgart, Mainz 1982, S. 86 und S. 91 sowie Wissenschaftlicher Beirat beim Bundesministerium der Finanzen: Gutachten zum Begriff der öffentlichen Investitionen, Schriftenreihe des Bundesministeriums der Finanzen, Heft 29, Bonn 1980, S. $44 \mathrm{f}$. 
in dem Gebäude gebundenen Kapitals ein. Steht dieses Gebäude dagegen im Eigentum des Staates, so enthält das Budget keine kalkulatorischen Kosten für die Verzinsung des vom Staat eingesetzten Kapitals.

Während der erste Aspekt eine in zeitlicher Hinsicht unkorrekte Verteilung von Kosten darstellt, impliziert der zweite eine totale Vernachlässigung von opportunitätskosten. Speziell aus dem Ansatz der Anschaffungsausgaben im Budget resultiert eine systematische Verzerrung. Unter der entscheidungstheoretischen Fragestellung erscheinen zwar die Auszahlungen als das geeignete Untersuchungsobjekt, falls die Politiker bei den Budgetberatungen jährlich neu über die geplanten Auszahlungen und nicht über die Kosten entscheiden. Wenn sie beschließen, in einem bestimmten Haushaltsjahr eine bestimmte Investition $z u$ tätigen und die finanziellen Mittel dafür bereitzustellen, so setzen sie damit einen schwerpunkt, der bei Betrachtung lediglich der Abschreibungen möglicherweise nivelliert würde. Es handelt sich dabei jedoch nur um einen Schwerpunkt auf der Ebene der monetären Inputs; in bezug auf die Staatstätigkeit stellt sich ein anderes Ergebnis ein. Die Staatstätigkeit in dem betreffenden Aufgabenbereich erhöht sich im Jahr der Anschaffung eines Investitionsgutes und verbleibt (ceteris paribus) bis zum Ende der Nutzungsdauer dieses Gutes auf dem neuen Niveau. Diesen Verlauf würden bereits die sachlichen und personellen Kapazitäten zutreffend anzeigen, während die ausgabenorientierten Kennziffern im Anschaffungsjahr (zu stark) hochschnellen und in den Folgejahren auf ein niedrigeres Niveau zurückfallen. Derartige Verzerrungen lassen sich nur bei kontinuierlicher öffentlicher Investitionspolitik vermeiden. 
Das öffentliche Geldvermögen betreffend stellt die staatliche Kreditvergabe neben öffentlichen Schuldendiensthilfen und Gewährleistungen ein wesentliches Instrument im Rahmen der öffentlichen Finanzierungshilfen dar. Der staat gewährt Kredite häufig zu zinsen, die unter dem Marktpreis liegen, oder zu günstigen Tilgungskonditionen. Aus dem Unterschied zwischen den Konditionen eines staatlichen und eines am Kapitalmarkt aufgenommenen Darlehens sowie in bestimmten Fällen aus dem Umstand, daß der Kreditnehmer auf dem Kapitalmarkt einen Kredit überhaupt nicht erhalten hätte, resultiert ein Subventionselement $^{1)}$. Es wird jedoch, im Gegensatz etwa zu schuldendiensthilfen, weder auf der Ausgaben- noch auf der Einnahmenseite des staatlichen Budgets sichtbar. Das Budget enthält in der Kapitalrechnung die Darlehensgewährung sowie die Tilgungsrückflüsse, die zinseinnahmen gehen dagegen in die laufende Rechnung ein. Die staatliche Vermögensrechnung erfaßt den Forderungsbestand nicht in einer einzigen, sondern nur unter verschiedenen Positionen; die Bewertung erfolgt zu Nominalwerten, obgleich in bestimmten Fällen vorauszusehen ist, daß die Darlehen nicht in voller Höhe zurückgezahlt werden ${ }^{2)}$.

Bei der Festlegung der Höhe eines Darlehens orientiert sich der staat grundsätzlich am Subsidiaritätsprinzip, so daß er ein Projekt nur in Ausnahmefällen in voller Höhe finanziert ${ }^{3)}$. Die mit

1) Vgl. G. Zeitel: Theoretische und technische Aspekte öffentlicher Darlehen und Gewährleistungen, in: Handbuch der Finanzwissenschaft, hrsg. von F. Neumark, Bd. 1, 3. Aufl., Tübingen 1977, S. 997-1024, S. 997 ff. sowie D. Dickertmann: రffentliche Finanzierungshilfen. Darlehen, Schuldendiensthilfen und Bürgschaften als Instrumente des finanzwirtschaftlichen Interventionismus, Baden-Baden 1980, S. $49 \mathrm{ff}$. und S. $64 \mathrm{ff}$.

2) Vgl. D. Dickertmann: రffentliche Finanzierungshilfen... a.a.O., S. $214 \mathrm{ff}$.

3) Vgl. ebenda, S. 60 f. Zu den Gestaltungsmöglichkeiten der Darlehensverträge im Detail siehe ebenda, S. $258 \mathrm{ff}$. 
der staatlichen Darlehensgewährung verfolgten Ziele liegen - soweit sie nicht fiskalisch motiviert ist - binnenwirtschaftlich im Bereich der Förderung von Wirtschaftszweigen (wobei der Anteil der Wohnungsbauförderung zunehmende Tendenz aufweist) und Regionen, im internationalen Bereich sind insbesondere die Hilfen für Entwicklungsländer zu nennen ${ }^{1)}$. Der Neunte Subventionsbericht gibt die Höhe der versteckten Subventionierung mittels der staatlichen Darlehensgewährung allein durch den Bund für das Haushaltsjahr $1982 \mathrm{mit}$ 1,47 Mrd. DM bei einem Bestand von 40,2 Mrd. DM und einer Neugewährung an Darlehen in Höhe von 1,5 Mrd. DM an ${ }^{2)}$.

Im Hinblick auf die Schwerpunktbestimmung lassen sich bei der staatlichen Kreditvergabe analoge Verzerrungen in intertemporaler Hinsicht aufzeigen wie bei den öffentlichen Investitionen. Darüber hinaus können Verzerrungen entstehen, wenn die öffentliche Hand die verschiedenen Finanzierungshilfearten gegeneinander substituiert. Würde der staat anstelle der Kreditvergabe an die potentiellen Kreditnehmer Zinszuschüsse in Höhe des geplanten Subventionselementes zahlen oder Gewährleistungen zu ihren Gunsten übernehmen, könnte möglicherweise

1) Vgl. G. Zeitel: Staatliche Darlehensgewährung als Mittel der Finanz- und Wirtschaftspolitik, in: Finanzarchiv N.F., Bd. 26 (1967), S. 193-214, S. 195 ff.; G. Zeitel: Theoretische und technische Aspekte... a.a.0., s. 1000 ff.; D. Dickertmann: offentliche Finanzierungshilfen... a.a.o., S. $81 \mathrm{ff}$.

2) Vg1. Bundesregierung: Bericht der Bundesregierung über die Entwicklung der Finanzhilfen des Bundes und der Steuervergünstigungen $f u ̈ r$ die Jahre 1981 bis 1984 gemäß $\$ 12$ des Gesetzes zur Förderung der stabilität und des Wachstums der Wirtschaft (StWG) vom 8. Juni 1967 (Neunter Subventionsbericht), Bundestags-Drucksache 10/352, Bonn, 6.9.1983, S. $27 \mathrm{ff}$. Zahlen zur Entwicklung der staatlichen Darlehensgewährung ab 1961 finden sich bei D. Dickertmann: öffentliche Finanzierungshilfen... a.a.0., s. $552 \mathrm{ff}$. 
der gleiche $\mathrm{Zweck}$ mit erheblich geringeren Ausgaben erreicht werden ${ }^{1)}$.

Die bereits erwähnten staatlichen Gewährleistungen nehmen ebenso wie die staatliche Kreditvergabe eine $\mathrm{zwischenstellung}$ insofern ein, als sie sich zwar teilweise in den staatsausgaben niederschlagen, die Budgetposten jedoch ein unzutreffendes Bild von der damit verbundenen staatstätigkeit liefern. Bei den Gewährleistungen handelt es sich um verschiedene Formen von Bürgschaften und Garantien, die eine Haftungsübernahme des Staates zugunsten privater oder öffentlicher Unternehmen sowie privater Haushalte implizieren ${ }^{2)}$. Die Bandbreite der mit staatlichen Gewährleistungen verfolgten $\mathrm{Zwecke}$ reicht von der Ubernahme eines Teils des Risikos, das mit langfristigen Investitionen verbunden ist, bis zur Befähigung des Empfängers, Kreditmarktmittel überhaupt $z u$ erhalten. Die Begünstigten erhalten die Möglichkeit, sich liquide Mittel zu verschaffen, die sie anderenfalls nur zu ungünstigeren Bedingungen oder gar nicht bekommen hätten. In der Regel sind die Gewährleistungen

1) Vgl. auch W. Albers: Der Umfang der staatlichen Tätigkeit und ihre wirtschaftlichen Wirkungen, in: Weltwirtschaftliches Archiv, Bd. 77 (1956 II), S. 176-234, S. 190 ff. Die Instrumente sind allerdings aufgrund haushaltsrechtlicher Vorschriften nicht beliebig substituierbar. Der Bund darf nur dann Kredite vergeben oder Schuldendiensthilfen leisten, wenn der angestrebte Zweck nicht durch die Ubernahme von Gewährleistungen erreicht werden kann. Vgl. hierzu R. Bergsträsser: Die Bürgschaften und Garantien des Bundes als Mittel der Wirtschaftsförderung, in: Zeitschrift für das gesamte Kreditwesen, 28. Jg. (1975), S. $146-148$, S. $182-184$, S. $218-222$, S. 146 .

2) $\mathrm{Zu}$ den Ausgestaltungsmöglichkeiten siehe $\mathrm{K}$. von Wysocki: öffentliche Finanzierungshilfen, Forschungsberichte des Landes Nordrhein-Westfalen, Nr. 946, Köln, Opladen 1961 , S. $63 \mathrm{ff}$. sowie E.-A. Conrad: Bürgschaften und Garantien als Mittel der Wirtschaftspolitik, Berlin 1967, S. 20 ff. 
mit Entgelten verbunden, die jedoch niedriger sind als vergleichbare private Sätze, soweit uberhaupt privatwirtschaftliche Absicherungsmöglichkeiten existieren. Daher enthalten diese Sicherheitsleistungen häufig eine subventionskomponente ${ }^{1)}$.

Die Ubernahme von Gewährleistungen bedarf in der Bundesrepublik gemäB Art. 115 GG, \ 23 HGrG und \$ 39 BHO einer Ermächtigung durch Gesetz. Während diese bis zum Rechnungsjahr 1958 in Einzelgesetzen oder in den betreffenden Wirtschaftsförderungsgesetzen enthalten waren, werden sie inzwischen auf Initiative des Haushaltsausschusses zum überwiegenden Teil (Ausnahmen sind nach wie vor in Einzelgesetzen geregelt) jeweils im Haushaltsgesetz zusammengefaBt, so daB das Parlament nun jährlich über die Ermächtigungen beschließt. Der von der Exekutive ausfüllbare Spielraum für Neuengagements ergibt sich nach Abzug der bereits bestehenden Gewährleistungsverpflichtungen von diesem Plafonds. Allerdings darf der Plafonds revolvierend belegt werden, d.h., daß frei gewordene Beträge aus alten Verpflichtungen bei schadensfreiem Verlauf wieder zur Verfügung stehen. Daher kann das Umsatzvolumen weit über dem Plafonds liegen ${ }^{2)}$.

Im Bundeshaushaltsplan wird jährlich für sämtliche Gewährleistungen des Bundes im Einzelplan 32 (Bundesschuld) ein sogenannter "Schadenstitel" für die "Inanspruchnahme aus Bürgschaften, Garantien und sonstigen Gewährleistungen" vorgesehen. Er erfaßt die Ausgaben für die Deckung von Schadensfällen und die Kosten der Mandatare (mit der Vergabe von Gewährleistungen beauftragte Kreditinstitute oder Treuhandstellen) einerseits

1) Vgl. E.-A. Conrad: Bürgschaften und Garantien... a.a.0., S. $86 \mathrm{ff.;} \mathrm{G.} \mathrm{Zeitel:} \mathrm{Theoretische} \mathrm{und} \mathrm{technische} \mathrm{Aspekte...}$ a.a.O., S. 1005 f.; D. Dickertmann: రffentliche Finanzierungshilfen... a.a.O., S. 58 f. und S. $268 \mathrm{ff}$.

2) Vgl. E.-A. Conrad: Bürgschaften und Garantien... a.a.o., S. $40 \mathrm{ff.}$. D. Dickertmann: offentliche Finanzierungshilfen... a.a.O., S. $196 \mathrm{ff}$. 
und die Entgelte für die Ubernahme von Gewährleistungen sowie Zinsen und Schadensrückzahlungen andererseits, Regelmäßig enthält der Titel den Vermerk "Einnahmen fließen den Mitteln zu", zudem sind die Ausgabemittel übertragbar"). Eine Bürgschaftssicherungsrücklage, wie sie haushaltsrechtlich zulässig wäre und auf der Ebene der Gemeinden und einiger Länder zeitweilig praktiziert wurde, gibt es dagegen im Bundeshaushaltsplan nicht ${ }^{2)}$.

Im Haushaltsjahr 1983 betrug die Summe der Ermächtigungen zur Ubernahme von Gewährleistungen $283 \mathrm{Mrd}$. DM oder $111 \mathrm{v}$.H. des Volumens des Bundeshaushalts und war am 30.6.1983 mit 230,5 Mrd. DM bzw. zu 81 v.H. belegt. Sowohl bei den Ermächtigungen als auch beim tatsächlich eingegangenen Obligo entfallen zwei Drittel auf den AuBenwirtschaftsverkehr ${ }^{3)}$. Die Bundesregierung hält die mit der Ubbernahme von Gewährleistungen verbundenen Subventionswirkungen für nicht quantifizierbar und weist darauf hin, daB sich seit 1950 insgesamt im Bereich der Bundesbürgschaften ein rechnerischer GesamtüberschuB der Einnahmen über die Ausgaben von ca. 1,8 Mrd. DM ergeben hat (1980 standen Ausgaben in Höhe von 945,1 Mio. DM Einnahmen in Höhe von 863,4 Mio. DM gegenüber), daher könne langfristig und über den gesamten Bereich betrachtet die Inanspruchnahme

1) Vgl. E.-A. Conrad: Bürgschaften und Garantien... a.a.o., S. 53 f.; D. Dickertmann: Öffentliche Finanzierungshilfen... a.a.0., S. 205 und S. 213.

2) Vgl. E.-A. Conrad: Bürgschaften und Garantien... a.a.0., S. 66 f.; D. Dickertmann: Öffentliche Finanzierungshilfen... a.a.0., S. 56 f., S. 205 und S. $481 \mathrm{ff}$.

3) Vgl. Bundesministerium der Finanzen (Hrsg.): Finanzbericht 1984, Bonn 1983, S. 241 ff. 
aus Bürgschaften nicht als Subvention angesehen werden ${ }^{1)}$. Das bedeutet allerdings nicht, daß für einzelne Begünstigte bzw. Gruppen die Ubernahme von Gewährleistungen durch den Bund keine Subventionselemente enthält ${ }^{2}$.

Im Hinblick auf die Bestimmung der Schwerpunkte öffentlicher Aktivitäten gehen von den staatlichen Gewährleistungen gravierende Einflüsse aus. Das in einer Haushaltsperiode mit der tbernahme von Gewährleistungen induzierte Transaktionsvolumen spiegelt sich weder in den vom Parlament bewilligten Plafonds noch im tatsächlich belegten Obligo wider. Einerseits können die revolvierende Belegung der Ermächtigungsbeträge und die Möglichkeit, Kredite zu weniger als $100 \% \mathrm{zu}$ besichern, eine Vergrößerung des Transaktionsvolumens hervorrufen, andererseits können Mitnehmereffekte auftreten. Ein Teil der besicherten Transaktionen wäre möglicherweise auch ohne staatliche Gewährleistungen vorgenommen worden.

Auch der "Schadenstitel" im Budget weist kaum Aussagekraft auf. Zum einen spielt sich die staatliche Aufgabenerfüllung mit Hilfe von Gewährleistungen zu einem guten Teil außerhalb des Budgets $a b$, während dies etwa bei der staatlichen Kreditvergabe und bei Finanzierungshilfen nicht in demselben MaB der Fall ist.

1) Vgl. Bundesregierung: Bericht der Bundesregierung über die Entwicklung der $F$ inanzhilfen und Steuervergünstigungen $f$ ür die Jahre 1979 bis 1982 gemäß $\$ 12$ des Gesetzes zur Förderung der Stabilität und des Wachstums der Wirtschaft (StWG) vom 8. Juni 1967 (Achter Subventionsbericht), BundestagsDrucksache 9/986, Bonn, 6.11.1981, S. 9. Ähnlich äuBert sich die Bundesregierung auch im Neunten Subventionsbericht (a.a.0.), S. 308. Zur Entwicklung in den einzelnen Haushaltsjahren seit 1950 siehe E.-A. Conrad: Bürgschaften und Garantien... a.a.O., S. $167 \mathrm{ff}$. sowie D. Dickertmann: bffentliche Finanzierungshilfen... a.a.0., S. $123 \mathrm{ff}$. und S. $571 \mathrm{ff}$.

2) Vgl. auch R. Bergsträsser: Die Bürgschaften und Garantien... a.a.O., S. $220 \mathrm{f}$. 
Insofern entsteht durch Gewährleistungen ein "Schattenhaushalt". Die staatlicherseits gewährten Hilfen werden im Budget lediglich in Höhe der eingetretenen schäden sichtbar ${ }^{1}$. Zum anderen ist der Ansatz der in einem Haushaltsjahr erwarteten Schäden mit erheblichen Unsicherheiten behaftet. Hieraus können Verzerrungen zwischen den einzelnen Aufgabenbereichen resultieren, da insbesondere die politischen Risiken im AuBenwirtschaftsverkehr nur schwer abzuschätzen sind. Im Extremfall müssen Aufgaben mit höherer Priorität aufgrund massiert eingetretener Schäden zurückgestellt werden, so daß die Schwerpunkte der Staatstätigkeit am Ende der Haushaltsperiode anders verteilt sind als ex ante geplant ${ }^{2}$.

Im Hinblick auf eine periodengerechte zurechnung von Ausgaben und Aktivitäten weist der Ausweis der Gewährleistungen im Haushalt ebenfalls Mängel auf. Da sich die Laufzeit der Gewährleistungen häufig über mehrere Jahre erstreckt, belasten die während eines Haushaltsjahres eingegangenen verpflichtungen (auch) zukünftige Budgets mit Risiken. Insbesondere die Exekutive hat hier die Möglichkeit, Ausgaben bewußt in die folgenden Perioden zu verlagern, wodurch sie sich unter Umständen selbst zukünftige Handlungsspielräume beschneidet ${ }^{3)}$. Hieraus können Verzerrungen in intertemporaler Hinsicht resultieren, da der "Schadenstitel" eines einzelnen Budgets auch Ausgaben für Verpflichtungen vorsieht, die in früheren Perioden eingegangen wurden.

1) Vgl. E.-A. Conrad: Bürgschaften und Garantien... a.a.O., S. $58 \mathrm{f}$.

2) Vgl. ebenda, S. $54 \mathrm{ff}$.

3) Vgl. ebenda, S. $57 \mathrm{f}$. 


\subsubsection{Staatliche Normsetzung}

Die staatliche Normsetzung ist das gängige Beispiel für Unterschiede zwischen der Erfüllung öffentlicher Aufgaben mit Hilfe von Staatsausgaben einerseits und der Staatstätigkeit andererseits. Der Staat bedient sich zur Erfüllung von Aufgaben wie Ordnungs-, insbesondere Wettbewerbspolitik, AuBenpolitik oder einer Reform des Justizwesens vorwiegend der Normsetzung. Der Wirkungsbereich dieser im englischsprachigen Schrifttum als "Government by Regulation" bezeichneten Gesetzgebungs- und Verordnungstätigkeit erstreckt sich sowohl auf den staat selbst als auch auf den privaten Sektor. Einerseits nimmt der staat mit Hilfe der Normsetzung bestimmte Aufgaben selbst in Angriff (z.B. die Strafgesetzgebung); hinzu kommen Normen, deren Geltungsbereich sich auf die Gebietskörperschaften beschränkt (wie etwa die Regelungen über den Finanzausgleich). Andererseits überträgt der Staat eine Fülle von Aufgaben mit Hilfe von Zwang auf nicht-staatliche Entscheidungseinheiten. Dies setzt - zumindest in demokratischen Gemeinwesen - in den meisten Fällen eine Gesetzgebungs- und Verordnungstätigkeit voraus. Wir beschränken uns hier auf den erstgenannten Wirkungsbereich, dem zweiten Aspekt ist der folgende Abschnitt 1.2.3. gewidmet.

Die staatliche Normsetzung schlägt sich im Budget lediglich in Höhe der "Gemeinkosten" für die Erhaltung des Staatsapparates nieder. Hierzu gehören Anteile der Ausgaben für Parlament und Exekutive sowie für die Rechtsprechung, falls sie die Normen fortentwickelt ${ }^{1)}$.

Die Schwerpunktbildung auf der Basis der Staatsausgaben vernachlässigt die staatliche Normsetzung im Prinzip völlig und

1) Vgl. H. Zimmermann: Die Ausgabenintensität... a.a.০., S. 1 ff. 
läßt daher Verzerrungen befürchten. Zwar existiert kaum ein staatlicher Aufgabenbereich, in dem die Normsetzung nicht zur Anwendung kommt, ihre relative Bedelitung (im Verhältnis zur Erfüllung von Staatsaufgaben mit Hilfe von Ausgaben) kann jedoch in den verschiedenen Aufgabenbereichen unterschiedlich groß sein. Wenn die öffentliche Hand etwa für die auswärtigen Beziehungen wesentlich weniger Ausgaben tätigt als für die Verteidigung, nimmt die AuBenpolitik nicht notwendigerweise auch einen niedrigeren Rang in der Prioritätenskala der politischen Entscheidungsträger ein, ebensowenig impliziert dies notwendigerweise eine geringere staatstätigkeit.

1.2.3. Verlagerung von Aufgaben auf nicht-staatliche Entscheidungseinheiten

Der staat überträgt eine Reihe von öffentlichen Aufgaben auf nicht-staatliche Entscheidungseinheiten. Teils wendet er Zwang an, um sie zur Erbringung bestimmter Leistungen zu veranlassen, teils entfalten sie freiwillig Aktivitäten zur Erfüllung öffentlicher Aufgaben. Dieser Abschnitt beschränkt sich auf die erzwungenen Aktivitäten nicht-staatlicher Entscheidungseinheiten, da nur hier ein unmittelbarer zusammenhang zur staatstätigkeit gegeben ist ${ }^{1}$. Dabei spielt zwar meistens - wie bereits erwähnt - die staatliche Normsetzung eine Rolle, sie deckt diesen Bereich jedoch nicht vollständig ab. Der staat kann etwa ein Unternehmen mit Hilfe von Rechtsnormen, aber auch - bei geeigneten Beteiligungsverhältnissen - durch Ausübung seiner Rechte als (Mit-) Eigentümer zu einem von ihm gewünschten Verhalten veranlassen. Zudem schlagen sich die Normsetzung und die Verlagerung von Aufgaben auf nicht-staatliche Entscheidungseinheiten in teilweise sehr unterschiedlicher weise im

1) Zur freiwilligen Erfüllung öffentlicher Aufgaben durch nicht-staatliche Entscheidungseinheiten siehe Abschnitt 2 . in diesem Kapitel. 
Budget nieder. In institutioneller Hinsicht betrifft diese Art der Erfüllung öffentlicher Aufgaben die intermediären Entscheidungseinheiten und aus dem Bereich der privaten Entscheidungseinheiten vor allem die privaten Unternehmen und Haushalte ${ }^{1)}$.

1.2.3.1. Intermediäre Entscheidungseinheiten

1.2.3.1.1. Parafisci

Die Parafisci befinden sich, wie auch der synonyme Begriff "intermediäre Finanzgewalten" ${ }^{2}$ andeutet, zwischen dem privaten und dem öffentlichen Bereich und genießen eine mehr oder weniger starke Unabhängigkeit von den Gebietskörperschaften. Sie bereiten bereits in systematischer Hinsicht schwierigkeiten, da ihre juristische Zugehörigkeit vielfach keinen Schluß auf die Art der Aufgabenerfüllung erlaubt. So gehören manche, wie z.B. die gesetzliche Krankenversicherung, nach juristischen Kriterien eindeutig zum öffentlichen Bereich, erfüllen jedoch ähnliche Aufgaben wie private Unternehmen. Diese Ambivalenz mag auf die Entstehungsgeschichte der Parafisci zurückzuführen sein: Teils wurden bereits bestehenden unternehmerischen Organisationen öffentliche Aufgaben übertragen (z.B. die berufsständischen Kammern), teils gründete der staat juristisch

1) Ähnliche Verlagerungen lassen sich auch im internationalen Vergleich feststellen. Zahlenangaben existieren primär für die öffentlichen Unternehmen und die Parafisci. Vgl. für die Staaten der Europäischen Gemeinschaft CEEP: Die öffentliche Wirtschaft in der Europäischen Gemeinschaft. Jahrbuch 1984 der Europäischen Zentrale der öffentlichen Wirtschaft (CEEP), Brüssel, Berlin 1984; für Österreich C. Smekal: Die Flucht aus dem Budget, a.a.0.; für die USA die Budgetanalysen der Brookings Institution, zuletzt J.A. Pechman (Ed.): Setting National Priorities. The 1982 Budget, Washington D.C. 1981 , S. 261 ff.

2) Diese Bezeichnung geht wohl zurück auf F.K. Mann (Die intermediären Finanzgewalten... a.a.O.) Vgl. auch W. Herrmann: Intermediäre Finanzgewalten... a.a.0. 
selbständige Institutionen (z.B. die Sozialfisci) ${ }^{1}$. Die Parafisci weisen nach der üblichen Terminologie ${ }^{2)}$ zwei Hauptmerkmale auf: Sie erfüllen öffentliche Aufgaben, und sie verfügen ïber eigene Finanzquellen mit Zwangscharakter, so daß sie ihren Aufgaben weitgehend unabhängig von den Haushalten der Gebietskörperschaften nachkommen können. Als ergänzende Merkmale werden häufig die beamtenähnliche stellung ihrer Beschäftigten und ihre selbständige Rechnungslegung genannt. Aufgrund dieser Merkmale ließen sich den Parafisci die Sozialfisci (Träger der Sozialversicherung), die ständefisci (öffentlich-rechtlich organisierte Berufsvertretungen) sowie (zumindest von ihrer ursprünglichen. Finanzierung her) das ERP-Sondervermögen und Sonderfonds wie der Lastenausgleichsfonds schwergewichtig zuordnen ${ }^{3)}$.

1) Vgl. C. Smekal: Finanzen intermediärer Gewalten (Parafisci), in: Handwörterbuch der Wirtschaftswissenschaft (HdWW), hrsg. von W. Albers et al., Bd. 3, stuttgart et al. 1980, S. 1-17, S. $1 \mathrm{ff}$. sowie C. Scheer: Sozialstaat und öffentliche Finanzen. Theorie und Realität der sozialen Komponente der öffentlichen Finanzen im Wandel vom liberalen Rechtsstaat zum Sozialstaat, Köln 1975, S. $40 \mathrm{ff.} \mathrm{Zu}$ Vorläufern und Entstehung der gesetzlichen Krankenversicherung siehe L. Hajen: Bestimungsgründe... a.a.0., s. $91 \mathrm{ff}$.

2) Vgl. etwa K. Tiepelmann: Parafiski, in: Das Wirtschaftsstudium (WiSu), Jg. 1975, S. 295-300, S. 295 ff. sowie H. Zimmermann und K.-D. Henke: Finanzwissenschaft. Eine Einführung in die Lehre von der öffentlichen Finanzwirtschaft, 3. Aufl., München 1982, S. 7 ff. Eine abweichende, eher zielbezogene Merkmalsbildung verwendet C. Smekal: Die Finanzwirtschaft intermediärer Gruppen, Veröffentlichungen der Universität Innsbruck, Bd. 10, Innsbruck 1969, S. 50 ff.

3) Die Kirchen wollen wir im Rahmen dieser Untersuchung nicht unter die Parafisci, sondern unter die privaten Entscheidungseinheiten subsumieren, da die Kirchensteuer keinen Zwangscharakter aufweist. 
Die (Brutto-) Ausgaben der wichtigsten Parafisci betrugen im Jahre 1982 bei der Sozialversicherung insgesamt (ohne die Zahlung von Kindergeld) rund $318 \mathrm{Mrd}$. DM, beim ERP-Sondervermögen 3,47 Mrd. DM und beim Lastenausgleichsfonds 1,95 Mrd. $\mathrm{DM}^{1)}$. Das Budget des staates enthält lediglich Zuschüsse an bzw. abgeführte Uberschüsse der Parafisci. Dies mindert scheinbar den Umfang der Staatstätigkeit und die Bedeutung der von Parafisci erfüllten öffentlichen Aufgaben gegenüber den Aufgaben, die der staat vollständig in eigene Regie ubernimmt.

\subsubsection{2. Öffentliche Unternehmen}

Die begriffliche Abgrenzung der öffentlichen Unternehmen fällt wesentlich schwerer als die der Parafisci. Hier existiert eine Begriffsvielfalt, da sich Vertreter der Disziplinen Rechtswissenschaft, Finanzwissenschaft und Betriebswirtschaftslehre um Definitionen der Begriffe "Offentliche Unternehmen bzw. Betriebe" sowie "Gemeinwirtschaftlichkeit" bemühen ${ }^{2)}$. Im Rahmen dieser Untersuchung genügt vorläufig eine weite Abgrenzung:

1) Zu detaillierten Zahlenangaben siehe Bundesministerium der Finanzen (Hrsg.): Finanzbericht 1984, a.a.O., S. $256 \mathrm{ff}$.; Bundesminister für Arbeit und Sozialordnung (Hrsg.): Sozialbericht 1983, Stuttgart 1984, S. 75 ff.; O. Dietz: Darstellung der Sozialversicherung in der Finanzstatistik, in: Wirtschaft und Statistik, Jahrgang 1984, S. 185-193.

2) Überblicke geben J. Backhaus: Offentliche Unternehmen. Zum Wirtschaftsrecht, den Funktionen und Rechtsformen öffentlicher Unternehmen, 2. Auf 1., Frankfurt/Main 1980, S. $14 \mathrm{ff}$. und S. $91 \mathrm{ff.;}$ T. Thiemeyer: Betriebswirtschaftslehre der öffentlichen Betriebe, in: Wirtschaftswissenschaftliches Studium (WiSt), Jg. 1981, S. 367-373 und S. 417-423, S. $369 \mathrm{ff.;} \mathrm{D.} \mathrm{Budäus:} \mathrm{Betriebswirtschaftslehre} \mathrm{öffentlicher}$ Unternehmen und Verwaltungen - Ansätze und Probleme eines Bezugsrahmens einzelwirtschaftlicher Analyse und Gestaltung öffentlicher Leistungsprozesse, in: Zeitschrift für öffentliche und gemeinwirtschaftliche Unternehmen, Bd. 4 (1981), S. 395-419, S. 409 ff. 
Eine Unternehmung ist als öffentlich anzusehen, wenn sie im Eigentum der öffentlichen Hand steht ${ }^{1)}$.

Nach der Rechtsform lassen sich die öffentlichen Unternehmen wie folgt einteilen ${ }^{2)}$ :

a) Öffentlich-rechtliche Unternehmen bzw. Betriebe ohne eigene Rechtspersönlichkeit

Hierzu gehören die reinen Regiebetriebe und die verselbständigten Regiebetriebe (Eigenbetriebe). Die reinen Regiebetriebe weisen als unmittelbarer Teil der allgemeinen Verwaltung das geringste $M a B$ an rechtlicher Verselbständigung auf. Sie verfügen über kein rechnerisch abgegrenztes Betriebskapital, daher erscheinen ihre Ausgaben und Einnahmen (kameralistisches Rechnungswesen) in voller Höhe im Trägerhaushalt (sog. Bruttobetriebe). Diese Rechtsform ist häufig bei Theatern, Wasserwerken, Hafenbetrieben und Krankenhäusern vorzufinden. Die Eigenbetriebe, deren Rechtsverhältnisse durch die verschiedenen Eigenbetriebsverordnungen oder -gesetze der Länder und die Betriebssatzungen geregelt sind, weisen

1) So auch die Abgrenzung in den primär funktional orientierten Beiträgen von D. Bös: Öffentliche Unternehmungen, a.a.O., S. 4 und D. Bös: Economic Theory of Public Enterprise, Berlin, Heidelberg, New York 1981, S. $1 \mathrm{f}$.

Bei gemeinsamem Eigentum von öffentlicher Hand und von Privaten an einem Unternehmen können zusätzliche Definitionsmerkmale erforderlich werden. So gehören nach $\$ 8$ Abs. 2 des Gesetzes über die Finanzstatistik alle Unternehmen, an deren Nennkapital oder Stimmrecht die öffentliche Hand mit mehr als 50 \% beteiligt ist, zu den öffentlichen Unternehmen.

2) Vgl. zum Folgenden T. Thiemeyer: Wirtschaftslehre öffentlicher Betriebe, Reinbek 1975, S. 34 ff.; T. Thiemeyer: Betriebswirtschaftslehre der öffentlichen Betriebe, a.a.o., S. 368 f.; V. Emmerich: Das Wirtschaftsrecht der öffentlichen Unternehmen, Bad Homburg 1969, S. 183 ff.; V. Emmerich: öffentliche Produktion II: Rechtsformen (einschlieBlich Bundesbahn und Bundespost), in: Handwörterbuch der Wirtschaftswissenschaft (HdWW), hrsg. von W. Albers et al., Bd. 5, stuttgart et al. 1980, S. 457-464, S. 459 f. 
eine wesentlich größere Verselbständigung als die reinen Regieunternehmen auf. Bei Eigenbetrieben handelt es sich um aus dem übrigen öffentlichen Vermögen ausgegliederte Sondervermögen. Sie verfügen über eine eigenverantwortliche Werkleitung und ein kaufmännisches Rechnungswesen. Im Trägerhaushalt erscheint nur der Differenzbetrag zwischen ihren Ausgaben und Einnahmen (sog. Nettobetriebe). Träger von Eigenbetrieben können nur Gemeinden sein, diese betreiben in dieser Rechtsform häufig ihre Verkehrs- und Versorgungsunternehmen.

Verwandt mit den Eigenbetrieben sind die wirtschaftlichen Sondervermögen des Bundes (Bundesbahn und Bundespost), deren Rechtsverhältnisse durch Sondergesetze geregelt sind. Die grundsätzliche zulässigkeit von Nettobetrieben mit eigenem Wirtschaftsplan und doppelter Buchführung mit kaufmännischem Jahresabschluß ergibt sich aus den $\$ \$ 26$ und 74 BHO; entsprechende Regelungen finden sich im AnschluB daran im Haushaltsrecht der Länder.

b) Öffentlich-rechtliche Unternehmen mit eigener Rechtspersönlichkeit

Die öffentlichen Unternehmen in der Rechtsform einer juristischen Person des öffentlichen Rechts sind in der Regel als Anstalten organisiert; daneben finden sich öffentlich-rechtliche Körperschaften und Stiftungen. Zu diesen Unternehmen, deren Rechtsverhältnisse sich ausschließlich aus den verschiedenen Errichtungsgesetzen ergeben, gehören die Bundesbank ${ }^{1)}$, die Kreditanstalt für Wiederaufbau und die Sparkassen.

1) Die Bundesbank klammern wir im folgenden aus der Untersuchung aus, da sie zwar ausschließlich öffentliche Aufgaben erfüllt, jedoch - trotz ihrer in jüngster zeit entdeckten fiskalischen Bedeutung - eher der Stabilisierungs- als der Allokationsabteilung zuzurechnen ist. 
c) Öffentliche Unternehmen in privatrechtlichen Rechtsformen Der öffentlichen Hand steht zwar die Gründung von oder die Beteiligung an Unternehmen in privatrechtlichen Formen frei, $\$ 65$ BHO und das Gemeindewirtschaftsrecht beschränken jedoch Gründung von und Beteiligung an Unternehmen in privat-rechtlicher Form auf solche, bei denen die Haftung des staates auf einen bestimmten Betrag begrenzt ist. Daher kommen als privatrechtliche Formen praktisch nur die Aktiengesellschaft und die $\mathrm{GmbH}$ sowie die Beteiligung an bestimmten Genossenschaften in Betracht.

Uber die Gesamtheit der öffentlichen Unternehmen in der Bundesrepublik liegt keine vollständige amtliche statistik vor. Das Statistische Bundesamt weist in den Volkswirtschaftlichen Gesamtrechnungen die öffentlichen Unternehmen nicht gesondert aus. Die europäische Zentrale der öffentlichen Wirtschaft (CEEP) legt jedoch in ihren Jahrbüchern umfassendes $\mathrm{Z}$ ahlenmaterial vor. Demnach belief sich die Zahl der öffentlichen Unternehmen $^{1)}$ in der Bundesrepublik Ende 1982 auf 4070 . Der öffentliche Anteil am Nennkapital dieser Unternehmen (insgesamt 138,7 Mrd. DM) betrug 132,6 Mrd. DM oder 95,6 \%. Die Schwerpunkte der öffentlichen Wirtschaft insgesamt liegen in den Bereichen Versorgung und Verkehr, Wohnungswesen und Kreditinstitute; bei den Unternehmen der Länder und Gemeinden liegen sie in den Bereichen Kreditwesen, Raumordnung und Industrieansiedlung, Wohnungsbau und Siedlungswesen, Versorgung mit Strom, Gas, Wasser und Fernwärme sowie Verkehr.

1) Ohne reine Regiebetriebe und ohne Unternehmen, an deren Nennkapital oder Stimmrecht die öffentliche Hand zu weniger als 508 beteiligt war. Vgl. CEEP: Die öffentliche Wirtschaft... a.a.0., s. $25 \mathrm{ff}$. 
Speziell uber die Unternehmen im Bundesbesitz informieren die Berichte ubber die Beteiligungen des Bundes ${ }^{1)}$. Ende 1982 waren der Bund und seine Sondervermögen (ERP-Sondervermögen, Lastenausgleichsfonds, Bundespost und Bundesbahn) an insgesamt 170 Unternehmen des öffentlichen und privaten Rechts unmittelbar beteiligt. Mit mindestens 258 und einem Nennkapital von wenigstens 100000 DM waren diese Träger an 102 dieser Unternehmen unmittelbar und an 856 weiteren mittelbar beteiligt. Das Nennkapital der Unternehmen, an denen der Bund und seine Sondervermögen unmittelbar beteiligt sind, betrug am 31.12 .1982 insgesamt 12,17 Mrd. DM. Hiervon entfallen auf den Bund 5,91 Mrd. DM $(48,68)$ und auf die Sondervermögen $1,07 \mathrm{Mrd}$. $\operatorname{DM}(8,88)$. Als Schwerpunkte der Beteiligungen nennt der Bericht das industrielle Bundesvermögen, den Bankenbereich, das Verkehrswesen, die Wohnungswirtschaft, den Forschungsbereich sowie den entwicklungspolitischen Bereich.

In jüngerer zeit gewannen primär die öffentlichen Unternehmen wachsende Bedeutung, da insbesondere die übergeordneten Gebietskörperschaften "die Flucht aus dem Budget"2) ergriffen. Um die Kreditmarktverschuldung des Staatshaushalts - vor allem optisch - niedrig zu halten, wurden Aufgaben aus dem Bereich der Verwaltung auf privat-oder öffentlich-rechtlich organisierte Institutionen übertragen, denen dann auch die Finanzierung obliegt. So gründeten zur Durchführung der staatlichen

1) Die folgenden Angaben sind entnommen aus: Bundesministerium der Finanzen (Hrsg.): Beteiligungen des Bundes im Jahre 1982, Bonn 1983, S. 1 ff. Sie umfassen nicht die Sondervermögen selbst und die Beteiligungen an internationalen Einrichtungen wie z.B. der Weltbank.

2) So auch der treffende Titel einer Studie von C. Smekal (a.a.0.) über entsprechende Entwicklungen in Österreich. Der Wissenschaftliche Beirat beim Bundesministerium der Finanzen (Gutachten zur Lage und Entwicklung der Staatsfinanzen in der Bundesrepublik Deutschland, in: Bulletin des Presse- und Informationsamtes der Bundesregierung, Bonn, $\mathrm{Nr} .103$ vom 16.8.1975, S. 1001-1016, S. $1007 \mathrm{f.}$ ) spricht hier von einer "formalen Verlagerung" öffentlicher Aufgaben. 
Wohnungsbauprogramme verschiedene Bundesländer Anstalten bzw. betrauten bereits bestehende Anstalten mit diesen Programmen. Diese so aus dem Budget ausgegliederten Wohnungsbauförderungsanstalten refinanzieren die von ihnen zu gewährenden öffentlichen Baudarlehen auf eigene Rechnung und im eigenen Namen am Kapitalmarkt ${ }^{1)}$. Ungewöhnliche Wege beschritten Niedersachsen und Nordrhein-Westfalen. Sie gründeten die Niedersächsische Hochschulbaugesellschaft mbH bzw. die Nordrhein-Westfälische Hochschulbau- und Finanzierungsgesellschaft. Ihnen fällt unter anderem die Aufgabe $z u$, die Investitionen im Hochschulbau zu finanzieren, daher erscheinen die hierfür erforderlichen Kapitalmarktmittel nicht im staatshaushalt ${ }^{2)}$. Ein weiteres Beispiel liefert der Bund, der im Frühjahr 1981 ein Sonderprogramm beschloß, nach dem die Kreditanstalt für Wiederaufbau (KfW) Investitionskredite insbesondere mit den zielbereichen Energieeinsparung, ölsubstitution und neue Energietechnologie in Höhe von 6,3 Mrd. DM vergibt. Die erforderlichen Mittel sollte die KfW auf den internationalen Kapitalmärkten aufnehmen. Der Bundeshaushalt ist von diesem Programm nur insofern betroffen, als über ihn die zur verbilligung der Kredite um bis zu 2,25 Prozentpunkte pro Jahr benötigten Mittel bereitgestellt werden. (Insgesamt nahezu 600 Mio. DM; 1982 waren erstmals Mittel in Höhe von 130 Mio. DM eingeplant.) ${ }^{3)}$

1) Vgl. G. Götz: Die Schuldenpolitik der Länder, Meisenheim am Glan 1970, S. 42 ff. Kritisch zur "Flucht aus dem Budget" auch schon F.K. Mann: Die intermediären Finanzgewalten... a.a.O., S. 222 f. Zu Maßnahmen und Institutionen zur Förderung des Wohnungsbaus in Frankreich und in der Bundesrepublik siehe $W$. Kitterer: Das moderne Budget... a.a.O., S. $158 \mathrm{ff}$.

2) Die Nordrhein-Westfälische Hochschulbau- und Finanzierungsgesellschaft wurde zum 31.12.1977 aufgelöst. Vgl. Statistisches Bundesamt: Hochschulfinanzen 1976, in: Wirtschaft und Statistik, Jg. 1978, S. 590-593, S. $590 \mathrm{ff}$.

3) Vgl. Bundesministerium der Finanzen (Hrsg.): Finanzbericht 1982 , Bonn 1981, S. 61 . 
Die Verlagerung von öffentlichen Aufgaben auf öffentliche Unternehmen schlägt sich im staatlichen Budget - wie bereits angedeutet - in unterschiedlicher Weise nieder. Die Einnahmen und Ausgaben der reinen Regiebetriebe, die Bestandteil der allgemeinen Verwaltung sind, erscheinen in voller Höhe im Budget des jeweiligen Trägers. Handelt es sich dagegen um Nettobetriebe oder um juristisch verselbständigte öffentliche Unternehmen, so enthält das Budget allenfalls Zuschüsse bzw. die abgeführten Uberschüsse. Verzerrungen bei der Feststellung von Schwerpunkten können schon aus dem Umstand resultieren, daß Betriebe mit gleichen öffentlichen Aufgaben in den verschiedensten Rechtsformen geführt werden. Beispielsweise können die Gemeinden bestimmte Aufgaben wie die Müllabfuhr in eigener Regie mit Hilfe eines Nettobetriebes oder eines Bruttobetriebes erfüllen oder sie privaten Unternehmen überlassen ${ }^{1)}$. Wie das Beispiel der Hochschulbau-Gesellschaften besonders deutlich illustriert, beeinträchtigt die "Flucht aus dem Budget" die Vergleichbarkeit der Ausgaben in einem bestimmten Aufgabenbereich schon für eine einzelne Gebietskörperschaft, da bei der Betrachtung über mehrere Jahre hinweg zumindest ein sprung auftritt. Zudem führt der Vergleich mehrerer Gebietskörperschaften der selben Ebene untereinander, sofern sie nicht alle in gleichem Maße Funktionen verlagert haben, nicht zu sinnvollen Ergebnissen.

\subsection{Rundfunkanstalten}

Die Rundfunkanstalten sind in der Bundesrepublik Deutschland als öffentlich-rechtliche Institutionen organisiert, die der Staat mit der Aufgabe der Rundfunkversorgung betraute. Sie stehen zwischen dem öffentlichen und dem privaten Sektor, stellen jedoch einerseits keine Parafisci dar, da die Rundfunkgebühren keinen Zwangscharakter aufweisen. Das Bereithalten von

1) Vgl. auch K. Littmann: Definition und Entwicklung der staatsquote... a.a.0., s. 13 . 
Empfangsgeräten, an das die Gebührenpflicht anknüpft, steht im freien Ermessen der Individuen. Andererseits lassen sie sich auch nicht unter die öffentlichen Unternehmen subsumieren. Zwar handelt es sich um Leistungen produzierende und abgebende wirtschaftseinheiten, sie weisen jedoch allenfalls im Bereich der Werbesendungen unternehmensähnliche Elemente auf ${ }^{1)}$. Zudem fehlt das Merkmal staatlichen Eigentums.

Die in der Bundesrepublik bestehende Aufgabenverteilung im Rundfunkwesen leitet sich aus der Garantie der Freiheit der Berichterstattung durch Presse, Rundfunk und Film in Art. 5 des Grundgesetzes ab. Hieraus folgt zwar nicht zwingend die Organisation des Rundfunks in öffentlich-rechtlicher Form, sondern lediglich das Gebot einer pluralistischen Organisation, der staat entschied sich jedoch für die Gründung intermediärer Entscheidungseinheiten und den Ausschluß privater Anbieter. Einher mit dieser Beschränkung auf wenige, öffentlich-rechtlich organisierte Anbieter geht das Gebot der Ausgewogenheit der Programme. Um sie zu gewährleisten, sollen in den Aufsichtsgremien der Sender alle gesellschaftlich relevanten Gruppen repräsentiert sein, wobei der EinfluB keiner Gruppe dominieren darf. zudem erfordert das Gebot der staatsfreiheit, daß staatsorgane keinen entscheidenden oder beherrschenden EinfluB innerhalb der Rundfunkorganisation erhalten ${ }^{2)}$.

Die neun Landesrundfunkanstalten entstanden durch Landesgesetze, das Zweite Deutsche Fernsehen durch einen Staatsvertrag. Hinzu kommen die zwei Bundesanstalten Deutschlandfunk und Deutsche welle. Die Anstalten sind mit dem Recht der Selbstverwaltung ausgestattet. Die verschiedenen Errichtungsgesetze umschreiben die Aufgaben der Rundfunkanstalten sehr allgemein als Gestal-

1) Vgl. zu diesem Aspekt V. Emmerich: Das Wirtschaftsrecht der öffentlichen Unternehmen, a.a.O., S. $271 \mathrm{ff}$.

2) Vgl. I. von Münch: Randnummern $34 \mathrm{ff.}$ zu Art. 5, in: I. von Münch (Hrsg.): Grundgesetz-Kommentar, Bd. 1, 2. Aufl., München 1981 . 
tung und Vertreibung von Programmen des Hör- und Fernsehfunks. Inhaltliche Programmgrundsätze sind kaum festgelegt, als gemeinsamer Nenner läßt sich die Vermittlung von Information, Unterhaltung und Bildung feststellen. Die nähere Bestimmung ihres Programmauftrages obliegt den Rundfunkanstalten selbst ${ }^{1)}$.

Zur Finanzierung ihrer Aufgaben stehen den Rundfunkanstalten neben ihren Einnahmen aus Werbesendungen - primär die bundeseinheitlich gleich hohen Rundfunkgebühren zur Verfügung, deren Höhe mit zustimmung der Länderparlamente durch staatsvertrag festgelegt wird. (1980 betrug das Gebührenaufkommen rund 3,1 Mrd. DM.) Die Anstalten selbst können lediglich an der Entscheidung über die Befreiung bestimmter Teilnehmer von dieser Gebühr mitwirken. Sodann existiert ein Finanzausgleich zwischen fast allen Rundfunkanstalten. (Auf diesem Wege wurden 1980122 Mio. DM umverteilt.) ${ }^{2)}$ Der Deutschlandfunk erhält seine Mittel teils im zuge des Finanzausgleichs von den Länderanstalten, teils vom Bund, während der Bund die Deutsche Welle allein finanziert ${ }^{3}$ ). Nach den mittelfristigen Finanzvorschauen beliefen sich die betrieblichen Aufwendungen der ARD-Anstalten im Jahre 1982 auf $3,8 \mathrm{Mrd}$. DM und beim ZDF auf 1,19 Mrd. DM ${ }^{4)}$.

1) Vgl. W. Schreckenberger: Probleme bei der Ermittlung des Finanzbedarfs der Rundfunkanstalten, in: Zeitschrift für öffentliche und gemeinwirtschaftliche Unternehmen, Bd. 4 (1981), S. 100-107, S. 102 .

2) Vgl. ebenda, S. $100 \mathrm{ff}$. sowie O. Maier: Zur Ertrags- und Vermögenslage der Rundfunkanstalten in der Bundesrepublik Deutschland, in: Zeitschrift für öffentliche und gemeinwirtschaftliche Unternehmen, Bd. 3 (1980), S. 415-430, S. $418 \mathrm{ff}$.

3) Vgl. H.J. Kleinsteuber: Rundfunkpolitik. Der Kampf um die Macht über Hörfunk und Fernsehen, Opladen 1982, S. 35 f.

4) Vgl. W. Schreckenberger: Probleme bei der Ermittlung... a.a.0., S. 102. Zur Struktur dieser Aufwendungen siehe o. Maier: Zur Ertrags- und Vermögenslage... a.a.0., S. $420 \mathrm{ff}$. 
Der staat ist von den Aktivitäten der Rundfunkanstalten allgemein nur insoweit betroffen, als deren Finanzgebaren der Kontrolle durch die Rechnungshöfe unterliegt. Direkte Bezüge zu den Staatsausgaben bestehen lediglich bei den beiden Bundesanstalten Deutschlandfunk und Deutsche Welle, die der Bund teilweise bzw. ganz aus seinem Budget finanziert.

In bezug auf die staatstätigkeit resultieren hieraus keine Verzerrungen. Die Aktivitäten der Rundfunkanstalten stellen - obgleich ihre Leistungen starke Elemente reiner öffentlicher Güter aufweisen - nicht eine an sich staatliche Aufgabe dar, die der Staat auf intermediäre Entscheidungseinheiten verlagert hat. Vielmehr obliegt ihm auf diesem Gebiet lediglich die tendenziell ordnungspolitische Aufgabe, die Freiheit der Berichterstattung durch Schaffung geeigneter Rahmenbedingungen zu gewährleisten. Die Zuschüsse an die beiden Bundesanstalten spiegeln insoweit die staatstätigkeit im Rundfunkwesen zutreffend wider.

\subsubsection{Private Entscheidungseinheiten}

Bei der Ubertragung von öffentlichen Aufgaben auf private Entscheidungseinheiten bedient sich der staat einer Politik der Ge- und Verbote, um private Unternehmen und private Haushalte $\mathrm{zu}$ bestimmten Verhaltensänderungen $\mathrm{zu}$ zwingen. Hierbei handelt es sich zum einen um den sogenannten "versteckten öffentlichen Bedarf"1) bzw. "versteckten Staatsbedarf"2) , bei dem die Privaten unentgeltlich Leistungen für den Staat erbringen müssen, beispielsweise der Militärdienst bei allgemeiner Wehrpflicht, die ehrenamtliche Tätigkeit als Schöffe oder die Verpflichtung der Unternehmen, die Lohnsteuer und die Sozialversicherungsbeiträge ihrer Beschäftigten einzubehalten und abzuführen. Diese Art der Erfüllung öffentlicher Aufgaben gleicht den aus der

1) Vgl. G. Schmölders: Finanzpolitik, 3. Aufl,, Berlin, Heidelberg, New York 1970, S. 176.

2) Vgl. F.K. Mann: Die intermediären Finanzgewalten... a.a.0., S. 221 . 
griechischen Geschichte bekannten Leiturgien ${ }^{1)}$. Zum anderen zwingt der staat die Privaten in vielen Fällen dazu, unentgeltlich Leistungen zugunsten Dritter zu erbringen. Hierzu gehören die Lohnfortzahlung für Arbeitnehmer im Krankheitsfalle, die gesetzliche Auflage für Arbeitgeber, daB ein bestimmter Anteil ihrer Belegschaft aus Schwerbehinderten bestehen muB ${ }^{2}$, sowie bestimmte versteckte subventionen für einzelne Unternehmen bzw. Branchen wie Beimischungszwang oder Lizensierung ${ }^{3)}$.

Sowohl die Leistungen für den staat als auch diejenigen zugunsten Dritter könnte grundsätzlich auch der staat selbst mit entsprechenden Auswirkungen auf die Ausgabenseite seines Budgets erbringen ${ }^{4}$ ). Der staat bürdet den privaten Haushalten und Unternehmen mit der tbertragung von öffentlichen Aufgaben auch die Kosten der Aufgabenerfüllung auf. Die so verlagerten Ausgaben stellen Folgekosten staatlicher Aktivität dar, da hier private Aktivitäten unter staatlicher Direktive ablaufen ${ }^{5)}$. Insoweit kann in diesem zusammenhang von "Staatsaufgaben ohne staatsausgaben" gesprochen werden, die es in einer "unsicht-

1) Vgl. hierzu R. Nöll von der Nahmer: Lehrbuch der Finanzwissenschaft. Band I Allgemeine Finanzwissenschaft, Köln, Opladen 1964, S. 23 f. sowie H. Kolms: Finanzwissenschaft, Bd. I... a.a.O., s. $13 \mathrm{f}$.

2) Von beiden Maßnahmen ist der staat in seiner Eigenschaft als Arbeitgeber ebenfalls betroffen.

3) Vgl. N. Andel: Subventionen, in: Handwörterbuch der Wirtschaftswissenschaft (HdWW), hrsg. von W. Albers et al., Bd. 7, Stuttgart et al. 1977, S. 491-510, S. 492 .

4) $\mathrm{Ob}$ der staat oder Private eine Aufgabe effizienter erfüllen können, bleibe hier vorläufig offen. Diese Frage spielt eine wesentliche Rolle in der Debatte über die Privatisierung öffentlicher Leistungen. $\mathrm{Zu}$ diesem Problemkreis siehe vor allem M. Zumbühl: Privatisierung staatlicher Wirtschaftstätigkeit - Notwendigkeit und Möglichkeiten?, Zürich 1978 und die Bibliographie in H. Hanusch (Hrsg.): Reform öffentlicher Leistungen. Beiträge zur Entstaatlichung. Ein Kolloquium der Universität Augsburg in Memmingen, Baden-Baden 1978 , S. $127 \mathrm{ff}$.

5) Vgl. H. Zimmermann: Die Ausgabenintensität... a.a.O., S. 3 . 
baren Staatsquote" zu erfassen gelte ${ }^{1)}$. Beim staat selbst fallen neben den "Gemeinkosten" für die Erhaltung des Staatsapparats, der die Normen aufstellt, durchsetzt und ihre Einhaltung kontrolliert, Ausgaben für den Sold der Wehrpflichtigen oder bestimmte Vergütungen für (gezwungenermaßen) ehrenamtlich tätige Bürger an. Letztere Ausgaben sind jedoch nicht als Entgelt oder Entschädigung anzusehen oder sollen gar eine marktmäßige Entlohnung ersetzen, sondern besitzen schon von ihrer Höhe her eher Symbolcharakter. Daher sind die ins staatliche Budget eingehenden Ausgaben gering im Verhältnis zu den Kosten, die die privaten Entscheidungseinheiten zu tragen haben. Die hieraus resultierende fiskalische Entlastung für die öffentliche Hand dokumentiert sich auch in dem von verschiedenen Seiten vorgebrachten Vorschlag, der staat solle gezielt und verstärkt Aufgaben und damit Ausgaben auf private Träger verlagern, um mittelfristig finanzielle Handlungsspielräume zu gewinnen ${ }^{2)}$.

Empirische Untersuchungen zu diesem Problemkreis legen insbesondere die Unternehmerverbände vor. Eine jüngst erschienene Studie aus dem Institut der deutschen Wirtschaft beziffert die Kosten des privaten Sektors aufgrund des Lohnfortzahlungsgesetzes im Jahre 1980 auf 22,5 Mrd. DM und schätzt die sogenannte "Bürokratieüberwälzung" (sozialrechts-, steuer-, statistikund betriebsbedingter Verwaltungsaufwand) auf die privaten Unternehmen auf durchschnittlich 1,4 \& des Umsatzes, woraus sich

1) So C. Folkers: Staatswirtschaftliche Quoten und Beziehungszahlen, in: Das Wirtschaftsstudium (WiSu), Jg. 1979, S. 405-409 und S. 457-463, S. 406.

2) Vgl. Wissenschaftlicher Beirat beim Bundesministerium der Finanzen: Gutachten zur Lage und Entwicklung der Staatsfinanzen... a.a.O., S. 1010 f. sowie D. Ewringmann: Die Flexibilität öffentlicher Ausgaben. Eine Analyse der Restriktionen ausgabenpolitischer Handlungsspielräume, Göttingen 1975, S. $101 \mathrm{f}$. 
für 1980 ein Kostenvolumen von 42,8 Mrd. DM ergibt ${ }^{1)}$. Diese Zahlen enthalten allerdings nur Primäreffekte. Die Entlastung des Staates ist wesentlich geringer, da hierbei bereits die aus der Verlagerung von Ausgaben auf die privaten Unternehmen resultierenden steuermindereinnahmen nicht berücksichtigt sind.

Wie schon der Terminus "unsichtbare Staatsquote" andeutet, beeinfluBt die zwangsweise Utbertragung von öffentlichen Aufgaben auf private Entscheidungseinheiten zunächst einmal das Niveau der staatstätigkeit. Strukturelle verzerrungen treten dann auf, wenn derartige Verlagerungen ungleichmäßig über die verschiedenen staatlichen Aufgabenbereiche verteilt sind. Hierfür gibt es zumindest Anhaltspunkte. So dürften die Ausgaben im staatlichen Budget $f u ̈ r$ das Verkehrswesen oder für Innere Sicherheit die Aktivitäten in diesem Bereich besser widerspiegeln als die Ausgaben im Verteidigungshaushalt. Dies läßt vermuten, daß die Schwerpunkte der staatstätigkeit anders verteilt sind, als es in den auf funktional gegliederten staatsausgaben beruhenden Kennziffern zum Ausdruck kommt.

1.2.4 Aufgabenerfüllung über öffentliche Einnahmen

Im Budget finden finanzwirksame Maßnahmen nur insoweit zahlenmäBigen Ausdruck, als es sich bei Einnahmen und Ausgaben um positive Zahlungsströme handelt.Weder Ausgabensenkungen noch Einnahmenminderungen lassen sich aus einem einzelnen Budget erkennen, obwohl sie von Umfang und wirkung her eine wichtige Rolle spielen können ${ }^{2}$. Von besonderer Bedeutung für die Bestimmung

1) Vgl. R. Kroker: Der Staat als Wirtschaftsfaktor. Zur Aussagefähigkeit der staatsquote, Beiträge zur Wirtschafts- und Sozialpolitik, Bd. 93, Institut der deutschen Wirtschaft, Köln 1981 , S. $32 \mathrm{ff}$. Die erste $\mathrm{Zahl}$ schließt auch die von privaten Haushalten geleisteten Entgeltfortzahlungen ein. Vgl. in diesem Zusammenhang auch E. Hamer: Bürokratieüberwälzung auf die Wirtschaft. Analyse und Therapie, in: D. Dickertmann, H. König und G.W. Wittkämper (Hrsg.): Bürokratieüberwälzung. Stand, Ursachen, Folgen und Abbau, Regensburg 1982, S. 15-26.

2) Vgl. W. Kitterer: Das moderne Budget... a.a.0., S. $165 \mathrm{f}$. Man denke nur an stabilisierungspolitisch motivierte Variationen der Ausqaben und Einnahmenkh Ring-978-3-631-75590-7 
von Schwerpunkten der Staatstätigkeit ist die vollständige Vernachlässigung der öffentlichen Einnahmen. Da diese ebenfalls zur Erreichung öffentlicher Planungsziele eingesetzt werden können, vermittelt die Betrachtung nur der Ausgaben kein zutreffendes Bild ${ }^{1)}$. So ist es möglich, daß die Einkommensposition einer bestimmten Bevölkerungsgruppe durch Transferzahlungen schwerpunktartig gefördert wird, die steuerliche Belastung eben dieser Gruppe diese Maßnahmen jedoch wieder (teilweise) kompensiert ${ }^{2}$.

Neben den Einnahmen selbst spielen staatliche Einnahmenverzichte eine wesentliche Rolle bei der Erfüllung öffentlicher Aufgaben. Den größten Teil machen Steuervergünstigungen aus, die die öffentliche Hand Unternehmen und privaten Haushalten gewährt. Ihre Einsatzzwecke liegen in der Bundesrepublik auf den gleichen Gebieten wie diejenigen der Finanzhilfen, es handelt sich überwiegend um Ziele der Sozialpolitik und der Unternehmensförde$\operatorname{rung}^{3 \text { ) }}$.

Steuertechnisch erfolgt die Entlastung in Gestalt von Ausnahmen von der steuerpflicht, Sondertarifen, Abzügen von der Bemessungsgrundlage oder von der steuerschuld, die nicht aus dem wesen der jeweiligen steuer heraus erklärt werden können. Die Steuervergünstigungen, für die die angelsächsische Literatur die prägnante Bezeichnung "Tax Expenditures" prägte, besitzen fast ausschließlich den Charakter von Finanzhilfen. Von offenen Subventionen unterscheiden sie sich dadurch, daß sie nicht im Budget ausgewiesen werden ${ }^{4}$. In den meisten Fällen könnten sie durch

1) Vgl. E. Wille: Offentlicher Haushalt IV... a.a.0., S. 607.

2) Vgl. B.M. Blechmann et al.: Setting National Priorities. The 1976 Budget, a.a.O., S. 9 ff.

3) Vgl. H. Zimmermann: Instrumente der Finanzpolitik, in: Handbuch der Finanzwissenschaft, hrsg. von F. Neumark, Bd. 1, 3. Aufl., Tübingen 1977, S. 165-192, S. 182; Bundesregierung: Bericht... (Neunter Subventionsbericht)... a.a.0., S. 6 .

4) Vgl. F. Neumark: "Tax Expenditures", in: Finanzarchiv N.F., Bd. $33(1974 / 75)$, S. $139-142$, S. $139 \mathrm{ff}$. 
offene Ausgabenprogramme ersetzt werden. Der Tendenz nach erfolgte eine solche Umstellung im Jahre 1975 im Bereich des Familienlastenausgleichs, als die Kinderfreibeträge des Einkommensteuergesetzes durch die Zahlung von Kindergeld ersetzt wurden ${ }^{1}$ ).

Wesentlich schwieriger als bei Finanzhilfen gestaltet sich die Ermittlung der absoluten Höhe der "Tax Expenditures". Das erste Problem besteht darin, zu definieren, was als steuerliche Normalregelung anzusehen ist. "Diese Norm wird man jeweils in der Grundidee der einzelnen steuer und ihrer allgemeinen, für die überwiegende $\mathrm{Z}$ ahl der Steuerschuldner gültigen Ausgestaltung finden müssen..."2). Im nächsten schritt sind die als Subventionen $z u$ betrachtenden partiellen Abweichungen von dieser Norm $z u$ ermitteln. Hierbei dürfen Maßnahmen im Interesse einer Verwirklichung der Besteuerung nach der Leistungsfähigkeit oder verwaltungstechnische Differenzierungen nicht berücksichtigt werden. Merkmal des Subventionsbegriffs ist das privilegierende bzw. diskriminierende Element. Er umfaßt partielle Vergünstigungen für einige Unternehmen oder einen wirtschaftszweig, nicht aber allgemeine - beispielsweise konjunkturpolitisch motivierte Begünstigungen für alle Unternehmen oder privaten Haushalte ${ }^{3)}$. Bei der Quantifizierung der so eingegrenzten Abweichungen von der allgemeinen Norm schlieblich bedient sich die Analyse in der Regel zweier restriktiver Annahmen. Es wird unterstellt,

1) Mit dieser Umstellung waren allerdings geänderte Verteilungswirkungen verbunden.

2) N. Andel: Subventionen als Instrument des finanzwirtschaftlichen Interventionismus, Tübingen 1970, S. 6; vgl. auch S.S. Surrey: The Concept of Tax Reliefs. Its Relation to Tax Policy and Budget Policy, in: K. Häuser (Ed.): Subsidies, Tax Reliefs and Prices. Proceedings of the 33 rd Congress of the International Institute of Public Finance (Varna 1977), Paris 1981 , S. 35-55, S. $36 \mathrm{ff}$.

3) Vgl. N. Andel: Subventionen als Instrument... a.a.0., S. 6. Ähnlich F. Neumark: "Tax Expenditures", a.a.O., S. 140; W. Kitterer: Das moderne Budget... a.a.0., s. 167; Wissenschaftlicher Beirat beim Bundesministerium der Finanzen: Gutachten zum Begriff der öffentlichen Investitionen, a.a.o., S. 38 f.; S.S. Surrey: The Concept of Tax Reliefs... a.a.0., S. 37 . 
alle ökonomischen Aggregatgrößen blieben bei der Aufhebung der Vergünstigungen unverändert; zudem bleiben eventuell daraus resultierende Verhaltensänderungen der Steuerzahler auBer Betracht ${ }^{1)}$.

Der Neunte Subventionsbericht der Bundesregierung definiert "Steuervergünstigungen" im Einklang mit den oben genannten Merkmalen als "spezielle steuerliche Ausnahmeregelungen, die für die öffentliche Hand zu Mindereinnahmen führen"2), wobei Begünstigungen, die die (weit) überwiegende Mehrzahl der Steuerpflichtigen betreffen, (seit dem Sechsten Subventionsbericht) nicht (mehr) berücksichtigt werden ${ }^{3)}$. Diese Differenz zwischen "normaler" und tatsächlicher steuerbelastung betrug im Jahre 1982 rund 29,5 Mrd. DM, wovon 14 Mrd. DM auf den Bund entfielen. Im selben Jahr gewährte der Bund Finanzhilfen in Höhe von 13,2 Mrd. DM (einschlieBlich 1,5 Mrd. DM Darlehensgewährung) ${ }^{4}$ ).

Für die Bestimmung von Schwerpunkten der staatstätigkeit sind die Kritikpunkte besonders relevant, die sich gegen die mangelnde (politische) Transparenz dieser Form der versteckten Subvention richten. Aus der sicht der Begünstigten und ihrer Fürsprecher besteht der wesentliche Vorzug der steuervergünstigungen gegenuber offenen Subventionen in der mit der geringeren Merklichkeit sinkenden Gefahr, daß Vergünstigungen dem Rotstift zum Opfer fallen. Die Kontrolle ist hier schwächer, weil Steuervergünstigungen nicht automatisch Gegenstand der jährlichen

1) Vgl. S.S. Surrey: The Concept of Tax Reliefs... a.a.0., S.39.

2) Bundesregierung: Bericht... (Neunter Subventionsbericht)... a.a.0., S. 6 .

3) Vgl. Bundesregierung: Bericht... (Achter Subventionsbericht) ...a.a.0., S. 9. Die Einengung des Begriffs "Steuervergünstigungen" wurde mit einer Anpassung an den Begriff der Finanzhilfen begründet. Vgl. D. Albrecht: Subventionen. Problematik und Entwicklungen, Schriftenreihe des Bundesministeriums der Finanzen, Heft 25, Bonn 1978, S. 15 f.

4) Vgl. Bundesregierung: Bericht... (Neunter Subventionsbericht) ... a.a.O., S. $57 \mathrm{ff}$. und S. $179 \mathrm{ff}$. 
Haushaltsberatungen sind. Zudem unterschätzen Legislative und Exekutive möglicherweise den Vorteil aus der Begünstigung. Für die Regierung weisen Steuervergünstigungen ebenfalls mehrere Vorzüge auf: Ihre Erhöhungen tangieren weder die zuwachsrate des Budgetvolumens noch die in der tagespolitischen Diskussion dominierenden globalen Staatsquoten; die administrative Abwicklung kann über eine bereits bestehende stelle erfolgen, was die Gefahr eines von den Gegnern der Subvention vorgebrachten Vorwurfs der Aufblähung des Verwaltungsapparates verringert; und schließlich gestatten Vergünstigungen bei Gemeinschaftssteuern eine nahezu geräuschlose Beteiligung der Länder und der Gemeinden an der Finanzierung der Subvention ${ }^{1)}$. Selbst wenn die politischen Entscheidungsträger versuchen, für mehr Transparenz zu sorgen, indem sie die Höhe der steuervergünstigungen offenlegen, steht dem, wie ein Blick in die Subventionsberichte der Bundesregierung zeigt, in einigen Fällen die unzureichende Datenlage entgegen ${ }^{2}$. Ungeachtet dieser Lucken in der Berichterstattung ließe sich die Transparenz schon dadurch verbessern, daB die steuervergïnstigungen nicht (nur) in einem gesonderten und zudem nur alle zwei Jahre erscheinenden Subventionsbericht, sondern (auch) im Haushaltsplanentwurf offengelegt werden, wie es der US-amerikanische "Congressional Budget and Impoundment

1) Vgl. N. Andel: Subventionen, a.a.O., S. 498 sowie N. Andel: Subventionen als Instrument... a.a.O., S. 149 ff.; S.S. Surrey: The Concept of Tax Reliefs... a.a.O., S. 41 ff.

2) So zählt etwa der Neunte Subventionsbericht insgesamt 124 Steuervergünstigungen (in der engeren Begriffsfassung) gesondert auf. Bei 17 Begünstigungen davon heiBt es: "Bezifferung des Steuerausfalls wegen besonderer Schwierigkeiten kaum möglich", bei weiteren 3: "Genauere Berechnung des Steuerausfalls wegen unzureichender Schätzungsunterlagen kaum möglich, nach grober Schätzung jedoch steuerausfall unter 5 bis 10 Millionen DM" und in 11 Fällen erfolgt "Keine Angabe wegen Wahrung des Steuergeheimnisses". Vgl. Bundesregierung: Bericht ... (Neunter Subventionsbericht)... a.a.0., s. $179 \mathrm{ff}$. 
Control Act" von 1974 vorschreibt" und in Frankreich ansatzweise erfolgt ${ }^{2)}$.

Weitere Kritikpunkte, auf die hier nicht näher eingegangen werden soll, stellen auf die Minderung der Effizienz und die unnötige Komplizierung des steuersystems sowie die mangelnde Erfolgskontrolle bei dieser subventionsform ab ${ }^{3)}$.

Für die Bestimmung von Schwerpunkten der staatstätigkeit kommt es auf die absolute Höhe der bestehenden "Tax Expenditures" an. Sie können unter ökonomischen Aspekten in den verschiedenen Aufgabenbereichen neben den eigentlichen öffentlichen Ausgaben als indirekte monetäre Inputs mit einbezogen werden. "Materiell gesehen ist der Verzicht auf Einnahmen durchaus einer Ausgabe gleichzusetzen"4). Die Nichtberücksichtigung der steuervergünstigungen bei der Schwerpunktbestimmung läßt gravierende Verzerrungen befürchten, da sie ungleichmäßig über die einzelnen Aufgabenbereiche verteilt sind. Während die öffentliche Hand etwa in den Bereichen Bildungs- und Gesundheitswesen (auch) von der steuerlichen seite her in erheblichem Umfang Förderungen gewährt, fehlen solche Begünstigungen - mit einer geringfügigen Ausnahme - 5) im Verteidigungsbereich.

1) Vgl. N. Andel: Subventionen, a.a.O.., S. 506, J.A. Pechman (Ed.) : Setting National Priorities. The 1984 Budget, a.a.0., S. $243 \mathrm{ff}$.

2) Vgl. W. Kitterer: Das moderne Budget... a.a.0., S. $166 \mathrm{f}$.

3) Vgl. S.S. Surrey: The Concept of Tax Reliefs... a.a.O., S. $41 \mathrm{ff}$. Im Achten Subventionsbericht der Bundesregierung (a.a.o., s. $11 \mathrm{ff.})$ finden sich erstmals Uberlegungen $\mathrm{zu}$ einer Erfolgskontrolle.

4) K. Littmann: Definition und Entwicklung der Staatsquote, a.a.0., S. 104, ähnlich: Wissenschaftlicher Beirat beim Bundesministerium der Finanzen: Gutachten zum Begriff der öffentlichen Investitionen, a.a.0., S. $38 \mathrm{f}$.

5) Für privat erstellte Schutzräume können gemäB Abschnitt $42 b$ der Einkommensteuerrichtlinien erhöhte Abschreibungen in Anspruch genommen werden. 
1.2.5. Zur Ausgabenintensität der öffentlichen Aufgabenerfüllung

Wenngleich die Instrumente zur Erfüllung öffentlicher Aufgaben hier getrennt dargestellt wurden, treten in der Realität durch kombinierten Einsatz vielfältige Uberschneidungen auf. So gewähren nicht nur Gebietskörperschaften, sondern im staatlichen Auftrag auch bestimmte öffentliche Unternehmen wie die Landeskreditanstalten zinsgünstige Darlehen sowie Bürgschaften und Garantien ${ }^{1)}$, und die Subventionierung einer privaten Entscheidungseinheit auf direktem Wege sowie mittels steuervergünstigungen schließen sich nicht gegenseitig aus.

Auch von daher bietet sich für eine zusammenfassende würdigung der Wirkungen der beschriebenen Instrumente auf die Kennziffern zur Schwerpunktbestimmung ein Rückgriff auf das Konzept der Ausgabenintensität der öffentlichen Aufgabenerfüllung an. Dieses Konzept geht davon aus, daß dem Staat zur Erfüllung seiner Aufgaben eine Reihe von unterschiedlichen Instrumenten zur Verfügung steht, die man sich auf einer skala abgetragen vorstellen kann. Am einen Ende dieser Skala stehen rein finanzwirtschaftliche Instrumente, z.B. Transferzahlungen, die ohne Auflagen gewährt werden, das andere Ende bilden Normsetzungen, die im Budget nur als "Gemeinkosten" auftauchen. Zwischen diesen beiden Extremen liegen verschiedene Kombinationen von finanzwirtschaftlichen und normsetzenden Instrumenten. Je weniger finanzwirtschaftliche Elemente eine Maßnahme enthält, um so unvollkommener bildet das staatliche Budget sie $a^{2}{ }^{2}$.

1) Vgl. etwa D. Dickertmann: Öffentliche Finanzierungshilfen... a.a.O., S. $138 \mathrm{ff}$.

2) Vgl. H. Zimmermann: Die Ausgabenintensität... a.a.O., s. 7. 
Zimmermann definiert die Ausgabenintensität als "das Verhältnis der aufgewendeten öffentlichen Ausgaben zur gesamten Erfüllung einer einzelnen öffentlichen Aufgabe"1). Rein formal scheint es sich dabei um den Kehrwert der Produktivität des Staates zu handeln. Die Produktivität indes enthält im Nenner Inputs bzw. Kosten der Aufgabenerfüllung, und diese sind, wie bereits erwähnt, nur teilweise mit den budgetären Ausgaben identisch. Die Ausgabenintensität zeigt unter anderem deshalb nicht die gesamten Inputs bzw. Kosten zur Erfüllung einer öffentlichen Aufgabe an, weil der zähler dieser Relation lediglich die aufgewendeten staatsausgaben, nicht aber den nicht-staatlichen Entscheidungseinheiten aufgebürdeten Teil der Kosten enthält. tberdies gehen - anders als bei der Produktivität - in die Bezugsgröße sowohl der staatliche als auch der Beitrag nichtstaatlicher Entscheidungseinheiten zur Erfüllung einer Aufgabe ein.

Bei weiter Auslegung dieses Konzeptes lassen sich unterschiedlich hohe Ausgabenintensitäten in den verschiedenen öffentlichen Aufgabenbereichen auf dreierlei Ursachen zurückführen:

(1) Der staat verlagert mit Hilfe der Normsetzung Aufgaben und damit Ausgaben auf nicht-staatliche Entscheidungseinheiten. Je gröBer das (relative) Ausmaß der Verlagerung bzw. ihr Beitrag zur Erfüllung einer Aufgabe ist, um so niedriger ist tendenziell die Ausgabenintensität. Auf diesen Einflußfaktor scheint $\mathrm{z}$ immermann in seiner Argumentation primär abzustellen ${ }^{2)}$.

1) Ebenda, S. 8

2) Zimmermann (ebenda, S. 3) spricht ganz allgemein davon, "daß private Aktivität unter öffentlicher Direktive abläuft." 
(2) Selbst wenn der staat auf die Verlagerung von Aufgaben auf nicht-staatliche Entscheidungseinheiten verzichtet, stehen ihm Instrumente mit ganz unterschiedlichem Niederschlag im Budget zur Erreichung eines bestimmten Zwecks zur Verfügung, wie ein Blick auf die öffentlichen Finanzierungshilfen erhellt.

(3) Unabhängig von den beiden bisher genannten Einflußfaktoren können Unterschiede der Ausgabenintensität schließlich durch produktionstechnische Spezifika der Aufgabenbereiche bedingt sein. Hierbei handelt es sich um Produktivitätsdifferenzen, die etwa aus Unterschieden in der X-(In-) Effizienz oder aus den unterschiedlich hohen Anfälligkeiten der Aufgabenbereiche für den EinfluB exogener störgrößen resultieren können ${ }^{1)}$.

Die schwierigsten Probleme beim Konzept der Ausgabenintensität wirft die Messung der Erfüllung einer öffentlichen Aufgabe auf. $\mathrm{Z}$ immermann schlägt hierfür, da die Messung an den öffentlichen Ausgaben per se ausscheidet, drei Hilfsgrößen vor: Gewonnene Wählerstimmen; einen Index aus Größen wie Durchlaufzeit eines Gesetzes, $\mathrm{Zahl}$ der damit befaßten stellen und Häufigkeit der politischen Reaktionen der Opposition etc.; Vergleich von Rechtsnormen ${ }^{2)}$.

Die Hilfsgröße Wählerstimmen erscheint zwar im Prinzip als geeignetes MaB, da sie die Bewertung der staatlichen Leistungen durch deren Empfänger ausdrückt, dieser Vorschlag scheitert

1) Zum ursprünglich für den Bereich der privaten Unternehmen entwickelten Konzept der X-Effizienz siehe H. Leibenstein: Allocative Efficiency vs. "X-Efficiency", in: American Economic Review, Vol. 56 (1966), S. 392-415. Man könnte hier mit K. Gresser (Probleme... a.a.O., S. 82) von "spezifischen Kosten der Aufgabenerfüllung" sprechen. Gresser versteht allerdings darunter offenbar ganz allgemein die Ausgabenintensität.

2) Vgl. H. Zimmermann: Die Ausgabenintensität... a.a.0., S. 9. 
indes daran, daB sich stimmengewinne bzw. -verluste nur in den seltensten Fällen vollständig auf bestimmte Aktivitäten in den einzelnen staatlichen Aufgabenbereichen zurückführen und zurechnen lassen. Die beiden Meßgrößen Vergleich von Rechtsnormen sowie der Index aus Durchlaufzeit eines Gesetzes etc. repräsentieren dagegen Inputindikatoren. Sie können zwar signalisieren, wieviel Aufmerksamkeit und Bedeutung eine Aufgabe in der politischen Diskussion genieBt ${ }^{1}$, keinesfalls jedoch den Grad der Aufgabenerfüllung anzeigen. Im übrigen erscheint a priori zweifelhaft, ob ein Ansteigen der Zahl der Rechtsnormen eine Verbesserung darstellt oder, wenn die Normen der Kompensation bereits eingetretener bzw. der Verhinderung drohender unerwünschter Veränderungen dienen, sich deshalb insgesamt nicht eher als Ausdruck einer Verschlechterung der Lage in dem betroffenen Aufgabenbereich interpretieren $1 a ̈ B t$.

Ungeachtet der Schwächen des Konzeptes der Ausgabenintensität hinsichtlich der vorgeschlagenen Maße für die Aufgabenerfüllung deutet die vorangegangene Analyse in diesem Kapitel darauf hin, daß aus dem Einsatz der verschiedenen Instrumente - bei jeweils gleichem Grad der Aufgabenerfüllung - je nach Instrument unterschiedlich hohe Ausgaben resultieren und auch die Ausgabenintensitäten in den verschiedenen Aufgabenbereichen unterschiedliche Höhen aufweisen können; daher wollen wir es für eine zusammenfassende Würdigung nutzen. Bei der Analyse des Einflusses der Ausgabenintensität auf die Kennziffern zur Schwerpunktbestimmung muß unterschieden werden zwischen der Wirkung unterschiedlicher Niveaus der Ausgabenintensität in verschiedenen Aufgabenbereichen und der Wirkung von Veränderungen der Ausgabenintensität.

1) Zimmermann verwendet die Begriffe "Gewicht einer Aufgabe", "Bedeutung einer Aufgabe" sowie "Maß der Zielerreichung" synonym. Vgl. H. Zimmermann: Die Ausgabenintensität... a.a.0., s. 9 . 
Unter der entscheidungstheoretischen Fragestellung, d.h. bei der Identifizierung derjenigen Aufgabenbereiche, die die politischen Entscheidungsträger für die wichtigsten halten, können die absolute Höhe der Ausgaben in einem Aufgabenbereich (Kennziffer $1=\mathrm{K} 1$ ) oder deren Veränderung (K2) nur dann zur Kennzeichnung von Schwerpunkten dienen, wenn in den verschiedenen Aufgabenbereichen gleiche Niveaus der Ausgabenintensität gegeben sind. Diese Voraussetzung dürfte, wie der Uberblick über verschiedene staatliche Instrumente zur Erfüllung öffentlicher Aufgaben zeigt, in der Realität kaum erfüllt sein. Der gleiche Einwand gilt für das Kriterium Anteil an den Gesamtausgaben (K3) sowie, in abgemilderter Form, für die kombinierte Kennziffer 5. Die Höhe dieser vier Kennziffern kann ihren Grund sowohl im mehr oder minder großen Schwerpunktcharakter eines Aufgabenbereichs als auch im Niveau der Ausgabenintensität haben. Die genannten Kennziffern ermöglichen allenfalls, in einem intertemporalen Vergleich der Ausgaben eines einzelnen Aufgabenbereiches festzustellen, ob dieser im Lauf der zeit (absolut) an Bedeutung gewonnen oder verloren hat. Voraussetzung hierfür ist allerdings, $d a ß$ bei der betreffenden Aufgabe im Beobachtungszeitraum das Niveau der Ausgabenintensität unverändert bleibt.

Das Kriterium Zuwachsrate der Ausgaben für einen Aufgabenbereich (K4) scheint zunächst ein geeigneter Indikator für den Schwerpunktcharakter von Aufgaben zu sein, da unterschiedliche Niveaus der Ausgabenintensität in den verschiedenen Aufgabenbereichen sowohl im zähler als auch in der Bezugsgröße (Plafonds im Ausgangszeitpunkt) enthalten sind ${ }^{1)}$. Nach diesem Kriterium haben Aufgabenbereiche mit gleich hohen Zuwachsraten den gleichen Schwerpunktcharakter, unabhängig davon, ob sie eine hohe oder niedrige Ausgabenintensität aufweisen. Hier ist

1) Vgl. K. Gresser: Probleme der mehrjährigen öffentlichen Finanzplanung, a.a.O., S. 80 
jedoch eine wesentliche Einschränkung vorzunehmen. Die Kennziffer 4 erfaßt, wie auch die übrigen Kriterien, lediglich diejenigen staatlichen Aktivitäten zur Erfüllung öffentlicher Aufgaben mit einer positiven Ausgabenintensität, während sich etwa monetäre Inputs in Form von Steuervergünstigungen überhaupt nicht auswirken.

Damit eignet sich bei unterschiedlichen Niveaus der Ausgabenintensität in den verschiedenen Aufgabenbereichen keine der Kennziffern zur Bestimmung der Prioritätsvorstellungen der politischen Entscheidungsträger. Hiergegen läßt sich, betrachtet man speziell das Instrument der Verlagerung von Aufgaben auf nicht-staatliche Entscheidungseinheiten, einwenden, der Staat erfülle die Aufgaben, die die Politiker für wichtig halten, auch in eigener Regie, also ausgabenintensiv, und verlagere nur die weniger wichtigen. Demnach würden die Kennziffern die Prioritätsvorstellungen der Politiker tendenziell richtig wiedergeben. Dieses Argument kann jedoch nur für Bereiche Gültigkeit beanspruchen, in denen der Staat überhaupt die Möglichkeit der Verlagerung von Aufgaben auf nicht-staatliche Entscheidungseinheiten besitzt. In klassischen Bereichen wie Äußere und Innere Sicherheit oder Rechtspolitik steht ihm dieses Instrument kaum oder gar nicht zur Verfügung, während es etwa bei der Sozialen Sicherung eine große Variationsbreite aufweist. Eine vollständige Rangordnung läBt sich auf der Basis der ausgabenorientierten Kennziffern nur aufstellen, falls in allen Aufgabenbereichen dieselben Möglichkeiten der Verlagerung auf nicht-staatliche Entscheidungseinheiten bestehen.

Fragt man nach dem Einfluß von Veränderungen der Ausgabenintensität, so sind unter der entscheidungstheoretischen Fragestellung die Auswirkungen auf die Aussagefähigkeit der Kennziffern unklar. Erhöht sich die Ausgabenintensität in einem Aufgabenbereich, beispielsweise indem der Staat eine Aufgabe in eigene Regie übernimmt, die er bisher nicht-staatlichen Entscheidungseinheiten übertragen hatte, so weist der betref- 
fende Aufgabenbereich eine hohe Zuwachsrate auf, die übrigen Kennziffern reagieren entsprechend ${ }^{1)}$. Nun können die politischen Entscheidungsträger diese (Rück-) Verlagerung vornehmen, weil sie der betreffenden Aufgabe plötzlich erhöhte Bedeutung zumessen. Die Kennziffern würden dies zumindest in der Tendenz zutreffend signalisieren. Falls freilich die Politiker bei unveränderter Priorität der Aufgabe die (Rück-)Verlagerung deshalb veranlassen, weil die nicht-staatlichen Entscheidungseinheiten sie, etwa aufgrund gesunkener Produktivität, schlechter als bisher erfüllen, verleitet der Anstieg der Kennziffern zu falschen Schlüssen. Ohne zusatzinformationen eignen sich daher die Kennziffern bei sich verändernden Ausgabenintensitäten ebenfalls nicht zur Feststellung der zielvorstellungen der politischen Entscheidungsträger.

Durchgehend versagen die Kennziffern dagegen als Indikatoren von Schwerpunkten der staatstätigkeit, d.h. bei der Frage, in welchen Bereichen die meisten Aktivitäten, seien sie staatlich oder seitens des Staates veranlaßt, zur Erfüllung öffentlicher Aufgaben entfaltet werden. Für unterschiedliche Niveaus der Ausgabenintensität in den verschiedenen Aufgabenbereichen, also unter statischen Bedingungen, gelten analoge Argumente wie oben: Mit Ausnahme des Kriteriums Zuwachsrate bewältigt keine der Kennziffern das Problem der unterschiedlichen Gewichtung von Inputs zur Erfüllung öffentlicher Aufgaben im staatlichen Budget. Auch dieses Kriterium vernachlässigt indes Inputs, die sich in den staatsausgaben überhaupt nicht widerspiegeln (steuervergünstigungen). Veränderungen in der staatlichen Instrumentenwahl schlieblich, die in der Form der "Flucht aus dem Budget" große Aktualität besitzen, erweist

1) Dieser Effekt ist für die Schwerpunktbestimmung nur in dem unwahrscheinlichen Fall bedeutungslos, daß eine gleiche relative Erhöhung dex Ausgabenintensität gleichzeitig in allen Aufgabenbereichen erfolgt. 
sich keine der Kennziffern als gewachsen. Wenn der Staat eine bisher von ihm selbst übernommene Aufgabe auf nicht-staatliche Entscheidungseinheiten verlagert, signalisieren sämtliche Kennziffern fälschlicherweise ein Absinken der staatstätigkeit, obwohl lediglich staatliche durch nicht-staatliche Aktivitäten bzw. Inputs substituiert werden ${ }^{1)}$.

1.3. Verzerrungen durch exogene Störgrößen

1.3.1. Relativpreisveränderungen

Veränderungen der relativen Preise auf den Beschaffungsmärkten des Staates tangieren die staatlichen "Produktionsfunktionen" direkt auf der Inputseite. Die budgetären Ausgaben geben keine Auskunft daruber, ob sie zur Erweiterung sachlicher und personeller Kapazitäten oder lediglich zum Ausgleich von Preissteigerungen auf den Beschaffungsmärkten des Staates dienen. Weichen hier die preisänderungen von denjenigen auf den übrigen Märkten ab, so liefert die Ausgabenbetrachtung bei Deflationierung der Staatsausgaben und der Aggregate des privaten Sektors mit demselben Preisindex ein unzutreffendes Bild von der rea-

1) Bei gleicher Produktivität des Staates und der nicht-staatlichen Entscheidungseinheiten bleibt sogar das Leistungsangebot in dem betreffenden Aufgabenbereich konstant. 
len gesamtwirtschaftlichen Allokation ${ }^{1)}$. Ein derartiger Niveaueffekt muB jedoch noch keine Auswirkungen auf die struktur der Staatstätigkeit haben. Verzerrungen innerhalb des öffentlichen Sektors treten erst dann auf, wenn die Preisentwicklungen auf den staatlichen Beschaffungsmärkten die verschiedenen öffentlichen Aufgabenbereiche in unterschiedlichem Ausmaß treffen. Hieraus resultieren unterschiedliche Verhältnisse der monetären zu den sachlichen und personellen Inputs in den verschiedenen Aufgabenbereichen ${ }^{2)}$. Hinter einem gleich hohen Ausgabenzuwachs in verschiedenen Aufgabenbereichen kann sich je nach Richtung und AusmaB der Preisänderungen ein steigendes, konstantes oder sinkendes Volumen an sachlichen und personellen

1) Zur Preisbereinigung im öffentlichen Sektor vgl. etwa K. Littmann: Definition und Entwicklung der staatsquote, a.a.O., S. $133 \mathrm{ff.;} \mathrm{B.} \mathrm{Görzig:} \mathrm{Die} \mathrm{Verteilungswirkungen}$ der Inflation auf den privaten und öffentlichen sektor. Ermittlung von Inflationswirkungen nach alternativen Berechnungsansätzen unter besonderer Berücksichtigung des Problems der Produktivitätsmessung im öffentlichen sektor, Göttingen 1975; B. Felderer: Die reale Staatsquote, in: Finanzarchiv N.F., Bd. 35 (1976), S. 405-434; D. Fricke: Verteilungswirkungen der Inflation, Baden-Baden 1981, S. $207 \mathrm{ff.;} \mathrm{E.} \mathrm{Wille:} \mathrm{Öffentliche} \mathrm{Budgets} \mathrm{im} \mathrm{Inflations-}$ prozeB, in: Das Wirtschaftsstudium (WiSu), Jg. 1982, S. 197-202 und S. 249-255, insbesondere S. 250 ff.; M. Klein: Deflationierung der staatlichen Leistungen und Preisentwicklungen im öffentlichen Sektor, in: E. Wille (Hrsg.): Konzeptionelle Probleme öffentlicher Planung, Frankfurt et al. 1983, S. 85-126; H. -W. Sinn: Die Inflationsgewinne des Staates, in: E. Wille (Hrsg.): Beiträge zur gesamtwirtschaftlichen Allokation. Allokationsprobleme im intermediären Bereich zwischen öffentlichem und privatem Wirtschaftssektor, Frankfurt et al. 1983, S. 111-166, S. $153 \mathrm{ff}$.

2) Falls beim Staat keine kompensierenden Produktivitätssteigerungen auftreten, erhöhen steigerungen seiner Beschaffungsmarktpreise grundsätzlich auch die Ausgabenintensität der öffentlichen Aufgabenerfüllung, da immer höhere öffentliche Ausgaben nötig sind, um ein bestimmtes Leistungsniveau aufrechtzuerhalten. Ähnlich H. Zimmermann: Die Ausgabenintensität... a.a.0., s. 6 . 
Inputs verbergen ${ }^{1)}$.

Unter entscheidungstheoretischen Gesichtspunkten hängt die Aussagekraft der Kennziffern, läßt man alle anderen Einf lußfaktoren beiseite, davon $a b$, ob die politischen Entscheidungsträger bei ihren Budgetberatungen die Preisänderungen berücksichtigen oder nicht. Antizipieren sie die Preisänderungen nicht, so geben im Prinzip die aufgrund der nominalen staatsausgaben errechneten Kennziffern die richtige Ausgangsbasis für die Ermittlung ihrer zielvorstellungen $a b$. Da die Politiker in diesem Falle ihre Prioritäten ausschließlich auf der Ebene der Staatsausgaben setzen, ist diese Vorgehensweise unter entscheidungstheoretischen Aspekten sinnvoll, bezüglich der Schwerpunkte der faktischen staatstätigkeit dagegen führt sie zu falschen Ergebnissen. Bereits auf der Ebene der sachlichen und personellen Inputs wird bei unterschiedlichen Preisänderungen in den verschiedenen Aufgabenbereichen die tatsächlich realisierte struktur der staatlichen Aktivitäten von derjenigen struktur abweichen, die die Politiker mit ihren Budgetansätzen verwirklichen wollen. Für den Nachweis der Schwerpunkte der staatstätigkeit müßte man daher auf Kennziffernreihen zurückgreifen, die auf der Basis von mittels spezifischer Deflatoren preisbereinigten staatsausgaben errechnet wurden ${ }^{2)}$. Hieraus muß nicht notwendigerweise auch eine veränderte Rangordnung resultieren, zumindest aber ändern sich die Abstände zwischen den Aufgabenbereichen in einer Kennziffernreihe.

Antizipieren die politischen Entscheidungsträger hingegen die Preisänderungen, so sind zur Bestimmung ihrer zielvorstellungen

1) Der staat beeinfluBt allerdings - ob gewollt oder nicht - sowohl über die Ausgaben- als auch über die Einnahmenseite seines Budgets die Preisentwicklung auf seinen Beschaffungsmärkten; insofern handelt es sich bei diesen Relativpreisveränderungen nicht um eine rein exogene störgröße. Vgl. auch E. Wille: రffentliche Budgets im InflationsprozeB, a.a.O., S. 254 f.; M. Klein: Deflationierung der staatlichen Leistungen... a.a.0., S. 120.

2) Mit der Ermittlung staatlichen Outputs hat diese Preisbereinigung nichts zu tun. Es handelt sich lediglich um eine Deflationierung von nominalen Inputgrößen. 
ebenfalls Kennziffernreihen auf der Basis preisbereinigter Budgetansätze erforderlich, da eine auf nominalen staatsausgaben beruhende Kennziffernreihe falsche Aussagen liefern würde. Falls die Politiker die unterschiedlichen Preisniveauveränderungen in den verschiedenen Aufgabenbereichen gerade kompensieren, bleibt die geplante Rangfolge der Aufgaben konstant, während die unbereinigten Kennziffern eine gegenüber dem Vorjahr (zumindest in den Abständen) geänderte Rangordnung anzeigen. Dabei kommt es unter entscheidungstheoretischen Aspekten nicht darauf an, ob die Politiker die Preisänderungen richtig oder fehlerhaft antizipieren; relevant für die Bereinigung sind vielmehr die von ihnen erwarteten Veränderungsraten. Für die Kennzeichnung der staatstätigkeit im Sinne der struktur der Aktivitäten bzw. (realen) Inputs zur Erfüllung öffentlicher Aufgaben andererseits müßte man auf die um die tatsächlichen Preisänderungen bereinigten staatsausgaben rekurrieren.

\subsubsection{Bevölkerungsentwicklung}

Veränderungen der Bevölkerungsgröße und -struktur beeinflussen sowohl die Entscheidungen iber die Staatsausgaben als auch die outputseite des staatlichen Leistungserstellungsprozesses. Eine Zunahme der Bevölkerung kann beispielsweise in höheren staatlichen Aufwendungen für die Wohnungsbauförderung resultieren, ohne daB sich hierdurch die Pro-Kopf-Versorgung verbessern mu. Veränderungen im Altersaufbau der Bevölkerung können einerseits zu einem höheren Bedarf an Altenheimplätzen, andererseits zu niedrigeren Kindergeldzahlungen und geringeren Ausgaben im Bildungsbereich führen. In diesen Fällen läßt sich die individuelle Versorgung auch bei konstantem oder gar sinkendem Ausgabenvolumen verbessern, indem der Staat Kindergeldsätze und LehrerSchüler-Relationen anhebt bzw. ansteigen läßt ${ }^{1)}$.

1) Vgl. E. Wille: Offentlicher Haushalt IV... a.a.0., S. 607 und Karl-Bräuer-Institut des Bundes der Steuerzahler (Hrsg.): Bevölkerungsentwicklung und Staatsausgaben, Schriften des KarlBräuer-Instituts des Bundes der Steuerzahler, Heft 43 , Wiesbaden 1979 , S. $18 \mathrm{ff}$. 
Der Zusammenhang zwischen Veränderungen der Bevölkerungsgröße oder -struktur und Veränderungen bestimmter staatsausgaben besteht allerdings nicht immer in so strenger Form. Je mehr eine staatliche Leistung den Charakter eines reinen öffentlichen Gutes hat, desto weniger muB der staat auf Veränderungen der Bevölkerung reagieren, will er die Versorgung auf konstantem Niveau halten. Dies dürfte etwa bei der Äußeren Sicherheit der Fall sein. Bei Leistungen, die eher den Charakter eines privaten Gutes aufweisen, führt eine erhöhte Nachfrage nur zu unterproportional zunehmenden öffentlichen Ausgaben, solange freie Kapazitäten, beispielsweise freie Altenheimplätze, bestehen ${ }^{1)}$. Diese Umstände deuten darauf hin, daß Bevölkerungsveränderungen die verschiedenen öffentlichen Aufgabenbereiche in unterschiedlichem Ausmaß beeinflussen.

Relativ wenig Probleme im Hinblick auf die Bestimmung der zielvorstellungen der politischen Entscheidungsträger sowie der Schwerpunkte der staatstätigkeit bereiten Veränderungen der Bevölkerung, solange die Politiker nicht deren Richtung entgegengesetzt handeln. Steigen beispielsweise die Bevölkerungsgröße und die Subventionen für den Wohnungsbau an, so signalisieren die Kennziffern einen höheren Rang dieser Aufgabe. Dies entspricht im Prinzip auch den veränderten zielvorstellungen der politischen Entscheidungsträger, denn der Umstand, daß sie nicht den Kreis der Anspruchsberechtigten einschränken oder/und die Förderungssätze senken, ist ein Indiz für die erhöhte Priorität der betreffenden Aufgabe. Entsprechendes gilt auch für den Nachweis von Schwerpunkten der Staatstätigkeit. In beiden Fällen signalisieren die Kennziffern zwar nur der Tendenz, nicht aber notwendigerweise auch dem Umfang nach zutreffend, da sie die zunahme der gerade im Wohnungsbau wichtigen Förderung auf dem Wege von Steuervergünstigungen nicht erfassen; dies ist jedoch kein spezifisches Problem der Bevölkerungsentwicklung.

1) Vgl. C.V. Brown und P.M. Jackson: Public Sector Economics, a.a.o., s. $110 \mathrm{f}$. 
Zu anderen Ergebnissen gelangt man in bestimmten Extremfällen, wenn die Politiker den Trends bei der Bevölkerungsentwicklung entgegenzuwirken versuchen. Erhöhen sie etwa aus Sorge über eine sinkende Geburtenrate "Gebärprämien" und ähnliche finanzielle Anreize, so läßt dies auf gestiegene priorität schliessen. Gleichwohl können die Aufwendungen für diesen Zweck konstant bleiben oder gar weiterhin absinken, so daB die Kennziffern falsche Signale bezüglich der Zielvorstellungen der Politiker geben. Die relative Bedeutung der staatstätigkeit in diesem Bereich hingegen spiegeln sie zutreffend wider.

Ähnliche Divergenzen lassen sich feststellen, wenn man die staatlichen Outputs pro Kopf der Bevölkerung betrachtet oder die politischen Entscheidungsträger sich an diesen Größen orientieren. Die ausgaben- und somit inputorientierten Kennziffern erlauben zwar ohnehin kaum Aussagen über staatliche Outputs, bei bestimmten oben diskutierten Entwicklungen der Bevölkerung jedoch müssen Inputs und Outputs pro Kopf bzw. pro Leistungsempfänger in einem Aufgabenbereich sich nicht einmal in dieselbe Richtung entwickeln. Es ist nicht nur möglich, daß die ausgabenorientierten Kennziffern auf Veränderungen der Leistungen pro Kopf überhaupt nicht reagieren, teilweise signalisieren sie sogar Entwicklungen bei den (monetären) Inputs, die denen beim Leistungsangebot pro Kopf und damit beim Versorgungsniveau genau entgegengesetzt verlaufen. Die Eignung der Kennziffern als Indikatoren der Staatstätigkeit beeinträchtigt dies zwar nicht, denn für diesen Zweck kommt es auf die tatsächliche Allokation der Inputs an, wohl aber ihre Aussagefähigkeit bezüglich der zielvorstellungen der politischen Entscheidungsträger, falls diese das Versorgungsniveau der Bevölkerung als Kriterium verwenden. 
2. Budgetäre Ausgabenschwerpunkte und Angebot an öffentlichen Gütern

Erweitert man im zweiten Schritt den Blickwinkel von der staatstätigkeit auf die in einer Volkswirtschaft insgesamt entfalteten Aktivitäten zur Erstellung bzw. Bereitstellung öffentlicher Güter $^{1)}$, so rücken als zusätzliche Komponente die freiwilligen Aktivitäten nicht-staatlicher Entscheidungseinheiten in den Brennpunkt des Interesses. Es handelt sich um die Erfüllung von öffentlichen Aufgaben durch die Kirchen einschließlich ihrer Hilfswerke, freie Wohlfahrtsverbände, private wissenschaftliche und kulturelle Vereinigungen sowie private Unternehmen aus eigener Initiative. Die zuerst genannten karitativen Organisationen stellen einen großen Teil der sozialen Infrastruktur (Kindergärten, Schulen, Altersheime, Einrichtungen der Gesundheitsfürsorge und -vorsorge in freier Trägerschaft) bereit, darüber hinaus erbringen sie Leistungen auf dem Gebiet der Entwicklungshil$\mathrm{fe}^{2)}$. Private Unternehmen beteiligen sich freiwillig an der Erfüllung öffentlicher Aufgaben beispielsweise auf dem Bildungssektor (Privatschulen) und im Gesundheitswesen (Sanatorien,

1) Unter "öffentlichen Gütern" wollen wir hier nicht reine öffentliche Güter im technischen Sinne verstehen, sondern sämtliche Leistungen, die der Erfüllung öffentlicher Aufgaben dienen. (Siehe dazu auch weiter unten in diesem $\mathrm{Ab}-$ schnitt.) Deshalb bleiben hier auch diejenigen möglicherweise von nicht-staatlichen Entscheidungseinheiten bereitgestellten öffentlichen Güter im technischen Sinne aus der Betrachtung ausgeschlossen, die keine öffentliche Aufgabe betreffen. Zur Bestimmung öffentlicher Aufgaben siehe Abschnitt 3.2.1. in diesem Kapitel.

2) Vgl. K. Littmann: Definition und Entwicklung der Staatsquote... a.a.O., S. $17 \mathrm{ff.} \mathrm{und} \mathrm{S.} 99 \mathrm{ff.;}$ Wissenschaftlicher Beirat beim Bundesministerium der Finanzen: Gutachten zur Aussagefähigkeit staatswirtschaftlicher Quoten, a.a.0., S. 852. Úber Ziele und Aktivitäten ausgewählter karitativer Organisationen berichtet $\mathrm{R}$. Bauer: Wohlfahrtsverbände in der Bundesrepublik. Materialien und Analysen zu Organisation, Programmatik und Praxis. Ein Handb'ch, Weinheim, Basel 1978, S. $117 \mathrm{ff.;}$ teilweise auch W. Kirberger: Staatsentlastung durch private Verbände... a.a.O., S. $117 \mathrm{ff}$. 
Werkskrankenstationen, firmeneigene Erholungsheime u.ä.) . Letztlich ließe sich auch die Einbeziehung bestimmter freiwillig entfalteter Aktivitäten der privaten Haushalte rechtfertigen, soweit von ihnen Einflüsse auf staatliche planungsiele ausgehen. So kann etwa ein erhöhtes GesundheitsbewuBtsein der Bevölkerung $z u$ einer steigenden Nachfrage nach Gesundheitsleistungen wie Vorsorgeuntersuchungen und $\mathrm{zu}$ einer Einschränkung gesundheitsschädlicher Verhaltensweisen führen, wodurch ceteris paribus das Gesundheitsniveau steigen wirde. Eine Erfassung derartiger Aktivitäten scheitert jedoch, vor allem in der Realität, an unüberwindbaren Abgrenzungsproblemen.

Obgleich diese Aktivitäten nicht-staatlicher Entscheidungseinheiten sich auf die Erfüllung öffentlicher Aufgaben richten, können ihre Leistungen verschiedene Gütereigenschaften aufweisen. Aktivitäten mit distributionspolitischer zielsetzung, vor allem die Sachleistungen im Rahmen der Entwicklungshilfe, konzentrieren sich notwendigerweise auf Güter, die die Möglichkeit des Ausschlusses bieten. Auf welcher Seite des Spektrums von reinen öffentlichen bis hin zu reinen privaten Gütern ${ }^{1}{ }^{1}$ hingegen das allokationspolitisch motivierte Leistungsangebot der nicht-staatlichen Entscheidungseinheiten angesiedelt ist, steht in engem Zusammenhang mit ihren Finanzierungsmöglichkeiten. Organisationen, die bei der Erfüllung öffentlicher Aufgaben primär auf die Finanzierung aus Entgelt angewiesen sind, werden deshalb tendenziell private Güter bereitstellen. Der Umstand, daß Privatschulen und Sanatorien, soweit private Unternehmen sie betreiben, in der Regel ihre Leistungen am Markt anbieten und Gewinne erzielen wollen, steht jedoch der Qualifikation als Maßnahmen zur Erfüllung öffentlicher Aufgaben nicht entgegen. Es handelt sich hier zwar - ebenso wie bei vielen Entwicklungshilfeleistungen - nicht um die Bereitstellung von reinen öffent-

1) Vgl. zu diesem Spektrum etwa H. Hanusch: Theorie des öffentlichen Gutes. Allokative und distributive Aspekte, Göttingen 1972 , S. 86 ff. 
lichen Gütern (social goods) im Sinne der Theorie der öffentlichen Güter, wohl aber um Aktivitäten, die in praxi auf das Angebot meritorischer Güter abzielen und insofern staatlichen Aktivitäten vergleichbar sind. Demgegenüber sind Organisationen ohne Erwerbscharakter, die sich weitgehend aus Beiträgen freiwilliger Mitglieder, Spenden und staatlichen zuschüssen refinanzieren, in der Lage, Leistungen bereitzustellen, welche sich verstärkt durch Merkmale eines social good auszeichnen ${ }^{1)}$. Diese theoretischen Uberlegungen erfahren in empirischer Hinsicht Unterstützung durch von B.A. Weisbrod durchgeführte bzw. angeregte Untersuchungen. Es handelt sich dabei um typische Aufgabenbereiche, in denen der staat, private organisationen ohne Erwerbscharakter und private Unternehmen tätig sind (Schul- und Krankenhauswesen). Die studien kommen zu dem Ergebnis, daB das Leistungsprogramm der privaten Unternehmen deutlich mehr tendenziell private Güter enthält als das der anderen Anbieter, während sich die Leistungsprogramme des staates und der privaten Organisationen ohne Erwerbscharakter insofern gleichen, als beide relativ viele Leistungen mit Kollektivguteigenschaften enthalten ${ }^{2)}$.

1) Zu den Interdependenzen zwischen Gütereigenschaften des Leistungsangebots privater Organisationen ohne Erwerbscharakter und dessen Finanzierung siehe vor allem U. Schmoltzi: Zur Finanzierung privater Organisationen ohne Erwerbscharakter... a.a.O., S. 73 ff.; H.H. Nachtkamp: Idealtypen von Fiskalsystemen. Ein Versuch, in: K. Häuser (Hrsg.): Finanzsysteme: Ideal- und Realtypen - Gesundheitswesen und Hochschulbildung, Schriften des Vereins für Socialpolitik N.F., Bd. 135, Berlin 1983, S. 11-55, S. $41 \mathrm{ff.;} \mathrm{W.J.} \mathrm{van} \mathrm{Braband:} \mathrm{General} \mathrm{Survey}$ of Subsidizing Non-Profit Institutions, in: K. Häuser (Ed.): Subsidies, Tax Reliefs and Prices. Proceedings of the 33rd Congress of the International Institute of Public Finance (Varna 1977), Paris 1981, S. 133-149.

2) Vgl. B.A. Weisbrod: Some Collective-Good Aspects of NonGovernment Activities: Not-for-Profit Organizations, in: H.C. Recktenwald (Ed.): Secular Trends of the Public Sector. Proceedings of the 32 nd Congress of the International Institute of Public Finance (Edimbourg 1976), Paris 1978, S. 163-174; A.J. Lee und B.A. Weisbrod: Collective Goods and the Voluntary Sector: The Case of the Hospital Industry, in: B.A. Weisbrod: The Voluntary Nonprofit Sector. An Economic Analysis, Lexington 1977, S. 77-100; M. Bendick Jr.: Education as a Three-Sector-Industry, in: B.A. Weisbrod: The

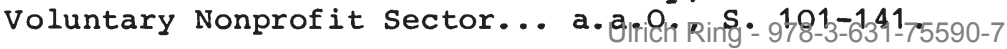


Wenngleich diese Argumente und Ergebnisse empirischer Untersuchungen Ahnlichkeiten in qualitativer Hinsicht zwischen den Leistungsprogrammen des staates und der nicht-staatlichen Entscheidungseinheiten erwarten lassen, erlauben sie nicht den SchluB auf entsprechende Affinitäten in quantitativer Hinsicht. Die nicht-staatlichen Entscheidungseinheiten können die Schwerpunkte ihrer aus eigener Initiative entfalteten Aktivitäten in anderen öffentlichen Aufgabenbereichen setzen als der Staat. Für ein derartiges Verhalten spricht bereits die Motivation potentieller Gründer und Förderer vor allem von privaten Organisationen ohne Erwerbscharakter. Eine notwendige Bedingung für die Entfaltung von Aktivitäten solcher Organisationen besteht darin, daß ihre Initiatoren das staatliche Angebot in einem Aufgabenbereich für zu gering oder für qualitativ unzureichend halten ${ }^{1)}$; sie werden daher andere ziel- bzw. Prioritätsvorstellungen verfolgen als der staat.

Auch ohne eine umfassende empirische Analyse der Erfüllung öffentlicher Aufgaben durch nicht-staatliche Entscheidungseinheiten lassen sich zumindest Indizien für eine von der des staates abweichende struktur ihrer Aktivitäten finden. So dürften die freiwilligen Aktivitäten nicht-staatlicher Entscheidungseinheiten im Gesundheits - und Bildungswesen sowie bei der Entwicklungshilfe eine erheblich größere Rolle spielen als etwa im Verkehrswesen. Solche Abweichungen in den Angebotsstrukturen des Staates und der nicht-staatlichen Entscheidungseinheiten existieren nicht nur zwischen den öffentlichen Aufgabenbereichen, sondern auch innerhalb der Bereiche, in denen beide Anbieter tätig sind. Im Bereich des Bildungswesens etwa existieren neben den staatlichen Schulen und Hochschulen zwar eine

1) Vgl. in diesem Zusammenhang auch die Definition des Kollektivgutes bei P.O. Steiner: Public Expenditure Budgeting, in: A.S. Blinder et al.: The Economics of Public Finance. Studies of Government Finance, Washington D.C. 1974, S. 241-357, S. $244 \mathrm{ff}$. Sowie die tberlegungen von U. Schmoltzi: Zur Finanzierung privater Organisationen ohne Erwerbscharakter... a.a.0., S. 67 ff. 
Reihe von Schulen in freier, bisher jedoch nur wenige Universitäten in privater Trägerschaft ${ }^{1)}$. Während hier staatliche Aktivitäten dominieren, liegen die Verhältnisse in einem Teilbereich des Gesundheitswesens umgekehrt. Als Anbieter von Krankentransportleistungen treten fast ausschlieBlich private Organisationen auf ${ }^{2)}$.

Der staat nimmt auf die aus eigener Initiative entfalteten Aktivitäten nicht-staatlicher Entscheidungseinheiten zur Erfüllung öffentlicher Aufgaben zwar keinen unmittelbaren Einfluß, insofern handelt es sich hier nicht um eine Form von staatstätigkeit, er fördert sie jedoch - mit entsprechenden Niederschlägen im Budget - in ganz unterschiedlicher Weise. Die Palette reicht von der Ubernahme der Investitionsausgaben freier Träger für Krankenhausneubauten in voller Höhe, sofern diese in den Krankenhausbedarfsplan eines Bundeslandes aufgenommen sind ${ }^{3)}$, bis hin zum Fehlen jeglicher staatlicher Förderung bei bestimmten freiwilligen Aktivitäten privater Unternehmen ${ }^{4)}$.

1) Erwähnt seien hier die Katholische Universität Eichstätt, die Hochschule für jüdische studien, Heidelberg, sowie die Universität Witten-Herdecke.

2) Der Staat unterhält entsprechende Dienste beispielsweise bei der Bundeswehr.

3) Zur Krankenhausfinanzierung in der Bundesrepublik vgl. S. Eichhorn: Preisbildung im Krankenhaus nach dem Kostendeckungsprinzip, in: Krankenhäuser als Unternehmen, Beiheft 2 (1979) der Zeitschrift für öffentliche und gemeinwirtschaftliche Unternehmen, Baden-Baden 1979, S. 39-55, S. $42 \mathrm{ff.;}$. Sauerzapf : Das Krankenhauswesen in der Bundesrepublik Deutschland. Institutionelle Regelungen aus ökonomischer Sicht, Baden-Baden 1980, S. $112 \mathrm{ff.;} \mathrm{J}$. Wiemeyer: Krankenhausfinanzierung und Krankenhausplanung in der Bundesrepublik Deutschland, Berlin 1984, s. $78 \mathrm{ff}$.

4) Die entsprechenden Kosten etwa einer Werkskrankenstation kann das Unternehmen zwar wie die Aufwendungen für die eigentlichen betrieblichen Zwecke im Rahmen der Ertragsbesteuerung als Betriebsausgaben geltend machen, hierbei handelt es sich jedoch nicht um eine Förderung durch den staat, da das privilegierende Element fehlt. 
Darüber hinaus erlangten die karitativen Organisationen in der Regel steuerrechtlich den Status der Gemeinnützigkeit ${ }^{1)}$. Dies hat einerseits die Befreiung von den wichtigsten Steuern, andererseits die Abzugsfähigkeit von spenden bei den Spendern zur Folge, so daß auch hier die Problematik der "Tax Expenditures" zum Tragen kommt.

Abschließend wollen wir prüfen, wie sich die freiwilligen Aktivitäten nicht-staatlicher Entscheidungseinheiten zur Erfüllung öffentlicher Aufgaben auf die Aussagefähigkeit der Kennziffern zur Schwerpunktbestimmung auf der Basis der budgetären Ausgaben auswirken. Diese Aktivitäten lassen sich, wie bereits erwähnt, nicht unter die staatstätigkeit subsumieren. Wenn die Kennziffern sie nicht in vollem Umfang widerspiegeln, können wir ihnen deshalb kein Versagen vorwerfen, da dies nicht zu ihrer Zweckbestimmung gehört. "Haften" müssen sie indes insoweit, als der staat diese Aktivitäten unterstützt.

Trotz ihrer beschränkten Reichweite eignen sich die Kennziffern zunächst relativ gut zur Bestimmung der Prioritätsvorstellungen der politischen Entscheidungsträger, falls diese den nichtstaatlichen Entscheidungseinheiten finanzielle Unterstützung nur für jene Aktivitäten gewähren, die sie für wichtig halten. Hierbei handelt es sich allerdings lediglich um einen möglichen Extremfall; die Aussagekraft der Kennziffern läßt sich bereits relativieren, wenn es den politischen Entscheidungsträgern gleichgültig ist, ob der staat selbst oder nicht-staatliche Entscheidungseinheiten eine Aufgabe erfüllen. In diesem Falle würden die Politiker die Aktivitäten nicht-staatlicher Entscheidungseinheiten bei der Planung der staatlichen Aktivitäten berücksichtigen und letztere im Hinblick auf Umfang und Struk-

1) Die grundsätzlichen steuerrechtlichen Regelungen finden sich seit dem 1.1.1977 in den $\$ \$ 51$ ff.der Abgabenordnung. Zu diesem Zeitpunkt verloren die Regelungen der Gemeinnützigkeits-Verordnung und des Steueranpassungsgesetzes ihre Gültigkeit. 
tur anpassen. Entfaltet der Staat in einem Aufgabenbereich nur wenige Aktivitäten, so kann dies auf den geringen stellenwert der Aufgabe zurückzuführen sein. Sie kann jedoch auch hohe Priorität genießen, wobei indes nicht-staatliche Entscheidungseinheiten für ein aus der Sicht der politischen Entscheidungsträger quantitativ und qualitativ ausreichendes Leistungsangebot sorgen. Die staatlichen Zuschüsse allein würden dann zur Bestimmung der zielvorstellungen der Politiker nicht ausreichen. Im anderen Extremfall schlieblich übernehmen die politischen Entscheidungsträger Aufgaben, die sie für wichtig halten, auch in staatliche Regie. Bereits der Umstand, daß der staat eine Aufgabe nicht-staatlichen Entscheidungseinheiten überläßt, würde dann auf den geringen stellenwert dieser Aufgabe hindeuten ${ }^{1)}$.

Unabhängig von der Frage der Einstellung der politischen Entscheidungsträger gegenüber den nicht-staatlichen Entscheidungseinheiten erleidet die Aussagekraft der Kennziffern bezüglich der Prioritätsvorstellungen der Politiker wesentliche Einbußen, sobald der staat den konventionellen Weg der Förderung mittels Zuschüssen verläßt. Die Subventionierung der nicht-staatlichen Entscheidungseinheiten durch Steuervergünstigungen, Käufe von Vorleistungen vom Staat zu herabgesetzten Preisen $u . \ddot{a}$. findet in den ausgabenorientierten Kennziffern keinen Widerhall und sorgt insoweit für Verzerrungen.

Derselbe Einwand richtet sich gegen die Eignung der Kennziffern als Ausdruck der Struktur der Staatstätigkeit, da diese nicht nur die direkten, sondern auch sämtliche indirekten bzw. verdeckten Subventionen zugunsten nicht-staatlicher Entscheidungseinheiten umfaßt.

1) Insofern wäre auch zu fragen, ob die von Kirberger definierte "Entlastungsintensität" tatsächlich eine Entlastung des Staates durch die Aktivitäten nicht-staatlicher Entscheidungseinheiten in einem öffentlichen Aufgabenbereich widerspiegelt. Vgl. hierzu W. Kirberger: Staatsentlastung durch private Verbände... a.a.O., S. 291 ff. 
Noch weniger eignen sich die Kennziffern, was allerdings auch nicht ihrer Zweckbestimmung entspricht, als Ausdruck der struktur der Aktivitäten zur Erfüllung öffentlicher Aufgaben bzw. zur Bereitstellung öffentlicher Leistungen in gesamtwirtschaftlicher Betrachtungsweise. Zunächst einmal vermitteln sie ein unzureichendes Bild vom Umfang dieser Aktivitäten, da diese Fragestellung nicht nur den staatlich geförderten Teil, sondern die gesamten Aktivitäten nicht-staatlicher Entscheidungseinheiten zur Erfüllung öffentlicher Aufgaben einbezieht. Darüber hinaus treten strukturelle Verzerrungen auf, wenn der Staat diese Aktivitäten indirekt subventioniert oder die Initiatoren und Förderer der nicht-staatlichen Entscheidungseinheiten andere zielvorstellungen besitzen und deshalb andere Schwerpunkte setzen als die staatlichen Entscheidungsträger.

\section{Verbesserungsmöglichkeiten auf der Inputebene}

\subsection{Die Notwendigkeit von Korrekturen}

Die Ergebnisse der vorangegangenen Analysen erwecken zumindest beträchtliche Zweifel an der Eignung der diskutierten Kennziffern und generell der budgetären Ausgaben des staates zur Kennzeichnung der zielvorstellungen der politischen Entscheidungsträger oder/und von schwerpunkten der staatstätigkeit. Wir wollen im folgenden die entscheidungstheoretische Fragestellung beiseite lassen und uns auf die Schwerpunktbestimmung in eher produktionstheoretischer Hinsicht konzentrieren, also der Frage nach der Allokation von Inputs zur Erfüllung öffentlicher Aufgaben nachgehen. Hierfür ist die Betrachtung der Opportunitätskosten der Erfüllung einer öffentlichen Aufgabe auf der Inputseite des Leistungserstellungsprozesses erforderlich. Die budgetären Ausgaben erlauben nur bedingt Aussagen über Opportunitätskosten bzw. Inputs. Selbst wenn die exogenen Störgrößen (und die freiwilligen Aktivitäten nichtstaatlicher Entscheidungseinheiten) auBer Betracht bleiben, 
spiegeln die Staatsausgaben die Inputs zur Erfüllung öffentlicher Aufgaben nicht korrekt, sondern unvollständig und mit unterschiedlicher Gewichtung wider. Dies beeinträchtigt nicht nur die Schwerpunktbestimmung, sondern auch Produktivitätsbzw. Effizienzanalysen. Verwendet man bei der Untersuchung der. Produktivität in einem öffentlichen Aufgabenbereich als Bezugsgröße (Nenner) statt der verbrauchten Einsatzfaktoren einfach die Staatsausgaben, so stehen verzerrte Ergebnisse zu erwarten.

Aus dem ziel, die tatsächlich zur Erfüllung einer öffentlichen Aufgabe aufgewendeten Inputs bzw. Kosten zu messen, ergibt sich ein Korrekturbedarf bei den budgetären Ausgaben in zweierlei Hinsicht: Zum einen müssen, um eine einheitliche Gewichtung zu gewährleisten, beim staat und bei den nicht-staatlichen Entscheidungseinheiten anstelle von Auszahlungen die zur Erfüllung einer Aufgabe aufgewendeten Kosten ausgewiesen werden. $\mathrm{zu}$ diesem $\mathrm{Zweck}$ ist jeweils eine Ermittlung und eine Periodenzurechnung, die der Nutzungsdauer sämtlicher von diesen Institutionen eingesetzter Inputs entspricht, erforderlich. Dabei ziehen wir der Aggregierbarkeit halber eine monetäre Bewertung vor. Zum anderen müssen im Hinblick auf die staatstätigkeit die Inputs einbezogen werden, die der staat nichtstaatliche Entscheidungseinheiten auf die Erfüllung öffentlicher Aufgaben zu verwenden zwingt; die Betrachtung lediglich der staatlichen Inputs in einem Aufgabenbereich greift bereits insoweit zu kurz. zielt man hingegen auf die Aktivitäten zur Erfüllung öffentlicher Aufgaben insgesamt ab, so müssen zusätzlich auch die freiwillig eingesetzten Inputs der nichtstaatlichen Entscheidungseinheiten in die Rechnung eingehen.

Zumindest bei den nicht-staatlichen Entscheidungseinheiten setzt die Lösung beider Probleme die Bestimung und Abgrenzung öffentlicher Aufgaben voraus. Bevor wir die primär technischen Fragen der Berechnung der Inputs bzw. Kosten der Erfüllung öffentlicher Aufgaben sowie der Aggregation der Inputs von 
Staat und nicht-staatlichen Entscheidungseinheiten diskutieren können, ist zu untersuchen, welche Aktivitäten der nicht-staatlichen Entscheidungseinheiten sich auf die Erfüllung öffentlicher Aufgaben richten und daher in ein Inputmaß einzugehen haben. Wir versuchen zunächst, ein allgemeingültiges Kriterium zur Abgrenzung öffentlicher Aufgaben zu finden und analysieren danach spezielle Ansätze zur Bestimmung der diesbezüglichen Aktivitäten öffentlicher Unternehmen.

3.2. Korrekturmöglichkeiten

\subsubsection{Zur Bestimmung öffentlicher Aufgaben}

Wir hatten bisher unterstellt, daß nicht-staatliche Entscheidungseinheiten sich mit allen oder Teilen ihrer Aktivitäten mit der Erfüllung öffentlicher Aufgaben befassen, die Frage nach der Bestimmung der diesbezüglichen Aktivitäten jedoch ausgeklammert. Der grundsätzliche Bedarf einer Differenzierung zwischen Aktivitäten zur Erfüllung öffentlicher und solchen zur Erfüllung privater Aufgaben entsteht primär aus folgenden Kombinationen:

- Es existieren Aufgaben, an deren Erfüllung sowohl der Staat als auch nicht-staatliche Entscheidungseinheiten mitwirken.

- Es gibt möglicherweise öffentliche Aufgaben, die ausschließlich nicht-staatliche Entscheidungseinheiten in Angriff nehmen, und private Aufgaben, bei denen ausschließlich der staat aktiv ist.

Die einfachste Lösung der Frage, welche Aktivitäten eigentlich zu aggregieren sind, besteht wohl darin, die Aktivitäten der herausragendsten Institutionen, auf die der staat Aufgaben verlagert, vollständig unter staatliche Direktive zu subsumieren und entsprechend $\mathrm{zu}$ den Kosten des Staates sämtliche Kosten 
der Parafisci und der öffentlichen Unternehmen zu addieren ${ }^{1) 2) . ~}$

Möglicherweise wäre damit für internationale Vergleiche des Niveaus der Staatstätigkeit, die durch historisch geprägte $\mathrm{Zu-}$ fälle Verzerrungen unterliegen, etwas gewonnen. Beispielsweise fallen Aufgaben, die in der Bundesrepublik die Sozialversicherung wahrnimmt, in Schweden tendenziell bei der zentralen Gebietskörperschaft an ${ }^{3)}$. Unter dem Aspekt der Erfüllung öffentlicher Aufgaben erscheint uns dieser Weg vor allem vertretbar für die Sozialversicherung, den Lastenausgleichsfonds und das ERP-Sondervermögen, deren Ausgabenverhalten dem staatswirtschaftlichen Kalkül entspricht und die hoheitliche Funktionen wahrnehmen. Bei den Ständefisci und den öffentlichen Unternehmen verbietet sich eine derartige Vorgehensweise jedoch, da diese Institutionen kaum ausschlieBlich öffentliche Aufgaben erfüllen dürften. So betreiben die ständefisci neben den ihnen vom staat übertragenen Aufgaben auch reine Interessenvertretung, und ein Teil der öffentlichen Unternehmen unterscheidet sich von privaten Unternehmen lediglich dadurch, daß als Eigentümer eine Gebietskörperschaft agiert ${ }^{4}$. Während sich

1) In ähnlicher Weise gehen Carlberg sowie Kroker vor, wenn sie den Umsatz der öffentlichen Unternehmen und die Staatsausgaben addieren, um eine erweiterte staatsquote zu errechnen. Vgl. M. Carlberg: రffentliche Unternehmen und Staatsquote, in: Zeitschrift für öffentliche und gemeinwirtschaftliche Unternehmen, Bd. $3(1980)$, S. 1-11, S. 6 sowie R. Kroker: Der staat als wirtschaftsfaktor... a.a.o., S. 28 ff. Zu einer ausführlichen Kritik an der Aussagefähigkeit dieser erweiterten Staatsquote siehe W. Leetz: Zur Einbeziehung der öffentlichen Unternehmen in die Staatsquote, in: Zeitschrift für öffentliche und gemeinwirtschaftliche Unternehmen, Bd. 4 (1981), S. 420-455, S. $438 \mathrm{ff}$.

2) Dabei sind bestimmte Bereinigungen zur Vermeidung von Doppelzählungen erforderlich. Siehe hierzu unten Abschnitt 3.2.3. in diesem Kapitel.

3) Vgl. K. Littmann: Definition und Entwicklung der Staatsquote... a.a.0., s. 16 .

4) Vgl. auch ebenda, S. 14; Wissenschaftlicher Beirat beim Bundesministerium der Finanzen: Gutachten zur Aussagefähigkeit staatswirtschaftlicher Quoten, a.a.O., S. 852 f.; V. Emmerich: రffentliche Produktion II ... a.a.0., S. $457 \mathrm{f}$. 
etwa für viele Leistungen und Tarife von Bundesbahn und Bundespost allokations- und verteilungspolitische Argumente anführen lassen, ist bei der Beteiligung des Bundes am VW-Konzern ein öffentlicher Zweck kaum $z$ u erkennen ${ }^{1)}$. Der weite Bereich der öffentlichen Unternehmen erstreckt sich von Betriebseinheiten, die ausschließlich öffentliche Aufgaben wahrnehmen und sich grundsätzlich anders verhalten als private Unternehmen bis hin zu rein erwerbswirtschaftlich orientierten Unternehmen.

Bei den freiwilligen Aktivitäten nicht-staatlicher Entscheidungseinheiten andererseits steht nicht einmal das relativ vage Kriterium der staatlichen Lenkung zur Verfügung. Während etwa einiges dafür spricht, die Unterhaltung eines Krankenhauses als Erfüllung einer öffentlichen Aufgabe anzusehen, die Aktivitäten eines skatklubs hingegen nicht, erhebt sich bereits bei Sportvereinen die Frage nach der Grenze zwischen öffentlichen und privaten Aufgaben.

Die Abgrenzung zwischen dem öffentlichen und dem privaten Bereich kann sich demnach nicht lediglich auf Instutionen beziehen, sondern muB anhand geeigneter Kriterien einzelne Aktivitäten zuordnen. Die darauf aufbauende Aggregation von Aktivitäten zur Erfüllung öffentlicher Aufgaben gleicht der funktionalen Abgrenzung des Staatssektors. Hierbei zählen die Aktivitäten nicht-staatlicher Entscheidungseinheiten, soweit sie Funktionen dominierend öffentlicher Art freiwillig oder gezwungenermaßen wahrnehmen, zum sektor staat, während die Erfüllung privater Aufgaben durch den staat dem privaten Sektor zugeordnet wird ${ }^{2}$. Qualifiziert man beispielsweise das

1) Auch der Beteiligungsbericht macht hierzu keine Angaben. Vgl. Bundesministerium der Finanzen (Hrsg.): Beteiligungen des Bundes im Jahre 1982, a.a.0., s. $160 \mathrm{ff}$.

2) Vgl. K. Littmann: Definition und Entwicklung der Staatsquote... a.a.0., s. 20 
Gesundheitswesen als öffentlichen Aufgabenbereich, so zählen bei einer funktionalen Abgrenzung Krankenhäuser in kirchlicher Trägerschaft zum Sektor staat. Fällt die Entscheidung entgegengesetzt aus, sind die staatlichen Aktivitäten im Gesundheitswesen dem privaten Sektor zuzurechnen.

Die von der Finanzwissenschaft entwickelten Ansätze zur Bestimmung öffentlicher Aufgaben bzw. des staatlichen Zuständigkeitsbereiches lassen sich in zwei große Kategorien einteilen ${ }^{1)}$ : Normative (wohlfahrtstheoretische) Ansätze gehen von vorgegebenen zielen aus und suchen nach einer zieladäquaten Aufteilung der vorhandenen Ressourcen auf die Produktion öffentlicher und privater Güter, so z.B. die Theorien von Samuelson ${ }^{2)}$ und Musgrave ${ }^{3)}$. Positive (pragmatische) Ansätze hingegen versuchen, die ziele der politischen Entscheidungsträger aus der Beobachtung konkreten staatlichen Handelns zu ermitteln und

1) Die Grenzen zwischen den beiden Kategorien sind allerdings in einigen Fällen fließend, da bestimmte positive Ansätze zur Bestimmung der staatstätigkeit auch normative Elemente enthalten. Siehe hierzu E. Wille: Die öffentlichen Ausgaben und die Entwicklung der Staatsquote. $\mathrm{Zu}$ entsprechenden Beiträgen im neuen "Handwörterbuch der Wirtschaftswissenschaft (HdWW)", in: ORDO, Bd. 32 (1981), S. 297-311, S. 299 ff.

2) Vgl. P.A. Samuelson: The Pure Theory of Public Expenditure, in: Review of Economics and Statistics, Vol. 36 (1954), S. 387-389; P.A. Samuelson: Diagrammatic Exposition of a Theory of Public Expenditure, in: Review of Economics and Statistics, Vol. 37 (1955), S. 350-356.

3) Vgl. R.A. Musgrave: The Theory of Public Finance. A Study in Public Economy, New York et al. 1959, S. 6 ff. und S. 61 ff., deutsch: Finanztheorie, 2. Auf 1., Tübingen 1969, S. $6 \mathrm{ff}$. und S. $49 \mathrm{ff.;}$ R.A. Musgrave und P.B. Musgrave: Public Finance in Theory and Practice, 3rd Ed. (4th Printing), Auckland et al. 1982, S. 7 ff. und S. 54 ff., deutsch: R.A. Musgrave, P.B. Musgrave und L. Kullmer: Die öffentlichen Finanzen in Theorie und Praxis, Bd. 1, 2. Aufl., Tübingen 1978 , S. $5 \mathrm{ff}$. und $\mathrm{S} .53 \mathrm{ff}$. 
daraus Erklärungsversuche abzuleiten, so vor allem die Arbeiten von A. Wagner ${ }^{1)}$, Downs ${ }^{2)}$, Harding ${ }^{3)}$ und Peacock/Wiseman ${ }^{4}$. Wir wollen hier auf diese Ansätze nicht näher eingehen, da sie in der Literatur intensiv diskutiert wurden. Wenngleich die Argumente durchaus voneinander abweichen, herrscht doch weitgehende Einigkeit darüber, daß weder die normativen noch die positiven Theorien der Staatstätigkeit Ergebnisse liefern, die in der Realität eine eindeutige zuordnung von Aufgaben zum öffentlichen bzw. zum privaten Sektor gestatten. Die normativen Ansätze beruhen auf so realitätsfernen Annahmen, daß eine Anwendung in der Praxis ausscheidet. Musgrave selbst bezeichnet seine Theorie als "somewhat utopian scheme", das einen Beitrag "to orderly thinking about the basic issues of budget policy" ${ }^{5)}$ leisten soll. Die positiven Theorien der Staatstätigkeit zeigen zwar Faktoren staatlichen Handelns auf, eine klare Grenze zwischen öffentlichen und privaten Aufgaben

1) Vgl. A. Wagner: Das Gesetz der zunehmenden Staatstätigkeit (Auszug aus: Staat in nationalökonomischer Hinsicht), in: H. C. Recktenwald (Hrsg.) : Finanztheorie, 2. Aufl., Köln, Berlin 1970, S. 241-243.

2) Vg1. A. Downs: An Economic Theory of Democracy, New York 1957, deutsch: రkonomische Theorie der Demokratie, Tübingen 1968 .

3) Vgl. F. O. Harding: Politisches Modell zur Wirtschaftstheorie. Theorie der Bestimmungsfaktoren finanzwirtschaftlicher Staatstätigkeit, Freiburg 1959. (F.O. Harding ist das Pseudonym für P. Herder-Dorneich.)

4) Vgl. A.T. Peacock und J. Wiseman: The Growth of Public Expenditure in the United Kingdom, National Bureau of Economic Research, Princeton 1961, insbes. S. $20 \mathrm{ff}$.

5) R.A. Musgrave: The Theory of Public Finance... a.a.o., S. VII. 
vermögen sie jedoch nicht abzustecken. 1) Als wenig hilfreich erweist sich auch ein Blick in die juristische Literatur zu diesem Problemkreis. Zwar ist die Zulässigkeit staatlicher Aktivitäten in formaler Hinsicht an eine institutionelle $\mathrm{Ab}-$ sicherung durch objektives Recht gebunden ${ }^{2)}$, woraus sich auch in materieller Hinsicht Grenzen der Staatstätigkeit ziehen lassen. Andererseits jedoch determiniert die Verfassung die staatlichen Aufgaben nicht abschließend, sondern überläßt die genaue Grenzziehung zum privaten Bereich dem staatlichen

1) Zu einer kritischen Würdigung normativer und positiver Theorien der staatstätigkeit vgl. etwa $\mathrm{H}$. Timm: Das Gesetz der wachsenden Staatsausgaben, in: Finanzarchiv N.F., Bd. 21 (1961), S. 201-247; K. Schmidt: Zur Geschichte der Lehre von den Kollektivbedürfnissen, in: N. Kloten et al. (Hrsg.): systeme und Methoden in den Wirtschafts- und Sozialwissenschaften. Erwin von Beckerath zum 75. Geburtstag, Tübingen 1964, S. 335-362; K. Häuser: Uber Ansätze zur Theorie der Staatsausgaben, in: H. Timm und H. Haller (Hrsg.): Beiträge zur Theorie der öffentlichen Ausgaben, Schriften des Vereins $f$ ür Socialpolitik N.F., Bd. 47, Berlin 1967, S. 36-65; K. Schmidt: Kollektivbedürfnisse und Staatstätigkeit, in: H. Haller et al. (Hrsg.): Theorie und Praxis des finanzpolitischen Interventionismus. Fritz Neumark zum 70. Geburtstag, Tübingen 1970, S. 3-27; G. Krause-Junk: Probleme der Berechnung und Schätzung öffentlicher Ausgaben, in: Public Finance, Vol. 27 (1972), S. 127-144; K. Mackscheidt: Zur Theorie des optimalen Budgets, Tübingen, Zürich 1973; K. Littmann, L. Hajen und R. Ulbrich: Argumente für eine Aufgabenabgrenzung $z$ wischen privatem und öffentlichem Sektor und Mischformen. Literaturanalyse. Projekt 90 der Kommission für wirtschaftlichen und sozialen Wandel, Manuskript, 0.0., O.J., (Hamburg 1974); H. Fecher: Ausgaben, öffentliche, I: Ansätze zu ihrer Analyse, a.a.O., S. 339 ff.; K. Littmann: Ausgaben, öffentliche, II: Die "Gesetze" ihrer langfristigen Entwicklung, in: Handwörterbuch der Wirtschaftswissenschaft (HdWW), hrsg. von W. Albers et al., Bd. 1, stuttgart et al. 1977, S. 349-363, S. $350 \mathrm{ff.;}$ G. Hesse: Staatsaufgaben. Zur Theorie der Legitimation und Identifikation staatlicher Aufgaben, Baden-Baden 1979, S. $261 \mathrm{ff.;}$. Wille: Die öffentlichen Ausgaben und die Entwicklung der Staatsquote... a.a.O., S. $299 \mathrm{ff}$.

2) Vgl. H.P. Bull: Die Staatsaufgaben nach dem Grundgesetz, Frankfurt 1973, S. $99 \mathrm{ff}$. 
WillensbildungsprozeB ${ }^{1)}$.

Unter ökonomischen Aspekten erscheint das Kriterium der Prozeßsteuerung prima vista als sinnvoll für eine Aufgabenabgrenzung $^{2)}$. Danach ist entscheidend, ob der Marktmechanismus den wirtschaftlichen Ablauf steuert oder nicht. Der staat nimmt öffentliche Aufgaben wahr, wenn und soweit er anstelle des Marktmechanismus eigene Mechanismen setzt oder den Marktmechanismus mittels wirtschafts- oder finanzpolitischer Instrumente beeinfluBt. Private Aufgabenbereiche liegen vor, wo der Marktmechanismus die wirtschaftlichen Aktivitäten koordiniert. Für eine anzustrebende Aufgabenzuordnung kommt es darauf an, ob der Marktmechanismus oder staatswirtschaftliche Planungsmechanismen die Erfüllung einer bestimmten Aufgabe besser gewährleisten. Der Vorzug des Kriteriums Prozeßsteuerung besteht darin, daß die Abgrenzung der Aufgabenbereiche von der Vorstellung einer ausschlieBlich an Güter gebundenen zuordnung abstrahiert. öffentliche Aufgaben bestehen nicht nur in der Bereitstellung von Gütern wie Innere und Äußere Sicherheit und private Aufgaben nicht nur in der Produktion von Autos, Kartoffeln etc. Der Verzicht auf eine rein gütermäßige Zuordnung von Aufgabenbereichen erlaubt die Einbeziehung auch der in der Realität existierenden engen Verflechtungen von öffentlichen und privaten Aufgaben. Der Staat nimmt häufig Einfluß auf die Produktion im privaten sektor, beispielsweise bei der Subventionierung von Agrarprodukten oder dem Verbot der Beimischung von Blei-Additiven in Vergaserkraftstoffen. Derartige Interdependenzen zwischen Aktivitäten des Staates und Privater indes führen andererseits dazu, daß beim Kriterium Prozeßsteuerung ebenfalls ein Bereich der Unbestimmtheit der Aufgabenabgrenzung verbleibt.

1) Vgl. ebenda, S. $211 \mathrm{ff}$. sowie M. Krautzberger: Die Erfüllung öfentlicher Aufgaben durch Private. Zum Begriff des staatlichen Bereichs, Berlin 1971, S. $97 \mathrm{ff}$.

2) Vgl. zum Folgenden K. Littmann, L. Hajen und R. Ulbrich: Argumente für eine Aufgabenabgrenzung... a.a.O., S. $141 \mathrm{ff}$. 
Probleme der Zuordnung ergeben sich da, wo private Aktivitäten der steuerung durch den Marktmechanismus nur beschränkt unterliegen. Man könnte einen dritten Sektor "Mischformen" einführen, der Phänomene aufnimmt, die sich nicht eindeutig dem privaten oder dem öffentlichen Sektor zuordnen lassen. Er würde beispielsweise gemeinwirtschaftliche Unternehmen, gemeinnützige Organisationen und die Delegation anscheinend öffentlicher Aufgaben an den privaten Bereich wie etwa die gesetzlich erzwungene Durchführung des Quellenabzugsverfahrens oder Umweltschutzauflagen für Unternehmen umfassen. Die Einführung eines Sektors "Mischformen" könnte das Abgrenzungsproblem zwar formal lösen, doch wäre damit konkret wenig gewonnen, da sich ein öffentlicher und ein privater Aufgabenbereich definieren lassen, die "Mischformen", die im Verlauf des zwanzigsten Jahrhunderts in Volkswirtschaften westlichen Typs in immer stärkerem MaBe auftreten, jedoch nur als Residualgröße konstituiert werden können.

Die ProzeBsteuerung scheitert als Kriterium für eine anzustrebende Aufgabenzuordnung letztlich daran, daß keine grundsätzlichen Aussagen darüber möglich sind, ob und inwieweit der Marktmechanismus auf bestimmten Gebieten staatswirtschaftlichen Allokationsmechanismen überlegen ist. Vielmehr hängt das Ausmaß der staatlichen Aufgabenerfüllung von politischen, kulturellen, sozialen und ökonomischen Faktoren ab ${ }^{1)}$. Es gibt "- abgesehen von der Rechtsprechung - keine Tätigkeit des Staates..., die historisch nicht auch einmal von Privaten ausgeübt worden wäre"2) ; ebenso lassen sich im internationalen Vergleich starke Abweichungen im Aufgabenbereich des staates feststellen. Bei einer positiven Analyse der Staatstätigkeit

1) Vgl. K. Littmann: Definition und Entwicklung der staatsquote... a.a.0., s. $17 \mathrm{ff}$.

2) K. Littmann: Zunehmende staatstätigkeit und wirtschaftliche Entwicklung. Versuche über die Wirkungen staatswirtschaftlicher Prozesse in der spätkapitalistischen Periode, Köln Opladen 1957, S. 35, Fn. 70. 
weist das Kriterium Prozeßsteuerung nicht nur den Mangel eines verbleibenden Unbestimmtheitsbereichs auf, sondern führt auch in funktionaler Hinsicht zu willkürlich anmutenden Differenzierungen. So wären etwa Privatschulen und Sanatorien, die ihre Leistungen am Markt anbieten, dem privaten, daneben bestehende Institutionen, die vergleichbare Leistungen in staatlicher Regie erstellen, jedoch dem öffentlichen Aufgabenbereich zuzuordnen.

Da sich die Frage, was als öffentliche Aufgabe anzusehen ist, nicht grundsätzlich beantworten läßt, wollen wir eine pragmatische, zeit- und ortsgebundene (Not-) Lösung wählen: Aktivitäten zur Erfüllung öffentlicher Aufgaben stellen (neben denjenigen des Staates) zunächst alle Aktivitäten nicht-staatlicher Entscheidungseinheiten dar, die auf einer Verlagerung von Aufgaben beruhen, welche der staat vorwiegend mit Hilfe der Normsetzung vornimmt. Zum anderen umfassen sie die freiwillig entfalteten Aktivitäten nicht-staatlicher Entscheidungseinheiten, die auf die Produktion bzw. Bereitstellung von i.d.R. meritorischen Gütern abzielen. Es handelt sich hier entweder um die subsidiäre Erstellung von den staatlichen Leistungen vergleichbaren Gütern oder aber, falls ein staatliches Angebot fehlt, um eine substitutive Produktion, die der staat zumindest in einigen Aufgabenbereichen, beispielsweise im Krankenhauswesen, einplant und in entsprechendem Maße unterstützt. Zur Rechtfertigung dieser bereits bisher implizit verwendeten - rein positivistischen Abgrenzung verweisen wir auf die in dieser Arbeit zugrundegelegte orientierung an den in der Realität existierenden Zielen der öffentlichen Planung. Sie stehen nicht unverrückbar fest, sondern können selbst innerhalb relativ kurzer Zeiträume von den politischen Entscheidungsträgern neu formuliert werden. 
3.2.2. Spezielle Korrekturansätze für öffentliche Unternehmen

Bei den in diesem Abschnitt diskutierten Ansätzen zur Bestimmung der Aktivitäten bzw. Inputs öffentlicher Unternehmen zur Erfüllung öffentlicher Aufgaben handelt es sich im Grunde lediglich um eine Anwendung der oben gewählten Abgrenzung des öffentlichen Aufgabenbereichs auf eine bestimmte Art von Trägern der Aufgabenerfüllung. Diese Ansätze enthalten sich jeglicher normativer Aussagen, sie dienen vielmehr zu einer streng positiven Analyse des real existierenden systems der Verlagerung von Aufgaben auf öffentliche Unternehmen.

Entsprechend der unterschiedlich stark ausgeprägten öffentlichen Komponente an den zielen bzw. Aktivitäten eines öffentlichen Unternehmens unterscheidet Neumark zwischen "den Unternehmungen, die mehr oder minder den Charakter eines 'service public' angenommen haben (wie namentlich Bahn, Post und Versorgungsbetriebe)... einerseits, eigentlichen Industrie- und Handelsunternehmungen nach Art der 'nationalisierten' Industriebetriebe, Rohstoffämter und dgl. anderseits"1). Bei der Untersuchung, ob und gegebenenfalls wie die Einnahmen und Ausgaben der öffentlichen Unternehmen in das staatliche Budget integriert werden sollen, lehnt er für beide Gruppen sowohl die Bruttobudgetierung, u.a. mit dem Hinweis auf die Unvereinbarkeit der Grundsätze budgetmäßiger Haushaltsführung mit einer kommerziellen Tätigkeit, als auch eine vollkommene finanzielle Autonomie der öffentlichen Unternehmen ab. Stattdessen plädiert er für die Erfassung der Einnahmen und Ausgaben öffentlicher Unternehmen in mit dem allgemeinen Haushalt verbundenen Anhangbudgets, damit diese der Bewilligung des Parlaments, der Beaufsichtigung des Finanzministers und der Kontrolle

1) F. Neumark: Theorie und Praxis der Budgetgestaltung, in: Handbuch der Finanzwissenschaft, hrsg. von W. Gerloff und F. Neumark, Bd. 1, 2. Aufl., Tübingen 1952, S. 554-605, S. 597 . 
durch den Rechnungshof unterliegen. Diese Budgetierungspraxis hält er für um so notwendiger, je weniger ein öffentliches Unternehmen sich von erwerbswirtschaftlichen Prinzipien leiten läßt ${ }^{1)}$ - Zudem müßten, sofern der staat ein öffentliches Unternehmen zu Abweichungen vom rein rentabilitätsorientierten Verhalten zwingt, die hieraus resultierenden Mehrausgaben oder Einnahmenausfälle beim Unternehmen rechnerisch kompensiert und stattdessen dem Etat des jeweils auftraggebenden Ressortministers angelastet werden. Dadurch werde transparent, was die Allgemeinheit insgesamt auf direktem und indirektem Wege für eine bestimmte öffentliche Aufgabe aufwendet ${ }^{2}$.

Der letzte Gedanke entspricht genau dem hier angestrebten $\mathrm{ziel}$. Letztlich geht es darum, aus den teils erwerbswirtschaftlich, teils gemeinwirtschaftlich motivierten Aktivitäten bzw. Inputs der öffentlichen Unternehmen diejenigen herauszufiltern, die durch die Erfüllung öffentlicher Aufgaben veranlaßt sind ${ }^{3)}$. In der Literatur ist der Gedanke, daß der staat die mit der Erfüllung öffentlicher Aufgaben verbundenen Abweichungen von einem rein erwerbswirtschaftlichen Verhalten buchstäblich erkauft, indem er den öffentlichen Unternehmen die hieraus resultierenden Mehrausgaben bzw. Mindereinnahmen ersetzt, unter der

1) Vgl. ebenda, S. 597 f.; ebenso P. Senf: Kurzfristige Haushaltsplanung, a.a.0., S. $396 \mathrm{f}$.

2) Vgl. F. Neumark: Theorie und Praxis der Budgetgestaltung, a.a.0., S. 600 .

3) Mit einem verwandten Problem beschäftigt sich der Wissenschaftliche Beirat beim Bundesministerium der Finanzen. Er wirft die Frage auf, welche der Investitionen der öffentlichen Unternehmen der Produktion öffentlicher Güter dienen und deshalb zu den öffentlichen Investitionen zu zählen seien. Vgl. Wissenschaftlicher Beirat beim Bundesministerium der Finanzen: Gutachten zum Begriff der öffentlichen Investitionen, a.a.0., S. 35 f. 
Bezeichnung "Abgeltungsprinzip" bekannt" . Zur konkreten Durchführung dieser rechnerischen Trennung von Eigenwirtschaft und Gemeinwirtschaft dient die sogenannte "Normalisierung der Konten". Dieses Verfahren, dessen Einführung der Ministerrat (Verkehr) der Europäischen Wirtschaftsgemeinschaft 1965 anstrebte ${ }^{2)}$, zielte beispielsweise bei den Eisenbahnen darauf ab,

"a) die Geschäftsergebnisse der Eisenbahnen so auszuweisen, wie sie aussehen würden, wenn die Eisenbahnen nicht von außen her einwirkenden Einflüssen auf ihre Geschäftsführung unterliegen würden. Dies sollte dadurch erreicht werden, daß bei einzelnen Einnahmekonten bestimmte rechnerische Zusetzungen und bei einzelnen Ausgabekonten bestimmte rechnerische Absetzungen dann durchgeführt werden sollten, wenn bei einem kaufmännisch geführten Eisenbahnunternehmen diese Konten einen anderen Inhalt haben würden, d.h. wenn die betreffenden Einnahmen höher und die betreffenden Ausgaben niedriger sein würden;

b) sodann die offiziellen Konten der Eisenbahnen, bei denen die erwähnten rechnerischen operationen durchzuführen waren, durch die normalisierten Konten zu ersetzen, wobei die Differenz zwischen den offiziellen und den normalisierten Konten vom staat übernommen werden sollte" ${ }^{3}$.

1) Vgl. W. Kitterer: Das moderne Budget... a.a.0., S. $65 \mathrm{f}$. Zur Diskussion um das Abgeltungsprinzip siehe vor allem T. Thiemeyer: Die Abgeltung gemeinwirtschaftlicher Lasten als Mittel der Finanzierung öffentlicher Unternehmen, in: P. Eichhorn und T. Thiemeyer (Hrsg.): Finanzierung öffentlicher Unternehmen. Festschrift für Dr. Paul Münch, BadenBaden 1979, S. 127-151.

2) Vgl. R. Eiermann: Die Berechnung und Abgeltung einseitiger Belastungen der Eisenbahnen (sogenannte Normalisierung der Konten), in: Die Bundesbahn, 39. Jg. (1965), S. 397-405, S. 397 .

3) Ebenda, S. 397. "Den Vorschlag zu a) bezeichnete man als rechnerische Normalisierung (normalisation calculée), den $z u$ b) als tatsächliche Normalisierung (normalisation effective)" Ebenda. 
Korrekturtatbestände sind u.a. die aufgrund staatlicher Auflagen erhöhten Personalausgaben (z.B. bei der Altersversorgung der Bahnbediensteten), die Wegekosten (die Eisenbahnen haben ihren Fahrweg selbst vorzuhalten, während die straßen und Binnenwasserwege aus allgemeinen Steuermitteln finanziert werden), Belastungen aus Tarifauflagen sowie aus der staatlich erzwungenen Beibehaltung verlustbringender Strecken und Anlagen ${ }^{1)}$.

Dieses Verfahren erscheint, obwohl wir die Behandlung etwa der Steuerbelastungen der Eisenbahnen für problematisch halten, im Prinzip besser als die häufig praktizierte staatliche Verlustabdeckung geeignet, die Kosten der Erfüllung öffentlicher Aufgaben durch öffentliche Unternehmen aufzuzeigen. Die Verluste öffentlicher Unternehmen, welche manche Interessenvertreter mit der Aufzählung möglichst vieler gemeinwirtschaftlicher zielsetzungen zu begründen suchen, können sowohl durch die Verfolgung öffentlicher Aufgaben als auch durch Unwirtschaftlichkeit verursacht sein. Solange die gemeinwirtschaftlichen zwecke der öffentlichen Unternehmen nicht operationalisiert und die Kosten ihrer Erfüllung nicht nachprüfbar ausgewiesen werden, ist die parlamentarische Kontrolle und die leistungsorientierte Planung und Erfüllung öffentlicher Aufgaben zumindest erschwert. Insofern macht es auch keinen Unterschied, ob das staatliche Budget alle Ausgaben und Einnahmen eines öffentlichen Unternehmens in einem Anhangbudget enthält oder nur zuführungen und Ablieferungen erfaBt ${ }^{2}$.

Im Hinblick auf die periodengerechte zurechnung der Aufwendungen öffentlicher Unternehmen zur Erfüllung öffentlicher Aufgaben weist das Verfahren der Normalisierung der Konten allerdings den Nachteil auf, daB es für eine Anwendung ex post konzipiert ist. Grundlage der Normalisierung im Einzelfall ist die offizielle Erfolgrechnung, in der sich die gemeinwirt-

1) Vgl. ebenda, S. $401 \mathrm{ff}$.

2) Vgl. auch W. Kitterer: Das moderne Budget... a.a.0., S. 67 . 
schaftlichen Belastungen niedergeschlagen haben. Diesem Mangel helfen - sofern man nicht eine Normalisierung ex ante mit Hilfe von Erfahrungswerten versuchen will - im Prinzip die in zunehmendem Maße in Frankreich und teilweise in der Bundesrepublik angewandten Programmerträge (contrats de programme) zwischen dem Staat und den öffentlichen Unternehmen ab. Sie sind gekennzeichnet durch

"(1) einen mittelfristigen Zeithorizont;

(2) die Festlegung der Maßnahmen für die Erhaltung des finanziellen Gleichgewichts des Unternehmens und

(3) die Quantifizierung

(a) der technischen, kommerziellen und finanziellen Zielsetzungen, die der staat und das Unternehmen für den betreffenden Zeitraum festlegen sowie

(b) der Mittel, die von beiden seiten zu ihrer Verwirklichung eingesetzt werden" ${ }^{1)}$.

Der staat zwingt sich mit einem Programmvertrag selbst, die auf ein öffentliches Unternehmen delegierten öffentlichen Aufgaben abzugrenzen, in Form von Programmen zu operationalisieren und somit das politisch Gewollte transparent zu machen. Das öffentliche Unternehmen ist erst dann zur Erbringung der gemeinwirtschaftlichen Leistungen verpflichtet, wenn beide Vertragspartner über die Kosten eines Programms Einigung erzielt haben und die entsprechenden Ausgleichszahlungen im staatlichen Budget veranschlagt sind.

Derartige Verträge implizieren tendenziell eine selbständigere kaufmännische Unternehmensführung und die Verwirklichung des Abgeltungsprinzips. Das Verhältnis zwischen staat und öffentlichen Unternehmen gewinnt dadurch klarere Konturen. Die Vorzüge der Programmerträge liegen sowohl in der anspruchsvolleren, programmorientierten Planung als auch in einer Verbesserung der

1) Ebenda, S. 75 . 
parlamentarischen Kontrollmöglichkeiten bezüglich der Kosten und der Erfolge der Verlagerung von Aufgaben auf öffentliche Unternehmen ${ }^{1)}$.

\subsubsection{Die Kosten der Erfüllung öffentlicher Aufgaben}

Im ersten Schritt sind die Inputs von staat und nicht-staatlichen Entscheidungseinheiten voneinander getrennt und für jeden öffentlichen Aufgabenbereich zu ermitteln. Der hier angestrebte Ubergang von den budgetären Ausgaben zu den Kosten der Erfüllung einer Aufgabe erfordert ${ }^{2)}$

- die Verteilung der Anschaffungskosten von Investitionsgütern auf die Jahre der Nutzung mit Hilfe von Abschreibungen

- bei der Darlehensgewährung die Einbeziehung lediglich der Subventionskomponente

- bei den Gewährleistungen die Einbeziehung der zu erwartenden bzw. geplanten Aufwendungen, die den Erwartungswert der Netto-Inanspruchnahme aus den in einer periode neu eingegangenen Verpflichtungen wiedergeben ${ }^{3)}$

- die Einbeziehung der Steuervergünstigungen und ähnlicher Verzichte auf Ressourcentransfers.

Diese Korrekturen betreffen auch nicht-staatliche Entscheidungseinheiten, da der staat etwa die Vergabe von Darlehen und Gewährleistungen teilweise auf Parafisci und öffentliche Unternehmen verlagert. Speziell bei den nicht-staatlichen Entscheidungseinheiten sind neben den "normalen" Aufwendungen für die

1) Vgl. ebenda, S. $70 \mathrm{ff}$.

2) Diese Aufzählung von Korrekturen erhebt keinen Anspruch auf Vollständigkeit, sondern berücksichtigt lediglich die in Abschnitt 1.2. dieses Kapitels beschriebenen Verzerrungsfaktoren.

3) Für empirische Untersuchungen dürften indes die vom theoretischen standpunkt aus gebotenen Forderungen nach einer exante-Betrachtung und einer exakten Periodenzurechnung unüberwindliche Hindernisse darstellen, sie werden sich daher mit dem Saldo des "Schadenstitels" am Ende einer Abrechnungsperiode begnügen müssen. 
Erfüllung öffentlicher Aufgaben auch Verrechnungskosten für Inputs zu ermitteln, die der staat direkt bei seinen Aktivitäten einsetzt, die jedoch Private aufbringen müssen, und denen weder Ausgaben der Privaten noch Mindereinnahmen des Staates gegenüberstehen (z.B. die allgemeine Wehrpflicht, ehrenamtliche Tätigkeiten) ${ }^{1)}$. Hinzu kommen - vor allem bei den privaten Organisationen ohne Erwerbscharakter - Verrechnungskosten für die von ihren Mitgliedern erbrachten ehrenamtlichen Leistungen und für empfangene Sachspenden, die sie unmittelbar als Inputs in ihrem Leistungserstellungsproze $B$ verwenden.

Für die eigentliche Aggregation der Inputs des Staates und der nicht-staatlichen Entscheidungseinheiten in jedem öffentlichen Aufgabenbereich stehen im Prinzip zwei Wege offen ${ }^{2}$. Zum einen kann man die beim staat anfallenden Kosten der Erfüllung einer öffentlichen Aufgabe ansetzen. Diese Größe schließt auch die Zuschüsse und spezifischen Steuervergünstigungen ein, die der Staat den nicht-staatlichen Entscheidungseinheiten in dem betreffenden Aufgabenbereich gewährt. Steuervergünstigungen stellen - wie bereits erwähnt - als indirekte bzw. verdeckte Subventionen Inputs zur Erfüllung öffentlicher Aufgaben dar. In vielen Fällen gewährt sie der staat für ein seinen zielsetzungen entsprechendes Verhalten; als aktuelles Beispiel sei auf die Befreiung von der Ergänzungsabgabe zur Einkommensteuer für Investoren verwiesen. Dieses Aggregat gibt jedoch eher die Inputs des Staates in einem Aufgabenbereich wieder und vernachlässigt weitgehend diejenigen der nicht-staatlichen Entscheidungseinheiten. Die Inputs bzw. Kosten letzterer gehen nur insoweit ein, als der staat ihnen auf direktem und indirektem Wege Unterstützung gewährt.

1) Bei der Wehrpflicht bemessen sich die Verrechnungskosten im Prinzip nach dem Verdienstausfall der Wehrpflichtigen in ihrem erlernten Beruf abzüglich des Wehrsoldes.

2) Der Grundgedanke zu diesen beiden Lösungswegen findet sich bei K. Littmann: Definition und Entwicklung der Staatsquote... a.a.0., s. $100 \mathrm{ff}$. Dort werden allerdings nicht Kosten, sondern Ausgaben aggregiert. 
Unter dem Aspekt der Vollständigkeit erweist sich daher die zweite Meßgröße als überlegen. Sie umfaßt im Prinzip die Kosten des Staates zuzüglich der Kosten der nicht-staatlichen Entscheidungseinheiten in einem Aufgabenbereich ${ }^{1}$. Diese summe würde indes zunächst allenfalls "die für die Erfüllung dieser Aufgabe bewegten Finanzströme (bzw. Ressourcen, U.R.) erfassen" ${ }^{2)}$. Um Mehrfachzählungen zu vermeiden, sind mehrere Bereinigungen erforderlich. Zur Vereinfachung der Argumentation können wir in jedem Aufgabenbereich die Akteure gedanklich zu einem "öffentlichen Teilsektor" zusammenfassen. Er umfaßt die einschlägigen Aktivitäten des staates und derjenigen nichtstaatlichen Entscheidungseinheiten, die als eigenverantwortliche Träger an der Erfüllung der betreffenden Aufgabe mitwirken. Eigenverantwortlich bedeutet hier nicht notwendigerweise freiwillig, sondern signalisiert einen gewissen spielraum bei der Entscheidung uber die Verwendung von Ressourcen für eine Aufgabe, wobei die $\mathrm{ziele}$ vom staat vorgegeben oder selbstgesteckt sein können. Diese Bedingung ist i.d.R. bei Parafisci, öffentlichen Unternehmen, Kirchen und Wohlfahrtsverbänden erfüllt, auch soweit sie im Auftrag des Staates handeln. Zum "privaten Sektor" zählen hingegen die privaten Haushalte und größtenteils die privaten Unternehmen. Sie wirken zwar teilweise ebenfalls an der Erfüllung öffentlicher Aufgaben mit, jedoch i.d.R. nicht eigenverantwortlich, sondern aufgrund staatlicher vorschriften, die ihnen keinen Entscheidungsspielraum bezüglich des Einsatzes von Inputfaktoren lassen. Bei der Wehrpflicht und bei ehrenamtlichen Leistungen beschränkt sich ihre Mitwirkung - der Terminus "verdeckter staatsbedarf" bringt dies schon zum Ausdruck - faktisch darauf, dem "öffentlichen Sektor" Ressourcen zur Verfügung zu stellen. Wir fragen hier jedoch

1) Da wir jetzt nicht mehr die entscheidungstheoretische, sondern die produktionstheoretische Fragestellung verfolgen, halten wir hier die Zusammenfassung von Inputs verschiedener Gebietskörperschaften und nicht-staatlicher Entscheidungseinheiten in einem Aufgabenbereich für zulässig.

2) H. Zimmermann: Die Ausgabenintensität... a.a.0., s. 8 . 
nicht nach der Beschaffung, sondern nach der Verwendung von Ressourcen, die sie erst - unabhängig von ihrer Herkunft - als Inputs im öffentlichen Leistungserstellungsprozeß qualifiziert. Grundsätzlich wollen wir bei derartigen Ressourcentransfers vom "privaten" zum "öffentlichen Sektor", die sich dadurch auszeichnen, daß die Ressourcen direkt als Inputs in die öffentliche Leistungserstellung eingehen, die zurechnung auf einen Träger beim Empfänger vornehmen, da er als Produzent letztlich über deren Verwendung entscheidet ${ }^{1)}$.

Die erste Bereinigungskomponente betrifft die Geld- und Realtransfers innerhalb des "öffentlichen Sektors". Herausgerechnet werden müssen sämtliche Transfers zwischen dem Staat und den zum "öffentlichen Sektor" gezählten nicht-staatlichen Entscheidungseinheiten (direkte staatliche Zuschüsse, an den staat abgeführte Ưberschüsse, steuervergünstigungen), zwischen den Gebietskörperschaften (neben den Zahlungen im Rahmen des allgemeinen Finanzausgleichs, die jedoch nicht auf spezifische Aufgaben abzielen, gehört hierzu vor allem die Mischfinanzierung im Rahmen der Gemeinschaftsaufgaben) sowie verschiedener, zum "öffentlichen Sektor" gezählter, nicht-staatlicher Entscheidungseinheiten untereinander (z.B. Ausgleichszahlungen ohne spezielle Gegenleistung von der gesetzlichen Krankenversicherung an die Träger der Rentenversicherung). Bei diesen Transfers handelt es sich noch nicht um Inputs, sondern lediglich um eine Umverteilung von Ressourcen innerhalb des "öffent-

1) So würden wir etwa die Verrechnungskosten für die Leistungen der Wehrpflichtigen als Inputs des Staates im Aufgabenbereich Verteidigung und die Verrechnungskosten für ehrenamtliche Leistungen und Sachspenden zugunsten privater Organisationen ohne Erwerbscharakter als Inputs dieser Organisationen betrachten. Bei Steuern, Sozialversicherungsbeiträgen, Geldspenden an karitative Organisationen etc. hingegen stellt sich die Frage der zuordnung auf einen Träger nicht. Es handelt sich dabei zwar um einen Ressourcentransfer vom "privaten" zum "öffentlichen Sektor", jedoch - mit Ausnahme der Erhebung von Abgaben zu anderen als fiskalischen Zwecken - noch nicht um Inputs des "öffentlichen Sektors". 
lichen Sektors", die keinen verstärkten Einsatz von Inputs impliziert ${ }^{1)}$. Bei der gebenden Institution stellen sie zwar Kosten der Erfüllung der Aufgabe dar, beim Empfänger betreffen sie jedoch zunächst lediglich die Beschaffung von Ressourcen, über deren Verwendung direkt als Inputs oder zum Tausch gegen Inputfaktoren er noch entscheiden kann. Wenn der staat etwa karitative Organisationen mittels direkter Zuschüsse und steuervergünstigungen fördert, handelt es sich aus deren sicht bei der Unterstützung lediglich um eine Finanzierungsquelle. Die Staatshilfen fließen in ihre Ausgaben ein ${ }^{2)}$, die Inputs des "öffentlichen Sektors" insgesamt verändern sich deshalb allein jedoch nicht.

Keine Bereinigungen dürfen hingegen um Transfers vorgenommen werden, die vom "öffentlichen" in den "privaten Sektor" fliessen, beispielsweise die distributionspolitisch begründeten Steuervergünstigungen und Ausgaben des Staates wie Wohngeld, Sozialhilfe usw. oder zuschüsse an private Unternehmen im Rahmen der strukturpolitik, da sie als Inputs des "öffentlichen sektors" zu qualifizieren sind.

Die zweite Bereinigungskomponente umfaßt die Käufe bzw. Verkäufe von Produktionsfaktoren innerhalb des "öffentlichen sektors", beispielsweise der Verkauf eines Grundstücks vom Staat an das Rote Kreuz. Auch hier erfolgt lediglich eine Verlagerung von Ressourcen - allerdings gegen ein spezielles Entgelt - zwischen verschiedenen Trägern der öffentlichen Aufgabenerfüllung, der

1) Primär bei Realtransfers kann es vorkommen, daß eine Ressource nach der Verlagerung in einem anderen Aufgabenbereich Verwendung findet als vorher. Die Bereinigung kann dann erst beim Ubergang zur Betrachtung aller Aufgabenbereiche erfolgen.

2) Dies übersieht beispielsweise Henke, der bei der Berechnung von Gesundheitsquoten in bezug auf das Sozialprodukt Ausgaben des staates, der Privaten und Steuervergünstigungen addiert, ohne Bereinigungen vorzunehmen. Vgl. K. -D. Henke: Öffentliche Gesundheitsausgaben und Verteilung... a.a.o., S. $29 \mathrm{ff}$. 
"Öffentliche sektor" insgesamt setzt jedoch nicht mehr Inputs ein"1). Bei den intrasektoralen Ressourcenverlagerungen wollen wir ebenfalls die Zurechnung bei dem Träger vornehmen, der über ihre Verwendung entscheidet.

Diese Summe der Inputs bzw. Kosten der Erfüllung öffentlicher Aufgaben enthält - zumindest nach der Aggregation aller Aufgabenbereiche - sowohl Ressourcen, die in Produktionsprozesse zur Erstellung öffentlicher Leistungen eingehen, als auch Ressourcen, die, etwa aus distributionspolitischen Gründen, ohne Gegenleistung in den "privaten Sektor" fließen. Eine derartige Ambivalenz läßt sich zwar rechtfertigen, geht es uns doch hier lediglich um die Kosten der Erfüllung öffentlicher Aufgaben; indes würde der Informationsgehalt der Rechnung durch eine Aufschlüsselung in reine Transfers und in Produktionsinputs beträchtlich steigen.

AuBer der Vollständigkeit weist dieser zweite Lösungsweg den Vorzug auf, daß die schwierige Ermittlung von steuervergünstigungen wenigstens insoweit unterbleiben kann, als der staat sie eigenverantwortlich tätigen nicht-staatlichen Entscheidungseinheiten gewährt. Andererseits erscheint die wünschenswerte Aufspaltung der Inputs in reine Transfers und Produktionsinputs primär bei den Sozialfisci als schwer durchführbar. Zudem tauchen im Zusammenhang mit vor allem personellen Inputs (beim "verdeckten Staatsbedarf") Bewertungsprobleme auf.

\subsubsection{Die Aussagefähigkeit der korrigierten Daten}

Ausgangspunkt für unsere Suche nach Verbesserungsmöglichkeiten war der Umstand, daß die budgetären Ausgaben ein zwar praktikables, zumal von Bewertungsproblemen freies, jedoch unvoll-

1) Diese Bereinigung ist nicht mit der Unterscheidung zwischen Vorleistungen und Endprodukten zu verwechseln. Wir stellen hier nicht auf die Wertschöpfung ab, sondern verbleiben ausschließlich auf der Inputebene. 
ständiges Maß für die Kosten der Erfüllung öffentlicher Aufgaben abgeben. Die vorgeschlagenen Korrekturen dienen dazu, die tatsächlich aufgewendeten Kosten bzw. Inputs der öffentlichen Aufgabenerfüllung $z u$ ermitteln und so Transparenz über deren Opportunitätskosten auf der Inputebene zu schaffen. Dabei kam es insbesondere darauf an, die Rolle der nicht-staatlichen Entscheidungseinheiten als teils subsidiäre, teils eigenständige Träger der öffentlichen Aufgabenerfüllung zu verdeutlichen. Die korrigierten Daten eignen sich dazu, die Schwerpunkte der öffentlichen Aufgabenerfüllung - vor allem bei produktionstheoretischer Betrachtungsweise - ohne die Verzerrungen der aufgezeigten Art, entweder für die Volkswirtschaft insgesamt oder aufgeschlüsselt in die verschiedenen an einer Aufgabe beteiligten Akteure, zu bestimmen. Gleichzeitig offenbaren sie die vom privaten sektor zu tragende effektive Belastung durch den Entzug von Ressourcen, die für die Erfüllung öffentlicher Aufgaben verwendet werden, besser als die staatsausgaben ${ }^{1)}$. SchlieBlich bieten die korrigierten Daten auch eine tragfähige Bezugsbasis für Produktivitätsanalysen. Gerade auch unter diesem Aspekt erweist sich die Zurechnung von Ressourcen als Inputs auf den Träger, der letztlich über ihre Verwendung entscheidet, sowie die Aufspaltung in Transfers und Produktionsinputs als zweckmäßig. Möglicherweise produzieren der staat und nicht-staatliche Entscheidungseinheiten die gleichen oder zumindest ähnliche Leistungen unterschiedlich effizient.

Es sei in diesem Zusammenhang noch einmal darauf hingewiesen, daß die korrigierten Daten ebensowenig wie die budgetären Ausgaben Aussagen über den in öffentlichen Produktionsprozessen erstellten Output - hier gemessen auf der Ebene der öffent-

1) Bei dieser Belastung handelt es sich allerdings lediglich um einen Primäreffekt, da ein Teil der entzogenen Ressourcen, etwa in Form von Transferzahlungen, direkt wieder in den privaten sektor zurückfließt. 
lichen Produkte ${ }^{1)}$ - erlauben; wirbeschäftigten uns in diesem Kapitel ausschließlich mit den Inputebenen öffentlicher Leistungserstellungsprozesse. Diese Einschränkung gilt nicht nur bezüglich des Niveaus, sondern auch hinsichtlich Veränderungen des öffentlichen Outputs. A priori ist die Behauptung, bei einer Erhöhung der Inputs in einem Aufgabenbereich um 5\% steige auch die produzierte Menge der betreffenden Leistung um 5\%, nicht zu rechtfertigen, da bereits offenbleibt, ob vor und nach der Erhöhung der Inputs die gleiche X-(In-)Effizienz der Produktion herrscht ${ }^{2}$. Wir werden im vierten Kapitel dieser Arbeit Ansätze zur Outputmessung auf ihre Eignung zur Bestimmung der (öffentlichen) Leistungen nicht-staatlicher Entscheidungseinheiten hin untersuchen.

Abschließend wollen wir noch kurz auf die Frage eingehen, welche Anwendungsmöglichkeiten für die (nicht korrigierten) budgetären Ausgaben verbleiben. Zunächst ist die empirische Forschung weitgehend auf die Ausgabenbetrachtung angewiesen, denn auf dieser Ebene liegt noch am ehesten hinreichendes Datenmaterial vor. Ferner erscheinen zwar für produktionstheoretische

1) Vgl. Schaubild 1 .

2) Vgl. D. Bös: Effizienz des öffentlichen Sektors aus volkswirtschaftlicher Sicht, in: Schweizerische zeitschrift für Volkswirtschaft und statistik, 114. Jahrgang (1978), S. 287-314, S. 303. Bös verwendet als Hilfsgröße für die Inputs allerdings deflationierte Staatsausgaben. $\mathrm{Zu}$ weiteren Ubertragungen des Leibensteinschen Konzeptes der X-(In-) Effizienz auf den öffentlichen Sektor siehe P. Friedrich: Regional Aspects of X-Inefficiency in the Public Sector, in: E. Wille (Hrsg.): Konzeptionelle Probleme öffentlicher Planung, Frankfurt et al. 1983, S. 189-243 und E. Wille: öffentliche Sachausgaben versus öffentliche Personalausgaben, erweiterte Fassung eines Referates auf der 26. Arbeitstagung des finanzwissenschaftlichen Ausschusses des Vereins für Socialpolitik in Hannover, erscheint in: K. Häuser (Hrsg.): Die Produktivitätsentwicklung staatlicher Leistungen, Manuskript, Mannheim 1983, S. $44 \mathrm{ff}$. 
Fragestellungen Daten, wie sie tendenziell die Volkswirtschaftlichen Gesamtrechnungen liefern, geeignet, für entscheidungstheoretisch begründete Aussagen über das Verhalten des Staates besitzen jedoch die Daten der Finanzstatistik relative Vorzüge. 
Kapitel III: DIE AKTIVITÄTEN NICHT-STAATLICHER ENTSCHEIDUNGSEINHEITEN IN DEN VOLKSWIRTSCHAFTLICHEN GESAMTRECHNUNGEN

1. Die Zuordnung der nicht-staatlichen Entscheidungseinheiten auf die Sektoren der Volkswirtschaftlichen Gesamtrechnungen

Bevor wir die sektorale Zuordnung der nicht-staatlichen Entscheidungseinheiten darstellen, wollen wir kurz auf die den Volkswirtschaftlichen Gesamtrechnungen (VGR) in der Bundesrepublik zugrundeliegenden Konzeptionen eingehen, um im übernächsten Abschnitt im Zuge einer kritischen Würdigung Konflikte zwischen diesen Leitideen und der tatsächlichen Vorgehensweise aufzeigen zu können.

Die VGR zielen darauf ab, ein umfassendes, gegliedertes, quantitatives Gesamtbild des wirtschaftlichen Geschehens in einem abgelaufenen Zeitraum zu zeichnen. Sie weisen die von den am Wirtschaftsablauf beteiligten Einheiten (Wirtschaftseinheiten) ausgeübten wirtschaftlichen Tätigkeiten Produktion und Verteilung von Gütern, ihre Verwendung für Verbrauchs- und Investitionszwecke, die Entstehung, Verteilung und Umverteilung der Einkommen sowie deren letzte Verwendung für den Verbrauch oder die Bildung von Ersparnissen und schlieblich die Kreditgewährung und -aufnahme nach ${ }^{1)}$. Wesentliche Bedeutung kommt der Wahl der zweckmäBigsten Darstellungseinheit zu. Das Statistische Bundesamt verwendet als Darstellungseinheit "die kleinsten Institutionen, die selbst bilanzieren (Unternehmen) bzw. die eine eigene Haushalts- und ggf. Vermögensrechnung aufstel-

1) Vgl. H. Bartels: Das Kontensystem für die Volkswirtschaftlichen Gesamtrechnungen der Bundesrepublik Deutschland. Erster Teil: Das angestrebte Kontensystem, in: Wirtschaft und Statistik, Jahrgang 1960 , S. 317-344, S. 317; Statistisches Bundesamt: Fachserie 18 Volkswirtschaftliche Gesamtrechnungen, Reihe 1 Konten und Standardtabellen 1981, a.a.0., S. 56, im folgenden zitiert als: Statistisches Bundesamt: FS 18, Reihe 1... 1981. 
len (z.B. Gebietskörperschaften, Kirchen, Wohlfahrtsverbände, private Haushalte)"1). Um das Gesamtbild überschaubar zu halten, werden die kleinsten Darstellungseinheiten nach der Art ihres wirtschaftlichen Verhaltens gruppiert und zu Sektoren zusammengefaBt. Maßgebend für die zuordnung einer Institution $\mathrm{zu}$ einem bestimmten Sektor sind zwei Kriterien ${ }^{2)}$ : Zunächst differenzieren die VGR nach der Art der von einer Wirtschaftseinheit vorwiegend ausgeübten Tätigkeit oder nach der besonderen Kombination der bei einer Wirtschaftseinheit vereinigten Tätigkeiten. Hieraus resultiert u.a. die Unterscheidung zwischen Produzenten und letzten Verbrauchern. Als zweites Kriterium, welches zur weiteren Aufteilung der Produzenten in Unternehmen und andere produzierende Institutionen dient, fungiert die stellung einer Wirtschaftseinheit zum Markt und die daraus resultierenden Finanzierungsmöglichkeiten. Entscheidend ist, ob ein Produzent die von ihm erzeugten Güter überwiegend gegen Entgelt auf dem Markt verkauft (Qualifikation als Unternehmen) oder nicht. Die Sektorbildung nach dem "Prinzip der institutionellen Abgrenzung und der zuordnung von Institutionen nach dem Schwerpunkt der wirtschaftlichen Tätigkeit" ${ }^{3}$ ) erfolgt unabhängig von Rechtsform und Eigentumsverhältnissen. Aufgrund dieser Kriterien teilen die VGR die Wirtschaftseinheiten den drei Sektoren Unternehmen (mit den Untersektoren Produktionsunternehmen, Kreditinstitute, Versicherungsunternehmen), Staat (mit den Untersektoren Gebietskörperschaften, Sozialversicherung) sowie

1) Statistisches Bundesamt: FS 18, Reihe 1... 1981, S. 61. Hervorhebungen im Original.

2) Vgl. H. Bartels: Das Kontensystem..., a.a.O., S. 318; Statistisches Bundesamt: FS 18, Reihe 1... 1981, S. 61.

3) H. Bartels: Das Kontensystem..., a.a.0., S. 322. Eine teilweise Durchbrechung dieses Prinzips stellt allerdings die Bildung des Wirtschaftszweiges Wohnungsvermietung (einschlieblich der Nutzung von Eigentümerwohnungen) im Unternehmenssektor dar. Vgl. ebenda. 
private Haushalte und private Organisationen ohne Erwerbscharakter ${ }^{1)}$ (mit den Untersektoren private Haushalte, private Organisationen ohne Erwerbscharakter) zu.

Unternehmen sind dadurch charakterisiert, da B sie in erster Linie waren und Dienstleistungen produzieren (und verteilen) und sie "gegen spezielles Entgelt verkaufen, das in der Regel Uberschüsse abwirft, zumindest jedoch annähernd die Kosten deckt" 2 ).

Der Staat erfüllt seine Aufgaben zumeist unabhängig vom Marktgeschehen. Zu diesem Sektor zählen Institutionen, die überwiegend Dienstleistungen für die Allgemeinheit erbringen und sich hauptsächlich aus Zwangsabgaben finanzieren. Innerhalb des Staatssektors unterscheiden sich Gebietskörperschaften und Sozialversicherung in ihrer Aufgabenstellung und bilden daher getrennte Untersektoren.

Der Untersektor Gebietskörperschaften enthält den Bund einschlieBlich Lastenausgleichsfonds und ERP-Sondervermögen, die Länder einschlieblich Stadtstaaten, die Gemeinden und Gemeindeverbände sowie kommunale Zweckverbände. Unternehmen im Eigentum der Gebietskörperschaften rechnen unabhängig von ihrer Rechtsform zum Sektor Unternehmen, wobei die Abgrenzung zu den Einrichtungen der Gebietskörperschaften der in der

1) Die privaten Organisationen ohne Erwerbscharakter werden in den VGR zwar als Produzenten angesehen, jedoch u.a. wegen Mängeln des statistischen Ausgangsmaterials entgegen den Zuordnungskriterien mit den privaten Haushalten zu einem Sektor zusammengefaBt. Vgl. dazu auch Abschnitt 3 in diesem Kapitel. Ferner ist hier eine Veränderung der Terminologie zu konstatieren. Während das Statistische Bundesamt bisher von "privaten Organisationen ohne Erwerbscharakter" sprach, bezeichnet es diese Institutionen seit der Revision der VGR 1982 als "private Organisationen ohne Erwerbszweck". Wenngleich wir die neue Bezeichnung für besser erachten, möchten wir hier doch den früheren Terminus beibehalten, da er sich in der Literatur eingebürgert hat.

2) Statistisches Bundesamt: FS 18, Reihe 1... 1981, S. 62 . 
deutschen Finanzstatistik ublichen Trennungslinie folgt ${ }^{1)}$. Sie beruht auf dem Prinzip, daB jene Institutionen ihrem Wesen nach zum Unternehmenssektor gehören, die Güter anbieten, welche im Prinzip auch die Privatwirtschaft anbieten könnte, und die Kostendeckung oder Gewinnerzielung anstreben ${ }^{2)}$. Demnach rechnen zu den Einrichtungen der Gebietskörperschaften u.a. öffentliche Einrichtungen der Sozialhilfe (Alten-, Blinden-, Obdachlosenheime u.ä.), Einrichtungen der Jugendhilfe (Erziehungs-, Jugendbildungsheime u.ä.), Einrichtungen des Gesundheitswesens (Krankenhäuser für Akutkranke einschlieBlich der kaufmännisch buchenden Krankenhäuser, Anstalten für Nervenund Geisteskranke u.ä.), ferner sonstige Einrichtungen wie Vieh- und Schlachthöfe, Markthallen, Feuerwehren, Bestattungseinrichtungen u.a.m. $\mathrm{Zu}$ den Produktionsunternehmen zählen hingegen u.a. die Bundesmonopolverwaltung für Branntwein, die Bundesanstalt für landwirtschaftliche Marktordnung, kommunale Versorgungs- und Verkehrsunternehmen sowie Hafenbetriebe ${ }^{3)}$. Weiterhin enthält der Untersektor Produktionsunternehmen die Hochschulbau-Gesellschaften der Länder (diese Zuordnung erfolgt in Anlehnung an die Finanzstatistik) sowie die Rundfunkanstalten, da sie nach Ansicht des Statistischen Bundesamtes marktbestimmte Dienstleistungen erstellen und sich überwiegend aus Gebühreneinnahmen finanzieren ${ }^{4}$, ferner neben sämtlichen Beteiligungen des Staates in der Industrie auch Bundesbahn und Bundespost ${ }^{5}$. Die Lastenausgleichsbank und die

1) Vgl. Statistisches Bundesamt: FS 18, Reihe 1... 1981, S.63.

2) Vgl. G. Bombach: Die öffentliche Finanzwirtschaft im Wirtschaftskreislauf, in: Handbuch der Finanzwissenschaft, hrsg. von F. Neumark, Bd. 1, 3. Aufl., Tübingen 1977, S. 53-75, S. 59 .

3) Vgl. Statistisches Bundesamt: FS 18, Reihe 1...1981, S. 63 f.

$4)$ So das Statistische Bundesamt in einem Schreiben an den Verfasser vom 22.12.1980.

5) Dabei wird die Bundespost vollständig den Produktionsunternehmen zugeordnet, da ein gesonderter Ausweis der Postscheck- und Postsparkassendienste im Untersektor Kreditinstitute mangels statistischer Unterlagen nicht möglich ist. Vgl. Statistisches Bundesamt: FS 18, Reihe 1... 1981, S. 63 . 
übrigen Kreditinstitute mit Sonderaufgaben rechnen die VGR ebenso wie öffentlich-rechtliche Grundkreditanstalten und die Wohnungsbauförderungsanstalten der Länder in Anlehnung an die Bankenstatistik der Deutschen Bundesbank im Unternehmenssektor zu den Kreditinstituten ${ }^{1)}$.

Bei der Sozialversicherung entschied sich das Statistische Bundesamt dafür, sie nicht zusammen mit den privaten Versicherungsunternehmen, sondern als Untersektor des Staates auszuweisen. Als Gründe für diese zuordnung wurden genannt, die Sozialversicherung sei eines der wichtigsten Instrumente der öffentlichen Sozialpolitik, und Einrichtungen und Tätigkeiten der Sozialversicherung seien in besonders starkem MaBe gesetzlich geregelt und in ihrem Charakter den Gebietskörperschaften sehr ähnlich. Letzteres zeige sich u.a. in der Aufbringung der Mittel durch Zwangsbeiträge und der Lockerung der Zusammenhänge zwischen Rentenzahlungen und früheren Beitragsleistungen und dem dadurch bedingten stärkeren Ubergang zum Umlageverfahren $^{2}$. Die Sozialversicherung umfaBt die Rentenversicherungen der Arbeiter und Angestellten, die knappschaftliche Rentenversicherung, Zusatzversorgungseinrichtungen für Angestellte und Arbeiter des Bundes, der Länder, der Gemeinden und anderer öffentlicher Körperschaften, die, Altershilfe für Landwirte, die gesetzliche Krankenversicherung leinschlieblich der Ersatzkassen, auch soweit es sich um ihr Geschäft mit freiwilligen Mitgliedern handelt), die gesetzliche Unfallversicherung und die Arbeitslosenversicherung. Unternehmen im Eigentum der Sozialversicherung dagegen zählen unabhängig von ihrer Rechtsform zum Unternehmenssektor ${ }^{3)}$.

1) Vgl. Statistisches Bundesamt: FS 18, Reihe 1... 1981, S. $62 \mathrm{f}$.

2) Vgl.H. Bartels: Das Kontensystem... a.a.O., S. 322.

3) Vgl. Statistisches Bundesamt: FS 18, Reihe 1... 1981, S. $63 \mathrm{f}$. 
Der Sektor private Haushalte und private Organisationen ohne Erwerbscharakter enthält im Untersektor private Haushalte alle Institutionen, die auf dem Markt primär als Anbieter von Arbeitskraft, letzte Kăufer von Ver- und Gebrauchsgütern sowie als Anleger von Ersparnissen auftreten ${ }^{1)}$. Zu den privaten Organisationen ohne Erwerbscharakter gehören "alle Organisationen, Verbände, Vereine, Institute usw., deren Leistungen vorwiegend bestimmten Gruppen von privaten Haushalten dienen und die diese Leistungen ihrer Zwecksetzung entsprechend zu wesentlichen Teilen nicht gegen spezielles Entgelt - mit dem erwerbswirtschaftlichen streben nach Gewinnerzielung -, sondern unentgeltlich oder zu nicht voll die Kosten deckenden Preisen abgeben. Sie finanzieren sich in erheblichem Umfang durch freiwillige Zahlungen (Beiträge, Spenden usw.) der privaten Haushalte und nur zu einem geringeren Teil aus öffentlichen Zuwendungen; dazu kommen - in mehr oder weniger groBem Umfang - Einnahmen aus dem Verkauf ihrer Leistungen"2). Es handelt sich hierbei um Kirchen, religiöse und weltanschauliche Vereinigungen, karitative, kulturelle (soweit ubberwiegend von privaten Haushalten finanziert) und im Erziehungswesen tätige Organisationen, politische Parteien, Gewerkschaften, Sportvereine, gesellige Vereine, Gartenbauvereine usw. Dazu gehören auch - unabhängig von der Art der Finanzierung einzelner Einrichtungen - die von den privaten Organisationen ohne Erwerbscharakter betriebenen Anstalten und Einrichtungen, beispielsweise Krankenhäuser, Heime, Schulen und Forschungsinstitute $^{3)}$.

1) Vgl. Statistisches Bundesamt: FS 18, Reihe 1... 1981, S. 64 .

2) N. Hartmann ("Har.") : Private Organisationen ohne Erwerbscharakter als Teil des. Haushaltssektors in den Volkswirtschaftlichen Gesamtrechnungen, in: Wirtschaft und statistik, Jahrgang 1976, S. 638-643, S. 638 .

3) Vgl. ebenda sowie Statistisches Bundesamt: FS 18, Reihe 1... 1981, S. 64 f. 
Tabelle 1: Die Zuordnung nicht-staatlicher Entscheidungseinheiten auf die Sektoren der Volkswirtschaftlichen Gesamtrechnungen

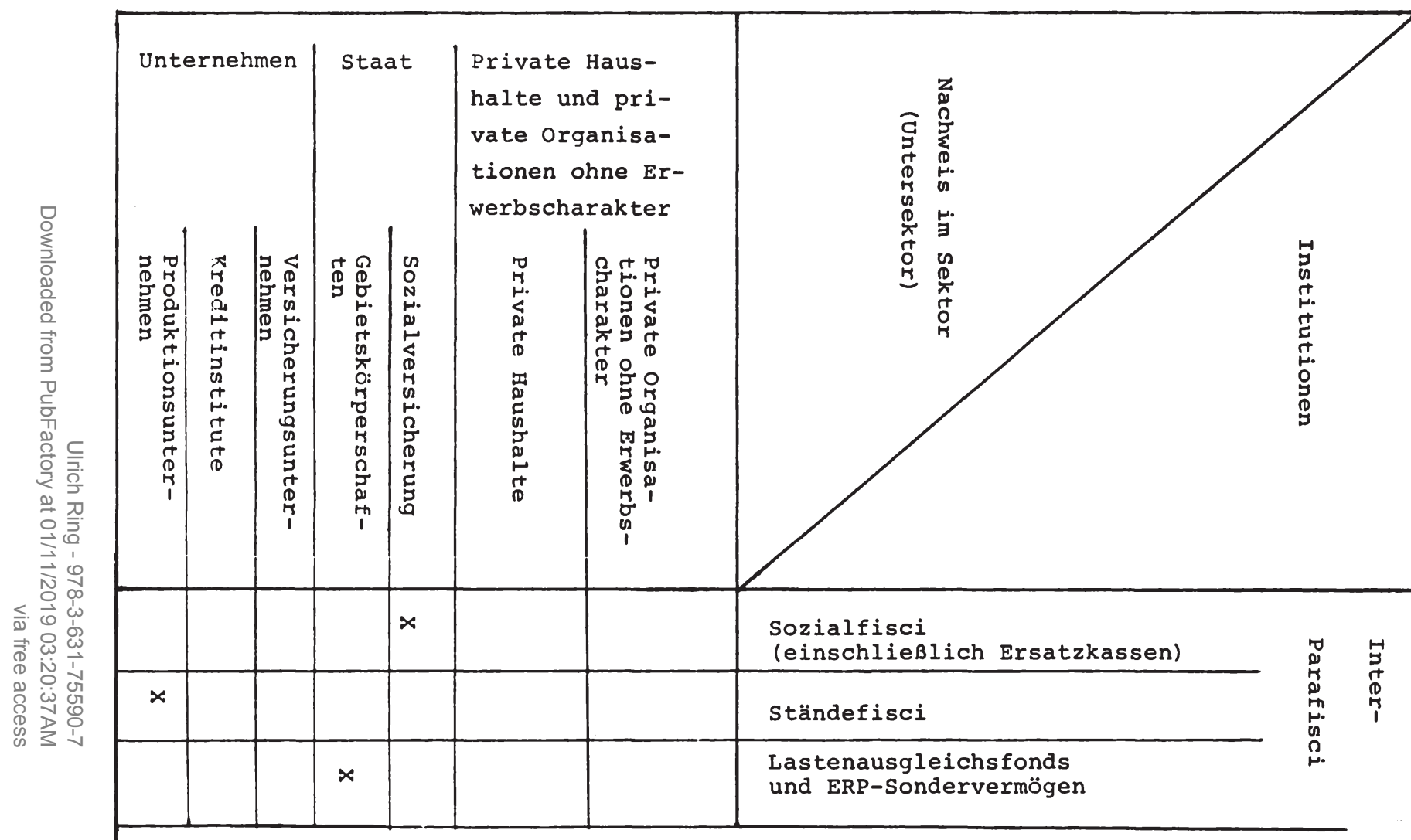


Die VGR unterscheiden neben den privaten Organisationen ohne Erwerbscharakter noch zwei weitere Gruppen von Organisationen ohne Erwerbscharakter. Zum einen rechnen sie zu den (Produktions-) Unternehmen auch Organisationen ohne Erwerbscharakter (mit den dazugehörigen Anstalten und Einrichtungen), die ihre Leistungen vorwiegend den Unternehmen ohne spezielles Entgelt zur Verfügung stellen und von diesen finanziert werden. Hierzu gehören Arbeitgeberverbände, Berufsorganisationen und Wirtschaftsverbände (ohne Gewerkschaften), öffentlich-rechtliche Wirtschafts- und Berufsvertretungen (die Kammern bzw. Ständefisci) sowie uberwiegend von Unternehmen finanzierte wissenschaftliche Institute ${ }^{1)}$.

Die dritte Gruppe beinhaltet Organisationen ohne Erwerbscharakter, die überwiegend vom staat finanziert werden. Hierbei handelt es sich vor allem um organisationen und Institutionen, die ihre Aktivitäten im Bereich von Wissenschaft und Forschung entfalten, so etwa die Institute der Max-Planck-Gesellschaft ${ }^{2)}$. Während diese Gruppe von Organisationen ohne Erwerbscharakter bisher, ihrer hauptsächlichen Finanzierungsquelle entsprechend, zum Staat (Untersektor Gebietskörperschaften) gerechnet wur$\mathrm{de}^{3)}$, erfassen sie die VGR seit der Revision 1982 "aus statistischen Gründen"4) zusammen mit den privaten Organisationen ohne Erwerbscharakter in deren Untersektor.

Die bisher beschriebenen Zuordnungen tangieren, da sie auf Institutionen abstellen, jeweils nur einen einzigen (Unter-)Sektor; sie sind in einer synoptischen Darstellung zusammengefabt (Tabelle 1). Die folgenden Vorgänge hingegen betreffen mehrere

1) Vgl. Statistisches Bundesamt: FS 18, Reihe 1... 1981, S.62.

2) Vgl. ebenda, S. 65 sowie E. Freund: Die Neugestaltung der Finanzstatistik ab 1970, in: Wirtschaft und Statistik, Jahrgang 1970, S. 543-546, S. 546.

3) Vgl. etwa Statistisches Bundesamt: Fachserie 18 Volkswirtschaftliche Gesamtrechnungen, Reihe 1 Konten und Standardtabellen 1980, Stuttgart, Mainz 1981, S. 57.

4) Statistisches Bundesamt: FS 18, Reihe 1... 1981, S. 65 . 
Sektoren gleichzeitig und entziehen sich daher einer Integration in die Tabelle 1. Die Verlagerung von Aufgaben auf Unternehmen und private Haushalte mit Hilfe staatlicher Vorschriften erfassen die VGR nicht als Aktivitäten besonderer Qualität, sondern weisen sie zusammen mit den übrigen Aktivitäten dieser Wirtschaftseinheiten in deren (Unter-) Sektoren nach. Entsprechendes gilt auch für die freiwillig erbrachten meritorischen Leistungen von Unternehmen und anderen Arbeitgebern wie betriebseigene Krankenstationen, Erholungsheime u.ä. Der dritte Vorgang betrifft die Altersversorgung der Beamten bei staat, Sozialversicherung und öffentlichen Unternehmen sowie der in beamtenähnlicher Stellung bei den Kirchen Beschäftigten. Um die Vergleichbarkeit der Einkommen und Pensionen der Beamten mit denen anderer Arbeitnehmer zu gewăhrleisten, weisen die VGR unterstellte Zahlungen von und an fiktive Pensionsfonds nach. Seit der Revision von 1977 konzentrieren die VGR diese fiktiven Pensionsfonds nicht mehr bei der Sozialversicherung, sondern siedeln sie jeweils bei der als Arbeitgeber fungierenden Institution, also bei den Gebietskörperschaften, der Sozialversicherung, Bundesbahn, Bundespost und Bundesbank sowie den Kirchen, an ${ }^{1)}$.

2. Produktionskonto und Bewertung der Aktivitäten der nicht-staatlichen Entscheidungseinheiten

Die Darstellung in diesem Abschnitt beschränkt sich im wesentlichen auf die Produktionskonten der nicht-staatlichen Entscheidungseinheiten, da hier die Unterschiede im Nachweis der Aktivitäten verschiedener Institutionen in den VGR am deutlichsten zutage treten.

1) Vgl. Statistisches Bundesamt: Fachserie 18 Volkswirtschaftliche Gesamtrechnungen, Reihe S. 2 Revidierte Ergebnisse 1960 bis 1976, Stuttgart, Mainz 1977, s. 19 ff.; Statistisches Bundesamt: Fachserie 18 Volkswirtschaftliche Gesamtrechnungen, Reihe S. 5 Revidierte Ergebnisse 1960 bis 1981, Stuttgart, Mainz 1982, S. 23 und S. 28 ff.; Statistisches Bundesamt: FS 18, Reihe 1... 1981, S. 95 und S. $100 \mathrm{f}$. 
Das derzeitige Kontensystem der VGR stellt in erster Linie auf Marktvorgänge ab. Zusätzlich erfaBt es jedoch auch Vorgänge, die sich auBerhalb des Marktes vollziehen. So beziehen die VGR auch etwa die von Staat, Sozialversicherung und Organisationen ohne Erwerbscharakter produzierten und ohne spezielles Entgelt abgegebenen Dienstleistungen in die Darstellung ein, damit ein möglichst vollständiges Bild der Produktion entsteht. Das in den VGR verwendete Produktionskonzept geht damit über das auf den Markt beschränkte Konzept (restricted market production concept) hinaus und tendiert zu einem umfassenden Produktionskonzept (comprehensive production concept) hin ${ }^{1}$ ).

Nicht nur der Umfang der dargestellten Vorgänge, sondern auch deren Bewertung orientiert sich weitgehend am Marktgeschehen. Deshalb bewerten die VGR über den Markt abgesetzte Güter grundsätzlich mit ihren Marktpreisen. Bei den Leistungen von Staat, Sozialversicherung und Organisationen ohne Erwerbscharakter gehen sie hingegen im Prinzip von den Kosten aus ${ }^{21}$.

Der Produktionswert der Unternehmen leinschl. der öffentlichen Unternehmen) errechnet sich anhand der Verkäufe von Waren und Dienstleistungen (incl. Eigenverbrauch der Unternehmer) zuzüglich des Wertes der Bestandsveränderungen an halbfertigen und fertigen Erzeugnissen aus eigener Produktion so-

1) $\mathrm{Zu}$ den verschiedenen Produktionskonzepten vgl. P. Studenski: The Income of Nations. Part Two: Theory and Methodology, New York, London 1958, S. $15 \mathrm{ff}$; C. Leipert: Unzulänglichkeiten des Sozialprodukts in seiner Eigenschaft als WohlstandsmaB, Tübingen 1975, S. 56 ff.; D. Brümmerhoff: Gesamtwirtschaftliches Rechnungswesen. Eine problemorientierte Einführung mit einem Kompendium wichtiger Begriffe des gesamtwirtschaftlichen Rechnungswesens, Köln 1975, S. $52 \mathrm{ff}$. und A. Stobbe: Volkswirtschaftliche Gesamtrechnung, in: Handwörterbuch der Wirtschaftswissenschaft (HdWW), hrsg. von W. Albers et al., Bd. 8, Stuttgart et al. 1980, S. 368-405, S. $370 \mathrm{f}$.

2) Vgl. Statistisches Bundesamt: FS 18, Reihe 1... 1981, S. 68 . 
wie des Wertes der in der Betrachtungsperiode selbsterstellten Anlagen ${ }^{1)}$; bei den Kreditinstituten werden teilweise Transaktionen unterstellt ${ }^{2}$. Dem Produktionswert stehen auf der linken Seite des Produktionskontos die Vorleistungen und die Bruttowertschöpfung (Lohn- und Gehaltszahlungen, Abschreibungen, gezahlte Produktionssteuern abzüglich Subventionen, Fremdkapitalzinsen und als ResidualgröBe der Gewinn) gegenüber. Den Produktionswert von Staat, Sozialversicherung und Organisationen ohne Erwerbscharakter ermitteln die VGR dagegen, da diese ihre Leistungen überwiegend ohne spezielles Entgelt abgeben, auf der linken Seite ihrer Produktionskonten jeweils durch Addition der Aufwandsposten Lohn- und Gehaltszahlungen, Produktionssteuern, Abschreibungen (mit einer Ausnahme) und Vorleistungen ${ }^{3)}$. Anders als bei den Unternehmen kommen hier Schuldzinsen, unterstellte Eigentümermieten und Gewinnzuschläge nicht in Ansatz ${ }^{4}$. Die Nicht-Einbeziehung dieser Posten erklärt sich daraus, daB bei staat, Sozialversicherung, organisationen ohne Erwerbscharakter und privaten Haushalten ex definitione keine Einkommen aus Unternehmertätigkeit und Vermögen entstehen, da diese Einkommensart in den VGR eine ResidualgröBe bildet. Diese Institutionen können allerdings Einkommen aus Unternehmertätigkeit und Vermögen empfangen, so der staat etwa die Ausschüttungen bzw. Uberschüsse der öffentlichen Unternehmen. Umgekehrt leisten sie auch Vermögenseinkommen, beispielsweise der Staat die Zinsen auf öffentliche Schulden. Die entsprechenden, Transaktionen werden jedoch jeweils auf dem Einkommensverteilungskonto verbucht ${ }^{5)}$. Der Verbrauch von Vorleistungen bemiBt sich bei staat, Sozialversicherung und or-

1) Vgl. Statistisches Bundesamt: FS 18, Reihe 1... 1981, s. 82 .

2) Vgl. Statistisches Bundesamt, Fachserie 18..., Reihe S. 2 Revidierte Ergebnisse 1960 bis 1976, a.a.O., S. 12 f. sowie Statistisches Bundesamt: FS 18, Reihe 1... 1981, S. 83.

3) Vgl. Statistisches Bundesamt: FS 18, Reihe 1... 1981, S. $83 \mathrm{f}$.

4) Vgl. N. Hartmann: Private Organisationen... a.a.0., S. 639.

5) Vgl. Statistisches Bundesamt: FS 18, Reihe 1... 1981, S. $96 \mathrm{f}$. 
ganisationen ohne Erwerbscharakter nach den Käufen von Vorleistungen, da das statistische Ausgangsmaterial keine Aufschlüsse über Lagerbestandsveränderungen gibt ${ }^{1)}$. Die oben erwähnte Ausnahme schlieblich betrifft die privaten Organisationen ohne Erwerbscharakter. Ihre Anlageinvestitionen lassen sich wegen Lücken im Ausgangsmaterial nicht sicher ermitteln und werden deshalb mit denen der Produktionsunternehmen zusammen dargestellt. Entsprechend erfolgt der Nachweis der Abschreibungen auf diese Anlageinvestitionen im Unternehmenssektor ${ }^{2}$.

Dem so über die linke Seite des Produktionskontos ermittelten Produktionswert stehen der Wert der Verkäufe von laufenden Produktionsleistungen, ggf. der selbsterstellten Anlagen und als ResidualgröBe der staatsverbrauch bzw. bei den Organisationen ohne Erwerbscharakter der Eigenverbrauch gegenüber.

Der Wert der Verkäufe machte bei den privaten Organisationen ohne Erwerbscharakter im Jahre $1975 \mathrm{ca} .70$ \& und beim Staat inclusive Sozialversicherung ca. 11 \& jeweils des Produktionswertes aus ${ }^{3)}$; für 1981 belaufen sich diese Anteile auf $70 \%$ bei den privaten Organisationen ohne Erwerbscharakter, 16,6 \& bei den Gebietskörperschaften, 0,08 \& bei der Sozialversicherung und 12,1 \& beim staatssektor insgesamt ${ }^{4)}$. Die Verkäufe der privaten Organisationen ohne Erwerbscharakter bestehen zu einem erheblichen Teil aus Krankenhausleistungen u.ä., die sie gegen Entgelt an die Sozialversicherung zur Abgabe an deren Mitglieder bzw. an die Gebietskörperschaften zur Abgabe an Kriegsopfer, Sozialhilfeempfänger und dgl. liefern. Daneben sind Verkäufe an private Haushalte enthalten, insbesondere Leistungen der Krankenhäuser und Heime dieser Organisationen,

1) Vgl. Statistisches Bundesamt: FS 18, Reihe 1... 1981, S. 84 .

2) Vgl. ebenda, S. 81.

3) Vgl. N. Hartmann: Private Organisationen... a.a.0., S. 641.

4) Dies ergaben Berechnungen des Verfassers anhand der Tabellen in: Statistisches Bundesamt: FS 18, Reihe 1... 1981, S. 226 und S. $258 \mathrm{ff}$. 
die sie den privaten Haushalten unmittelbar in Rechnung stellen, ferner Einnahmen aus Veranstaltungen von Sportvereinen usw. Aufgrund dieser Art der Verbuchung leisten die Sozialversicherungsträger in den VGR an die privaten organisationen ohne Erwerbscharakter keine Zuschüsse, sondern ausschlieblich Entgelte für den Kauf von Leistungen ${ }^{1)}$.

Auf die Verkäufe der Gebietskörperschaften brauchen wir hier nicht einzugehen, da sie kaum Bezüge zu unserem Thema aufwei$\operatorname{sen}^{2}$ ). Die vernachlässigbar geringe Höhe der Verkäufe (von Verwaltungsleistungen) der Sozialversicherung beruht darauf, daB die VGR die Sozialversicherungsbeiträge nicht als Entgelt für Versicherungsleistungen, sondern als laufende Ubertragungen über die Einkommensumverteilungskonten verbuchen ${ }^{3)}$.

Die ResidualgröBe Staats- bzw. Eigenverbrauch enthält auch die von der staatlichen Sozialhilfe, der Sozialversicherung und privaten Organisationen ohne Erwerbscharakter unentgeltlich abgegebenen Sachleistungen wie Medikamente, Arzt- und Krankenhausleistungen. Diese Realtransfers werden nicht jeweils über das Einkommensverwendungskonto dem Privaten Verbrauch zugerechnet, sondern über das Produktionskonto verbucht. Sie gehen in die Vorleistungen der betreffenden Institutionen ein und erhöhen in gleichem Umfang deren Eigen- bzw. Staatsverbrauch ${ }^{4}$. Aus dieser Art der Verbuchung von unentgeltlich abgegebenen

1) Vgl. N. Hartmann: Private Organisationen... a.a.0., S.640 f. und Statistisches Bundesamt: FS 18, Reihe 1... 1981, S. 83 ff.

2) Hier ergaben sich bei der Revision 1970 im Zuge einer Angleichung an neue internationale systeme Volkswirtschafticher Gesamtrechnungen einige Veränderungen. Vgl. dazu G. Hamer: Revision der Volkswirtschaftlichen Gesamtrechnungen, in: Wirtschaft und Statistik, Jahrgang 1970, S. 57-65, S. 58 .

3) Vgl. Statistisches Bundesamt, FS 18, Reihe 1...1981, S. 79.

4) Vgl. ebenda, S. 85 . 
Sachleistungen folgt, $d a \beta$ es sich bei den von Gebietskörperschaften, Sozialversicherung und privaten Organisationen ohne Erwerbscharakter geleisteten und in den VGR nachgewiesenen laufenden tubertragungen und Vermögensübertragungen im Prinzip ausschließlich um Geldleistungen handelt. Die einzige Ausnahme hiervon stellen unentgeltlich erbrachte Sachleistungen und Lieferungen der genannten Institutionen an das Ausland dar, beispielsweise im Rahmen der Entwicklungs- oder Nahrungsmittelhilfe. Die Ausgaben der leistenden Institutionen verbuchen die VGR seit der Revision 1977 nicht mehr auf deren Produktionskonten als Vorleistungen/Eigen- bzw. Staatsverbrauch, sondern als Ausfuhr/laufende Ubertragungen an die übrige welt ${ }^{1}$.

Die Aufwendungen von Arbeitgebern für meritorische Leistungen zugunsten ihrer Beschäftigten wie betriebseigene Unfallstationen, Kindergärten und Erholungsheime behandeln die VGR nicht als Bestandteil der Einkommen aus unselbständiger Arbeit, sondern als Vorleistungen. Dagegen sind Aufwendungen für betriebliche Altersversorgung u.ä. in den Einkommen aus unselbständiger Arbeit enthalten ${ }^{2)}$.

\section{Kritische Würdigung}

Die VGR beschränken ihre Berichterstattung, wie oben dargestellt, nicht auf den Marktbereich, sondern beziehen auch den Staat und die nicht-staatlichen Entscheidungseinheiten (soweit sie nicht als Anbieter von Gütern am Markt ohnehin enthalten sind) mit ihren unentgeltlich abgegebenen Leistungen ein. Diese Erweiterung führt jedoch zu Konflikten mit folgenden drei Konventionen, auf denen die Sozialproduktsberechnung beruht ${ }^{3)}$ :

1) Vg1. Statistisches Bundesamt: Fachserie 18..., Reihe S. 2 Revidierte Ergebnisse 1960 bis 1976, a.a.0., S. 13 f. und Statistisches Bundesamt: FS 18, Reihe 1... 1981, S. $88 \mathrm{f}$.

2) Vgl. Statistisches Bundesamt: FS 18, Reihe 1... 1981, S. 94 ff.

3) Vgl. hierzu G. Bombach: Die öffentliche Finanzwirtschaft... a.a.0., s. 56 . 
- Die Sozialproduktsberechnung beschränkt sich auf Marktvorgänge mit gewissen, genau umrissenen Ausnahmen (Eigenverbrauch der Landwirtschaft, Wohnen in Eigenheimen, selbsterstellte Anlagen).

- Die Güter- und Leistungsströme werden zu Marktpreisen bewertet.

- Alle Doppelzählungen werden durch Wertschöpfungsrechnung bzw. durch Erfassung des reinen Endverbrauchs ("final output") ausgeschaltet.

Der staat und ein Teil der nicht-staatlichen Entscheidungseinheiten jedoch "sind unter dem Aspekt der Wohlstandsmessung ein Fremdkörper im System Volkswirtschaftlicher Gesamtrechnungen, indem alle drei Konventionen durchbrochen werden"1). Staat und nicht-staatliche Entscheidungseinheiten bieten ihre Leistungen zu einem erheblichen Teil nicht auf Märkten an. Bei ihrer unentgeltlichen Leistungsabgabe fehlt die Analogie zu Marktprozessen, die etwa beim Eigenverbrauch der Landwirtschaft besteht. Diese mangelnde Vergleichbarkeit zwingt zu einem Rekurs auf ein systemfremdes, willkürliches Bewertungsverfahren. Ferner wird - außer bei den zum Unternehmenssektor gezählten nicht-staatlichen Entscheidungseinheiten - das Prinzip der Nettorechnung durchbrochen, da die unentgeltlich abgegebenen Leistungen von staat und nicht-staatlichen Entscheidungseinheiten dem Endverbrauch zugeschlagen werden, woraus Doppelzählungen resultieren können. Darüber hinaus messen die VGR bei der Abgabe von Leistungen ohne spezielles Entgelt nicht, wie im Marktbereich, Outputs ${ }^{2}$.

1) Ebenda, S. 56 (im Original hervorgehoben). Ähnlich auch H.W.Holub: Probleme der Erfassung der Staatstätigkeit, in: Wirtschaftswissenschaftliches Studium (WiSt), Jahrgang 1977, S. 324-329.

2) Vgl. G.Bombach: Die öffentliche Finanzwirtschaft... a.a.o., S. 56 . 
Diesen Nachteilen der Erweiterung des Berichtskreises um zumindest einen Teil des Nicht-Marktbereiches steht andererseits ein Informationsgewinn gegenüber. Zudem spiegeln, da die VGR die als produzenten qualifizierten Wirtschaftseinheiten danach einteilen, ob diese ihre Güter überwiegend auf dem Markt verkaufen oder nicht, die Beiträge der verschiedenen produzierenden Sektoren zum gesamtwirtschaftlichen Produktionsergebnis im Prinzip das relative Gewicht des Marktmechanismus bzw. anderer Koordinationsmechanismen ${ }^{1)}$ wider. Eine Einschränkung ergibt sich allerdings bereits daraus, daB die VGR die Einflüsse der staatlichen Verordnungstätigkeit auf die nachgewiesenen Marktprozesse nicht offenlegen.

Es ist jedoch nicht ohne weiteres möglich - und von der zielsetzung der VGR her auch gar nicht beabsichtigt - an den Anteilen von Markt-Produktion und Produktion auBerhalb des Marktes das Verhältnis zwischen der Erfüllung privater Aufgaben und der Erfüllung öffentlicher Aufgaben abzulesen. Verzerrungen dieser Relation entstehen aus dreierlei Gründen:

Im Marktsektor werden teilweise auch öffentliche Aufgaben erfüllt, und nicht jede Aktivität auBerhalb des Marktes richtet sich auf die Erfüllung privater Aufgaben. Das in den VGR verwendete Kriterium der Prozeßsteuerung vermag diesbezüglich selbst dann nicht zu differenzieren, wenn eine wirtschaftseinheit ausschließlich private oder ausschließlich öffentliche zielsetzungen verfolgt.

In den Fällen, in denen eine Wirtschaftseinheit sowohl öffentliche als auch private Aufgaben erfüllt, erweist sich die kleinste Darstellungseinheit der VGR als nicht klein genug für

1) Da die VGR primär auf Marktvorgänge abstellen, spezifizieren sie die in den Sektoren auBerhalb des Marktes wirkenden Koordinationsmechanismen nicht. 
eine Analyse der tatsächlichen Aufgabenverteilung. Hierfür wäre die zuordnung von Aktivitäten anstatt von Institutionen auf verschiedene sektoren erforderlich.

SchlieBlich werden Aussagen der VGR dadurch relativiert, daB bei der Berechnung der Produktionswerte der verschiedenen Sektoren bzw. produzierenden Wirtschaftseinheiten je nach ihrer Stellung zum Markt sowohl die erfaBten Vorgänge als auch die Bewertungsverfahren voneinander abweichen.

Die ersten beiden Gründe betreffen die sektorale zuordnung der nicht-staatlichen Entscheidungseinheiten in den VGR. Eine detaillierte Analyse erweckt Zweifel insbesondere bezüglich der Zuordnung derjenigen Institutionen, die ihre Entstehung der "Flucht aus dem Budget" verdanken. Die VGR rechnen sie ubberwiegend zu den Unternehmen. Es bleibe dahingestellt, ob "es den Gesamtrechnern u.a. darauf ankam, den schwer zu handhabenden staatssektor möglichst klein zu halten"1), die zuordnungskriterien des Statistischen Bundesamtes nicht scharf genug sind, oder $\mathrm{ob}$ es sich, der Abgrenzung im statistischen Ausgangsmaterial (Finanzstatistik, Bankenstatistik der Deutschen Bundesbank) folgend, über seine eigenen Kriterien hinwegsetzt. Der Nachweis der Wohnungsbauförderungsanstalten und der Hochschulbau-Gesellschaften sowie bestimmter Kreditinstitute mit Sonderaufgaben im Unternehmenssektor trägt jedenfalls, obgleich die Zuordnungskriterien tendenziell funktional angelegt sind, eher juristischen bzw. institutionellen Gegebenheiten Rechnung.

$\mathrm{Zu}$ welch fragwürdigen Ergebnissen der Nachweis von Institutionen, die ausschließlich in staatlichem Auftrag handeln, aber formal verselbständigt sind, im Unternehmenssektor führt, läBt

1) H.W. Holub: Probleme der Erfassung der Staatstätigkeit, a.a.O., S. 326 . 
sich besonders gut am Beispiel der Hochschulbau-Gesellschaften verdeutlichen. Während bei den übrigen Bundesländern die VGR die mit dem Hochschulbau verbundenen Aktivitäten im Sektor Staat erfassen, werden bzw. wurden sie für Niedersachsen und Nordrhein-Westfalen aufgrund einer rein optischen Veränderung und in Anlehnung an die Finanzstatistik plötzlich dem Unternehmenssektor zugerechnet. Hieraus resultiert - abgesehen von der Beeinträchtigung der intertemporalen Vergleichbarkeit - nicht nur eine Verzerrung der Beiträge von Staat und Unternehmen zum gesamtwirtschaftlichen Produktionsergebnis, darüber hinaus ist die Vergleichbarkeit der Beiträge der Bundesländer nicht mehr gewährleistet $^{1)}$. Das Statistische Bundesamt kündigte allerdings für die Revision 1982 der VGR eine wenigstens teilweise Anderung der bisherigen Verbuchungspraxis an, indem die Bauinvestitionen der Hochschulbau-Gesellschaften in den kassenmäBigen Nachweis der staatlichen Bauten eingehen sollen. Das Amt begründet diese Anderung damit, "daß die Hochschulbauten der Produktion staatlicher Leistungen dienen und deshalb auch im Staatssektor ausgewiesen werden müssen" ${ }^{2)}$.

Der Nachweis der Wohnungsbauförderungsanstalten der Länder und derjenigen Kreditinstitute mit Sonderaufgaben, die staatliche Kreditaktionen aus Mitteln der öffentlichen Hand bankmäßig durchzuführen haben, im Unternehmenssektor (Untersektor Kreditinstitute), kann aus denselben Gründen nicht überzeugen. Diese Zuordnung entspricht derjenigen der Bankenstatistik, die anderen zielsetzungen dient als die VGR.

1) Konsequenterweise müssen die VGR die zuschüsse der Länder Niedersachsen und Nordrhein-Westfalen an ihre HochschulbauGesellschaften als Subventionen oder Vermögensübertragungen an den Unternehmenssektor qualifizieren.

2) So das Statistische Bundesamt in einem Schreiben an den Verfasser vom 22.12.1980. 
Spricht bei den bisher genannten Institutionen die Qualifizierung ihrer Aufgaben und Aktivitäten als rein öffentlich für den Nachweis im staatssektor, so sind bei Institutionen mit Kombinationen von öffentlichen und privaten Aufgaben keine eindeutigen Aussagen über deren optimale zuordnung möglich. Wenn die VGR diese Gruppe von nicht-staatlichen Entscheidungseinheiten - im Einklang mit den zuordnungskriterien - i.d.R. im privaten sektor nachweisen, verdecken sie zwangsläufig die öffentliche Komponente ihrer Aktivitäten. Die ziele bestimmter, bei den Produktionsunternehmen nachgewiesener, öffentlicher Unternehmen erschöpfen sich jedoch keineswegs darin, ihre Leistungen gegen Entgelt zu verkaufen und möglichst hohe tberschüsse zu erwirtschaften, man denke etwa an die allokations- und distributionspolitischen Aufgaben von Bundesbahn und Bundespost. Kombinationen von öffentlichen und privaten Aufgaben finden sich auch bei den zum Unternehmenssektor gerechneten berufsständischen Kammern und bei einem Teil der privaten Organisationen ohne Erwerbscharakter (z.B. Kirchen). Die Aufspaltung dieser Aktivitäten in eine eher öffentliche und eine eher private Komponente verhindert jedoch bereits die in den VGR gewählte Größe der kleinsten Darstellungseinheit.

Auch ohne eine Veränderung der Größe der Darstellungseinheit ließe sich der Informationswert der VGR indes dadurch erhöhen, daß wenigstens die Unternehmen getrennt nach privaten und öffentlichen ausgewiesen werden. Das Statistische Bundesamt hielt 1960 diese Aufteilung für "wahrscheinlich für alle Konten des Teilsektors Produktionsunternehmen von Bedeutung, da sie Anhaltspunkte dafür gibt, wieweit der staat - auBerhalb seines eigentlichen Aufgabengebietes - am Produktions- 
proze $\beta$ beteiligt ist" ${ }^{1)}$, verfolgte diese Frage aber wegen der Schwierigkeit der Zuordnung gemischt-wirtschaftlicher Unternehmen nicht mehr weiter.

In mehrfacher Hinsicht unbefriedigend ist die Art der Verbuchung der Aktivitäten der Organisationen ohne Erwerbscharakter. Bei den privaten Organisationen ohne Erwerbscharakter erweckt bereits der Umstand, daß der Wert der Verkäufe 70 \& ihres Produktionswertes ausmacht, Zweifel an der Qualifikation als Organisation ohne Erwerbscharakter. Dieser scheinbare Widerspruch erklärt sich daraus, daß sich die Abgrenzungskriterien der VGR nur auf die Trägerorganisationen beziehen ${ }^{2)}$, während die von

1) H. Bartels: Das Kontensystem.... a.a.O., s. 344 . Dagegen ist für die Finanzstatistik die Erfassung der öffentlichen Unternehmen unabhängig von der Rechtsform vorgeschrieben. GemäB \$ 8 Abs.2 des Gesetzes über die Finanzstatistik gehören - wie bereits erwähnt - zu den öffentlichen Unternehmen alle Unternehmen, an deren Nennkapital oder Stimmrecht die öffentliche Hand zu mehr als $50 \mathrm{v} . \mathrm{H}$. beteiligt ist. Dies ist zwar ein im Hinblick auf die Erfüllung öffentlicher Aufgaben wenig aussagekräftiges, jedoch praktikables Abgrenzungskriterium. Die Empfehlungen internationaler Organisationen zur Behandlung der öffentlichen Unternehmen in den VGR sind unterschiedlich. Während das OECD-Rahmenschema der Volkswirtschaftlichen Gesamtrechnung und das Europäische System Volkswirtschaftlicher Gesamtrechnungen (ESVG) auf den getrennten Ausweis öffentlicher Unternehmen verzichten, sieht ihn das Schema der Vereinten Nationen (SNA) vor. Öffentliche Unternehmen sind im SNA durch staatliches Eigentum (der Staat muB mindestens $50 \mathrm{v} . \mathrm{H}$. der Unternehmensanteile besitzen) und/oder staatliche Kontrolle gekennzeichnet. GroBbritannien weist in seinen VGR, insofern dem Vorschlag der Vereinten Nationen folgend, die öffentlichen Unternehmen gesondert aus. Vgl. D. Brümmerhoff: Die öffentlichen Unternehmen in der volkswirtschaftlichen Gesamtrechnung. Gegenwärtiger stand und Verbesserungsmöglichkeiten, in: Zeitschrift für öffentliche und gemeinwirtschaftliche Unternehmen, Bd. 1 (1978), Heft 1, s. 5-11, S. 8 ff.

2) Offenbar verwendet das Statistische Bundesamt zusätzlich zu den in den Systematikbänden genannten weitere Kriterien zur Abgrenzung der Trägerorganisationen. So findet sich etwa bei Hartmann (Private Organisationen... a.a.O., S. 641) die Aussage, die freiwilligen ehrenamtlichen Leistungen der Mitglieder privater Organisationen ohne Erwerbscharakter $f$ ür ihre Organisationen seien "in vielen Institutionen typisch und begründe(te) $\mathrm{n}$ zu einem wesentlichen Teil deren nichterwerbswirtschaftlichen Charakter". 
einer privaten Organisation ohne Erwerbscharakter betriebenen Einrichtungen unabhängig von der Art ihrer Finanzierung wie ihre Trägerorganisation zugeordnet werden. Uberträgt man die vom Statistischen Bundesamt in seinen Erläuterungen zur systematik der VGR angegebenen Abgrenzungskriterien auf die von privaten Organisationen ohne Erwerbscharakter betriebenen Einrichtungen, so ließe sich ein Teil dieser Institutionen den Unternehmen zurechnen. Zumindest ein Teil dieser Organisationen gibt (gemäß obigem Anteilswert) seine Leistungen überwiegend gegen ein spezielles Entgelt ab. Zudem liegt dieses Entgelt, da es sich bei den Verkäufen der privaten Organisationen ohne Erwerbscharakter zu einem erheblichen Teil um Krankenhauslei-

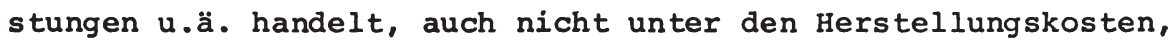
sondern die Pflegesätze werden gemäB Krankenhausfinanzierungsgesetz und Bundespflegesatzverordnung auf der Grundlage der Selbstkosten festgesetzt. Der Umstand, daß die Pflegesätze lediglich die laufenden Betriebskosten decken, während der Staat die Investitionskosten trägt ${ }^{1)}$, ändert an dieser Argumentation nichts.

Die VGR wiesen bis zur Revision 1982 Organisationen ohne Erwerbscharakter in demjenigen sektor nach, der sie überwiegend finanziert. Bei den privaten und den dem Unternehmenssektor zugerechneten Organisationen ohne Erwerbscharakter besteht ein weiteres Kriterium darin, daß ihre Leistungen vorwiegend privaten Haushalten bzw. den Unternehmen zugute kommen. Diese Vorgehensweise ist sinnvoll, solange die VGR für die Organisationen ohne Erwerbscharakter keine.(n) eigenen Sektor(en) konstruieren. Sie impliziert jedoch den Nachweis von unter funktionalen Aspekten vergleichbaren Aktivitäten in (bisher drei, nach der Revision) zwei verschiedenen Sektoren. So werden die wis-

1) Vgl. hierzu S. Eichhorn: Preisbildung im Krankenhaus nach dem Kostendeckungsprinzip, a.a.0., s. 42 ff; M. Sauerzapf: Das Krankenhauswesen in der Bundesrepublik Deutschland... a.a.O., S. $112 \mathrm{ff.;}$ J. Wiemeyer: Krankenhausfinanzierung... a.a.0., s. 78 ff. 
senschaftlichen Forschungsinstitute der Unternehmerverbände dem Unternehmenssektor zugerechnet, während diejenigen der Gewerkschaften im Sektor der privaten Haushalte statistischen Niederschlag finden. Die Revision 1982 führte diesbezüglich insofern zu einer Verringerung der Bandbreite, als die überwiegend staatlich finanzierten Forschungsinstitute jetzt nicht mehr zum Staat, sondern zum Haushaltssektor zählen.

Der Sektor private Haushalte und private Organisationen ohne Erwerbscharakter enthält - entgegen der zielsetzung und den Prinzipien der VGR - sowohl in erster Linie konsumierende als auch mit den privaten Organisationen ohne Erwerbscharakter in erster Linie produzierende Wirtschaftseinheiten. Das Statistische Bundesamt sieht diesen Widerspruch selbst und nennt als Rechtfertigungsgründe die geringe quantitative Bedeutung der Organisationen ohne Erwerbscharakter insgesamt, ein bisher nicht vordringliches wirtschaftspolitisches Interesse an umfassenden Statistiken über Einnahmen und Ausgaben der privaten Organisationen ohne Erwerbscharakter sowie Lücken im statistischen Ausgangsmaterial ${ }^{1)}$. Aus dieser Zusammenfassung resultiert - zumindest für die laufende Berichterstattung - 2) auBerdem ein Informationsverlust. Wichtige Transaktionen zwischen den Untersektoren, insbesondere laufende Ubertragungen wie Kirchensteuern, Kirchgeld, Gewerkschaftsbeiträge u.ä. werden bei der Konsolidierung gegenseitig aufgerechnet ${ }^{3)}$.

Abweichungen in den erfaBten Transaktionen bei der Berechnung der Produktionswerte der verschiedenen Sektoren ergeben sich in zweierlei Hinsicht. Zunächst erhalten beim staat und bei den privaten Organisationen ohne Erwerbscharakter - anders als

1) Vgl. H. Bartels: Das Kontensystem... a.a.O., S. 323; N. Hartmann: Private Organisationen... a.a.O., S. 638 sowie Statistisches Bundesamt: FS 18, Reihe 1... 1981, S. 62 .

2) Für ausgewählte Jahre liegen die Daten vollständig vor.

3) Vgl. N. Hartmann: Private Organisationen... a.a.0., S.639. 
bei den Unternehmen - die Produktionskonten auf der Aufwandsseite weder Schuldzinsen noch eine unterstellte Eigentümermiete. Ihre Produktionswerte schließen somit kein Entgelt für produktive Leistungen der Gebäude und eines Teils des übrigen Sachkapitals ein ${ }^{1)}$.

Die zweite Auslassung betrifft primär die privaten Organisationen ohne Erwerbscharakter. Soweit ihnen Organe, Mitglieder und auch Nichtmitglieder Dienste ehrenamtlich, unentgeltlich oder gegen verhältnismäßig geringes Entgelt zur verfügung stellen, erfassen die VGR sie wegen Bewertungsschwierigkeiten nicht zahlenmäßig 2). Hinzu kommt, daß die VGR die Vorleistungen der privaten Organisationen ohne Erwerbscharakter wegen unentgeltlicher oder verbilligter Bezüge, empfangener Sachspenden u.ä. unter ihrem Marktwert ausweisen. Daher liegen der Eigenverbrauch und der Produktionswert dieser Institutionen in Wirklichkeit über den in den VGR errechneten Werten ${ }^{3)}$.

1) Bombach sieht in der Weglassung der Eigentümermieten des Staates die Beseitigung eines Elementes der Willkür, da die Unterstellung von Eigentümermieten nur für einen sehr kleinen Teil des öffentlichen Vermögens einen Ertragsansatz. implizieren würde. Vgl. G. Bombach: Die öffentliche Finanzwirtschaft... a.a.0., S. 66. Die Verbuchungspraxis bezüglich der staatlichen Zinsausgaben ist in der Literatur nicht unumstritten. So plädiert Bombach dafür, zinsen nicht nur beim Staat, sondern auch bei den Unternehmen über die Redistributionskonten zu verbuchen, da sie kein Maßstab für das in einem Sektor investierte Sachkapital, sondern ein Spiegelbild der intersektoralen Verschuldungsstruktur seien. Vgl. G. Bombach: Staatshaushalt und Volkswirtschaftliche Gesamtrechnung, in: Finanzarchiv N.F., Bd. 17 (1956/57), S.344383, S. 356. Demgegenüber scheinen Littmann und Brümmerhoff die Einbeziehung von $\mathrm{Zinsen}$ in die staatliche Wertschöpfung im Prinzip zu befürworten. Vgl. K. Littmann: Definition und Entwicklung der Staatsquote... a.a.0., S. 72 ff.; D. Brümmerhoff: Gesamtwirtschaftliches Rechnungswesen... a.a.0., S. 57 f. und S. 218 .

2) Dasselbe gilt auch für die ehrenamtlichen Tätigkeiten für die Gebietskörperschaften. Vgl. H. Bartels: Das Kontensystem... a.a.0., s. 332 .

3) Vgl. N. Hartmann: Private Organisationen... a.a.0., S.641. 
Auf der rechten Seite der Produktionskonten der verschiedenen Produzenten treten gravierende Unterschiede in der Qualität der dort erfaßten Posten auf. Die VGR benötigen beim Ubergang von der Aufwands- zur Verwendungsseite der Produktionskonten des Staates und eines Teils der nicht-staatlichen Entseheidungseinheiten einige gedankliche Hilfskonstruktionen, um neben den Verkäufen auch die unentgeltlich abgegebenen Leistungen dieser Institutionen als Bestandteil ihrer Produktion ausweisen zu können.

Im ersten Schritt wird angenommen, daß sämtliche Güter- und Faktoreinsätze beim staat und den betroffenen nicht-staatlichen Entscheidungseinheiten auch zu einem Output führen. Sodann wird unterstellt, daB dieser output ausnahmslos entweder anderen Wirtschaftseinheiten zufließt oder den eigenen Kapitalstock vergröBert. Als dritter schritt erfolgt schließlich die Gleichsetzung (sog. Bewertung) der unentgeltlich abgegebenen Leistungen mit ihren (Herstell-) Kosten ${ }^{1)}$.

Faktisch verzichten die VGR auf jegliche Messung der unentgeltlich abgegebenen Leistungen von staat und nicht-staatlichen Entscheidungseinheiten und weisen - mangels besserer Methoden stellvertretend für die Leistungen Produktionskosten bzw. Inputs, "d.h. praktisch... die Zahl der Beschäftigten multipliziert mit ihren Löhnen"2), aus. Diese "verblüffend einfache Lösung"3) mag im Hinblick auf kreislauftheoretische Fragestellungen noch als tolerabel erachtet werden, sie vermag jedoch

1) Vgl. die Argumentation zur Erfassung und Bewertung unentgeltlich abgegebener Leistungen des Staates bei K. Littmann: Definition und Entwicklung der Staatsquote... a.a.o., S. $40 \mathrm{f}$.

2) D. Brümerhoff: Produktivität des öffentlichen Sektors, in: Finanzarchiv N.F., Bd. 34 (1975/76), S. 226-243, S. 232.

3) K. Littmann: Definition und Entwicklung der Staatsquote... a.a.0., s. 41 . 
für produktionstheoretische Analysen wenig zu leisten. Die ResidualgröBe Eigen-bzw.Staatsverbrauch spiegelt nämlich lediglich den Teil der nach den Konventionen der VGR abgegrenzten Produktionskosten wider, der nicht durch den Wert der verkäufe abgedeckt ist. Es handelt sich bei diesen Inputs zwar um einen Teil der von einer wirtschaftseinheit verbrauchten Inputs, jedoch nicht notwendigerweise auch um diejenigen, die für die Erstellung der Leistungen eingesetzt werden, die sie repräsentieren sollen. So läßt sich nicht ohne weiteres behaupten, eine karitative Organisation, bei der die verkäufe $10 \%$ ihres Produktionswertes ausmachen, setze folglich $90 \%$ der auf der linken Seite ihres produktionskontos enthaltenen Inputs $f u ̈ r$ die Produktion unentgeltlich abgegebener Leistungen ein. Bereits bei den Faktormengen können diesbezügliche Abweichungen auftreten, da die VGR keinen zusammenhang zwischen den verkauften Leistungen und den zu ihrer Erstellung benötigten Inputs über eine Produktionsfunktion generieren und deshalb den Eigenverbrauch nur als Differenz zwischen der summe aller von einem Produzenten aufgewendeten bewerteten Inputs (Produktionswert) und seinen Verkäufen errechnen. Die Aufschlüsselung der Inputs anhand des Anteils der Verkäufe am Produktionswert würde jedoch voraussetzen, daB bei allen Aktivitäten der betrachteten Institution die gleiche produktivität herrscht. Speziell bei den privaten Organisationen ohne Erwerbscharakter bewirkt der Umstand, daB ihre Inputs aufgrund der Vernachlässigung von Sachspenden und ehrenamtlichen Tätigkeiten unvollständig erfaßt sind, eine zusätzliche Vergrößerung der potentiellen Diskrepanzen. Geht man schließlich zur wertmäBigen Betrachtung über, so kommt hinzu, daß die Verkäufe zu Marktpreisen, die durchaus über oder unter den Herstellungskosten liegen können, bewertet werden, der Eigenverbrauch jedoch grundsätzlich Herstellungskosten widerspiegelt.

Damit setzt sich das Sozialprodukt aus dreierlei qualitativ verschiedenen Komponenten zusammen: Bei den Verkäufen der Unternehmen, aber auch der übrigen produzenten, handelt es sich 
um zu Marktpreisen bewerteten Output. Anstelle der unentgeltlich abgegebenen Leistungen des staates und der nicht-staatlichen Entscheidungseinheiten dagegen weisen die VGR Inputs aus, die mit ihren Einstandspreisen bewertet werden. Neben diesen beiden wichtigsten Komponenten existieren in den VGR noch Leistungen, die Outputs darstellen, jedoch zu Herstellkosten bewertet werden, so die selbsterstellten Anlagen und die Lagerbestandsveränderungen an Halb- und Fertigfabrikaten der Unternehmen. Zwischen diesen verschiedenen Komponenten besteht nur insofern - rein rechnerisch - Komparabilität, als sie dieselbe Dimension aufweisen ${ }^{1)}$. Den methodischen Bedenken gegen die in den VGR praktizierte Behandlung von Inputs als unentgeltlich abgegebene Leistungen und damit als Output steht allein der Vorzug der Praktikabilität dieses Verfahrens gegenüber. Zu ihrer Abschwächung kann keinesfalls der Hinweis auf die Bewertung des Faktoreinsatzes zu Einstandspreisen, folglich Marktpreisen, dienen, da er verkennt, daß es sich um die Bewertung von Inputs statt von Outputs handelt ${ }^{2}$. Zudem sind die Preise, die nicht-staatliche Entscheidungseinheiten für ihre Inputs entrichten, nicht in jedem Falle echte Marktpreise. So empfangen karitative organisationen Spenden teilweise auch in Form von herabgesetzten Preisen für Vorleistungen.

Eine unzureichende Aussagefähigkeit bezüglich der Erfüllung öffentlicher und privater Aufgaben läßt sich selbst bei der Erfassung der gegen ein spezifisches Entgelt abgegebenen Leistungen nicht-staatlicher Entscheidungseinheiten konstatieren. Die VGR behandeln diese Leistungen im Prinzip ebenso wie die Verkäufe privater Unternehmen und tragen damit kreislauf theoretischen Fragestellungen Rechnung. Auch im Hinblick auf die

1) Littmann hält die Addition der ersten beiden Sozialproduktskomponenten $f$ ür "methodisch ebenso fragwürdig... wie die Summierung von Äpfeln und Birnen auf der Basis einer'stück'Dimension". K. Littmann: Definition und Entwicklung der Staatsquote... a.a.0., s. 43 .

2) Vgl. ebenda. 
Ziele der öffentlichen Planung entstehen keine Probleme, solange der Staat mit öffentlichen Unternehmen ausschlieBlich fiskalische Interessen verfolgt. Sobald der staat jedoch öffentliche Unternehmen oder andere nicht-staatliche Entscheidungseinheiten zur Verfolgung allokativer und/oder distributiver Ziele einsetzt oder nicht-staatliche Entscheidungseinheiten von sich aus an der Erfullung öffentlicher Planungsziele mitwirken, erweist sich der Wert der Verkäufe allein als ein unzureichendes Erfolgsmaß. In diesen Fällen müBten zusätzliche outputkategorien die mit den Verkäufen oder auch bereits mit den Produktionsaktivitäten, beispielsweise der Unterhaltung bestimmter Eisenbahnstrecken aus regionalpolitischen Gründen, der nicht-staatlichen Entscheidungseinheiten bewirkten Effekte abbilden. Ein eindimensionales Outputmaß vermag derartige Wirkungen nicht oder nur unzureichend zum Ausdruck zu bringen. Dieselben Einwände lassen sich auch gegen die Aussagekraft der unentgeltlich abgegebenen Leistungen nicht-staatlicher Entscheidungseinheiten bezüglich einer mehrdimensionalen staatlichen zielfunktion hervorbringen. Sie gelten hier allerdings noch in verstärktem Maße, da es sich bei den Verkäufen wenigstens tatsächlich um Outputs handelt, von denen aus im Prinzip der SchluB auf die letztlich angestrebten öffentlichen Planungsziele eher möglich ist als vom statistisch nachgewiesenen Eigenverbrauch aus.

Die von nicht-staatlichen Entscheidungseinheiten zur Erfüllung öffentlicher Aufgaben eingesetzten Inputs sind den VGR in ihrer Gesamtheit ebenfalls nicht ohne weiteres zu entnehmen. So schlägt sich die Verfolgung staatlicher ziele teilweise in der Preispolitik bestimmter Anbieter nieder (z.B. die Sozialtarife der Bundesbahn oder die Kreditkonditionen der Wohnungsbauförderungsanstalten). Setzt ein öffentliches Unternehmen seine Preise bewußt niedriger als die Herstellungskosten oder die am Markt erzielbaren Preise, so fliebt faktisch ein in 
den VGR nicht ausgewiesener Transferstrom an die Nachfrager ${ }^{1)}$. Am EinfluB des Staates auf die Preise öffentlicher Unternehmen entzündet sich die Kontroverse, ob deren Uberschüsse bzw. Defizite in den VGR dem Aggregat "Gewinn" zugeschlagen oder als indirekte steuern bzw. Subventionen betrachtet werden sollen ${ }^{2}$. Das Statistische Bundesamt realisiert offenbar die erste Alternative. Die VGR weisen - ebenso wie die Haushaltsrechnung - nur positive, tatsächlich erfolgte oder unterstellte $\mathrm{Z}$ ahlungsströme explizit aus, jedoch keine teilweisen Verzichte auf Einnahmen, aus denen u.a. die Defizite öffentlicher Unternehmen resultieren ${ }^{3)}$.

Unabhängig von der Frage nach der Erfüllung öffentlicher und privater Aufgaben fällt auf, daß das Produktionsergebnis von Gebietskörperschaften, Sozialversicherung und privaten organisationen ohne Erwerbscharakter wegen der erwähnten Art der Verbuchung von unentgeltlich abgegebenen Sachleistungen sowohl Verwaltungs- als auch Sachleistungen enthält. Damit rechnen die VGR die vom staatssektor unentgeltlich abgegebenen Sachleistungen nicht dem Privaten Verbrauch $\mathrm{zu}$, obgleich sie letztlich privaten Haushalten zugute kommen ${ }^{4)}$. Das statistische Bundesamt begründet seine Vorgehensweise damit, daß Höhe und Art der Käufe dieser Sachleistungen in hohem Maße von staatlichen Gesichtspunkten bestimmt würden, und daB die Zurechnung auf einzelne Haushaltsgruppen kaum möglich sei ${ }^{5}$ ). Ebenfalls nicht Bestandteil des Privaten Verbrauchs und auch

1) Ähnlich N. Andel: Subventionen, a.a.O., S. 492.

2) Vgl. G. Bombach: Die öffentliche Finanzwirtschaft... a.a.0., S. 59 .

3) Vgl. A. Stobbe: Volkswirtschaftslehre I. Volkswirtschaftliches Rechnungswesen, 5. Aufl., Berlin, Heidelberg, New York 1980, S. 127.

4) Bei den privaten Organisationen ohne Erwerbscharakter spielt dieses Problem materiell keine Rolle, da ihr Eigenverbrauch Bestandteil des Privaten Verbrauchs ist.

5) Vgl. Statistisches Bundesamt: FS 18, Reihe 1... 1981, S. 85; ähnlich H. Bartels: Das Kontensystem... a.a.O., S. 332 . 
nicht der Einkommen aus unselbständiger Tätigkeit bilden bestimmte meritorische Leistungen der Arbeitgeber für ihre Beschäftigten wie betriebliche Krankenstationen u.ä.

Vergleicht man die VGR mit der im vorhergehenden Kapitel dargestellten Haushaltsrechnung, so weisen sie einige wesentliche Vorzüge auf. Das Problem der Vollständigkeit lösen die VGR gleichsam automatisch, da sie die gesamten wirtschaftlichen Aktivitäten in einer Volkswirtschaft und somit auch diejenigen der nicht-staatlichen Entscheidungseinheiten erfassen. Die unterschiedliche Gewichtung von Inputs vermeiden die VGR von vornherein, indem sie auf einer konzeptionell anderen Ebene als die Haushaltsrechnung ansetzen. Während das staatliche Budget Auszahlungen auflistet, weisen die VGR auf den Produktionskonten der Gebietskörperschaften und der nicht-staatlichen Entscheidungseinheiten im Prinzip - mit den aufgezeigten Lücken die Kosten der Leistungserstellung aus ${ }^{1)}$. Dies manifestiert sich beispielsweise in der zeitlichen Verteilung von Ausgaben für Investitionen mit Hilfe von Abschreibungen und der Einbeziehung von fiktiven $\mathrm{z}$ ahlungsströmen im zusammenhang mit den Altersbezügen der Beamten. Sowohl von der Vollständigkeit als auch von der konzeptionellen Ebene her eignen sich deshalb die VGR besser als die budgetären Ausgaben zur Feststellung von Schwerpunkten (in produktionstheoretischer Hinsicht) der Aktivitäten bzw. Inputs zur Erfüllung öffentlicher Aufgaben. Hierzu trägt auch bei, daß die VGR die am Ende des letzten Kapitels vorgeschlagene Aufspaltung der Kosten der Erfüllung öffentlicher Aufgaben in Produktionsinputs und Transferzahlungen vornehmen. Darüber hinaus weisen die VGR den Vorteil auf, anders als das staatliche Budget neben den Inputs bei einem Teil der nicht-staatlichen Entscheidungseinheiten auch die damit erstellten Leistungen zu messen.

1) Vgl. in diesem Zusammenhang auch H. Hanusch: Verteilung öffentlicher Leistungen. Eine Studie zur personalen Inzidenz, Göttingen 1976, S. $124 \mathrm{ff}$. 
Andererseits verbleiben auch bei der Bestimmung von Schwerpunkten von Aktivitäten zur Erfüllung öffentlicher Aufgaben auf der Basis der Daten der VGR erhebliche Probleme. Die VGR dienen nicht dem Zweck, die Aktivitäten zur Erfüllung öffentlicher denen zur Erfüllung privater Aufgaben explizit gegenüberzustellen. Deshalb versuchen sie, der Heterogenität der Aktivitäten nicht-staatlicher Entscheidungseinheiten Rechnung zu tragen, nicht etwa, indem sie hierfür einen oder mehrere eigene Sektoren konstruieren, sondern indem sie diese Aktivitäten - in zum Teil fragwürdiger Weise - auf die drei vorhandenen Sektoren zuordnen. Dabei soll im Prinzip jeweils zumindest ein großer Teil der Aktivitäten der nicht-staatlichen Entscheidungseinheiten mit denen der übrigen Institutionen des betroffenen Sektors im Hinblick auf die Stellung zum Markt und die Leistungsempfänger vergleichbar sein. Dieses "Einpressen" in ein bereits vorhandenes, enges Schema, verbunden mit dem Nachweis von Institutionen statt Aktivitäten, führt zwangsläufig zu Informationsverlusten und erlaubt es allenfalls annäherungsweise, den Umfang der Aktivitäten nicht-staatlicher Entscheidungseinheiten zur Erfüllung öffentlicher Aufgaben abzuschätzen. Geben sie aus den am Anfang dieses Abschnittes diskutierten Gründen bereits die Relation zwischen den Aktivitäten zur Erfüllung öffentlicher und denjenigen zur Erfüllung privater Aufgaben nicht unverzerrt wieder, so läßt sich die Struktur der Aktivitäten zur Erfüllung öffentlicher Aufgaben den VGR ohne Zusatzinformationen noch weniger entnehmen. Das Statistische Bundesamt veröffentlichte erstmals 1980 Ergebnisse der VGR über die Ausgaben des Staates nach Aufgabenbereichen und kündigte eine jährliche Publikation dieser Daten an ${ }^{1)}$. Erst eine entsprechende Auf-

1) Vgl. G. Kopsch: Ausgaben des Staates nach Aufgabenbereichen in den Volkswirtschaftlichen Gesamtrechnungen, in: Wirtschaft und Statistik, Jahrgang 1980, S. 155-169 sowie Statistisches Bundesamt, Fachserie 18 Volkswirtschaftliche Gesamtrechnungen, Reihe S. 4 Ausgaben des Staates nach Aufgabenbereichen in den Volkswirtschaftlichen Gesamtrechnungen 1970 bis 1978, stuttgart, Mainz 1981. 
stellung für die nicht-staatlichen Entscheidungseinheiten würde es indes erlauben, aus beiden Rechenwerken die Schwerpunkte der Erfüllung öffentlicher Aufgaben auf der Inputebene zu ermitteln.

Der Nachweis der von nicht-staatlichen Entscheidungseinheiten erstellten bzw. bereitgestellten Leistungen in den VGR wurde oben bereits gewïrdigt. Die Beschränkung auf ein eindimensionales Meßkonzept erklärt zwar die möglicherweise unzureichende Aussagefähigkeit der Verkaufswerte im Hinblick auf die öffentlichen Planungsiele, rechtfertigt jedoch keinesfalls die beim Nachweis der unentgeltlich abgegebenen Leistungen erfolgende Behandlung von Inputs als Output. Die Daten der VGR bringen hier lediglich insofern eine geringfügige Verbesserung gegenüber den budgetären Ausgaben, als sie Kosten statt Auszahlungen repräsentieren. Vor allem jedoch resultieren aus der Verwendung unterschiedlicher $M e B-$ und Bewertungsverfahren in den VGR neue Verzerrungen. Die auf der rechten Seite der Produktionskonten erfaßten Vorgänge innerhalb des Marktes sind nicht mit jenen außerhalb des Marktes vergleichbar und somit auch nicht die Aktivitäten des staates mit denen bestimmter nicht-staatlicher Entscheidungseinheiten, so daB sich die VGR nicht zur Bestimmung von Schwerpunkten der Erfüllung öffentlicher Aufgaben auf der Outputebene eignen ${ }^{1)}$. Wir werden deshalb im anschließenden Kapitel verschiedene Ansätze zur Messung der Leistungen nicht-staatlicher Entscheidungseinheiten untersuchen.

1) Hinzu kommt, daB die VGR das Problem der Aufspaltung der Leistungen nicht-staatlicher Entscheidungseinheiten in Endprodukte und intermediäre Leistungen nur bei den gegen ein spezielles Entgelt abgegebenen Leistungen zu lösen vermögen. 
Kapitel IV: ANSÄTZE ZUR MESSUNG DER OFFENTLICHEN LEISTUNGEN NICHT-STAATLICHER ENTSCHEIDUNGSEINHEITEN

Die Messung der (öffentlichen) Leistungen der nicht-staatlichen Entscheidungseinheiten ist eine unumgängliche Voraussetzung für die Beurteilung ihrer Aktivitäten unter ökonomischen Gesichtspunkten sowie für deren eventuelle Integration in eine öffentliche Aufgabenplanung. In der Literatur existieren für die Messung der Leistungen nicht-staatlicher Entscheidungseinheiten mit wenigen Ausnahmen keine eigenständigen Ansätze. Die offenkundigen Ähnlichkeiten ihrer Aktivitäten mit bestimmten Aktivitäten des Staates lassen es jedoch als naheliegend erscheinen, Ansätze zur Messung der Leistungen des Staates auf ihre Eignung für die nicht-staatlichen Entscheidungseinheiten hin zu überprüfen.

Je nachdem, ob verschiedene Leistungseinheiten mit Hilfe einer monetären Bewertung aggregiert werden oder physische Maßeinheiten aufweisen, lassen sich die Ansätze zur Leistungsmessung in eindimensionale und mehrdimensionale einteilen; diese Grobgliederung behalten wir im folgenden bei. Zuvor wollen wir jedoch eine kurze Skizzierung des Begriffs "(öffentliche) Leistungen" vornehmen, die die Grundlage für die Einordnung und die Beurteilung der diskutierten Ansätze abgeben soll.

1. Zum Leistungsbegriff bei nicht-staatlichen Entscheidungseinheiten

Im Rahmen dieser Untersuchung analysieren wir lediglich diejenigen Aktivitäten der nicht-staatlichen Entscheidungseinheiten, die sich auf die Erfüllung öffentlicher Aufgaben richten; insofern handelt es sich bei den von ihnen erstellten bzw. bereitgestellten Leistungen um öffentliche Leistungen. Der Begriff "öffentliche Leistungen" scheint in der $\Gamma$ inanzwissenschaft lange 
Zeit so eindeutig gewesen zu sein, daß er zunächst kaum einer intensiven Analyse für wert befunden wurde. Zentrale Bedeutung erlangte er in der Theorie der Nutzen-Kosten-Analyse sowie - zunächst auBerhalb der Finanzwissenschaft - in der Theorie der nationalen Buchhaltung im Rahmen der Diskussion um die drei Produktionskonzepte comprehensive production concept, restricted market production concept und restricted material production concept ${ }^{1)}$. Wenn heute die Frage, ob die - insbesondere staatlichen - Aktivitäten zur Erbringung öffentlicher Leistungen als Produktion im Sinne der Volkswirtschaftlichen Gesamtrechnungen anzusehen sind, auch nicht mehr umstritten ist, so erscheint doch das Problem, wie diese Leistungen charakterisiert und gemessen werden sollen, keineswegs als gelöst. Im zuge der Kritik an der herkömmlichen Sozialproduktsberechnung und der Tendenz zu ihrer Erweiterung in Richtung auf eine umfassende Sozialberichterstattung, wobei die Sozialindikatorenbewegung eine wesentliche Rolle spielte, entstand auch eine Fülle von Vorschlägen zur Messung öffentlicher Leistungen, auf deren wichtigste wir im Verlauf dieses Kapitels eingehen wollen.

Grundsätzlich lassen sich öffentliche Leistungen auf drei Wegen charakterisieren ${ }^{2)}$ : input- bzw. mittelbezogen, output- bzw. zielbezogen und schließlich als eine Kombination dieser beiden Möglichkeiten in einem Nettokonzept.

Das inputbezogene Konzept verbindet öffentliche Leistungen nicht mit dem Ergebnis der Produktion, sondern mit der Tätigkeit, die $z u$ diesem Ergebnis führt. Die öffentlichen Leistungen sind hier identisch mit den Anstrengungen, mit denen ein AusstoB erbracht

1) Vgl. hierzu vor allem P. Studenski: The Income of Nations ...a.a.O., S. $15 \mathrm{ff}$.

2) Vgl. zum Folgenden H. Hanusch: Verteilung öffentlicher Leistungen... a.a.0., s. $33 \mathrm{ff}$. 
werden soll; sie liegen bereits bei einem Einsatz von Produktionsfaktoren vor und zwar unabhängig von Quantität und Qualität des Produktionsergebnisses, selbst wenn diesem ein negativer Wert beigemessen werden sollte. Für die Messung der Leistungshöhe sind ausschließlich Menge, Dauer und Intensität des Faktoreinsatzes ausschlaggebend. Bewertet man diesen zu Anschaffungspreisen, so erhält man die (Real-) Ausgaben des Staates bzw. der nicht-staatlichen Entscheidungseinheiten.

Das outputbezogene Konzept stellt dagegen auf die Realisierung vorgegebener Ziele ab. Öffentliche Leistungen liegen in dem Grad vor, in dem der staat bzw. nicht-staatliche Entscheidungseinheiten ihre Funktionen, Aufgaben oder Programme erfüllen. Leistung orientiert sich hier an der zielerfüllung bzw. dem Ergebnis oder AusstoB. Sie liegt um so höher, je höher der Grad der Zielrealisation ist, auf den Mitteleinsatz kommt es dabei nicht an.

Das Nettokonzept verbindet das input- und das outputbezogene Konzept miteinander. Öffentliche Leistungen lassen sich einerseits als Verbesserungen bei den Zielen der öffentlichen Planung interpretieren, andererseits erfordert dies den Einsatz von Ressourcen, denen zumindest bei Vollauslastung der Produktionsfaktoren nutzbringende Verwendungsmöglichkeiten in anderen Bereichen der Volkswirtschaft offenstehen. Das Nettokonzept betrachtet die Differenz zwischen dem (Brutto-)Ergebnis des öffentlichen Produktionsprozesses und dem hierfür erforderlichen Ressourcenaufwand als öffentliche Leistung und gibt somit Auskunft darüber, wie hoch per saldo die von staat und nichtstaatlichen Entscheidungseinheiten verursachten Wohlfahrtsgewinne sind. Im Prinzip findet sich eine derartige Vorgehensweise bei der Nutzen-Kosten-Analyse sowie innerhalb der Volkswirtschaftlichen Gesamtrechnungen beim Ausweis von Gewinnen und verlusten. 
Im folgenden wollen wir nur noch das outputbezogene Konzept öffentlicher Leistungen näher betrachten. Das inputbezogene Konzept scheidet aus, weil wir jetzt die Erfüllung öffentlicher Aufgaben durch nicht-staatliche Entscheidungseinheiten und letztlich ihren Beitrag zur Realisation der öffentlichen Planungsziele analysieren. Zudem entsprechen "Leistungen" schon rein intuitiv bzw. semantisch eher dem Ergebnis als dem Aufwand eines Produktionsprozesses, und in diesem Sinne gebrauchten wir den Begriff auch im Verlauf dieser Untersuchung. Sodann scheint uns das Bruttokonzept eine bessere Ausgangsbasis für die Bestimmung des Beitrags eines einzelnen Anbieters öffentlicher Leistungen zur Erreichung der öffentlichen Planungsziele abzugeben als das Nettokonzept. Wăhrend das Bruttokonzept die getrennte Betrachtung von Input und Output bei einem Anbieter erlaubt, führt das Nettokonzept diesbezüglich zu einem Informationsverlust. Es vermag nicht offenzulegen, ob ein möglicherweise geringer Beitrag eines Anbieters öffentlicher Leistungen zur zielrealisation mit einem absolut niedrigen output, oder aber, trotz hohem (Brutto-) Output, mit einer relativ geringen Produktivität dieses Anbieters $z u$ erklären ist.

Zur Konkretisierung des outputbezogenen Leistungskonzepts stehen folgende drei Wege offen ${ }^{1)}$ :

a) das Verfügungsmachtkonzept

b) das mengenmäBige Nutzungskonzept

c) das Konzept der bewerteten Vorteile (und Nachteile)

Nach dem Verfügungsmachtkonzept erbringt der staat Leistungen, wenn er den persönlichen Verfügungsspielraum der Bürger über Sachgüter oder Dienste durch den Einsatz von Ressourcen, durch Transferzahlungen oder durch gesetzgeberische Aktivitäten vergrößert. Diese potentialorientierte Auffassung sieht eine Leistung in der Bereitstellung von bzw. dem Angebot an Gütern.

1) Vgl. H. Hanusch: Verteilung öffentlicher Leistungen... a.a.O., S. $44 \mathrm{ff}$. 
Bereits die Möglichkeit der Bürger, darüber zu verfügen, begründet die Leistung, auf die tatsächliche Inanspruchnahme des vergrößerten Spielraums durch die Begünstigten kommt es nicht an. Hier wie auch bei den beiden anderen Konzepten bestehen Parallelen zu bestimmten Ebenen des im ersten Kapitel dieser Arbeit dargestellten Ziel-Mittel-Schemas. Das Verfügungsmachtkonzept entspricht der Ebene der öffentlichen Produkte einerseits und, folgt man der Interpretation, die in einer bereitgestellten Gütermenge nicht einfach ein quantitatives Angebot sieht, sondern bereits in einer option eine Wohlfahrtssteigerung als verwirklicht betrachtet, der Ebene der quasi-Endprodukte andererseits. Das Verfügungsmachtkonzept erlaubt die Beurteilung der Effizienz bzw. Produktivität, mit der der staat oder nicht-staatliche Entscheidungseinheiten öffentliche Leistungen produzieren oder bereitstellen. Es gibt jedoch keinerlei Auskünfte über die Effektivität dieser Aktivitäten, weil es die Reaktionen der Nachfrager bzw. Nutzer nicht einbezieht ${ }^{1)}$. Im Markt- ebenso wie im Nicht-Marktbereich stellt eine Erhöhung des Angebotes an Gütern noch keine hinreichende Bedingung für eine Wohlfahrtssteigerung dar, sondern es kommt darauf an, ob Nachfrager das Mehrangebot zu den verlangten Konditionen abzunehmen bereit sind ${ }^{2)}$. Die Bestimmung öffentlicher Leistungen nach dem Verfügungsmachtkonzept würde jedoch implizieren, daß die potentiell Begünstigten jedwedes öffentliche Angebot in voller Höhe in Anspruch nehmen und daraus einen Nutzen ziehen.

Das mengenmäßige Nutzungskonzept dagegen konzentriert sich auf die tatsächliche Inanspruchnahme von Gütern. Die öffent-

1) Vgl. in diesem Zusammenhang auch D. Bös (Effizienz des öffentlichen sektors aus volkswirtschaftlicher Sicht, a.a.0.), der statt von "Produktivität" von "X-Effizienz" spricht (S. $299 \mathrm{ff.})$ und bezüglich der Effektivität zwischen einer "allokativen Effizienz" (S. $288 \mathrm{ff.}$ ) und einer "Qualitätseffizienz" (S. $306 \mathrm{ff.}$ ) unterscheidet.

2) Vgl. H. Brüngger und C. Orga: Ansätze zur Messung des Outputs des Staates, a.a.0., S. $371 \mathrm{f}$. 
liche Leistung liegt erst bei der Nutzung eines Angebotes an Gïtern vor, noch nicht bei dessen Existenz allein. Bei der Messung der Leistungshöhe beschränkt man sich nicht auf den Umfang bzw. die Angebotskapazität der öffentlichen Einrichtungen, sondern erfaßt Häufigkeit, Dauer und Intensität der Nutzung. Dieses Konzept entspricht der Ebene der output-objectives im Ziel-Mittel-Schema. Hier besteht Gleichbehandlung mit dem Marktbereich insofern, als von Leistung erst dann gesprochen wird, wenn bereitgestellte Kapazitäten auch tatsächIich in Anspruch genommen werden. Zudem ist die Nutzung eines öffentlichen Angebotes bei Fehlen staatlichen Nachfragezwangs eine notwendige Voraussetzung für die Realisation der öffentlichen Planungsziele, es sei denn, der staat sieht den Erfolg bereits durch das Eröffnen möglichst vieler optionen gewährleistet. Unter bestimmten Voraussetzungen liefert die Messung der mengenmäßigen Nutzung auch Anhaltspunkte für die Beurteilung eines öffentlichen Angebots durch die Zielgruppen, die in seinen GenuB kommen sollen. Falls Individuen die betreffende Leistung freiwillig und aktiv nachfragen, geben sie bereits damit $z u$ erkennen, daB sie die Leistung positiv beurteilen, denn auch bei unentgeltlicher Abgabe ist für die Nachfrager die Nutzung mit bestimmten Kosten, etwa in Form von Zeitverlusten, verbunden. Diese Information allein genügt freilich noch nicht für die Ermittlung einer quantifizierten Bewertung ${ }^{1)}$.

Das - theoretisch anspruchsvollste - Konzept der bewerteten Vorteile schließlich begnịt sich nicht mit der Inanspruchnahme der staatlicherseits bereitgestellten Gïter allein, vielmehr sieht es die Leistung erst dann als gegeben an, wenn die Nachfrager den Gütern einen positiven vert bzw. einen Nutzen beimessen. Das Leistungsniveau liegt um so höher, je

1) Im Rahmen von Nutzen-Kosten-Analysen wird allerdings teilweise versucht, aus der Messung der mengenmäßigen Nutzung in Verbindung mit zusätzlichen Daten Informationen über die Bewertung einer Leistung zu gewinnen. Vgl. dazu unten Abschnitt 2.5.5. in diesem Kapitel. 
besser die Bewertung durch die Benutzer ausfällt, es hängt damit von den Präferenzen der Individuen ab. Eine vollständige Leistungsmessung erfordert allerdings auch analog die Berücksichtigung negativer Bewertungen bzw. bewerteter Nachteile der Betroffenen, die etwa bei unerwünschten Nebenwirkungen der eigentlichen Leistung in Form negativer externer Effekte entstehen können. Beim Konzept der bewerteten Vorteile (und Nachteile) bleibt noch offen, ob die Bewertung durch die Individuen in monetären oder anderen Größen erfolgt. Hier besteht keine Identität, sondern allenfalls eine verwandtschaftliche Beziehung zur Ebene der impact-objectives in der Ziel-Mittel-Hierarchie. Sowohl das Konzept der bewerteten Vorteile als auch die impact-objectives gehen über die bloße Nutzung eines öffentlichen Angebotes hinaus, insofern betrachten beide den öffentlichen output "als intermediäres Zwischenprodukt ('throughput') im Hinblick auf die Erfüllung individueller Bedürfnisse und gesellschaftlicher Ziele"1). Dabei stellen impact-objectives nicht auf die subjektive Bewertung der empfangenen Leistungen oder ihrer Wirkungen durch die betroffenen Individuen $a b$, sondern sie repräsentieren objektive Wirkungen der Leistungen auf die Empfänger. Das Konzept der bewerteten Vorteile hingegen setzt unmittelbar bei den betroffenen Individuen an; allein ihre Bewertung einer Leistung oder ihrer wirkungen ist maßgeblich. Seine operationalisierung stößt allerdings, wie noch $\mathrm{zu}$ begründen ist, in der Realität auf erhebliche Probleme.

Nach diesen Vorüberlegungen können wir Ansprüche an ein Maß für die (öffentlichen) Leistungen nicht-staatlicher Entscheidungseinheiten formulieren. Es sollte grundsätzlich Outputsowie Nachfrageorientierung aufweisen, indem es folgende Voraussetzungen erfüllt:

1) H. Brüngger und C. Orga: Ansätze zur Messung des Outputs des Staates, a.a.0., S. 367 . 
(1) Es soll nur echte Outputeinheiten im Sinne des zielbezogenen Konzeptes als öffentliche Leistungen ausweisen.

(2) Für die Beurteilung der abgegebenen Outputeinheiten sollen ausschlieBlich die Bewertungen der Nachfrager bzw. Empfänger maBgeblich sein.

In idealer Weise wird diesen Ansprüchen das Konzept der bewerteten Vorteile gerecht, wir wollen es daher als ReferenzgröBe für die Beurteilung der im folgenden diskutierten Ansätze zur Outputmessung verwenden. Die Messung echten Outputs sowie die Kontrolle von der Nachfrageseite her ist, wenigstens soweit es die Mengenkomponente betrifft, auch beim Konzept der mengenmässigen Nutzung gewährleistet, es kann somit als "Second-BestLösung" dienen. Eine derartige Senkung des Anspruchsniveaus in bezug auf ein Leistungsma $B$ ist allerdings nur bei Leistungen möglich, die aktive Konsumtion erlauben, oder anders ausgedrückt: bei denen die potentiellen Nachfrager sich selbst von der Nutzung ausschließen können ${ }^{1)}$. Die Möglichkeit des Selbstausschlusses steht dem Empfänger von externen Effekten jedoch grundsätzlich nicht offen ${ }^{2)}$. Erfolgt die Erfüllung öffentlicher Aufgaben durch die Bereitstellung von Gütern, die auf dem Spektrum von reinen privaten bis hin zu reinen öffentlichen Gütern

1) Zur Unterscheidung zwischen aktiver und passiver Konsumtion vgl. etwa K. Schmidt: Zur Geschichte der Lehre von den Kollektivbedürfnissen, a.a.0., S. 353 ff. Zur Frage der AusschluBmöglichkeit durch den potentiellen Nachfrager selbst siehe U. Schmoltzi: Zur Finanzierung privater Organisationen ohne Erwerbscharakter... a.a.0., S. 73 ff.

2) Vgl. hierzu auch die Definition externer Effekte bei T.P. Hill: On Goods and Services, in: The Review of Income and Wealth, Series 23, 1977, S. 315-338, S. 331 f. Demnach unterscheiden sich externe Effekte von Dienstleistungen dadurch, daB letztere mit dem Einverständnis des Empfängers erbracht werden, während dieses bei externen Effekten fehlt. 
(im Sinne der Theorie der öffentlichen Güter) eher beim öffentlichen Pol angesiedelt sind, so externalisiert die Leistung zu einem großen Teil. Demzufolge werden Güter wie Innere und Außere Sicherheit passiv konsumiert. Allerdings existieren auch externalisierte Güter mit aktiver Konsumtion, ein Beispiel bilden Rundfunksendungen. In allen Fällen passiver Konsumtion besagt die mengenmäBige Nutzung noch nichts über die Wohlfahrtsrelevanz der betreffenden Leistungen. Es handelt sich hier faktisch um Zwangskonsum, wobei der Empfänger der Leistung einen positiven, keinen oder gar einen negativen Wert beimessen kann. Ein aussagekräftiges LeistungsmaB muB dann die bewerteten Vorteile anzuvisieren versuchen.

Eine Einschränkung ist auch im Hinblick auf alle meritorischen Güter erforderlich. Hierbei weicht die Bewertung durch die Nachfrager ex definitione von derjenigen durch den staat ab, so daß die Ermittlung der bewerteten Vorteile bei den betroffenen Individuen sich nicht zur Beurteilung der mit der Inanspruchnahme dieser Leistungen verbundenen Auswirkungen auf die Realisation der öffentlichen Planungsziele eignet. Gleichwohl wollen wir auch an ein Leistungsmaß für meritorische Güter die oben beschriebenen Ansprüche stellen. Dem Staat kommt es bei meritorischen Gütern vor allem darauf an, daß sie in größerem (bzw. bei demeritorischen Gütern in geringerem) Umfang nachgefragt werden, als es ohne seine Intervention geschehen würde. Deshalb erlaubt die Messung der mengenmäBigen Nutzung Aussagen über die Realisation der öffentlichen Planungsziele. Die Bewertung durch den staat hingegen ist nicht feststellbar. Mit Sicherheit läBt sich nur sagen, daB sie höher (bzw. niedriger) liegt als diejenige durch die Nachfrager. Ein MaB für die bewerteten Vorteile kann deshalb auch bei meritorischen Gütern nur bei den nachfragenden Individuen ansetzen, es liefert gleichzeitig Informationen über die Mengenkomponente. 
2. Eindimensionale MeBverfahren zur Bestimmung des Leistungsumfangs

2.1. Die Willingness-to-Pay

Die Willingness-to-Pay bzw. Zahlungsbereitschaft repräsentiert eine Form des Konzeptes der bewerteten Vorteile (und Nachteile), bei der die Bewertung in monetären GröBen erfolgt. Sie versucht abzuschätzen, welchen Vorteil Individuen einem Mehr an Gütern bzw. welchen Nachteil sie einem Weniger an Gütern beimessen. Die maximale Zahlungsbereitschaft gibt an, welche Menge an Gütern, ausgedrückt in Geldeinheiten, der Empfänger einer Leistung maximal hinzugeben bereit wäre, um sich den mit dieser Leistung verbundenen Vorteil zu sichern. Sie läBt sich gedanklich ermitteln, indem man ein Wirtschaftssubjekt fragt, "was es bei gegebenem Einkommen und gegebenen sonstigen Preisen für die erste Einheit des zu bewertenden Gutes zu zahlen bereit ist, nach Zahlung des genannten Betrages für die zweite Einheit, nach zahlung dieses Betrages für die dritte Einheit usw."1). Die individuelle marginale maximale Zahlungsbereitschaft nimmt mit wachsender Menge der betreffenden Leistung $a b$, so daB folgender Kurvenverlauf entsteht:

1) N. Andel: Nutzen-Kosten-Analysen, in: Handbuch der Finanzwissenschaft, hrsg. von F. Neumark, Bd. 1, 3. Aufl., Tübingen 1977 , S. 475-518, S. 487 . 
Schaubild 3: Die individuelle marginale maximale Zahlungsbereitschaft (MMZB)

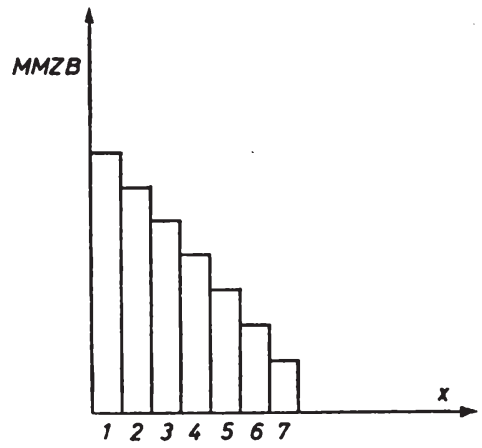

Quelle: N. Andel: Nutzen-Kosten-Analysen, a.a.0., S. 487

Falls die individuellen marginalen maximalen Zahlungsbereitschaften voneinander unabhängig sind, lassen sie sich durch horizontale Addition zur marginalen maximalen Zahlungsbereitschaft auf dem Gesamtmarkt aggregieren. Die maximale Zahlungsbereitschaft für die betreffende Leistung entspricht dann der Fläche unter der resultierenden Kurve.

Die Konstruktion der Kurve der marginalen maximalen Zahlungsbereitschaft weist Ähnlichkeiten mit der Nachfragekurve auf. Beide Kurven stimmen jedoch grundsätzlich nur im Schnittpunkt mit der Ordinatenachse überein, in Punkten rechts davon verläuft die Kurve der marginalen maximalen Zahlungsbereitschaft unterhalb der Nachfragekurve. Dies beruht darauf, daß bei der Nachfragekurve für alle intramarginalen Einheiten derselbe Betrag entrichtet wird, während bei der marginalen maximalen Zahlungsbereitschaft für die intramarginalen Einheiten jeweils entsprechend dieser Zahlungsbereitschaft gezahlt wurde. Bei der Kurve der marginalen maximalen Zahlungsbereitschaft handelt es sich um eine kompensierte Nachfragekurve, die sich durch die Eliminierung des Einkommenseffektes auszeichnet. Beide Kur- 
ven sind identisch, wenn die Einkommenselastizität der Nachfrage nach dem betreffenden Gut Null ist ${ }^{1)}$.

Die maximale Zahlungsbereitschaft, der u.a. im Rahmen von Nutzen-Kosten-Analysen zentrale Bedeutung zukommt, stellt ein Idealkonzept zur Leistungsmessung dar, das erst noch einer Operationalisierung bedarf. Der naheliegende Weg zu ihrer Bestimmung besteht darin, die Leistungsempfänger zur offenbarung ihrer Präferenzen zu bewegen, indem man sie über ihre maximale Zahlungsbereitschaft befragt und die Ergebnisse aggregiert, um so das Leistungsvolumen $z u$ erhalten.

Gegen die Befragung der NutznieBer öffentlicher Leistungen über ihre Zahlungsbereitschaft lassen sich jedoch schwerwiegende konzeptionelle Argumente anführen. Der am häufigsten und bereits von Wicksell vorgebrachte Einwand stellt ab auf die mangelnde Bereitschaft rational handelnder Wirtschaftssubjekte, ihre wahren Präferenzen zu offenbaren, weil jeder einzelne erwarte, auch ohne eigenen finanziellen Beitrag in den GenuB der Leistung $\mathrm{zu}$ kommen ${ }^{2)}$. Andererseits wird teilweise bezweifelt, daB Wirtschaftssubjekte ausschlieBlich ihre eigenen selbstsüchtigen Interessen verfolgen. So behauptet etwa Margolis, als verantwortungsbewuBtes Mitglied einer sozialen Gruppe verhalte sich der einzelne anders als bei seinen alltäglichen rein in-

1) Vgl. ebenda, S. 487 f. Siehe auch M. Friedman: The Marshallian Demand Curve, in: Journal of Political Economy, Vol. 57 (1949), S. 463-495 sowie die ausführliche Darstellung bei H. Hesse: Nutzen-Kosten-Analyse I: Theorie, in: Handwörterbuch der Wirtschaftswissenschaft (HdWW), hrsg. von W. Albers et al., Bd. 5, Stuttgart et al. 1980, S. 361-382, s. $362 \mathrm{ff}$.

2) Vgl. K. Wicksell: Finanztheoretische Untersuchungen nebst Darstellung und Kritik des Steuerwesens Schwedens, Neudruck der Ausgabe Jena 1896, Aalen 1969, S. 100 sowie P.A. Samuelson: The Pure Theory of Public Expenditure, a.a.0., s. $388 \mathrm{f}$. Ausführlich behandelt dieses Argument auch G. Krause-Junk: Probleme der Berechnung und Schätzung öffentlicher Ausgaben, a.a.O., S. $137 \mathrm{ff}$. 
dividuellen Aktivitäten ${ }^{1)}$. Empirische Uberprüungen der FreeRider-Hypothese liegen zwar inzwischen vor, vermochten jedoch die Frage nach der Relevanz strategischen Verhaltens bisher nicht eindeutig zu klären ${ }^{2}$. Daneben trachtet die Forschung, dem Problem des Freifahrerverhaltens zu begegnen, indem sie Verfahren $z u$ entwickeln sucht, die den Befragten keine Anreize zu strategischem Verhalten bieten ${ }^{3)}$. Ein seit einigen Jahren als Methode der Entscheidungsfindung über die Allokation reiner öffentlicher Güter diskutiertes Verfahren, der sog. DemandRevealing Process bzw. die "Clarke-Steuer", erfüllt diese Be-

1) Vgl. J. Margolis: A Comment on the Pure Theory of Public Expenditure, in : Review of Economics and Statistics, Vol. 37 (1955), S. 347-349, S. 348 f.

2) Am bekanntesten dürfte das Experiment von Bohm sein. Vgl. P. Bohm: An Approach to the Problem of Estimating Demand for Public Goods, in: Swedish Journal of Economics, Vol. 73 (1971), S. 55-66 und P. Bohm: Estimating Demand for Public Goods: An Experiment, in: European Economic Review, Vol. 3 (1972), S. 111-130. Zu einer kritischen Würdigung siehe H.-D. Stolper: Zur Leistungsfähigkeit der konsumtheoretischen Analyse der Nachfrage nach öffentlichen Gütern, Berlin 1982 , S. 76 ff. Einen tberblick über die zu diesem Problemkreis durchgeführten empirischen Untersuchungen geben W.W. Pommerehne und F. Schneider: Wie steht's mit dem Trittbrettfahren? - Eine experimentelle Untersuchung -, in: Zeitschrift für die gesamte staatswissenschaft, Bd. 136 (1980), S. 286308 , S. $288 \mathrm{ff}$. und H. Hanusch: Aquivalenzprinzip und kollektive Güter - Allokationstheoretische Aspekte -, in: D. Pohmer (Hrsg.): Beiträge zum Äquivalenzprinzip und zur Zweckbindung öffentlicher Einnahmen, Schriften des Vereins für Socialpolitik N.F., Bd. 121, Berlin 1981, S. 37-91, S. $62 \mathrm{ff}$.

3) Bedingungen zur Ausgestaltung entsprechender Experimente diskutiert M. Kurz: Experimental Approach to the Determination of the Demand for Public Goods, in: Journal of Public Economics, Vol. 3 (1974), S. 329-348. Vgl. auch H. Johann: Theorie der Nachfrage nach öffentlichen Gütern, Frankfurt, Bern, Las Vegas 1977, S. $112 \mathrm{ff}$. und H.-D. Stolper: Zur Leistungsfähigkeit der konsumtheoretischen Analyse... a.a.0., S. 58 ff. 
dingung $^{1)}$. Bei diesem Verfahren stimmen die Bürger über ein oder mehrere öffentliche Projekte ab, indem sie ihre Zahlungsbereitschaft für die verschiedenen Alternativen angeben. Jeder Beteiligte kann das Ergebnis, das sich ohne seine Stimme einstellen wïrde, seinen Vorstellungen entsprechend verändern, falls er einen bestimmten Betrag (die "Clarke-Steuer") an den Staat entrichtet. Er entspricht den Netto-Kosten, die den übrigen Wählern dadurch entstehen, daB die von ihnen präferierte Alternative nicht zum Zuge kommt. Die Erhebung der "Clarke-Steuer" bewirkt, daB sich rational handelnde Individuen am besten stehen, wenn sie ihre wahren Präferenzen offenbaren. Allerdings fehlt bei diesem bisher lediglich auf theoretischer Ebene diskutierten Verfahren bei großen Gruppen für den einzelnen der Anreiz, sich überhaupt an der Abstimmung $z u$ beteiligen, und der zur Ermittlung des Ergebnisses erforderliche Aufwand ist relativ $\operatorname{groB}^{2)}$.

Unabhängig davon, ob die Wirtschaftssubjekte die Free-RiderPosition tatsächlich anstreben oder nicht, betont ein weiterer Einwand, sie seien gar nicht fähig, öffentliche Leistungen in der gleichen Weise wie private Güter zu bewerten. Die Argumentation verläuft hier auf zwei unterschiedlichen Ebenen. So sieht K. Schmidt bei der Bewertung öffentlicher Güter primär

1) Vgl. hierzu T.N. Tideman und G. Tullock: A New and Superior Process for Making Social Choices, in: Journal of Political Economy, Vol. 84 (1976), S. 1145-1159; T.N. Tideman: Introduction, in: Public Choice, Vol. 29-2, Special Supplement to Spring 1977, S. 1-13 und die Diskussion in jenem Sonderband; H. Hanusch: Äquivalenzprinzip und kollektive Güter... a.a.0., S. 65 ff.

2) Vgl. W. Pfähler: Free-Rider-Problem bei öffentlichen Gütern und Clarke-Groves-Steuermechanismus, in: Das Wirtschaftsstudium (WiSu), Jg. 1981, S. 403-408, S. 453-458 und S. 505508, S. 458 . 
Probleme der Wahrnehmung einer Leistung als gegeben an ${ }^{1)}$. Der Nutzen vieler öffentlicher Leistungen trete den Individuen teils wegen der unentgeltlichen Bereitstellung, teils wegen der weitgehend passiven Art der Konsumtion, gar nicht ins BewuBtsein. Besonders schwierig sei die individuelle Bewertung, wenn, wie etwa die Verteidigung, eine Leistung präventiver Natur ist. Überdies werde die individuelle Nutzenschätzung bei öffentlichen Gütern durch unzureichende Informationen erschwert, wenn nicht gar verhindert. Wenigstens dem unzureichenden individuellen Informationsstand lieBe sich zwar im Prinzip durch Aufklärungsmaßnahmen über konkrete Projekte abhelfen, dem stünden jedoch Kostengründe entgegen. Zudem verbiete sich das Weitergeben von Informationen bei vielen Aktivitäten in den Bereichen AuBenpolitik, Verteidigung und Geheimdienste von selbst, weil dies die Leistungen wertlos mache.

Eher produktionstheoretisch argumentiert dagegen olson ${ }^{2}$. Seiner Ansicht nach können die Individuen ihre Präferenzen für öffentliche Leistungen schon deshalb nicht offenbaren, weil sie (und das gilt auch für die Regierung), anders als bei privaten Gütern, bei öffentlichen Gütern über keine oder nur unzureichende Informationen bezüglich der vom staat angebotenen Mengen verfügen. Wenn der Bürger sich bei einer Wahl zwischen Kandidaten entscheiden soll, die für höhere oder niedrigere Ausgaben in einem bestimmten öffentlichen Aufgabenbereich eintreten, sei er auf seine Vermutungen über die staatliche Pro-

1) Vgl. K. Schmidt: Kollektivbedürfnisse und Staatstätigkeit, a.a.O., S. $24 \mathrm{ff}$. Änlich argumentieren auch G. Colm: National Goals Analysis and Marginal Utility Economics. Some Non-Technical Comments on a Highly Technical Topic, in: Finanzarchiv N.F., Bd. 24 (1965), S. 209-224, S. 215 und R.M. Reichhardt: Gesellschaftliche Bedarfsanalyse. Ein Ansatz zur Ermittlung der Bürger-Präferenzen für öffentliche Güter, Berlin 1979, S. $162 \mathrm{ff}$.

2) Vgl. M. Olson: Evaluating Performance in the Public Sector, a.a.O., S. $405 \mathrm{ff}$. 
duktionsfunktion für das betreffende Gut angewiesen. So müsse er etwa abschätzen, in welchem AusmaB erhöhte Ausgaben für die Polizei die Wahrscheinlichkeit reduzieren, Opfer eines Verbrechens $\mathrm{zu}$ werden. Erst im AnschluB daran könne er über die Bewertung der Leistung entscheiden. Uberdies sieht Olson allenfalls beschränkte Möglichkeiten zur Verbesserung des Informationsstandes über den Output an öffentlichen Leistungen. Während er intertemporalen und internationalen Vergleichen nur geringen Informationswert beimiBt, hält er Experimente, beispielsweise Variationen der Höhe der Staatsausgaben für einen bestimmten Aufgabenbereich, im Prinzip für einen erfolgversprechenderen Weg. Da jedoch öffentliche Güter allen Mitgliedern einer Nation oder einer Gruppe zugute kommen, seien die Zahl der möglichen Experimente geringer und die damit verbundenen Kosten höher als bei privaten Gütern.

Diese Einwände erwecken beträchtliche Zweifel an der Eignung der Befragung zur Ermittlung der Leistungen nicht-staatlicher Entscheidungseinheiten. Obgleich sie sich gegen die Befragung über die Zahlungsbereitschaft für reine öffentliche Güter richten, gelten sie teilweise auch gegen Mischgüter. Hier sind die Nachfrager zwar eher als bei reinen öffentlichen Gütern fähig und bereit, ihre Präferenzen zu offenbaren, ihre Bewertung kann sich jedoch lediglich auf den internalisierten Nutzenanteil beziehen. Um allfällige externe Effekte in die Leistungsmessung einzubeziehen, müBte man die Befragung auf einen möglichst großen Empfängerkreis ausdehnen. Dabei entstehen jedoch wiederum die oben beschriebenen Probleme, denn bei den externalisierten Leistungskomponenten handelt es sich um (i.d.R. passiv konsumierte) reine öffentliche Güter.

Auf eher indirektem Wege gehen demoskopische Ansätze vor, bei denen tatsächlich durchgeführte Referenden ausgewertet werden. Die Bürger stimmen dabei über alternative öffentliche projekte und programme $a b$ und kennen häufig auch den auf sie jeweils entfallenden Kosten- bzw. Steueranteil. Das Ziel der Analyse 
eines Abstimmungsergebnisses besteht darin, Erkenntnisse über die politischen Attitüden und ökonomischen Präferenzen der Bürger zu gewinnen. Einige empirische Studien versuchen, anhand nach Wahlbezirken aufgeschlüsselter Abstimmungsergebnisse Zusammenhänge zwischen der Zustimmung zu einem Projekt und vorherrschenden Merkmalen der wähler in einem Bezirk wie z.B. Einkommenshöhe, Bildungsniveau etc. festzustellen. Die Analyse dient dem Zweck, Determinanten der Nachfrage nach staatlichen Angeboten zu identifizieren. Derartige studien wurden für einige Bundesstaaten und Gemeinden in den USA und der Schweiz durchgeführt ${ }^{1)}$. Für die Bestimmung des Umfangs an öffentlichen Leistungen eignen sich diese Ansätze allerdings nur, soweit sie zusätzlich versuchen, Aussagen über die Nachfrage nach den betreffenden Leistungen abzuleiten. Dabei bestehen relativ weite Interpretationsspielräume, da sich demoskopische Ansätze schon von der Konzeption her mit wesentlich weniger Informationen begnügen als die Befragung nach der individuellen Zahlungsbereitschaft. Zwar trifft der einzelne seine Entscheidung über ein öffentliches Projekt in beiden Fällen aufgrund eines individuellen Nutzen-Kosten-Kalküls. Bei einem Referendum kann er jedoch - abgesehen von der Möglichkeit der Stimmenthaltung lediglich seine Zustimmung oder Ablehnung offenbaren. Unterschiedliche Intensitäten der Befürwortung oder Ablehnung eines projektes kommen bei der Abstimmung nicht zum Ausdruck, jede abgegebene Stimme hat das gleiche Gewicht. Der Analytiker kann nur von der Höhe bzw. den Anteilen der Ja- und der Nein-Stimmen bei der Bestimmung der Präferenzen der Bürger ausgehen. Der

1) Vgl. W.C. Birdall: A Study of the Demand for Public Goods, in: R.A. Musgrave (Ed.): Essays in Fiscal Federalism, Washington D.C. 1965, S. 235-294, S. 246 ff.; R.L. Frey und L. Kohn: An Economic Interpretation of Voting Behaviour on Public Finance Issues, in: Kyklos, Vol. 23 (1970), S. 792805; R. Deacon und P. Shapiro: Private Preference for Collective Goods Revealed through Voting on Referenda, in: American Economic Review, Vol. 65 (1975), S. 943-955; E.M. Noam: Demand Functions and the Valuation of Public Goods, in: Public Choice, Vol. 38 (1982), S. 271-280. 
Anteil der Ja-Stimmen läBt sich als grobes MaB für die Intensität des Wunsches der Wahlberechtigten insgesamt nach der zur Diskussion gestellten Änderung gegenüber dem status-quo, und der tuberschuB der Ja- über die Nein-Stimmen als Indikator der Netto-Nachfrage der Wählergesamtheit nach der öffentlichen Leistung interpretieren. Aussagen über die zahlungsbereitschaft der Bürger setzen voraus, daB den Wählern bei der Abstimmung ihr Finanzierungsanteil bekannt war ${ }^{1)}$.

Bei diesem Verfahren spricht vor allem die Anonymität der Stimmabgabe dafür, daß die wähler sich nicht strategisch verhalten. Andererseits ist auch hier nicht auszuschließen, daß die Bürger den (erwarteten) Output eines Projektes nicht oder nur unzureichend abschätzen können. Als Leistungsmaß eignen sich die Daten der demoskopischen Ansätze nur bedingt, Birdsall selbst verweist auf erhebliche Unsicherheitsfaktoren ${ }^{2)}$. Zudem erlaubt die Analyse allenfalls Aussagen über die Nachfrage der Wähler insgesamt. Auf die Präferenzen eines einzelnen Bürgers kann selbst dann nicht geschlossen werden, wenn zusätzliche Daten über sozioökonomische Merkmale von Wählergruppen vorliegen. Schließlich vermag der Anteil der Ja-Stimmen in Verbindung mit den Finanzierungsanteilen lediglich die Untergrenze der bewerteten Vorteile aus einem öffentlichen Angebot zu markieren.

\subsection{Marktpreise}

Mit der Existenz von Marktpreisen sind nahezu ideale Voraussetzungen für die Leistungsmessung gegeben. Die Messung setzt am Output eines produktionsprozesses an und erfaßt nur das als Leistung, was die Nachfrager auch tatsächlich abnehmen, wobei

1) Vgl. W.C. Birdsall: A Study of the Demand for Public Goods, a.a.O., S. 279 ff.

2) Vgl. ebenda, S. 292 ff. 
sie ihre Präferenzen offenbaren. Zudem erlaubt die Bewertung in Geldeinheiten eine einfache Aggregation. Der Leistungsumfang ergibt sich schlicht durch Multiplikation der nachgefragten Mengen mit den dazugehörigen Preisen.

Staat und nicht-staatliche Entscheidungseinheiten geben jedoch nur einen Teil ihrer Leistungen gegen ein spezielles Entgelt $a b$. Marktpreise bilden sich grundsätzlich nur für Güter mit hohem Anteil internalisierter Nutzeneffekte. Die spezifischen Eigenschaften reiner öffentlicher Güter (Nicht-Rivalität, Nicht-AusschlieBbarkeit) bilden allerdings nicht die einzige Ursache für das Fehlen von Marktpreisen für bestimmte öffentliche Leistungen. Der staat etwa stellt - u.a. aus verteilungspolitischen Gründen - auch solche Güter unentgeltlich bereit, die, wie z.B. Bildungsleistungen, grundsätzlich einer Abgabe gegen ein spezielles Entgelt zugänglich wären.

Wenngleich die Marktpreise bzw. die Verkaufswerte die oben skizzierten Anforderungen an ein Leistungsmaß erfüllen, sind doch einige Kritikpunkte anzubringen. Zunächst einmal spiegeln Marktpreise, außer bei den Grenz-Nachfragern, nicht die maximale Zahlungsbereitschaft, sondern eine Untergrenze der Bewertung des betreffenden Gutes durch die Nachfrager wider, da die Konsumentenrenten der intramarginalen Nachfrager nicht in das Leistungsmaß eingehen.

Wie bereits im letzten Kapitel dargestellt, können vor allem bei öffentlichen Unternehmen neben den am Markt abgesetzten Leistungen zusätzliche Outputkategorien in Form externer Effekte auftreten. So beschränkt sich etwa der Kreis der NutznieBer des öffentlichen Personenverkehrs nicht auf die Nachfrager der Transportleistungen. Darüber hinaus kommen auch Nicht-Benutzer in den GenuB externer Vorteile in Form entlasteter StraBen, 
verminderter Lärmbelästigung u.ä. ${ }^{1)}$. In diesem Fall positiver externer Effekte ist der Marktpreis zu niedrig, der Verkaufswert müBte um den Betrag nach oben korrigiert werden, den die Begünstigten maximal für den GenuB der externen Vorteile entrichten würden; analoge Korrekturen wären im Falle negativer externer Effekte vorzunehmen ${ }^{2)}$. Um technologische externe Effekte in ein umfassendes LeistungsmaB für Unternehmen einbeziehen zu können, entwickelte die Betriebswirtschaftslehre in den letzten Jahren sowohl eindimensionale als auch mehrdimensionale Ansätze einer erweiterten Erfolgsrechnung für Unternehmen, die wir unten analysieren wollen.

Speziell bei den nicht-staatlichen Entscheidungseinheiten, die Leistungen im Bereich Gesundheitswesen gegen ein spezielles Entgelt abgeben, kommen weitere Einschränkungen hinzu. Hier besteht die Besonderheit, $\mathrm{da} B$ an den Entscheidungen der Individuen über ihre Nachfrage nach medizinischen Leistungen zumeist Ärzte maBgeblich beteiligt sind ${ }^{3)}$. Im Prinzip gewährleistet zwar die freiwillige Nachfrage der Patienten auch bei ärztlicherseits verordneten Leistungen, daB ihr (erwarteter) Vorteil den preis zumindest abdeckt. Andererseits können mit zunehmender Hilfsbedürftigkeit der Patienten aufgrund des Informationsvorsprungs der Ärzte und ihrer daraus resultierenden Rolle als "Bedarfsfixierer" primär die entrichteten Preise, in Grenzfällen auch die mengenmäBige Nachfrage, an Aussagekraft im Hinblick auf die Beurteilung der Leistungen durch die Em-

1) Zudem weist das öffentliche Angebot an Transportleistungen für aktuelle Nicht-Benutzer möglicherweise den Charakter eines Optionsgutes auf, für dessen Existenz sie einen Preis $\mathrm{zu}$ entrichten bereit sind.

2) Vgl. dazu etwa N. Andel: Nutzen-Kosten-Analysen, a.a.O., S. 490.

3) Vgl. hierzu L. Hajen: Bestimmungsgründe für die parafiskalische Organisation von Krankenversicherung, a.a.0., S. $66 \mathrm{ff}$. und $W$. Krämer: Gesundheit um jeden Preis?, in: Die Zeit, $\mathrm{Nr} .27$ vom 27.6 .1980 , S. 20 . 
pfänger verlieren. Wesentlicher ist jedoch der Umstand, daB im Gesundheitswesen zwei verschiedene Abrechnungssysteme nebeneinander existieren. Einesteils tätigen nicht-staatliche Entscheidungseinheiten Verkäufe, für die die nachfragenden Individuen unmittelbar bezahlen. Im Regelfalle dagegen sind Individuen zwar Empfänger der Leistung, die Bezahlung obliegt jedoch einer anderen nicht-staatlichen Entscheidungseinheit, so etwa bei der Abrechnung der Leistungen von Krankenhäusern in der Trägerschaft karitativer Organisationen mit der Sozialversicherung. Nur im ersten Fall offenbaren die Nachfrager ihre Präferenzen, hier können die entrichteten Beträge - allerdings u.U. mit der oben genannten Einschränkung - als Ausdruck der bewerteten Vorteile dienen. Im zweiten Fall dagegen erhalten die Nachfrager die Leistungen scheinbar gratis. Die Anbieter medizinischer Leistungen und die Träger der gesetzlichen Krankenversicherung legen in Verhandlungen die Preise fest, die Patienten erfahren sie in der Regel jedoch nicht. Deshalb eignet sich hier allenfalls die Mengenkomponente der Verkaufswerte als Ausdruck der mengenmäBigen Nutzung.

Diese Kritikpunkte betreffen den Preis primär in seiner Funktion als Ausdruck der mit der Verwendung des betrachteten Gutes selbst verbundenen Wertschätzung. Hiervon zu unterscheiden ist die Funktion des Preises als Ausdruck der Wertschätzung alternativ herstellbarer Güter. Beide GröBen reflektiert genau der Preis, der den Grenzkosten entspricht ${ }^{1)}$. Abweichungen zwischen dem Marktpreis und der Wertschätzung alternativ herstellbarer Güter bzw. den Opportunitätskosten des betrachteten Gutes können entstehen durch Marktunvollkommenheiten sowie durch staatliche Subventionen und nicht nach dem kostenmäßigen Aqquivalenzprinzip erhobene Abgaben, welche die Anbieter im Preis weitergeben. Derartige Interventionen des Staates betreffen auch viele am Markt abgegebene Leistungen nicht-staatlicher Entscheidungseinheiten. Sie stören zwar nicht die Funktion des Marktpreises als Ausdruck der Wertschätzung des erworbenen Gutes

1) Vgl. N. Andel: Nutzen-Kosten-Analysen, a.a.0.0. 5 s. 490. 
und damit seine Eignung für die Leistungsmessung bei dem Anbieter am Markt. Dieser Marktpreis eignet sich jedoch nicht als Ausdruck der Wertschätzung alternativ herstellbarer Güter, da er Opportunitätskosten verzerrt widerspiegelt. Komplikationen verursacht diese Abweichung insbesondere dann, wenn vorhandene Marktpreise zur Bewertung anderer Güter herangezogen werden.

Trotz dieser Einschränkungen geben die Marktpreise bzw. Verkaufserlöse der nicht-staatlichen Entscheidungseinheiten ein gutes MaB für die Beurteilung ihrer Leistungen durch die Nachfrager $a b$. Daher erscheint es als naheliegend, wenn u.a. in Nutzen-Kosten-Analysen versucht wird, beim Fehlen von Marktpreisen vorhandene Marktpreise ähnlicher Güter als ReferenzgröBe zur Bewertung der unentgeltlich abgegebenen Leistungen heranzuziehen. Bei dieser bereits vor Jahrzehnten diskutierten Methode sucht der Analytiker den öffentlichen Leistungen weitgehend vergleichbare Güter aus privater Produktion und verwendet die dafür entrichteten Marktpreise zur Bewertung abgegrenzter Mengeneinheiten des öffentlichen Angebotes. Schon Colm untersuchte z.B. die Möglichkeit, Verrechnungspreise für die Leistungen des staatlichen Schulsystems in Anlehnung an das Schulgeld von privaten, nicht unterstützten Schulen zu bilden, die den Wert des Unterrichts je Schüler widerspiegeln sollen ${ }^{1)}$.

So theoretisch bestechend dieses Verfahren prima facie erscheint, so einhellig wird es andererseits in der Literatur abgelehnt. Die Einwände beruhen zumeist auf zweifeln daran, ob sich überhaupt äquivalente Leistungen im privaten Sektor finden lassen. Colm verwirft das Verfahren, weil offenbleiben müsse, ob die qualität der Leistungen bei beiden Schultypen gleich ist. Nur bei Leistungen einheitlicher Qualität könne mit derartigen Verrechnungspreisen gearbeitet werden ${ }^{2}$. Nach

1) Vgl. G. Colm: Volkswirtschaftliche Theorie der Staatsausgaben. Ein Beitrag zur Finanztheorie, Tübingen 1927, S. 38 .

2) Vgl. ebenda. 
Ansicht von Kuznets lassen sich auf dem Markt zwar ähnliche Angebote, keinesfalls jedoch spezifische Substitute zu den zu bewertenden öffentlichen Leistungen finden ${ }^{1)}$. Zudem könne dieses Verfahren zu völlig falschen Ergebnissen führen. Er unterstellt, auf dem Markt existiere tatsächlich ein spezifisches Pendant $z u$ einem bestimmten öffentlichen Angebot. Dann liege eine Situation vor, in der Nachfrager freiwillig für eine Leistung bezahlen, die sie auch unentgeltlich vom staat beziehen könnten. Offensichtlich bewerteten die Bürger, die lieber die privat angebotene Leistung kaufen, die staatliche Leistung anders als diejenigen, die das unentgeltliche staatliche Angebot in Anspruch nehmen ${ }^{2)}$. Eine derartige Fehlinterpretation ist nicht nur bezüglich des Niveaus eines Schattenpreises, sondern sogar bezüglich der Richtung seiner Veränderung möglich. Verlagert sich etwa die Nachfrage weg von den staatlichen hin zu Privatschulen und steigen dort in der Folge die Preise, so widerspricht eine Höherbewertung der Leistungen staatlicher Schulen deren Bewertung durch die Nachfrager.

Ein potentieller Anwender dieses Verfahrens sieht sich damit vor ein unlösbares Problem gestellt. Einerseits eignen sich die Marktpreise von lediglich ähnlichen privat angebotenen Gütern nicht zur Bewertung öffentlicher Leistungen, eben weil diese Güter zwar ähnlich, aber nicht hinreichend vergleichbar sind. Andererseits scheiden auch die Preise spezifischer Pendants als Bewertungsmaßstab aus, da es sich selbst bei vollkommener Identität des öffentlichen und des privaten Angebotes in technischer Hinsicht in den Augen der Nachfrager um zwei verschiedene Güter handelt. Der Umstand, daB Individuen für das private Gut freiwillig Marktpreise entrichten, ist - mag es

1) Vgl. S. Kuznets: Government Product and National Income, in: E. Lundberg (Ed.): Income and Wealth, Series 1, Cambridge 1951 , S. 178-244, S. 204 .

2) Vgl. S. Kuznets: On the Valuation of Social Income - Reflections on Professor Hicks' Article, in: Economica, Vol. 15 $(1948)$, S. 1-16 und S. 116-131, S. 11 . 
der öffentlichen Leistung auch noch so ähneln - per se der Indikator für das Fehlen der notwendigen Vergleichbarkeit, denn diese Marktpreise "werden gerade für das gezahlt, was die öffentlichen Einrichtungen typischerweise nicht bieten"1) . Nur noch eine untergeordnete Rolle spielen dann die beiden weiteren Einwände gegen dieses Verfahren: Zweifel an der Eignung der Marktpreise vergleichbarer Güter als BewertungsmaBstab bestehen auch deshalb, weil diese Preise häufig seitens des Staates beeinfluBt sind, beispielsweise durch Zuschüsse an Privatschu$\operatorname{len}^{2)}$. Diese Subventionswirkungen beschränken, wie oben erläutert, die Tauglichkeit der Marktpreise als Ausdruck der Opportunitätskosten, da der Preis eines subventionierten Gutes nur einen Teil der Kosten der Faktoren reflektiert, die bei einem Verzicht auf das Gut für die Produktion anderer Güter zur Verfügung stünden. Sie lassen lediglich seine Funktion als Indikator der Bewertung des erworbenen Gutes durch die Nachfrager und damit seine Verwendungsmöglichkeit für die Leistungsmessung bei dem Anbieter am Markt unberührt. Sodann handelt es sich bei den zu messenden öffentlichen Angeboten i.d.R. um Dienstleistungen, bei denen sich naturgemäB die Aufspaltung in eine Mengen- und eine Preiskomponente auBerordentlich schwierig gestaltet $^{3)}$.

Einen ganz anderen theoretischen Ansatzpunkt zur Bewertung der staatlichen Leistungsabgabe mit Marktpreisen wählt Krause-Junk. Er geht davon aus, daB in einer Marktwirtschaft im privaten Sektor andere WertmaBstäbe herrschen als bei den staatlichen Entscheidungsträgern. Ein einheitlicher BewertungsmaBstab für

1) N. Andel: Nutzen-Kosten-Analysen, a.a.O., S. 493.

2) Implizit scheint dieses Argument bereits bei G. Colm (Volkswirtschaftliche Theorie der Staatsausgaben... a.a.0., S. 38) enthalten $\mathrm{zu}$ sein. Vgl. ferner $\mathrm{K}$. Littmann: Definition und Entwicklung der Staatsquote... a.a.0., S. 45.

3) Vgl. K. Littmann: Definition und Entwicklung der Staatsquote... a.a.0., s. 45 . 
die Leistungsströme beider sektoren läßt sich nur finden, indem die Bewertungen des Marktes auf den staat übertragen werden; für die umgekehrte Vorgehensweise fehlt ein geeignetes Aggregationsverfahren ${ }^{1)}$. Die theoretisch exakte Lösung der geforderten "Als-ob-privat"-Bewertung der vom und zum staat kommenden Leistungsströme besteht in einem "Als-ob-ohne-Staat"-Bewertungsprinzip. Demnach wäre die staatliche Leistungsabgabe nach dem "opportunity-returns-Prinzip" zu bewerten bzw. gewichten. Hierbei handelt es sich nicht um einen Vergleich zwischen staatlicherseits bereitgestellten und von Privaten am Markt angebotenen Gütern, sondern um eine Simulation der Situation ohne staat. Die staatlichen Leistungen sollen mit den Preisen bewertet werden, die sie erzielen würden, wenn Private sie am Markt anbieten würden. In der Praxis scheitert die Bewertung nach dem "opportunity-returns-Prinzip" indes daran, daB die typischen öffentlichen Leistungen sich durch mangelnde Teilbarkeit und zurechenbarkeit auszeichnen und folglich auch bei privater Produktion keine Marktpreise erzielen können ${ }^{2}$.

\subsection{Kosten der Leistungserstellung}

Der in der Literatur üblicherweise unter der irreführenden Bezeichnung "Bewertung der öffentlichen Leistungen zu Kosten" 3 ) firmierende Ansatz verdankt seine Entstehung primär den Bestrebungen, die staatlichen Aktivitäten in das eindimensionale Rechenwerk der Volkswirtschaftlichen Gesamtrechnungen zu integrieren. Die international praktizierte, im letzten Kapitel

1) Vgl. G. Krause-Junk: Staatsausgaben, Einkommensniveau und Wirtschaftsstruktur. Kreislauftheoretische Betrachtungen über Wirkungen öffentlicher Ausgaben, Diss. Berlin 1966, S. $29 \mathrm{ff}$.

2) Vgl. ebenda, S. 34 ff. Für die staatliche Leistungsaufnahme hingegen empfiehlt Krause-Junk die Bewertung nach dem "opportunity-cost-Prinzip". Siehe dazu den folgenden Abschnitt.

3) Vgl. beispielsweise P. Studenski: The Income of Nations... a.a.0., s. 35 . 
bereits analysierte Methode besteht darin, in Ermangelung von Marktpreisen als Wert der unentgeltlich abgegebenen Leistungen von staat und Organisationen ohne Erwerbscharakter die Herstellungskosten dieser Leistungen anzusetzen. Nur wenige Autoren sehen allerdings dieses Verfahren unter theoretischen Gesichtspunkten als befriedigend an, die meisten tolerieren es lediglich aus Gründen der Praktikabilität. Ihre Protagonisten hingegen halten die Kostenbewertung im öffentlichen sektor für ein vollkommenes oder zumindest annäherndes Äquivalent zur Marktpreisbewertung im privaten sektor. Während im privaten Sektor die Bürger ihre Präferenzen für ein Gut am Markt zum Ausdruck bringen können, übernähmen in bezug auf öffentliche Leistungen diese Funktion stellvertretend die staatlichen Entscheidungsträger. So begründet etwa Colm die Analogie zwischen Marktpreis- und Kostenbewertung wie folgt: "...in den Preisrelationen der verschiedenen Leistungen und Waren zueinander kommen die in einem Wirtschaftssystem geltenden Wertrelationen zum verhältnismäBig besten Ausdruck. Die Kosten spielen hierbei nur mittelbar eine Rolle. In der öffentlichen wirtschaft werden die Leistungen jedoch danach gegeneinander abgewogen, was sie kosten. Das Ergebnis dieses Abwägens findet im 'Etat' seinen objektiven Niederschlag. Man muB annehmen, daB beim Ansatz der öffentlichen Leistung die Bewertung der Leistung mit den Kosten in Ubereinstimmung steht, daB das Parlament z.B. Kosten für eine bestimmte Aufgabe nur dann bewilligt, wenn ihm die Erfüllung der betreffenden Aufgabe entsprechend wertvoll erscheint. Unter dieser Voraussetzung können die Kosten als Maßstab für den 'Wert' der Leistung nach dem herrschenden Wertungssystem gelten." 1 )

Mit ganz ähnlichen Argumenten plädiert Hicks für die Kostenbewertung. Die staatlichen Leistungen seien der Gesellschaft min-

1) G. Colm: Volkswirtschaftliche Theorie der Staatsausgaben... a.a.O., S. 74. Hervorhebung im Original. 
destens so viel wert, wie sie kosten. Daher hält er - auBer im Falle einer Produktion mit sinkenden Kosten - die Durchschnittskosten der staatlichen Produktion für ein grobes MaB im Sinne einer unteren Grenze für die von den staatlichen Leistungen gestifteten Grenznutzen ${ }^{1)}$. Die Unterschiede zwischen beiden Axgumentationen sind graduell. Während für Colm die Herstellungskosten öffentlicher Leistungen exakt deren Bewertung widerzuspiegeln scheinen, markieren sie für Hicks lediglich eine Untergrenze. Dabei strebt Colm eine gesamtwirtschaftliche Opportunitätskostenbetrachtung an. Die staatliche Leistungserstellung geht - zumindest bei Vollbeschäftigung - mit einem Ressourcenentzug für den privaten sektor einher. Colm nennt daher als Bedingung für die Aufnahme einer neuen staatstätigkeit, daB die staatlichen Entscheidungsträger den Wert dieser Leistung höher veranschlagen als den Wert der Leistungen, die mit den in der staatlichen Produktion gebundenen Ressourcen im privaten Sektor hergestellt und am Markt abgesetzt werden könnten $^{2)}$. Da sich der Wert der staatsleistungen nach ihren Produktionskosten bemiBt, sind die opportunitätskosten i.d.R. identisch mit den Nominalkosten. Abweichungen treten jedoch auf, sobald der Staat Ressourcen nicht am Markt kauft, sondern kraft hoheitlicher Gewalt in Dienst stellt, wie etwa bei allgemeiner Wehrpflicht. Um die Opportunitätskostenbetrachtung konsequent durchzuhalten, schlägt Colm für derartige Fälle den Ansatz fiktiver Verrechnungskosten statt der Nominalkosten $\operatorname{vor}^{3)}$.

1) Vgl. J.R. Hicks: The Valuation of the Social Income, in: Economica, Vol. 7 (1940), S. 105-124, S. $115 \mathrm{ff}$.

2) Vgl. G. Colm: Volkswirtschaftliche Theorie der Staatsausgaben.... a.a.0., s. 36 .

3) Vgl. ebenda, S. 39.

Eine ähnliche Vorgehensweise scheint auch in einem neueren Revisionsansatz der Sozialproduktsberechnung beabsichtigt zu sein, bei dem sich der Wert bestimmter staatsleistungen durch den Aufwand an Vorleistungen und primären Inputs ergibt. Er soll ausdrücken, "welche Preise theoretisch verlangt werden müBten, um die Knappheit der eingesetzten Ressourcen wiederzugeben...". P. Walser: Volkswirtschaftliche Gesamtrechnung - Revision und Erweiterung, Göttingen 1975, S. 99. 
Einen diametral entgegengesetzten Standpunkt nimmt Krause-Junk ein. Zunächst unterscheidet er zwischen der Input- und der Outputseite des staatlichen Leistungserstellungsprozesses und schlägt für beide unterschiedliche Bewertungsverfahren vor ${ }^{1)}$. $\mathrm{Da}$ er die staatlichen Realausgaben ausschlieBlich auf die Inputseite staatlicher Produktion bezieht, eignen sie sich schon aus diesem Grunde nicht als Ausdruck der Bewertung der staatlichen Leistungsabgabe durch die Bürger. Darüber hinaus bestreitet er jedoch auch die Aquivalenz der von Individuen und der vom Staat am Markt entrichteten Preise als Ausdruck der Bewertung der staatlichen Leistungsaufnahme. Während der Marktmechanismus individuelle Nutzenerklärungen nach dem quantitativen Anteil der einzelnen Wirtschaftssubjekte an den marktwirtschaftlichen Transaktionen gewichte und zu Marktpreisen aggregiere, würden bei Transaktionen des Staates die Nutzenschätzungen der individuellen wertträger anders, nämlich nach ihrem Anteil an der politischen Willensbildung, gewichtet. Als Folge dieser unterschiedlichen Gewichtungssysteme zahle der staat i.d.R. Preise, die jenen auf staatsfreien Märkten nicht entsprächen. Ein einheitlicher Bewertungsmaßstab erfordere vielmehr die Bewertung der staatlichen Leistungsaufnahme nach dem "opportunity-cost-Prinzip", d.h. die vom staat nachgefragten Ressourcen müssen mit den Preisen bewertet werden, die sich für sie am Markt ohne die staatlichen Transaktionen einstellen würden ${ }^{2)}$. Allerdings sieht Krause-Junk selbst die Vergleichbarkeit der Gewichtungssysteme bei einem Teil der staatlichen Leistungsaufnahmen als gegeben an und akzeptiert die vom staat am Markt entrichteten Preise als annähernden Ausdruck der opportunity-costs auf der Inputseite. Bezüglich der staatlichen Lei-

1) Vgl. G. Krause-Junk: Staatsausgaben, Einkommensniveau und Wirtschaftsstruktur... a.a.O., S. $29 \mathrm{ff} . \mathrm{Zu}$ dem von KrauseJunk diskutierten Verfahren zur Bewertung der staatlichen Leistungsabgabe siehe den vorhergehenden Abschnitt.

2) Vgl. G. Krause-Junk: Staatsausgaben, Einkommensniveau und wirtschaftsstruktur... a.a.0., S. $29 \mathrm{ff}$. 
stungsabgabe schlieblich entscheidet er sich für die Kostenbewertung als das einzige in der Realität praktikable verfahren ${ }^{1)}$.

Kuznets stellt im Rahmen der sog. Economica-Debatte die Stellvertreterfunktion der staatlichen Entscheidungsträger in Frage. Seiner Meinung nach ist die Aqquivalenz von Marktpreis- und Kostenbewertung schon deshalb nicht gewährleistet, weil über den Nutzen der Konsumenten aus den öffentlichen Leistungen nichts bekannt sei. Gleichwohl befürwortet er die Kostenbewertung für die Zwecke der Volkswirtschaftlichen Gesamtrechnungen aus Gründen der Praktikabilität ${ }^{2)}$. Operationalitätsgesichtspunkte dürften auch die Erklärung dafür sein, da $B$ in einigen neueren Revisionsansätzen der Sozialproduktsberechnung die Staatsausgaben als MaBstab zumindest für die als direkt konsumwirksam eingestuften staatlichen Leistungen dienen ${ }^{3)}$.

Für die hier vorliegende Fragestellung bildet indes die Kompa-

1) Vgl. ebenda, S. $36 \mathrm{ff}$.

2) Vgl. S. Kuznets: On the Valuation of Social Income... a.a.0., S. 11 f.; ähnlich S. Kuznets: Government Product and National Income... a.a.0., S. 204 f.

3) Vgl. A.W. Sametz: Production of Goods and Services. The Measurement of Economic Growth, in: E.B. Sheldon und W.E. Moore (Eds.): Indicators of Social Change. Concepts and Measurements, New York 1968, S. 77-96, S. 85 ff.; W. Nordhaus und J. Tobin: Is Growth Obsolete?, in: Economic Research: Retrospect and Prospect. Economic Growth, Fiftieth Anniversary Colloquium $v$. National Bureau of Economic Research, New York 1972 , S. 1-80, S. 26 ff.

$\mathrm{Zu}$ dem in Japan entwickelten Ansatz siehe U.E. Simonis: "Nettowohlfahrtsindikator" - ein japanischer Ansatz, in: W. Zapf (Hrsg.): Soziale Indikatoren. Konzepte und Forschungsansätze III, Frankfurt, New York 1975, S. 296-317, S. $298 \mathrm{ff}$. und C. Leipert: Gesellschaftliche Berichterstattung.... a.a.0., s. 220 ff. Der arbeitsorientierte Ansatz verwendet ebenfalls die Kostenbewertung. Vgl. U.-P. Reich, P. Sonntag und H. -W. Holub: Arbeit-Konsum-Rechnung. Axiomatische Kritik und Erweiterung der Volkswirtschaftlichen Gesamtrechnung, Köln 1977, S. 145 ff. Einen tberblick über einige Revisionsansätze der Sozialproduktsberechnung gibt C. Leipert: Unzulänglichkeiten des Sozialprodukts... a.a.O., S. $153 \mathrm{ff}$. 
tibilität mit den Volkswirtschaftlichen Gesamtrechnungen kein relevantes Kriterium, sondern die Kostenbewertung muB sich an den oben skizzierten Anforderungen an ein LeistungsmaB messen lassen. Dabei ist zunächst festzustellen, daB es sich bei der Kostenbewertung, wie im letzten Kapitel aufgezeigt, um ein Verfahren zur Messung von Inputs handelt. Zwar setzen Colm und Hicks offenbar voraus, der reale staatliche output sei den staatlichen Entscheidungsträgern bekannt, insofern stellt sich aus ihrer Sicht lediglich die Frage der richtigen Bewertung, tatsächlich jedoch fehlen den Politikern Informationen nicht nur über den bei den Bürgern anfallenden Nutzen der staatlichen Leistungen, sondern bereits über die Mengenkomponente. Bei der Kostenbewertung beschränkt sich die Bestimmung des realen Outputs auf qualitative Definitionen wie Bildungs-, Gesundheitsleistungen usw., eine eingehendere Messung einzelner Outputeinheiten unterbleibt ebenso wie eine Eingrenzung des Empfängerkreises. Die Auswirkungen auf die Realitätsbezogenheit des Verfahrens sind fatal. Wenn der Output definitionsgemäB gleich dem Input ist, impliziert dies die gleiche Produktivität bzw. Ausgabenintensität der öffentlichen Aufgabenerfüllung in allen staatlichen Aufgabenbereichen (nämlich in Höhe von Eins). Unabhängig davon sind die Implikationen, die sich bei Einbeziehung der Empfänger der unentgeltlich abgegebenen Leistungen ergeben. Bei der Kostenbewertung findet jede vom Staat produzierte oder bereitgestellte Leistungseinheit annahmegemä $B$ auch Abnehmer, wobei sich deren Bewertung der empfangenen Leistungen nach der Höhe der Staatsausgaben bemiBt. Dies bedeutet, daB das staatliche Leistungsangebot stets in Art und Umfang genau den Präferenzen der Bürger entspricht. Der Staat kann ex definitione keine "Ladenhüter" bereitstellen, welche keinen oder gar einen negativen Nutzen stiften. Darüber hinaus vermag dieses LeistungsmaB nicht, allfällige Qualitätsunterschiede zwischen gleichartigen Leistungseinheiten zum Ausdruck zu bringen. Eine Schulstunde etwa weist die Kostenbewertung an jeder beliebigen Schule als qualitativ gleichwertig aus, solange sie nur dasselbe kostet. 
Damit erfüllt die Kostenbewertung trotz der postulierten Analogie zur Marktpreisbewertung keines der Kriterien an ein Leistungsmaß. Weder handelt es sich um die Messung von Outputs, noch spielt die Nachfrage bzw. Nutzung eine Rolle. Beide Schwachpunkte sind nicht etwa eine Folge der Bewertung zu Herstellungskosten ${ }^{1)}$, sondern der unterstellten Identität von Input und Output und des Umstandes, daB jegliches Angebot auch als sofort nachgefragt gilt. Während die erste Annahme theoretisch unhaltbar ist, läBt sich die zweite bis zu einem gewissen Grad noch damit rechtfertigen, daB bei einem Teil des staatlichen Leistungsangebots passive Konsumtion vorliegt. Ob freilich die Bewertung der Leistungen durch die Empfänger mit deren Herstellungskosten übereinstimmt, halten wir für zweifelhaft. Für das Angebot der nicht-staatlichen Entscheidungseinheiten scheidet daher die Kostenbewertung als Leistungsma $B$ aus.

Ein ebenfalls kostenorientiertes Verfahren, das ausschlieblich auf Opportunitätskosten abstellt, kommt im Rahmen von NutzenKosten-Analysen zur Anwendung. Dieses Verfahren verzichtet von vornherein auf die Ermittlung der Zahlungsbereitschaft der Nutznießer einer staatlichen Maßnahme und verwendet stattdessen als Substitut für den Nutzen eines öffentlichen Projekts die Kosten eines alternativen Projekts, das die gleichen Wirkungen erzielen würde und nunmehr überflüssig wird. Das Verfahren hat den Vorteil, daB für die Kosten eher als für die Nutzen Marktpreise zur Verfügung stehen. Es läßt sich jedoch nur unter zwei Bedingungen anwenden: Zum einen müssen die verglichenen Projekte in bezug auf die Nutzenseite identisch sein. Dies setzt u.a. voraus, daB. das alternative Projekt nicht auch andere ziele als das $z u$ bewertende Projekt beeinfluBt. Ein Projekt, das beispielsweise auf Ziel A dieselbe Wirkung ausübt wie das Projekt, dessen Nutzen gemessen werden soll, zusätzlich jedoch auch Ziel B beeinfluBt, stellt keine geeignete Referenz-

1) Auch echte Outputeinheiten können zu Herstellungskosten bewertet werden. 
größe dar. Zum anderen muß entschieden sein, daß die für die Evaluation relevanten Effekte der Nutzenseite in jedem Falle verwirklicht werden sollen. Dies ist dann sichergestellt, wenn als ReferenzgröBe ein privates Projekt dient, das bei Nichtdurchführung des öffentlichen Projekts mit Sicherheit realisiert würde, beispielsweise der Einbau von Doppelfenstern statt der Errichtung von Lärmschutzwällen. Es bietet die Gewähr, daB die Nutzen des Projekts die Kosten auch übersteigen ${ }^{1)}$

Sind schon an die Verwendbarkeit der Alternativkostenmethode strenge Bedingungen geknüpft, so erfährt sie eine weitere Begrenzung von der Aussagekraft ihrer Ergebnisse her. Zwar zielt die Alternativkostenmethode, anders als die Kostenbewertung, nicht auf Inputs, sondern auf die Wirkungen einer Maßnahme, jedoch wird der Output lediglich qualitativ beschrieben. Uber seine Bewertung durch die Empfänger ist nur bekannt, daß sie seine Herstellungskosten übersteigt. Ergibt etwa ein Vergleich einen Kostenvorteil eines öffentlichen gegenüber einem privaten Projekt, so markiert die Kostendifferenz lediglich die Untergrenze für den Nettonutzen des öffentlichen Projekts, entsprechendes gilt für die Kosten des privaten Projekts selbst ${ }^{2}$.

2.4. Nicht-marktmäßige Einnahmen

\subsubsection{Der Payment-Price Approach}

Der von Kuznets ursprünglich im Jahre 1941 zur Erfassung der staatlichen Leistungen im Rahmen der Volkswirtschaftlichen Gesamtrechnungen vorgeschlagene Payment-Price Approach stellt

1) Vgl. N. Andel: Nutzen-Kosten-Analysen, a.a.0., S. 495 und V. Arnold: Nutzen-Kosten-Analyse II: Anwendung, in: Handwörterbuch der Wirtschaftswissenschaft (HdWW), hrsg. von W. Albers et al., Bd. 5, Stuttgart et al. 1980, S. 382-399, S. $396 \mathrm{ff}$.

2) Vgl. V. Arnold: Nutzen-Kosten-Analyse II... a.a.O., S. 396 . 
faktisch ebenfalls einen Ansatz zur Messung des staatlichen Outputs dar ${ }^{1)}$. Kuznets bezeichnet ihn zwar als Bewertungsverfahren, nennt jedoch kein Verfahren, mit dem unabhängig von dieser Bewertung der mengenmäBige Output des staates gemessen werden kann. Vielmehr dient der Payment-Price-Approach beiden Zwecken zugleich. Dieser Ansatz unterstellt, der staat gebe seine Leistungen wie ein Unternehmen gegen Entgelt ab, welches sich in den Zahlungen von Steuern, Gebühren, Beiträgen u.ä. manifestiert. Die von den privaten Haushalten und den Unternehmen entrichteten Zahlungen an den Staat entsprechen dem Wert der ihnen jeweils zugeflossenen staatlichen Leistungen. Zur Begründung verweist kuznets auf den politischen ProzeB, in dem die Gesellschaft uber demokratisch legitimierte Vertreter den Wert der staatlichen Leistungen und entsprechend die $\mathrm{Zah}-$ lungsverpflichtungen der Haushalte und Unternehmen festlege. Die Analogie zu marktwirtschaftlichen Austauschbeziehungen beschränkt sich allerdings auf die Ebene der Sektoren, sie gilt nicht für einzelne Haushalte oder Unternehmen ${ }^{2)}$.

Die Entscheidung zugunsten der Kostenbewertung oder zugunsten des Payment-Price Approach hält Kuznets zwar für eine Wahl zwischen zwei tbeln, dabei weist jedoch seiner Ansicht nach der Payment-Price Approach zwei relative Vorzüge auf ${ }^{3)}$. Zum einen reagierten die Einnahmen des Staates empfindlicher und

1) Vgl. zum Folgenden S. Kuznets: National Income and Its Composition, 1919-1938. Volume I, National Bureau of Economic Research, New York 1954, S. 31 ff.

2) Demgegenüber strebten U.a. E. Sax, K. Wicksell und E. Lindahl eine Form der Besteuerung an, bei der der vom Individuum $z u$ entrichtende Steuerbetrag seiner Bewertung der öffentlichen Leistungen entspricht. Vgl. hierzu die Darstellung bei H. Haller: Die Steuern. Grundinien eines rationalen Systems öffentlicher Abgaben, 2. Aufl., Tübingen 1971, S. $16 \mathrm{f}$.

3) Später verwarf Kuznets den Paymeni-Price Approach wieder zugunsten der Kostenbewertung. Vgl. etwa S. Kuznets: Government Product and National Income... a.a.0., S. 204 f. 
schneller auf kurzfristige Schwankungen im Marktsystem als seine Kosten. Daher sei dieser Ansatz für Analysen mit kurzem Zeithorizont wie die Sozialproduktsberechnung besser geeignet als die Kostenbewertung. Zum anderen träten in Depressionsphasen Haushaltsdefizite auf, weil die staatlichen Einnahmen unter die Kosten sinken würden. Dieser Vorgang finde seine Entsprechung in den Verlusten privater Unternehmen; beide Phänomene lieBen sich als Ausdruck verminderter Wertschätzung der Gesellschaft für die vom Staat bzw. den Unternehmen angebotenen Leistungen interpretieren.

In den USA wurde der auf dem Payment-Price Approach beruhende sogenannte "Tax-Payment Approach" vorübergehend zur Aufspaltung des (unterstellten) Outputs des staates in Vorleistungen an Unternehmen, Leistungen des Endkonsums sowie Kapitalbildung im Rahmen der Sozialproduktsberechnung herangezogen ${ }^{1)}$. Auf dieses Verfahren soll jedoch hier nicht näher eingegangen werden. Vielmehr erhebt sich die Frage nach der Brauchbarkeit des Payment-Price Approach zur Leistungsmessung bei nicht-staatilchen Entscheidungseinheiten. Demnach würde sich bei nicht-staatlichen Entscheidungseinheiten, die ihre Leistungen nicht gegen in freier Vereinbarung ausgehandelte Marktpreise abgeben, das (öffentliche) Leistungsvolumen nach ihren nicht-marktmäßigen Einnahmen bemessen. Wir konzentrieren uns zunächst auf die von nicht-staatlichen Entscheidungseinheiten erhobenen Zwangsabgaben und analysieren im AnschluB daran die Aussagekraft von freiwillig entrichteten Mitgliedsbeiträgen und Spenden.

Das Recht zur Erhebung von Zwangsabgaben ist ein Qualifikationsmerkmal der Parafisci. Wir argumentieren im folgenden am Bei-

1) Eine ausführliche Darstellung dieses Ansatzes gibt er in: S. Kuznets: Government Product and National Income... a.a.o., S. $188 \mathrm{ff}$. Vgl. auch D. Schiele: Die Dienstleistungen des Staates in der Volkswirtschaftlichen Gesamtrechnung, in: Schmollers Jahrbuch für Gesetzgebung, Verwaltung und Volkswirtschaft, 78. Jahrgang (1958), s. 167-197, S. 177 ff.; C. Leipert: Unzulänglichkeiten des Sozialprodukts... a.a.0., S. $87 \mathrm{ff}$. 
spiel der Sozialversicherung, denn sie agiert ausschlieblich in staatlichem Auftrag, so daB sich (im Gegensatz zu den Ständefisci) die Abgrenzung ihrer öffentlichen Leistungskomponente erübrigt, und ihre Aufgabenstellung beschränkt sich nicht (wie beim Lastenausgleichsfonds) auf distributionspolitische ziele. Zudem weisen von allen Zwangsabgaben an Parafisci die Sozialversicherungsbeiträge noch am ehesten Ähnlichkeiten mit Marktpreisen auf. Anders als bei der Zahlung von Steuern hat bei den Sozialversicherungsbeiträgen der Versicherte Anspruch auf eine Gegenleistung. Allerdings besteht zwischen den Beiträgen und der Versicherungsleistung keine marktmäBige Äquivalenz, sondern in die Beitragsgestaltung flieBen auch Elemente des Leistungsfähigkeitsprinzips ein ${ }^{1)}$.

Betrachten wir zum Vergleich den Fall, in dem ein Individuum eine Risikoversicherung bei einem privaten Unternehmen abschliebt. Mit dem freiwilligen VertragsabschluB gibt der Versicherungsnehmer zu erkennen, daB ihm die Versicherungsleistung mindestens soviel wert ist wie die vereinbarte Prämie. Diese entspricht dem Erwartungswert des Schadens sowie den anteiligen Verwaltungs- und Vertriebskosten des Versicherungsunternehmens. Bei "richtiger" Kalkulation decken die Risikoanteile der Prämien aller Versicherten gerade die Auszahlungen für Schadenersatz ab, so daB die Versicherung - bei Betrachtung ex post - lediglich die Schäden auf die Gesamtheit der Versicherten verteilt ${ }^{2}$. Aus der Sicht der Versicherungsnehmer be-

1) Bei Abwesenheit staatlicher Zuschüsse an die Sozialversicherung liegt eine gruppenmäBige Aquivalenz vor.

$\mathrm{Zu}$ den verschiedenen Formen des Aqquivalenzprinzips vgl. vor allem H. Haller: Die Steuern... a.a.O., s. $13 \mathrm{ff}$. und ergänzend K. Mackscheidt und J. Steinhausen: Finanzpolitik I. Grundfragen fiskalpolitischer Lenkung, 3. Aufl., Tübingen, Düsseldorf 1978, S. $16 \mathrm{f}$.

2) Entsprechend weisen die Volkswirtschaftlichen Gesamtrechnungen als Produktionswert der Versicherungsunternehmen im Prinzip lediglich die Verwaltungs- und Vertriebskosten aus. Vgl. Statistisches Bundesamt: Fachserie 18, Reihe 1... 1981, a.a.o., s. 83 . 
schränkt sich die Leistung des Unternehmens jedoch nicht auf die Organisation dieser versicherungsimmanenten Umverteilung, sondern sie besteht (ex ante) in der permanenten Bereitstellung des immateriellen Gutes Versicherungsschutz. Ihn genieBen sie auch dann, wenn kein Schaden auftritt, und sie entrichten dafür, falls ihre Risikoaversion genügend groB ist, einen Preis in Form der Prämie ${ }^{1)}$. Demnach läBt sich die Leistung eines Versicherungsunternehmen's aus der sicht der Nachfrager - auBer bei Kapitallebensversicherungen, deren Prämien als dritte Komponente einen Ersparnisanteil enthalten - im Prinzip nach dem Prämienaufkommen bemessen.

Dieses Ergebnis kann freilich nicht auf die Sozialversicherung übertragen werden. Die Untauglichkeit der Beiträge als LeistungsmaB beruht weniger auf ihrer Zwitterfunktion. Mit Ausnahme der gesetzlichen Unfallversicherung stellen alle Zweige der Sozialversicherung nicht lediglich Versicherungen dar, vielmehr benutzt sie der staat gleichzeitig als Instrument der Redistributionspolitik ${ }^{2}$. Es handelt sich dabei nicht um die versicherungsimmanente Umverteilung, wie sie ex post etwa von den Gesunden zu den Kranken im Rahmen der Krankenversicherung erfolgt, sondern um eine von vornherein beabsichtigte, auf Vorschriften des Gesetzgebers beruhende interpersonelle Umverteilung. Das Sozialversicherungsrecht enthält - vor allem bei der gesetzlichen Kranken- und Rentenversicherung - eine Fülle

1) Zur Bedeutung der Versicherungen aus einzel- und gesamtwirtschaftlicher Sicht vgl. etwa W. Mahr: Einführung in die Versicherungswirtschaft. Allgemeine Versicherungslehre, Berlin 1951, S. $80 \mathrm{ff.;}$ D. Farny: Entwicklungslinien, Stand und wirtschaftliche Bedeutung des Versicherungswesens in Deutschland, in: F.-W. Henning (Hrsg.): Entwicklung und Aufgaben von Versicherungen und Banken in der Industrialisierung, Schriften des Vereins für Socialpolitik N.F., Bd. 105, Berlin 1980, S. 7-28, S. $24 \mathrm{ff}$.

2) Vgl. etwa H. Meinhold: Fiskalpolitik durch sozialpolitische Parafisci, a.a.0., S. $23 \mathrm{ff.;} \mathrm{D.} \mathrm{Lepelmeier:} \mathrm{Soziale} \mathrm{Siche-}$ rung und Parafiskalität... a.a.0., s. 179 ff. 
von Regelungen, die zu beabsichtigten Abweichungen der Beitragsgestaltung vom versicherungstechnischen Aquivalenzprinzip fuhren. Beispielsweise erkennt die Rentenversicherung bestimmte beitragslose Jahre als rentensteigernde Ausfallzeiten an, und die Beiträge in der gesetzlichen Krankenversicherung richten sich weder nach Alter und Geschlecht eines Versicherten noch nach der Zahl der versicherten Familienmitglieder, sondern nach dem Einkommen des Beitragszahlers ${ }^{1)}$. Diese tberlagerung mit verteilungspolitischen Aufgaben wäre für die Zwecke einer reinen Leistungsmessung anhand des Beitragsaufkommens möglicherweise nicht gravierend. Es ist denkbar, daB die von der Umverteilungspolitik benachteiligten Mitglieder ihren Beitrag trotz des distributionspolitischen "Zuschlags" freiwillig

1) Zur Umverteilung über die Sozialversicherung vgl. etwa $\mathrm{N}$. Andel: Verteilungswirkungen der Sozialversicherung am Beispiel der gesetzlichen Krankenversicherung der Bundesrepublik Deutschland, in: W. DreiBig (Hrsg.): రffentliche Finanzwirtschaft und Verteilung III, Schriften des Vereins für Socialpolitik N.F., Bd. 75/III, Berlin 1975, S. 39-82, S. $43 \mathrm{ff.;} \mathrm{W.} \mathrm{Schmähl:} \mathrm{Alterssicherung} \mathrm{und} \mathrm{Einkommensver-}$ teilung. Theoretische und empirische Untersuchungen zur Finanzierung, Leistungsgewährung und zur Verteilung zwischen Generationen, Tübingen 1977, S. 23 ff.; W. Schmähl: Einkommensumverteilung im Rahmen von Einrichtungen der Sozialen Sicherung - Einige Probleme ihrer Ermittlung und Ausgestaltung am Beispiel der gesetzlichen Rentenversicherung -, in: B. Külp und H. D. Haas (Hrsg.): Soziale Probleme der modernen Industriegesellschaft, Schriften des Vereins für Socialpolitik N.F., Bd. 92/II, Berlin 1977, S. 519576 , S. 541 ff.: D. Lepelmeier: Soziale Sicherung und Parafiskalität... a.a.O., S. $179 \mathrm{ff.;}$ C. Helberger und G. Wagner: Beitragsäquivalenz oder interpersonelle Umverteilung in der gesetzlichen Rentenversicherung? - Eine Analyse auf der Grundlage von Lebenseinkommen, in: P. Herder-Dorneich (Hrsg.): Dynamische Theorie der Sozialpolitik, Schriften des Vereins für Socialpolitik N.F., Bd. 123, Berlin 1981 , S. 331-392, S. $337 \mathrm{ff.;} \mathrm{G.} \mathrm{Ott:} \mathrm{Einkommensumvertei-}$ lungen in der gesetzlichen Krankenversicherung. Eine quantitative Analyse, Frankfurt, Bern 1981, S. 64 ff.; W. Albers: Soziale Sicherung. Konstruktionen für die zukunft, stuttgart 1982, S. 58 ff.; R. Kössler: Sozialversicherungsprinzip und Staatszuschüsse in der gesetzlichen Rentenversicherung, Frankfurt, Bern 1982, S. 161 ff.; N. Andel: Finanzwissenschaft, Tübingen 1983, S. $429 \mathrm{ff}$. 
entrichten würden, weil sie die reine Versicherungsleistung aufgrund ihrer Risikoaversion hoch genug einschätzen. Die Leistungsmessung anhand der Sozialversicherungsbeiträge scheitert vielmehr an der Art der Mitgliedschaft und der Beitragserhebung. Der staat läßt die Beiträge, u.a. wegen der Uberlagerung mit verteilungspolitischen Aufgaben, in Form von Zwangsabgaben erheben. Zwangsabgaben vermögen jedoch keinerlei Aufschlüsse über die Zahlungsbereitschaft bzw. Bewertung der Leistungsempfänger $z u$ geben. Uberdies sind die Beiträge zur Hälfte von den Arbeitgebern $z u$ entrichten, insofern unterschätzen die Mitglieder möglicherweise die Kosten ihrer Versicherung. Letztlich führt die mangelnde Nachfragebezogenheit des Payment-Price Approach auch zu einer fehlenden Outputorientierung. Bei der Gleichsetzung von Beitragseinnahmen und Leistungsvolumen wird ein Output nicht gemessen, sondern lediglich unterstellt.

Die gesetzliche Unfallversicherung unterscheidet sich in zweierlei Hinsicht von den übrigen Zweigen der Sozialversicherung. Zum einen entfällt bei ihr die Redistributionskomponente, da sich ihre Beiträge ausschließlich nach versicherungstechnischen Kriterien bestimmen. Zum anderen sind hier weitgehend die Arbeitgeber als Leistungsempfänger anzusehen. Die gesetzliche Unfallversicherung löst im Prinzip die Haftpflicht des Arbeitgebers für Arbeitsunfälle $a b$, und er allein entrichtet die Beiträge ${ }^{1)}$. Gleichwohl verhindert auch hier die Ausgestaltung als Zwangsgemeinschaft mit Zwangsabgaben die Leistungsmessung auf der Basis der Beitragseinnahmen. Es handelt sich dabei weder um die Messung von Output, noch spielt die Bewertung durch die Nachfrager eine Rolle.

1) Vgl. D. Lepelmeier: Soziale Sicherung und Parafiskalität... a.a.O., S. $127 \mathrm{ff}$. 
2.4.2. Freiwillig entrichtete Beiträge und Spenden

Neben den nicht-staatlichen Entscheidungseinheiten mit Zwangsmitgliedschaft und -beiträgen existiert eine Vielzahl von organisationen, die sich aus den Beiträgen ausschließlich freiwillig inkorporierter Mitglieder oder/und Spenden finanzieren. Hier scheint zumindest das Beitragsaufkommen ein gutes MaB für das Leistungsvolumen einer Organisation abzugeben, da diese freiwillig entrichteten Zahlungen einem marktmäßigen Tausch nahekommen. Die Aussagekraft der Mitgliedsbeiträge als Leistungsmaß hängt jedoch - unabhängig davon, ob die Aktivitäten einer Organisation auf die Erfüllung öffentlicher Aufgaben gerichtet sind oder nicht - davon ab, welchem Empfängerkreis die Organisation ihre Leistungen zugute kommen lassen will. Verfolgt ein privater ZusammenschluB ausschlieBlich die Interessen seiner Mitglieder, so gibt der Mitgliedsbeitrag des einzelnen die Untergrenze dafür $a b$, wie er die von ihm empfangenen oder zumindest erwarteten Leistungen der Organisation bewertet. Ein geeignetes Leistungsma $B$ ist dies jedoch nur, wenn sich der Kreis der Mitglieder tatsächlich mit dem der Leistungsbezieher deckt. Selbst wenn die Organisation nur im Interesse ihrer Mitglieder tätig sein will, schliebt das nicht aus, daB aufgrund von Spillovers auch Nicht-Mitglieder an ihren Leistungen teilhaben. In diesem Falle erweist sich das Leistungsmaß Mitgliederbeiträge als unzureichend. Die Mitglieder beziehen in ihre Bewertung nur den auf sie entfallenden Teil der Leistungen ein, nicht jedoch die "Nebenleistungen".

Verfolgt eine Organisation dagegen zumindest teilweise karitative, philanthropische u.ä. Ziele, so versagt das Leistungsmaß Mitgliederbeiträge grundsätzlich. Die Mitglieder beziehen zwar in ihre Bewertung der Organisationsleistungen den Anteil ein, in dessen GenuB AuBenstehende kommen sollen, insoweit handelt es sich hier um eine umfassende Bewertung. Für die nach außen abfließenden Leistungen kommt es jedoch nicht auf die Bewertung durch die Mitglieder, sondern durch deren Empfänger an. 
Noch deutlicher tritt diese Problematik bei den Spenden zutage. Die Literatur beschäftigt sich neuerdings verstärkt mit altruistischen Handlungsweisen einzelner, insbesondere mit Geldspenden sowohl an karitative Organisationen als auch direkt an Individuen. Als Erklärungsansatz für Spendentätigkeit dienen i.d.R. interdependente Nutzenfunktionen von Spender und Empfänger $^{1)}$. Charakteristikum jeder spende an eine nicht-staatliche Entscheidungseinheit mit karitativer zielsetzung ist es, daB der spender sich damit eine Leistung nicht für sich, sondern für einen Dritten erhofft. Die Höhe der Spende - gegebenenfalls korrigiert um die steuerersparnis des spenders - gibt zwar möglicherweise Auskunft über den Nutzen, den der Spender aus seiner Handlungsweise bzw. den damit ermöglichten Aktivitäten der karitativen Organisationen $z u$ ziehen erwartet. Sie gibt jedoch keinerlei Anhaltspunkte dafür, welche Leistungen diese Organisation mit Hilfe der zusätzlichen finanziellen Mittel erbringt oder gar, welchen Wert die Begünstigten ihnen beimessen ${ }^{21}$.

Somit können wir Spenden als LeistungsmaB verwerfen, während die Mitgliedsbeiträge nur unter recht restriktiven Bedingungen die Untergrenze der bewerteten Vorteile aus den Leistungen einer nicht-staatlichen Entscheidungseinheit zu markieren vermögen .

1) Vgl. etwa H.M. Hochman und J.D. Rodgers: Pareto Optimal Redistribution, in: American Economic Review, Vol. 59 (1969), S. 542-557; D. Collard: Altruism and Economy. A Study in Non-Selfish Economics, Oxford 1978.

2) Letzteres gilt auch für Sachspenden. 
2.5. Wirkungen auf privaten Konsum und private Investitionen: Verfahren der Nutzen-Kosten-Analyse

2.5.1. Zur konzeptionellen Eignung der Nutzen-Kosten-Analyse für die Leistungsmessung

Nutzen-Kosten-Analysen versuchen, günstige und ungünstige Folgen eines i.d.R. staatlichen Projektes im Hinblick auf bestimmte Ziele systematisch zu erfassen und (nach Möglichkeit monetär) $\mathrm{zu}$ bewerten. Sie beruhen auf einem Vorteilhaftigkeitsvergleich zwischen den erwarteten künftigen Entwicklungen, die sich bei der Realisierung alternativer konkurrierender Projekte (einschlieblich der Alternative Unterlassen) einstellen; insofern handelt es sich um die Messung projektspezifischer Outputs. Welche Projektwirkungen im Rahmen des Vergleichs als Nutzen und Kosten zu berücksichtigen sind, hängt von den angestrebten zielen $a b$. Ganz allgemein repräsentieren Nutzen (Kosten) positiv (negativ) bewertete Wirkungen eines Projekts auf die verfolgten Ziele. Sofern man die Kosten hier konsequent als Opportunitätskosten begreift, verlangen Nutzen-Kosten-Analysen letztlich einen Nutzen-Nutzen-Vergleich. Eine nähere Definition von Nutzen und Kosten setzt voraus, daB die Zielfunktion, die relevante(n) Alternative(n), die Indikatoren für den Grad der Zielerfüllung sowie die betroffenen Personen festgelegt sind. Uberwiegend steht bei Nutzen-Kosten-Analysen das ziel "effizienter Faktoreinsatz" bzw. die Maximierung der gesellschaftlichen Wohlfahrt im Vordergrund. Unter effizienzanalytischen Kriterien schlagen sich die aus einem bestimmten Projekt resultierenden Nutzen und Kosten in der Summe der in einer Periode produzierten Sachgüter und Dienstleistungen nieder, letztlich manifestieren sie sich in Zuwächsen bzw. EinbuBen der Individuen an Konsummöglichkeiten ${ }^{1)}$. Ihre Ermittlung geht

1) Vgl. N. Andel: Nutzen-Kosten-Analysen, a.a.O., S. 482 ff.; J. Hofmann: Erweiterte Nutzen-Kosten-Analyse. Zur Bewertung und Auswahl öffentlicher Projekte, Göttingen 1981, S. $12 \mathrm{ff}$. 
im Prinzip in zwei Stufen vor sich: Zunächst werden die positiven und negativen Veränderungen der verfügbaren Konsumgütermengen bestimmt, im AnschluB daran erfolgt deren monetäre Bewertung ${ }^{1)}$. Soweit sie nicht auf Marktpreise zurückgreifen kann, sei es, weil diese nicht existieren, sei es, weil die vorhandenen Marktpreise sich im Hinblick auf das Zielsystem nicht eignen, stützt sich die Bewertung auf Schattenpreise, welche die maximale Zahlungsbereitschaft der Individuen repräsentieren sollen. Die maximale Zahlungsbereitschaft bemiBt die Vorteile eines öffentlichen Projektes nach dem Betrag, den die Betroffenen maximal für die positiven Projektwirkungen zu zahlen bereit wären, und entsprechend die Nachteile nach dem Betrag, den die Betroffenen freiwillig maximal aufwenden würden, um das Eintreten der Kosten $z$ verhindern ${ }^{2}$ ). Somit geben aus-

1) Vgl. H. Hesse: Nutzen-Kosten-Analyse I... a.a.O., S. 371.

2) Vgl. N. Andel: Nutzen-Kosten-Analysen, a.a.O., S. 486 sowie Abschnitt 2.1. in diesem Kapitel.

Das Bewertungsprinzip Zahlungsbereitschaft für die Wirkungen eines öffentlichen Projektes, wie es in Nutzen-KostenAnalysen zur Anwendung kommt, läßt sich wohlfahrtstheoretisch auf zwei unterschiedlichen Wegen interpretieren. Nach dem ersten Ansatz spiegelt die maximale Zahlungsbereitschaft für die Wirkungen eines öffentlichen Projekts die Änderung des Nutzenniveaus in der Einschätzung der Individuen genau wider. Dabei handelt es sich letztlich um den Versuch einer kardinalen Nutzenmessung. Sie setzt voraus, daB jedem ermittelten Geldbetrag eine bestimmte Nutzenänderung entspricht. Der zweite Ansatz verzichtet auf eine kardinale Nutzenmessung. Er unterstellt stattdessen ein konstantes Nutzenniveau und ermittelt sog. Kompensationsvariationen. Hierbei handelt es sich um Geldbeträge, die ein Individuum erhalten oder bezahlen müBte, um die Wirkungen eines öffentlichen Projektes auf sein Nutzenniveau genau zu kompensieren. Zahlt bzw. erhält das Individuum diesen Betrag nicht, so realisiert es einen entsprechenden Vorteil ("benefit") bzw. Nachteil. Obgleich sich zunehmend die zweite Interpretation der Zahlungsbereitschaft durchgesetzt hat, bezeichnet die deutschsprachige Literatur zur Nutzen-Kosten-Analyse die Vorteile eines Projektes als "Nutzen". Vgl. H. Hesse: Nutzen-Kosten-Analyse I... a.a.O., S. $362 \mathrm{ff}$. Zum zweiten Ansatz siehe auch E.J. Mishan: CostBenefit Analysis. An Informal Introduction, 2nd Ed., London 1975, S. $24 \mathrm{ff}$. 
schlieBlich die von den Wirkungen eines Projektes betroffenen Individuen die Instanz für deren Bewertung $a b^{1)}$.

Die Eignung vorhandener Marktpreise zur Bewertung öffentlicher Projekte erleidet grundsätzlich Einschränkungen, wenn ein Preis - etwa aufgrund technologischer externer Effekte - nicht die Wertschätzung des betrachteten Gutes oder - u.a. aufgrund von Subventionen - nicht die Wertschätzung alternativ herstellbarer Güter bzw. Opportunitätskosten ausdrückt. In diesen Fällen müssen korrigierte Marktpreise (Schattenpreise) zur Bewertung herangezogen werden, um die Diskrepanzen zwischen Preis und Wertschätzung $\mathrm{zu}$ beseitigen ${ }^{2)}$.

Die Bewertung öffentlicher Leistungen im Rahmen der NutzenKosten-Analyse mit Hilfe von Markt- oder Schattenpreisen als Ausdruck der Zahlungsbereitschaft stöBt in der Literatur teilweise auf heftige Kritik. Verschiedene Autoren sehen in einer sich letztlich an Marktpreisen orientierenden Bewertung eine Inkonsistenz, weil sie die Ubertragung von Kriterien des Marktes auf einen Bereich impliziere, der auBerhalb des Marktes liege und seine Existenz gerade einem Versagen des Marktes verdanke. Die Nutzen-Kosten-Analyse strebe somit eine optimale Allokation im öffentlichen Sektor mit Hilfe von Kriterien an,

1) Vgl. H. Hesse: Nutzen-Kosten-Analyse I... a.a.O., S. 362.

2) Vgl. N. Andel: Nutzen-Kosten-Analysen, a.a.O., S. $490 \mathrm{ff}$. 
die auf einem mängelbehafteten Markt gewonnen würden ${ }^{1)}$. Einige Kritiker plädieren deshalb für eine andere (eindimensionale) Bewertung $^{2)}$ oder für den Verzicht auf eine monetäre Bewertung überhaupt ${ }^{3)}$. Wir halten diese Einwände allerdings für nicht zutreffend. Idealtypisch erfaBt die Nutzen-Kosten-Analyse - im Gegensatz zu privaten Wirtschaftlichkeitsrechnungen - die entstehenden Nutzen und Kosten vollständig, um Aussagen über eine Verbesserung der Allokationseffizienz machen zu können. Bei unvollkommenen Märkten stützt sie sich deshalb gerade nicht auf Marktpreise, sondern auf Schattenpreise.

Kriterium für die Entscheidung zwischen mehreren alternativen Projekten ist i.d.R. der Netto-Nutzen, der sich im Prinzip errechnet, indem die Differenzen zwischen Nutzen und Kosten

1) Vgl. hierzu H.C. Recktenwald: Möglichkeiten und Grenzen der Methode der Nutzen-Kosten-Analyse, in: H. Arndt und D. Swatek (Hrsg.): Grundfragen der Infrastrukturpolitik für wachsende Wirtschaften, Schriften des Vereins für Socialpolitik N.F., Bd. 58, Berlin 1971, S. 233-262 (Diskussion S. 263-288), S. 258 und aus der Diskussion S. 264, S. 274, S. 277 und S. $285 \mathrm{ff.;} \mathrm{G.} \mathrm{Kirsch:} \mathrm{Die} \mathrm{Cost-Benefit-Analyse:}$ Zur Kritik ihrer theoretischen Grundlagen, in: Das Wirtschaftsstudium (WiSu), Jg. 1972, S. 531-535 und S. 581-585, wiederabgedruckt in: G. Kirsch und W. Wittmann (Hrsg.): Nationale Ziele und Soziale Indikatoren, Stuttgart 1975, S. 69-80, insbes. S. $74 \mathrm{ff}$; G. Hesse: Kosten-Nutzen-Analy-. se und souveränes Individuum, in: Jahrbücher für Nationalökonomie und Statistik, Bd. 189 (1975), S. 498-521, S. 500 f. und C. Leipert: Staatskonsum, staatliche Investitionen und die Produktivität staatlichen Handelns, in: Wirtschaftsdienst, Jg. 1979, S. 147-152, S. 151 f. Eher auf die Bewertung staatlicher Leistungen im Rahmen der Outputmessung beziehen dieses Argument $\mathrm{H}$. Brüngger und C. Orga: Ansätze zur Messung des Outputs des Staates, a.a.O., S. 379 .

2) Ein entsprechender Vorschlag findet sich bei G: Hesse: Kosten-Nutzen-Analyse und souveränes Individuum, a.a.O., S. $503 \mathrm{ff}$.

3) So schlagen Leipert und Brüngger/Orga für die Outputmessung die Verwendung sozialer Indikatoren vor. Vgl. C. Leipert: Staatskonsum... a.a.O., S. 152; H. Brüngger und C. Orga: Ansätze zur Messung des Outputs des Staates, a.a.0., s. 379 . 
einer jeden Periode der Laufzeit eines Projekts auf einen bestimmten Zeitpunkt abdiskontiert und aufsummiert werden ${ }^{1)}$. Das Netto-Konzept von öffentlicher Leistung, das die Nutzen-KostenAnalyse damit letztlich verwirklicht, würde zwar grundsätzlich auch für die Leistungsmessung bei nicht-staatlichen Entscheidungseinheiten genügen, doch bringt es erhebliche Informationsverluste mit sich. Wesentlich aussagekräftiger für die Leistungsmessung bei einem einzelnen Projekt sind getrennte Angaben über die Brutto-Nutzen und -Kosten des projekts sowie ihr Anfallen in jeder einzelnen Periode; Daten, die bei der Berechnung des Netto-Nutzens ohnehin vorliegen. Sie informieren nicht nur über die erwünschten, sondern daneben auch über die als negativ eingestuften Outputkomponenten. Zwar beschränken sich Nutzen-Kosten-Analysen primär auf das Ziel Allokationseffizienz, dies gilt jedoch für die anderen bisher diskutierten Ansätze zur Leistungsmessung ebenfalls. Uberdies schlieBt die Nutzen-Kosten-Analyse die Berücksichtigung distributionspolitischer ziele nicht aus ${ }^{2)}$. Ferner erlaubt das MaB Brutto-Nutzen und -Kosten im Gegensatz zum Netto-Nutzen, gegebenenfalls diejenigen Outputkomponenten bzw. Projektwirkungen aus der Betrachtung auszuschließen, die nicht die Erfüllung öffentlicher Aufgaben betreffen.

1) Vgl. N. Andel: Nutzen-Kosten-Analysen, a.a.0., S. $515 \mathrm{ff}$. Zur Diskussion über die im Hinblick auf das Ziel der Allokationseffizienz $\mathrm{zu}$ berücksichtigenden Nutzen- und Kostenelemente siehe vor allem A.R. Prest und R. Turvey: CostBenefit Analysis: A Survey, in: The Economic Journal, Vol. 75 (1965), S. 683-735, S. $687 \mathrm{ff}$; B.A. Weisbrod: Concepts of Costs and Benefits, in: S.B. Chase Jr. (Ed.): Problems in Public Expenditure Analysis, Washington D.C. 1968, S. 257262 ; R.H. Haveman und B.A. Weisbrod: Defining Benefits of Public Programs: Some Guidance for Policy Analysts, in: R.H. Haveman und J. Margolis (Eds.): Public Expenditure and Policy Analysis, 2nd Ed., Chicago 1977, S. 135-160, S. 145 ff.; J. Hofmann: Erweiterte Nutzen-Kosten-Analyse... a.a.O., S. $41 \mathrm{ff}$.

2) Die Einbeziehung von Verteilungsaspekten diskutieren etwa N. Andel: Nutzen-Kosten-Analysen, a.a.0., S. $509 \mathrm{ff}$. sowie J. Hofmann: Erweiterte Nutzen-Kosten-Analyse...a.a.0., S. $56 \mathrm{ff}$. 
Nutzen-Kosten-Analysen kommen, soweit sie die Grundlage für Allokationsentscheidungen abgeben sollen, vor der Durchführung eines Projektes zur Anwendung. Es handelt sich dann um, sich allerdings auch auf Erfahrungswerte stützende, ex ante abgeschätzte Projektfolgen. Für die Zwecke der Leistungsmessung hingegen genügt im Sinne einer Wirksamkeitsanalyse eine Anwendung ex post. Die Einflüsse, die von Einstellungen und persönlichen Ansichten der Analytiker auf das Ergebnis ausgehen können, lassen sich zwar durch die nachträgliche Messung nicht eliminieren, doch bietet sie wenigstens die Chance für eine erhöhte Verläßlichkeit der Ergebnisse.

Die folgenden Abschnitte 2.5.2. bis 2.5.5. stellen einige grundlegende Verfahren der Nutzen-Kosten-Analyse zur Messung und Bewertung der Wirkungen öffentlicher Projekte sowie deren Anwendungsbereiche dar und versuchen, jeweils spezifische Schwächen und Unsicherheitsfaktoren aufzuzeigen. Der Abschnitt 2.5.6. schließlich untersucht die Eignung eines oder mehrerer dieser Verfahren für die Leistungsmessung bei nicht-staatlichen Entscheidungseinheiten ${ }^{1)}$.

2.5.2. Qualitätsverbesserungen privater Produktionsfaktoren

Viele öffentliche Leistungen gehen nicht direkt in den privaten Konsum ein, sondern erhöhen auf dem Wege über Qualitätsverbesserungen die Produktivität im privaten sektor eingesetzter Produktionsfaktoren. Der Nutzen der öffentlichen Leistung bemiBt sich hier nach dem Wert des durch die Produktivitätssteigerung erzielbaren Mehrprodukts. Solche Qualitätsverbesserungen lassen

1) Zu zwei weiteren Verfahren der Nutzen-Kosten-Analyse, der Bewertung öffentlicher Leistungen mit Marktpreisen vergleichbarer Güter und der Alternativkostenmethode, siehe die Abschnitte 2.2 . und 2.3. oben in diesem Kapitel. 
sich vor allem bei den Faktoren Boden und Arbeit erzielen. An MaBnahmen zur Verbesserung des Bodens seien staatliche Be- und Entwässerungsprojekte, Hochwasserschutzprojekte usw. erwähnt; die Produktivität des Faktors Arbeit läßt sich wesentlich beeinflussen durch nahezu alle Aktivitäten des Bildungswesens sowie eine Reihe von MaBnahmen im Gesundheitswesen.

Zur Erfassung und Bewertung der Qualitätsverbesserungen stehen zwei Methoden zur Verfügung. Die erste versucht, das durch die Produktivitätssteigerungen bewirkte Mehrprodukt zu ermitteln und mit den dafür auf dem Markt erzielten Preisen zu bewerten ${ }^{1)}$. Sie findet bevorzugt Anwendung bei MaBnahmen zur Verbesserung landwirtschaftlich genutzter Flächen. So führen Bewässerungsprojekte zu einem landwirtschaftlichen Mehrertrag und folglich Einkommenssteigerungen bei den betroffenen Bauern. Der bewertete Mehrertrag pro Einheit Boden dient als Schattenpreis einer Einheit Wasser aufgrund der Annahme, der Bauer sei bereit, einen seinem Nutzen aus dem Projekt entsprechenden Preis zu entrichten ${ }^{2)}$.

Soweit diese Methode bei Projekten zur Verbesserung des Faktors Boden zum Einsatz kommt, richten sich Einwände weniger gegen die Ermittlung des realen Mehrertrags als vielmehr gegen die Berechnung der Schattenpreise. Diese können insbesondere dann über der tatsächlichen Zahlungsbereitschaft der Bauern liegen, wenn die Analyse den Landwirten zur Verfügung stehende alternative Maßnahmen zur Ertragssteigerung und deren Kosten nicht berücksichtigt ${ }^{3)}$. Bei Projekten im

1) Vgl. V. Arnold: Nutzen-Kosten-Analyse II... a.a.O., S. 383.

2) Vgl. J. Margolis: Shadow Prices for Incorrect or Nonexistent Market Values, in: R.H. Haveman und J. Margolis (Eds.): Public Expediture and Policy Analysis, 2nd Ed., Chicago 1977, S. 204-220, S. 214 .

3) Vgl. ebenda, S. $214 \mathrm{f}$. 
Bildungs- und Gesundheitswesen stöBt dagegen bereits die Bestimmung des Mehrertrags auf Schwierigkeiten. Zum einen ist kaum vorhersehbar, bei der Produktion welchen Gutes die NutznieBer dieser Leistungen zukünftig Verwendung finden, zum anderen wird der Faktor Arbeit i.d.R. in Kombination mit weiteren Faktoren genutzt, was die Abschätzung des Netto-Erfolgs der öffentlichen Maßnahme erschwert ${ }^{1)}$.

Die zweite Methode zur Erfassung und Bewertung der Qualitätsverbesserungen der Faktoren Boden und Arbeit beruht auf dem Rentenkonzept. Führt beispielsweise ein staatliches projekt zur Verbesserung des Bodens zu Gewinnen bel den betroffenen Bauern, so werden andere Bauern den Eigentümern dieser Grundstücke höhere als die bisher bezahlten Pachtpreise bieten. Bei vollkommener Konkurrenz steigen die Pachtpreise solange, bis keine Gewinne mehr vorhanden sind. Der Nutzen des staatlichen Projektes zur Verbesserung der Bodenqualität schlägt sich schließlich in erhöhten Renten bei den Bodeneigentümern nieder. Der Perioden-Nutzen läßt sich ermitteln, Indem die Differenz zwischen dem neuen und dem alten Pachtpreis mit der Menge des verbesserten Bodens multipliziert wird. Dagegen ergibt sich der Gesamtnutzen des Projektes entweder aus der Summe aller abdiskontierten zukünftigen Rentenerhöhungen oder aber, da bei vollkommener Konkurrenz die Bodenpreise dem Gegenwartswert aller zukünftigen Nettoerträge entsprechen, aus der Differenz der Bodenpreise ${ }^{2)}$.

Auf analoge Weise lassen sich staatliche Blldungsaktivitäten behandeln. Sie erhöhen die Qualifikation der Betroffenen und damit in der Regel ihre Einkommenserzielungschancen. Der Nutzen der Maßnahme besteht in dem zusätzlich erzielbaren kapitalisierten Einkommen der Ausgebildeten abzüglich ihrer während der

1) Vgl. V. Arnold: Nutzen-Kosten-Analyse II... a.a.0., s. 383.

2) Vgl. ebenda, S. $383 \mathrm{f}$. 
Ausbildungszeit entgangenen Einkommen. Die Ermittlung dieser Elnkommensdifferenz erfolgt primär auf der Basis von statistischen Querschnittsanalysen üher den Zusammenhang zwischen Ausbildung und Arbeitseinkommen ${ }^{1)}$.

Gegen diese zweite Methode bestehen erhebliche theoretische Einwände. Es läßt sich zeigen, daß nur unter recht restriktiven Bedingungen der Einkommenszuwachs eines Faktors dem durch die Produktivitätssteigerung bewirkten bewerteten Mehrertrag entspricht; i.d.R. wird die Einkommenssteigerung dieses Faktors kleiner $\operatorname{sein}^{2}$. Speziell bei Maßnahmen im Bildungsbereich ist in diesem zusammenhang auf durch Tradition und institutionelle Hemmnisse hervorgerufene Marktunvollkommenheiten $\mathrm{zu}$ verweisen $^{3)}$. Beim Staat als Arbeitgeber kommt erschwerend hinzu, daB er seine Beschäftigten gar nicht nach dem Wertgrenzprodukt entlohnen kann ${ }^{4}$ ) Zudem verfolgt er mit Bildungsmaßnahmen nicht ausschließlich das Ziel, die Einkommen der Begünstigten zu verbessern ${ }^{5)}$. Mit einer derartigen Zielsetzung wären Bildungsaktivitäten zugunsten von Personen, die voraussichtlich niemals erwerbstätig sein werden, nicht zu vereinbaren. Auch aus der Sicht der Ausgebildeten erweist sich diese eindimensionale Betrachtungsweise als zu eng, weil sie den möglicherweise ebenfalls vorhandenen konsumtiven Charakter von Bildung verleugnet ${ }^{6)}$. Darüber hinaus ist zumindest in Ländern mit einem vielfältigen Angebot an Ausbildungsmöglichkeiten nicht von vornherein auszuschließen, daß bestimmte Personen auch ohne die staatlichen

1) Vgl. J. Margolis: Shadow Prices... a.a.O., S. 215 f.

2) Vgl. V. Arnold: Nutzen-Kosten-Analyse II... a.a.O., S. 384 ff.

3) Vgl. N. Andel: Nutzen-Kosten-Analysen, a.a.O., S. 497.

4) Vgl. V. Arnold: Nutzen-Kosten-Analyse II... a.a.0., S. 387.

5) Vgl. J. Margolis: Shadow Prices... a.a.O., S. 215 f.

6) Vgl. M. Weiß: Effizienzforschung im Bildungsbereich. Aufgabenfelder, Methoden und empirische Befunde, Berlin 1982, S. $194 \mathrm{f}$. 
MaBnahmen eine entsprechende Qualifikation erwerben. Sodann läßt sich auch die Ausgangsbasis der Nutzenberechnung, die Querschnittsanalysen über den Zusammenhang zwischen Ausbildung und Entlohnung, angreifen. Ferner müssen solche Analysen neben der Ausbildung auch weitere Einflubfaktoren auf das Arbeitseinkommen wie Intelligenz und Lebensalter berücksichtigen, was die Ubertragung der Ergebnisse nur auf weitgehend vergleichbare Gruppen zuläBt ${ }^{1)}$. SchlieBlich sind diese Untersuchungen primär angebotsorientiert, da sie die Nachfrageseite auf dem Arbeitsmarkt nur unzureichend berücksichtigen ${ }^{2)}$.

Noch gravierendere Probleme entstehen bei der Anwendung dieses Verfahrens auf MaBnahmen im Bereich des Gesundheitswesens. Der Nutzen medizinischer Leistungen bestimmt sich nach dieser Methode gemäß der dadurch ermöglichten zusätzlichen Arbeitszeit und erhöhten Produktivität, die mittels des geltenden Lohnsatzes in monetäre Größen überführt werden. Der Wert eines erhaltenen Lebens entspricht demnach dem kapitalisierten Einkommensstrom, den der Gerettete in Zukunft erwartungsgemäB erzielen wird. Gegen diese Betrachtungsweise läßt sich - zusätzlich zu einigen oben bereits angeführten Argumenten - vor allem einwenden, $d a B$ Gesundheit in weitaus gröBerem MaBe Merkmale eines Konsumgutes aufweist als Bildung. Die Sorge um verlorene Arbeitszeit dürfte kaum das alleinige Motiv für das Bedürfnis des einzelnen nach MaBnahmen zur Erhaltung seiner Gesundheit sein. Würde sich der staat bei der Planung von MaBnahmen im Gesundheitswesen ausschlieblich am Kriterium des Einkommenszuwachses orientieren, so dürfte er den Krankheiten von Alteren und Hausfrauen kaum noch Beachtung schenken ${ }^{3)}$.

1) Vgl. N. Andel: Kosten-Nutzen-Analysen, a.a.O., S. 497.

2) Vgl.M. WeiB: Effizienzforschung im Bildungsbereich... a.a.O., S. $194 \mathrm{f}$.

3) Vgl. J. Margolis: Shadow Prices... a.a.o., S. 216. Siehe auch E.J. Mishan: Cost-Benefit Analysis... a.a.0., S. 298 ff. 
2.5.3. Qualitätsverbesserungen privater Konsumgüter

Konsumgüter lassen sich zumeist durch mehrere Eigenschaften und deren unterschiedliche Ausprägungen kennzeichnen. Maßgebend für die Qualität von Wohnungen etwa sind neben Ausstattungsmerkmalen Komponenten wie das Ausma $B$ an Lärmbelästigung, der Grad der Luftverschmutzung oder die Erreichbarkeit öffentlicher und privater Einrichtungen. Insbesondere diese letztgenannten Eigenschaften kann der Staat durch bestimmte Maßnahmen, beispielsweise Straßenbauprojekte, den Bau und die Wahl des Standortes von Flugplätzen, Behörden, Schulen, Schwimmbädern, Parks usw, beeinflussen. Nutzen-Kosten-Analysen, die sich mit solchen projekten befassen, gehen davon aus, daß die meisten Menschen ein Bedürfnis nach Ruhe, sauberer Luft und leichter Erreichbarkeit von öffentlichen Einrichtungen der genannten Art empfinden. Zwar existieren keine Märkte, auf denen die Individuen ihre Zahlungsbereitschaft für die Erfüllung dieser Bedürfnisse unmittelbar offenbaren können, wenn jedoch das Ausma $B$ an Lärm- und Abgasbelästigung sowie die Erreichbarkeit öffentlicher Einrichtungen Komponenten des Eigenschaftsvektors von Wohnungen sind, liegt es nahe, die durch öffentliche Projekte bewirkten Veränderungen dieser Komponenten anhand ihrer Auswirkungen auf die Mieten sowie die Haus- und Grundstückspreise zu bemessen. So existieren Analysen, die, i.d.R. mit Hilfe der multiplen Regressionsrechnung, den Einfluß des Grades der Luftverschmutzung sowie von Parks auf die Immobilienpreise zu beziffern suchen ${ }^{1)}$.

Derartige Analysen geben jedoch ein in theoretischer Hinsicht schwaches Fundament für Nutzen-Kosten-Analysen ab. Sie lassen sowohl den Einfluß der Angebotsseite auf die Preise für Wohnungen ${ }^{2)}$ als auch die Möglichkeit, daß Nachfrageverschiebungen

1) Vgl. V. Arnold: Nutzen-Kosten-Analyse II... a.a.O., S. 387.

2) Vgl. ebenda, S. $387 \mathrm{f}$. 
aus Veränderungen der Präferenzen resultieren ${ }^{1)}$, außer acht. zudem ermöglichen sie allenfalls Aussagen über die marginale Zahlungsbereitschaft, die Schätzung der gesamten Zahlungsbereitschaft dagegen würde Informationen über die Konsumentenrente erfordern. Auch zieht die Maßgröße Veränderung der Wohnungspreise die Grenzen der Reichweite der Wirkungen eines öffentlichen Projekts zu eng. Zum einen berühren Lärm und Umweltverschmutzung die Menschen nicht nur in der Aktivität Wohnen, sondern auch am Arbeitsplatz, in der Schule usw., zum anderen nutzen nicht nur Anlieger Parks und andere Freizeiteinrichtungen. Bei Umweltschutzmaßnahmen ist auch zu fragen, ob die Betroffenen marginale veränderungen überhaupt wahrnehmen, und ob sie gegebenenfalls über genügend Informationen verfügen, um ihre Auswirkungen abschätzen zu können ${ }^{2}$ ). Schließlich begnügt sich eine Analyse, die sich auf Grundstückspreisänderungen stützt, auch mit weniger Informationen. Während in der Nutzen-Kosten-Analyse üblicherweise Nutzen für die einzelnen Perioden ermittelt und diskontiert werden, reflektieren Grundstückspreisänderungen unmittelbar den Barwert, ohne dessen Komponenten offenzulegen ${ }^{3)}$.

\subsubsection{Kostensenkungen durch öffentliche Aktivitäten}

Viele staatliche Leistungen ermöglichen die Durchführung privater oder auch anderer öffentlicher Aktivitäten mit geringeren Kosten als bisher. So erlauben Investitionen in das Verkehrswegenetz häufig die Bewältigung eines gegebenen Verkehrsaufkommens zu gesunkenen Kosten. Die Einsparungen können z.B. aus Zeitersparnissen, Betriebskostensenkungen bei den Benutzern der Verkehrswege sowie sinkenden Unfallfolgekosten entstehen.

1) Vgl. J. Margolis: Shadow Prices... a.a.O., S. 219.

2) Vgl. V. Arnold: Nutzen-Kosten-Analyse II... a.a.O., S. 388 f.

3) Vgl. N. Andel: Nutzen-Kosten-Analysen, a.a.O., S. $496 \mathrm{f}$. 
Im Bereich des Gesundheitswesens lassen sich Senkungen der Behandlungs- und Pflegekosten durch Programme vorbeugenden Charakters wie Schutzimpfungen und Vorsorgeuntersuchungen erzielen; zudem vermindern sich hierdurch die krankheitsbedingten Arbeitsunfähigkeitszeiten. Hochwasserschutzmaßnahmen schließlich vermindern oder vermeiden gar die Kosten der Schadensbeseiti$\operatorname{gung}^{1)}$.

Der Nutzen solcher kostensenkender MaBnahmen und Projekte ist der Wert der Mehrproduktion, die die freigesetzten Faktoren in ihrer alternativen Verwendung erzielen. Bei vollkommener Konkurrenz, Vollbeschäftigung und völliger Faktormobilität sowie Abwesenheit von indirekten Steuern wird ein Faktor in allen Verwendungen gleich, und zwar jeweils nach dem Wertgrenzprodukt, entlohnt. Dann sind die Faktorkosten in der bisherigen Verwendung und der Wert der alternativ produzierbaren Güter äquivalent, so daß der Nutzen des Projektes sich nach den Kostensenkungen bemessen $1 \ddot{B B} t^{2)}$.

Allerdings sind diese Voraussetzungen in der Bundesrepublik weder im Gesundheits- noch im Verkehrswesen erfüllt. Das Gesundheitswesen ist größtenteils nach anderen als marktwirtschaftlichen Grundsätzen organisiert. Hier freiwerdende Faktoren verbleiben in der Regel im Gesundheitswesen. Da jedoch die Patienten meistens für die neue ebensowenig wie für die bisherige Produktion dieser Faktoren Marktpreise entrichten, die ihre Zahlungsbereitschaft widerspiegeln würden, vermitteln die Kostensenkungen keine Informationen über ihre Zahlungsbereitschaft. Bei Verkehrsprojekten bereitet neben der Existenz von indirekten Steuern wie der Mineralölsteuer der Umstand Probleme, daß Zeitersparnisse nicht nur für produktive Zwecke,

1) Vgl. J. Margolis: Shadow Prices... a.a.o., S. $216 \mathrm{ff}$;

V. Arnold: Nutzen-Kosten-Analyse II... a.a.O., S. 389.

2) Vgl. V. Arnold: Nutzen-Kosten-Analyse II... a.a.0., S. 389. 
sondern zum Teil auch für Freizeit genutzt werden, woraus erhebliche Bewertungsprobleme resultieren ${ }^{1)}$.

\subsubsection{Nachfrageschätzungen für öffentliche Leistungen}

Die Ermittlung der Zahlungsbereitschaft für öffentliche Leistungen auf dem Wege der Befragung der NutznieBer über ihre Präferenzen haben wir oben bereits diskutiert. Eine eher indirekte Methode versucht, Nachfragekurven für öffentliche Leistungen aus Wahlhandlungen der Bevölkerung abzuleiten. Sie findet Anwendung bei Erholungseinrichtungen (Stauseen, Parks, Skigelände, wälder, natürliche wildnis usw.) und konzentriert sich darauf, wie sich die Bürger verhalten, um in den GenuB von Leistungen solcher Anlagen zu kommen. Auch wenn der Staat sie bereitstellt, ohne Eintrittspreise zu erheben, erfordert ihre Nutzung regelmäBig Ausgaben der Nachfrager, beispielsweise Anfahrtskosten. Die wahlhandlungsorientierten Untersuchungen ermitteln den Zusammenhang zwischen den Besuchskosten einer Anlage und der Häufigkeit der Besuche aus dem Einzugsgebiet. Hieraus leiten sie Nachfragekurven für die Anlagen ab, indem sie die Reaktion der Bevölkerung auf steigende Besuchskosten als eine Reaktion auf steigende Eintrittspreise interpretieren. Der Nutzen einer Erholungseinrichtung bemiBt sich dann nach der Fläche unter der Nachfragekurve ${ }^{2}$.

Das Untersuchungsergebnis hängt bei dieser Methode wesentlich davon ab, welche Besuchskosten in welcher Höhe als Ausdruck des Preises bzw. der Zahlungsbereitschaft in die Analyse eingehen. Die folgenden Beispiele zeigen, daB hier eine Fülle von EinfluBfaktoren erhebliche Ermessensspielräume schafft. Ausga-

1) Vgl. ebenda, S. 389 ff. Zu Problemen der Bewertung von Freizeit siehe auch $\mathrm{N}$. Andel: Nutzen-Kosten-Analysen, a.a.O., S. 494 .

2) Vgl. N. Andel: Nutzen-Kosten-Analysen, a.a.0., S. 495 f.: V. Arnold: Nutzen-Kosten-Analyse II... a.a.O., S. 393 ff. 
ben der Besucher eines Stausees für Verpflegung können nicht einfach zu den Anfahrtskosten addiert werden, wenn diese das Essen am See höher als das alternative Essen zu Hause bewerten. Die Anfahrtskosten dürfen nicht in voller Höhe angesetzt werden, wenn die Besucher der Fahrt selbst wegen landschaftlicher Reize ebenfalls einen Wert beimessen. Die relevanten Anfahrtskosten übersteigen die faktischen Ausgaben der Nachfrager, falls diese den Zeitaufwand für die Anfahrt als Nachteil empfinden ${ }^{1)}$. Bei Fahrten mit dem eigenen Wagen ist zu berücksichtigen, daß die Besucher möglicherweise nicht die tatsächlichen Betriebskosten in ihr Kalkül aufnehmen. Existieren zwischen mehreren Erholungseinrichtungen substitutive Beziehungen, so spielen wechselseitige externe Effekte in Form von Uberfullungskosten eine Rolle. So profitieren von einem neueröffneten Park nicht nur dessen Besucher, sondern auch die Personen, die weiterhin einen bereits bestehenden und bisher überfüllten Park besuchen ${ }^{2)}$.

\subsubsection{Kritische Würdigung}

Unterwirft man die Verfahren der Nutzen-Kosten-Analyse einer Prüfung im Hinblick auf die Kriterien für ein LeistungsmaB, Outputs unabhängig von Inputs sowie nachfrage- bzw. empfängerorientiert $\mathrm{zu}$ messen, so fällt das Urteil zunächst weitgehend positiv aus. Die meisten Verfahren erfassen von der Konzeption her projektspezifische Outputs, sehen nur diejenigen Outputeinheiten als Leistung an, die die Empfänger in Anspruch nehmen, und versuchen, sich auf deren Bewertung der Projektwirkungen zu stützen. Von vornherein können wir lediglich die Leistungsmessung mit Hilfe der Marktpreise vergleichbarer privat angebotener Güter ausscheiden, da sie sich als theoretisch undurchführbar erweist.

1) Vgl. N. Andel: Nutzen-Kosten-Analysen, a.a.O., S. 496.

2) Vgl. V. Arnold: Nutzen-Kosten-Analyse II...a.a.O., S. 395 f. 
Das weitgehend positive Urteil läßt sich relativieren, berücksichtigt man die verfahrensspezifischen Mängel der beschriebenen Methoden. Bei allen Verfahren kommen in mehr oder weniger groBem Umfang Unsicherheitsfaktoren ins Spiel, die sich gröBtenteils auch nicht durch den Ubergang von der Schätzung zur Messung ex post eliminieren lassen, da sie zumeist nicht auf Schätzproblemen, sondern auf konzeptionellen Schwächen beruhen. Wenngleich die Verfahren der Nutzen-Kosten-Analyse im Prinzip output- und nachfrageorientiert sind, ist doch bei einigen Methoden zu fragen, was die dort verwendeten Indikatoren eigentlich messen. Die öffentlichen Leistungen errechnen sich teilweise mangels geeigneter Indikatoren anhand von Veränderungen von ErsatzgröBen, die den Output eines Projekts und seine Bewertung nur unzureichend erfassen oder/und auBer dem Output auch projektfremde EinfluBgröBen widerspiegeln. Angreifbar ist in dieser Hinsicht vor allem die Bemessung des Leistungsumfangs von Schulen, Parks usw. nach der Veränderung von Mieten und Immobilienpreisen. In diesem Indikator mag sich zwar ein Teil der Wirkungen der öffentlichen Projekte niederschlagen, der Kreis der Mieter und Hauseigentümer muB jedoch nicht mit dem der Leistungsempfänger, auf deren Bewertung es uns ankommt, übereinstimmen. Bei den unter dem Aspekt der Anwendung bei nicht-staatlichen Entscheidungseinheiten besonders interessanten Verfahren zur Messung von Leistungen im Bildungs- und Gesundheitswesen erscheint der Vorwurf mangelhafter Indikatoren ebenfalls als berechtigt, impliziert doch die Quantifizierung der Leistungen anhand der zukünftig erzielbaren Mehreinkommen eine Reduktion der Betrachtungsweise auf ausschlieblich ökonomische Kategorien ${ }^{1)}$. Dies mag man bei Leistungen des Bildungswesens im Interesse der Operationalität, und weil hier investive Charakterelemente überwiegen dürften, noch in Kauf nehmen, bei Aktivitäten im Gesundheitswesen indes zeitigt das

1) In bezug auf das Unterrichtswesen und die Forschung findet sich dieses Argument beispielsweise bei F. Neumark: Planung in der öffentlichen Finanzwirtschaft, a.a.0., s. $200 \mathrm{f}$. 
Verfahren unbefriedigende Ergebnisse. Als das am ehesten gegen diese Kritik gewappnete Verfahren kristallisiert sich von der Konzeption her die Methode der Kostensenkungen heraus. Diesem Vorzug stehen andererseits bei der konkreten Durchführung Probleme der Messung und der Bewertung gegenüber.

Damit erweist sich keines der Verfahren der Nutzen-Kosten-Analyse als ideal für die Leistungsmessung bei nicht-staatlichen Entscheidungseinheiten. Festgehalten $\mathrm{zu}$ werden verdient der Umstand, daB die Nutzen-Kosten-Analyse von der Konzeption her die bewerteten Vorteile öffentlicher Projekte zu messen beabsichtigt und dies in der konkreten Durchfürung teilweise auch zu leisten vermag. Dies hebt die Nutzen-Kosten-Analyse in positiver Hinsicht von den bisher diskutierten Ansätzen zur Messung unentgeltlich abgegebener Leistungen ab. Ob freilich dieser Vorzug allein die Anwendung insbesondere des Verfahrens für das Bildungswesen trotz dessen spezifischer Mängel zu rechtfertigen vermag, muB vorläufig noch offenbleiben.

2.6. Die Messung der Leistungsabgabe von Vermögensbeständen

Einer der Hauptkritikpunkte am herkömmlichen Sozialproduktsbegriff ist die begrenzte Reichweite des zugrundeliegenden Kapital- und Produktionskonzeptes. Mehrere Revisionsansätze enthalten daher neben den bereits im konventionellen Rechnungssystem erfaßten Vermögensheständen weitere Bestandsqrößen und legen Interdependenzen zwischen diesen und den Strömungsgrößen offen. Einen konsequenten Schritt in dieser Richtung unternahm das National Bureau of Economic Research mit seinem Vermögensbestandsansatz ${ }^{1}$, der die eigentliche Quelle des volkswirt-

1) Vg1. F.T. Juster: A Framework for the Measurement of Economic and Social Performance, in: M. Moss (Ed.): The Measurement of Economic and Social Performance, Studies in Income and Wealth, Vol. 38, New York 1973, S. 25-84 (Diskussion: S. 84-109) sowie die Darstellung bei C. Leipert: Unzulänglichkeiten des Sozialprodukts... a.a.o., S. $212 \mathrm{ff}$. 
schaftlichen Leistungsstroms in den wirtschaftlichen und sozialen Vermögensbeständen liegen sieht. Der Entwurf für ein erweitertes Rechnungssystem unterscheidet folgende fünf Vermögenskategorien $^{1 \text { ) : }}$

- Reproduzierbares tangibles Vermögen. Hierunter fallen Anlageinvestitionen der Unternehmen und des Staates sowie Bauten und langlebige Konsumgüter der privaten Haushalte.

- Reproduzierbares intangibles Vermögen. Dieser Bestand an nicht-personengebundenen Fähigkeiten und Kenntnissen resultiert aus den Aufwendungen von Staat und Unternehmen für Forschung und Entwicklung.

- Humanvermögen. Es spiegelt den Bestand an personengebundenen Fähigkeiten und Kenntnissen wider, der sich als Folge von individueller Begabung, Aus- und Weiterbildung sowie Lernvorgängen im ArbeitsprozeB akkumuliert.

- Natürliches Umweltvermögen. Es enthält neben mineralischen und agrarischen Rohstoffen Umweltbestände wie Wasser, Luft, Boden, Klima.

- Sozio-politisches Vermögen. Hierzu gehören äußerst subjektive und wenig tangible Größen wie persönliche und nationale Sicherheit, Freiheit, Gleichheit.

Während dieser Ansatz bisher über konzeptionelle Uberlegungen nicht hinauskam, findet der Grundgedanke einer verstärkten Einbeziehung von Vermögensbeständen im japanischen ${ }^{2}$ ), dem

1) Vgl. F.T. Juster: A Framework... a.a.0., S. $42 \mathrm{ff.}$

2) Zum japanischen Revisionsansatz vgl. U.E. Simonis:"Nettowohlfahrtsindikator" - ein japanischer Ansatz, a.a.o., S. $298 \mathrm{ff}$; C C. Leipert: Gesellschaftliche Berichterstattung... a.a.0., s. $220 \mathrm{ff}$. 
von Ruggles/Ruggles ${ }^{1)}$ sowie dem von Nordhaus/Tobin ${ }^{2)}$ konzipierten Revisionsvorschlag eine konkrete, wenn auch nicht sämtliche fünf Vermögenskategorien umfassende Ausgestaltung. Es mag hier, da es lediglich um die Prinziplen der Berechnung von Kapitalbeständen sowie ihrer Leistungsabgabe geht, genügen, auf die einschlägigen Verfahrensweisen des prominentesten Revisionsansatzes, desjenigen von Nordhaus/Tobin, einzugehen.

Nordhaus/Tobin ${ }^{3)}$ gruppieren zunächst die privaten und öffentlichen Gesamtausgaben um in direkt konsumwirksame Ausgaben, Ausgaben für Investitionen und Ausgaben für intermediäre Leistungen. Beim Staat stufen sie lediglich die Ausgaben für das Postwesen und für Erholung als direkt konsumwirksam ein; hier kommt die Kostenbewertung zum Ansatz. Zu den staatlichen Investitionen rechnen sie neben konventionellen Bereichen wie Wohnungsbau und Transportwesen die Ausgaben für Bildung, Gesundheit, Handel, Entwicklung und Erhaltung natürlicher Ressourcen, Landwirtschaft sowie $50 \%$ der Ausgaben zur Entwicklung der Atomenergie. Bei den privaten Haushalten klassifizieren sie neben den bereits im herkömmlichen System der VGR als Vermögensaufbau erfaBten Ausgaben für den Erwerb von Eigentümerwohnungen sämtliche Ausgaben für Bildung, Gesundheit sowie für langlebige Konsumgüter als Investitionen.

Die Ermittlung der verschiedenen Kapitalbestände erfolgt i.d.R. anhand der getätigten Investitionsausgaben abzüglich Abschreibungen. Das Gesundheitskapital etwa errechnet sich durch Kumulation der staatlichen und privaten Ausgaben für Gesundheit

1) Vgl. N. Ruggles und R. Ruggles: The Design of Economic Accounts, National Bureau of Economic Research, New York. 1970, insbes. S. $38 \mathrm{ff}$.

2) Vgl. W. Nordhaus und J. Tobin: Is Growth Obsolete?, a.a.O.

3) Vgl. zum Folgenden ebenda, S. 5 ff. und S. $26 \mathrm{ff}$. 
bei einer unterstellten jährlichen Abschreibungsrate von $20 \%$. Das Bildungskapital dagegen ergibt sich aufgrund von Schätzungen der Ausbildungskosten pro Schiller.

Im nächsten Schritt berechnen Nordhaus/Tobin die monetären Aquivalente der von den verschiedenen Kapitalbeständen in einer Periode abgegebenen Leistungen. Diese gehen direkt in den Endkonsum oder/und als Vorleistungen in die Produktion ein. Beim Bildungs- und beim Gesundheitskapital unterstellen Nordhaus/Tobin allerdings, daB deren Leistungen keinerlei direkte Konsumeffekte zeitigen, sondern sich ausschlieBlich in einem Anstieg der Produktivität und der Einkommen niederschlagen. Dadurch können sie auf eine Bestimmung der monetären Åquivalente dieser Leistungsabgaben verzichten. Bei Vermögensbeständen dagegen, die überwiegend Nutzen im Konsum stiften, wie etwa langlebige Konsumgüter und zivile öffentliche Bauten, ist die Schätzung fiktiver Werte für die jährlich abgegebenen Nutzungen unerläBlich. Hierfür stehen grundsätzlich zwei Wege offen: Der Wert der Leistungsabgabe von Vermögensbeständen läßt sich entweder nach den Aufwendungen, die für den Erwerb äquivalenter Leistungen auf dem Wege des Mietens entsprechender Güter erforderlich sind, oder aber nach den Kosten des Besitzes dauerhafter Güter, die sich im Prinzip aus Kapitalverzehr und einer unterstellten Verzinsung zusammensetzen, bemessen ${ }^{1)}$. Die adäquate Lösung scheint zwar in der Verwendung von Marktpreisen als Bewertungsmaßstab zu liegen, in vielen Fällen fehlen jedoch geeignete Daten über die Mietpreise für äquivalente Leistungen, so daB nur der Weg der kostenorientierten Bewertung offenbleibt. Immerhin läßt sich ein enger Zusammenhang zwischen beiden Maßgrößen nachwelsen. Der Bruttomietwert bzw. die Bruttoertragsrate eines Kapitalgutes deckt die Abschreibungen und die Verzinsung bzw. die Nettoertragsrate sowie u.U.

1) Vgl. N. Ruggles und R. Ruggles: The Design of Economic Accounts, a.a.0., s. $40 \mathrm{ff}$. 
Aufwendungen für Steuern, Versicherungen und für Erhaltung und Reparaturen ab. Die Nettoertragsrate schätzt Júster, auf dessen Berechnungen sich Nordhaus/Tobin stützen, in Anlehnung an den Marktzins ${ }^{1)}$. Grundlage für diese Vorgehensweise ist die Uberlegung, daB im Marktgleichgewicht die privaten Haushalte ihre Käufe dauerhafter Güter so lange ausdehnen, bis der Grenzertrag aus der Nutzung langlebiger Güter den Opportunitätskosten entspricht. Diese manifestieren sich in den für die Finanzierung erforderlichen Zinsaufwendungen oder/und in entgangenen $\mathrm{Zinserträgen.} \mathrm{In} \mathrm{Verbindung} \mathrm{mit} \mathrm{Annahmen} \mathrm{über} \mathrm{die}$ Lebensdauer der langlebigen Güter läßt sich dann der wert der Nutzung als Summe aus unterstellter Nettoertragsrate und unterstelltem Kapitalverzehr errechnen.

Die Beurteilung des dargestellten Umweges über Vermögensbestände im Hinblick auf die Messung vorzugsweise der unentgeltlich abgegebenen Leistungen nicht-staatlicher Entscheidungseinheiten fällt zwiespältig aus. Grundsätzlich erscheint die Qualifikation bestimmter Ausgaben, insbesondere für Bildung und Gesundheit, als Investitionen sinnvoller als ihre Zurechnung ausschlieBlich zum Endkonsum. Zudem weist die vorgeschlagene Art der Ermittlung und Bewertung der Vermögensbestände bzw. ihrer Zuwächse den Vorteil der Einfachheit und Praktikabilität auf. Die einschlägigen Aktivitäten nicht-staatlicher Entscheidungseinheiten lassen sich bereits nach Maßgabe ihrer Ausgaben in den entsprechenden Aufgabenbereichen als Beitrag zum Aufbau eines Humankapitalstocks zuordnen und quantitativ erfassen. Gerade in dieser rein kostenorientierten Bewertung von Vermögensbeständen liegt jedoch eine Problematik dieses Ansatzes. Die Bewertung einer Investition zu ihren Kosten hat ihre Berechtigung, wenn der Investor identisch ist mit demjenigen, der die Leistungen des Vermögensbestandes empfängt

1) Vgl. F.T. Juster: Household Capital Formation and Financing 1897-1962, National Bureau of Economic Research, New York, London 1966 , S. $115 \mathrm{ff}$. 
und verwertet. Diese Identität ist bei den Investitionsausgaben der privaten Haushalte und der Unternehmen gegeben, nicht jedoch bel den als Investitionen qualifizierten Ausgaben nichtstaatlicher Entscheidungseinheiten für die Produktion bzw. Bereitstellung unentgeltlich abgegebener Leistungen. Abgesehen davon, daß die kostenorientierte Bewertung den Einfluß von Unterschieden in Preis- und Produktivitätsniveau auf die Höhe einer Investitionsausgabe ignoriert, kann in diesem Falle die Bewertung der Investition durch die sie tätigende nichtstaatliche Entscheidungseinheit durchaus von der Bewertung durch die tatsächlichen oder potentiellen Nutznießer abweichen; im Extremfall mögen letztere die Investition für völlig überflüssig halten. Unabhängig davon besteht ein entscheidendes Problem in der Wahl einer geeigneten Abschreibungsrate, die auch die positiven und negativen Auswirkungen des technischen Fortschritts auf den Vermögensbestand integriert.

Noch schwerwiegender wirkt sich die Frage der Identität von Investor und NutznieBer auf die Aussagekraft der monetären squivalente der Leistungsabgabe eines Vermögensbestandes pro Periode aus. Bei der von Juster vorgeschlagenen Berechnungsart handelt es sich nicht um eine Messung, sondern sowohl beim Kapitalverzehr als auch bei der Nettoertragsrate lediglich um unterstellte Beträge. Die mangels geeigneter Daten über die Marktpreise für äquivalente Leistungen angestellte Opportunitätskostenbetrachtung läßt sich nur dann vertreten, wenn der Investor auch Nutznießer der Leistungen seines Vermögensbestandes ist. Sie markiert dann die Untergrenze für die Leistungen, die der Investor für sich im Durchschnitt aus seiner Investition erwartet; exakte Aussagen sind ohnehin erst ex post möglich. Wenn jedoch, wie bei den Investitionsausgaben des staates und der nicht-staatlichen Entscheidungseinheiten, die der unentgeltlichen Leistungsabgabe dienen, die potentiellen Leistungsempfänger direkt weder an der Entscheidung über die Investition mitwirken noch an der Finanzierung betelligt sind, verliert die opportunitätskostenbe- 
trachtung ihre Gültigkeit. Der Leistungsstrom wird dann lediglich kostenorientiert ermittelt, es handelt sich dabei allenfalls um ein unterstelltes Nutzungspotential. Tatsächlich bleibt offen, ob und welche Leistungsströme bei den potentiellen Empfängern ankommen und welchen Wert sie diesen beimessen.

Fragt man abschließend nach den konkreten Anwendungsmöglichkeiten des dargestellten Leistungsmaßes, so verbleiben im Bereich der nicht-staatlichen Entscheidungseinheiten nur geringe Spielräume. So bestechend der Gedanke auch sein mag, ihre Aktivitäten im Bildungs- und Gesundheitswesen als Investitionen in Humankapital zu behandeln, zwingt er auch andererseits zur Inkaufnahme gravierender Nachteile. Gerade in diesen Bereichen geben die nicht-staatlichen Entscheidungseinheiten ihre Leistungen teilweise auch gegen Entgelt ab. Auf die Mängel des Leistungsmaßes Entgelt bei nicht-staatlichen Entscheidungseinheiten wurde im Abschnitt über Marktpreise schon hingewiesen, sie sind jedoch vergleichsweise gering gegenüber denen des Vermögensbestandsansatzes. Der Umweg über Vermögensbestände erweist sich in der konkreten Durchführung bei den unentgeltlich und erst recht bei den gegen Entgelt abgegebenen Leistungen nicht-staatlicher Entscheidungseinheiten als Rückschritt in Richtung auf die Kostenbewertung.

2.7. Indizes der realen Leistungen des staates

Die beiden Ansätze von Hill und Franz ${ }^{1)}$ setzen sich eine mög-

1) Vgl. T.P. Hill: Price and Volume Measures for Non-Market Services, Statistical office of the European Communities, Brüssel 1975; A. Franz: The Measurement of Real Output of Non-Market Services, in: Der öffentliche Sektor - Forschungsmemoranden, hrsg. von E. Matzner, 3. Jg. (1977) Heft 1, S. 55-121. Eine kurze Darstellung beider Ansätze geben H. Brüngger und C. Orga: Ansätze zur Messung des Outputs des Staates, a.a.0.. s. $367 \mathrm{ff}$. 
lichst weitgehende getrennte Bestimmung der Input- und OutputgröBen des Staates zu konstanten Preisen zum ziel. Auf dieser Basis sollen Produktivitätsveränderungen beim Staat für Zwecke der Volkswirtschaftlichen Gesamtrechnungen nicht vorgegeben, sondern residual bestimmt werden. Das Produktionskonto des Staates zu laufenden Preisen lassen beide Ansätze unberührt; Kernstück für die Bestimmung der Entwicklung der Outputseite zu konstanten Preisen ist ein direkt ermittelter Mengenindex.

Hill teilt die staatlichen Leistungen in die zwei Kategorien halböffentliche und reine öffentliche (Dienst-)Leistungen ein. Erstere lassen sich primär kennzeichnen durch die Unterscheidungsmöglichkeit zwischen Benutzern und Nicht-Benutzern und sind im Gegensatz zu den reinen öffentlichen Leistungen zuteilbar auf Haushalte oder Unternehmen. Ferner weisen die halböffentlichen Leistungen, $z u$ denen Hill Transportleistungen, Freizeiteinrichtungen, Bildungs- und bestimmte medizinische Leistungen zählt, das Merkmal der Existenz von Kapazitätsgrenzen bei gleichzeitiger Benutzung auf. Für die Messung des Outputs an halböffentlichen Leistungen empfiehlt Hill Benutzungsziffern, welche er zusätzlich um die zeitdimension erweitert, wo diese, wie etwa bei Bildungsleistungen, eine wesentliche Rolle spielt ${ }^{1)}$. Konkret handelt es sich um MaßgröBen wie Krankenhaustage und Schülerstunden. Bei reinen öffentlichen Leistungen dagegen schlägt er als Outputindikator die dafür eingesetzten Arbeitsstunden vor. Die Aggregation zu einem Gesamtindex erfolgt, indem die verschiedenen Leistungsindikatoren mit den Durchschnittskosten pro Leistungseinheit im Basisjahr gewichtet werden.

Während der Ansatz von Hill einen Output erst dann vorliegen sieht, wenn bereitgestellte Kapazitäten tatsächlich benutzt

1) Vgl. T.P. Hill: Price and Volume Measures... a.a.0., S. 21 ff. und ergänzend T.P. Hill: On Goods and Services, a.a.O., S. $321 \mathrm{ff}$. 
werden, will der Vorschlag von Franz auch den Optionsgutcharakter bestimmter öffentlicher Leistungsangebote berücksichtigen $^{1)}$. Der Output bemiBt sich hier nicht nur nach der Benutzung, sondern zusätzlich auch nach den unabhängig von der Benutzung bereitgestellten Kapazitäten. Der Mengenindex im Vorschlag von Franz setzt sich demnach aus Benutzungsund Kapazitätskennziffern zusammen, wobei als Gewichte für die Benutzungskomponenten die variablen (d.h. benutzungsabhänglgen) Kosten und für die Kapazitätskomponenten die Fixkosten des Basisjahres dienen. Da Franz jedoch variable Kosten sehr restriktiv definiert, führt seln Vorschlag faktisch zu einem gewichteten Angebotsindex, in dem MaBgröBen wie Krankenhausbetten und Lehrerstunden dominieren. Bei reinen öffentlichen Leistungen schlieblich verwendet Franz die Zahl der dafür eingesetzten Erwerbstätigen als Kapazitätsindikator und schlägt als Benutzungsindikator z.B. die Zahl der behandelten Gesuche vor.

Beide Ansätze erheben den Anspruch, ein von Inputs unabhängiges OutputmaB zu konzipieren. Dieses ziel erreichen sie jedoch nur teilweise. Am besten wird der Ansatz von Hill, soweit es die halböffentlichen Leistungen betrifft, den selbstgestellten Anforderungen gerecht. Die vorgeschlagenen Benutzungsziffern erfüllen, solange sie physische MaBgröBen repräsentieren, die eingangs aufgestellten Kriterien an ein Outputmaß: Sie sind sowohl output- als auch nachfrageorientiert. Geradezu einen Rückschritt stellt dann jedoch die aus Rücksicht auf die angestrebte Kompatibilität mit den Volkswirtschaftlichen Gesamtrechnungen empfohlene Gewichtung mit Hilfe von Kosten dar. Bei den reinen öffentlichen Leistungen dagegen handelt es sich bereits bei den vorgeschlagenen physischen Maßgrößen um typische Inputindikatoren.

1) Vgl. A. Franz: The Measurement...a.a.0., s. $78 \mathrm{ff}$. 
Der Ansatz von Franz erfüllt die Kriterien für ein Leistungsma $B$ ebenfalls nur teilweise. Zum einen beruht dies auf der inputbezogenen Bewertung, zum anderen spielt die Nachfrage bzw. Nutzung eine untergeordnete Rolle, da MaBzahlen für das Angebot an öffentlichen Leistungen dominieren. Uberdies vermischt er mit der additiven Verknüpfung von Benutzungs- und Kapazitätskennziffern die verschiedenen Ebenen der output-objectives und der öffentlichen Produkte im Sinne der im ersten Kapitel dargestellten Ziel-Mittel-Hierarchie.

2.8. Spezielle Verfahren der Leistungsmessung für Unternehmen

2.8.1. Die gesellschaftsbezogene Unternehmensrechnung

Seit etwa einem Jahrzehnt diskutiert die Betriebswirtschaftslehre, zunächst in den USA und mit einer gewissen Verzögerung auch in der Bundesrepublik, unter den Oberbegriffen "Sozialbilanz", "gesellschaftsbezogene Unternehmensrechnung", "Corporate Social Accounting" u.ä. Möglichkeiten einer erweiterten Rechnungslegung für Unternehmen. Parallel zur Kritik am Sozialprodukt als Wohlstandsindikator und der damit einhergehenden Entwicklung von Revisionsansätzen, die der Ausdehnung des engen Bezugsrahmens der Volkswirtschaftlichen Gesamtrechnungen auch auf nicht-ökonomische Tatbestände der Gesellschaft dienen ${ }^{1)}$, laufen Bestrebungen zur Konzipierung von Rechnungssystemen auf der mikroökonomischen Ebene, die das herkömmliche betriebliche Rechnungswesen um gesellschaftliche Bezüge ergänzen bzw. erweitern sollen. Während die konventionelle betriebliche Bilanz und Erfolgsrechnung sich lediglich an der unternehmensindividuellen Wirtschaftlichkeit orientieren und ausschlieblich jene Beziehungen des Unternehmens zu seiner (sozialen und physischen) Umwelt erfassen, die auf Zahlungsvorgängen beruhen,

1) Zur Kritik am Sozialprodukt als Wohlstandsindikator sowie zu den wichtigsten Revisionsansätzen vgl. C. Leipert: Unzulänglichkeiten des Sozialprodukts... a.a.o. 
setzt sich die gesellschaftsbezogene Unternehmensrechnung zum ziel, zusätzlich primär die positiven, teilweise auch die negativen gesellschaftlichen Auswirkungen der Aktivitäten eines einzelnen Unternehmens nachzuweisen. Bei diesen gesellschaftlichen Auswirkungen kann es sich sowohl um marktmäBige Beziehungen, die jedoch, wie etwa Konsumentenrenten, nicht in das betriebliche Rechnungswesen Eingang finden, als auch um nichtmarktmäBige Beziehungen in der Form (technologischer) externer Effekte handeln. Die anspruchsvollen Konzepte der gesellschaftsbezogenen Unternehmensrechnung legen Wert auf die Erfassung der positiven ebenso wie der negativen externen Effekte und orientieren sich damit letztlich an den durch die Aktivitäten eines Unternehmens entstehenden sozialen Nutzen und Kosten ${ }^{1)}$. Insofern erscheint diese Art der Rechnungslegung als

1) Aus der Fülle der deutschsprachigen Literatur zu Theorie und Ansätzen der gesellschaftsbezogenen Unternehmensrechnung vgl. etwa M. Dierkes: Die Sozialbilanz. Ein gesellschaftsbezogenes Informations- und Rechnungssystem, Frankfurt 1974; P. Eichhorn: Gesellschaftsbezogene Unternehmensrechnung, Göttingen 1974; K. Faltlhauser: Unternehmen und Gesellschaft. Theorie und Praxis der Sozialbilanz, Schriftenreihe des Deutschen Instituts für Betriebswirtschaft e.V., Bd. 5, Berlin 1978; $\mathrm{K}$. von Wysocki: Das Unternehmen in seiner Umwelt: Möglichkeiten und Grenzen der Sozialbilanz, in: E. Pieroth (Hrsg.): Sozialbilanzen in der Bundesrepublik Deutschland. Ansätze - Entwicklungen - Beispiele, Wien, Düsseldorf 1978, S. 15-33; E. Hemmer: Sozialbilanzen. Konzepte und Realität, Beiträge zur Wirtschafts- und Sozialpolitik Heft 62, hrsg. vom Institut der deutschen Wirtschaft, Köln 1979; G.K. Piller: Sozialbilanz. Unternehmungspolitische Ziele in der gesellschaftsbezogenen Rechnungslegung, Thun, Frankfurt 1980, S. $108 \mathrm{ff.;} \mathrm{M.} \mathrm{Dierkes} \mathrm{und} \mathrm{A.} \mathrm{Hoff:}$ Sozialbilanzen und gesellschaftsbezogene Rechnungslegung in der Bundesrepublik Deutschland - Eine Analyse der bisherigen Experimente, in: H.-J. Hoffmann-Nowotny (Hrsg.): Sozialbilanzierung. Soziale Indikatoren VIII. Konzepte und Forschungsansätze, Frankfurt, New York 1981, S. 9-67; H.-H. Heymann: Die Sozialbilanz als Instrument der Unternehmensführung. Das gesellschaftsbezogene Rechnungswesen der Unternehmung in der Sozialen Marktwirtschaft, Frankfurt 1981, S. $113 \mathrm{ff}$; K. von Wysocki: Sozialbilanzen. Inhalt und Formen gesellschaftsbezogener Berichterstattung, stuttgart, New York 1981. 
besonders interessant im Hinblick auf die Messung der Leistungen öffentlicher Unternehmen, da hier die Verkaufswerte, wie erwähnt, häufig eine eingeschränkte Ạussagekraft aufweisen, und bei denen nach ihrem selbstverständnis der erwerbswirtschaftliche Erfolg keinesfalls eine rationale Würdigung ihrer Leistungen und ihres Verhaltens zuläBt ${ }^{1)}$.

Je nachdem, ob die Berichterstattung im Prinzip sämtliche günstigen und ungünstigen Folgen unternehmerischer Betätigung für die Umwelt erfaBt, oder ob sie sich auf einzelne mehr oder weniger willkürlich ausgewählte unternehmerische Aktivitäten oder umweltbezogene Wirkungen beschränkt, liegt eine gesellschaftsbezogene Vollrechnung oder eine gesellschaftsbezogene Teilrechnung vor. Ferner läbt sich die Fülle der existierenden Vorschläge zur Sozialbilanzierung in eindimensionale und mehrdimensionale Ansätze einteilen 2). Im folgenden sei stellvertretend für die eindimensionalen Ansätze P. Eichhorns Vorschlag einer gesellschaftsbezogenen Unternehmensrechnung dargestellt. Er kann aus zwei Gründen zu den anspruchsvollsten Konzepten der Sozialbilanzierung gezählt werden: Zum einen handelt es sich dabei um eine Vollrechnung, zum anderen will er die Auswirkungen unternehmerischer Aktivitäten nicht, wie die meisten anderen Ansätze, inputorientiert, nämlich anhand getätigter oder unterlassener Aufwendungen, sondern outputorientiert, d.h. auf der Ebene der Betroffenen, messen.

Eichhorn stellt neben der Wirtschaftlichkeit folgende drei grundsätzliche Anforderungen an eine gesellschaftsbezogene Unternehmensrechnung $^{3)}$ :

1) Vgl. dazu auch P. Eichhorn: Gesellschaftsbezogene Unternehmensrechnung, a.a.O., S. 18; H. -H. Heymann: Die Sozialbilanz als Instrument... a.a.0., S. $158 \mathrm{ff}$.

2) Vgl. K. von Wysocki: Sozialbilanzen... a.a.O., S. 9 ff.

3) Vgl. P. Eichhorn: Gesellschaftsbezogene Unternehmensrechnung, a.a.O., S. $19 \mathrm{ff}$. Ergänzend zu diesem Ansatz siehe auch P. Eichhorn: Grundlagen einer gemeinwirtschaftlichen Erfolgsrechnung für Unternehmen, Schriftenreihe Gemeinwirtschaft Nr. 15, hrsg. von der Bank für Gemeinwirtschaft, Frankfurt, Köln 1974. 
(1) Vollständigkeit: Eine gesellschaftsbezogene Unternehmensrechnung soll im Prinzip alle jene günstigen und ungünstigen Folgen der Aktivitäten eines Unternehmens für seine (soziale und physische) Umwelt erfassen, die in das herkömmliche betriebliche Rechnungswesen keinen Eingang finden. Diese Forderung dient dazu, interessengebundene, einseitig geschönte Darstellungen zu verhindern.

(2) Uberprüfbarkeit: Die Uberprüfbarkeit der Ergebnisse einer gesellschaftsbezogenen Unternehmensrechnung erfordert zum einen eine widerspruchsfreie und eindeutige Begriffshildung, zum anderen das Vorhandensein von Richtlinien für die qualitative, quantitative und möglichst monetäre Erfassung der gesellschaftsbezogenen wirkungen betrieblicher Betätigung. Besondere Schwierigkeiten diesbezüglich resultieren aus dem Anspruch, nicht die unternehmerischen Maßnahmen (Inputs), sondern den aus ihnen resultierenden gesellschaftsbezogenen Erfolg (Outputs) zu messen.

Formal lehnt sich die gesellschaftsbezogene Unternehmensrechnung an den herkömmlichen Jahresabschluß an. Sie besteht aus einer gesellschaftsbezogenen Erfolgsrechnung, welche die relevanten stromgröBen erfaBt, und einer gesellschaftsbezogenen Bestandsrechnung, die die an einem bestimmten Stichtag vorhandenen Forderungen und Verbindlichkeiten des Unternehmens gegenüber den verschiedenen gesellschaftlichen Bezugsgruppen ausweist. Beide Rechnungen schlieBen die korrespondierende Aufwands- und Ertragsrechnung bzw. Bilanz nicht ein, sondern bilden eine Nebenrechnung bzw. Ergänzung um die Strom- und BestandsgröBen, die das herkömmliche betriebliche Rechnungswesen vernachlässigt.

(3) Vergleichbarkeit: Hier plädiert Eichhorn für eine möglichst weitgehende monetäre Bewertung der Wohlfahrtsbeiträge eines Unternehmens und entsprechend für den Verzicht auf reale Indikatoren. Erreicht werden soll dies durch die Verwendung des Zahlungsbereitschaftsansatzes der Nutzen-Kosten-Analyse. 
Abweichend von der in der Volkswirtschaftslehre gebräuchlichen Terminologie bezelchnet Eichhorn lediglich die Nutzen bzw. Kosten unternehmerischer Aktivitäten als "sozial" bzw. "gemeinwirtschaftlich", die nicht bereits im herkömmlichen betrieblichen Rechnungswesen enthalten sind ${ }^{1)}$. Den Oberbegriff bilden für ihn der Gesamtnutzen bzw. die Gesamtkosten, die sich folgendermaßen errechnen ${ }^{2)}$ :

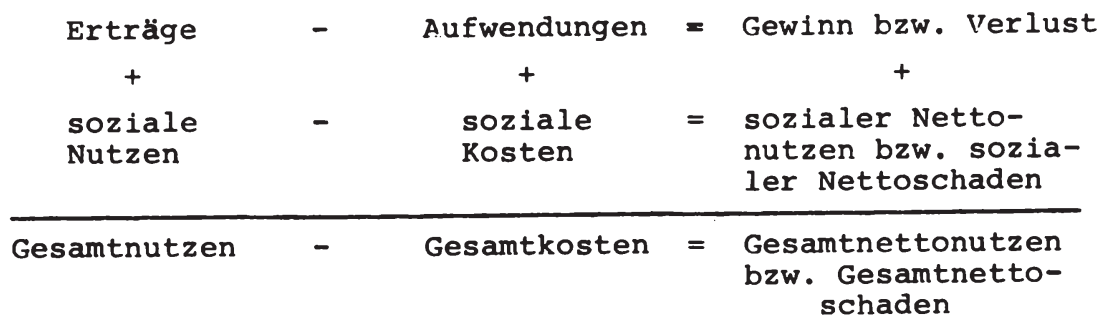

Die gesellschaftsbezogene Erfolgsrechnung beinhaltet die sozialen Nutzen und Kosten; sie setzen sich aus der sog. Absatzrente und dem Wert der positiven externen Effekte einerseits, der sog. Beschaffungsrente und dem Wert der negativen externen Effekte andererseits, zusammen ${ }^{3)}$. Unter einer "Absatzrente" versteht Eichhorn die Konsumentenrente. Sie repräsentiere auch deshalb einen sozialen Nutzen, weil die Nachfrager nicht gezwungen seien, auf ungünstigere alternative Angebote auszuweichen. Entsprechend führe eine negative Absatzrente, die etwa bei öffentlıchen Monopolbetrieben auftreten könne, zu Abzügen bei den sozialen Nutzen. Die Beschaffungsrente spiegelt den Betrag wider, den das Unternehmen über das tatsächlich entrichtete Entgelt hinaus für einen Faktor zu zahlen bereit wăre, bevor es auf den Kauf verzichtet. Sie stelle soziale

1) Vgl. P. Eichhorn: Gesellschaftsbezogene Unternehmensrechnung, a.a.0., s. 38 .

2) Vgl. ebenda, S. 81 .

3) Vgl. ebenda, S. 85 ff. 
Kosten dar, weil der Faktor in alternativen Verwendungen eine höhere Entlohnung erzielen könne. Analog entlaste eine positive Beschaffungsrente, etwa bei einem Unternehmen, das aufgrund von Tarifverträgen höhere Löhne als andere vergleichbare Unternehmen zu zahlen verpflichtet sei, die sozialen Kosten.

Die positiven und negativen externen Effekte repräsentieren alle durch die betrieblichen Aktivitäten verursachten, jedoch sich nicht über den Markt vollziehenden Verbesserungen bzw. Beeinträchtigungen bei den Bezugsgruppen Betriebsangehörige, Bevölkerung, andere Unternehmen und öffentliche Haushalte. Sie entstehen u.a. durch Aus- und Fortbildung, Gesundheitsvorsorge etc. einerseits und Luftverunreinigung, Abfälle, Lärm, Unfallgefahren etc. andererseits. Kennzeichen aller gesellschaftsbezogenen Erfolgskomponenten ist das Fehlen einer Gegenleistung ("Einseitigkeit"). Unter externen Effekten versteht Eichhorn nicht nur bei der Leistungserstellung oder der Produktverwendung anfallende Kuppelprodukte, sondern auch die Wirkungen eigenständig erstellter bzw. bereitgestellter, von einem Unternehmen ohne Gegenleistung erbrachter oder empfangener Leistungen. Da diese sog. sozial relevanten Leistungen vielfach bereits direkt die Aufwands- und Ertragsrechnung betreffen, grenzt Eichhorn folgendermaßen zwischen erwerbswirtschaftlichen und gesellschaftsbezogenen Erfolgskomponenten ab: Freiwillig oder zwangsweise erbringt ein Unternehmen sozial relevante Leistungen vor allem in Form von Sonderzahlungen (z.B. Beteiligung der Belegschaft am Gewinn), Naturalleistungen (z.B. Kohlendeputate für Betriebsangehörige), Spenden und Beiträgen für gemeinnützige $\mathrm{Zwecke} \mathrm{sowie} \mathrm{öffentlichen}$ Abgaben. Den aus den entsprechenden Aufwendungen resultierenden sozialen Nutzen will Eichhorn erfassen, indem er "mindestens in gleicher Höhe"1) positive externe Effekte, verteilt auf die Bezugsgruppen, in die gesellschaftsbezogene Erfolgsrechnung einstellt. Dies gilt jedoch nur, falls "die

1) Ebenda, S. 89 . 
Allgemeinheit (eine bestimmte Aktivität, U.R.) als eine relativ und absolut überdurchschnittliche sozialnutzenstiftende Leistung anerkennt" ${ }^{1)}$. Diese Einschränkung soll verhindern, daB ein Unternehmen für MaBnahmen, die nach allgemeiner Auffassung zum selbstverständichen Bestandtell der Leistungserstellung gehören, wie z.B. die Einbehaltung der Lohnsteuer, einen sozialen Nutzen ausweist. Analog entstehen soziale Kosten, wenn ein Unternehmen sozial relevante Leistungen seiner Umwelt (ohne Gegenleistung) in Gestalt von Sonderzahlungen (z.B. Subventionen) oder Naturalleistungen (z.B. Standortvorteile) empfängt oder öffentliche Dienste (Infrastruktureinrichtungen, Verwaltungsleistungen, Rechtsprechung usw.) in Anspruch nimmt. Negative externe Effekte gehen auch hier nur dann in die gesellschaftsbezogene Erfolgsrechnung ein, solange gewisse Vorrechte nicht, wie z.B. Steuervergünstigungen für öffentliche Unternehmen, allgemein als selbstverständlich erachtet werden.

Die gesellschaftsbezogene Erfolgsrechnung, die letztlich auf der Nutzenseite tatsächliche oder fiktive Mehreinnahmen und Minderausgaben und auf der Kostenseite tatsächliche oder fiktive Mehrausgaben und Mindereinnahmen der durch die Unternehmenstätigkeit Betroffenen enthält ${ }^{2}$, nimmt damit folgende Gestalt an:

1) Ebenda, S. 90 .

2) Vgl. K. von Wysocki: Sozialbilanzen....a.a.O., S. 74 . 
Schaubild 4:

Gesellschaftsbezogene Erfolgsrechnung

Soziale Kosten

Soziale Nutzen

I. Beschaffungsrenten für

1. Arbeitsleistungen

2. Betriebsmittel

3. Werkstoffe

4. Kapital

5. Unternehmerleistungen

6. Vorleistungen

II.Wert der negativen externen Effekte auf

1. Betriebsangehörige

2. Bevölkerung

3. Unternehmen

4. Offentliche Haushalte

III. Sozialer Nettonutzen als Saldo
I. Absatzrenten für

1. Produkt $A$

2. Produkt $B$

3. Produkt C

4. Produkt $D$

II.Wert der positiven externen Effekte auf

1. Betriebsangehörige

2. Bevölkerung

3. Unternehmen

4. Offentliche Haushalte

III.Sozialer Nettoschaden als als Saldo

Quelle: P. Eichhorn: Gesellschaftsbezogene Unternehmensrechnung, a.a.o., s. 94 .

Relativ wenig Aufmerksamkeit schenkt Eichhorn der Frage, wie die einzelnen gesellschaftlichen Vor- und Nachteile zu ermitteln und $z u$ bewerten sind ${ }^{1)}$. Eine Aktivitäten-BezugsgruppenMatrix, die ähnlich dem Betriebsabrechnungsbogen in den Zeilen die wichtigsten betrieblichen Funktionen und in den Spalten die Bezugsgruppen auflistet, soll in ihren Feldern die sozialen Nutzen und Kosten aufnehmen. Bei der eigentlichen Ermittlung der gesellschaftsbezogenen Wirkungen orientiert sich Eichhorn

1) Vgl. P. Eichhorn: Gesellschaftsbezogene Unternehmensrechnung, a.a.0., S. $107 \mathrm{ff}$. 
am Konzept der Zahlungshereitschaft. Aus unternehmerischen Aktivitäten resultierende Marktveränderungen bemessen sich nach der Verschiebung der Umsätze und Absatzrenten bzw. der Kosten und der Beschaffungsrenten; hinsichtlich der Messung der (technologischen) externen Effekte schließlich begnügt sich Eichhorn mit einer Aufzählung von Methoden der NutzenKosten-Analyse wie etwa Kosteneinsparungen, Sekundärgrößenverschiebung oder die Befragung.

Bei einer kritischen Würdigung dieses Ansatzes zur Leistungsmessung bei Unternehmen ${ }^{1)}$ bleibt zunächst festzustellen, daB er im Grunde weitgehend eine Ubertragung der Nutzen-KostenAnalyse auf betriebliche Aktivitäten darstellt. Die wesentlichen Unterschiede zur Nutzen-Kosten-Analyse sind der engere Bezugsrahmen, weshalb der Ansatz auch pekuniäre Nutzen und Kosten enthält, die Beschränkung auf eine einzige Periode und die Anwendung ex post ${ }^{2}$. Auch bei der gesellschaftsbezogenen Erfolgsrechnung vermag der Saldo (der sog. Sozialsaldo) allein wesentlich weniger Informationen für die Leistungsmessung $\mathrm{zu}$ liefern als seine einzelnen Bestandteile. Zudem kommt es für den hier zugrundeliegenden Untersuchungszweck nicht auf sämtliche gesellschaftsbezogenen Erfolgskomponenten an, sondern - und diese Unterscheidung ist gerade bei Unternehmen von wesentlicher Bedeutung - auf diejenigen, die der Erfüllung öffentlicher Aufgaben dienen. Diese lassen sich nur aus der Gesamtheit aller Vor- und Nachtelle, nicht jedoch aus dem saldo herausfiltern.

1) Auf die Darstellung der zweiten Komponente, der gesellschaftsbezogenen Bestandsrechnung, wollen wir hier verzichten, da sie kaum Bezüge zur Leistungsmessung aufweist und Eichhorn sich auf äuBerst knappe Ausführungen beschränkt.

2) Vgl. in diesem Zusammenhang auch Eichhorns Aufzählung von Unterschieden zur Nutzen-Kosten-Analyse in P. Eichhorn: Grundlagen einer gemeinwirtschaftlichen Erfolgsrechnung... a.a.0., s. 10 . 
Von der Konzeption her verdient der Vorschlag einer gesellschaftsbezogenen Erfolgsrechnung durchaus Beachtung. Neben der Vollständigkeit strebt Eichhorn die Messung von Outputs statt Inputs an. Zugleich ist das Kriterium der Nachfrageorientierung erfüllt, denn als soziale Nutzen bzw. Kosten sollen nicht die Angebote des Unternehmens, sondern die Bewertung deren Wirkungen durch die Betroffenen ausgewiesen werden. Damit orientiert sich der Ansatz ebenso wie die Nutzen-Kosten-Analyse am Konzept der bewerteten Vorteile.

Eine eingehende Würdigung der gesellschaftsbezogenen Erfolgsrechnung im Hinblick auf ihre Eignung zur Leistungsmessung wird durch den Allgemeinheitsgrad von Eichhorns Ausführungen erschwert, zumal er eine relativ weite Abgrenzung der gesellschaftlichen Bezugsgruppen wählt. So bezieht Eichhorn nicht nur die Mitarbeiter, sondern auch deren Familien in die Betrachtung ein, wenn er die Auswirkungen von Arbeitsunfällen nach den Einkommenseinbußen des Mitarbeiters sowie "etwaigen Gesundheitsschäden bis hin zur Invalidität mit den entsprechenden Folgen für die Arbeitnehmerfamilie wie geringere Bildungschancen der Kinder, weil sie früher mitverdienen müssen"1), bemiBt. Bei den sog. sozial relevanten Leistungen führt er zwar das Kriterium der "allgemeinen Auffassung" über eine bestimmte Aktivität ein, behandelt jedoch die Steuerzahlungen des Unternehmens anders als seine Aktivitäten für die Einbehaltung der Lohnsteuer, obwohl beide Leistungen allgemein als selbstverständlich erachtet werden dürften. Darüber hinaus bemiBt er den sozialen Nutzen dieser beiden Leistungskomponenten nach der Höhe der Aufwendungen und rekurriert insoweit entgegen seinem Anspruch auf Inputs $^{2)}$.

Der Nachweis von "Absatz- und Beschaffungsrenten" erscheint

1) Ebenda, s. 37.

2) Vgl. die Beispiele ebenda, S. 39. 
bei einem LeistungsmaB für ein einzelnes Unternehmen zwar als zulässig, wirft aber doch einige Fragen auf. Zunächst einmal sind, wenngleich Eichhorn dessen Absatz- und Beschaffungsmärkte völlig getrennt betrachtet, auch bei einem einzelnen Unternehmen die Absatz- und die Beschaffungsrenten nicht voneinander unabhängig, da in gesamtwirtschaftlicher Hinsicht die Höhe der Faktoreinkommen die Zahlungsbereitschaft maBgeblich beeinfluBt ${ }^{1)}$. Sodann bleibt unklar, wie eine negative Absatzrente entstehen kann, falls man staatlich verordneten Zwangskonsum ausschliebt. Ferner mag ein Unternehmen zwar seine eigene Zahlungsbereitschaft kennen, dürfte freilich an einer Veröffentlichung seiner Beschaffungsrenten ebensowenig Interesse haben wie an der der übrigen negativen Erfolgskomponenten. Zudem konkretisiert Eichhorn nicht, wie die Absatzrente in der Realität zu ermitteln ist.

Der Ansatz genügt allerdings - mit wenigen Ausnahmen - den Anforderungen der Output- und der Nachfrageorientierung. Soweit er konkrete Vorschläge macht, nennt Eichhorn Indikatoren, die Wirkungen bei den Betroffenen reflektieren, und er empfiehlt ausschlieBlich outputorientierte Verfahren der Nutzen-KostenAnalyse zur Messung und Bewertung. Dieser konzeptionelle Vorzug wird allerdings durch mangelnde Praktikabilität erkauft. Wenn Eichhorn beispielsweise die Auswirkungen einer Gewinnbeteiligung der Belegschaftsmitglieder in einem verbesserten Betriebsklima sieht und an den Indikatoren "niedriger Krankenstand und unterbliebener Produktionsausfall" messen will2), erscheint bereits die Zurechnung von beobachteten Indikatorveränderungen

1) $\mathrm{Zu}$ einer sehr ausführlichen Kritik an der Einbeziehung von Absatz- und Beschaffungsrenten in die gesellschaftsbezogene Erfolgsrechnung siehe A. Picot: Betriebswirtschaftliche Umweltbeziehungen und Umweltinformationen. Grundlagen einer erweiterten Erfolgsanalyse für Unternehmungen, Berlin 1977, S. $69 \mathrm{ff}$.

2) Vgl. P. Eichhorn: Grundlagen einer gemeinwirtschaftlichen Erfolgsrechnung... a.a.0., S. 28 . 
auf eine bestimmte Ursache als kaum lösbares Problem. Bei der eigentlichen Messung schlieblich treten die bereits diskutierten Probleme der Verfahren der Nutzen-Kosten-Analyse auf, teilweise noch verschärft durch den Umstand, daB die gesellschaftsbezogene Erfolgsrechnung stärker als die Nutzen-Kosten-Analyse auBerökonomische, personengebundene Wirkungen einbeziehen will. Insgesamt kann Eichhorns Ansatz als Modell für eine gesellschaftsbezogene Unternehmensberichterstattung dienen, zumal er in der vorliegenden, allgemein gehaltenen Form eher den Charakter eines Orientierungsrahmens als einer konkreten Lösung aufweist.

Abschließend ist $z u$ prüfen, ob Eichhorns Ansatz der gesellschaftsbezogenen Unternehmensrechnung sich prinzipiell zum Nachweis der Erfüllung öffentlicher Aufgaben durch ein Unternehmen eignet. Sie liegt dort vor, wo der staat einem Unternehmen das Erbringen einer Leistung für ihn oder für Dritte vorschreibt oder wenn ein Unternehmen freiwillig meritorische Güter anbietet ${ }^{1)}$. Daher muB die gesellschaftsbezogene Erfolgsrechnung um einige Komponenten ergänzt, um andere bereinigt werden. Einerseits bedarf es für den Nachweis der Erfüllung öffentlicher Aufgaben der Berücksichtigung erwerbswirtschaftlicher Erfolgskomponenten, soweit ein Unternehmen (wie z.B. Privatschulen, Sanatorien) meritorische Güter gegen Entgelt abgibt. Die gesellschaftsbezogene Erfolgsrechnung allein ist unter diesem Aspekt aus einem weiteren Grund nicht ausreichend, denn sie weist zwar den Nutzen von freiwillig entfalteten betrieblichen Aktivitäten mit meritorischem Charakter wie Maßnahmen der Berufsaus- und -fortbildung und Gesundheitsvorsorge nach, schließt jedoch mit Hilfe des Kriteriums der "allgemeinen Auffassung" den sog. verdeckten Staatsbedarf, etwa die Einbehaltung der Lohnsteuer, aus dem Bericht aus. Andererseits müssen unter dem Aspekt der Er-

1) Siehe hierzu auch Abschnitt 3.2. in Kapitel II dieser Arbeit. 
füllung öffentlicher Aufgaben Aktivitäten wie die Entrichtung von Steuern, die Beteiligung der Belegschaft am Gewinn oder die Unterhaltung einer Werkskantine wegfallen. Während die Steuern des Unternehmens und die Gewinnbetelligung keinen Output, sondern lediglich Transfers darstellen ${ }^{1)}$, handelt es sich bei der Kantine nicht um eine meritorische Leistung. Die Gesamtheit der Absatz- und Beschaffungsrenten kann in den Nachweis der Erfüllung öffentlicher Aufgaben durch ein Unternehmen nur dann eingehen, wenn das relevante zielsystem (auch) verteilungspolitische Aufgabenstellungen umfaßt. Ist dies nicht der Fall, läBt sich die Einbeziehung von Absatzrenten nur insoweit rechtfertigen, als es sich bei den am Markt abgesetzten Leistungen um meritorische Güter handelt. Analog wäre der Nachweis von Beschaffungsrenten nur bei den Faktoren bzw. Vorleistungen vertretbar, die ausschließlich bei der Erstellung von als öffentlich qualifizierten Leistungen Verwendung finden.

Im Prinzip eignet sich also die gesellschaftsbezogene - gegebenenfalls in Verbindung mit der erwerbswirtschaftlichen Erfolgsrechnung zum Nachweis der Erfüllung öffentlicher Aufgaben durch ein Unternehmen. Da sie hierfür jedoch lediglich die Ausgangsbasis abgeben kann, die um $\mathrm{Zu}$ - und Abrechnungen korrigiert werden muß, ließe sich dasselbe Ergebnis auch mit Hilfe einer Teilrechnung erzielen ${ }^{2}$ ).

1) Eine Einbeziehung dieser beiden Komponenten ließe sich allerdings unter verteilungspolitischen Aspekten rechtfertigen.

2) Vgl. in diesem Zusammenhang auch $\mathrm{K}$. von Wysocki ( Meß- und Bewertungsprobleme in der sozialen Rechnungslegung, in: H.R. Haeseler (Hrsg.): Gemeinwirtschaftliche Betriebe und öffentliche Verwaltungen, Schmalenbachs Zeitschrift für betriebswirtschaftliche Forschung, Sonderheft 5, Opladen 1976, S. 171-180), der sich gegen eine ausschließlich monetäre Bewertung und für gesellschaftsbezogene Tellrechnungen ausspricht. 
2.8.2. Exkurs: Die gesellschaftsbezogene Berichterstattung der Deutschen Bundesbahn

Die Geschäftsberichte der Deutschen Bundesbahn (DB) enthalten seit einigen Jahren jeweils unter der Uberschrift "Deutsche Bundesbahn und Gesellschaft" - vermutlich auch als Reaktion auf die wachsenden Defizite des Unternehmens - verschiedene Versuche einer gesellschaftsbezogenen Berichterstattung. Dabei läßt sich eine zwar sprunghafte, dennoch deutlich in eine einzige Richtung weisende Entwicklung, nämlich eine Senkung des Anspruchsniveaus, feststellen.

Im Geschäftsbericht 1977 setzte sich die DB zum ziel, die betriebswirtschaftliche Rechnungslegung um gesellschaftliche Nutzen und Kosten der Tätigkeit des Unternehmens zu ergänzen und stellte als ersten Schritt in dieser Richtung "die Inanspruchnahme volkswirtschaftlicher Mittel durch die DB einerseits und Leistungen der DB für Wirtschaft und Gesellschaft andererseits" folgendermaBen einander gegenüber ${ }^{1)}$ :

1) Deutsche Bundesbahn: Geschäftsbericht der Deutschen Bundesbahn. Geschäftsjahr 1977, 0.0., 0.J., S. 65 . 
Verbrauch

(Inanspruchnahme volks-

wirtschaftlicher Mittel)
Ergebnis

(Leistungen für Wirtschaft und Gesellschaft)
Bundesleistungen

Betriebswirtschaftliche Unterdeckung der Gewinn- und Verlustrechnung

(Mehrverbrauch von Mitteln des Unternehmens)

Umwel tbelastungen

- Lärm

- Luftverschmutzung

- Flächenbedarf

- Energie

- Verkehrsunfälle
Leistungen zugunsten der staatlichen Gemeinschaft

- Vorhaltung des Fahrwegs

- politische Gesamtheiten

- private Haushalte

- Wirtschaft

Umweltentlastungen

- Lärm

- Luftverschmutzung

- Flächenbedarf

- Energie

- Verkehrssicherheit

Leistungen zugunsten der Mitarbeiter

- Humanisierung der Arbeitswelt

- Aus - und Fortbildung

- Fahrvergünstigungen

- Krankheits- und Altersfürsorge

- Wohnungsbau- und Familienheimförderung

- Selbsthilfeeinrichtungen

Anders als die Tabellenform vermuten läßt, handelt es sich nicht um ein geschlossenes Rechnungssystem mit einem einheitlichen Bewertungsmaßstab, sondern der Bericht listet die einzelnen Erfolgskomponenten lediglich auf und erläutert sie; eine Aggregation oder gar saldierung beabsichtigt er nicht. Dabei begnügt er sich vorwiegend mit verbalen Darlegungen (so bei den Umweltbelastungen), teilweise kombiniert mit physischen Maßgrößen. Soweit der Bericht monetäre Größen enthält, 
handelt es sich um Aufwendungen der DB (die allerdings i.d.R. nicht als MaB für den Nutzen bestimmter Aktivitäten dienen sollen) oder um Vermeidungskosten ${ }^{1)}$.

Die Hauptrolle spielt ein Vermeidungskostenansatz dann im Geschäftsbericht 1978. Dieser Bericht soll aufzeigen, "wie unsere Volkswirtschaft als 'Gesellschaft ohne DB' aussähe"2) "indem er den tatsächlichen Aufwendungen der DB die Kosten gegenüberstellt, die eine Verlagerung des gesamten Verkehrsaufkommens der DB auf Straßen und Binnenwasserwege verursachen wïrde. Diese Rechnung bezleht folgende Kostenelemente ein: Kosten der Wege, Kosten des Betriebs auf den Wegen, Kosten der Stauungen, der Unfälle sowie der Umweltbelastungen durch Lärm und Luftverunreinigung. Die wesentlichen Annahmen sind:

(1) Die Gesamttransportleistung der DB im Güter- und Personenverkehr verlagert sich auf straßen und Binnenschiffahrt.

(2) Die Gesellschaft nimmt den hieraus resultierenden Anstieg der Umweltbelastung durch Lärm und Abgase nicht in Kauf; in die Rechnung gehen daher die zur Vermeidung dieses Anstiegs erforderlichen Aufwendungen ein.

(3) Die Anpassungsprozesse bei Beschäftigung und Produktion verlaufen reibungslos und sehr schnell.

Der Bericht errechnet aus der Verlagerung resultierende (fiktive) Mehrkosten für die Volkswirtschaft insgesamt im Jahre 1978 in Höhe von 10,8 Mrd. DM, die sich aus einer Minderbelastung bei den Wegekosten und Mehrbelastungen bei allen übrigen Kostenelementen zusammensetzen. Ergänzt wird diese Rechnung durch überwiegend verbale Beschreihungen der Beziehungen der DB zu verschiedenen Bezugsgruppen ihrer physischen und sozialen Umwelt ${ }^{3)}$.

1) Vgl. ebenda, S. $65 \mathrm{ff}$.

2) Deutsche Bundesbahn: Geschäftsbericht der Deutschen Bundesbahn. Geschäftsjahr 1978, 0.0., 0.J., S. 61 .

3) Vgl. ebenda, S. $61 \mathrm{ff}$. 
Der Geschäftsbericht 1979 enthält überhaupt keine gesellschaftsbezogene Berichterstattung, sondern kündigt lediglich für die Zukunft eine Sozialbilanz an, die gesondert veröffentlicht werden soll'1). Dieses Vorhaben scheint schnell wieder aufgegeben worden $\mathrm{zu}$ sein, denn in den Geschäftsberichten 1980 und 1981 findet sich kein Hinweis auf eine Sozialbilanz der DB. Stattdessen führt sie jetzt eine sog. Trennungsrechnung durch, die sie im Geschäftsbericht 1981 um einige primär verbale Ausführungen zu Flächenbedarf, Umweltbelastung, Ernergieverbrauch und Verkehrssicherheit der DB im Vergleich zu anderen Verkehrstrăgern ergänzt ${ }^{2)}$. Hinter der Bezeichnung "Trennungsrechnung" verbirgt sich lediglich eine - unzureichend erläuterte - Aufspaltung der betriebswirtschaftlichen Gewinn- und Verlustrechnung der DB auf Unternehmensbereiche mit eigenwirtschaftlichen zielsetzungen, solche mit gemeinwirtschaftlichen zielsetzungen sowie Unternehmensbereiche "mit staatlicher $\mathrm{Z}$ ielsetzung Infrastruk$\operatorname{tur}^{31}$ ).

Insgesamt weist die gesellschaftsbezogene Berichterstattung der DB eine gewisse Einseitigkeit auf. Alle Berichte geben sich sehr zurückhaltend bei den Ausführungen zu den Umweltbelastungen; i.d.R. finden sich hier keine Angaben eigener Daten, sondern lediglich Verweise auf andere, schlechter abschneidende Verkehrsträger. Eine Outputmessung in der hier relevanten monetären Form erfolgt nur bei den Verkaufswerten. Weist die im Geschäftsbericht 1978 enthaltene Alternativkostenrechnung, wenngleich sie schon von ihrer Konzeption her sich auf InputgröBen stützt, noch den Vorzug auf, externe Effekte zu berücksichtigen,

1) Vgl. Deutsche Bundesbahn: Geschäftsbericht der Deutschen Bundesbahn. Geschäftsjahr 1979, 0.0., 0.J., S. 65.

2) Vgl. Deutsche Bundesbahn: Geschäftsbericht der Deutschen Bundesbahn. Geschäftsjahr 1980, 0.0., 0.J., S. 59 ff.i Deutsche Bundesbahn: Geschäftsbericht der Deutschen Bundesbahn. Geschäftsjahr 1981, 0.0., 0.J., s. 57 ff.

3) Wie nicht anders zu erwarten, weist die "Trennungsrechnung" in beiden Berichten für die Unternehmensbereiche mit eigenwirtschaftlichen $\mathrm{Zielsetzungen} \mathrm{beträchtliche} \mathrm{Uberschüsse}$ aus. 
so stellt die "Trennungsrechnung" nichts anderes als den Verzicht auf die Erfassung externer Erfolgskomponenten dar. Die DB erhebt jetzt nicht einmal mehr den Anspruch, derartige Effekte nachzuweisen.

3. Mehrdimensionale MeBverfahren zur Bestimmung des Leistungsumfangs

3.1. Soziale Indikatoren

3.1.1. Quellen und Ansatzpunkte der Sozialindikatorenforschung

In der einschlägigen Literatur existiert keine allgemein akzeptierte Definition des Begriffs "sozialer Indikator", sondern je nach Verwendungszweck und Fragestellung mehr oder weniger weite Begriffsfassungen. "Als Zusammenfassung von verschiedenen Definitionsversuchen läßt sich die erste Hälfte einer Definition formulieren: Ein Sozialer Indikator ist ein statistisches (quantitatives) Konstrukt, das als zusammenfassende Messung benützt werden kann. Das Objekt der Messung und somit auch der zweite Teil der Definition hängen von der Zweckformulierung ab"1). Das Beiwort "sozial" meint "die Gesellschaft betreffend". Es handelt sich bei sozialen (oder besser: gesellschaftlichen) Indikatoren um Abbildungen bzw. Messungen gesellschaftlich "relevanter" Lebensbedingungen, sie sollen den abstrakten Begriff "Lebensqualität" konkretisieren. Der folgende tberblick über die Entstehungsgeschichte dient u.a. dazu, über die verschiedenen mit sozialen Indikatoren verfolgten ziele und somit über ihre gesellschaftliche zielrelevanz $\mathrm{zu}$ informieren.

1) So das Sekretariat der Vereinten Nationen, zitiert nach M. Peters und P. Zeugin: Sozialindikatorenforschung. Eine Einführung, Stuttgart 1979, s. 26. Peters/Zeugin führen (ebenda, S. $22 \mathrm{ff.}$ ) mehr als 30 unterschiedliche Definitionen verschiedener Autoren auf. 
Obschon der Begriff "soziale Indikatoren" erst 1966 in Umlauf kam ${ }^{1)}$, lassen sich Vorläufer der statistischen Erfassung menschlicher Lebensbedingungen bereits im 17. Jahrhundert nachweisen ${ }^{2)}$. Etablieren konnte sich die Sozialindikatorenbewegung Ende der 60 er Jahre unseres Jahrhunderts. Die AnstöBe zur Entwicklung sozialer Indikatoren kamen damals aus drei Richtungen. Zum einen wurde zunehmend die Eignung des Sozialprodukts als Wohlfahrtsindikator bestritten, zum anderen erwiesen sich die herkömmlichen Planungs-, Entscheidungs- und Informationsinstrumente als den drängenden gesellschaftlichen Problemen immer weniger angemessen. Hinzu kam ein wachsendes Interesse an bisher kaum vorhandenen langen Zeitreihen und adäquaten Verteilungsdaten, die zur Analyse langfristiger gesellschaftlicher Entwicklungen dienen sollten ${ }^{3)}$.

Die Kritik an den Volkswirtschaftlichen Gesamtrechnungen und ihrem zentralen Derivat Sozialprodukt als Wohlfahrtsindikator findet in der ökonomischen Literatur schon seit Jahrzehnten breite Resonanz und gehört inzwischen zum standardrepertoire einschlägiger Lehrbücher ${ }^{4)}$. Zweifel an der Stärke des Zusammen-

1) Als "Erfinder" dieses Begriffs gilt allgemein Bauer mit dem Werk R.A. Bauer (Ed.): Social Indicators, Cambridge, London 1966.

2) Vgl. die Beispiele bei E. Tuchtfeldt: Soziale Indikatoren: Ansätze und kontroverse Fragen, in: Hamburger Jahrbuch für Wirtschafts- und Gesellschaftspolitik, 21. Jahr (1976), S. $63-85$, S. 66 f. und C. Leipert: Soziale Indikatoren. Uberblick über den Stand der Diskussion, in: Konjunkturpolitik, 19. Jg. (1973), S. 204-256, S. 211.

3) Vgl. C. Leipert: Gesellschaftliche Berichterstattung... a.a.O., S. 2 ff., ähnlich auch C. Leipert: Soziale Indikatoren... a.a.0.. S. 204 ff. Teilweise abweichend gliedert die Quellen der Sozialindikatorenbewegung R.C. Bartholomäi: Das Social Indicator Movement in den USA - eine kritische würdigung, in: G. Kirsch und W. Wittmann (Hrsg.): Nationale Ziele und Soziale Indikatoren, Stuttgart 1975, S. 33-56.

4) Uberblicke über verschiedene Phasen unterschiedlicher Interpretation der Marktergebnisse geben $W$. Elsner: Mehrdimensionale Bestimmung und Ermittlung von Wohlfahrt mit Hilfe von Sozialindikatoren-Systemen. Theoretische und methodologische Probleme, Diskussionspapiere der Fakultät für Wirtschaftswissenschaften der Universität Bielefeld, $\mathrm{Nr}$. 53, Dezember 1978, S. $3 \mathrm{ff.} \mathrm{sowie} \mathrm{C.} \mathrm{Leipert:} \mathrm{Unzulänglichkeiten} \mathrm{des}$ Sozialprodukts... a.a.0., s. $21 \mathrm{ff}$. 
hangs zwischen Sozialprodukt und Wohlfahrt nährten bereits die Ausklammerung des Distributionsaspekts in der Wohlfahrtsökonomie und die Infragestellung der Marktpreise als Bewertungsmaßstab. Einige Autoren bestreiten die Parallelitätsannahme nicht nur, sondern stellen ihr als bewußten Gegensatz die These von der gegenläufigen Entwicklung von Wachstum und Wohlfahrt gegenüber, die sich u.a. auf den Hinweis auf dem wirtschaftlichen WachstumsprozeB immanente "kompensatorische Ausgaben" bzw. "regrettable necessities" stützt" . Wir wollen die einzelnen Kritikpunkte an der Funktion des Sozialprodukts als Wohlfahrtsindikator hier nicht im einzelnen auflisten, da sie in der Literatur ausführlich behandelt werden ${ }^{2)}$. Sie lassen sich $z u$ zwei Gruppen zusammenfassen. Einesteils macht die Kritik auf Mängel innerhalb des vorliegenden systems Volkswirtschaftlicher Gesamtrechnungen aufmerksam, so etwa die Behandlung des staates oder die sozialproduktssteigernde Wirkung "kompensatorischer Ausgaben", obwohl diese lediglich der Wiederherstellung des (wohlfahrtsmäßigen) Status quo ante dienen. Die zweite Gruppe von Argumenten zählt Wohlfahrtskomponenten auf, die, wie etwa Freizeit und Umweltqualität, im System der Volkswirtschaftlichen Gesamtrechnungen keinen Niederschlag finden.

Aus dieser, auch von den meisten okonomen nicht bestrittenen und teilweise vorweggenommenen, Kritik an der Parallelitätsannahme entstanden zwei grundlegende Richtungen zur Konzipierung von Verbesserungsvorschlägen. Eindimensionale Revisionsansätze versuchen, das geschlossene Rechnungssystem der Volkswirtschaftlichen Gesamtrechnungen im Prinzip beizubehalten und mit Hilfe einer Reihe von Korrekturen $z u$ einem monetären Wohlfahrtsindikator auszubauen, so etwa die Konzepte von Sametz und Nordhaus/Tobin. Demgegenüber verzichtet die zweite Forschungsrich-

1) Vgl. zu dieser These etwa C. Leipert: Gesellschaftliche Berichterstattung... a.a.0., s. 3 ff.

2) Vgl. vor allem C. Leipert: Unzulänglichkeiten des Sozialprodukts... a.a.0., s. $50 \mathrm{ff}$. Gegenargumente liefert

E. Wille: Soziale Indikatoren als Ansatzpunkte... a.a.o., S. $131 \mathrm{f}$. 
tung bewuBt auf eine einheitliche monetäre Bewertung aller wohlfahrtsrelevanten Endprodukte und damit (zunächst) auch auf eine Aggregation. Stattdessen wird versucht, den Begriff "Lebensqualität" zunächst in wichtige Teilaspekte aufzuspalten und dann die resultierenden Wohlfahrtskomponenten mit Hilfe umfassender Systeme bzw. Kataloge sozialer Indikatoren zu konkretisieren und in physischen Kategorien zu messen. Ein Beispiel hierfür stellt das seit Anfang der 70er Jahre laufende Programm der OECD zur Entwicklung eines Systems sozialer Indikatoren dar ${ }^{1)}$.

Obschon beide Forschungsrichtungen im Prinzip völlig unterschiedliche Wege einschlagen, lassen sich doch Teilüberschneidungen konstatieren. Für eine Ergänzung der herkömmlichen Volkswirtschaftlichen Gesamtrechnungen um physische MaBgröBen, die wohlfahrtsrelevante Komponenten abbilden, sprechen sich grundsätzlich selbst Theoretiker der Sozialproduktsberechnung aus, die ansonsten monetäre Größen bevorzugen. So schlägt Sametz die Ergänzung seines Netto-Output-Indikators, der ausschlieBlich ökonomische Wohlfahrtskomponenten erfaBt, um Indizes vor, die soziale Wohlfahrtsbestandteile ausdrücken sollen und nennt als Indikatoren u.a. die Lebenserwartung und die Anzahl an Jahren des Schulbesuchs ${ }^{2)}$. Nordhaus/Tobin halten ebenfalls Sachgüter- und Dienstleistungsströme für einen unzureichenden Ausdruck der Wohl-

1) Vgl. hierzu C. Leipert: Soziale Indikatoren... a.a.o., S. $238 \mathrm{ff}$. und H. Brüngger: Das Programm der O.E.C.D. zur Entwicklung Sozialer Indikatoren, in: H. $J$. Hoffmann-Nowotny (Hrsg. unter Mitarbeit von M.U. Peters und P.G. Zeugin): Soziale Indikatoren. Internationale Beiträge zu einer neuen praxisorientierten Forschungsrichtung, Frauenfeld, stuttgart 1976, S. 247-266.

Zur Berichterstattung über einen einzelnen Lebensbereich siehe etwa G. Betz: Einstieg in die Gesellschaftsberichterstattung - ein Versuch der praktischen Gestaltung, in: H.-J. Hoffmann-Nowotny (Hrsg.): Gesellschaftliche Berichterstattung zwischen Theorie und politischer Praxis. Soziale Indikatoren $x$. Konzepte und Forschungsansätze, Frankfurt, New York 1983, S. 17-26.

2) Vgl. A.W. Sametz: Production of Goods and Services... a.a.0., s. $91 \mathrm{f}$. 
fahrt und plädieren grundsätzlich für andere MaBgröBen. Die Verwendung sozialer Indikatoren lehnen sie allerdings ab, weil bisher noch (Stand: 1972) kein kohärenter Indikatorenkatalog vorliege ${ }^{1)}$. Bombach diskutiert einen wesentlich weitergehenden Vorschlag zur Lösung der Probleme, die bei der Integration der staatlichen Aktivitäten in die Volkswirtschaftlichen Gesamtrechnungen entstehen. Danach bleiben für Zwecke der Wohlstandsmessung die öffentlichen Ausgaben aus dem Sozialprodukt völlig ausgeklammert. Die Versorgung mit öffentlichen Gütern soll stattdessen in einem Satz von spezifischen, erfolgsbezogenen sozialen Indikatoren zum Ausdruck kommen ${ }^{2)}$.

Den zweiten wesentlichen Impuls der Sozialindikatorenforschung lieferten die Bemühungen zur Verbesserung des staatlichen Entscheidungsprozesses, wobei soziale Indikatoren einen rapide gestiegenen Informationsbedarf befriedigen sollen. Einerseits fehlt es an Informationen über Art, Umfang und Qualität staatlicher Leistungen. Die herkömmliche "Kostenbewertung" offenbart weder die mit Hilfe öffentlicher Ausgaben bewirkten Leistungen und damit die Veränderungen der Wohlfahrtsposition der Gesellschaftsmitglieder, noch, wie die staatlichen Leistungen die Verteilung verändern. Outputorientierte soziale Indikatoren sollen anstelle monetärer Inputindikatoren zur Messung öffentlicher Leistungen dienen und so zunächst die Kontrolle des realisierten Zielerreichungsgrades ermöglichen und darüber hinaus durch die Gegenüberstellung mit gesellschaftlichen Normvorstellungen über eine anzustrebende Verteilung Versorgungsmängel aufdecken. Hier liefern soziale Indikatoren primär Kontrollinformationen und kommen erst gegen Ende staatlicher Entscheidungsprozesse zum Zuge. Andererseits weist ihnen die Sozialindikatorenforschung auch bereits am Anfang eines Entscheidungsprozesses eine tragende Rolle zu. Soziale Indikatoren sollen zur Operatio-

1) Vgl. W. Nordhaus und J. Tobin: Is Growth Obsolete?, a.a.0., S. 9 .

2) Vgl. G. Bombach: Die öffentliche Finanzwirtschaft im Wirtschaftskreislauf, a.a.0., s. $73 \mathrm{ff}$. 
nalisierung staatlicher Ziele und damit zur Präzisierung und Objektivierung vager $\mathrm{zielvorstellungen} \mathrm{der} \mathrm{politischen} \mathrm{Entschei-}$ dungsträger dienen. Neue, anspruchsvolle Planungstechniken wie etwa das Planning-Programming-Budgeting system mit seinem gegenüber der traditionellen Planungspraxis wesentlich erhöhten Informationsbedarf bieten sowohl bei der Zielvorgabe als auch bei der zielerreichungskontrolle Einsatzmöglichkeiten für soziale Indikatoren ${ }^{1)}$.

Der dritte AnstoBfaktor schlieBlich, das spezifisch sozialwissenschaftliche Interesse an sozialen Indikatoren, die den sozialen Wandel langfristig wiedergeben, ist stark theoretisch motiviert. Eine verbesserte Berichterstattung über gesellschaftliche Entwicklungen wird als ein Instrument angesehen, mit dessen Hilfe sich Theorien über die Struktur und/oder Funktionsweise sozialer Systeme operational formulieren und damit spezifische Hypothesen empirisch überprüfbar machen lassen ${ }^{2}$.

In teilweiser Ubereinstimmung mit diesen Quellen verfolgt die Sozialindikatorenbewegung heute drei unterschiedliche und "soziale Indikatoren" verschieden definierende Forschungsansätze"

1) Vgl. C. Leipert: Gesellschaftliche Berichterstattung... a.a.O., s. $30 \mathrm{ff.} \mathrm{Zum} \mathrm{Planning-Programming-Budgeting} \mathrm{System}$ siehe unten, Abschnitt 3.3. in diesem Kapitel.

2) Vgl. C. Leipert: Gesellschaftliche Berichterstattung... a.a.0., S. 61 ff. Ein Beispiel für eine langfristige Analyse bildet $W$. Zapf: Die Wohlfahrtsentwicklung in Deutschland seit der Mitte des 19. Jahrhunderts, in: $W$. Conze und M.R. Lepsius (Hrsg.): Sozialgeschichte der Bundesrepublik Deutschland. Beiträge zum Kontinuitätsproblem, Stuttgart 1983, S. 46-65, S. 48 ff.

3) Vgl. zum Folgenden W. Zapf: Soziale Indikatoren - Eine Zwischenbilanz, in: H. -J. Krupp und W. Zapf: Sozialpolitik und Sozialberichterstattung, Frankfurt, New York 1977, S. 231246 , S. $234 \mathrm{ff.;} \mathrm{H.-J.} \mathrm{Krupp} \mathrm{und} \mathrm{W.} \mathrm{Zapf:} \mathrm{Indikatoren} \mathrm{II:}$ soziale, in: Handwörterbuch der Wirtschaftswissenschaft (HdWW), hrsg. von W. Albers et al., Bd. 4, stuttgart et al. 1978 , S. 119-133, S. $121 \mathrm{f}$. 
Wohlfahrtsmessung: Dieser Ansatz "geht von gesellschaftlichen Wohlfahrtszielen aus und sucht das Ausmaß sozialer Probleme bzw. den zielerreichungsgrad wohlfahrtsrelevanter Maßnahmen zu bestimmen"1). Er versucht, das als unzureichend empfundene eindimensionale Sozialprodukt durch eine mehrdimensionale Wohlfahrtsanalyse zu überwinden. Soziale Indikatoren sollen neben den externen Folgen des Wirtschaftsprozesses selbst auch Wohlfahrtserträge in den nicht-ökonomischen Lebensbereichen messen und im Hinblick auf ziele bewerten. Der Ansatz faßt soziale Indikatoren auf als statistiken von direktem normativem Interesse, die eine umfassende und ausgewogene Beurteilung zentraler gesellschaftlicher Lebensbedingungen erlauben. Sie sollen Endprodukte messen, individuenbezogen sein und sich auf gesellschaftliche Probleme beziehen.

Dauerbeobachtung des sozialen Wandels: Dieser zweite Ansatz der Sozialindikatorenbewegung geht davon aus, daB die Gesellschaft sich weder an einem allgemein anerkannten Satz von Wohlfahrtszielen orientiert, noch sich auf absehbare zeit in theoretischen Modellen simulieren läßt. Er faßt die Gesellschaft auf als ein komplexes Geflecht von strukturen und Prozessen, über die zunächst grundlegende Informationen vorliegen müssen, bevor man die Aufgaben Wohlfahrtssteigerung und politische steuerung in Angriff nehmen kann. Dauerbeobachtung will unter weitgehendem Verzicht auf normative Elemente den sozialen Wandel kontinuierlich analysieren, der Ansatz sieht daher als soziale Indikatoren alle jene Daten an, die Informationen liefern über Strukturen, Prozesse, ziele, Leistungen, Werte und Meinungen.

Prognose und Steuerung: Vertreter dieses Ansatzes sehen die wichtigste Aufgabe der Sozialindikatorenforschung darin, theoretische Modelle zu entwickeln, die in der Lage sind, Wohlfahrtsveränderungen und soziale Prozesse überhaupt erst zu er-

1) W. Zapf : Soziale Indikatoren - Eine Zwischenbilanz, a.a.0., s. 234 . 
klären. Sie suchen primär Indikatoren, die Variablen expliziter theoretischer (Kausal-) Modelle darstellen. Handelt es sich um exogene, nicht beeinfluBbare Variablen solcher Modelle, so gestattet ihre regelmäBige Beobachtung wenigstens die Prognose und die Frühwarnung. Sind sie hingegen politisch beeinfluBbar, so kann man Verfahren (Programme) der gezielten Gesellschaftssteuerung entwickeln.

Die Forschungsbereiche Wohlfahrtsmessung und Dauerbeobachtung des sozialen Wandels besitzen ex post-Charakter, ihr gemeinsames Programm läßt sich als "Sozialberichterstattung" bzw. "gesellschaftliche Berichterstattung" bezeichnen" ${ }^{1)}$. Soziale Indikatoren übernehmen im Rahmen der gesellschaftlichen Berichterstattung grundsätzlich nur eine Beobachtungs- und Bewertungsfunktion. Die dritte Forschungsrichtung hingegen versucht, ein gesellschaftliches Indikatoren- und Entscheidungssystem zum Zwecke politischer Gestaltung zu errichten. Hier bilden soziale Indikatoren "vornehmlich als ex ante-Informationen eine Entscheidungsgrundlage öffentlicher Planung" 2$)$.

\subsubsection{Zum Selektionsproblem}

Soziale Indikatoren unterscheiden sich grundsätzlich von Sozialstatistiken dadurch, daß sie für etwas stehen bzw. "einen Sachverhalt repräsentieren, der logisch auf einer höheren stufe steht und den sie als solchen unmittelbar nicht ausdrücken. Sie indizieren ein Phänomen, das nicht direkt gemessen werden kann. Erst eine Theorie, eine Plausibilitätsüberlegung oder eine empirische Regelmäßigkeit, die den stellenwert einer statistischen Maßzahl als empirisches Korrelat eines Konzeptes begründen, machen eine meBbare Sozialvariable zu einem Sozialindika-

1) Vgl. H.-J. Krupp und W. Zapf: Indikatoren II: soziale, a.a.O., S. 121 und E. Wille: Soziale Indikatoren als Ansatzpunkte... a.a.O., s. 129 .

2) E. Wille: Soziale Indikatoren als Ansatzpunkte... a.a.0., S. 129 . 
tor"1). Die Zusammenhänge zwischen Indikandum und Indikator lassen sich an einem Schema zur wissenschaftlichen Informationsgewinnung verdeutlichen ${ }^{2)}$. Am Anfang des Informationsgewinnungsprozesses steht ein theoretisches konstrukt (Indikandum), das jedoch keine unmittelbaren empirischen Korrelate besitzt und als solches nicht meBbar ist. Deshalb muB es durch Indikatoren operationalisiert werden. Die Brücke zwischen der abstrakten Symbolebene, auf der Konstrukte und Indikatoren angesiedelt sind, und der Realitätsebene stellt der Meßvorgang dar, der reale Phänomene auf einer Skala abbildet.

Zentrale Bedeutung kommt bei diesem Informationsgewinnungsproze $\beta$ der Operationalisierungsproblematik zu. Ein Indikator soll das interessierende Phänomen möglichst gut repräsentieren. Das Kriterium der Gültigkeit (Validität) fordert daher, daß Veränderungen eines Indikators gleichgerichtete Variationen des übergeordneten Indikandums entsprechen (und umgekehrt) ${ }^{3}$ ) . Grundsätzlich ist die Validität eines Indikators raum- und zeitbezogen $^{4)}$. Eines der größten Probleme bei der operationalisierung von Konstrukten ist die bruchstückhafte (Teil-)Messung. Ein Indikator bildet nur selten sämtliche Dimensionen eines Konstruktes $a b$, und die einzelnen Dimensionen können sich durchaus in verschiedene Richtungen entwickeln. Der hieraus resultierenden Gefahr einer verzerrenden, nicht repräsentativen Messung läßt sich im Prinzip durch die Konstruktion von systemen sozialer

1) C. Leipert: Gesellschaftliche Berichterstattung... a.a.0., S. $68 \mathrm{f}$.

2) Vgl. hierzu R. Werner: Soziale Indikatoren und politische Planung. Einführung in Anwendungen der Makrosoziologie, Reinbek 1975, S. 56 ff.

3) Vgl. E. Wille: Soziale Indikatoren als Ansatzpunkte... a.a.0., S. 137 .

4) Vgl. C. Leipert: Gesellschaftliche Berichterstattung... a.a.O., S. 74; zu entsprechenden Beispielen siehe auch E. Tuchtfeldt: Soziale Indikatoren... a.a.O., S. 79 f. 
Indikatoren begegnen, die möglichst alle Dimensionen des fraglichen Konzeptes erfassen ${ }^{1)}$.

3.1.3. Objektive und subjektive Indikatoren

Objektive und subjektive Indikatoren unterscheiden sich grundsätzlich hinsichtlich der Beteiligung der betroffenen Individuen als Bewertungsinstanz. Subjektive Indikatoren geben die Bewertungen der Individuen selbst wieder, sie offenbaren in Umfragen ihre Beurteilung vorgegebener Sachverhalte. Demgegenüber enthalten objektive Indikatoren keine Wertungen von Betroffenen. Sie informieren über beobachtbare, zählbare Phänomene und verwenden die Individuen allenfalls als Beobachtungseinheiten" " "Objektivität" bedeutet dabei nicht, daß es sich um wissenschaftlich unangreifbare und durch Wertungen unverzerrte Indikatoren handelt, da nur in wenigen Fällen gesicherte theoretische Ufberlegungen zum Konstrukt-Indikator-Verhältnis vorliegen ${ }^{3)}$. Da einerseits objektive und subjektive Indikatoren für einen bestimmten Sachverhalt unterschiedliche Ergebnisse signalisieren können ${ }^{4)}$, und andererseits die politischen Entscheidungsträger unschwer durch die Auswahl geeigneter Indikatoren Erfolge "nachzuweisen" vermögen" ${ }^{5)}$, hat sich heute weitgehend die Einsicht durchgesetzt, daß beide Indikatortypen

1) Vgl. C. Leipert: Gesellschaftliche Berichterstattung... a.a.0., S. $69 \mathrm{ff}$. und R. Werner: Soziale Indikatoren... a.a.O., s. $95 \mathrm{ff}$. Allerdings ist der systembegriff der Sozialindikatorenforschung weniger anspruchsvoll als der herkömmliche. Vgl. hierzu E. Wille: Soziale Indikatoren als Ansatzpunkte... a.a.0., s. 137.

2) Vgl. C. Leipert: Gesellschaftliche Berichterstattung... a.a.0., S. 101; R. Werner: Zur Problematik subjektiver Indikatoren, in: W. Zapf (Hrsg.): Soziale Indikatoren. Konzepte und Forschungsansätze II, Frankfurt, New York 1974, s. 264-275.

3) Vgl. C. Leipert: Gesellschaftliche Berichterstattung... a.a.O., S. 112 f.

4) Beispiele finden sich ebenda, S. 113.

5) Vgl. J. Steinhausen: Soziale Indikatoren als Elemente eines gesellschaftlichen Planungs- und Steuerungssystems, Meisenheim am Glan 1975, S. 180 |fifh Ring-978-3-631-75590-7 
nebeneinander zum Zuge kommen sollen ${ }^{1)}$. Den subjektiven Indikatoren kommt dabei die Aufgabe zu, Kontrollinformationen über die Validität der objektiven Indikatoren zu liefern, weil erstere die größere Nähe zu den betroffenen Individuen besitzen und somit für die Wohlfahrtsmessung das theoretisch adäquate Instrument darstellen ${ }^{2)}$.

3.1.4. Leistungsmessung mit Hilfe sozialer Indikatoren

Eines der am häufigsten genannten Merkmale sozialer Indikatoren ist deren outputorientierung ${ }^{3)}$, insofern könnten sie zur Messung der Leistungen nicht-staatlicher Entscheidungseinheiten dienen. Da wir von einem Leistungsma $B$ auBerdem Nachfragebezogenheit verlangen, können wir die Messung des Angebots an öffentlichen Leistungen mit Angebots- oder Kapazitätsindikatoren $^{4)}$ sowie die Messung von quasi-Endprodukten mit Hilfe von Erreichbarkeitsindikatoren von vornherein ausschließen. Die Leistungsmessung kann somit entweder auf Leistungs- bzw. Nutzungsindikatoren, die auf der Ebene der output-objectives angesiedelt sind, oder auf individuenbezogenen Resultatindikatoren, die der Ebene der impact-objectives zuzuordnen sind, basieren. Bezogen auf die konzeptionelle Basis sozialer Indi-

1) Vgl. H.-J. Krupp und W. Zapf: Indikatoren II: soziale, a.a.O., S. 124; ähnlich C. Leipert: Gesellschaftliche Berichterstattung... a.a.0., s. $113 \mathrm{f}$.

2) Siehe zu diesem Aspekt auch den folgenden Abschnitt.

3) Vgl. C. Leipert: Gesellschaftliche Berichterstattung... a.a.0., S. $96 \mathrm{f}$.

4) Angebotsindikatoren als Abbild der Ausstattung mit sozialen Infrastruktureinrichtungen verwendet beispielsweise die "Systemanalyse Baden-Wurttemberg". Vgl. Arbeitsgemeinschaft Systemanalyse Baden-Württemberg (Dornier-System, Prognos, Arbeitsgruppe Landespflege): Systemanalyse zur Landesentwicklung Baden-Württemberg, im Auftrag des Landes Baden-Württemberg, stuttgart 1975, S. 8 und S. 34 ff. 
katoren handelt es sich bei ihrer Verwendung zur Leistungsmessung um den sog. Performanzansatz. Er faBt staatliche Maßnahmen als Vorgang der Leistungserstellung (Performanz) auf und fragt nach deren Erfolg bzw. Wirkungen auf die zielgruppen sowie nach eventuellen Nebenwirkungen ${ }^{1)}$.

Nutzungsindikatoren messen die mengenmäßige Nutzung bzw. die Inanspruchnahme bestimmter Einrichtungen. Sie reflektieren einen Leistungsbegriff, der primär die Versorgungssituation der Individuen charakterisiert und deren Bewertungen der in Anspruch genommenen Leistungseinheiten bzw. deren Wirkungen auf die individuellen Wohlfahrtspositionen außer acht läßt ${ }^{2)}$. Es handelt sich um Maßzahlen wie etwa die Zahl der Schuller und studenten auf verschiedenen Stufen des Bildungssystems; Verweiltage im Krankenhaus, Zahl der ärztlichen Konsultationen, Impfungen, Röntgenaufnahmen; Zahl der Benutzer öffentlicher Verkehrsmittel; Theaterbesucher u.ä. ${ }^{3)}$. Wie die beiden letzten Beispiele zeigen, wird die Verwendung von Nutzungsindikatoren nicht nur für unentgeltlich abgegebene Leistungen diskutiert, sondern teilweise auch für Güter, bei denen zwar Marktpreise vorliegen, der staat jedoch in erheblichem Umfang Subventionen gewährt ${ }^{4}$. In jedem Falle setzt die Konstruktion von Nutzungsindikatoren voraus, daß sich die Individuen von der Nachfrage selbst ausschließen können, folglich eignen sich Nutzungsindikatoren grundsätzlich nicht für passiv konsumierte reine öffentliche Güter. Selbst in manchen Fällen aktiver Konsumtion ist ihre Aussagekraft eingeschränkt.

1) Vgl. zum Performanzansatz R. Werner: Soziale Indikatoren... a.a.O., S. $70 \mathrm{ff}$. und M. Peters und P. Zeugin: Sozialindikatorenforschung... a.a.0., S. $73 \mathrm{ff}$.

2) Ähnlich C. Leipert: Gesellschaftliche Berichterstattung... a.a.0., S. $98 \mathrm{f}$. und S. $103 \mathrm{f}$.

3) Vgl. ebenda, S. $103 \mathrm{f}$. und H. Brüngger und C. Orga: Ansätze zur Messung des Outputs des Staates, a.a.0., s. 370 .

4) Vgl. H. Brüngger und C. Orga: Ansätze zur Messung des Outputs des Staates, a.a.0., S. 370 . 
Handelt es sich um Leistungen, die sich durch einen externalisierten Nutzenanteil auszeichnen, so vernachlässigt ein Nutzungsindikator ex definitione die externalisierten Leistungskomponenten. Andererseits ist durch die aktive (und freiwillige) Inanspruchnahme gewährleistet, daß die Individuen zumindest dem internalisierten Teil der beanspruchten Leistungen einen positiven Wert beimessen.

An Nutzungsindikatoren wird hauptsächlich bemängelt, sie liefen Gefahr, Ungleiches gleich zu behandeln, nur weil es dieselbe Dimension besitzt. So macht etwa der Indikator "Verweiltage im Krankenhaus" keine Unterschiede zwischen verschiedenen Arten der Behandlung. Zudem nivelliere die einheitliche Dimension allfällige Qualitätsunterschiede. Anders als der Indikator "Verweildauer", der nicht zwischen verschiedenen Krankenhäusern unterscheidet, würde eine Marktpreisbewertung auch eine Qualitätskomponente einschließen ${ }^{1)}$. Während sich dem ersten Kritikpunkt im Prinzip durch die Wahl genügend ausdifferenzierter Indikatoren begegnen läßt, beruht das zweite Argument auf einer rein statischen Betrachtung. Eine Beobachtung über mehrere Perioden hinweg könnte Wanderungen der Nachfrager zwischen verschiedenen Anbietern gleichartiger Leistungen offenbaren und - ggf. in Verbindung mit Informationen über Kapazitätsauslastungsgrade, Wartelisten u.ä. Tendenzaussagen über die Bewertung der Leistungen einzelner Anbieter durch die Nachfrager erlauben.

Ein weiterer Kritikpunkt stellt auf eine latente Manipulationsgefahr ab. Auf die u.U. eingeschränkte Konsumentensouveränität bei medizinischen Leistungen haben wir bereits hingewiesen $^{2}$. Vor allem in diesem Bereich kann die Fähigkeit der zu-

1) Vgl. ebenda, S. 371

2) Vgl. Abschnitt 2.2 in diesem Kapitel. 
ständigen Stellen, die Nutzungsindikatoren künstlich aufzublähen, zu falschen Anreizen führen. Wenn die verantwortlichen Leiter wissen, nach welchen Kriterien Output und Effizienz ihrer Abteilungen beurteilt werden, werden sie diejenigen Tätigkeiten maximieren, die sich auch in den entsprechenden Indikatoren niederschlagen, wobei sie letztlich Qualität zugunsten der Quantität opfern ${ }^{1)}$.

Die Resultatindikatoren auf individueller Ebene verfolgen einen wesentlich höheren Anspruch als die Nutzungsindikatoren. Sie sollen reflektieren, wie die Individuen die Leistungen von Staat und nicht-staatlichen Entscheidungseinheiten verarbeiten bzw. welche Wirkungen diese Leistungen auf die Wohlfahrtsposition der Individuen ausüben. Als Beispiele seien Indikatoren wie Mütter- und Säuglingssterblichkeiten, die Lebenserwartung für verschiedene Altersgruppen, Sterberaten, Rekonvaleszenzzeiten, Pendelzeiten, Unfallhäufigkeiten, die $\mathrm{Z}$ ahl der Verkehrstoten und -verletzten u.ä. genannt ${ }^{2)}$. Gegenüber den Nutzungsindikatoren weisen die Resultatindikatoren den Vorzug eines größeren potentiellen Anwendungsbereiches auf. Letztere vermögen im Prinzip, da sie von der aktiven Konsumtion einer Leistung abstrahieren, auch die wirkungen externer Effekte zu erfassen und stellen somit grundsätzlich ein vollständiges Leistungsma $B$ dar. Allerdings lassen sich vorläufig noch nicht für alle Bereiche geeignete Resultatindikatoren konstruieren bzw. empirische Daten beschaffen, so daß die Forschung teilweise auf Hilfsgrößen angewiesen ist, die einer analytisch niedrigeren Ebene zuzuordnen sind ${ }^{3)}$.

1) Vgl. H. Brüngger und C. Orga: Ansätze zur Messung des Outputs des Staates, a.a.0., S. 371 .

2) Vgl. C. Leipert: Gesellschaftliche Berichterstattung... a.a.O., S. $104 \mathrm{ff.i}$ E. Wille: Soziale Indikatoren als Ansatzpunkte... a.a.O., S. 138 und S. 142 .

3) Vgl. C. Leipert: Gesellschaftliche Berichterstattung... a.a.0., S. 106 . 
Unstrittig ist die Outputorientierung der Resultatindikatoren. Indes bringt die Konstruktion von Resultatindikatoren, die auf der höchsten operationalisierbaren Ebene der Ziel-Mittel-Hierarchie angesiedelt sind, gravierende Evaluationsprobleme mit sich. Das Konzept der bewerteten Vorteile, das uns als Referenzgröße für die Beurteilung eines Ansatzes zur Leistungsmessung dient, setzt idealiter direkt beim einzelnen an und beschränkt sich auf den Nutzen, den dieser aus der Inanspruchnahme eines bestimmten öffentlichen Angebots zieht, ohne den Einfluß eventuell vorhandener störgrößen zu berücksichtigen. Die als operationalisierung der impact-objectives fungierenden Resultatindikatoren sollen demgegenüber die Wohlfahrtswirkungen bei der Gesamtheit der von einer staatlichen MaBnahme betroffenen Individuen abbilden. Sie können demnach nicht einzelne Leistungseinheiten, sondern lediglich das von einem Anbieter insgesamt abgegebene Leistungsvolumen bzw. die Auswirkungen auf die Betroffenen im Durchschnitt messen. Zudem vermögen Resultatindikatoren in der Realität nicht die Auswirkungen einer einzelnen staatlichen Maßnahme isoliert nachzuweisen. Sie reflektieren grundsätzlich ein Endergebnis, das als summe der positiven und negativen Wirkungen sämtlicher beteiligter Einflußgrößen auf das betreffende ziel entsteht. So bleibt zunächst offen, ob ein Anstieg der Lebenserwartung auf erhöhten Aktivitäten von Staat und nicht-staatlichen Entscheidungseinheiten im Gesundheitswesen oder/und beispielsweise auf aus einem wachsenden Gesundheitsbewußtsein resultierenden Veränderungen des Ernährungsverhaltens und der Einschränkung gesundheitsschädlicher Verhaltensweisen beruht. Für die zwecke der Leistungsmessung reicht die Information lediglich über ein Endergebnis nicht aus. Hierfür kommt es vielmehr darauf an, Indikatorveränderungen auch eindeutig auf die verschiedenen Ursachen zurückzuführen. Letztlich müßte der Beitrag jedes einzelnen Leistungsanbieters zu einer gemessenen Indikatorveränderung eruiert werden. Die Fvaluationsforschung, die versucht, Informationen über "soziale Produktionsfunktionen" zu erhalten, vermag bisher jedoch lediglich in Ausnahme- 
fällen eine schlüssige Wirksamkeitsanalyse vorzulegen ${ }^{1)}$.

Strenge Nachfrageorientierung weisen die Resultatindikatoren nicht auf, da sie nicht nur die Wirkungen aktiv konsumierter Leistungen auf die individuelle Wohlfahrtsposition zu erfassen suchen. Es ist daher zu prüfen, ob ihre Individuenbezogenheit einen hinreichenden Ersatz für die Nachfrageorientierung bieten kann. Sowohl die Nutzungs- als auch die Resultatindikatoren sind objektive Indikatoren. Während bei Nutzungsindikatoren - die Abwesenheit von Zwangskonsum vorausgesetzt die Individuen in ihren Nachfrageentscheidungen offenbart haben, daß die betreffenden Leistungen für sie überhaupt Wohlfahrtsrelevanz besitzen, fehlt bei den Resultatindikatoren ein derart enger Bezug $z u$ den Individuen. Sie partizipieren weder an der Konstruktion eines Resultatindikators, noch nehmen sie beim eigentlichen Meßvorgang als Bewertungsinstanz teil. Uber die (vermutete) Wohlfahrtsrelevanz der mit Hilfe von Resultatindikatoren abgebildeten Wirkungen entscheidet vielmehr der einzelne Forscher oder allenfalls der allgemeine Konsens unter Experten.

Im Prinzip hängt die Nachfrageorientierung eines Resultatindikators davon $a b$, ob er Wirkungen aktiv oder passiv konsumierter Leistungen abbildet. Im ersten Falle ermöglichen vorgelagerte Nutzungsindikatoren grundsätzlich eine Kontrolle, wie die betroffenen Individuen eine von einem Resultatindikator wiedergegebene Leistungsverbesserung einschätzen. Eine gleichgerichtete Entwicklung zusammengehörender Nutzungs- und Resultatindikatoren in einem Aufgabenbereich läBt ceteris paribus auf die Wohlfahrtsrelevanz der abgebildeten Wirkungen schließen. Freilich besteht dabei das problem, im Rahmen einer

1) Vgl. etwa C.H. Weiss: Evaluierungsforschung. Methoden zur Einschätzung von sozialen Reformprogrammen, Opladen 1974, S. $124 \mathrm{ff}$. und C. Leipert: Gesellschaftliche Berichterstattung... a.a.O., s. $123 \mathrm{ff}$. 
Wirksamkeitsanalyse eindeutige Kausalbeziehungen zwischen Veränderungen der Nutzung einer Leistung und den Resultatindikatoren zu generieren.

Eine derartige Kontrollmöglichkeit fehlt schon konzeptionell, falls ein Resultatindikator Wirkungen passiv konsumierter Leistungen bzw. externe Effekte abbildet, da dann keine vorgelagerten Nutzungsindikatoren existieren. Hier gewährleistet die Individuenbezogenheit eines Resultatindikators lediglich, "daB die betreffenden Effekte die Individuen auch erreichen und nicht, daß die Betroffenen diese auch wünschen"1) oder auch nur registrieren. Wenn jedoch nicht die betroffenen Individuen, sondern Dritte über die Wohlfahrtsrelevanz bestimmter Leistungen entscheiden und deren Bewertung vornehmen, können bereits bei der Konstruktion von Resultatindikatoren paternalistische Elemente ins Spiel kommen ${ }^{2)}$. Dies wird nicht nur dann offenbar, wenn objektive und subjektive Indikatoren einander widersprechen, sondern bereits dann, wenn verschiedene objektive Resultatindikatoren für einen bestimmten Aufgabenbereich divergierende Entwicklungen anzeigen. So ist es vorstellbar, daß ein Anstieg der Lebenserwartung mit einer Verlängerung der wahrscheinlichen zukünftigen Krankheitsdauer einhergeht. Die Einführung von Gewichtungsfaktoren, die auch in diesem Extremfall eindeutige Aussagen über die Wohlfahrtsentwicklung erlauben sollen ${ }^{3)}$, halten wir für äußerst fragwürdig, solange sich die Gewichtung nicht auf ein gesichertes Meinungsbild der betroffenen Individuen stützt. Derselbe Einwand richtet sich gegen alle Bestrebungen, mehrere Resultatindikatoren

1) E. Wille: Soziale Indikatoren als Ansatzpunkte... a.a.0., S. 146 .

2) Vgl. hierzu ebenda, S. 145 ff. und E. Tuchtfeldt: Soziale Indikatoren... a.a.O..S. $82 \mathrm{ff}$.

3) Diese Lösung diskutiert Leipert, von dem das Beispiel stammt. Vgl. C. Leipert: Gesellschaftliche Berichterstattung... a.a.0.. s. 106 . 
eines Aufgabenbereichs zu normieren, zu gewichten und zu einem einzigen Bereichsindikator, beispielsweise für den Bildungsstand oder die Gesundheit, zusammenzufassen. Zum einen fiihrt die Aggregation zu einem Informationsverlust, zum anderen beruht die Gewichtung auf Wertvorstellungen des Forschers, die jedoch an einem Bereichsindikator nicht zu erkennen sind.

Dem Problem der mangelnden Nachfrageorientierung ließe sich im Prinzip durch die Zuhilfenahme subjektiver Indikatoren begegnen. Die in Befragungen ermittelte zufriedenheit der Individuen mit der objektiven Situation vermag sowohl Informationen über die als wohlfahrtsrelevant empfundenen Wirkungen öffentlicher Leistungen als auch Anhaltspunkte für eine eventuelle Gewichtung objektiver Indikatoren zu liefern. Zwar erlauben die bisherigen Erkenntnisse auf dem Gebiet der Befragungstechnik und der skalierung noch nicht die Konstruktion subjektiver Indikatoren, die hinreichend aussagefähig im Hinblick auf die individuelle Wohlfahrt sind ${ }^{1)}$ und objektive Indikatoren ersetzen könnten, gleichwohl können subjektive Indikatoren als "Korrekturfaktoren" dienen, die Aufschluß über die Wohlfahrtsrelevanz der von objektiven Indikatoren abgebildeten Lebensbereiche geben ${ }^{2}$.

Somit eignen sich zur Messung der Leistungen nicht-staatlicher Entscheidungseinheiten Nutzungsindikatoren aus zwei Gründen besser als Resultatindikatoren. Zum einen erfüllen, wenngleich beide Indikatortypen outputorientiert sind, die Nutzungsindikatoren das Kriterium der Nachfrageorientierung besser als die Resultatindikatoren. Zum anderen gestatten vorläufig noch allein die Nutzungsindikatoren, den Beitrag jeden einzelnen Anbieters zum gesamten Leistungsvolumen zu ermitteln. Diesen Vorteilen steht allerdings vor allem die begrenzte Reichweite von Nutzungsindikatoren, die sich aus ihrem Versagen bei passiver Konsumtion ergibt, gegenüber.

1) Siehe auch Abschnitt 2.1. in diesem Kapitel.

2) Vgl. E. Wille: Soziale Indikatoren als Ansatzpunkte... a.a.0., s. 147 . 
3.1.5. Gesellschaftsbezogene Berichterstattung von Unternehmen mit Hilfe sozialer Indikatoren

Vertreter der Betriebswirtschaftslehre und Praktiker diskutieren seit einigen Jahren im Rahmen der gesellschaftsbezogenen Berichterstattung die Einsatzmöglichkeiten sozialer Indikatoren zum Nachweis der Beziehungen zwischen einem Unternehmen und seiner Umwelt. Gegen die eindimensionalen Vollrechnungen vom Typ der oben dargestellten Eichhornschen gesellschaftsbezogenen Unternehmensrechnung ${ }^{1)}$ bringen die Protagonisten mehrdimensionaler Berichtsysteme im wesentlichen zwei Argumente vor. Zum einen existierten keine allgemein anerkannten Kriterien dafür, wie weit der Kreis der durch die Unternehmensaktivitäten betroffenen Individuen $\mathrm{zu}$ ziehen sei. Je nachdem, ob man nur die direkten Wirkungen auf die unmittelbar Betroffenen, oder auch indirekte Wirkungen erfasse, ergebe sich ein unterschiedlicher Sozialsaldo. Verschiedene Ansätze umgehen dieses strittige Problem der Vollständigkeit, indem sie die soziale Rechnungslegung auf einzelne Teilbereiche beschränken ${ }^{2}$. Zum anderen entziehe sich eine Vielzahl von positiven und negativen externen Effekten, insbes. die Verursachung von Beeinträchtigungen der Gesundheit, einer monetären Bewertung, die die Beurteilung durch die Betroffenen widerspiegelt. Deshalb sollten vor allem physische MaßgröBen, teilweise auch nur verbale Beschreibungen, die Umweltbeziehungen eines Unternehmens abbil$\operatorname{den}^{3)}$.

Die meisten bisher vorliegenden Ansätze einer mehrdimensionalen gesellschaftsbezogenen Berichterstattung zeichnen sich durch eine Beschränkung auf ausgewählte Teilbereiche und die

1) Vgl. dazu Abschnitt 2.8.1. in diesem Kapitel.

2) Vgl. etwa $\mathrm{K}$. von Wysocki: Meß- und Bewertungsprobleme in der sozialen Rechnungslegung, a.a.0., S. 173.

3) Vgl. etwa P. Jäger: Probleme und Möglichkeiten einer mit sozialen Kosten und Nutzen erweiterten Erfolgswïrdigung öffentlicher Betriebe des Personen-Nahverkehrs, Diss. München 1974 , S. $183 \mathrm{ff}$. und A. Picot: Betriebswirtschaftliche Umweltbeziehungen und Umweltinformationegn -078-9-69p-955905\% $178 \mathrm{ff}$. 
Dominanz von (objektiven) Inputindikatoren aus. Das in Frankreich seit 1977 gesetzlich vorgeschriebene Sozialindikatorensystem begnügt sich mit der Beschreibung von Beziehungen zwischen einem Unternehmen und seinen Mitarbeitern und enthält überwiegend Aktivitäten bzw. Inputs des Unternehmens. Demgegenüber geht der Vorschlag des Deutschen Gewerkschaftsbundes über das "innere Beziehungsfeld" hinaus. Er enthält auch Indikatoren für die Beziehungen eines Unternehmens zur öffentlichen Hand, die verursachten Umweltbelastungen und die Erfolge von Maßnahmen zu ihrer Vermeidung bzw. Beseitigung sowie die Beiträge des Unternehmens zur Erreichung von den Gewerkschaften als wichtig erachteter ziele. AuBer bei den Umweltbelastungen finden sich im äuBeren Beziehungsfeld jedoch keine Outputindikatoren. Uberwiegend Inputindikatoren enthält auch der Indikatorenkatalog des Verbandes der Chemischen Industrie, der sich auf relativ viele AuBenbeziehungen eines Unternehmens erstreckt ${ }^{1)}$. Wir wollen auf diese Vorschläge nicht näher eingehen, da sie sich schon aufgrund ihrer weitgehenden Inputorientierung und ihrer begrenzten Reichweite nicht für zwecke der Leistungsmessung eignen.

Wesentlich vielversprechender erscheint uns ein 1974 entwickelter Ansatz zur Erfolgswürdigung öffentlicher Betriebe des Personen-Nahverkehrs ${ }^{2}$ ). Dieser Vorschlag dient dazu, die von den genannten Betrieben verursachten sozialen Nutzen und Kosten möglichst vollständig zu quantifizieren und konzentriert sich so von vornherein auf das äuBere Beziehungsfeld eines Unternehmens. Den Ausgangspunkt bildet folgender Katalog von Oberzielen bzw. Aufgaben der öffentlichen Nahverkehrspolitik ${ }^{3)}$ :

1) Vgl. zu diesen und weiteren Ansätzen K. von Wysocki: Sozialbilanzen... a.a.0., S. $132 \mathrm{ff}$. und ergänzend H.J. Gärtner: Indikatoren in der gesellschaftsbezogenen Unternehmensberichterstattung, in: H. -J. Hoffmann-Nowotny (Hrsg.): Sozialbilanzierung. Soziale Indikatoren VIII. Konzepte und Forschungsansätze, Frankfurt, New York 1981, S. 69-86.

2) Vgl. P. Jäger: Probleme und Möglichkeiten... a.a.0.

3) Ebenda, S. 112. 
"(1) Schutzpolitische Erhaltungsaufgaben

(a) Sicherung des menschlichen Lebens und der menschlichen Gesundheit

(aa) Sicherung gegen Verkehrsunfälle

(ab) Sicherung gegen Lärm

(ac) Sicherung gegen Luftverschmutzung

(b) Schutz der natürlichen Hilfskräfte des Menschen (ba) Sicherung gegen Raumvergeudung

(bb) Sicherung gegen Energievergeudung

(c) Schutz gegen verkehrsbedingte Eingriffe in privates und öffentliches Eigentum

(d) Schutz des historisch gewachsenen Stadtbildes

(2) Aufgabe der Unterstützung von Bemühungen, Stadtund Regionalentwicklungsziele zu erreichen

(3) Aufgabe der straßenentlastung

(4) Aufgabe der Sicherung des Verkehrszuganges und der Verkehrsbedienung."

Diese Oberziele werden in einen umfangreichen Katalog von Erfolgskomponenten untergliedert. Die Erfolgskomponenten geben die von öffentlichen Unternehmen des Personen-Nahverkehrs verursachten sozialen Kosten (u.a. Verkehrsunfälle und deren Folgekosten, Belastungen der Umwelt durch Lärm und Luftverschmutzung) einerseits und die sozialen Nutzen, die überwiegend als vermiedene soziale Kosten definiert sind (z.B. vermiedene Unfälle, vermiedene Umweltbelastungen; jeweils im Vergleich zum Individualverkehr), andererseits wieder ${ }^{1)}$. Die Operationalisierung der einzelnen Erfolgskomponenten erfolgt mit Hilfe einiger monetärer, größtenteils jedoch physischer Maßgrößen (z.B. Emissions- und Immissionswerte), nur in wenigen Fällen bleibt es

1) Der vollständige Katalog von Erfolgskomponenten ist im Anhang dieser Arbeit wiedergegeben. 
bei einer verbalen Beschreibung ${ }^{1)}$. Grundsätzlich geht der Anspruch dahin, Erfolgsindikatoren zu konstruieren, die die Ermittlung von zielerreichungsgraden erlauben ${ }^{2)}$.

Dieser Vorschlag, der die herkömmliche betriebliche Erfolgsrechnung ergänzen soll, weist, wenngleich er aufgrund seiner Konzipierung für öffentliche Betriebe des Personen-Nahverkehrs lediglich Beispielcharakter besitzt, mehrere positive Aspekte im Hinblick auf die Leistungsmessung bei Unternehmen auf. Zunächst einmal strebt der Ansatz die vollständige Erfassung der Auswirkungen der Unternehmensaktivitäten auf dessen physische und soziale Umwelt an, indem er die sozialen Nutzen und Kosten sehr weit abgrenzt. Sodann ist das Kriterium der Outputorientierung weitgehend erfüllt, da zur Operationalisierung der Erfolgskomponenten gröBtenteils Outputindikatoren dienen. Andererseits impliziert der bei den sozialen Nutzen liberwiegend zum zuge kommende Ansatz von im Vergleich zum Individualverkehr vermiedenen Kosten, daß teilweise nicht gemessene, sondern hypothetische Outputkomponenten in die Rechnung eingehen. Die zur Operationalisierung der sozialen Nutzen und Kosten vorgeschlagenen Indikatoren können freilich - ähnlich wie die im letzten Abschnitt diskutierten Resultatindikatoren - nicht in jedem Fall gewährleisten, daß die abgebildeten wirkungen für die betroffenen Individuen auch wohlfahrtsrelevant sind.

\subsection{Kosten-Wirksamkeits-Analysen}

Kosten-Wirksamkeits-Analysen (KWA) sind Verfahren der Identifikation des besten aus mehreren alternativen Projekten zur Lösung einer fest umrissenen Aufgabe. Die Auswahl beruht auf einem Vergleich der verschiedenen Projekte bezüglich ihrer Kosten und ihrer Wirksamkeit. Unter "Kosten" versteht man im

\footnotetext{
1) Vgl. P. Jäger: Probleme und Möglichkeiten... a.a.0., S. $218 \mathrm{ff}$.

2) Vgl. ebenda, S. $118 \mathrm{ff}$.
} 
Rahmen der KWA ebenso wie bei Nutzen-Kosten-Analysen die i.d.R. in Geldeinheiten gemessenen Opportunitätskosten eines Projektes $^{1)}$. Sie setzen sich zusammen aus Investitionskosten, den während der Laufzeit eines Projektes anfallenden laufenden Kosten sowie ggf. den durch das Projekt verursachten Schäden für die Gesellschaft wie etwa Lärm, Luftverschmutzung etc. ${ }^{2)}$. Die Wirksamkeit der betrachteten Alternativen wird im Hinblick auf i.d.R. mehrere Anforderungen beurteilt, die die politischen Entscheidungsträger an die Lösung einer Aufgabe stellen. So können die Entscheidungsträger etwa mit Maßnahmen zur Verbesserung der innerstädtischen Verkehrsverhältnisse die zielvorstellungen "Verkürzung der Fahrtzeiten" sowie "Erhöhung der sicherheit" und "Erhöhung der Bequemlichkeit" verbinden. Die Aufgabe der Analytiker besteht zunächst darin, die ihnen vorgegebenen Beurteilungskriterien zu operationalisieren, indem sie so weit wie möglich quantitative (primär physische, teilweise auch monetäre) Maßstäbe ableiten. Diese geben die Auswirkungen (Wirksamkeit) eines Projekts auf die erwünschten Teilziele wieder. Die Auswahl geeigneter Projekte obliegt ebenfalls den Experten. Sie haben für jede Alternative die Kosten zu ermitteln und die Wirksamkeiten auf die Teilziele zu quantifizieren oder zumindest so exakt zu beschreiben, daß sie einem Projekt hinsichtlich eines jeden Kriteriums eine Rangziffer zuordnen können $^{3)}$. Den Bezugspunkt, von dem aus die Konsequenzen gemessen

1) Vgl. E. S. Quade: Kosten-Wirksamkeits-Analyse, in: H.C. Recktenwald (Hrsg.): Nutzen-Kosten-Analyse und Programmbudget. Grundlage staatlicher Entscheidung und Planung, Tübingen 1970, S. 235-242, S. 238 .

2) Vgl. U. Meyke: Cost-Effectiveness-Analysis als Planungsinstrument. Unter besonderer Berücksichtigung von Infrastrukturinvestitionen im Verkehr, Göttingen 1973, s. $82 \mathrm{ff}$. und H. Hesse: Die Kostenwirksamkeitsanalyse, in: Verwaltung und Fortbildung, 3. Jg. (1975), S. 79-90, S. 82.

3) Vgl. H. Hesse: Die Kostenwirksamkeitsanalyse, a.a.0., S. 81 ff. und V. Arnold: Methoden der Entscheidungsfindung bei staatlichen Allokationsaktivitäten - ein kritischer Vergleich, in: Finanzarchiv N.F., Bd. 33 (1974/75), S.418434 , S. 420 ff. 
werden, gibt dabei die Situation ab, in der keine der betrachteten Alternativen realisiert wird ${ }^{1)}$. Um die Vergleichbarkeit der Alternativen zu gewährleisten, legen die Analytiker die Projekte nach Größe und Struktur so fest, daß sie entweder die gleichen Kosten verursachen (fixed cost approach) oder aber hinsichtlich ihrer Wirksamkeit nur unerheblich differieren (fixed effectiveness approach) ${ }^{2}$ ). Die Ergebnisse werden i.d.R. in Tabellen zusammengefaßt, die etwa folgende Form haben: ${ }^{3}$ )

\begin{tabular}{|c|c|c|c|c|c|c|c|}
\hline $\begin{array}{l}\text { Alter- } \\
\text { nativen }\end{array}$ & $\begin{array}{l}\text { Investi- } \\
\text { tions- } \\
\text { ausgaben }\end{array}$ & 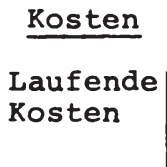 & $\mid \begin{array}{l}\text { Sonstige } \\
\text { Kosten }\end{array}$ & $\begin{array}{l}\text { Krite- } \\
\text { rium } \\
1\end{array}$ & $\begin{array}{l}\text { Nirksamk } \\
\text { Krite- } \\
\text { rium } \\
\quad 2\end{array}$ & $\begin{array}{l}\text { reit } \\
\left|\begin{array}{c}\text { Krite- } \\
\text { rium } \\
3\end{array}\right|\end{array}$ & etc. \\
\hline Projekt A & & & & & & & \\
\hline Projekt B & & & & & & & \\
\hline Projekt C & & & & & & & \\
\hline etc. & & & & & & & \\
\hline
\end{tabular}

Die Auswahl der "besten" Alternative mit Hilfe dieser Ubersicht ist weitgehend Aufgabe der politischen Entscheidungsträger. Beim fixed effectiveness approach gilt jenes Projekt als das beste, bei dem die geringsten Kosten anfallen. Beim fixed cost approach zeichnet sich die beste Alternative durch die höchsten wirksamkeiten in bezug auf die vorgegebenen Teilziele aus. Schwierigkeiten entstehen vor allem in folgenden Fällen ${ }^{4)}$ :

1) Vgl. V. Arnold: Methoden der Entscheidungsfindung... a.a.0., S. 420.

2) Vgl. A.D. Kazanowski: A Standardized Approach to Cost-Effectiveness Evaluations, in: J.M. English (Ed.): Cost-Effectiveness. The Economic Evaluation of Engineered Systems, New York et al. 1968, S. 113-150, S. 126 ff.; H. Hesse: Die Kostenwirksamkeitsanalyse, a.a.0., s. 83 .

3) Vgl. H. Hesse: Die Kostenwirksamkeitsanalyse, a.a.0., S. 82.

4) Vgl. ebenda, S. 83. 
- Kein Projekt weist hinsichtlich aller Kriterien die höchsten Wirksamkeiten auf, so daß im Hinblick auf ein Teilziel einer Alternative der Vorzug zu geben wäre, im Hinblick auf ein anderes Teilziel sich jedoch eine andere Alternative als überlegen erweist.

- Es gelingt den Analytikern nicht, die Projekte so auszuwählen bzw. zu dimensionieren, daß alle die annähernd gleichen Kosten verursachen. Zudem ist das Projekt mit den niedrigsten Kosten nicht zugleich dasjenige mit den größten wirksamkeiten.

Vor allem für den zweiten Fall wurde vorgeschlagen, die Alternativen mit Hilfe von Kosten-Wirksamkeits-Quotienten zu vergleichen. Eine derartige Vorgehensweise stößt jedoch in der Literatur weitgehend auf Ablehnung. Das Hauptargument gegen solche Quotienten weist darauf hin, daB sie die absolute Höhe der positiven Projektfolgen vernachlässigen ${ }^{1)}$. Die KWA selbst bietet zur Uberwindung dieser Probleme keine Lösungsmöglichkeiten. Die politischen Entscheidungsträger haben ihre wahl allein auf der Basis der von den Analytikern ermittelten Daten uber Kosten und Wirksamkeiten der Projekte zu treffen; es handelt sich bei der KWA lediglich um ein Verfahren zur Unterstützung politischer Entscheidungen ${ }^{2)}$. Es existieren zwar Verfahren, die die wirksamkeiten eines Projektes hinsichtlich der verschiedenen Teilziele mittels einer Standardisierung und Gewichtung zu einer

1) Vgl. U. Meyke: Cost-Effectiveness Analysis... a.a.o., S. $131 \mathrm{ff}$.

2) Vgl. H. Hesse: Die Kostenwirksamkeitsanalyse, a.a.0., s. 84 und E.S. Quade: Kosten-Wirksamkeits-Analyse, a.a.0., s. $239 \mathrm{f}$. 
einzigen Kennziffer, dem sog. Nutzwert, verdichten ${ }^{1)}$, diese Nutzwertanalysen gehen jedoch über den Bereich der kWA hinaus und sollen deshalb hier nicht diskutiert werden.

Vor einer Würdigung der KWA im Hinblick auf ihre Eignung zur Leistungsmessung möchten wir die Unterschiede zwischen KWA und Nutzen-Kosten-Analysen herausarbeiten. Bei der KWA handelt es sich nicht lediglich um eine Form der Nutzen-Kosten-Analyse, bei der die monetäre Bewertung der erfaßten Projektoutputs zugunsten physischer Maßgrößen weitgehend unterbleibt ${ }^{2)}$, sondern beide Verfahren weichen in Konzeption und Aussagefähigkeit wesentlich voneinander ab. Der grundlegende Unterschied betrifft die Frage, wessen Präferenzen bzw. Zielsysteme für die Entscheidung über die Durchführung eines Projektes relevant sind. Nutzen-Kosten-Analysen orientieren sich an den Präferenzen der durch ein Projekt positiv oder negativ Betroffenen selbst; ihre Zahlungsbereitschaft als Ausdruck ihrer Einschätzung der Projektfolgen dient als Bewertungsmaßstab. Bei KWA hingegen bewerten die politischen oder administrativen Entscheidungsträger die Konsequenzen alternativer Maßnahmen. Sie geben den Analytikern die von ihnen für relevant gehaltenen ziele bzw. Kriterien vor, und sie bewerten nach Abschluß der Analyse die

1) Zur Nutzwertanalyse siehe vor allem C. Zangemeister: Nutzwertanalyse in der Systemtechnik. Eine Methodik zur multidimensionalen Bewertung und Auswahl von Projektalternativen, 4. Aufl., München 1976 und die Arbeiten von Koelle, beispielsweise H.H. Koelle: Ansätze für ein praktikables, zielorientiertes Modell der gegenwärtigen Gesellschaft, in: Analysen und Prognosen, 4. Jg. (1972), Heft 22, S. 22-28 und Heft 23 , s. 23-28, insbes. Heft 23, s. $27 \mathrm{f}$.

Die Erläuterungen des Bundesministeriums der Finanzen zur Durchführung von Nutzen-Kosten-Untersuchungen gem. 57 Abs. 2 der Bundeshaushaltsordnung schreiben eine Version der KWA vor, die in inkonsistenter Weise Elemente der Nutzwertanalyse enthält. Vgl. hierzu V. Arnold: Methoden der Entscheidungsfindung... a.a.O., S. $420 \mathrm{ff}$. und H. Hesse: Die Kostenwirksamkeitsanalyse, a.a.0., S. $85 \mathrm{ff}$.

2) Dies scheint für Meyke der gravierendste Unterschied zwischen beiden Methoden zu sein. Vgl. U. Meyke: Cost-Effectiveness Analysis... a.a.0., s. 63 . 
ermittelten Projektfolgen; auf die Präferenzen der Betroffenen kommt es dabei nicht an ${ }^{1}$.

Primär eine Folge der verwendeten Bewertungsmaßstäbe ist es, daß Nutzen-Kosten-Analysen über die absolute, KWA hingegen nur über die relative Vorteilhaftigkeit eines Projektes zu informieren vermögen. Wegen der unterschiedlichen Dimensionen von Inputs und Outputs können KWA im Idealfall die untersuchten Alternativen zwar in eine Rangfolge bringen, nicht aber auch die durch die Realisation eines Projektes bewirkte absolute (Netto-) Veränderung gegenüber dem Status quo quantifizieren. KWA versagen deshalb in folgenden Fällen, während NutzenKosten-Analysen jeweils grundsätzlich eine eindeutige Lösung liefern ${ }^{2)}$ :

- Offen bleibt bereits, ob die Verwirklichung einer mit Hilfe der KWA als "beste" identifizierten Alternative auch volkswirtschaftlich gerechtfertigt ist. Sicher ist allenfalls, daß es sich um die Maßnahme handelt, die die geringsten Verluste verursacht.

- KWA sind dann nicht anwendbar, wenn zur Lösung eines Problems nur ein einziges projekt zur Verfügung steht.

- KWA gestatten keinen Vergleich von MaBnahmen, die zur Lösung unterschiedlicher Aufgaben dienen. Sie können beispielsweise nicht zur Beantwortung der Frage beitragen, ob vorhandene Budgetmittel besser zur Beseitigung der Luft-oder der Wasserverschmutzung eingesetzt werden sollen.

- KWA eignen sich nicht zur Bestimmung der optimalen Ausbaugröße eines Projekts.

1) Vgl. V. Arnold: Methoden der Entscheidungsfindung... a.a.0., S. 427 sowie H. Hesse: Die Kostenwirksamkeitsanalyse, a.a.O., S. 81 und S. 84 f. Dieser konzeptionelle Unterschied schließt freilich nicht aus, daß sich die politischen Entscheidungsträger uber die Ergebnisse von NutzenKosten-Analysen hinwegsetzen und stattdessen ein ihren eigenen Präferenzen eher entsprechendes Projekt realisieren können.

2) Vgl. V. Arnold: Methoden der Entscheidungsfindung... a.a.0., S. 431 und H. Hesse: Die Kostenwirksamkeitsanalyse, a.a.O., S. 81 . 
Ein weiterer Unterschied liegt in der Berücksichtigung der Zeitdimension. Während Nutzen-Kosten-Analysen zu unterschiedlichen zeitpunkten anfallende Projektwirkungen durch Abdiskontieren vergleichbar machen, gelingt dies bei KWA allenfalls für die Kosten $^{1)}$.

Unter dem Aspekt der Leistungsmessung ist festzuhalten, daB die KWA ein outputorientiertes Verfahren darstellt, da sie zur Quantifizierung spezifischer Projektwirkungen bzw. -outputs dient. Dabei taucht jedoch die Frage auf, ob es sich um eine umfassende und vollständige Outputmessung handelt. Dies scheint nicht grundsätzlich gewährleistet, denn welche Projektwirkungen erfabt werden, richtet sich nach den von den Entscheidungsträgern vorgegebenen Kriterien. Völliges Versagen ist hingegen bei der KWA aufgrund der dort relevanten Bewertungsinstanz bezüglich der Nachfrageorientierung zu konstatieren. Die (politischen) Entscheidungsträger bestimmen nicht nur die in der Analyse zu berücksichtigenden Projektwirkungen, ihnen obliegt auch deren Bewertung, während die Präferenzen der von einem Projekt Betroffenen keinerlei Rolle spielen. Daher erfüllen KWA nicht die an ein Leistungsmaß für nicht-staatliche Entscheidungseinheiten gestellten Anforderungen.

3.3. Das Planning-Programming-Budgeting system

\subsubsection{Ziele und Charakteristika}

Hinter der Bezeichnung "Planning-Programing-Budgeting System" (PPBS) verbirgt sich kein eindeutig definiertes Planungssystem, sondern eine Reihe verschiedener Varianten. Die Grundzüge des PPBS wurden innerhalb der RAND-Corporation entwickelt; verbindlich eingeführt wurde es 1961 im Us-Verteidigungsministerium und 1965 für die gesamte US-Bundesverwaltung. Nachahmung fand es u.a. in Schweden, Kanada, Japan, Belgien, Israel und Frankreich sowie bei zahlreichen nachgeordneten Gebietskörper-

1) Vgl. V. Arnold: Methoden der Entscheidungsfindung... a.a.0., S. 431 . 
schaften der USA ${ }^{1)}$. Obschon das PPBS auf der US-Bundesebene bereits 1971 wieder abgeschafft wurde, wollen wir im folgenden auf die Version der US-Bundesregierung Bezug nehmen, die in der Literatur am meisten Beachtung fand.

Das PPBS bildete die dritte station der US-Budgetreformen ${ }^{2}$. Während in den USA ursprünglich die einzelnen Ressorts direkt mit dem Parlament über die Bewilligung von Haushaltsmitteln verhandelten, schrieb der "Budget and Accounting Act" von 1921 erstmals ein einheitliches und umfassendes Budget vor, das vom Präsidenten einzubringen war und vom neugeschaffenen Bureau of the Budget vorbereitet wurde. Die vorgeschriebene Klassifikation der öffentlichen Ausgaben nach Personal- und Sachausgaben usw. diente primär dem ziel der Kontrolle der Exekutive durch das Parlament im Hinblick auf eine juristisch ordnungsgemäße Verwendung der bewilligten Mittel. Diesem kontrollorientierten Planungsabschnitt folgte eine managementorientierte Phase, die im 1949 von der Hoover-Commission vorgeschlagenen "performance budgeting" ihren Höhepunkt fand. Danach sollte die Haushaltsklassifikation nach Ausgabenarten zugunsten einer Klassifikation nach den Aktivitäten der Regierungs- und Verwaltungseinheiten aufgegeben werden und die weitere Aufteilung der einer Funktionseinheit bewilligten Mittel auf die verschiedenen Ausgabenarten dieser selbst überlassen bleiben. Im Vordergrund stand nicht mehr die traditionelle Inputkontrolle, sondern die

1) Vgl. H. Reinermann: Programmbudgets in Regierung und Verwaltung. Möglichkeiten und Grenzen von Planungs- und Entscheidungssystemen, Baden-Baden 1975, S. 93 ff.; P. Senf: Kurzfristige Haushaltsplanung, a.a.O., S. 418 ff.; E. Wille: öffentlicher Haushalt IV... a.a.O., S. $613 \mathrm{ff}$. Berichte über die Erfahrungen verschiedener Länder mit dem PPBS finden sich in J.H. Kaiser (Hrsg.): Planung VI. Integrierte Systeme der Planung und Budgetierung, Baden-Baden 1972 und in D. Novick (Ed.): Current Practice in Program Budgeting (PPBS). Analysis and Case Studies Covering Government and Business, London 1973.

2) Vgl. zum Folgenden A. Schick: The Road to PPB: The Stages of Budget Reform, in: F. J. Lyden und E. G. Miller (Eds.): Public Budgeting: Program Planning and Evaluation, 3rd Ed., Chicago 1978, S. 17-42, S. 21 ff und H. Reinermann: Programmbudqets... a.a.O. S. 109 fowniloadé from PubFactory at 01/11/2019 03:20:37AM 
Beurteilung der Aktivitäten der Funktionseinheiten im Hinblick auf ihre Produktionseffizienz, wobei als Kriterien Aktivitätskennziffern oder allenfalls Maßgrößen für das öffentliche Angebot dienten ${ }^{1)}$. Diese beiden Stufen der US-Haushaltsreformen gingen von existierenden Aktivitäten der Verwaltung aus und konzentrierten sich auf die Kontrolle der Vorschriftsmäßigkeit der Ausgabengebarung bzw. auf die Minimierung der Inputs bei der Entfaltung bestimmter Aktivitäten, ohne die staatlichen $\mathrm{ziele}$ selbst $\mathrm{zu}$ analysieren.

Das PPBS sollte die Hauptmängel der herkömmlichen Budgetierungspraxis $^{2)}$ überwinden. Uber die Intentionen und die Grundstruktur des Planungskonzeptes bzw. organisierten Entscheidungsprozesses PPBS geben bereits seine vier begrifflichen Elemente Auskunft. Im Rahmen des "Planning" möchte man die Ziele der staatlichen Aktivitäten outputorientiert, d.h. möglichst auf den objectives-Ebenen, operationalisieren und quantifizieren. Das "Programming" umfaßt die systematische Analyse von Maßnahmen, u.a. mit Hilfe von Nutzen-Kosten-Analysen, und die Auswahl derjenigen Alternativen, die sich am besten zur Erreichung der konkretisierten Ziele eignen. Im AnschluB an die Maßnahmenplanung erfolgt die Ubertragung der ausgewählten Programme in

1) Die Niederlande führten 1976 ein Performanz-Budget ein. Vgl. H. M. van de Kar: User Charges, Privatization and Performance Budgeting in the Social Services, Manuskript für den 39. Kongress des International Institute of Public Finance in Budapest, 22.-26. Aug. 1983.

2) Die budgetären Entscheidungen orientierten sich nicht an Zielen, die als konsumierbare Endprodukte konkretisiert wurden, sondern bezogen sich auf laufende Aktivitäten und deren marginale Veränderungen; es erfolgte keine systematische Analyse aller Aktivitäten, verbunden mit einer Gegenüberstellung der Nutzen und Kosten jeder Alternative; der Zeithorizont der Budgetperiode erwies sich in vielen Fällen als zu eng. Vgl. hierzu P. Senf: Kurzfristige Haushaltsplanung, a.a.O., S. 419; ähnlich D. Brümmerhoff: Das "Planning-Programming-Budgeting System" (PPBS), in: Finanzarchiv N.F., Bd. 29 (1970), S. 64-74, S. $64 \mathrm{f}$. 
Geldeinheiten und in die Systematik des herkömmlichen Exekutivbudgets ("Budgeting"). Diese Elemente bilden, da sie inhaltlich und organisatorisch in vielfältiger Weise miteinander verzahnt sind, integrale Bestandteile eines ganzheitlichen Planungskonzepts bzw. -systems ${ }^{1)}$.

Das hervorragendste Merkmal des PPBS ist seine Outputorientierung. Es eignet sich dazu, verschiedene Alternativen zur Erreichung spezifischer ziele zu vergleichen und zu beurteilen. Dies impliziert jedoch nicht notwendigerweise eine Präjudizierung der politischen Entscheidungsträger durch deterministische Ergebnisse. Nach Ansicht seiner geistigen Väter soll das PPBS nicht dazu dienen, eine eindeutige Lösung des Allokationsproblems im Sinne von Maximierungs- und Optimierungspostulaten zu liefern, indem es die ziele selbst setzt. Vielmehr ist es Sache der politischen Entscheidungsträger, die ziele und deren Rangordnung festzulegen. Das PPBS hilft dabei, die so vorgegebenen ziele zu operationalisieren, auf Widersprüche aufmerksam zu machen und die relative Erreichbarkeit der ziele im Hinblick auf die begrenzten Ressourcen zu prüfen. Es soll die politische Entscheidung nicht ersetzen, sondern sie als sachlich-analytische Informationsbasis unterstüzen ${ }^{2)}$.

In formaler Hinsicht basiert die PPBS-Version der US-Bundesregierung auf fünf Instrumenten: program structure, issue letters, program memoranda, special analytic studies, program and financial plan. Wir beschränken uns hier auf die Grundkonzeptionen der für die Leistungsmessung wichtigsten Instrumente.

1) Vgl. E. Wille: రffentlicher Haushalt IV... a.a.0., S. 614; B. Rürup: Die Programmfunktion des Bundeshaushaltsplanes... a.a.O., S. 58; C. Böhret: Entscheidungshilfen für die Regierung. Modelle, Instrumente, Probleme, Opladen 1970, S. 180.

2) Vgl. A. Schick: The Road to PPB... a.a.O., S. 29 f.;

C. Böhret: Entscheidungshilfen für die Regierung... a.a.0.,

S. $180 ;$ H. Reinermann: Programmbudgets... a.a.0., S. $119 \mathrm{f}$;

P. Senf: Kurzfristige Haushaltsplanung, a.a.O., S. 420;

E. Wille: రffentlicher Haushalt IV... a.a.O., S. $614 \mathrm{f}$. 
Die Programmstrukturen ${ }^{1)}$ nehmen eine zentrale stellung im Rahmen des PPBS ein, weil sie in wesentlichen Teilen längerfristigen Bestand haben und sich an ihnen die übrigen Instrumente ausrichten. Eine Programmstruktur beinhaltet eine hierarchische Aktivitäten- bzw. Aufgabenstruktur für ein einzelnes Ressort oder eine Behörde. Sie weist auf der untersten Ebene sämtliche Aktivitäten einer Verwaltungseinheit aus und faBt sie auf den darübergelagerten Stufen zielorientiert zusammen; außerdem enthält sie auf jeder Ebene die mit einer Aktivitätengruppe angestrebten Ziele. Die Budgetansätze weist eine Programmstruktur allerdings nicht aus, sondern sie stellt lediglich deren Klassifikation zur Verfügung. Sie läßt sich sowohl deduktiv als auch induktiv aufstellen und interpretieren; in der Praxis setzte sich bei der Ableitung von Programmstrukturen eine Mischung aus beiden Vorgehensweisen durch. Meistens enthält eine Programmstruktur drei bis vier Ebenen: Programmategorien, Programmsubkategorien, Programmelemente und ggf. -subelemente. An der Spitze rangieren die (pro Ressort liblicherweise fünf bis zehn) Programmkategorien. Eine Programmkategorie beschreibt in vager Form eine bestimmte Gruppe von Ressortaktivitäten, z.B. "Aufrechterhaltung einer physischen Umwelt, die möglichst wenig Gesundheits- und Vermögensschäden verursacht". Die ziele der Aktivitätengruppen auf der Ebene der Programmategorien lassen sich meistens nur verbal als Goals oder Leitbilder ${ }^{2}$ formulieren.

Jede Programmkategorie wird auf der nachgelagerten Ebene in Programmsubkategorien oder Unterprogramme unterteilt, die als Mittel zur Erreichung der ziele der Programmategorien dienen. Programmsubkategorien gruppieren Ressortaktivitäten nach weniger abstrakten Kriterien als Programmkategorien, auf dieser Ebene finden sich beispielsweise Maßnahmen zur "Kontrolle der

1) Vgl. zum Folgenden H. Reinermann: Programmbudgets... a.a.0., S. $120 \mathrm{ff.;} \mathrm{E.} \mathrm{Wille:} \mathrm{రffentlicher} \mathrm{Haushalt} \mathrm{IV...} \mathrm{a.a.O.,}$ S. $608 \mathrm{ff.;} \mathrm{C.} \mathrm{Böhret:} \mathrm{Entscheidungshilfen} \mathrm{für} \mathrm{die} \mathrm{Regie-}$ rung... a.a.O., S. 182 ff.

2) Vgl. hierzu auch Schaubild 1. 
Luftverschmutzung". Die dazugehörenden Ziele sind besser operationalisierbar als diejenigen auf der Ebene der Programmategorien und lassen sich häufig quantifizieren. Grundsätzlich handelt es sich um impact-objectives, die die angestrebten Auswirkungen der Aktivitätengruppen auf die Leistungsempfänger wiedergeben. Die herausragende Bedeutung der Programmsubkategorien im Rahmen einer Programmstruktur liegt darin begründet, da $B$ sie eine Brücke zwischen den übergeordneten Goals oder Leitbildern und den auf der dritten Hierarchieebene angesiedelten konkreten Ressortaktivitäten schlagen und bei deduktiver Betrachtung die erste Hierarchieebene bilden, die eine Quantifizierung von zielen erlaubt.

Die Ebene der Programmelemente enthält die spezifischen, identifizierbaren Aktivitäten eines Ressorts oder einer Behörde, die zu einem bestimmten Output führen. Sie stellen wiederum Mittel zur Erreichung der ziele der jeweils übergeordneten Programmsubkategorien dar. Beispielsweise kann die Subkategorie "Kontrolle der Luftverschmutzung" u.a. das Element "Inspektion von Fabrikanlagen" enthalten. Programmelemente stellen die Grundbausteine (building blocks) einer Programmstruktur dar. zur Formulierung der mit Programmelementen verbundenen zielsetzungen dienen Ausbringungsziele (output-objectives), im obigen Beispiel etwa die zahl der Inspektionen pro zeiteinheit.

Somit bildet eine Programmstruktur eine aktivitätenbezogene Ziel-Mittel-Hierarchie mit mehreren, durch Kausalrelationen verbundenen, Ebenen. Sie beruht auf einer Funktionsanalyse für ein Ressort, die die Beiträge der verschiedenen Aktivitäten zu bestimmten zielen ermittelt. Auf jeder Ebene weist sie Aktivitäten mit komplementärem oder substitutivem Charakter, d.h. vom ziel her zusammengehörende Aktivitäten, unabhängig von organisatorischen Begrenzungslinien nebeneinander aus.

Eine zweckmäßige Programmstruktur genügt folgenden Kriterien ${ }^{1)}$ :

1) Vgl. H. Reinermann: Programmbudgets... a.a.0., S. $125 \mathrm{f}$. 
- Sie enthält ausschließlich konsumierbare Endprodukte staatlichen Handelns bzw. bezieht alle identifizierbaren Aktivitäten über Ziel-Mittel-Relationen auf solche Endprodukte.

- In deduktiver Hinsicht führt sie hin zu identifizierbaren Aktivitäten von Ressorts und Behörden, über die Programmund Ausgabenniveauentscheidungen getroffen werden können.

- Bei den auf jeder Ebene nebeneinanderstehenden (substitutiven, nicht jedoch komplementären) Aktivitäten(-gruppen) handelt es sich um solche, zwischen denen die politischen Entscheidungsträger tatsächlich zu wählen haben.

- Die ausgewiesenen ziele sind operational, jedoch nicht so eng formuliert, daB sie den Blick auf Alternativen verstellen. Zudem erfolgt die zielformulierung in einer Weise, die die Aufstellung von den Grad der Zielerreichung messenden Kriterien gestattet.

Von der Konzeption her würde sich eine einheitliche, ressortübergreifende Programmstruktur dazu eignen, die vielfältigen interministeriellen Aufgabenüberschneidungen, beispielsweise im Gesundheitswesen, Verkehrswesen und Umweltschutz, offenzulegen und auf Planungslücken und Doppelplanungen aufmerksam zu machen ${ }^{1)}$. Beim Vollzug der Programmstrukturierung in der USBundesregierung blieben allerdings die Ressortgrenzen gewahrtvor allem aufgrund antizipierter Widerstände der Ressorts gegen eine Programmstrukturierung, die ihre zuständigkeitsbereiche überschreiten würde ${ }^{2)}$.

In den analytischen Spezialstudien versuchten die Ressorts, für vorgegebene Fragenkreise alternative zielsetzungen und zielerreichungskriterien sowie alternative Programme zur Erreichung der zielsetzungen zu finden und jeweils deren Auswirkungen auf die $z$ iele $z u$ beschreiben. Indem für jede betrachtete Alternative Aufwendungen und Auswirkungen für unterschiedliche Aktivitätsniveaus abgeschätzt werden sollten, zielten diese Analysen

1) Vgl. E. Wille: రffentlicher Haushalt IV... a.a.O., S. 609.

2) Vgl. H. Reinermann: Programmbudgets... a.a.O., S. 127. 
letztlich auf die Konstruktion staatlicher Produktionsfunktionen ab. Dabei waren sowohl die Programmaufwendungen als auch die -wirkungen möglichst umfassend und ohne Berücksichtigung von Ressortgrenzen zu ermitteln. Als analytische Hilfsmittel dienten u.a. Nutzen-Kosten- und Kosten-Wirksamkeits-Analysen ${ }^{1)}$.

Die Verbindung von Aufgaben- und Finanzplanung dokumentierte sich in den Programm- und Finanzplänen (PFP), die jedes Ressort bzw. jede Behörde zu erstellen hatte. Sie sollten eine zielorientierte Totalübersicht über sämtliche Regierungsaktivitäten ermöglichen. Ein PFP enthält die Finanzplanung eines Ressorts in der Ordnung der Programmstruktur unter Angabe quantifizierter Programmwirkungen, indem er neben den geplanten Programmwirkungen (outputs) die für die Durchführung erforderlichen Ausgaben ausweist ${ }^{2}$. Die Transformation der Programmausgaben in die traditionelle Bewilligungsstruktur des Jahreshaushaltsplans erfolgte durch eine Matrix, den sog. "crosswalk"3).

\subsubsection{Leistungsmessung im Rahmen des PPBS}

Im Rahmen dieser Arbeit wollen wir keine würdigung des Planungskonzeptes PPBS vornehmen oder die Gründe seines Scheiterns in der US-Bundesadministration analysieren ${ }^{4)}$, sondern der Frage

1) Vgl. C. Böhret: Entscheidungshilfen für die Regierung... a.a.O., S. 189,ff.; H. Reinermann: Programmbudgets... a.a.o., S. $131 \mathrm{ff}$. und $\mathrm{K}$. Gresser: Das Planning-Programming-Budgeting System. Probleme der Anwendung bei der staatlichen Aufgabenund Finanzplanung, München-Pullach, Berlin 1972, S. $17 \mathrm{ff}$.

2) Vgl. C. Böhret: Entscheidungshilfen für die Regierung... a.a.O., S. $197 \mathrm{ff}$.; K. Gresser: Das Planning-ProgrammingBudgeting System... a.a.O., S. $19 \mathrm{ff.;}$ H. Reinermann: Programmbudgets... a.a.0., S. $139 \mathrm{ff}$.

3) Vgl. zum crosswalk E. Wille: రffentlicher Haushalt IV... a.a.0., S. $615 \mathrm{f}$.

4) Vgl. zu diesen Fragestellungen vor allem C. Böhret: Entscheidungshilfen für die Regierung... a.a.O., S. $204 \mathrm{ff} . ; \mathrm{H}$. Reinermann: Zehn Jahre Systembudgetierung in der US-Bundesregierung. Analyse eines Experiments, in: J.H. Kaiser (Hrsg.): Planung VI. Integrierte Systeme der Planung und Budgetierung, Baden-Baden 1972, S. 301-342, S. 318 ff.; H. Reinermann: Programmbudgets... a.a.0., s. $169 \mathrm{ff}$. 
nachgehen, ob das PPBS Impulse für die Leistungsmessung auch bei nicht-staatlichen Entscheidungseinheiten zu geben vermag. Von seiner Konzeption her erfüllt das PPBS das für die Leistungsmessung unerläßliche Kriterium der Outputorientierung ${ }^{1)}$, die sich vor allem in den Instrumenten Programmstruktur, analytische Spezialstudien und Programm- und Finanzpläne manifestiert. Gestützt auf die Spezialstudien, die teilweise sogar impact-objectives ausweisen, sollen die Programm- und Finanzpläne quantifizierte ziele auf der Ebene der output-objectives enthalten. Diese Zielvorgaben eignen sich im Prinzip auch für eine ex post vorgenommene Kontrolle des Leistungsvolumens, welches ein Anbieter öffentlicher Leistungen abgibt und damit auch für die Beurteilung der Effizienz und der Effektivität öffentlicher Leistungen.

Obschon der programmatische Aspekt ein konstitutives Element des PPBS bildet, bietet es zur Ermittlung dieser ziele bzw. Outputs keine neuen, eigenständigen Verfahren. Es stellt vielmehr einen konzeptionellen und organisatorischen Rahmen dar, in dem verschiedene Verfahren der Outputmessung zum zuge kommen können. Dabei handelt es sich vor allem um Nutzen-Kostensowie um Kosten-Wirksamkeits-Analysen. Im Prinzip bietet das PPBS auch Einsatzmöglichkeiten für soziale Indikatoren. Sie könnten grundsätzlich den erheblichen Informationsbedarf des organisierten Entscheidungsprozesses PPBS befriedigen und insbesondere zur Operationalisierung von $\mathrm{zielen}$ dienen. Einige Politiker in den USA erkannten relativ früh den Mangel an politischen und sozialen Daten und bemühten sich um die Institutionalisierung von Sozialberichten, die über erreichte Erfolge informieren und die Grundlage für das setzen programmatischer Prioritäten abgeben sollten ${ }^{2)}$. Bei der Einführung des PPBS befand sich die Sozialindikatorenforschung jedoch noch in embryonalem Zustand.

1) Vgl. auch E. Wille: రffentlicher Haushalt IV... a.a.O.,S.615.

2) Vgl. C. Böhret: Entscheidungshilfen für die Regierung... a.a.o., S. $148 \mathrm{ff}$ und S. $171 \mathrm{ff}$. 
Unsere bisherigen Untersuchungen ergaben, daß diese drei Ansätze konzeptionell zwar das Kriterium der Outputorientierung erfüllen, indes lediglich Nutzen-Kosten-Analysen und Nutzungsindikatoren auch Nachfragebezogenheit aufweisen; während letztere bei den Resultatindikatoren auf individueller Ebene nicht in jedem Falle vorliegt. Kosten-Wirksamkeits-Analysen hingegen verzichten grundsätzlich auf die Bewertung von Outputeinheiten durch die Betroffenen.

Den hohen konzeptionellen Ansprüchen genügte das PPBS in der US-amerikanischen Praxis nicht durchgehend. In den Programmund Finanzplänen konnten nur eine oder zwei Auswirkungen öffentlicher Aktivitäten aufgeführt werden, und i.d.R. enthielten sie nur unmittelbar zurechenbare Projektoutputs wie etwa die $\mathrm{Zahl}$ der Auszubildenden oder der zu Impfenden ${ }^{1)}$. Eine derartig eingeengte Betrachtungsweise wird der Multidimensionalität öffentlicher Aktivitäten nicht gerecht; sie läßt vor allem eine Vernachlässigung externer Effekte bzw. eine unvollständige Erfassung von Programmoutputs befürchten. Vielfach beruhten die entsprechenden Angaben in den Programm- und Finanzplänen auch nicht auf analytischen Spezialstudien, sondern lediglich auf Schätzungen ${ }^{2)}$, so daß insoweit die Konstruktion staatlicher Produktionsfunktionen unterblieb. Die analytischen Spezialstudien orientierten sich in manchen Fällen nicht an impact-, sondern an output-objectives und miBachteten teilweise Interdependenzen zwischen verschiedenen Ressorts, so etwa bei bestimmten Ausbildungsprojekten, die verschiedene Ministerien jeweils isoliert durchführten und auch analysierten ${ }^{3)}$. Insgesamt erwies sich das PPBS am ehesten für solche Entscheidungen als geeignet, die direkte Budgetkonsequenzen hatten; es führte

1) Vgl. H. Reinermann: Programmbudgets... a.a.0., S. 277.

2) Vgl. ebenda, S. 277.

3) Vgl. ebenda, S. 275. 
dementsprechend im Gesundheits- und Verkehrswesen $\mathrm{zu}$ besseren Ergebnissen als etwa im Bereich der auswärtigen Beziehungen oder der Justiz ${ }^{1 \text { ). }}$

4. Ausgewählte Verfahren zur Messung der öffentlichen Leistungen nicht-staatlicher Entscheidungseinheiten

Die Untersuchungen in diesem Kapitel bezogen sich auf die von nicht-staatlichen Entscheidungseinheiten produzierten Güter (i.d.R. Dienstleistungen), weil dieser Produktionsoutput die gravierendsten Probleme im Hinblick auf die Leistungsmessung aufwirft. Nicht-staatliche Entscheidungseinheiten wirken indes an der Erfüllung öffentlicher Aufgaben nicht nur mit, indem sie Güter erstellen und absetzen. Sie erbringen weiterhin in Verfolgung distributions- oder/und allokationspolitischer ziele bestimmte Transfers an andere Wirtschaftseinheiten. Obschon zwischen diesen beiden Aktivitäten vielfältige Uberschneidungen existieren, wollen wir sie im folgenden getrennt analysieren. Zunächst versuchen wir, praktikable Verfahren zur Messung der Leistungen nicht-staatlicher Entscheidungseinheiten im Sinne eines abgesetzten Produktionsoutputs vorzuschlagen und untersuchen im anschließenden Abschnitt die von nicht-staatlichen Entscheidungseinheiten erbrachten Transfers.

4.1. Verfahren zur Messung der von nicht-staatlichen Entscheidungseinheiten produzierten Leistungen

Die von nicht-staatlichen Entscheidungseinheiten selbsterstellten Leistungen zeichnen sich dadurch aus, daß die Ressourcen einen Transformationsproze $B$ durchlaufen und erst ein Produktionsoutput zur Abgabe an Nachfrager zur Verfügung steht. Die Analyse ein- und mehrdimensionaler Ansätze zur Leistungsmessung

1) Vgl. C. Böhret: Entscheidungshilfen für die Regierung... a.a.o., S. $212 \mathrm{ff}$. 
in diesem Kapitel diente dazu, Outputmaße zu finden, die

- das von einem einzelnen Anbieter innerhalb eines bestimmten Zeitraums abgegebene Volumen an öffentlichen Leistungen ermitteln.

- die Bestimmung der Produktivität, mit der dieses Leistungsvolumen erstellt wird, erlauben.

Dabei begnügten wir uns nicht mit der Produktionseffizienz, für deren Ermittlung die Leistungsmessung sich an der Ebene der öffentlichen Produkte bzw. am Verfügungsmachtkonzept orientieren könnte. Vielmehr stellten wir mit Hilfe des Kriteriums der Nachfragebezogenheit eines Leistungsmaßes auf die Effektivität der Versorgung mit öffentlichen Leistungen ab.: Scheiterten von den untersuchten Verfahren viele an den Kriterien Output-oder/ und Nachfrageorientierung und muBten deshalb sofort verworfen werden, so lassen sich gleichwohl jene Ansätze, welche diese Kriterien weitgehend erfüllen, d.h. die Marktpreisbewertung, Nutzungs- und in bestimmten Fällen auch Resultatindikatoren sowie von der Konzeption her einige Verfahren der Nutzen-KostenAnalyse, nicht uneingeschränkt empfehlen. Trotz der aufgezeigten spezifischen Mängel kristallisierte sich die Marktpreisbewertung als das relativ beste der analysierten Verfahren heraus. Marktpreise informieren zwar, anders als im Prinzip Nutzen-Kosten-Analysen, nicht über die ganze, sondern lediglich über die Untergrenze der Zahlungsbereitschaft bzw. der bewerteten Vorteile, bedürfen jedoch keiner "künstlichen" Operationalisierung, die bei Nutzen-Kosten-Analysen häufig zu zweifelhaften Ergebnissen führt. Zudem liefern Marktpreise mehr Information als Nutzungsindikatoren, bei denen lediglich sichergestellt ist, daB die Nachfrager einer Leistung einen positiven Wert beimessen. In der Realität erzwingt die weitgehende Abwesenheit von Marktpreisen für die Leistungen nichtstaatlicher Entscheidungseinheiten Abstriche vor allem beim Kriterium Nachfrageorientierung, es läßt sich jedoch in den meisten Fällen wenigstens ansatzweise erfüllen. 
Die gröBten Probleme wirft die Leistungsmessung bei der Sozialversicherung auf. Sowohl die Gleichsetzung des Leistungsvolumens mit dem Beitragsaufkommen als auch eine Bewertung mit den Prämien vergleichbarer privater Versicherungen scheiden aus den oben dargestellten Gründen ${ }^{1)}$ aus, zumal ohnehin kein Pendant zur Arbeitslosenversicherung existiert. Die Ausgaben der Sozialversicherung für Zahlungen und die Abgabe von Sachgütern zugunsten ihrer Mitglieder andererseits spiegeln nicht die eigentliche Leistung, das Gewähren von Versicherungsschutz, sondern lediglich die Regelung von Schadensfällen wider. Letztlich verhindert die Ausgestaltung der Sozialversicherungsbeiträge als Zwangsabgaben eine Leistungsmessung, die auch nur rudimentär die Bewertung durch die Mitglieder berücksichtigt; jede Lösung muB sich auf die Messung eines Produktionsoutputs beschränken. Ein vages Abbild des von einem Sozialversicherungsträger gewährten Versicherungsschutzes vermittelt die $\mathrm{Zahl}$ der Mitglieder einschlieblich der mitversicherten Familienangehörigen. Diese Größe repräsentiert einen Output, denn jedes Mitglied kommt gezwungenermaßen in den GenuB des Versicherungsschutzes. Sie miBt ihn jedoch nicht tatsächlich und vermag deshalb auch nicht zwischen dem verschiedenen Mitgliedern gewährten (in Geldeinheiten ausgedrückt) unterschiedlich hohen Versicherungsschutz zu differenzieren. zudem liefert sie keine ausreichenden Informationen für eine Bestimmung der Produktionseffizienz. Ein theoretisch wesentlich aussagekräftigeres Outputmaß könnte die Summe der von einem Versicherungsträger abgedeckten Risiken, multipliziert mit der Schadenswahrscheinlichkeit, abgeben. Dieses fiktive Prämienaufkommen, das noch keinen Verwaltungskostenanteil enthält, entsteht durch Aggregation der individuellen Erwartungswerte der (versicherten) Schäden aller Mitglieder, läßt sich jedoch in der Realität kaum ermitteln. Eine weitere Möglichkeit besteht darin, nicht den output Versicherungsschutz selbst, sondern den zu seiner

1) Vgl. die Abschnitte 2.2. und 2.4.1. in diesem Kapitel. 
Erstellung erforderlichen intermediären Output Verwaltungsleistungen $z u$ erfassen. Hier müßte die zahl der in einer Periode bearbeiteten Fälle, gegliedert nach verschiedenen Vorgängen, gemessen werden. Allerdings hängt. diese Größe auch wesentlich von der $\mathrm{Zahl}$ der eingetretenen Schadensfälle ab.

Die Ständefisci (Kammern) finanzieren sich ebenfalls größtenteils aus Zwangsbeiträgen. Deshalb, und weil sie nur mit einem Teil ihrer Aktivitäten öffentliche Aufgaben erfüllen, lassen sich ihre öffentlichen Leistungen nicht nach ihren Beitragseinnahmen bemessen. Die bedeutsamsten Aktivitäten zur Erfüllung öffentlicher Aufgaben entfalten sie auf dem Gebiet der beruflichen Aus- und Fortbildung, einesteils in Form einer Erarbeitung von Vorschriften für die einzelnen Ausbildungsgänge, der Kontrolle von Ausbildung und Prüfungen, in zunehmendem Maße auch in Form der Einrichtung von überbetrieblichen Ausbildungszentren, ferner in Form der Erstattung von Gutachten für Behörden und Gerichte ${ }^{1)}$. Hierfür eignen sich als Leistungsmaße Nutzungsindikatoren wie die zahl der Auszubildenden in verschiedenen Ausbildungsgängen ${ }^{2)}$, die Zahl der abgenommenen Abschlußprüfungen und der erstatteten Gutachten.

1) Vgl. G. Frentzel und E. Jäkel: Die deutschen Industrieund Handelskammern und der Deutsche Industrie- und Handelstag, Frankfurt, Bonn 1967, S. 36 ff.; G. Kleinhenz: Wirtschaftskammern, in: Handwörterbuch der Wirtschaftswissenschaft (HdWW), hrsg. von W. Albers et al., Bd. 9, Stuttgart et al. 1982, S. 898-904, S. $900 \mathrm{ff}$.

2) Die Kammern verlangen für ihre Aus- und Fortbildungskurse i.d.R. ein Entgelt. Für Auszubildende sind diese Gebühren jedoch vom Lehrherren zu entrichten, so daB hier der Preis nicht die Bewertung der Leistung durch die NutznieBer ausdrückt. Bei Fortbildungsmaßnahmen hingegen tragen die Leistungsempfänger häufig die Gebühren selbst, insoweit kann die Leistungsmessung sogar auf Marktpreise zurückgreifen. Dabei ist allerdings zu berlucksichtigen, da $\beta$ diese Nachfrager zum Teil einen direkten Zuschuß von der Bundesanstalt für Arbeit erhalten. 
Der Lastenausgleichsfonds betreibt ausschließlich Umverteilungspolitik zum Ausgleich von kriegsbedingten Vermögensschäden. Als intermediären Produktionsoutput erstellt er lediglich Verwaltungsleistungen im Sinne einer Organisation der Redistribution, die sich im Prinzip nach der Anzahl der bearbeiteten Fälle bzw. Vorgänge bemessen lassen.

Das ERP-Sondervermögen einschließlich der Kreditanstalt für Wiederaufbau, die Wohnungsbauförderungsanstalten und ähnliche Institutionen erfüllen öffentliche Aufgaben primär, indem sie im Rahmen ihres gesetzlichen Auftrages Kredite und Gewährleistungen $z u$ häufig vergünstigten Konditionen vergeben. Ihre öffentlichen Leistungen lassen sich, da die Nachfrager dafür ein Entgelt zu entrichten haben, nach der summe der Effektivkosten der Kredite bzw. der entrichteten Gebühren für Gewährleistungen bemessen. Zumindest bei den Aktivitäten der Wohnungsbauförderungsanstalten spielen allerdings auch verteilungspolitische zielsetzungen herein, unter diesem Aspekt besitzt die Anzahl der vergebenen Kredite mehr Aussagekraft.

Die öffentliche Leistung der Hochschulbau-Gesellschaften besteht ausschlieBlich im Bau und der (Vor-)Finanzierung von Hochschulen. Physische Maßgrößen wie etwa die Fläche der fertiggesteliten Räume, unterteilt nach Verwendungszwecken, eignen sich besser als die entsprechenden Investitionsausgaben zur Leistungsmessung, weil sie vermeiden, daB das Leistungsvolumen mit steigenden Baupreisen zunimmt.

Bei vielen öffentlichen und allen privaten Unternehmen stellt sich zunächst das Problem der Abgrenzung ihrer öffentlichen Leistungen. Seine Lösung fällt am leichtesten, wenn Unternehmen mit dem staat Programmverträge abgeschlossen haben, da diese Vereinbarungen die zu erbringenden Leistungen bereits in operationaler Form enthalten, oder wenn ein Unternehmen in Befolgung staatlicher Normen handelt. Soweit die öffentlichen Leistungen am Markt abgesetzt werden, stellen die Verkaufserlöse ein nahe- 
zu ideales Leistungsmaß dar, da die Marktpreise die (Untergrenze der) Bewertung durch die Nachfrager ausdrücken. VeranlaBt etwa der staat die Bundesbahn zur Unterhaltung einer Bahnlinie, die sie andernfalls stillegen würde, so bemißt sich die öffentliche Leistung nach den auf dieser strecke erzielten Erlösen. Bei unentgeltlicher Leistungsabgabe hingegen mu $B$ sich die Messung so weit wie möglich auf Nutzungsindikatoren stützen.

Primär bei der Güterproduktion von Unternehmen fallen vielfach als zusätzliche positive oder negative Leistungskomponente externe Effekte an. Die Verhinderung des Entstehens oder die Beseitigung der Auswirkungen externer Effekte stellt nach dem Verursacherprinzip grundsätzlich eine private Aufgabe dar, es sei denn, die externen Effekte entstehen im zusammenhang mit der Erstellung öffentlicher Leistungen. Mangels entsprechender freiwilliger Aktivitäten der Unternehmen vor allem zur Vermeidung von Beeinträchtigungen der physischen Umwelt schreibt der staat diesen indes häufig bestimmte Aktivitäten oder aber Normen für die höchstzulässigen Emissionen an Schadstoffen, Lärm usw. vor und macht so die Verhinderung negativer externer Effekte auch bei der Produktion privater Leistungen zu einer teilweise öffentlichen, von den Unternehmen zu erfüllenden Aufgabe. Den diesbezüglichen Output eines einzelnen Unternehmens aufgrund vorgeschriebener oder auch in einem Programmertrag vereinbarter Aktivitäten stellt die Verringerung der Emissionen dar. Sie läßt sich mit Hilfe physischer Indikatoren messen, so etwa die Auswirkungen von Lärmschutzwällen an Bahnlinien. Gibt der Staat Höchstwerte für Emissionen vor, so besteht der Output derartiger Aktivitäten in jeder Periode in den vermiedenen Emissionen, die die Norm überschreiten würden. Noch verbleibende Emissionen unterhalb der Norm stellen im Prinzip negativ zu bewertende Outputkomponenten dar, können hier jedoch auBer acht bleiben, da der staat sie toleriert und insoweit keine öffentliche Aufgabe betroffen ist. 
Die öffentlichen Leistungen der Rundfunkanstalten bestehen im Konsum der ausgestrahlten Sendungen mit Ausnahme der kommerziellen Werbung. Im Prinzip läßt sich das Leistungsvolumen anhand des Aufkommens an Rundfunkgebühren bemessen, da diese keinen Zwangscharakter aufweisen. Diese Größe vermag jedoch nicht über die tatsächliche Nutzung der angebotenen Sendungen zu informieren. Unter diesem Aspekt erweist sich die Messung des Programmangebotes in Zeiteinheiten, gewichtet mit den Einschaltquoten, als über legen ${ }^{1)}$.

Der "versteckte Staatsbedarf" erfordert weitgehend keine Leistungsmessung bei einer nicht-staatlichen Entscheidungseinheit. Bei der allgemeinen Wehrpflicht und der Tätigkeit als Schöffe etwa handelt es sich lediglich um einen erzwungenen Ressourcentransfer zum Staat; erst dieser ist als Produzent einer öffentlichen Leistung anzusehen. Eine Ausnahme stellt hier die Pflicht der Arbeitgeber zur Einbehaltung und Abführung von Lohnsteuer und Sozialversicherungsbeiträgen dar. Die entsprechende Leistung läßt sich nach der $\mathrm{Zahl}$ der bearbeiteten Fälle bemessen.

Die freiwilligen Aktivitäten privater Entscheidungseinheiten zur Erfüllung öffentlicher Aufgaben konzentrieren sich auf das Erstellen meritorischer Güter. Zur Erfassung der öffentlichen Leistungen von Kirchen und Wohlfahrtsverbänden im Bereich der sozialen Infrastruktur sowie von Privatschulen und Sanatorien stehen teilweise Marktpreise zur Verfügung. Sie können allerdings nur dann verwendet werden, wenn die Nachfrager selbst, oder im Falle des Schulgeldes die Erziehungsberechtigten, und nicht etwa eine Krankenversicherung sie entrichten. In allen anderen Fällen kann die Leistungsmessung lediglich auf Nutzungsindikatoren (z.B. die Zahl der belegten Kindergartenplätze oder bestimmter Behandlungen in Werkskrankenstationen) basieren.

1) Vgl. auch M. Dittmers: Die optimale BetriebsgröBe von Rundfunkanstalten, in: Rundfunkökonomie. Wirtschaftliche Aspekte von Hörfunk und Fernsehen, Beiheft 5 (1983) der Zeitschrift für offentliche und gemeinwirtschaftliche Unternehmen, Baden-Baden 1983, S. 105-119, S. 109 ff. 
Bei den privaten Hochschulen sind die Leistungskomponenten Lehre und Forschung $\mathrm{zu}$ unterscheiden. Die Ausbildung von Studenten ließe sich nach den pro Periode bestandenen Abschlußprüfungen bzw. erteilten Diplomen bemessen, diese Indikatoren weisen indes mehrere Nachteile auf. Sie sind von der Anbieterseite manipulierbar, berücksichtigen nicht die von studienabbrechern genutzten Leistungen und hängen andererseits auch wesentlich von den Eigenleistungen der Diplomierten zur Erzielung des Studienerfolges ab. Derartige Verzerrungen vermeidet eine Leistungsmessung anhand der $\mathrm{zahl}$ der studenten in einem Ausbildungsgang. Allerdings kann diese Größe kaum Qualitätsunterschiede zwischen verschiedenen Hochschulen widerspiegeln. In der Diskussion um die Messung von Forschungsleistungen der Hochschulen kristallisiert sich die wissenschaftliche Reputation der an einer Institution tätigen Wissenschaftler als geeigneter Ansatzpunkt heraus ${ }^{1)}$, da diese Größe Output und Nachfrageorientierung aufweist. Zur Operationalisierung dieses Konstruktes dienen zitatenanalysen, bei denen die zitierhäufigkeit publizierter Forschungsarbeiten gemessen wird ${ }^{2}$.

Die Produktionsaktivitäten privater Entscheidungseinheiten, vor allem der Kirchen, im Rahmen der Entwicklungshilfe konzentrieren sich auf die Ausbildung und die medizinische Betreuung der Bevölkerung in den Entwicklungsländern. Diese unentgeltlich abgegebenen Leistungen lassen sich nachfrageorientiert mit Hilfe von Nutzungsindikatoren messen.

1) Vgl. H. Heiber: Messung von Forschungsleistungen der Hochschulen. Ein empirischer Ansatz auf der Basis von Zitatenanalysen, Baden-Baden 1983, S. $39 \mathrm{ff}$. und ergänzend den tberblick bei B. Blankart: Probleme der Messung und Bewertung von Forschungsresultaten - eine Anwendung auf die Okonomie in schweizerischen Hochschulen, in: Schweizerische Zeitschrift für Volkswirtschaft und Statistik, 110. Jg. (1974), S. 205-229.

2) Vgl. H. Heiber: Messung von Forschungsleistungen der Hochschulen... a.a.0., S. $49 \mathrm{ff}$. Diese Arbeit enthält auch eine empirische Analyse für den Bereich der Betriebswirtschaftslehre. 
Für die von Anbietern medizinischer Leistungen allgemein verabreichten Impfungen genügt auf der Leistungsebene eine Messung mit Nutzungsindikatoren. Impfungen verursachen insofern positive externe Effekte, als auch Individuen, die diese Leistung nicht nachfragen, einen erhöhten Schutz vor ansteckenden Krankheiten genießen. Unter diesem Aspekt besitzt der Anteil der Geimpften an der Bevölkerung mehr Aussagekraft als die absolute Nachfrage. Es handelt sich jedoch nicht um eine zusätzliche Leistung eines Anbieters, sondern als externer Effekt im Konsum bereits um eine Wirkung der eigentlichen Leistung.

\subsection{Von nicht-staatlichen Entscheidungseinheiten erbrachte Transfers}

Nicht-staatliche Entscheidungseinheiten erbringen im zuge der Erfüllung öffentlicher Aufgaben bewuBt auf direktem und indirektem Wege Transfers an bestimmte Wirtschaftseinheiten. Direkte Transfers erfolgen unabhängig von bzw. zusätzlich zu der Erstellung und Abgabe von Leistungen. Es handelt sich um den Transfer von Geld und von Sachgütern, soweit sie von einer anderen Institution produziert wurden. Die eingesetzten Ressourcen erfahren hier keine Transformation in einem Produktionsprozeß, sondern durchlaufen eine nicht-staatliche Entscheidungseinheit unverändert; allenfalls nimmt diese vor der $\mathrm{Ab}-$ gabe einen Tausch von Geldmitteln gegen Sachgüter vor. $\mathrm{zu}$ den direkten Transfers zählen die Ausgleichszahlungen des Lastenausgleichsfonds an Kriegsgeschädigte, die Aufwendungen der Arbeitgeber für die Lohnfortzahlung im Krankheitsfalle sowie die Geld- und Sachgütertransfers karitativer Organisationen, auch im Rahmen der Entwicklungshilfe. Einen Sonderfall bildet die gesetzliche Verpflichtung der Arbeitgeber zur Beschäftigung Schwerbehinderter. Sofern hier Transferelemente zugunsten der Arbeitnehmer enthalten sind, lassen sie sich kaum eingrenzen. 
Indirekte Transfers hingegen werden nicht unabhängig von den öffentlichen Leistungen, sondern im AnschluB an die Leistungserstellung im Zuge der Abgabe bzw. Verteilung des Produktionsoutputs erbracht. Die Leistungsnachfrager erhalten einen beabsichtigten Transfer über den Preis, den sie für den Bezug der eigentlichen Leistung zu entrichten haben. Das Instrumentarium nicht-staatlicher Entscheidungseinheiten reicht hier von der bewußten Einräumung von Vorzugskonditionen für bestimmte zielgruppen, wie etwa bei den Sozialtarifen von Bundesbahn und -post, bis hin zur gänzlich unentgeltlichen Abgabe selbsterstellter Leistungen. Indirekte Transfers und Leistungen nichtstaatlicher Entscheidungseinheiten können Interdependenzen aufweisen. Falls das Angebotsvolumen nicht von vornherein kontingentiert ist, resultiert aus der Einräumung von Vorzugskonditionen i.d.R. eine Erhöhung der nachgefragten Leistungseinheiten; die Entwicklung des wertmäBigen Leistungsvolumens hängt von der Preiselastizität der Nachfrage ab.

Die nähere Bestimmung dieser Art der Erfullung öffentlicher Aufgaben erfordert die Messung des von einer nicht-staatlichen Entscheidungseinheit erbrachten Volumens an direkten und indirekten Transfers. Dies kann zum einen rein inputbezogen aus der sicht der gebenden Institution erfolgen. Im Prinzip ließe sich das von einer nicht-staatlichen Entscheidungseinheit insgesamt abgegebene Transfervolumen sehr einfach berechnen, indem man von den Kosten (der Leistungserstellung und der Gewährung direkter Transfers) sämtliche Entgelte aus dem Verkauf von Leistungen subtrahiert. Die Differenz schlieBt allerdings auch unbeabsichtigte Transfers ein, die lediglich Verluste wegen eines unattraktiven Leistungsangebots oder wegen ineffizienter Produktion widerspiegeln, zudem bleiben die Transferempfänger unbekannt. Die von einer nicht-staatlichen Entscheidungseinheit gezielt erbrachten Transfers müssen daher einzeln bestimmt werden. Dies bereitet vor aliem bei direkten Transfers und bei völlig unentgeltlicher Leistungsabgabe keine Pro- 
bleme; die erforderlichen Aufwendungen bzw. Produktionskosten lassen sich leicht ermitteln. Bei den vom ERP-Sondervermögen, der Kreditanstalt für Wiederaufbau, den Wohnungsbauförderungsanstalten und anderen Kreditinstituten mit Sonderaufgaben vergebenen Krediten bzw. Gewährleistungen entspricht der indirekte Transfer der Subventionskomponente. Sie bestimmt sich im Prinzip nach der Differenz zwischen den zum zeitpunkt der Vergabe jeweils am Markt herrschenden Konditionen für vergleichbare Leistungen und den mit den Begünstigten vereinbarten Konditionen ${ }^{1)}$. Bei den Sozialtarifen von Bundesbahn und -post sowie der Befreiung von den Rundfunkgebühren für bestimmte Personenkreise basiert die Berechnung des Transfervolumens analog auf der Differenz zwischen dem normalen und dem von den Mitgliedern der Zielgruppen entrichteten Preis. Derartige Einnahmenverzichte eines Leistungsanbieters markieren jedoch allenfalls eine Obergrenze für den erbrachten Transfer, da die Berechnung auf der Annahme beruht, die $\mathrm{z}$ ielgruppen würden die betreffenden Leistungen auch zum normalen Preis nachfragen. Am schwierigsten gestaltet sich die Bestimmung des Transfervolumens bei der Sozialversicherung. Die im staatlichen Auftrag erfolgende interpersonelle Umverteilung zwischen den Versicherten erfolgt mit Hilfe indirekter Transfers. Aus der Sicht des Versicherungsträgers entspricht der Transfer an ein von der Umverteilungspolitik begünstigtes Mitglied grundsätzlich der Differenz zwischen dem nach dem versicherungstechnischen Aquivalenzprinzip errechneten und dem von ihm geforderten Beitrag ${ }^{2)}$. Das von einem Sozialversicherungsträger insgesamt gewährte Volumen an indirekten Transfers hängt allerdings wesentlich von der Höhe staatlicher zuschüsse ab. Die Umverteilung über die Sozialversicherung impliziert, daß die begünstigten Mitglieder einen im Verhältnis $\mathrm{zu}$ ihren Beiträgen zu großen Versicherungsschutz genießen bzw. im "Schadensfall" zu große Zahlungen erhalten. Bei Abwesenheit

1) Vgl. auch Abschnitt 1.2.1. in Kapitel II dieser Arbeit.

2) Vgl. Abschnitt 2.4.1. in diesem Kapitel. 
von Zuschüssen des Staates wird diese Mehrleistung, da die Umverteilung lediglich zwischen den Mitgliedern erfolgt, gerade kompensiert durch den relativ zu ihren Beiträgen zu geringen Versicherungsschutz der "Nettozahler". Würde die Sozialversicherung von der Umverteilungsaufgabe entlastet und die Beiträge ausschließlich risikoäquivalent gestalten, bliebe ceteris paribus das Beitragsaufkommen und der ingesamt gewährte Versicherungsschutz konstant, lediglich die Verteilung der Leistungen auf die Mitglieder änderte sich. Ein Nettotransfer an die Mitglieder entsteht erst, wenn der staat Zuschüsse an die Sozialversicherung leistet. Sie führen entweder zu einer erhöhten Produktion von Versicherungsschutz bei konstanten Beiträgen oder zu Beitragssenkungen bei gleichbleibendem Versicherungsschutz. In beiden Fällen kommt zu der Umverteilung zwischen den Mitgliedern noch ein Transfer von der Sozialversicherung an ihre Mitglieder in Höhe des Staatszuschusses hinzu. Nach wie vor handelt es sich um einen indirekten Transfer, da die Sozialversicherung die ihr zur Verfügung stehenden Ressourcen ausschließlich zur Leistungserstellung verwendet.

Eine inputbezogene Ermittlung eines Transfervolumens reicht in diesem Zusammenhang freilich nicht aus. Nicht Aktivitäten, sondern die damit erzielten Leistungen oder gar Wirkungen stehen im Mittelpunkt der Analyse. Ebenso wie bei der Messung selbsterstellter Leistungen wäre auch bei den von nicht-staatlichen Entscheidungseinheiten erbrachten Transfers zu untersuchen, wie die Empfänger das Erhaltene beurteilen. Die Beurteilung ihres Nachteils durch die Nettozahler bei Umverteilungsmaßnahmen hingegen muB hier - außer bei den Mitgliedern der Sozialversicherung - nicht analysiert werden, da wir die Herkunft der Transfers nicht weiter als bis zu den uber ihre Gewährung entscheidenden Institutionen zurückverfolgen. Die Bewertungen der Transferempfänger bleiben jedoch weitgehend im Dunkeln. Lediglich bei direkten monetären Transfers läßt sich die Annahme rechtfertigen, daß die "Zahlungsbereitschaft" bzw. Bewertung der Empfänger mit der erhaltenen Geldsume überein- 
stimmt. Bereits bei Sachgütertransfers und der unentgeltlichen Leistungsabgabe liegt aufgrund der aktiven Konsumtion dieser Güter nur noch die Information vor, daß die Einschätzung der Empfänger positiv ausfällt. Bei der Umverteilung liber die Sozialversicherung bleibt, selbst wenn sich positive und negative Transfers aus der sicht des Versicherungsträgers gerade kompensieren, grundsätzlich offen, ob die Begünstigten ihren Vorteil höher einschätzen als die Nettozahler ihren Nachteil. Der Vorteil eines Nachfragers schlie $\dot{B l i c h}$, der eine Leistung zu einem bewuBt herabgesetzten Preis bezieht, entspricht nicht zwangsläufig der Differenz zum normalen Preis, die begünstigten Leistungsnachfrager können ihren individuellen Vorteil höher oder niedriger als die Preisdifferenz einschätzen. Den Extremfall bildet ein Grenznachfrager in der Gruppe der Begünstigten. Für ihn entspricht der Wert der nachgefragten Leistung gerade dem Sozialtarif, so daß aus seiner sicht kein Transfer vorliegt.

Die Messung der von nicht-staatlichen Entscheidungseinheiten erbrachten Transfers aus der Sicht der Empfänger würde demnach grundsätzlich die Bestimmung der bewerteten Vorteile bzw. von Konsumentenrenten erfordern. Lediglich bei der Abgabe öffentlicher Leistungen gegen "normale" Marktpreise könnte hierauf verzichtet werden. Hier mag zwar eine Konsumentenrente entstehen, es fehlt jedoch der zusammenhang zu einem beabsichtigten Transfer. Das Konzept der bewerteten Vorteile freilich erweist sich als (vorläufig noch) nicht in befriedigender Weise operationalisierbar. 
Kapitel V: LEISTUNGEN NICHT-STAATLICHER ENTSCHEIDUNGSEINHEITEN UND OFFENTLICHE PLANUNG

1. Leistungen nicht-staatlicher Entscheidungseinheiten und des staates im Vergleich

Die Knappheit der in einer Volkswirtschaft insgesamt und insbesondere der zur Erfüllung öffentlicher Aufgaben zur Verfügung stehenden Ressourcen schafft unter dem Aspekt der Allokationseffizienz einen Bedarf an Koordination bzw. Integration der Aktivitäten des Staates und nicht-staatlicher Entscheidungseinheiten. Eine anspruchsvolle öffentliche Planung muß sowohl die unter staatlicher Direktive ablaufenden als auch die freiwillig entfalteten Aktivitäten nicht-staatlicher Entscheidungseinheiten zur Erfüllung öffentlicher Aufgaben berücksichtigen ${ }^{1)}$. Dies gilt zum einen für eine ex post vorgenommene zielerreichungs- und eine Wirksamkeitskontrolle (Evaluation). Die Vernachlässigung der Aktivitäten nichtstaatlicher Entscheidungseinheiten würde $z u$ fehlerhaften Aussagen über Ursache-Wirkungs-Zusammenhänge führen. Zum anderen muB der staat bei der Festlegung seiner eigenen zukünftigen Aktivitäten diejenigen der nicht-staatlichen Entscheidungseinheiten - und sei es auch nur im Sinne einer passiven Koordination ${ }^{2)}$ - einschließen, will er unerwünschte Fehlallokationen in Form von Uber- und Unterversorgungen vermeiden. Beide Arten der Integration implizieren den Vergleich verschiedener nicht-staatlicher Entscheidungseinheiten untereinander sowie mit dem Staat im Hinblick auf das absolute Leistungsvolumen einerseits und unter den Gesichtspunkten der

1) Vgl. E. Wille: Offentlicher Haushalt IV...a.a.O., S. 595 f. $\mathrm{zu}$ Integrationsbedarf und -problemen öffentlicher Planungssysteme vgl. E. Wille: Mittel- und langfristige Finanzplanung, in: Handbuch der Finanzwissenschaft, hrsg. von F. Neumark, Bd. 1, 3. Aufl., Tübingen 1977, S. 427-474, S. 463 ff. und F. Wegelin: Integrierte staatliche Planung. Probleme der Koordination, Führung und Willensbildung, Winterthur 1978 , S. $13 \mathrm{ff}$.

2) Vgl. hierzu Abschnitt 2.2. in Kapitel I dieser Arbeit. 
Effizienz und der Effektivität der Aufgabenerfüllung andererseits. Im folgenden wollen wir untersuchen, welche Informationen die Verfahren zur Leistungsmessung bei nicht-staatlichen Entscheidungseinheiten den öffentlichen Planungsträgern zur Befriedigung des skizzierten Koordinationsbedarfes $z u$ liefern vermögen. Wir beschränken uns dabei auf die von nichtstaatlichen Entscheidungseinheiten selbsterstellten Leistungen, klammern die Betrachtung unter dem Blickwinkel des Gewährens von Transfers hingegen aus, weil sich diese Arbeit primär mit der Allokationsabteilung im Musgraveschen Sinne beschäftigt.

Keines der im letzten Kapitel ausgewählten Verfahren zur Messung der öffentlichen Leistungen nicht-staatlicher Entscheidungseinheiten erfüllt die Idealvoraussetzungen des Konzeptes der bewerteten Vorteile. Marktpreise bzw. -erlöse kommen ihm am nächsten, sind jedoch bei öffentlichen Leistungen nur vereinzelt vorzufinden. Uberwiegend stützen wir uns daher auf Nutzungsindikatoren, die das Kriterium der Nachfragebezogenheit nur ansatzweise erfüllen. Hingegen weisen vor allem die Maße zur Erfassung externer Effekte und von Verwaltungsleistungen lediglich Outputorientierung auf. Die Leistungsmessung bei nicht-staatlichen Entscheidungseinheiten setzt damit größtenteils auf der Ebene der output-objectives an.

Die Messung des Outputs oder des Leistungsvolumens gestattet zunächst die Bestimmung der Effizienz oder/und der Effektivität, mit der eine einzelne nicht-staatliche Entscheidungseinheit öffentliche Leistungen erstellt bzw. eine öffentliche Aufgabe erfüllt. Beide Konzepte setzen das Output- oder Leistungsvolumen in Beziehung $z u$ den $z u$ seiner Erstellung aufge- 
wendeten Inputs bzw. ihrem monetären Äquivalent Kosten ${ }^{1)}$. Bei den lediglich outputbezogenen Meßverfahren entsteht auf diese Weise die Produktionseffizienz ${ }^{2}$, bei allen anspruchsvolleren Verfahren der Leistungsmessung hingegen eine Effektivität, da sie zumindest auf der Ebene der output-objectives ansetzen. Erfolgt die Leistungsabgabe unentgeltlich und die Konsumtion freiwillig und aktiv, läßt sich eine Effektivität 1 definieren und ermitteln, die im zähler die mit Hilfe von Nutzungs-

1) Die Begriffe "Effizienz" und "Effektivität" werden in der Literatur mit unterschiedlichen Inhalten belegt. Allen Effizienzkonzepten ist gemeinsam, daß sie ein Output-InputVerhältnis kennzeichnen. "Effektivität" definieren einige Autoren als Maß für den Zielerreichungsgrad, d.h. als Verhältnis zwischen tatsächlicher und angestrebter zielrealisation. Vgl. etwa P. Eichhorn und H. Siedentopf: Effizienzeffekte der Verwaltungsreform. Exemplarische Ansätze einer Wirkungsanalyse der territorialen und funktionalen Verwaltungsreform in Rheinland-Pfalz, Baden-Baden 1976, S. 26; K. -D. Henke: Bestimmung und Steigerung der Effizienz im offentlichen Sektor - Ein Uberblick, in: Das Wirtschaftsstudium (WiSu), Jg. 1978, S. 601-605, S. 601; M. Klausing: Effizienz und Effektivität im Gesundheitswesen. Der Beitrag der allgemeinen Systemtheorie für die Methode ihrer Ermittlung und Beurteilung, Karlsruhe 1981, S. 58 f.; G. Kirsch: Effizienz und Effektivität: Zielbestimmung in der Sozialpolitik, in: M. Pfaff (Hrsg.): Effizienz und Effektivität staatlicher Transferpolitik in der Wirtschaftskrise, Berlin 1983, S. 9-26, S. 10. Demgegenüber kennzeichnet "Effektivität" im Rahmen dieser Arbeit das Verhältnis der - auf der Ebene der output- oder der impact-objectives gemessenen $\mathrm{zielrealisierung} z \mathrm{u}$ den eingesetzten Mitteln und bezieht so auch Inputelemente ein. Vgl. hierzu Schaubild 1 in Kapitel I dieser Arbeit, E. Wille: Őffentlicher Haushalt IV... a.a.O., S. 605 und E. Wille: Rationalität, Effizienz und Effektivität aus der Sicht des ökonomen. Erweiterte Fassung eines Feferates auf dem Symposion "Effizienz und Effektivität medizinischer Diagnostik", erscheint in dem entsprechenden Tagungsband der "Internationalen Gesellschaft für Gesundheitsökonomie", Manuskript, Mannheim 1983, S. 25 ff.

2) Vgl. hierzu auch A. Kyrer: Effzienz und staatliche Aktivität, Wien 1972 , S. 125 f. 
indikatoren gemessene $\mathrm{Zahl}$ der abgegebenen Leistungseinheiten enthält ${ }^{1)}$ :

Effektivität $1=\frac{\text { zahl der nachgefragten Leistungseinheiten }}{\text { Inputs bzw. Kosten }}$

Diese Relation stellt zwar formal eine Effektivität dar, besitzt jedoch relativ wenig Aussagekraft im Hinblick auf die Wirksamkeit der Leistungen, da Nutzungsindikatoren noch nicht über deren (objektive) Wirkungen informieren. Andererseits bleibt auch die subjektive Beurteilung der Leistung durch die Nachfrager weitgehend offen; sicher ist nur, daB sie positiv ausfällt.

Wesentlich mehr Informationen liegen bei einer Leistungsabgabe gegen spezielles Entgelt vor. Hier läßt sich eine Effektivität 2 ermitteln, die die bei der Abgabe einer bestimmten öffentlichen Leistung erzielten Verkaufserlöse in Beziehung zu den Inputs setzt:

Effektivität $2=\frac{\text { Verkaufserlöse }}{\text { Inputs bzw. Kosten }}$

Effektivität 2 stellt im Prinzip eine Nutzen-Kosten-Relation dar, weil Marktpreise die Untergrenze der Zahlungsbereitschaft markieren. Sie vermittelt weniger. Erkenntnisse über die (objektive) Wirksamkeit einer Leistung als vielmehr über die individuelle Wertschätzung der Nachfrager. Marktpreise repräsentieren die Beurteilung der Leistung durch die Nachfrager ex ante. Die Bewertung kann sich auf eine lediglich erwartete Wirkung beziehen und von derjenigen der später tatsächlich eingetretenen Wirkungen abweichen. Demgegenüber

1) Zu derartigen Aufspaltungen von Effizienz und Effektivität siehe auch K. Reding: Die Effizienz staatlicher Aktivitäten. Probleme ihrer Messung und Kontrolle, Baden-Baden 1981, S. $26 \mathrm{ff}$. und E. Wille: Rationalität, Effizienz und Effektivität aus der Sicht des రokonomen, a.a.0., S. $25 \mathrm{ff}$. Im Nenner der verschiedenen Effektivitäten können jeweils die physischen Inputs oder - der besseren Aggregierbarkeit halber - die Kosten der Leistungserstellung stehen. 
sollen impact-objectives die tatsächlich anfallenden Wirkungen (in physischen Größen) messen. Bei Effektivität 2 handelt es sich allerdings primär um eine Referenzgröße, da in der Realität nur in wenigen Fällen Marktpreise für öffentliche Leistungen existieren.

Falls die von einer nicht-staatlichen Entscheidungseinheit erstellte öffentliche Leistung aus mehreren Komponenten besteht, für die unterschiedliche Meßverfahren zum Ansatz kommen (z.B. Nutzungsindikatoren und physische Outputindikatoren zur Erfassung externer Effekte) lassen sich aufgrund mangelnder Komparabilität lediglich Teileffizienzen bzw. -effektivitäten ermitteln. Dabei sind die Inputs bzw. Kosten entsprechend aufzuschlüsseln.

Wesentlich aufschluBreicher als die Betrachtung eines einzelnen Anbieters ist für die öffentliche Planung der Vergleich verschiedener, gleichartige öffentliche Leistungen erstellender Anbieter untereinander. Je nach der Art der Aufgabenverteilung kann sich dieser Vergleich auf verschiedene nichtstaatliche Entscheidungseinheiten beschränken oder auch entsprechende Aktivitäten des Staates selbst (z.B. die Grundschulausbildung) einbeziehen. Die Gleichartigkeit der betreffenden Leistung gewährleistet im zweiten Falle, daß insoweit auch beim Staat eine Leistungsmessung zumindest mit Hilfe von Nutzungsindikatoren erfolgen kann. Im Rahmen einer ex post vorgenommenen positiven Analyse dient der Vergleich der Bestimmung von Schwerpunkten der Bereitstellung einer bestimmten öffentlichen Leistung. Es wird untersucht, welcher Anbieter den größten Anteil am von der betreffenden Leistung insgesamt abgegebenen Leistungsvolumen erbringt und weitergehend, welcher die höchste Effizienz bzw. Effektivität erzielt. Ein Vergleich mit normativer Fragestellung hingegen liefert Anhaltspunkte dafür, welcher Anbieter unter dem Aspekt der Wohlfahrtsmehrung die betreffende Leistung in zukunft verstärkt erbringen soll, und, ob ggf. der staat seine Aktions- 
parameter zu verändern Anlaß hat, etwa indem er seine eigenen Aktivitäten in diesem Bereich reduziert und stattdessen bestimmten nicht-staatlichen Entscheidungseinheiten verstärkte Förderung angedeihen 1äBt. Bei der positiven Fragestellung entstehen vor allem Probleme der Vergleichbarkeit verschiedener Leistungsanbieter untereinander, die normative Analyse erschweren primär Probleme der Aussagefähigkeit des Leistungsvolumens und der Effektivität. Wir beschränken uns im ersten Schritt auf die positive Analyse und unterstellen zunächst, da $B$ die betrachteten Institutionen ihre Leistungen jeweils einheitlich entweder unentgeltlich oder gegen ein spezielles Entgelt abgeben.

Welcher Anbieter eine bestimmte öffentliche Leistung schwerpunktmäßig erbringt, läßt sich sehr einfach anhand eines Vergleichs der Leistungsvolumina ermitteln. Die Spitzenposition nimmt der Anbieter ein, dessen Leistungsvolumen den größten Anteil an den insgesamt abgegebenen Leistungen ausmacht. Dieser Vergleich ist sowohl für eine Abgabe gegen Entgelt anhand der Verkaufserlöse als auch bei unentgeltlicher Abgabe mit Hilfe von Nutzungsindikatoren durchführbar. Zusätzliche Informationen für die Beurteilung eines Anbieters vermag die Betrachtung von Effizienz und Effektivität zu vermitteln. Während beim Effizienzvergleich keine spezifischen Probleme auftreten, weist die Effektivität je nach Art der Leistungsabgabe unterschiedliche Aussagekraft auf. Bei einer Abgabe gegen spezielles Entgelt läBt sich bei allen Anbietern Effektivität 2 bestimmen. Handelt es sich um einen vollkommenen Markt, der sich insbesondere durch die Abwesenheit von externen Effekten, Subventionen und indirekten steuern auszeichnet, stellt Effektivität 2 mit den Verkaufserlösen und den Kosten eines Anbieters im Prinzip den (erwarteten) Wohlfahrtszuwachs aus dem Leistungskonsum dem aus dem Verbrauch von Inputfaktoren resultierenden Wohlfahrtsverlust gegenüber. Eine Effektivität 2 mit einem Wert von größer als Eins signalisiert, daß die Aktivitäten des betrachteten Anbieters der 
Gesellschaft insgesamt einen Nettowohlfahrtszuwachs verschaffen. Allerdings besagt Effektivität 2, da es sich um eine Relation handelt, nicht, $d a \beta$ bei dem Anbieter mit der höchsten Effektivität auch der absolut größte Nettowohlfahrtszuwachs entsteht ${ }^{1)}$. Auch sind selbst bei einer hohen Effektivität produktionstechnische $(\mathrm{X}-)$ Ineffizienzen noch nicht zwangsläufig ausgeschlossen. Probleme der Vergleichbarkeit entstehen bereits dann, wenn externe Effekte auftreten oder einzelne Anbieter den Markt mittels Sozialtarifen u.ä. spalten, da insoweit Effektivität 2 Wohlfahrtswirkungen unvollständig repräsentieren kann.

Lediglich Effektivität 1 läßt sich hingegen vergleichen, wenn alle Anbieter ihre Leistungen unentgeltlich abgeben. Diese Relation weist freilich unter Wohlfahrtsaspekten kaum Aussagekraft auf. Die im Zähler stehenden Nutzungsindikatoren lassen außer acht, daß die Nachfragergruppen die Leistungen der verschiedenen Anbieter möglicherweise unterschiedlich einschätzen und unterschiedlich hohe Zahlungsbereitschaften besitzen. Die absolute Zahl der bei einem Anbieter nachgefragten Leistungseinheiten bringt dies wenigstens ansatzweise noch zum Ausdruck, die Effektivität 1 hingegen beinhaltet als Relation diesbezüglich eine Tendenz zur Nivellierung.

Gravierende Probleme entstehen, läßt man die Annahme der einheitlichen Art der Leistungsabgabe fallen. Gibt etwa ein Anbieter eine bestimmte Leistung unentgeltlich ab und ein anderer gegen Marktpreise ${ }^{2)}$, so liegen als Leistungsma $B$ im ersten Falle Nutzungsindikatoren vor, im zweiten Verkaufserlöse; eine Schwerpunktbestimmung scheitert an der mangelnden Komparabilität der Leistungsmaße. Die im technischen Sinne gegebene Vergleichbarkeit zwischen den Leistungen beider An-

1) Aus Gründen der Vergleichbarkeit mit dem Kriterium Effektivität 1 argumentieren wir hier ebenfalls anhand von Relativgrößen und verzichten auf die in diesem spezialfall mögliche Bildung der aussagefähigeren Differenz zwischen Verkaufserlösen und Kosten.

2) Als Beispiel können öffentliche und private Schulen dienen. 
bieter kann unter ökonomischen Aspekten lediglich in formaler Hinsicht hergestellt werden. Zu diesem Zweck lassen sich aus den oben genannten Gründen nicht die Marktpreise des zweiten Anbieters auf die Leistungen des ersten übertragen ${ }^{1)}$, sondern es darf allenfalls eine Anpassung nach unten vorgenommen werden, indem bei dem Anbieter am Markt ebenfalls die Zahl der abgegebenen Leistungseinheiten zum Ansatz kommt. Dabei geht indes die Information verloren, daß die Individuen, die die Zutrittsbarriere Marktpreis überwinden, den Leistungen des zweiten Anbieters eine höhere Qualität zubilligen und deshalb offenbar eine höhere zahlungsbereitschaft besitzen als die Nutzer des unentgeltlichen Angebotes. Eine Schwerpunktbestimmung auf dieser Basis würde $z u$ einer Nivellierung $z u$ Lasten des Marktanbieters führen.

Entsprechend sind die Konsequenzen für die Betrachtung der Relativgrößen. Die mangelnde Komparabilität der Leistungsmaße hat keinen Einfluß auf den Vergleich der Produktionseffizienz, verhindert jedoch einen Effektivitätsvergleich. Bei dem am Markt agierenden Anbieter ließe sich Effektivität 2 ermitteln, beim anderen Effektivität 1 ; beide sind bereits aufgrund ihrer unterschiedlichen Dimensionen nicht vergleichbar. Der Ubergang auf die $\mathrm{Zahl}$ der abgegebenen Leistungseinheiten und damit Effektivität 1 auch beim zweiten Anbieter sorgt hier ebenfalls für lediglich formale Komparabilität. Indem diese Relation die unterschiedlich hohen zutrittsbarrieren und entsprechend unterschiedlich hohen Zahlungsbereitschaften der beiden Nachfragergruppen nicht berücksichtigt, enthält der Vergleich in noch stärkerem Maße als im oben diskutierten Fall eine systematische Verzerrung zu Lasten eines, und zwar des Marktanbieters.

1) Vgl. Abschnitt 2.2. in Kapitel IV dieser Arbeit. 
Im Rahmen einer normativen Analyse ist zu fragen, ob die Messung der Leistungen nicht-staatlicher Entscheidungseinheiten und des Staates der öffentlichen Planung ausreichende Informationen für im Hinblick auf allokative ziele erforderliche Veränderungen staatlicher Aktionsparameter zu liefern vermag. Das absolute Leistungsvolumen bietet hierfür unabhängig von der Art der Leistungsabgabe und -messung nicht genügend Anhaltspunkte, da es den Bezug zu den zu seiner Produktion aufgewendeten Ressourcen vermissen läBt. Ohne Berücksichtigung der Kosten ist keine Aussage darüber möglich, ob die Aktivitäten eines Produzenten der Gesellschaft insgesamt einen Nettowohlfahrtsgewinn verschaffen. Die Produktionseffizienz bildet ebenfalls kein hinreichendes Kriterium für staatliche Maßnahmen, da eine noch so hohe Wirtschaftlichkeit eines Leistungsanbieters in der Produktion noch nicht garantiert, daß das Angebot auch den Präferenzen potentieller Nachfrager entspricht. Das einzige Kriterium, das sowohl die Kosten als auch die Nachfrage nach einer Leistung integriert, bildet die Effektivität. Wie sich bereits im Rahmen der positiven Analyse ergab, sind Vergleiche der Effektivität verschiedener Anbieter einer bestimmten öffentlichen Leistung jedoch nur bei einheitlicher Art der Leistungsabgabe durchführbar und nur bei einer Abgabe gegen spezielles Entgelt prinzipiell aussagefähig im Hinblick auf Wohlfahrtswirkungen. Damit erweisen sich die Effektivitäten 1 und 2 als unzureichende Entscheidungsgrundlagen für die öffentliche Planung. Bei einheitlicher Leistungsabgabe auf einem (eher als Referenzsystem denn als Beschreibung der Realität dienenden) vollkommenen Markt liegt zwar mit der Effektivität 2 ein im Prinzip hinreichendes Kriterium vor, es fehlt jedoch im Hinblick auf allokationspolitische ziele ein staatlicher Handlungsbedarf, es sei denn, der staat setzt sich über die individuellen Präferenzen hinweg, indem er bestimmte Leistungen als (de-) meritorische Güter qualifiziert. Handelt es sich hingegen um einen durch das Vorhandensein externer Effekte oder von Sozialtarifen u.ä. gekennzeichneten 
unvollkommenen Markt, reicht Effektivität 2 nicht aus, vielmehr benötigt der staat zusatzinformationen, um einen Handlungsbedarf $\mathrm{zu}$ erkennen. Bei unentgeltlicher Leistungsabgabe schlieBlich mag zwar AnlaB für staatliche Aktivitäten gegeben sein, die Effektivität 1 bietet hierfür jedoch grundsätzlich keine hinreichende Entscheidungsbasis.

Im dritten Schritt ist $z u$ untersuchen, ob die Leistungsmessung bei nicht-staatlichen Entscheidungseinheiten eine Schwerpunktbestimmung über alle öffentlichen Aufgabenbereiche, in denen diese Aktivitäten entfalten, auf der Leistungsebene ermöglicht. Ermittelt werden soll nicht, welche Institution von einer bestimmten Leistung das größte Volumen erstellt und absetzt, sondern, in welchem öffentlichen Aufgabenbereich - unabhängig von welchem Anbieter - am meisten Leistungen erstellt und abgesetzt werden, um Informationen über opportunitätskosten auf der Leistungsebene $z u$ gewinnen. Eine derartige Schwerpunktbestimmung scheitert jedoch an der mangelnden Vergleichbarkeit verschiedener öffentlicher Leistungen. Auf der Inputebene war eine Schwerpunktbestimmung anhand der Kosten der Aufgabenerfüllung im Prinzip möglich. Die einheitliche Bewertung der Inputs in Geldeinheiten spiegelt letztlich individuelle Präferenzen wider und sorgt so für Komparabilität. Auf der Leistungsebene hingegen fehlt dieser einheitliche numéraire zumindest bei allen unentgeltlich abgegebenen Leistungen. Bereits der Vergleich im technischen Sinne identischer Leistungen erwies sich nur unter restriktiven Bedingungen als durchführbar. Vollends verloren geht die Komparabilität beim Vergleich unterschiedlicher Leistungen, sei es innerhalb eines Aufgabenbereiches (z.B. ambulante und stationäre Leistungen eines Krankenhauses), sei es zwischen verschiedenen Aufgabenbereichen (z.B. medizinische und Bildungsleistungen), solange ein Leistungsmaß nicht quantifizierte Bewertungen der Nutzer enthält. Zwar könnte man eine Standardisierung und Gewichtung einführen, damit setzt man sich jedoch über die individuellen Präferenzen der Leistungs- 
bezieher hinweg. Ohne eine solche Normierung kann allenfalls zwischen Aufgabenbereichen mit gegenüber der Vorperiode sinkenden, konstanten oder steigenden Leistungsvolumina unterschieden werden; diese Einteilung in drei Gruppen erlaubt jedoch nicht die Aufstellung einer durchgehenden Rangfolge über alle Aufgabenbereiche.

2. Leistungen nicht-staatlicher Entscheidungseinheiten und ziele der öffentlichen Planung

Die strikte Orientierung an den individuellen Präferenzen der Nutzer bei der Messung der Leistungen nicht-staatlicher Entscheidungseinheiten gestattet weitgehend lediglich die Anwendung von Verfahren, die die Ebene der output-objectives reräsentieren. In verstärktem Maße noch gilt dies für die vom Staat selbst bereitgestellten Güter, da sich seine Leistungen vielfach durch einen relativ hohen Anteil an externen Effekten auszeichnen. Für eine anspruchsvolle öffentliche Aufgabenplanung, die unter dem Aspekt der Wohlfahrtssteigerung die Aktivitäten sämtlicher an der Erfüllung öffentlicher Aufgaben beteiligter Institutionen integriert bzw. koordiniert, liefert eine Leistungsmessung auf der Ebene der output-objectives jedoch $z u$ wenig Informationen. Selbst wenn die Nachfrager ihre Präferenzen für einzelne öffentliche Leistungen offenbaren, läBt die Leistungsmessung einen Vergleich verschiedener Akteure, die an der Erfüllung einer öffentlichen Aufgabe mitwirken i.d.R. allenfalls hinsichtlich einer Effektivität $1 \mathrm{zu}$; diese bildet jedoch keine hinreichende Basis für Entscheidungen über die Entfaltung staatlicher Aktivitäten. Die öffentliche Aufgabenplanung bedarf zu ihrer orientierung auf einer höheren Ebene angesiedelter ziele, weil die Nutzung einer öffentlichen Leistung bzw. die output-objectives noch nicht hinreichend über Veränderungen der individuellen Wohlfahrtsposition informieren. In Ermangelung von Verfahren, die die 
aus Aktivitäten zur Erfüllung öffentlicher Aufgaben resultierenden Wolfahrtsveränderungen direkt abbilden, vermag die öffentliche Planung sich lediglich an den Wirkungen öffentlicher Leistungen bzw. impact-objectives $\mathrm{zu}$ orientieren.

Impact-objectives bilden die höchste Ebene der ziel-MittelHierarchie, die eine Formulierung der öffentlichen Planungsziele in konkreter und prüfbarer Form mit Hilfe von individuenbezogenen Resultatindikatoren gestattet. Resultatindikatoren lassen freilich in bestimmten Fällen offen, ob die Individuen die wiedergegebenen Wirkungen überhaupt bemerken und wie sie sie ggf. beurteilen ${ }^{1)}$. Insofern kann die Ausrichtung der öffentlichen Planung an den mit Hilfe von Resultatindikatoren operationalisierten impact-objectives im Prinzip eine Verlagerung der Bewertungsinstanz implizieren. Nicht die betroffenen Individuen, sondern stellvertretend für sie die politischen Entscheidungsträger befinden über die Wohlfahrtsrelevanz öffentlicher Leistungen, indem sie die Schwerpunkte der staatlichen Aktivitäten entsprechend den von ihnen vermuteten Präferenzen der Bürger oder ausschließlich nach ihren eigenen Vorstellungen setzen und die Aktivitäten nicht-staatlicher Entscheidungseinheiten entsprechend zu beeinflussen suchen ${ }^{2)}$. Zwar sollen die Betroffenen die Rolle der Bewertungs-

1) Vgl. hierzu Abschnitt 3.1.4. in Kapitel IV dieser Arbeit.

2) Ihre Vorstellungen über die Wohlfahrtsrelevanz bestimmter Aktivitäten bringen die politischen Entscheidungsträger allerdings nicht nur dann zur Geltung, wenn ihnen Informationen über die Präferenzen der Bürger nicht zur Verfügung stehen. Teilweise setzen sie sich auch explizit über die am Markt offenbarten Präferenzen der Bürger hinweg, indem sie bestimmte Güter als (de-)meritorisch qualifizieren und ihren Konsum einzuschränken bzw. auszuweiten suchen. 
instanz insofern auf indirektem Wege übernehmen, als von den Resultatindikatoren gefordert wird, daß sie nach allgemeinem Konsens wohlfahrtsrelevante ziele bzw. Lebensbereiche mit Endproduktcharakter abbilden, die letzte Entscheidungskompetenz liegt jedoch beim staat. Grundsätzlich kann die zielbildung auf der Ebene der impact-objectives einen integralen Bestandteil des Planungsprozesses darstellen oder diesem als isolierte Entscheidung vorgeschaltet $\operatorname{sein}^{1 \text { ). }}$

In diesem Sinne stellen die Leistungen des Staates und nichtstaatlicher Entscheidungseinheiten Mittel zur Erreichung der übergeordneten öffentlichen Planungsziele dar. So bestehen etwa im Bereich des Verkehrswesens die ziele nicht in einer möglichst intensiven Nutzung der Verkehrswege, sondern in der Verringerung von Transportwegen, -zeit und -kosten, einer Senkung der Unfallhäufigkeit u.ä. Einen Extremfall bildet in dieser Hinsicht das Gesundheitswesen. Ein erheblicher Teil der medizinischen Leistungen dient lediglich dazu, den status quo ante wieder herzustellen, man könnte hier mit gleicher Berechtigung wie bei Umweltschutzaktivitäten von "kompensatorischen Leistungen" sprechen. Die öffentliche Planung zielt jedoch nicht auf die Inanspruchnahme medizinischer Leistungen, sondern letztlich auf den Gesundheitsstand der Bevölkerung ab. Analoges gilt auch für die aus verteilungspolitischen Gründen gewährten Transfers. Die distributionspolitischen ziele des Staates erschöpfen sich nicht in der Bewegung eines möglichst großen Transfervolumens, sondern er versucht, eine gleichmäßigere Verteilung von Einkommen und Vermögen herzustellen. Da sich die mit der Gewährung und dem Empfang von Transfers verbundenen Wohlfahrtsveränderungen i.d.R. weder bei den Netto-

1) Vgl. hierzu E. Wille: Mittel- und langfristige Finanzplanung, a.a.0., s. $456 \mathrm{ff}$. 
zahlern noch bei den von Umverteilungsmaßnahmen Begünstigten ermitteln lassen, kann sich die öffentliche Planung allenfalls an Konzentrationskoeffizienten als Zielgrößen orientieren.

Resultatindikatoren stellen die bisher einzige Möglichkeit zur Operationalisierung der impact-objectives dar. Sie integrieren die Wirkungen der Aktivitäten und Leistungen verschiedener Mitwirkender an der Erfüllung öffentlicher Aufgaben und bilden ein Nettoergebnis ab, das aus einer Fülle verschiedener Einflußfaktoren resultiert. Zunächst stellen staat und nichtstaatliche Entscheidungseinheiten öffentliche Leistungen bereit, zudem hängt i.d.R. das Endergebnis auch von der Nachfrageseite bzw. dem Verhalten der Individuen $a b$. Aus dem Zusammenwirken verschiedener Anbieter öffentlicher Leistungen können Synergieeffekte entstehen, andererseits vermögen exogene Störgrößen die Wirkungen abgegebener Leistungen abzuschwächen. Sodann bestehen häufig mehrdimensionale Beziehungen zwischen öffentlichen Leistungen und Planungszielen. Verschiedenartige Leistungen können zur Verwirklichung desselben ziels beitragen, und eine bestimmte Leistung kann mehreren zielen zugleich dienen. Letzteres läBt sich sowohl für die Musgraveschen Abteilungen Allokation und Distribution konstatieren (man denke etwa an die Sozialversicherung), als auch innerhalb der Allokationsabteilung. Ferner schlagen sich bestimmte öffentliche Leistungen nicht direkt im Endergebnis nieder, weil sie eher den Charakter von Vorleistungen für die eigentliche Aufgabenerfüllung besitzen, so etwa die Aktivitäten der HochschulbauGesellschaften. Weiterhin dienen bestimmte öffentliche Leistungen, beispielsweise im Gesundheitswesen und im Bereich des Umweltschutzes, im Prinzip lediglich dazu, bereits eingetretene Schäden zu kompensieren, so daß das Endergebnis mit dem ursprünglichen zustand übereinstimmt. Schlieblich erweisen sich Resultatindikatoren als die einzigen operationalen Maßgrößen, die externe Effekte nicht lediglich als Output des Verursachers, sondern als Wirkungen auf die Empfänger bzw. nicht als Emissio- 
nen, sondern als Immissionen, abbilden. Resultatindikatoren kristallisieren aus diesen vielerlei positiven und negativen Einflußfaktoren ein als Durchschnittsgröße gemessenes (Netto-) Endergebnis heraus.

Diese Konzentrationsfähigkeit begründet ihre Eignung zur zielvorgabe und zielerreichungskontrolle durch die öffentliche Planung. Der staat trägt als oberste Instanz Verantwortung für die Erfüllung öffentlicher Aufgaben. Er kann sich demnach nicht damit begnügen, Leistungen bereitzustellen, deren positive Wirkungen unter Umständen durch den Einfluß von Störgrößen abgeschwächt, zunichte gemacht oder gar überkompensiert werden. Vielmehr muß sich die öffentliche Planung am Endergebnis als zielgröße orientieren, denn nur dieses kommt letztlich bei den Individuen an und vermag ihre Wohlfahrt zu erhöhen. Eine ex post vorgenommene zielerreichungskontrolle überprüft die Abweichungen zwischen der angestrebten und der tatsächlichen zielrealisation, verzichtet hingegen auf eine Kausalanalyse der Beziehungen zwischen Inputs und Zielwertveränderungen. Sie setzt lediglich eine Meß- und Prüfbarkeit der Wirkungsziele voraus, jedoch noch keine Kenntnis der die zielrealisation bewirkenden Faktoren ${ }^{1)}$.

Die öffentliche Planung benötigt freilich weitaus mehr als die für eine bloße zielerreichungskontrolle ausreichenden Informationen. Sowohl zur Erklärung des Erfolges bereits abgeschlossener Maßnahmen als auch zur Festlegung zukünftiger Aktivitäten ist sie auf Erkenntnisse über zusammenhänge zwischen Ressourceninputs und Veränderungen der Wirkungsziele angewiesen. Während die Zielerreichungskontrolle solche Veränderungen lediglich registriert, dient eine weitergehende

1) Vgl. E. Wille: Soziale Indikatoren als Ansatzpunkte..a.a.o., $\mathrm{S}$. $148 \mathrm{ff}$. sowie A. Hübener und R. Halberstadt: Erfolgskontrolle politischer Planung - Probleme und Ansätze in der Bundesrepublik Deutschland, Göttingen 1976, S. 68 ff. 
Wirksamkeitsanalyse (Evaluation) dazu, diese ursächlich auf bestimmte Aktivitäten oder Ressourceninputs zurückzuführen ${ }^{1}$ ! Erst aufgrund von Erkenntnissen über das Beziehungsgeflecht aller für die Erreichung eines bestimmten ziels relevanten Variablen kann die öffentliche planung etwa, falls es ihr nicht gelingt, den EinfluB unerwünschter störgrößen zu unterbinden, versuchen, ihn durch eine Variation der staatlichen Aktivitäten wenigstens $\mathrm{zu}$ kompensieren.

Setzt man die Informationen über die Zusammenhänge zwischen Ressourceninputs und Wirkungszielen als gegeben voraus, kann die öffentliche Planung - gesondert für jedes Planungsziel bzw. jeden Aufgabenbereich - die Aktivitäten verschiedener Mitwirkenden an einer bestimmten Aufgabe sowohl ex post vergleichen und im Hinblick auf ihren zielbeitrag beurteilen als auch die effektivsten Leistungsanbieter für zukünftige Aktivitäten auswählen. Der absolute Beitrag eines Mitwirkenden zur zielerreichung, gemessen als die ausschließlich aus seinen Aktivitäten resultierende Veränderung des betreffenden impact-objective, gestattet ihr die Beurteilung abgelaufener Maßnahmen, läßt jedoch noch den Bezug zu den aufgewendeten Inputs vermissen. Als wesentlich aufschluBreicher erweist sich ein Kriterium Effektivität 3, welche die aus den Aktivitäten eines Anbieters der betreffenden Leistung resultierende Veränderung des dazugehörigen Wirkungsziels in Beziehung zu den von ihm hierfür aufgewendeten Ressourcen setzt.

Effektivität $3=$ Beitrag zur zielrealisation jeweils für einen Inputs bzw. Kosten

Leistungsanbieter

Diese Relation erlaubt den Vergleich verschiedener Institutionen, die an der Erreichung eines bestimmten zieles mitwirken. Die öffentliche Planung vermag auf dieser Basis einerseits ex post den effektivsten Leistungsanbieter $z u$ ermitteln

1) Vgl. E. Wille: Soziale Indikatoren als Ansatzpunkte... a.a.O., s. 149 . 
und andererseits zukunftsbezogene Entscheidungen, etwa über eine verstärkte Verlagerung der Aufgabenerfüllung auf diesen Anbieter, zu fällen.

Bereits in theoretischer Hinsicht ist allerdings die Eignung von Effektivität 3 als Kriterium für Allokationsentscheidungen Einschränkungen unterworfen. Zunächst ist festzuhalten, daß sie - anders als Effektivität 2 - nicht notwendigerweise Wohlfahrtswirkungen der Aktivitäten eines Leistungsanbieters ausdrückt. Sie signalisiert häufig lediglich die Wirksamkeit dieser Aktivitäten im Hinblick auf ein von den politischen Entscheidungsträgern ausgewähltes und quantifiziertes $\mathrm{Ziel}$. Sodann gestattet dieses Kriterium zwar die Ermittlung des effektivsten Leistungsanbieters, vermag jedoch als Relativgröße nicht offenzulegen, welcher Anbieter die absolut größte Veränderung der Wohlfahrt oder ggf. bei dem betreffenden $\mathrm{Ziel}$ verursacht. Ferner kann die öffentliche Planung anhand von Effektivität 3 einen Vergleich nur innerhalb eines Aufgabenbereiches bzw. nur zwischen verschiedenen Leistungsanbietern, die Aktivitäten zur Erreichung ein und desselben zieles entfalten, vornehmen. Ein Vergleich von Institutionen, die an der Erreichung unterschiedlicher ziele mitwirken, führt hingegen zu keinen sinnvollen Ergebnissen.

Wesentlich gravierender ist jedoch, daß Effektivität 3 (vorläufig noch) eher eine theoretische Forderung als eine in der Realität ermittelbare Größe darstellt. Derzeit fehlen die für ihre Konstruktion unabdingbaren Erkenntnisse über die Kausalrelationen zwischen den relevanten Variablen weitgehend. Die Evaluierungsforschung vermag angesichts der eher zunehmenden Komplexität gesellschaftlicher zusammenhänge der öffentlichen Planung i.d.R.' noch keine Informationen vorzulegen, die den Beitrag der Aktivitäten des Staates bzw. nicht-staatlicher Entscheidungseinheiten $z u$ einer gemessenen Veränderung 
von Wirkungszielen hinreichend erklären ${ }^{1)}$. Auch eine sehr einfache Art von Wirksamkeitsanalyse, die vom Leistungsvolumen auf die Wirkungsziele bzw. von den output- auf die impactobjectives zu schlieBen versucht, kann diesem Informationsmangel kaum abhelfen. Es läßt sich zwar nicht behaupten, bei einem Anstieg des Leistungsvolumens in einem Aufgabenbereich um $x:$ erfahre auch das betreffende Wirkungsziel eine Verbesserung um denselben Prozentsatz. Prima facie spricht jedoch einiges dafür, daB beispielsweise bei einem Anteil der nichtstaatlichen Entscheidungseinheiten am Leistungsvolumen in einem Aufgabenbereich von 108 die Realisation des entsprechenden impact-objective ihre Begründung $z u 108$ in den Aktivitäten nicht-staatlicher Entscheidungseinheiten und zu 908 in denjenigen des Staates findet. Analoge Beziehungen lassen sich als Verfeinerung auch zwischen Veränderungen von Leistungsvolumen und Wirkungszielen herstellen, zudem sind weitere Aufschlüsselungen innerhalb der nicht-staatlichen Entscheidungseinheiten möglich. Indes beruhen solche simplen Plausibilitätsüberlegungen auf groben Vereinfachungen. zunächst implizieren sie, daß neben den Aktivitäten von staat und nicht-staatlichen Entscheidungseinheiten keine weiteren relevanten EinfluBfaktoren auf die Wirkungsziele zu berücksichtigen sind oder/und, daß diese beiden Leistungsanbietergruppen von Störgrößen in relativ gleichem Ausmaß betroffen werden. Sodann wird unterstellt, die Qualität der Leistungen und damit ihre

1) Vgl.E. Wille: Soziale Indikatoren als Ansatzpunkte...a.a.o., S. 150. Zu Problemen der Evaluation siehe vor allem S.A. Musto: Evaluierung sozialer Entwicklungsprojekte, Berlin 1972 , S. $11 \mathrm{ff.;}$ C.H. Weiss: Evaluierungsforschung...a.a.0., S. 88 ff.; D. Aderhold: Kybernetische Regierungstechnik in der Demokratie. Planung und Erfolgskontrolle, München, Wien 1973, S. 247 ff.; C. Leipert: Gesellschaftliche Berichterstattung...a.a.O., S. $123 \mathrm{ff}$. Eine empirische Untersuchung der Programmevaluation auf Regierungsebene in der Bundesrepublik bietet H.-U. Derlien: Die Erfolgskontrolle staatlicher Planung. Eine empirische Untersuchung über Organisation, Methode und Politik der Programmevaluation, BadenBaden, 1976, S. $43 \mathrm{ff}$. 
Wirksamkeit im Hinblick auf das übergeordnete impact-objective sei bei allen Mitwirkenden gleich. Hierdurch wird das, was letztlich Ergebnis der Analyse sein soll, implizit bereits vorgegeben. Schließlich ist auch beim Schluß von den Leistungen auf die Wirkungsziele zu berücksichtigen, daB eine bestimmte Leistung mehreren zielen zugleich dienen kann. Die Ermittlung einer Effektivität 3 erfordert in diesem Falle nicht nur eine Aufschlüsselung und Zurechnung von Zielwertveränderungen, sondern auch der Aktivitäten bzw. Kosten eines Leistungsanbieters auf die verschiedenen betroffenen ziele, hierfür fehlen jedoch geeignete Kriterien.

Im nächsten Schritt wollen wir analysieren, ob ein externer Beobachter auf der Ebene der impact-objectives die Schwerpunkte der Erfüllung öffentlicher Aufgaben bestimmen kann. Dieser Vergleich über alle öffentlichen Aufgabenbereiche abstrahiert von den einzelnen Aktivitäten des Staates oder nicht-staatlicher Entscheidungseinheiten und betrachtet nur noch deren aggregierte Wirkungen im Sinne eines Gesamterfolges dieser Leistungsanbieter. Entsprechend der Vorgehensweise auf der Inputebene ${ }^{1)}$ läßt sich auch auf der ziel-bzw. Wirkungsebene die Frage nach den Schwerpunkten bei öffentlichen Aufgaben in zwei Varianten aufspalten. Zum einen kann in eher produktionstheoretischer Hinsicht untersucht werden, in welchem öffentlichen Aufgabenbereich in der abgelaufenen Planungsperiode der höchste Grad der Aufgabenerfüllung unabhängig davon, ob er aus Aktivitäten des Staates oder aus erzwungenen und freiwilligen Aktivitäten nicht-staatlicher Entscheidungseinheiten resultiert - tatsächlich erreicht wurde, um eine entsprechende Rangfolge über alle öffentlichen Aufgabenbereiche aufzustellen. Vergleichsobjekte sind hier der realisierte absolute Wert der Wirkungsziele oder deren Veränderungen. Zum anderen läßt sich unter entscheidungstheoretischen Aspekten fragen, welche Aufgabenbereiche die politi-

1) Vgl. Abschnitt 1.1.2. in Kapitel II dieser Arbeit. 
schen Entscheidungsträger für die wichtigsten halten bzw. wo sie Prioritäten und Posterioritäten setzen. Als Vergleichsobjekte dienen dabei im Prinzip die von der öffentlichen Planung in der betrachteten Periode angestrebten absoluten Werte der Wirkungsziele oder ihre Veränderungen. Die sich bei beiden Fragestellungen ergebenden Rangfolgen können voneinander abweichen. Wir beschränken uns hier auf die erste Variante, da zwar die erzwungenen, nicht aber auch die freiwillig entfalteten Aktivitäten nicht-staatlicher Entscheidungseinheiten unmittelbarer Bestandteil der Staatstätigkeit sind. Obschon die Auswirkungen letzterer sich ebenfalls in den impact-objectives niederschlagen und bei der Planung staatlicher Maßnahmen zumindest im Sinne einer passiven Koordination berücksichtigt werden müssen, bleibt doch die Einstellung der politischen Entscheidungsträger dieser subsidiären Art der Aufgabenerfüllung gegenüber im Prinzip offen. Eine Gleichbehandlung mit den unter staatlicher Regie ablaufenden Aktivitäten halten wir unter entscheidungstheoretischen Gesichtspunkten für nicht ohne weiteres zulässig.

Die Schwerpunktbestimmung auf der Basis der realisierten Aufgabenerfüllung unterliegt zwar keinen derartigen entscheidungstheoretischen Imponderabilien, scheitert. jedoch gleichwohl aus zwei Gründen. Zum einen beruht sie auf einem Vergleich von Outputs des gesellschaftlichen Systems, die Ursachen für diese Ergebnisse sind indes allenfalls teilweise bekannt. Erst eine Evaluation könnte Aufschluß darüber geben, welche in der Realität festgestellten absoluten Werte oder Verănderungen der impact-objectives tatsächlich auch aus den Aktivitäten von staat und nicht-staatlichen Entscheidungseinheiten resultieren. Da diese Informationen fehlen, besteht die Gefahr, bei der Schwerpunktbestimmung auf der Ebene der impact-objectives diesen Institutionen Ergebnisse zuzurechnen, für die sie nicht verantwortlich zeichnen. 
Unabhängig davon sieht sich ein externer Beabachter vor das Problem der Inkomparabilität der Wirkungsziele gestellt. Selbst wenn eine Evaluation hinreichende Informationen über Ursache-Wirkungs-Beziehungen lieferte, bestünde aufgrund der unterschiedlichen Dimensionen der zur Operationalisierung der impact-objectives dienenden Resultatindikatoren keine Vergleichbarkeit zwischen Zielwerten verschiedener Aufgabenbereiche ${ }^{1)}$. Die absoluten Werte der realisierten Wirkungsziele vermitteln dem externen Beobachter keinerlei Anhaltspunkte zur Aufstellung einer Rangordnung. Er müßte so inkomparable Größen wie etwa das Niveau der säuglingssterblichkeit und die Unfallhäufigkeit im Straßenverkehr miteinander vergleichen. Er verfügt jedoch über keinen objektiven Bewertungsmaßstab, der ihm eine gegenseitige Abwägung der zielwerte erlaubt; allenfalls könnte er eine subjektive Bewertung vornehmen. Nur unwesentlich bessere Ergebnisse liefert der Vergleich von Veränderungen bei den Wirkungszielen. Augenscheinlich weist ein Aufgabenbereich, in dem die impact-objectives eine gegenüber der Vorperiode eingetretene Verbesserung signalisieren, einen höheren Grad der Aufgabenerfüllung auf als ein Bereich mit konstantem oder gar sinkendem Ergebnis. Abgesehen davon, daB das Kriterium Veränderung eines zielwertes die Ausgangsbasis, von der aus diese erfolgte, unberücksichtigt läßt, erlaubt dieser Spezialfall nur eine Zwischenlösung. Treten, was die Regel sein dürfte, in mehreren Aufgabenbereichen realisierte Verbeserungen bei den impact-objectives auf, sieht sich ein externer Beobachter aufgrund des mangelnden objektiven Bewer-

1) Dieses Argument kann bereits innerhalb eines Aufgabenbereiches Gültigkeit beanspruchen. Wir unterstellen jedoch aus Gründen der Vereinfachung, es existiere pro Aufgabenbereich nur ein impact-objective bzw. Resultatindikator. 
tungsmaßstabes, selbst wenn die Veränderungen prozentual unterschiedlich hoch ausfallen, nicht in der Lage, eine Rangordnung über alle öffentlichen Aufgabenbereiche zu erstellen.

In diesem Zusammenhang läßt sich schließlich auch fragen, in welchem öffentlichen Aufgabenbereich die höchste Effektivität erzielt wird. Hierfür wäre die Ermittlung einer Gesamteffektivität für jeden Aufgabenbereich erforderlich, die jeweils den Wert des betreffenden realisierten impact-objective oder seine Veränderung, soweit diese aus den Aktivitäten von staat und nicht-staatlichen Entscheidungseinheiten resultieren, in Beziehung setzt zu den entsprechenden Inputs bzw. Kosten ${ }^{1)}$. Eine derartige Betrachtung scheitert indes aus denselben Gründen wie die Schwerpunktbestimmung. Zum einen läßt sich zwar im Prinzip in verschiedenen Aufgabenbereichen jeweils eine Gesamteffektivität ermitteln, es existiert jedoch keine Evaluation, die sicherstellen könnte, daß diese Relationen auch einen ursächlichen zusammenhang zwischen Inputs und den Wirkungszielen oder ihren Veränderungen beschreiben. Sodann besteht keine Komparabilität zwischen den Gesamteffektivitäten verschiedener Aufgabenbereiche, vergleichbar sind allenfalls die Kosten. Selbst bei einer Reduktion etwa der Unfallzahlen im Straßenverkehr und der Säuglingssterblichkeit um unterschiedliche Prozentsätze, aber bei jeweils gleichen Kosten, verfügt ein externer Beobachter über keinen objektiven Bewertungsmaßstab, der ihm erst die erforderliche Abwägung gestattet.

Wenngleich nach dieser Analyse für die öffentliche Planung aus einer Ansiedlung ihrer ziele auf der Ebene der impactobjectives gravierende Probleme entstehen, repräsentieren diese Wirkungsziele die konzeptionell angemessene Ebene. Das gilt

1) Die Gesamteffektivität unterscheidet sich von der oben definierten Effektivität 3 nicht qualitativ, sondern lediglich bezüglich des Umfangs der erfaßten Wirkungen und Kosten. 
bereits, falls ausschließlich der staat Aktivitäten zur Erfüllung öffentlicher Aufgaben entfaltet. Würde die öffentliche Planung ihre ziele lediglich auf der Leistungsebene, d.h. vorwiegend auf der Ebene der output-objectives, fixieren, könnte sie zwar relativ leicht ursächliche und eindeutige Beziehungen zwischen In- und Outputs konstruieren. Output-objectives vermögen jedoch die im Hinblick auf die Evaluation abgelaufener und die Festlegung zukünftiger Aktivitäten erforderlichen Informationen über die Bewertung öffentlicher Leistungen durch die betroffenen Individuen nur in unzureichendem Maße zu liefern. Wenn deshalb anstelle der Einschätzungen der Betroffenen die (objektiven) Wirkungen der Leistungen auf die Empfänger, über deren Wohlfahrtsrelevanz sich freilich trefflich streiten läBt, als Kriterium für den Einsatz von Ressourcen zur Erfüllung öffentlicher Aufgaben dienen sollen, repräsentieren output-objectives eine konzeptionell zu niedrige Ebene. Erst die impact-objectives bilden dann die adäquate Ebene, auf der die öffentliche Planung ihre ziele bzw. FührungsgröBen ${ }^{1)}$ vorgeben und damit Schwerpunkte setzen kann. Unter dem Aspekt der Vollständigkeit erweisen sich impact-objectives ebenfalls als überlegen, da sie im Prinzip auch externe Effekte einschließen. Eine zielerreichungskontrolle kann auf beiden Ebenen durchgeführt werden; im Hinblick auf eine Wirksamkeitskontrolle besteht allerdings bei der operationalisierung von impact-objectives mit Hilfe von Resultatindikatoren die Gefahr, daß sich die öffentliche Planung Erfolge zurechnet, für die sie nicht die Ursache war. Für die Schwerpunktbestimmung durch einen externen Beobachter hingegen wäre bei einer Beschränkung auf die Leistungsebene nichts gewonnen; ihm fehlt sowohl auf der Ebene der impact- als auch der output-objectives der notwendige objektive Bewertungsmaßstab.

1) Zur Bedeutung von Führungsgrößen in Planungsprozessen vgl. E. Wille: Mittel- und langfristige Finanzplanung, a.a.0., S. $460 \mathrm{ff}$. 
Im-Hinblick auf die hier geforderte Integration der Aktivitäten nicht-staatlicher Entscheidungseinheiten in die öffentliche Planung erweist sich die zielsetzung auf der Ebene der impact- statt der output-objectives ebenfalls als vorteilhaft. Die Wirkungen einer bestimmten öffentlichen Leistung auf die Betroffenen sind, anders als i.d.R. die Leistungen verschiedener Anbieter selbst, miteinander komparabel. Die politischen Entscheidungsträger können Wirkungen von Leistungen besser als die Leistungseinheiten gegeneinander abwägen bzw. bewerten sowie aggregieren. Impact-objectives erlauben deshalb im Rahmen einer passiven Koordination im Prinzip den Vergleich und die Integration der Wirkungen gleichartiger Leistungen von staat und nicht-staatlichen Entscheidungseinheiten. Erst bei einer zielvorgabe auf dieser Ebene vermag die öffentliche Planung auf der Basis einer verbesserten Evaluation die unterschiedlichen Wirksamkeiten der Leistungen verschiedener Anbieter im Hinblick auf ihre ziele zu berücksichtigen und so eine effiziente Ressourcenallokation bei der Erfüllung öffentlicher Aufgaben herbeizuführen. 
Kapitel VI: ZUSAMMENFASSUNG

Neben dem Staat selbst erfüllen in der Bundesrepublik Deutschland nicht-staatliche Entscheidungseinheiten, teils aufgrund staatlichen Zwangs, teils freiwillig, öffentliche Aufgaben und tragen so zur Erreichung öffentlicher Planungsziele bei. Ihre diesbezüglichen Aktivitäten lassen sich als Bestandteil einer erweiterten Allokationsabteilung im Musgraveschen Sinne auffassen, den eine anspruchsvolle öffentliche Planung bei der Verfolgung ihrer ziele nicht ignorieren darf.

Die Untersuchung setzt zunächst auf der untersten Ebene einer ziel-Mittel-Hierarchie an, die den orientierungsrahmen bietet. Infolgedessen wird der Niederschlag der Aktivitäten nichtstaatlicher Entscheidungseinheiten auf der Ausgabenseite des staatlichen Budgets analysiert. Versteht man unter "staatstätigkeit" alle vom staat selbst oder auf staatliche Veranlassung hin zur Erfüllung öffentlicher Aufgaben entfalteten Aktivitäten bzw. eingesetzten Inputs, so erfährt die Eignung budgetärer Ausgabenschwerpunkte zur Kennzeichnung von Schwerpunkten der Staatstätigkeit aus zwei Gründen Einschränkungen: Zum einen repräsentieren budgetäre Ausgaben die vom staat eingesetzten Inputs nicht mit gleicher Gewichtung, da das Budget sowohl Ausgaben als auch Kosten enthält und beide gleich gewichtet. Für Verzerrungen in intertemporaler Hinsicht einerseits und zwischen verschiedenen öfentlichen Aufgabenbereichen andererseits können diesbezüglich vor allem der Ansatz von Investitionsausgaben statt ihrer Verteilung mit Hilfe von Abschreibungen, die staatliche Darlehensvergabe sowie die Ubernahme von Gewährleistungen sorgen. Zum anderen spiegeln Staatsausgaben die unter staatlicher Regie eingesetzten Inputs zur Erfüllung öffentlicher Aufgaben nicht vollständig wider. Neben der staatlichen Normsetzung allgemein und den in produktionstheoretischer Hinsicht den Staatsausgaben weitgehend äquivalenten indirekten monetären Inputs in Form von steuer- 
vergünstigungen sorgt hier vor allem die Verlagerung von Aufgaben und damit auch Ausgaben auf nicht-staatliche Entscheidungseinheiten für Verzerrungen. Die aufgrund einer Vielzahl von Gesetzen und anderen Vorschriften erfolgende Erfüllung öffentlicher Aufgaben durch Parafisci und öffentliche Unternehmen, wobei in den letzten Jahren eine "Flucht aus dem Budget" einsetzte, sowie durch private Haushalte und Unternehmen (insbesondere der "versteckte staatsbedarf") schlägt sich in den Staatsausgaben weitgehend lediglich in Höhe direkter staatlicher Zuschüsse nieder und führt insoweit zu einer Unterschätzung des Umfangs der Staatstätigkeit. Zudem kann sie strukturelle verzerrungen bewirken. Da der staat die untersuchten Instrumentvariablen in verschiedenen Aufgabenbereichen in unterschiedlichem Maße einsetzt, spiegeln budgetäre Ausgabenschwerpunkte die struktur bzw. die Schwerpunkte der staatstätigkeit unzutreffend wider. Unter dem Aspekt der Staatstätigkeit ergibt sich nicht notwendigerweise eine andere als die auf den budgetären Ausgaben basierende Rangfolge der öffentlichen Aufgabenbereiche, wohl aber ändern sich die Abstände zwischen verschiedenen Aufgabenbereichen.

In der Realität kombiniert der staat häufig mehrere der untersuchten Instrumentvariablen miteinander. Die Vergabe öffentlicher Darlehen und Gewährleistungen etwa überträgt er vielfach auf intermediäre Entscheidungseinheiten, und in den Genu $B$ von Steuervergünstigungen kommen typischerweise auch bestimmte nicht-staatliche Entscheidungseinheiten. Insofern eignet sich - ungeachtet seiner theoretischen Schwächen - das Konzept der Ausgabenintensität der öffentlichen Aufgabenerfüllung für eine zusammenfassende würdigung der Wirkungen, welche die analysierten staatlichen Instrumentvariablen auf die Eignung budgetärer Ausgabenschwerpunkte zur Kennzeichnung von Schwerpunkten der staatstätigkeit ausüben. Letztlich versagen hier jedoch sämtliche in der Literatur diskutierten ausgabenorientierten Kennziffern sowohl bei unterschiedlichen 
Niveaus der Ausgabenintensität in den verschiedenen öffentlichen Aufgabenbereichen als auch bei sich verändernden Ausgabenintensitäten.

Zusätzlich zu diesen endogenen, vom staat unmittelbar beeinflußbaren Faktoren vermögen exogene Störgrößen Verzerrungen bei der Bestimmung von Schwerpunkten der staatstätigkeit hervorzurufen. Hierbei handelt es sich um Faktoren, auf die der Staat allenfalls beschränkten EinfluB hat, deren Wirkungen sich jedoch ebenfalls in den Staatsausgaben niederschlagen können (Veränderungen von Relativpreisen und der Bevölkerung). Erweitert man schlieBlich den Blickwinkel von der staatstätigkeit auf die insgesamt entfalteten Aktivitäten zur Bereitstellung öffentlicher Güter bzw. zur Erfüllung öffentlicher Aufgaben, so sind die freiwillig entfalteten einschlägigen Aktivitäten nicht-staatlicher Entscheidungseinheiten (Kirchen, Wohlfahrtsverbände, teilweise auch private Unternehmen) zu berücksichtigen. Vor allem die unterschiedlichen zielvorstellungen der Initiatoren dieser Institutionen und der politischen Entscheidungsträger deuten darauf hin, daß diese primär auf das Angebot meritorischer Güter gerichteten und vom staat teilweise geförderten Aktivitäten sich anders als diejenigen des Staates über die verschiedenen Aufgabenbereiche verteilen.

Demnach indizieren auf der Basis budgetärer Ausgaben gebildete Schwerpunkte weder die struktur der staatstätigkeit, noch was allerdings auch nicht ihrer Zweckbestimmung entspricht die struktur der insgesamt entfalteten Aktivitäten zur Erfüllung öffentlicher Aufgaben verzerrungsfrei. Für beide Fragestellungen eignen sich vielmehr die von staat und nicht-staatlichen Entscheidungseinheiten aufgewendeten Kosten, definiert als vollständig erfaßte und verursachungsgerecht auf die verschiedenen Perioden zugerechnete Inputs, der öffentlichen Aufgabenerfüllung. Voraussetzung für die Ermittlung der Kosten, die die nicht-staatlichen Entscheidungseinheiten tragen, bildet die Abgrenzung derjenigen ihrer Aktivitäten, die sich auf 
die Erfüllung öffentlicher Aufgaben richten. Die in der Finanzwissenschaft diskutierten normativen und positiven Theorien der Staatstätigkeit können jedoch keine klare Grenze zwischen öffentlichem und privatem Aufgabenbereich abstecken. Auch das Kriterium der ProzeBsteuerung erlaubt keine grundsätzlichen Aussagen darüber, ob und inwieweit der Marktmechanismus auf bestimmten Gebieten staatswirtschaftlichen Allokationsmechanismen überlegen ist. In der Verfassung schließlich erscheint der Aufgabenbereich des Staates auch aus juristischer sicht nicht abschließend determiniert. Daher folgt diese Arbeit einer zeitund ortsgebundenen Abgrenzung: Nicht-staatliche Entscheidungseinheiten erfüllen öffentliche Aufgaben, soweit der staat bestimmte Aktivitäten auf sie verlagert, oder soweit sie freiwillig meritorische Güter bereitstellen. Zur Bestimmung der einschlägigen Kosten öffentlicher Unternehmen hingegen existieren in der Literatur spezielle Verfahren.

Bei der Aggregation der von staat und nicht-staatlichen Entscheidungseinheiten in einem Aufgabenbereich aufgewendeten Kosten sind zur Vermeidung von Mehrfachzählungen bestimmte Bereinigungen um Ressourcenströme zwischen den beteiligten Institutionen erforderlich. Zudem bietet sich unter produktionstheoretischen Aspekten eine Aufspaltung der ermittelten Summe in Kosten der Inputs, die in Produktionsprozesse einfließen, und in Kosten für unmittelbare Transfers an. Je nach Abgrenzung bilden die aggregierten Kosten von staat und nichtstaatlichen Entscheidungseinheiten eine tragfähige Basis für die Bestimmung von Schwerpunkten der staatstätigkeit einerseits oder der insgesamt entfalteten Aktivitäten zur Erfüllung öffentlicher Aufgaben andererseits; gleichzeitig eignen sie sich als Bezugsgröße für Produktivitätsanalysen. Die Kosten der öffentlichen Aufgabenerfüllung erlauben jedoch noch keine Aussagen über den mit Hilfe der eingesetzten Inputs erzielten output. 
In den Volkswirtschaftlichen Gesamtrechnungen (VGR) stellen der staat und ein Teil der nicht-staatlichen Entscheidungseinheiten weitgehend einen Fremdkörper dar. Einerseits resultiert aus ihrer Einbeziehung in die Berichterstattung ein Vorteil: Die VGR enthalten die Inputs zur Erfüllung öffentlicher Aufgaben annähernd vollständig. Zudem setzen sie auf der konzeptionell geeigneten Ebene an, indem sie auf der Aufwandsseite der Produktionskonten im Prinzip ausschließlich Kosten erfassen und so die Inputs einheitlich gewichten. Insofern geben sie eine bessere Basis zur Bestimmung der Schwerpunkte aller Aktivitäten zur Erfüllung öffentlicher Aufgaben ab als das staatliche Budget. Andererseits jedoch dienen die VGR nicht dem Zweck, die Aktivitäten zur Erfüllung öffentlicher und privater Aufgaben zu separieren. Sie versuchen, der Heterogenität der Aktivitäten nicht-staatlicher Entscheidungseinheiten Rechnung zu tragen, nicht indem sie hierfür einen oder mehrere eigene Sektoren konstituieren, sondern indem sie sie - in zum Teil fragwürdiger Weise - in ein vorhandenes Schema einpressen. Bereits im Hinblick auf die globale Relation zwischen den Aktivitäten zur Erfüllung öffentlicher und denjenigen zur Erfüllung privater Aufgaben entstehen aus drei Gründen Verzerrungen: Das in den VGR für die sektorale zuordnung einer Institution verwendete Kriterium Prozeßsteuerung differenziert nicht notwendigerweise gleichzeitig auch zwischen privaten und öffentlichen Aufgaben. Sodann ordnen die VGR Institutionen als Ganzes einem Sektor $z u$, gerade bei nicht-staatlichen Entscheidungseinheiten wäre jedoch häufig eine Aufspaltung in öffentliche und private Aktivitäten erforderlich. Schließlich weichen auf den Produktionskonten verschiedener Institutionen sowohl die erfaßten Vorgänge als auch die Bewertungsverfahren voneinander ab. Wenn demnach die VGR bereits bezüglich der globalen Relation zwischen öffentlichen und privaten Aktivitäten Verzerrungen enthalten, eignen sie sich ohne zusatzinformationen nicht zur Bestimmung von Schwerpunkten der Aktivi+äten zur Erfüllung öffentlicher Aufgaben. 
Ebenfalls als unzureichend stellen sich die von den VGR gelieferten Daten über den Output der nicht-staatlichen Entscheidungseinheiten heraus. Als angreifbar erscheint insbesondere der bei den unentgeltlich abgegebenen Leistungen der nichtstaatlichen Entscheidungseinheiten (und des Staates) erfolgende Nachweis von Inputs als Output, zumal es sich nicht notwendigerweise um diejenigen Inputs handelt, die für die Erstellung der von ihnen zu repräsentierenden Leistungen verbraucht werden.

Auch von daher erweist sich die Messung der öffentlichen Leistungen nicht-staatlicher Entscheidungseinheiten als notwendig. $\mathrm{Zu}$ diesem $\mathrm{Zweck}$ werden in der Literatur diskutierte Verfahren zur Messung des Outputs primär des staates auf ihre Eignung für nicht-staatliche Entscheidungseinheiten hin untersucht. Dabei dient ein Leistungsbegriff als Referenzgröße, der nur tatsächlich genutzte Outputeinheiten umfaBt und ausschlieBlich auf die Bewertung durch die betroffenen Individuen abstellt. Die untersuchten eindimensionalen (monetären) Verfahren zur Leistungsmessung scheitern weitgehend. In idealer Weise wird diesen Ansprüchen zwar das Konzept der individuellen Zahlungsbereitschaft gerecht, ihrer Ermittlung stehen jedoch bei öffentlichen Leistungen i.d.R. unüberwindliche Hindernisse im Wege, weil hier die Empfänger ihre Präferenzen nicht offenbaren können oder wollen. Auch die im Rahmen von Nutzen-KostenAnalysen erfolgende Operationalisierung der Zahlungsbereitschaft mit Hilfe von Schattenpreisen liefert in der konkreten Durchführung häufig angreifbare Ergebnisse. Fưr einen speziellen, auf Nutzen-Kosten-Analysen basierenden Ansatz einer gesellschaftsbezogenen Unternehmensrechnung gelten, soweit er konkrete Vorschläge zur Messung sozialer Nutzen und Kosten enthält, dieselben Einwände. Marktpreise andererseits markieren die Untergrenze der Zahlungsbereitschaft und eignen sich deshalb im Prinzip zur Leistungsmessung. Sie liegen jedoch für viele öffentliche Leistungen nicht-staatlicher Entscheidungseinheiten nicht vor, ggf. vorhandene Marktpreise weisen häufig 
nur begrenzte Aussagekraft auf, und die Marktpreise ähnlicher Güter lassen sich aufgrund mangelnder Vergleichbarkeit nicht auf unentgeltlich abgegebene Leistungen übertragen. Freiwillig entrichtete Mitgliedsbeiträge und spenden vor allem an karitative Organisationen schlieBlich drücken nur unter restriktiven Bedingungen die Bewertung der abgegebenen Leistungen durch die Empfänger aus. Als ausschließlich oder überwiegend inputorientiert müssen dagegen die sog. Kostenbewertung, die Anwendung des Payment-Price Approach auf die Sozialversicherung, der Vermögensbestandsansatz sowie Indizes der realen Leistungen des Staates verworfen werden.

Von den analysierten mehrdimensionalen Verfahren zur Bestimmung der öffentlichen Leistungen nicht-staatlicher Entscheidungseinheiten erweisen sich soziale Indikatoren als relativ erfolgversprechend. Das Kriterium der Outputorientierung erfüllen Nutzungsindikatoren, die die Ebene der output-objectives repräsentieren, sowie Resultatindikatoren, die zur Operationalisierung der impact-objectives dienen. Nutzungsindikatoren informieren insoweit über den EinfluB einer bestimmten öffentlichen Leistung auf die Wohlfahrtsposition der Individuen, als diese mit der aktiven und freiwilligen Inanspruchnahme offenbaren, daB sie die Leistung positiv beurteilen. Die Messung der nachgefragten Leistungseinheiten weist auch den Vorteil auf, daB relativ leicht ursächliche Beziehungen zwischen dem Output und den zu seiner Erstellung erforderlichen Inputs hergestellt werden können. Andererseits versagen Nutzungsindikatoren in allen Fällen passiver Konsumtion bzw. externer Effekte. Demgegenüber bilden Resultatindikatoren die Wirkungen öffentlicher Leistungen auf die Empfänger einschließlich der Wirkungen externer Effekte ab und stellen damit unter dem Aspekt der Vollständigkeit eines Leistungsmaßes das überlegene Konzept dar. Sie lassen jedoch in bestimmten Fällen offen, wie die betroffenen Individuen diese wirkungen bewerten, und ob sie sie überhaupt bemerken. Zudem reflektieren Resultatindikatoren ein Endergebnis, das sich (bisher noch) nur in wenigen Fällen vollständig auf verschiedene 
als Ursache in Frage kommende Aktivitäten bzw. Einflußfaktoren zurückführen $1 a ̈ B t$.

Als zur Leistungsmessung bei nicht-staatlichen Entscheidungseinheiten ungeeignet erweisen sich hingegen Kosten-WirsamkeitsAnalysen. Es handelt sich zwar um ein Verfahren zur Messung von Outputs, jedoch nicht notwendigerweise auch um eine vollständige Messung. Zudem befinden anstelle der betroffenen Individuen die politischen Entscheidungsträger über die Bewertung des erfaßten Outputs. Als vielversprechender erscheint von seiner Konzeption her das Planning-Programming-Budgeting system. Vor allem die Programmstrukturen als aktivitätenbezogene Ziel-Mittel-Hierarchien mit mehreren, durch Kausalrelationen verbundenen Ebenen und die analytischen Spezialstudien zielen letztlich auf die Konstruktion staatlicher Produktionsfunktionen $a b$ und liefern so im Prinzip auch geeignete Informationen für die Leistungsmessung bei nicht-staatlichen Entscheidungseinheiten. Das PlanningProgramming-Budgeting system enthält jedoch diesbezüglich keine zusätzlichen, eigenständigen Verfahren, vielmehr bietet es primär einen konzeptionellen und organisatorischen Rahmen für die Anwendung von Nutzen-Kosten- und Kosten-Wirksamkeits-Analysen.

Da die betroffenen Individuen ihre Präferenzen für die öffentlichen Leistungen nicht-staatlicher Entscheidungseinheiten nur selten in Marktpreisen quantifiziert offenbaren, repräsentieren die ausgewählten Verfahren zur Leistungsmessung überwiegend die Ebene der output-objectives, teilweise auch nur diejenige der öfentlichen Produkte. Fragt man andererseits nicht nach dem Produktionsoutput nicht-staatlicher Entscheidungseinheiten, sondern nach den von ihnen erbrachten direkten und indirekten Transfers, so lassen sich zwar ihre hierfür getätigten Aufwendungen, jedoch nur in Ausnahmefällen auch die Beurteilungen der Transfers durch die Empfänger ermitteln.

Die Knappheit der zur Erfüllung öffentlicher Aufgaben verfügbaren Ressourcen begründet einen Bedarf an Koordination bzw. In-

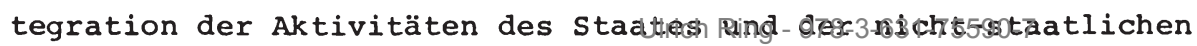


Entscheidungseinheiten zumindest insoweit, als der staat letztere bei der Planung eigener Maßnahmen berücksichtigt. Der Vergleich verschiedener Anbieter einer bestimmten öffentlichen Leistung bezüglich ihrer Effektivität vermag im Prinzip Kriterien für eine Beurteilung ihrer Aktivitäten unter Wohlfahrtsaspekten und für die Entscheidung über einen staatlichen Handlungsbedarf zu liefern, während die Betrachtung von Leistungsvolumina und Produktionseffizienzen für diese Fragestellungen nicht genügt. Bei einer Messung der Leistungen nicht-staatlicher Entscheidungseinheiten auf der Ebene der output-objectives mit Hilfe von Nutzungsindikatoren läßt sich zwar für jeden Leistungsanbieter eine Effektivität ermitteln, diese beinhaltet jedoch im Falle des Vergleichs verschiedener Anbieter einer bestimmten Leistung eine Tendenz zur Nivellierung. Ferner erlaubt die Leistungsmessung auf der Ebene der output-objectives keine Bestimmung von Schwerpunkten der öffentlichen Aufgabenerfülung bzw. von Leistungsschwerpunkten über alle öffentlichen Aufgabenbereiche, da ein externer Beobachter über keinen objektiven Bewertungsmaßstab verfügt.

Da die Nutzung öffentlicher Leistungen bzw. die output-objectives noch nicht über die Veränderungen der individuellen wohlfahrtsposition informieren und deshalb kaum Anhaltspunkte für die Beurteilung eines Leistungsanbieters liefern, muB eine anspruchsvolle öffentliche Planung ihre ziele auf einer übergeordneten Ebene ansiedeln. In Ermangelung von Verfahren zur Ermittlung der Bewertungen durch die betroffenen Individuen kann sie sich lediglich an den Wirkungen öffentlicher Leistungen auf die Empfänger bzw. impact-objectives orientieren. Unter diesem Aspekt stellen öffentliche Leistungen Mittel zur Erreichung der öffentlichen Planungsziele dar. Die Formulierung von Wirkungszielen kann allerdings eine Verlagerung der Bewertungsinstanz implizieren, da häufig nicht die betroffenen Individuen, sondern die politischen Entscheidungsträger über die Wohlfahrtsrelevanz der Wirkungen öffentlicher Leistungen entscheiden. 
Die zur Operationalisierung der impact-objectives dienenden Resultatindikatoren messen ein Endergebnis, das aus einer Vielzahl von EinfluBfaktoren entsteht. Deshalb eignen sie sich zwar zur Vorgabe von zielen und zur zielerreichungskontrolle, stellen die öffentliche Planung indes vor gravierende Probleme im Hinblick auf eine Wirksamkeitsanalyse (Evaluation), welche nach dem jeweiligen Beitrag eines Leistungsanbieters zu einem Wirkungsziel fragt. Der Vergleich von auf der Grundlage von Wirkungszielen ermittelten Effektivitäten verschiedener Anbieter einer bestimmten Leistung gestattet im Prinzip eine Beurteilung ihrer Aktivitäten im Hinblick auf die Planungsziele sowie ggf. die bewubte Verlagerung der betreffenden Aufgabe auf den effektivsten Anbieter, die Evaluationsforschung vermag jedoch bisher noch kaum die hierfür erforderlichen Informationen über die Ursache-Wirkungs-Beziehungen zu liefern. Auch der SchluB vom Leistungsvolumen eines Anbieters auf seinen Beitrag zur Realisation eines Wirkungszieles kann hier kaum weiterhelfen. Ein externer Beobachter schlieblich, der eine Bestimmung von Schwerpunkten der öffentlichen Aufgabenerfullung auf der Ebene der impact-objectives oder einen Vergleich von Gesamteffektivitäten über alle öffentlichen Aufgabenbereiche hinweg vornehmen will, scheitert an der fehlenden Komparabilität der zu vergleichenden Größen. Er verfügt über keinen objektiven Benutzungsmaßstab, der ihm erst die erforderliche Abwägung gestatten würde.

Obwohl für die öffentliche Planung aus einer Ansiedlung ihrer Ziele auf der Ebene der impact-objectives vielfältige Probleme erwachsen, repräsentieren diese Wirkungsziele die konzeptionell adăquate zielebene, denn bei öffentlichen Leistungen fehlen die Informationen über ihre Bewertung durch die betroffenen Individuen weitgehend. Auch im Hinblick auf die Integration der Aktivitäten nicht-staatlicher Entscheidungseinheiten in die öffentliche Planung besitzt diese Ebene Vorteile gegenüber den output-objectives, da die wirkungen der Leistungen verschiedener Anbieter eher komparabel sind als 
die Leistungen selbst. Insofern geben impact-objectives die geeigneten Führungsgrößen für die Koordination der Aktivitäten des Staates und nicht-staatlicher Entscheidungseinheiten $a b$. 


\section{ANHANG}

Katalog von Erfolgskomponenten einer gesellschaftsbezogenen Erfolgsrechnung für öffentliche Unternehmen des Personen-Nahverkehrs

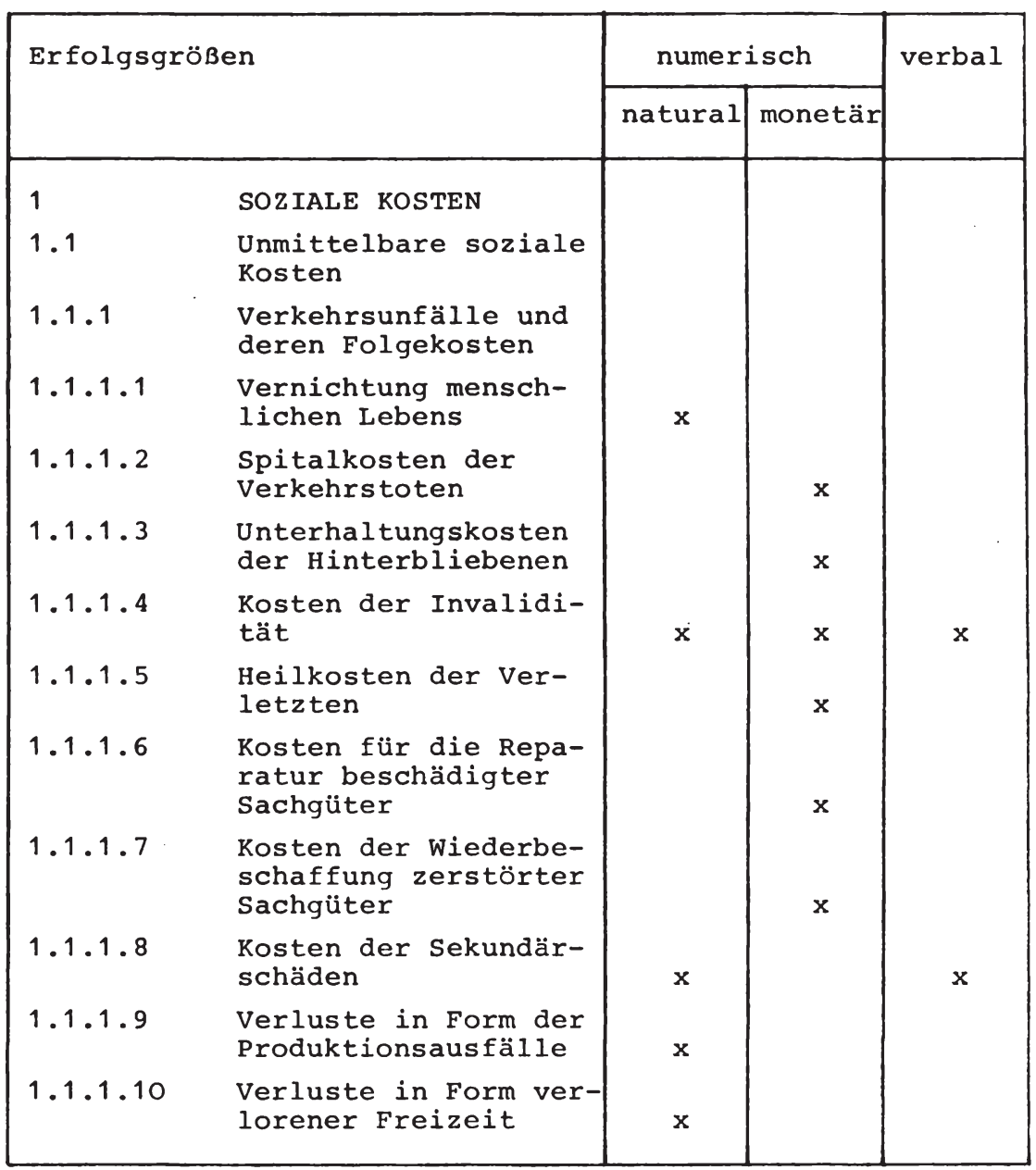




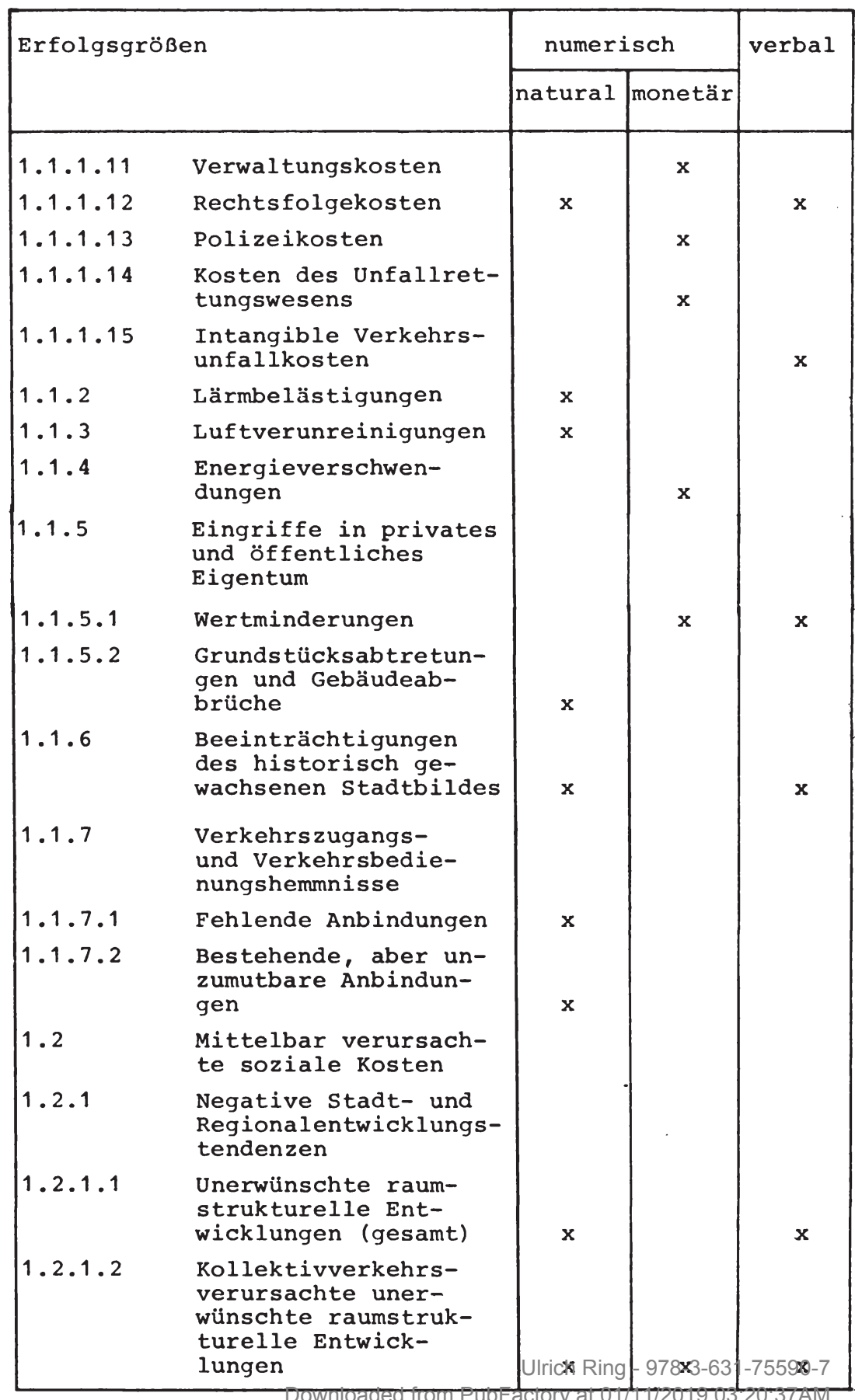




\begin{tabular}{|c|c|c|c|c|}
\hline \multicolumn{2}{|c|}{ Erfolgsgrößen } & \multicolumn{2}{|c|}{ numerisch } & \multirow[t]{2}{*}{ verbal } \\
\hline & & natural & monetär & \\
\hline $1.2 \cdot 2$ & $\begin{array}{l}\text { Straßenentlastungs- } \\
\text { hemmnisse }\end{array}$ & & & \\
\hline 1.2 .2 .1 & $\begin{array}{l}\text { Nicht erreichte } \\
\text { Verkehrsteilung }\end{array}$ & $\mathbf{x}$ & & \\
\hline 1.2 .2 .2 & $\begin{array}{l}\text { Angebotspolitische } \\
\text { Maßnahmen, die zu } \\
\text { einem nicht be- } \\
\text { darfsgerecht ausge- } \\
\text { stalteten öfentli- } \\
\text { chen Personen-Nah- } \\
\text { verkehrsangebot } \\
\text { füren }\end{array}$ & & & \\
\hline 1.2 .2 .2 .1 & $\begin{array}{l}\text { Leistungspolitische } \\
\text { Maßnahmen }\end{array}$ & $\mathbf{x}$ & & $\mathbf{x}$ \\
\hline 1.2 .2 .2 .2 & $\begin{array}{l}\text { Tarifpolitische } \\
\text { Maßnahmen }\end{array}$ & & $\mathbf{x}$ & $\mathrm{x}$ \\
\hline 1.2 .2 .3 & $\begin{array}{l}\text { Versäumnisse, ange- } \\
\text { botswirtschaftliche } \\
\text { Mängel zu beseitigen }\end{array}$ & & & \\
\hline $1.2 \cdot 2 \cdot 3 \cdot 1$ & $\begin{array}{l}\text { Unterlassene Kapazi- } \\
\text { tätsvorhaltung }\end{array}$ & $\mathbf{x}$ & & $\mathrm{x}$ \\
\hline $1.2 \cdot 2 \cdot 3 \cdot 2$ & $\begin{array}{l}\text { Unterlassene oder er- } \\
\text { folglos gebliebene } \\
\text { Kooperationsbemühun- } \\
\text { gen }\end{array}$ & $\mathbf{x}$ & $\mathbf{x}$ & $\mathbf{x}$ \\
\hline 2 & SOZIALE NUTZEN & & & \\
\hline 2.1 & $\begin{array}{l}\text { Unmittelbare soziale } \\
\text { Nutzen }\end{array}$ & & & \\
\hline 2.1 .1 & $\begin{array}{l}\text { Soziale Nutzen aus } \\
\text { der Sicherung des } \\
\text { Verkehrszuganges und } \\
\text { der Verkehrsbedienung } \\
\text { für den aktuellen und } \\
\text { potentiellen Verkehrs- } \\
\text { bedarf }\end{array}$ & $\mathbf{x}$ & & \\
\hline 2.1 .2 & $\begin{array}{l}\text { Durch den Einsatz } \\
\text { schutzpolitisch vor- } \\
\text { teilhafter kollekti- } \\
\text { ver Verkehrsmittel } \\
\text { vermiedene soziale } \\
\text { Kosten }\end{array}$ & & & \\
\hline
\end{tabular}




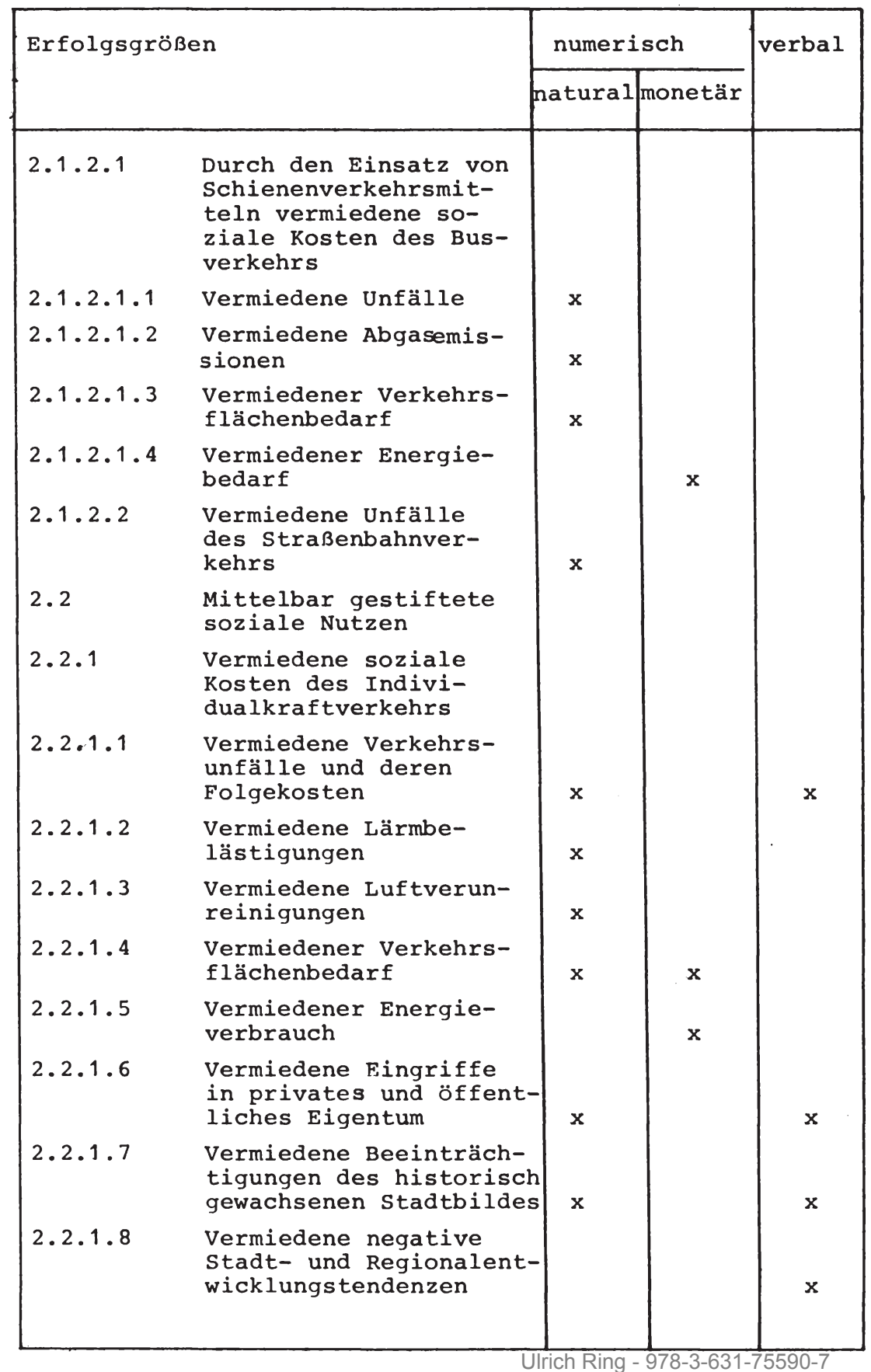




\begin{tabular}{|c|c|c|c|c|}
\hline \multicolumn{2}{|c|}{ Erfolgsgrößen } & \multicolumn{2}{|c|}{ numerisch } & \multirow{2}{*}{ verbal } \\
\hline & & natural & monetär & \\
\hline 2.2 .1 .9 & $\begin{array}{l}\text { Vermiedene Stauungs- } \\
\text { zeitverluste }\end{array}$ & $\mathbf{x}$ & & \\
\hline 2.2 .1 .10 & $\begin{array}{l}\text { Soziale Nutzen aus } \\
\text { der Unterstützung } \\
\text { von Bemühungen, } \\
\text { Stadt- und Regio- } \\
\text { nalentwicklungs- } \\
\text { ziele zu erreichen }\end{array}$ & & & \\
\hline $2.2 \cdot 1.10 .1$ & $\begin{array}{l}\text { Erwïnschte raum- } \\
\text { strukturelle Ent- } \\
\text { wicklungen (ge- } \\
\text { samt) }\end{array}$ & $\mathbf{x}$ & & $\mathbf{x}$ \\
\hline $2.2 \cdot 1 \cdot 10 \cdot 2$ & $\begin{array}{l}\text { Kollektivverkehrs- } \\
\text { bedingter Beitrag } \\
\text { zu erwüschten } \\
\text { raumstrukturellen } \\
\text { Entwicklungen }\end{array}$ & & & \\
\hline 2.2 .1 .10 .2 .1 & $\begin{array}{l}\text { Für den Bereich der } \\
\text { Kernstadt }\end{array}$ & $x$ & $\mathbf{x}$ & $\mathrm{x}$ \\
\hline 2.2 .1 .10 .2 .2 & $\begin{array}{l}\text { Für die Verkehrs- } \\
\text { region }\end{array}$ & $x$ & $\mathbf{x}$ & $\mathrm{x}$ \\
\hline
\end{tabular}

Quelle: P. Jäger: Probleme und Möglichkeiten einer mit sozialen Kosten und Nutzen erweiterten Erfolgswürdigung öffentlicher Betriebe des PersonenNahverkehrs, a.a.0., S. $366 \mathrm{ff}$. 


\section{LITERATURVERZEICHNIS}

Aderhold, Dieter: Kybernetische Regierungstechnik in der Demokratie. Planung und Erfolgskontrolle, München, Wien 1973.

Albers, Willi: Der Umfang der staatlichen Tätigkeit und ihre wirtschaftlichen Wirkungen, in: Weltwirtschaftliches Archiv, Bd. 77 (1956 II), S. 176-234.

- Soziale Sicherung. Konstruktionen für die zukunft, stuttgart 1982 .

Albrecht, Dietrich: Subventionen. Problematik und Entwicklungen, Schriftenreihe des Bundesministeriums der Finanzen, Heft 25. Bonn 1978.

Andel, Norbert: Subventionen als Instrument des finanzwirtschaftlichen Interventionismus, Tübingen 1970.

Verteilungswirkungen der Sozialversicherung am Beispiel der gesetzlichen Krankenversicherung der Bundesrepublik Deutschland, in: Wilhelmine DreiBig (Hrsg.): Uffentliche Finanzwirtschaft und Verteilung III, Schriften des Vereins für Socialpolitik N.F., Bd. 75/III, Berlin 1975, S. 39-82.

- Nutzen-Kosten-Analysen, in: Handbuch der Finanzwissenschaft, hrsg. von Fritz Neumark, Bd. 1, 3. Aufl., Tübingen 1977, S. 475-518.

Subventionen, in: Handwörterbuch der Wirtschaftswissenschaft (HdWW), hrsg. von Willi Albers et al., Bd. 7, stuttgart et al. 1977, S. 491-510.

- Finanzwissenschaft, Tübingen 1983.

Andreae, Clemens August: Die parafiskalischen Gebilde in finanzwissenschaftlicher Schau, in: Festschrift Walter Heinrich. Ein Beitrag. zur Ganzheitsforschung, Graz 1963, S. 333-344.

Arbeitsgemeinschaft Systemanalyse Baden-Württemberg (DornierSystem, Prognos, Arbeitsgruppe Landespflege): Systemanalyse zur Landesentwicklung Baden-Württemberg, im Auftrag des Landes Baden-Württemberg, Stuttgart 1975. 
Arnold, Volker: Methoden der Entscheidungsfindung bei staatlichen Allokationsaktivitäten - ein kritischer Vergleich, in: Finanzarchiv N.F., Bd. 33 (1974/75), S. 418-434.

Nutzen-Kosten-Analyse II: Anwendung, in: Handwörterbuch der Wirtschaftswissenschaft (HdWW), hrsg. von Willi

Albers et al., Bd. 5, Stuttgart et al. 1980, S. 382-399.

Backhaus, Jürgen: రffentliche Unternehmen. Zum Wirtschaftsrecht, den Funktionen und Rechtsformen öffentlicher Unternehmen, 2. Aufl., Frankfurt/Main 1980.

Bartels, Hildegard: Das Kontensystem für die Volkswirtschaftlichen Gesamtrechnungen der Bundesrepublik Deutschland. Erster Teil: Das angestrebte Kontensystem, in: Wirtschaft und Statistik, Jahrgang 1960, S. 317-344.

Bartholomäi, Reinhart Chr.: Das Social Indicator Movement in den USA - eine kritische Würdigung, in: Guy Kirsch und Walter Wittmann (Hrsg.): Nationale ziele und Soziale Indikatoren, Stuttgart 1975, s. 33-56.

Bauer, Raymond A. (Ed.) : Social Indicators, Cambridge, London 1966.

Bauer, Rudolph: Wohlfahrtsverbände in der Bundesrepublik. Materialien und Analysen zu Organisation, Programmatik und Praxis. Ein Handbuch, Weinheim, Basel 1978.

Bendick Jr., Marc: Education as a Three-Sector Industry, in: Burton A. Weisbrod: The Voluntary Nonprofit Sector. An Economic Analysis, Lexington 1977, S. 101-141.

Bergsträsser, Roland: Die Bürgschaften und Garantien des Bundes als Mittel der Wirtschaftsförderung, in: zeitschrift für das gesamte Kreditwesen, 28. Jahrgang (1975), S. 146-148, S. $182-184$, S. $218-222$.

Betz, Gerhard: Einstieg in die Gesellschaftsberichterstattung - ein Versuch der praktischen Gestaltung, in: Hans-Joachim.Hoffmann-Nowotny (Hrsg.): Gesellschaftliche Berichterstattung zwischen Theorie und politischer Praxis. Soziale Indikatoren $x$. Konzepte und Forschungsansätze, Frankfurt, New York 1983, S. 17-26.

Birdsall, William C.: A Study of the Demand for Public Goods, in: Richard A. Musgrave (Ed.): Essays in Fiscal Federalism, Washington D.C. 1965, S. 235-294.

Blankart, Beat: Probleme der Messung und Bewertung von Forschungsresultaten - eine Anwendung auf die 8konomie in schweizerischen Hochschulen, in: Schweizerische Zeitschrift für Volkswirtschaft und statistik, 110. Jahrgang (1974), S. 205-229. 
Blankart, Charles Beat: Ökonomie der öffentlichen Unternehmen. Eine institutionelle Analyse der Staatswirtschaft, München 1980.

Blechman, Barry M. et al.: Setting National Priorities. The 1976 Budget, Washington D.C. 1975.

Böhret, Carl: Entscheidungshilfen für die Regierung. Modelle, Instrumente, Probleme, Opladen 1970.

Bös, Dieter: Effizienz des öffentlichen Sektors aus volkswirtschaftlicher Sicht, in: Schweizerische Zeitschrift für Volkswirtschaft und Statistik, 114. Jahrgang (1978), S. 287-314.

- Öffentliche Unternehmungen, in: Handbuch der Finanzwissenschaft, hrsg. von Fritz Neumark, Bd. 2, 3. Auf1., Tübingen 1980 , S. $1-60$.

Economic Theory of Public Enterprise, Berlin, Heidelberg, New York 1981.

Bohm, Peter: An Approach to the Problem of Estimating Demand for Public Goods, in: Swedish Journal of Economics, Vol. 73 (1971), S. 55-66.

Estimating Demand for Public Goods: An Experiment, in: European Economic Review, Vol. 3 (1972), S. 111-130.

Bombach, Gottfried: Staatshaushalt und Volkswirtschaftliche Gesamtrechnung, in: Finanzarchiv N.F., Bd. 17 (1956/57), S. 344-383.

Die öffentliche Finanzwirtschaft im Wirtschaftskreislauf, in: Handbuch der Finanzwissenschaft, hrsg. von Fritz Neumark, Bd. 1, 3. Auf 1., Tübingen 1977, S. 53-75.

Braband, W.J. van: General Survey of Subsidizing Non-Profit Institutions, in: Karl Häuser (Ed.): Subsidies, Tax Reliefs and Prices. Proceedings of the 33 rd Congress of the International Institute of Public Finance (Varna 1977), Paris 1981, S. 133-149.

Brown, Charles V. und Jackson, Peter M.: Public Sector Economics, 2nd Ed., Oxford 1982.

Brümmerhoff, Dieter: Das "Planning-Programming-Budgeting System" (PPBS), in: Finanzarchiv N.F., Bd. 29 (1970), S. 64-74.

Gesamtwirtschaftliches Rechnungswesen. Eine problemorientierte Einführung mit einem Kompendium wichtiger Begriffe des gesamtwirtschaftlichen Rechnungswesens, Köln 1975. 
Brümmerhoff, Dieter: Produktivität das öffentlichen Sektors, in: Finanzarchiv N.F., Bd. $34(1975 / 76)$, S. 226-243.

- Die öffentlichen Unternehmen in der volkswirtschaftlichen Gesamtrechnung. Gegenwärtiger Stand und Verbesserungsmöglichkeiten, in: Zeitschrift für öffentliche und gemeinwirtschaftliche Unternehmen, Band 1 (1978), Heft 1 , S. 5-11.

Brüngger, Heinrich: Das Programm der O.E.C.D. zur Entwicklung Sozialer Indikatoren, in: Hans-Joachim Hoffmann-Nowotny (Hrsg. unter Mitarbeit von Matthias U. Peters und Peter G. Zeugin): Soziale Indikatoren. Internationale Beiträge zu einer neuen praxisorientierten Forschungsrichtung, Frauenfeld, stuttgart 1976, S. 247-266.

Brüngger, Heinrich und Orga, Carlos: Ansätze zur Messung des Outputs des Staates, in: Schweizerische Zeitschrift für Volkswirtschaft und statistik, 114. Jahrgang (1978), S. 357-388.

Budäus, Dietrich: Betriebswirtschaftslehre öffentlicher Unternehmen und Verwaltungen - Ansätze und Probleme eines Bezugsrahmens einzelwirtschaftlicher Analyse und Gestaltung öffentlicher Leistungsprozesse, in: Zeitschrift für öffentliche und gemeinwirtschaftliche Unternehmen, Bd. 4 $(1981)$, S. 395-419.

Bull, Hans Peter: Die Staatsaufgaben nach dem Grundgesetz, Frankfurt 1973.

Bundesminister für Arbeit und Sozialordnung (Hrsg.): Sozialbericht 1983 , Stuttgart 1984.

Bundesministerium der Finanzen (Hrsg.): Finanzbericht 1982, Bonn 1981.

- Finanzbericht 1984, Bonn 1983.

— Beteiligungen des Bundes im Jahre 1982, Bonn 1983.

Bundesregierung: Bericht der Bundesregierung über die Entwicklung der Finanzhilfen und steuervergünstigungen für die Jahre 1979 bis 1982 gemäß \$ 12 des Gesetzes zur Förderung der Stabilität und des Wachstums der Wirtschaft (StGW) vom 8. Juni 1967 (Achter Subventionsbericht), Bundestags-Drucksache 9/986, Bonn, 6.11.1981.

Bericht der Bundesregierung über die Entwicklung der Finanzhilfen des Bundes und der Steuervergünstigungen für die Jahre 1981 bis 1984 gemäß $\$ 12$ des Gesetzes zur Förderung der stabilität und des Wachstums der Wirtschaft (StGW) vom 8. Juni 1967 (Neunter Subventionsbericht), Bundestags-Drucksache 10/352, Bonn, 6.9.1983. 
Carlberg, Michael: రffentliche Unternehmen und Staatsquote, in: Zeitschrift für öffentliche und gemeinwirtschaftliche Unternehmen, Band 3 (1980), S. 1-11.

CEEP : Die öffentliche Wirtschaft in der Europäischen Gemeinschaft. Jahrbuch 1984 der Europäischen Zentrale der öffentlichen Wirtschaft (CEEP), Brüssel, Berlin 1984.

Collard, David: Altruism and Economy. A Study in Non-Selfish Economics, Oxford 1978.

Colm, Gerhard: Volkswirtschaftliche Theorie der Staatsausgaben. Ein Beitrag zur Finanztheorie, Tübingen 1927.

- National Goals Analysis and Marginal Utility Economics. Some Non-Technical Comments on a Highly Technical Topic, in: Finanzarchiv N.F., Bd. 24 (1965), S. 209-224.

Conrad, Ernst-Albrecht: Bürgschaften und Garantien als Mittel der Wirtschaftspolitik, Berlin 1967.

Deacon, Robert und Shapiro, Perry: Private Preference for Collective Goods Revealed through Voting on Referenda, in: American Economic Review, Vol. 65 (1975), S. 943-955.

Derlien, Hans-Ulrich: Die Erfolgskontrolle staatlicher Planung. Eine empirische Untersuchung über Organisation, Methode und Politik der Programmevaluation, Baden-Baden 1976.

Deutsche Bundesbahn: Geschäftsbericht der Deutschen Bundesbahn. Geschäftsjahr 1977, 0.0., 0.J.

- Geschäftsbericht der Deutschen Bundesbahn. Geschäftsjahr 1978, 0.0., 0.J.

- Geschäftsbericht der Deutschen Bundesbahn, Geschäftsjahr 1979, 0.0., 0.J.

- Geschäftsbericht der Deutschen Bundesbahn. Geschäftsjahr 1980, 0.0., 0.J.

- Geschäftsbericht der Deutschen Bundesbahn, Geschäftsjahr 1981, 0.0., 0.J.

Dickertmann, Dietrich: ర̈ffentliche Finanzierungshilfen. Darlehen, Schuldendiensthilfen und Bürgschaften als Instrumente des finanzwirtschaftlichen Interventionismus, Baden-Baden 1980 .

Dierkes, Meinolf: Die Sozialbilanz. Ein gesellschaftsbezogenes Informations- und Rechnungssystem, Frankfurt 1974. 
Dierkes, Meinolf und Hoff, Andreas: Sozialbilanzen und gesellschaftsbezogene Rechnungslegung in der Bundesrepublik Deutschland - Eine Analyse der bisherigen Experimente, in: Hans-Joachim Hoffmann-Nowotny (Hrsg.) : Sozialbilanzierung. Soziale Indikatoren VIII. Konzepte und Forschungsansätze, Frankfurt, New York 1981, S. 9-67.

Dietz, Otto: Darstellung der Sozialversicherung in der Finanzstatistik, in: Wirtschaft und Statistik, Jahrgang 1984, S. 185-193.

Dittmers, Manfred: Die optimale Betriebsgröße von Rundfunkanstalten, in: Rundfunkökonomie. Wirtschaftliche Aspekte von Hörfunk und Fernsehen, Beiheft 5 (1983) der Zeitschrift für öffentliche und gemeinwirtschaftliche Unternehmen, Baden-Baden 1983, S. 105-119.

Downs, Anthony: An Economic Theory of Democracy, New York 1957; deutsch: ठkonomische Theorie der Demokratie, Tibingen 1968.

Ehrlicher, Werner: Öffentliche Sachausgaben, in: Handbuch der Finanzwissenschaft, hrsg. von Fritz Neumark, Bd. 1, 3. Aufl., Tübingen 1977, S. 753-795.

Eichhorn, Peter: Gesellschaftsbezogene Unternehmensrechnung, Göttingen 1974 .

- Grundlagen einer gemeinwirtschaftlichen Erfolgsrechnung für Unternehmen, Schriftenreihe Gemeinwirtschaft Nr. 15, hrsg. von der Bank für Gemeinwirtschaft, Frankfurt, Köln 1974 .

Eichhorn, Peter und Siedentopf, Heinrich: Effizienzeffekte der Verwaltungsreform. Exemplarische Ansätze einer Wirkungsanalyse der territorialen und funktionalen Verwaltungsreform in Rheinland-Pfalz, Baden-Baden 1976.

Eichhorn, Siegfried: Preisbildung im Krankenhaus nach dem Kostendeckungsprinzip, in: Krankenhäuser als Unternehmen, Beiheft 2 (1979) der Zeitschrift für öffentliche und gemeinwirtschaftliche Unternehmen, Baden-Baden 1979, S. 39-55.

Eiermann, Rudolf: Die Berechnung und Abgeltung einseitiger Belastungen der Eisenbahnen (sogenannte Normalisierung der Konten), in: Die Bundesbahn, 39. Jahrgang (1965), S. 397-405.

Elsner, Wolfram: Mehrdimensionale Bestimmung und Ermittlung von Wohlfahrt mit Hilfe von Sozialindikatoren-Systemen. Theoretische und methodologische Probleme, Diskussionspapiere der Fakultät für Wirtschaftswissenschaften der Universität Bielefeld, Nr. 53, Dezember 1978. 
Emmerich, Volker: Das Wirtschaftsrecht der öffentlichen Unternehmen, Bad Homburg 1969.

Öffentliche Produktion II: Rechtsformen(einschließlich Bundesbahn und Bundespost), in: Handwörterbuch der Wirtschaftswissenschaft (HdWW), hrsg. von Willi Albers et al., Bd. 5, stuttgart et al. 1980, S. 457-464.

Ewringmann, Dieter: Die Flexibilität öffentlicher Ausgaben. Eine Analyse der Restriktionen ausgabenpolitischer Handlungsspielräume, Göttingen 1975.

Faltlhauser, Kurt: Unternehmen und Gesellschaft. Theorie und Praxis der Sozialbilanz, Schriftenreihe des Deutschen Instituts für Betriebswirtschaft e.v., Bd. 5, Berlin 1978.

Farny, Dieter: Entwicklungslinien, stand und wirtschaftliche Bedeutung des Versicherungswesens in Deutschland, in: Friedrich-Wilhelm Henning (Hrsg.): Entwicklung und Aufgaben von Versicherungen und Banken in der Industrialisierung, Schriften des Vereins für Socialpolitik N.F., Bd. 105, Berlin 1980, S. 7-28.

Fecher, Hans: Ausgaben, öffentliche, I: Ansätze zu ihrer Analyse, in: Handwörterbuch der Wirtschaftswissenschaft (HdWW), hrsg. von Willi Albers et al., Bd. 1, stuttgart et al. 1977 , S. 334-349.

Felderer, Bernhard: Die reale Staatsquote, in: Finanzarchiv N.F., Bd. 35 (1976), S. 405-434.

Folkers, Cay: Staatswirtschaftliche Quoten und Beziehungszahlen, in: Das Wirtschaftsstudium (Wisu), Jg. 1979, S. 405-409 und S. 457-463.

Franz, Alfred: The Measurement of Real Output of Non-Market Services, in: Der öffentliche Sektor - Forschungsmemoranden, hrsg. von Egon Matzner, 3. Jg. (1977), Heft 1, S. 55-121.

Frentzel, Gerhard und Jäkel, Ernst: Die deutschen Industrieund Handelskammern und der Deutsche Industrie- und Handelstag, Frankfurt, Bonn 1967.

Freund, Elmar: Die Neugestaltung der Finanzstatistik ab 1970, in: Wirtschaft und Statistik, Jahrgang 1970, S. 543-546.

Frey, René L. und Kohn, Leopold: An Economic Interpretation of Voting Behaviour on Public Finance Issues, in: Kyklos, Vol. 23 (1970), S. 792-805.

Fricke, Dieter: Verteilungswirkungen der Inflation, BadenBaden 1981. 
Friedman, Milton: The Marshallian Demand Curve, in: Journal of Political Economy, Vol. 57 (1949), S. 463-495.

Friedrich, Peter: Regional Aspects of X-Inefficiency in the Public Sector, in: Eberhard Wille (Hrsg.): Konzeptionelle Probleme öffentlicher Planung, Frankfurt et al. 1983 , S. 189-243.

Gäfgen, Gérard: Theorie der wirtschaftlichen Entscheidung. Untersuchungen zur Logik und Bedeutung des rationalen Handelns, 3. Aufl., Tübingen 1974.

Gärtner, Hans J.: Indikatoren in der gesellschaftsbezogenen Unternehmensberichterstattung, in: Hans-Joachim HoffmannNowotny (Hrsg.): Sozialbilanzierung. Soziale Indikatoren VIII. Konzepte und Forschungsansätze, Frankfurt, New York 1981 , S. 69-86.

Görzig, Bernd: Die Verteilungswirkungen der Inflation auf den privaten und öffentlichen Sektor. Ermittlung von Inflationswirkungen nach alternativen Berechnungsansätzen unter besonderer Berücksichtigung des Problems der Produktivitätsmessung im öffentlichen Sektor, Göttingen 1975.

Götz, Günter: Die Schuldenpolitik der Länder, Meisenheim am Glan 1970.

Gresser, Klaus: Das Planning-Programming-Budgeting System. Probleme der Anwendung bei der staatlichen Aufgabenund Finanzplanung, München-Pullach, Berlin 1972.

- Probleme der mehrjährigen öffentlichen Finanzplanung, Berlin 1974.

Häuser, Karl: Uber Ansätze zur Theorie der Staatsausgaben, in: Herbert Timm und Heinz Haller (Hrsg.): Beiträge zur Theorie der öffentlichen Ausgaben, Schriften des Vereins $f$ ür Socialpolitik N.F., Bd. 47, Berlin 1967, S. 36-65.

Hagemann, Günter: Beziehungen zwischen mittelfristiger Finanzplanung und Finanzverfassung im föderativen Staat unter besonderer Berücksichtigung der Verhältnisse in der Bundesrepublik Deutschland, in: Mittelfristige Finanzplanung, Beihefte der Konjunkturpolitik, Heft 15, Berlin 1968 , s. 47-56.

Hajen, Leonhard: Bestimmungsgrlinde für die parafiskalische Organisation von Krankenversicherung, Frankfurt, Bern, Cirencester 1979.

Haller, Heinz: Einige Gedanken zum Thema: öffentliche Finanzen im Wirtschaftswachstum, in: Erich Schneider (Hrsg.): Wirtschaftskreislauf und Wirtschaftswachstum. Carl Föhl zum 65. Geburtstag, Tübingen 1966, S. 57-74. 
Haller, Heinz: Die Steuern. Grundlinien eines rationalen Systems öffentlicher Abgaben, 2. Aufl., Tübingen 1971.

Hamer, Eberhard: Bürokratieüberwälzung auf die Wirtschaft. Analyse und Therapie, in: Dietrich Dickertmann, Herbert König und Gerhard W. Wittkämper (Hrsg.) : Bürokratieüberwälzung. Stand, Ursachen, Folgen und Abbau, Regensburg 1982, S. 15-26.

Hamer, Günter: Revision der Volkswirtschaftlichen Gesamtrechnungen, in: Wirtschaft und Statistik, Jahrgang 1970, S. 57-65.

Hanusch, Horst: Theorie des öffentlichen Gutes. Allokative und distributive Aspekte, Göttingen 1972.

- Verteilung öffentlicher Leistungen. Eine Studie zur personalen Inzidenz, Göttingen 1976.

Hanusch, Horst (Hrsg.) : Reform öffentlicher Leistungen. Beiträge zur Entstaatlichung. Ein Kolloquium der Universität Augsburg in Memmingen, Baden-Baden 1978.

Hanusch, Horst: Äquivalenzprinzip und kollektive Güter - Allokationstheoretische Aspekte -, in: Dieter Pohmer (Hrsg.): Beiträge zum Äquivalenzprinzip und zur Zweckbindung öffentlicher Einnahmen, Schriften des Vereins für Socialpolitik N.F., Bd. 121, Berlin 1981, S. 37-91.

Harding, Fred 0.: Politisches Modell zur Wirtschaftstheorie. Theorie der Bestimmungsfaktoren finanzwirtschaftlicher Staatstätigkeit, Freiburg 1959. (F.O. Harding ist das Pseudonym für Philipp Herder-Dorneich).

Hartmann, N. ("Har."): Private Organisationen ohne Erwerbscharakter als Teil des Haushaltssektors in den Volkswirtschaftichen Gesamtrechnungen, in: Wirtschaft und Statistik, Jahrgang 1976, S. 638-643.

Haveman, Robert H. und Weisbrod, Burton A.: Defining Benefits of Public Programs: Some Guidance for Policy Analysts, in: Robert H. Haveman und Julius Margolis (Eds.): Public Expenditure and Policy Analysis, 2nd Ed., Chicago 1977 , S. 135-160.

Heiber, Horst: Messung von Forschungsleistungen der Hochschulen. Ein empirischer Ansatz auf der Basis von Zitatenanalysen, Baden-Baden 1983.

Helberger, Christof und Wagner, Gert: Beitragsäquivalenz oder interpersonelle Umverteilung in der gesetzlichen Rentenversicherung? - Eine Analyse auf der Grundlage von Lebenseinkommen, in: Philipp Herder-Dorneich (Hrsg.): Dynamische Theorie der Sozialpolitik, Schriften des Vereins für Socialpolitik N.F. , Bd.ch 123, Berlin 1981, S. $331-392$. 
Hemmer, Edmund: Sozialbilanzen. Konzepte und Realität, Beiträge zur Wirtschafts- und Sozialpolitik, Heft 62, hrsg. vom Institut der deutschen Wirtschaft, Röln 1979.

Henke, Klaus-Dirk: రffentliche Gesundheitsausgaben und Verteilung. Ein Beitrag zur Messung und Beeinflussung des gruppenspezifischen Versorgungsniveaus im Gesundheitsbereich, Göttingen 1977.

- Bestimmung und Steigerung der Effizienz im öffentlichen Sektor - Ein Uberblick, in: Das Wirtschaftsstudium (WiSu), $\mathrm{Jg}$. 1978, S. 601-605.

Dezentralisierung im Gesundheitswesen. Föderalismustheoretische und andere Ansätze zur Bestimmung und Messung des Zentralitätsgrades im Gesundheitswesen, in: Klaus-Dirk Henke und Uwe Reinhardt (Hrsg.): Steuerung im Gesundheitswesen, Gerlingen 1983, S. 13-56.

Herrmann, Walther: Intermediäre Finanzgewalten. Eine Analyse deutscher hilfsfiskalischer Gebilde im ersten Jahrzehnt nach der Stabilisierung, Jena 1936.

Hesse, Günter: Kosten-Nutzen-Analyse und souveränes Individuum, in: Jahrbücher für Nationalökonomie und Statistik, Bd. 189 (1975), S. 498-521.

- Staatsaufgaben. Zur Theorie der Legitimation und IAentifikation staatlicher Aufgaben, Baden-Baden 1979.

Hesse, Helmut: Die Kostenwirksamkeitsanalyse, in: Verwaltung und Fortbildung, 3. Jg. (1975), S. 79-90.

- Nutzen-Kosten-Analyse I: Theorie, in: Handwörterbuch der Wirtschaftswissenschaft (HdWW), hrsg. von Willi

Albers et al., Bd. 5, stuttgart et al. 1980, S. 361-382.

Heymann, H.-Helmut: Die Sozialbilanz als Instrument der Unternehmensführung. Das gesellschaftsbezogene Rechnungswesen der Unternehmung in der Sozialen Marktwirtschaft, Frankfurt 1981 .

Hicks, John Richard: The Valuation of the Social Income, in: Economica, Vol. 7 (1940), S. 105-124.

Hill, Thomas Peter: Price and Volume Measures for Non-Market Services, Statistical office of the European Communities, Brüssel 1975.

On Goods and Services, in: The Review of Income and Wealth, Series 23, 1977, S. 315-338. 
Hochman, Harold M. und Rodgers, James D.: Pareto Optimal Redistribution, in: American Economic Review, Vol. 59 (1969), S. 542-557.

Hofmann, Jürgen: Erweiterte Nutzen-Kosten-Analyse. Zur Bewertung und Auswahl öffentlicher Projekte, Göttingen 1981.

Holub, Hans-Werner: Probleme der Erfassung der staatstätigkeit, in: Wirtschaftswissenschaftliches studium (Wist), Jahrgang 1977, S. 324-329.

Hübener, Arend und Halberstadt, Rudolf: Erfolgskontrolle politischer Planung - Probleme und Ansätze in der Bundesrepublik Deutschland -, Göttingen 1976.

Jäger, Peter: Probleme und Möglichkeiten einer mit sozialen Kosten und Nutzen erweiterten Erfolgswürdigung öffentlicher Betriebe des Personen-Nahverkehrs, Diss. München 1974.

Johann, Horst: Theorie der Nachfrage nach öffentlichen Gütern, Frankfurt, Bern, Las Vegas 1977.

Juster, F. Thomas: Household Capital Formation and Financing 1897-1962, National Bureau of Economic Research, New York, London 1966.

A Framework for the Measurement of Economic and Social Performance, in: Milton Moss (Ed.): The Measurement of Economic and Social Performance. Studies in Income and Wealth, Vol. 38, New York 1973, S. 25-84 (Diskussion: S. 84-109).

Kaiser, Joseph H. (Hrsg.): Planung VI. Integrierte Systeme der Planung und Budgetierung, Baden-Baden 1972.

Kar, Hans M. van de: User Charges, Privatization and Performance Budgeting in the Social Services, Manuskript für den 39. Kongress des International Institute of Public Finance in Budapest, 22.-26. Aug. 1983.

Karl-Bräuer-Institut des Bundes der Steuerzahler (Hrsg.): Bevölkerungsentwicklung und Staatsausgaben, Schriften des Karl-Bräuer-Instituts des Bundes der Steuerzahler, Heft 43, Wiesbaden 1979.

Kazanowski, A.D.: A Standardized Approach to Cost-Effectiveness Evaluations, in: J. Morley English (Ed.): Cost Effectiveness. The Economic Evaluation of Engineered Systems, New York et al. 1968, S. 113-150. 
Kirberger, Wolfgang: Staatsentlastung durch private Verbände. Die finanzpolitische Bedeutung der Mitwirkung privater Verbände bei der Erfüllung öffentlicher Aufgaben, BadenBaden 1978.

Kirsch, Guy: Die Cost-Benefit-Analyse: Zur Kritik ihrer theoretischen Grundlagen, in: Das Wirtschaftsstudium (WiSu), Jg. 1972, S. 531-535 und S. 581-585; wiederabgedruckt in: Guy Kirsch und Walter Wittmann (Hrsg.): Nationale Ziele und Soziale Indikatoren, Stuttgart 1975, S. 69-80.

- Einleitung, in: Guy Kirsch (Hrsg. unter Mitarbeit von Jürg Theiler): Föderalismus, Stuttgart, New York 1977, S. 1-14.

- Effizienz und Effektivität: Zielbestimmung in der Sozialpolitik, in Martin Pfaff (Hrsg.): Effizienz und Effektivität staatlicher Transferpolitik in der Wirtschaftskrise, Berlin 1983, S. 9-26.

Kirsch, Werner: Entscheidungsprozesse. Band 3: Entscheidungen in Organisationen, Wiesbaden 1971.

Kitterer, Wolfgang: Das moderne Budget. Eine vergleichende Analyse der zentralen Staatshaushalte Frankreichs und der BRD, Frankfurt, Bern 1976.

Klaas, Hermann: Aufgaben der funktionalen Haushaltsgliederung im Rahmen des Funktionenplanes, in: Der öffentliche Haushalt, 10. Jg. (1969/70), S. 150-158.

Klausing, Michael: Effizienz und Effektivität im Gesundheitswesen. Der Beitrag der allgemeinen Systemtheorie für die Methode ihrer Ermittlung und Beurteilung, Karlsruhe 1981 .

Klein, Marianne: Deflationierung der staatlichen Leistungen und Preisentwicklungen im öffentlichen Sektor, in: Eberhard Wille (Hrsg.): Konzeptionelle Probleme öffentlicher Planung, Frankfurt et al. 1983, S. 85-126.

Kleinhenz, Gerhard: Wirtschaftskammern, in: Handwörterbuch der Wirtschaftswissenschaft (HdWW), hrsg. von Willi Albers et al., Bd. 9, stuttgart et al. 1982, S. 898-904.

Kleinsteuber, Hans J.: Rundfunkpolitik. Der Kampf um die Macht über Hörfunk und Fernsehen, Opladen 1982.

Koelle, H.H.: Ansätze für ein praktikables, zielorientiertes Modell der gegenwärtigen Gesellschaft, in: Analysen und Prognosen, 4. Jg. (1972), Heft 22, S. 22-28 und Heft 23, S. 23-28. 
Kössler, Richard: Sozialversicherungsprinzip und Staatszuschlisse in der gesetzlichen Rentenversicherung, Frankfurt, Bern 1982 .

Kolms, Heinz: Finanzwissenschaft. Band I, Grundlegung. రffentliche Ausgaben, 4. Aufl., Berlin, New York 1974.

Kopsch, Günter: Ausgaben des Staates nach Aufgabenbereichen in den Volkswirtschaftlichen Gesamtrechnungen, in: Wirtschaft und Statistik, Jahrgang 1980, S. 155-169.

Krämer, Walter: Gesundheit um jeden Preis?, in: Die Zeit, Nr. 27 vom 27.6.1980, S. 20 .

Krause-Junk, Gerold: Staatsausgaben, Einkommensniveau und Wirtschaftsstruktur. Kreislauftheoretische Betrachtungen ubber Wirkungen öffentlicher Ausgaben, Diss. Berlin 1966.

- Probleme der Berechnung und Schätzung öffentlicher Ausgaben, in: Public Finance, Vol. 27 (1972), S. 127-144.

Krautzberger, Michael: Die Erfüllung öffentlicher Aufgaben durch Private. Zum Begriff des staatlichen Bereichs, Berlin 1971.

Kroker, Rolf: Der Staat als Wirtschaftsfaktor. Zur Aussagefähigkeit der Staatsquote, Beiträge zur Wirtschafts- und Sozialpolitik, Bd. 93, Institut der deutschen Wirtschaft, Köln 1981.

Krupp, Hans-Jürgen und Zapf, Wolfgang: Indikatoren II: soziale, in: Handwörterbuch der Wirtschaf tswissenschaft (HaWw), hrsg. von Willi Albers et al., Bd. 4, stuttgart et al. 1978 , S. 119-133.

Kurz, Mordecai: Experimental Approach to the Determination of the Demand for Public Goods, in: Journal of Public Economics, Vol. 3 (1974), S. 329-348.

Kuznets, Simon: On the Valuation of Social Income - Reflections on Professor Hicks' Article, in: Economica, Vol. 15 $(1948)$, S. 1-16 und S. 116-131.

- Government Product and National Income, in: Erik Lundberg (Ed.): Income and Wealth, Series 1, Cambridge 1951, s. $178-244$.

National Income and Its Composition, 1919-1938. Vol. I, National Bureau of Economic Research, New York 1954.

Kyrer, Alfred: Effizienz und staatliche Aktivität, Wien 1972. 
Lee, A. James und Weisbrod, Burton A.: Collective Goods and the Voluntary Sector: The Case of the Hospital Industry, in: Burton A. Weisbrod: The Voluntary Nonprofit Sector. An Economic Analysis, Lexington 1977, S. 77-100.

Leetz, Wolf: Zur Einbeziehung der öffentlichen Unternehmen in die Staatsquote, in: Zeitschrift für öffentliche und gemeinwirtschaftliche Unternehmen, Bd. 4 (1981), S. 420455.

Leibenstein, Harvey: Allocative Efficiency vs. "X-Efficiency", in: American Economic Review, Vol. 56 (1966), S. 392-415.

Leipert, Christian: Soziale Indikatoren. Uberblick über den Stand der Diskussion, in: Konjunkturpolitik, 19. Jg. (1973), S. 204-256.

- Unzulänglichkeiten des Sozialprodukts in seiner Eigenschaft als Wohlstandsmaß, Tübingen 1975.

- Gesellschaftliche Berichterstattung. Eine Einfürung in Theorie und Praxis sozialer Indikatoren, Berlin, Heidelberg, New York 1978.

- Staatskonsum, staatliche Investitionen und die Produktivität staatlichen Handelns, in: Wirtschaftsdienst, Jahrgang 1979, S. 147-152.

Lepelmeier, Dirk: Soziale Sicherung und Parafiskalität. Zur Einkommensumverteilungsproblematik im Bereich der Sozialversicherung, Frankfurt 1979.

Littmann, Konrad: Zunehmende staatstätigkeit und wirtschaftliche Entwicklung. Versuche über die Wirkungen staatswirtschaftlicher Prozesse in der spätkapitalistischen Periode, Köln, Opladen 1957.

- unter Mitarbeit von Berend Krüger: Definition und Entwicklung der Staatsquote. Abgrenzung, Aussagekraft und Anwendungsbereiche unterschiedlicher Typen von Staatsquoten, Göttingen 1975 (zitiert als: K. Littmann: Definition und Entwicklung der Staatsquote).

Littmann, Konrad: Ausgaben, öffentliche, II: Die "Gesetze" ihrer langfristigen Entwicklung, in: Handwörterbuch der Wirtschaftswissenschaft (HdWW), hrsg. von Willi Albers et al., Bd. 1, stuttgart et al. 1977, S. 349-363.

Littmann, Konrad; Hajen, Leonhard und Ulbrich, Rudi: Argumente für eine Aufgabenabgrenzung zwischen privatem und öffentlichem Sektor und Mischformen. Literaturanalyse. Projekt 90 der Kommission für wirtschaftlichen und sozialen Wandel, Manuskript, 0.0., 0.J., (Hamburg 1974). 
Mackscheidt, Klaus: Zur Theorie des optimalen Budgets, Tübingen 1973.

Mackscheidt, Klaus und Steinhausen, Jörg: Finanzpolitik I. Grundfragen fiskalpolitischer Lenkung, 3. Aufl., Tübingen, Düsseldorf 1978 .

Mahr, Werner: Einführung in die Versicherungswirtschaft. Allgemeine Versicherungslehre, Berlin 1951.

Maier, Oskar: Zur Ertrags- und Vermögenslage der Rundfunkanstalten in der Bundesrepublik Deutschland, in: Zeitschrift für öffentliche und gemeinwirtschaftliche Unternehmen, Bd. 3 (1980), S. 415-430.

Mann, Fritz Karl: Die intermediären Finanzgewalten und ihr Einflu $\beta$ auf Deutschlands finanzielle Belastung, in: Jahrbücher für Nationalökonomie und Statistik, Bd. 129 (1928), S. 219-237.

Margolis, Julius: A Comment on the Pure Theory of Public Expenditure, in: Review of Economics and Statistics, Vol. 37 (1955), S. 347-349.

- Shadow Prices for Incorrect or Nonexistent Market Values, in: Robert $\mathrm{H}$. Haveman und Julius Margolis (Eds.): Public Expenditure and Policy Analysis, 2nd Ed., Chicago 1977, S. 204-220.

Meier, Alfred: Öffentliches Vermögen II: Umfang und Funktionen, in: Handwörterbuch der Wirtschaftswissenschaft (HdWW), hrsg. von Willi Albers et al., Bd. 5, stuttgart et al. 1980 , S. 623-633.

Meinhold, Helmut: Fiskalpolitik durch sozialpolitische Parafisci, Tübingen 1976.

Meyke, Udo: Cost-Effectiveness-Analysis als Planungsinstrument. Unter besonderer Berücksichtigung von Infrastrukturinvestitionen im Verkehr, Göttingen 1973.

Mishan, Edward Joshua: Cost-Benefit Analysis. An Informal Introduction, 2nd Ed., London 1975.

Münch, Ingo von: Kommentar zu Art. 5, in: Ingo von Münch (Hrsg.): Grundgesetz-Kommentar, Bd. 1, 2. Auf 1., München 1981.

Musgrave, Richard A.: The Theory of Public Finance. A Study in Public Economy, New York et al. 1959; deutsch: Finanztheorie, 2. Auf1., Tübingen 1969. 
Musgrave, Richard A. und Musgrave, Peggy B.: Public Finance in Theory and Practice, 3rd Ed. (4th Printing), Auckland et al. 1982; deutsch: Musgrave, Richard A.; Musgrave, Peggy B. und Kullmer, Lore: Die öffentlichen Finanzen in Theorie und Praxis, Bd. 1, 2. Aufl., Tübingen 1978.

Musto, Stefan A.: Evaluierung sozialer Entwicklungsprojekte, Berlin 1972 .

Nachtkamp, Hans-Heinrich: Idealtypen von Fiskalsystemen. Ein Versuch, in: Karl Häuser (Hrsg.): Finanzsysteme: Idealund Realtypen - Gesundheitswesen und Hochschulbildung, Schriften des Vereins für Socialpolitik N.F., Bd. 135, Berlin 1983, S. 11-55.

Neumark, Fritz: Theorie und Praxis der Budgetgestaltung, in: Handbuch der Finanzwissenschaft, hrsg. von Wilhelm Gerloff und Fritz Neumark, Bd. 1, 2. Aufl., Tübingen 1952, S. 554-605.

- Planung in der öffentlichen Finanzwirtschaft, in: Erich Schneider (Hrsg.): Rationale Wirtschaftspolitik und Planung in der Wirtschaft von heute, Schriften des Vereins für Socialpolitik N.F., Bd. 45, Berlin 1967, S. 173-205 (Diskussion: S. 206-238).

- "Tax Expenditures", in: Finanzarchiv N.F., Bd. 33 (1974/ 75), S. 139-142.

Noam, Eli M.: Demand Functions and the Valuation of Public Goods, in: Public Choice, Vol. 38 (1982), S. 271-280.

Nöll von der Nahmer, Robert: Lehrbuch der Finanzwissenschaft. Band I Allgemeine Finanzwissenschaft, Köln, Opladen 1964.

Nordhaus, William und Tobin, James: Is Growth Obsolete?, in: Economic Research: Retrospect and Prospect. Economic Growth, Fiftieth Anniversary Colloquium V. National Bureau of Economic Research, New York 1972, S. 1-80.

Novick, David (Ed.): Current Practice in Program Budgeting (PPBS). Analysis and Case Studies Covering Government and Business, London 1973.

Olson, Mancur: Evaluating Performance in the Public Sector, in: Milton Moss (Ed.): The Measurement of Economic and Social Performance, Studies in Income and Wealth, Vol. 38, New York 1973, S. 355-384 (Diskussion: S. 384-409).

Ott, Günter: Einkommensumverteilungen in der gesetzlichen Krankenversicherung. Eine quantitative Analyse, Frankfurt, Bern 1981 . 
Peacock, Alan T. und Wiseman, Jack: The Growth of Public Expenditure in the United Kingdom, National Bureau of Economic Research, Princeton 1961.

Pechman, Joseph A. (Ed.) : Setting National Priorities. The 1982 Budget, Washington D.C. 1981.

- Setting National Priorities. The 1984 Budget, Washington D.C. 1983.

Peters, Matthias und Zeugin, Peter: Sozialindikatorenforschung. Eine Einführung, Stuttgart 1979.

Pfähler, Wilhelm: Free-Rider-Problem bei öffentlichen Gütern und Clarke-Groves-Steuermechanismus, in: Das Wirtschaftsstudium (WiSu), Jg. 1981, S. 403-408, S. 453-458 und S. 505-508.

Picot, Arnold: Betriebswirtschaftliche Umweltbeziehungen und Umweltinformationen. Grundlagen einer erweiterten Erfolgsanalyse für Unternehmungen, Berlin 1977.

Piller, Gerhard Klaus: Sozialbilanz. Unternehmungspolitische Ziele in der gesellschaftsbezogenen Rechnungslegung, Thun, Frankfurt 1980.

Pommerehne, Werner $W$. und Schneider, Friedrich: Wie steht's mit dem Trittbrettfahren? - Eine experimentelle Untersuchung -, in: Zeitschrift für die gesamte staatswissenschaft, Bd. $136(1980)$, S. 286-308.

Prest, Alan R. und Turvey, Ralph: Cost-Benefit Analysis: A Survey, in: The Economic Journal, Vol. 75 (1965), S. 683735.

Quade, Edward S.: Kosten-Wirksamkeits-Analyse, in: Horst Claus Recktenwald (Hrsg.): Nutzen-Kosten-Analyse und Programmbudget. Grundlage staatlicher Entscheidung und Planung, Tübingen 1970, S. 235-242.

Recktenwald, Horst Claus: Möglichkeiten und Grenzen der Methode der Nutzen-Kosten-Analyse, in: Helmut Arndt und Dieter Swatek (Hrsg.): Grundfragen der Infrastrukturplanung für wachsende Wirtschaften, Schriften des Vereins für Socialpolitik N.F., Bd. 58, Berlin 1971, S. 233-262 (Diskussion: S. 263-288).

Reding, Kurt: Die Effizienz staatlicher Aktivitäten. Probleme ihrer Messung und Kontrolle, Baden-Baden 1981.

Reich, Utz-Peter; Sonntag, Philipp und Holub, Hans-Werner: Arbeit-Konsum-Rechnung. Axiomatische Kritik und Erweiterung der Volkswirtschaftlichen Gesamtrechnung, Köln 1977. 
Reichhardt, Raimund M.: Gesellschaftliche Bedarfsanalyse. Ein Ansatz zur Ermittlung der Bürger-Präferenzen für öffentliche Güter, Berlin 1979.

Reinermann, Heinrich: Zehn Jahre Systembudgetierung in der USBundesregierung. Analyse eines Experiments, in: Joseph H. Kaiser (Hrsg.): Planung VI. Integrierte Systeme der Planung und Budgetierung, Baden-Baden 1972, S. 301-342.

- Programmbudgets in Regierung und Verwaltung. Möglichkeiten und Grenzen von Planungs- und Entscheidungssystemen, Baden-Baden 1975.

Ring, Ulrich: Die Aussagefähigkeit budgetärer Ausgabenschwerpunkte - ein tberblick, in: Eberhard Wille (Hrsg.): Konzeptionelle Probleme öffentlicher Planung, Frankfurt et al. 1983, S. 21-84.

Rürup, Bert: Die Programmfunktion des Bundeshaushaltsplanes. Die deutsche Haushaltsreform im Lichte der amerikanischen Erfahrungen mit dem Planning-Programming-Budgeting System, Berlin 1971.

Ruggles, Nancy und Ruggles, Richard: The Design of Economic Accounts, National Bureau of Economic Research, New York 1966.

Sametz, Arnold W.: Production of Goods and Services. The Measurement of Economic Growth, in: Eleanor Bernert Sheldon und Wilbert E. Moore (Eds.): Indicators of Social Change. Concepts and Measurements, New York 1968, S. 77-96.

Samuelson, Paul A.: The Pure Theory of Public Expenditure, in: Review of Economics and Statistics, Vol. 36 (1954), S. 387-389.

- Diagrammatic Exposition of a Theory of Public Expenditure, in: Review of Economics and Statistics, Vol. 37 (1955), S. 350-356.

Sauerzapf, Maria: Das Krankenhauswesen in der Bundesrepublik Deutschland. Institutionelle Regelungen aus ökonomischer Sicht, Baden-Baden 1980.

Scheer, Christian: Sozialstaat und öffentliche Finanzen. Theorie und Realität der sozialen Komponente der öffentlichen Finanzen im Wandel vom liberalen Rechtsstaat zum Sozialstaat, Köln 1975.

Schick, Allen: The Road to PPB: The Stages of Budget Reform, in: Fremont J. Lyden und Ernest G. Miller (Eds.): Public Budgeting: Program Planning and Evaluation, 3rd Ed., Chicago 1978, S. 17-42. 
Schiele, Dietmar: Die Dienstleistungen des Staates in der Volkswirtschaftlichen Gesamtrechnung, in: Schmollers Jahrbuch für Gesetzgebung, Verwaltung und Volkswirtschaft, 78 . Jahrgang (1958), S. 167-197.

Schmähl, Winfried: Alterssicherung und Einkommensverteilung. Theoretische und empirische Untersuchungen zur Finanzierung, Leistungsgewährung und zur Verteilung zwischen Generationen, Tübingen 1977.

- Einkommensumverteilung im Rahmen von Einrichtungen der sozialen Sicherung - Einige Probleme ihrer Ermittlung und Ausgestaltung am Beispiel der gesetzlichen Rentenversicherung -, in: Bernhard külp und Heinz-Dieter Haas (Hrsg.): Soziale Probleme der modernen Industriegesellschaft, Schriften des Vereins für Socialpolitik N.F., Bd. 92/II, Berlin 1977, S. 519-576.

Schmidt, Kurt: Zur Geschichte der Lehre von den Kollektivbedürfnissen, in: Norbert Kloten et al. (Hrsg.): Systeme und Methoden in den Wirtschafts- und Sozialwissenschaften. Erwin von Beckerath zum 75. Geburtstag, Tübingen 1964 , S. 335-362.

Kollektivbedürfnisse und staatstätigkeit, in: Heinz Haller et al. (Hrsg.): Theorie und Praxis des finanzpolitischen Interventionismus. Fritz Neumark zum 70. Geburtstag, Tübingen 1970, S. 3-27.

Schmidt, Kurt und Wille, Eberhard: Die mehrjährige Finanzplanung. Wunsch und Wirklichkeit, Tübingen 1970.

Schmölders, Günter: Finanzpolitik, 3. Aufl., Berlin, Heidelberg, New York 1970.

Schmoltzi, Ulrich: Zur Finanzierung privater Organisationen ohne Erwerbscharakter. Monolithisches staatliches Güterangebot versus vielfältiges Angebot, in: Eberhard Wille (Hrsg.): Beiträge zur gesamtwirtschaftlichen Allokation. Allokationsprobleme im intermediären Bereich zwischen öffentlichem und privatem Wirtschaftssektor, Frankfurt et al. 1983 , S. 65-87.

Schreckenberger, Waldemar: Probleme bei der Ermittlung des Finanzbedarfs der Rundfunkanstalten, in: Zeitschrift für öffentliche und gemeinwirtschaftliche Unternehmen, Bd. 4 $(1981)$, S. 100-107.

Schultze, Charles L. et al.: Setting National Priorities. The 1972 Budget, Washington D.C. 1971. 
Senf, Paul: Die Reform der öffentlichen Haushaltsgebarung zur Erhöhung der Transparenz, in: Heinz Haller (Hrsg.): Probleme der Haushalts- und Finanzplanung, Schriften des Vereins für Socialpolitik N.F., Bd. 52, Berlin 1969, S. 143-174.

- Kurzfristige Haushaltsplanung, in: Handbuch der Finanzwissenschaft, hrsg. von Fritz Neumark, Bd. 1, 3. Aufl., Tübingen 1977, S. 371-425.

Simonis, Udo Ernst: "Nettowohlfahrtsindikator" - ein japanischer Ansatz, in: Wolfgang zapf (Hrsg.): Soziale Indikatoren. Konzepte und Forschungsansätze III, Frankfurt, New York 1975 , S. 296-317.

Sinn, Hans-Werner: Die Inflationsgewinne des Staates, in: Eberhard Wille (Hrsg.): Beiträge zur gesamtwirtschaftlichen Allokation. Allokationsprobleme im intermediären Bereich zwischen öffentlichem und privatem Wirtschaftssektor, Frankfurt et al. 1983, S. 111-166.

Smekal, Christian: Die Finanzwirtschaft intermediärer Gruppen, Veröffentlichungen der Universität Innsbruck, Bd. 10, Innsbruck 1969.

- Die Flucht aus dem Budget, Wien 1977.

- Finanzen intermediärer Gewalten (Parafisci), in: Handwörterbuch der Wirtschaftswissenschaft (HdWW), hrsg. von Willi Albers et al., Bd. 3, stuttgart et al. 1980, S. $1-17$.

Statistisches Bundesamt: Fachserie 18 Volkswirtschaftliche Gesamtrechnungen, Reihe S. 2 Revidierte Ergebnisse 1960 bis 1976, Stuttgart, Mainz 1977.

- Hochschulfinanzen 1976, in: Wirtschaft und Statistik, Jahrgang 1978, S. 590-593.

Fachserie 18 Volkswirtschaftliche Gesamtrechnungen, Reihe 1 Konten und Standardtabellen 1980, stuttgart, Mainz 1981.

Fachserie 18 Volkswirtschaftliche Gesamtrechnungen, Reihe S. 4 Ausgaben des Staates nach Aufgabenbereichen in den Volkswirtschaftlichen Gesamtrechnungen 1970 bis 1978, Stuttgart, Mainz 1981.

Fachserie 18 Volkswirtschaftliche Gesamtrechnungen, Reihe 1 Konten und Standardtabellen 1981, stuttgart, Mainz 1982 . 
Statistisches Bundesamt: Fachserie 18 Volkswirtschaftliche Gesamtrechnungen, Reihe S. 5 Revidierte Ergebnisse 1960 bis 1981, stuttgart, Mainz 1982.

- Schreiben vom 22.12.1980 an den Verfasser.

Steiner, Peter 0.: Public Expenditure Budgeting, in: Alan S. Blinder et al.: The Economics of Public Finance, Studies of Government Finance, Washington D.C. 1974, S. 241-357.

Steinhausen, Jörg: Soziale Indikatoren als Elemente eines gesellschaftichen Planungs- und Steuerungssystems, Meisenheim am Glan 1975.

Stobbe, Alfred: Volkswirtschaftliche Gesamtrechnung, in: Handwörterbuch der Wirtschaftswissenschaft (HdWW), hrsg. von Willi Albers et al., Bd. 8, Stuttgart et al. 1980, S. 368-405.

- Volkswirtschaftslehre I. Volkswirtschaftliches Rechnungswesen, 5. Aufl., Berlin, Heidelberg, New York 1980.

Stolper, Hans-Dieter: Zur Leistungsfähigkeit der konsumtheoretischen Analyse der Nachfrage nach öffentlichen Gütern, Berlin 1982 .

Studenski, Paul: The Income of Nations. Part Two: Theory and Methodology, New York, London 1958.

Surrey, Stanley S.: The Concept of Tax Reliefs. Its Relation to Tax Policy and Budget Policy, in: Karl Häuser (Ed.): Subsidies, Tax Reliefs and Prices. Proceedings of the 33 rd Congress of the International Institute of Public Finance (Varna 1977), Paris 1981, S. 35-55.

Thiemeyer, Theo: Wirtschaftslehre öffentlicher Betriebe, Reinbek 1975.

Die Abgeltung gemeinwirtschaftlicher Lasten als Mittel der Finanzierung öffentlicher Unternehmen, in: Peter Eichhorn und Theo Thiemeyer (Hrsg.): Finanzierung öffentlicher Unternehmen. Festschrift für Dr. Paul Münch, Baden-Baden 1979, S. 127-151.

Betriebswirtschaftslehre der öffentlichen Betriebe, in: Wirtschaftswissenschaftliches studium (Wist), Jg. 1981, S. 367-373 und S. 417-423.

Tideman, T. Nicolaus: Introduction, in: Public Choice, Vol. 29-2, Special Supplement to Spring 1977, S. 1-13.

Tideman, T. Nicolaus und Tullock, Gordon: A New and Superior Process for Making Social Choices, in: Journal of Political Economy, Vol. 84 (1976), S. 1145-1159. 
Tiepelmann, Klaus: Parafiski, in: Das Wirtschaftsstudium (WiSu), Jg.1975, S. 295-300.

Timm, Herbert: Das Gesetz der wachsenden Staatsausgaben, in: Finanzarchiv N.F., Bd. 21 (1961), S. 201-247.

Tuchtfeld, Egon: Soziale Indikatoren: Ansätze und kontroverse Fragen, in: Hamburger Jahrbuch für Wirtschafts- und Gesellschaftspolitik, 21. Jahr (1976), S. 63-85.

Wagner, Adolph: Das Gesetz der zunehmenden Staatstätigkeit (Auszug aus: Staat in nationalökonomischer Hinsicht), in: Horst Claus Recktenwald (Hrsg.): Finanztheorie, 2. Aufl., Köln, Berlin 1970, S. 241-243.

Walser, Peter: Volkswirtschaftliche Gesamtrechnung - Revision und Erweiterung, Göttingen 1975.

Wegelin, Fritz: Integrierte staatliche Planung. Probleme der Koordination, Führung und Willensbildung, Winterthur 1978.

Weisbrod, Burton A.: Collective-Consumption Services of Individual-Consumption Goods, in: Quarterly Journal of Economics, Vol. 78 (1964), S. 471-477.

- Concepts of Costs and Benefits, in: Samuel B. Chase Jr. (Ed.): Problems in Public Expenditure Analysis, Washington D.C. 1968 , S. 257-262.

Toward a Theory of the Voluntary Nonprofit Sector in a Three-Sector Economy, in: Martin Pfaff (Ed.): Grants and Exchange, Amsterdam, New York, Oxford 1976, S. 227-243.

- The Voluntary Nonprofit Sector. An Economic Analysis, Lexington 1977.

Some Collective-Good Aspects of Non-Government Activities: Not-for-Profit Organizations, in: Horst Claus Recktenwald (Ed.): Secular Trends of the Public Sector. Proceedings of the 32 nd Congress of the International Institute of Public Finance (Edimbourg 1976), Paris 1978, S. 163-174.

Weiss, Carol H.: Evaluierungsforschung. Methoden zur Einschätzung von sozialen Reformprogrammen, Opladen 1974.

WeiB, Manfred: Effizienzforschung im Bildungsbereich. Aufgabenfelder, Methoden und empirische Befunde, Berlin 1982.

Werner, Rudolf: Zur Problematik subjektiver Indikatoren, in: Wolfgang Zapf (Hrsg.): Soziale Indikatoren. Konzepte und Forschungsansätze II, Frankfurt, New York 1974, S. 264-275. 
Werner, Rudolf: Soziale Indikatoren und politische Planung. Einführung in Anwendungen der Makrosoziologie, Reinbek 1975.

Wicksell, Knut: Finanztheoretische Untersuchungen nebst Darstellung und Kritik des Steuerwesens Schwedens, Neudruck der Ausgabe Jena 1896, Aalen 1969.

Wiemeyer, Joachim: Krankenhausfinanzierung und Krankenhausplanung in der Bundesrepublik Deutschland, Berlin 1984.

Wille, Eberhard: Mittel- und langfristige Finanzplanung, in: Handbuch der Finanzwissenschaft, hrsg. von Fritz Neumark, Bd. 1, 3. Aufl., Tübingen 1977, S. 427-474.

Öffentlicher Haushalt IV: Finanz- und Aufgabenplanung, in: Handwörterbuch der Wirtschaftswissenschaft (HdWW), hrsg. von Willi Albers et al., Band 5, stuttgart et al. 1980 , S. 591-620.

Soziale Indikatoren als Ansatzpunkte wirtschaftspolitischer Zielbildung und Kontrolle - Verbesserung oder Verschleierung politischer Entscheidungsgrundlagen?, in: ORDO, Bd. 31 (1980), S. 127-151.

Die öffentlichen Ausgaben und die Entwicklung der Staatsquote. $\mathrm{Zu}$ entsprechenden Beiträgen im neuen "Handwörterbuch der Wirtschaftswissenschaft (HdWW) ", in: ORDO, Bd. 32 (1981), S. 297-311.

Öffentliche Budgets im InflationsprozeB, in: Das Wirtschaftsstudium (WiSu), Jg. 1982, S. 197-202 und S. 249255 .

Gesamtwirtschaftliche Allokation zwischen "Markt- und Staatsversagen" - ein ordnungspolitischer Uberblick, in: Eberhard Wille (Hrsg.): Beiträge zur gesamtwirtschaftlichen Allokation. Allokationsprobleme im intermediären Bereich zwischen öffentlichem und privatem Wirtschaftssektor, Frankfurt et al. 1983, S. 1-28.

Öffentliche Sachausgaben versus öffentliche Personalausgaben. Erweiterte Fassung eines Referates auf der 26. Arbeitstagung des Finanzwissenschaftlichen Ausschusses des Vereins für Socialpolitik in Hannover, erscheint in: Karl Häuser (Hrsg.): Die Produktivitätsentwicklung staatlicher Leistungen, Manuskript, Mannheim 1983. 
Wille, Eberhard: Rationalität, Effizienz und Effektivität aus der Sicht des రkonomen. Erweiterte Fassung eines Referates auf dem Symposion "Effizienz und Effektivität medizinischer Diagnostik", erscheint in dem entsprechenden Tagungsband der "Internationalen Gesellschaft für Gesundheitsökonomie", Manuskript, Mannheim 1983.

Wissenschaftlicher Beirat beim Bundesministerium der Finanzen: Gutachten zur Lage und Entwicklung der Staatsfinanzen in der Bundesrepublik Deutschland, in: Bulletin des Presseund Informationsamtes der Bundesregierung, Bonn, Nr. 103 vom 16.8.1975, S. 1001-1016.

- Gutachten zur Aussagefähigkeit staatswirtschaftlicher Quoten, in: Bulletin des Presse- und Informationsamtes der Bundesregierung, Bonn, Nr. 90 vom 30.7.1976, S. 849862 .

- Gutachten zum Begriff der öffentlichen Investitionen, Schriftenreihe des Bundesministeriums der Finanzen, Heft 29, Bonn 1980.

Wöhe, Günter: Bilanzierung und Bilanzpolitik. Betriebswirtschaftlich-Handelsrechtlich-Steuerrechtlich. Mit einer Einführung in die verrechnungstechnischen Grundlagen, 4. Aufl., München 1976.

Wolkersdorf, Lorenz: Beziehungen zwischen Haushaltsplan und Finanzplanung unter besonderer Berücksichtigung politischer und administrativer Aspekte, in: Mittelfristige Finanzplanung, Beihefte der Konjunkturpolitik Heft 15, Berlin 1968, S. 31-46.

Wysocki, Klaus von: Öffentliche Finanzierungshilfen. Forschungsberichte des Landes Nordrhein-Westfalen, Nr. 946 , Köln, Opladen 1961.

MeB- und Bewertungsprobleme in der sozialen Rechnungslegung, in: Herbert R. Haeseler (Hrsg.): Gemeinwirtschaftliche Betriebe und öffentliche Verwaltungen, Schmalenbachs zeitschrift für betriebswirtschaftliche Forschung, Sonderheft 5, Opladen 1976, S. 171-180.

Das Unternehmen in seiner Umwelt: Möglichkeiten und Grenzen der Sozialbilanz, in: Elmar Pieroth (Hrsg.): Sozialbilanzen in der Bundesrepublik Deutschland. Ansätze - Entwicklungen - Beispiele, Wien, Düsseldorf 1978, S. 15-33.

Sozialbilanzen. Inhalt und Formen gesellschaftsbezogener Berichterstattung, Stuttgart, New York 1981. 
Zangemeister, Christof: Nutzwertanalyse in der systemtechnik. Eine Methodik zur multidimensionalen Bewertung und Auswahl von Projektalternativen, 4. Auf1., München 1976.

Zapf, Wolfgang: Soziale Indikatoren - Eine Zwischenbilanz, in: Hans-Jürgen Krupp und Wolfgang Zapf: Sozialpolitik und Sozialberichterstattung, Frankfurt, New York 1977, S. 231-246.

- Die Wohlfahrtsentwicklung in Deutschland seit der Mitte des 19. Jahrhunderts, in: Werner Conze und M. Rainer Lepsius (Hrsg.): Sozialgeschichte der Bundesrepublik Deutschland. Beiträge zum Kontinuitätsproblem, stuttgart 1983 , S. 46-65.

Zeitel, Gerhard: Staatliche Darlehensgewährung als Mittel der Finanz- und Wirtschaftspolitik, in: Finanzarchiv N.F., Bd. 26 (1967), S. 193-214.

Theoretische und technische Aspekte öffentlicher Darlehen und Gewährleistungen, in: Handbuch der Finanzwissenschaft, hrsg. von Fritz Neumark, Bd. 1, 3. Aufl., Tübingen 1977, S. 997-1024.

Zimmermann, Horst: Die Ausgabenintensität der öffentlichen Aufgabenerfüllung, in: Finanzarchiv N.F., Bd. 32 (1973/74), S. $1-20$.

- Instrumente der Finanzpolitik, in: Handbuch der Finanzwissenschaft, hrsg. von Fritz Neumark, Bd. 1, 3. Aufl., Tübingen 1977 , S. 165-192.

zimmermann, Horst und Henke, Klaus-Dirk: Finanzwissenschaft. Eine Einführung in die Lehre von der öffentlichen Finanzwirtschaft, 3. Aufl., München 1982.

Zumbühl, Marcel: Privatisierung staatlicher Wirtschaftstätigkeit - Notwendigkeit und Möglichkeiten?, Zürich 1978. 


\section{STAATLICHE ALLOKATIONSPOLITIK IM MARKTWIRTSCHAFTLICHEN SYSTEM}

Band 1 Horst Siebert (Hrsg.): Umweltallokation im Raum. 1982.

Band 2 Horst Siebert (Hrsg.): Global Environmental Resources. The Ozone Problem. 1982.

Band 3 Hans-Joachim Schulz: Steuerwirkungen in einem dynamischen Unternehmensmodell. Ein Beitrag zur Dynamisierung der Steuerüberwälzungsanalyse. 1981.

Band 4 Eberhard Wille (Hrsg.): Beiträge zur gesamtwirtschaftlichen Allokation. Allokationsprobleme im intermediären Bereich zwischen öffentlichem und privatem Wirtschaftssektor. 1983.

Band 5 Heinz König (Hrsg.): Ausbildung und Arbeitsmarkt. 1983.

Band 6 Horst Siebert (Hrsg.): Reaktionen auf Energiepreissteigerungen. 1982.

Band 7 Eberhard Wille (Hrsg.): Konzeptionelle Probleme öffentlicher Planung. 1983.

Band 8 Ingeborg Kiesewetter-Wrana: Exporterlösinstabilität. Kritische Analyse eines entwicklungspolitischen Problems. 1982.

Band 9 Ferdinand Dudenhöffer: Mehrheitswahl-Entscheidungen über Umweltnutzungen. Eine Untersuchung von Gleichgewichtszuständen in einem mikroökonomischen Marktund Abstimmungsmodell. 1983.

Band 10 Horst Siebert (Hrsg.): Intertemporale Allokation. 1984.

Band 11 Helmut Meder: Die intertemporale Allokation erschöpfbarer Naturressourcen bei fehlenden Zukunftsmärkten und institutionalisierten Marktsubstituten. 1984.

Band 12 Ulrich Ring: Offentliche Planungsziele und staatliche Budgets. Zur Erfüllung öffentlicher Aufgaben durch nicht-staatliche Entscheidungseinheiten. 1985.

Band 13 Ehrentraud Graw: Informationseffizienz von Terminkontraktmärkten für Währungen. Eine empirische Untersuchung. 1984. 
Ulrich Ring - 978-3-631-75590-7

Downloaded from PubFactory at 01/11/2019 03:20:37AM

via free access 